\title{
Le tre costituzioni pacifiste
}

Il rifiuto della guerra nelle costituzioni di Giappone, Italia e Germania

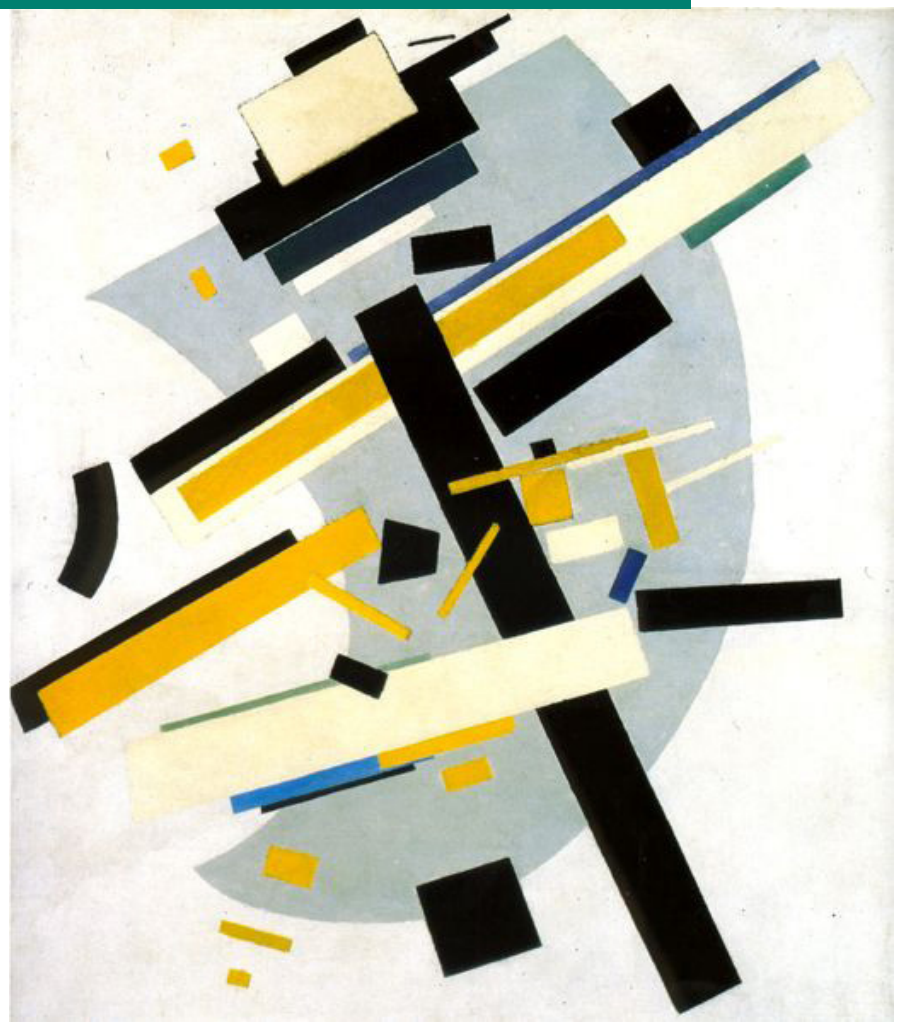



GLOBAL PERSPECTIVES

ON LEGAL HISTORY 


\title{
Global Perspectives on Legal History
}

\author{
A Max Planck Institute for European Legal History \\ Open Access Publication
}

http://global.rg.mpg.de

Series Editors: Thomas Duve, Stefan Vogenauer

\section{Volume 14}

Global Perspectives on Legal History is a book series edited and published by the Max Planck Institute for European Legal History, Frankfurt am Main, Germany.

As its title suggests, the series is designed to advance the scholarly research of legal historians worldwide who seek to transcend the established boundaries of national legal scholarship that typically sets the focus on a single, dominant modus of normativity and law. The series aims to privilege studies dedicated to reconstructing the historical evolution of normativity from a global perspective.

It includes monographs, editions of sources, and collaborative works. All titles in the series are available both as premium print-on-demand and in the open-access format. 
MARIO G. LOSANO

\section{Le tre costituzioni pacifiste}

Il rifiuto della guerra nelle costituzioni di Giappone, Italia e Germania

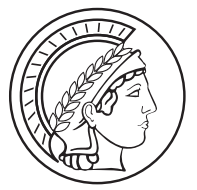

MAX PLANCK INSTITUTE

FOR EUROPEAN LEGAL HISTORY

2020 
ISBN 978-3-944773-26-1

eISBN 978-3-944773-27-8

ISSN 2196-9752

First published in 2020

Published by Max Planck Institute for European Legal History, Frankfurt am Main

Printed in Germany by epubli, Prinzessinnenstraße 20, 10969 Berlin, http://www.epubli.de

Max Planck Institute for European Legal History Open Access Publication

http://global.rg.mpg.de

Published under Creative Commons CC BY 4.0 International

https://creativecommons.org/licenses/by/4.0/

The Deutsche Nationalbibliothek lists this publication in the Deutsche Nationalbibliographie; detailed bibliographic data are available on the Internet at http://dnb.d-nb.de

Cover illustration:

Kazimir Malevich, Supremus \#58: Yellow and black, 1916

The State Russian Museum, Saint Petersburg

Public Domain: https://commons.wikimedia.org

Cover design by Elmar Lixenfeld, Frankfurt am Main

Recommended citation:

Losano, Mario G. (2020), Le tre costituzioni pacifiste. Il rifiuto della guerra nelle costituzioni di Giappone, Italia e Germania (Global Perspectives on Legal History 14), Max Planck Institute for European Legal History, Frankfurt am Main, http://dx.doi.org/10.12946/gplh14 


\section{Indice}

Un memento preliminare: Dulce bellum inexpertis ......... 1

\section{Capitolo I}

Tre costituzioni dalle dittature alle democrazie parlamentari. . . . . 7

1. L'intersecarsi di tre storie diverse........... 7

a) I punti di contatto fra i tre Stati del Patto Tripartito... 8

b) L'Italia in Giappone e il Giappone in Italia:

D'Annunzio e il "fratello samurai" Shimoi Harukichi . . 17

c) La Germania e il mito dei samurai . . . . . . . . . 26

2. L'internamento degli "alien enemies" durante la guerra ... 34

3. Dalla geopolitica dei "Grandi Spazi" al "Patto Tripartito” . . 37

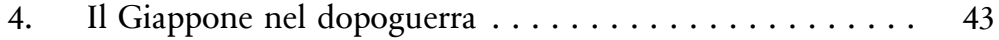

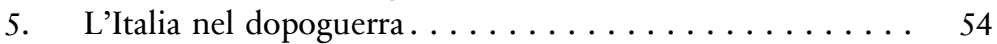

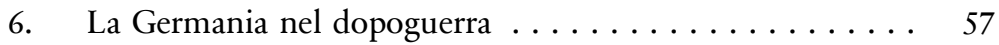

7. Il superamento del passato: i grandi processi......... 63

8. Il superamento del passato: le epurazioni . . . . . . . . 79

9. Il superamento del passato: le riparazioni belliche . . . . . 106

10. Il superamento del passato: i libri scolastici. . . . . . . . 128

11. Oltre mezzo secolo dopo la guerra: gli articoli costituzionali pacifisti e i nuovi conflitti . . . . . . . . 163

Capitolo II

Il rifiuto della guerra nella Costituzione giapponese del 1947 . . . . 169

Capitolo III

Il rifiuto della guerra nella Costituzione italiana del 1948. . . . . . 193

Capitolo IV

Il rifiuto della guerra nella Legge Fondamentale tedesca del 1949 . . 219 


\section{Appendici}

I. Giappone: Il disarmo totale in un'area dalle tensioni crescenti . . 235

I.1. Dichiarazione di Potsdam (Potsdam Declaration, 1945) . . . 235

I.2. Dopo la resa: le istruzioni per il Gen. MacArthur e la politica degli Stati Uniti verso il Giappone (1945) . . . . 237

a) Le istruzioni per il Generale Douglas MacArthur (1945) . . 238

b) L'iniziale politica degli Stati Uniti verso il Giappone dopo la resa (1945). . . . . . . . . . . . . 239

I.3. Come i giapponesi hanno percepito la costituzione del dopoguerra (1997) . . . . . . . . . . . . . . . . 247

I.4. Le radici storiche del pacifismo in Giappone: intervista a Karatani Kojin (2017) . . . . . . . . . . . 257

I.5. Il congresso del Partito Liberal-Democratico (LDP): verso un art. 9 bis $(2018) \ldots \ldots \ldots \ldots \ldots \ldots \ldots \ldots \ldots$

a) Shinzo Abe deciso a modificare la costituzione. . . . . . 263

b) Il tentativo di revisione costituzionale del LDP travolto dal "caso Moritomo" - Diminuisce l'influenza di Abe a causa dello scandalo. . . . . . . . . . . . 265

c) Testo del progetto dell'art. 9 bis. Proposta di modifica dell'articolo 9 della Costituzione da parte del Partito Liberal-Democratico . . . . . . . . . . . . . . 268

II. Italia: Tra guerra e pace: D’Annunzio, Shimoi Harukichi, Stalin e Bobbio . . . . . . . . . . . . . . . . . . . . 269

II.1. D'Annunzio tra l'Occidente "che non ci ama" e 1'“esempio inaudito di trasformazione" del Giappone . . . . 269

a) Dopo l'inerzia forzata, "Si vola!": e allora "a Tokio, in dieci o dodici tappe" . . . . . . . . . . . . . . . . . 269

b) Il saluto di Gabriele D'Annunzio al "fratello samurai" Shimoi Harukichi (1920) . . . . . . . . . . . . . . . 279

II.2. Stalin e Bobbio sulla propaganda di pace (1952-53).

Con due lettere inedite tra Mucchi e Bobbio . . . . . . . . 284

a) Stalin, i "Partigiani della Pace" e

l'imperialismo bellicista . . . . . . . . . . . . . 288 
b) Bobbio: qual è la pace dei "Partigiani della Pace"? . . . . 289

c) Mucchi e Bobbio: prospettive divergenti sui "Partigiani della Pace" . . . . . . . . . . . . . . . . . . 294

III. Germania: Il ripudio della guerra e il riarmo nelle costituzioni

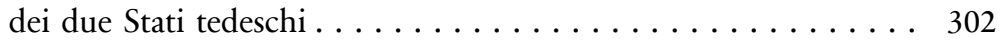

III. 1. Il ripudio della guerra nel dibattito costituente tedesco-federale $(1948) \ldots \ldots \ldots$. . . . . . . . . . 303

a) "Diritto internazionale e diritto federale". . . . . . . 306

b) I rapporti di diritto internazionale della Federazione. . . 309

c) Le Regole del diritto internazionale e Legge Fondamentale (art. 22 CHE [Chiemseer Entwurf]) . . . 310

d) La turbativa della pace fra i popoli (art. 31; art. $26 \mathrm{CHE}$ [Chiemseer Entwurf]) . . . . . . . . . . . . . 313

e) Discussione e decisione delle petizioni: l'obiezione di coscienza . . . . . . . . . . . . . . . 315

f) Incostituzionalità delle azioni che preparano una guerra; divieto delle armi (art. 29b e 29c) . . . . . . . . . . 322

g) La redazione finale degli articoli sul ripudio della guerra 325

III. 2. Repubblica Democratica Tedesca: la Costituzione (1949) e a Legge a tutela della pace $(1950) \ldots \ldots \ldots \ldots \ldots . \ldots 326$

III. 3. La legge istitutiva dell'Armata Popolare Nazionale (NVA) della DDR $(1956) \ldots \ldots \ldots \ldots \ldots \ldots \ldots \ldots \ldots$

Elenco degli scritti citati . . . . . . . . . . . . . 335

Indice analitico $\ldots \ldots \ldots \ldots \ldots \ldots \ldots \ldots \ldots \ldots \ldots \ldots \ldots$

Indice dei nomi. . . . . . . . . . . . . . . . . . . 389

About the Author ....................... 399 



\section{Un memento preliminare: Dulce bellum inexpertis}

I tre Stati dell'ex Patto Tripartito vengono qui presi in considerazione nella sequenza determinata dalla data di entrata in vigore delle rispettive costituzioni postbelliche: il 1947 per il Giappone, il 1948 per l'Italia, il 1949 per la Germania federale. ${ }^{1}$

Un esame della genesi, del contenuto e dell'attuale applicazione degli articoli pacifisti di queste tre costituzioni postbelliche esigerebbe la descrizione, almeno a grandi linee, della situazione geopolitica prima, durante e dopo la Seconda guerra mondiale, della transizione dalla dittatura alla democrazia nei tre Stati dell'ex Patto Tripartito e del risorgere delle loro rinnovate forze armate nel contesto della Guerra fredda. Bisognerebbe inoltre analizzare i dibattiti degli organi costituenti che portarono all'approvazione degli articoli pacifisti nella loro formulazione definitiva, per passare poi alla loro interpretazione e applicazione fino ai giorni nostri, nei quali si pone il problema della compatibilità di quegli articoli con i "conflitti asimmetrici", con le "guerre ibride", con le "guerre umanitarie" e con le "missioni di pace" o "di polizia" dell'ultimo ventennio.

Nell'impossibilità di assolvere un compito così vasto, queste pagine procedono per accenni evocativi su singoli temi. Esse vanno quindi viste come una piattaforma sinottica strutturata più per indirizzare che per esaurire, più per riassumere che per dettagliare, fornendo al tempo stesso qualche indicazione bibliografica in vista di eventuali approfondimenti su singoli temi. Infatti l'odierno contatto con gli studiosi delle due generazioni successive alla mia (nonché con gli studenti, la cui ultima generazione è nata nel 2000) $\mathrm{mi}$ ha rivelato che le aree dei nostri ricordi non sono coincidenti e, in particolare, che in loro molti ricordi e sensazioni legati agli anni successivi alla fine della guerra sono o molto diversi quelli della generazione nata durante la guerra (la mia), o del tutto assenti.

È per loro che mi è parso utile ricostruire gli eventi, nella misura del possibile, e, con essi, l'atmosfera che permeò gli anni della fine del conflitto e

1 Avevo affrontato questo tema nell'articolo Mario G. Losano (2017a), Il rifiuto della guerra. 
quelli immediatamente successivi dell'avvento della pace e della rinascita dell'economia. C'è infatti un'area che non è più affidata ai discorsi dell'ambito famigliare o culturale, ma che non sempre è narrata dai manuali e dai programmi scolastici, ed è proprio in quell'area che sono nate le tre costituzioni e, soprattutto, i loro articoli pacifisti. Se non si rievoca l'atmosfera di quegli anni risulta difficile capire quanto fosse forte, nei vinti, il desiderio di pace e, anche nei vincitori, il timore d'una terza guerra mondiale; quanto gravasse su tutti il terrore dell'annientamento atomico, mai prima sperimentato; e quanti timori suscitasse la guerra di Corea, oggi pressoché dimenticata ma allora temuta come la fiamma in una polveriera. Persino l'Italia, quando non faceva ancora parte delle Nazioni Unite, vi partecipò dal 1951 al 1955, sia pure soltanto con un ospedale da campo (chi ricorda oggi l'“Ospedale 68" alla periferia di Seul?) che serviva a curare i feriti, ma anche a segnalare la collocazione dell'Italia nel mondo bipolare. ${ }^{2}$

In conclusione, la pressione congiunta dei vincitori e della propria popolazione imponeva ai tre Stati sconfitti di accettare la rinuncia al diritto che costituisce l'essenza della sovranità: il "ius ad bellum", il diritto di dichiarare la guerra. È da questo contesto che nascono i tre articoli pacifisti delle costituzioni post-belliche degli Stati dell'ex Patto Tripartito.

La storia degli ultimi cinquanta-settant'anni è stata vissuta in modo diverso e frammentario da alcuni, mentre per altri è giunta filtrata attraverso narrazioni in famiglia o nella scuola, lasciando ricordi ora vividi ora lacunosi; pochi, infine, ne hanno ricevuto un insegnamento sistematico. Chi c'era, chi ricorda, chi ha dimenticato, chi non ne ha notizia può trovare aiuto in testi generali e sistematici, ${ }^{3}$ mentre invece nelle pagine che seguono troverà un insieme di microsistemi appena abbozzati o anche soltanto degli spunti

2 Gastone Breccia (2019), Corea, la guerra dimenticata (bibliografia, 381-383). Sull'“Ospedale 68" della Croce Rossa Italiana, 384.

3 Ad esempio: per il Giappone, Rosa Caroli, Francesco Gatti (2017), Storia del Giappone; Paolo Beonio-Brocchieri (1996), Storia del Giappone; Gary D. Allinson (1997), Japan's Postwar History; Masataka Kosaka (1982), A History of Postwar Japan; per l'Italia, Paul Ginsborg (2006), Storia d'Italia; per una descrizione giorno per giono che immerge il lettore nel flusso degli eventi: Enrico Deaglio (2009), Patria 1978-2008; e Enrico Deaglio (2017), Patria 1967-1977. Per la Germania: Merith Niehuss, Ulrike Lindner (eds.) (2012), Besatzungszeit, Bundesrepublik und DDR; Dieter Grosser et al. (eds.) (2015), Bundesrepublik und DDR. Infine, per una cronologia commentata non solo per i tre Stati in esame, ma per l'intero globo: Der große Ploetz (1998). 
illuminanti (una piattaforma, appunto), per ricostruire tre contesti sociali non lontani nel tempo ma ormai quasi assenti nella memoria: $i$ tre contesti in cui presero forma tre nuovi regimi politici e tre nuove costituzioni con $\mathrm{i}$ loro articoli pacifisti.

La piattaforma vera e propria è costituita dal primo capitolo: un sommario degli eventi che accompagnarono i tre Stati dell'ex Patto Tripartito dalla guerra alla sconfitta e, infine, a forme democratiche di regime politico. Dopo l'esame complessivo delle movimentate fasi del dopoguerra in ciascuno dei tre Stati, l'attenzione si concentra su quattro eventi che caratterizzarono quegli anni: i grandi processi di Norimberga e di Tokyo; le epurazioni dei responsabili dei crimini delle dittature; le riparazioni belliche imposte agli sconfitti; infine, le narrazioni degli eventi bellici e postbellici attraverso i libri scolastici destinati alle generazioni più giovani: narrazioni che, quando ci sono, si rivelano spesso di parte o reticenti.

Designo come "pacifiste" le tre costituzioni nate in questo contesto perché ciascuna di esse contiene un articolo che ripudia la guerra (insieme con qualche altra norma complementare per rafforzare quel rifiuto): tre capitoli analizzano, Stato per Stato, il contenuto di queste norme. La loro analisi letterale - insostituibile ma non esauriente - è accompagnata dai riferimenti politici essenziali per la sua comprensione.

A questo passaggio dal generale al particolare seguono tre appendici che si soffermano su specifici aspetti riconducibili a ciascuna delle norme pacifiste. Ogni appendice è un esempio settoriale di quell'approfondimento che sarebbe impossibile affrontare a tutto campo: è un case study che aiuta anche a comprendere l'atmosfera, oggi così lontana, in cui si discuteva sul rifiuto della guerra e sull'accettazione del pacifismo.

La prima appendice, sul Giappone, riporta alcuni dei testi con cui gli occupanti statunitensi diedero il primo impulso alla nuova costituzione (e, in particolare, al suo articolo pacifista). Seguono alcune analisi giapponesi di quell'articolo e del suo contesto sociale sino al dibattito del 2018, in cui il partito di maggioranza ha proposto la modifica, anche se non l'abrogazione, dell'articolo pacifista della costituzione, l'art. 9. Il mio ringraziamento va alla professoressa Hidemi Suzuki, dell'Università di Keio, che mi ha fornito i testi della polemica più recente, e a Pier Giorgio Girasole, cui sono debitore non solo del reperimento di alcuni testi, ma anche della loro traduzione in italiano. 
La seconda appendice, sull'Italia, si ricollega al movimento pacifista dei "Partigiani della pace", oggi pressoché dimenticato, e riproduce parte di uno scritto di Stalin su quel movimento, nonché la critica di Norberto Bobbio alla concezione unilaterale della pace in esso contenuta. Questa critica non sembrò condivisibile a un amico comunista di Bobbio, di cui si trascrive la lettera a Bobbio e la risposta di Bobbio stesso: queste lettere finora inedite costituiscono un esempio di civile polemica sulla pace in accesi tempi di guerra, ancorché fredda.

La terza appendice, sulla Germania, è quasi una lezione di tecnica legislativa, perché riporta in italiano alcuni passaggi decisivi nel dibattito politico della costituente tedesco-federale, dal quale prese forma l'articolo pacifista, cioè l'articolo 26 della Legge Fondamentale, oggi vigente in tutta la Germania unificata dal 1990. Però prima di questa data esistevano due Germanie, entrambe uscite dal nazismo e dalla guerra; entrambe occupate da potenze vincitrici fra loro sempre meno alleate, e infine frontalmente nemiche; entrambe terre di frontiera tra due blocchi ostili. Per questo mi è sembrato giusto affiancare alla costituzione tedesca vigente anche alcuni cenni sulla costituzione del 1949 (ormai soltanto storica) della Repubblica Democratica Tedesca. Quest'ultimo Stato oggi dissolto aveva approvato, oltre a un articolo costituzionale sulla pace, una Legge a tutela della pace nel 1950 e una legge istitutiva dell'“altro" esercito tedesco nel 1956: testi che vengono qui tradotti.

Quando vennero approvati gli articoli delle costituzioni pacifiste, le ferite della guerra erano ancora aperte, mentre oggi esse sono sempre più sbiadite nella memoria, cosí come lo sono i lutti e le distruzioni. Sempre più spesso la politica propone di abrogare o almeno di limitare gli articoli con cui le costituzioni pacifiste rifiutano la guerra. Per questo tornano d'attualità gli ammonimenti di Erasmo da Rotterdam, che condanna la guerra anzitutto dal punto di vista morale: "Cos'è la guerra se non un omicidio collettivo, di gruppo, una forma di brigantaggio tanto più infame quanto più ampia?”." Per Erasmo la guerra, oltre che immorale, non è neanche un affare: "Se vogliamo venire ai conti e valutare esattamente il costo della guerra e della pace, certamente scopriamo che la decima parte delle ansie, delle fatiche, dei

4 "Bellum quid aliud est, quam multorum commune homicidium et latrocinium, hoc sceleratius quo latius patens?”, Erasmo da Rotterdam (2013), Adagi, 1252-1253. 
disagi, dei pericoli, delle spese, e del sangue, con i quali la guerra è accesa, basterebbe a salvaguardare la pace. Metti in campo e in pericolo una grande moltitudine di uomini affinché rovesci una qualsiasi città; ma mettendoli al lavoro avresti potuto tirar su una città molto più bella. Ma tu vuoi nuocere al nemico. Questo è disumano. Tuttavia considera ciò: non puoi danneggiare lui se prima non danneggi i tuoi".5 Cinque secoli dopo, Erasmo avrebbe potuto constatare con ragione che il Piano Marshall era molto meno costoso delle distruzioni provocate dalla Seconda guerra mondiale.

Queste osservazioni fanno parte delle molte pagine con cui Erasmo commenta un adagio che acquista un significato ancora più profondo se oggi, a cinquecento anni di distanza, lo si ricorda a quelle generazioni che, in Europa, vivono in pace da più di settant'anni e che quindi possono aver dimenticato gli orrori della guerra:

\author{
Dulce bellum inexpertis ${ }^{6}$ \\ La guerra piace a chi non sa che cos'è.
}

5 "Quod si velimus rem ad calculum vocare et veris rationibus expendere quanti bellum constet, quanti pax, profecto comperimus hanc vel decima parte curarum, laborum, molestiarum, periculorum, sumptuum, denique sanguinis posse comparari, quibus bellum arcessitur. Tantam hominum turbam educis in periculum, ut oppidum aliquod evertas; at horum opera, vel citra periculum, aliud exstrui poterat multo praeclarius oppidum. Sed nocere vis hosti. Iam hoc ipsum inhumanum; attamen illud expende, num illi nocere non possis, nisi prius noceas tuis", Erasmo da Rotterdam (2013), Adagi, 1258-1259.

6 Erasmo da Rotterdam (2013), Adagi, 2142 (Centuria 31, Adagio 3001). Il commento di Erasmo a questo adagio, da cui sono tratte le precedenti citazioni, occupa più di quaranta pagine: $2142-2184$ (testo latino e italiano). 



\section{Capitolo I Tre costituzioni dalle dittature alle democrazie parlamentari}

\section{L'intersecarsi di tre storie diverse}

Gli anni tra il 1930 e il 1945 legarono strettamente fra loro tre Stati diversi ma non estranei l'uno all'altro: in Europa, i rapporti tra Italia e Germania duravano con alterne vicende almeno dai tempi dell'Impero Romano; invece i rarefatti rapporti del Giappone con l'Europa erano divenuti più stretti con la fine del XIX secolo. Infatti l'espansione degli imperi portoghese e spagnolo aveva lambito le isole giapponesi dalla metà del 1500, suscitando nei governanti nipponici il timore che l'Impero del Sol Levante potesse subire una colonizzazione analoga a quella degli imperi autoctoni dell'America latina. Di qui la persecuzione del cristianesimo, la chiusura quasi totale del Giappone per circa due secoli e mezzo, e la sua apertura all'Occidente solo a partire dal 1868. Da questa data si può far iniziare l'occidentalizzazione del Giappone, in cui ebbero peso non solo le grandi potenze di allora come la Gran Bretagna e la Francia, ma anche gli Stati Uniti e due Stati nuovi, il Regno d'Italia, sorto nel 1861, e l'Impero di Germania, nato nel 1871. Assenti invece i temuti mentori dei secoli anteriori, Spagna e Portogallo, che nell'Ottocento vivevano la fine della loro potenza coloniale. ${ }^{\mathbf{1}}$

Forse per non risuscitare i malintesi dei tempi passati, il Pontefice accompagnò l'apertura ottocentesca del Giappone con segnali cauti e concilianti. ${ }^{2}$ Inazo Nitobe ("un intellettuale giapponese aderente alla Società dei Fratelli",

1 Rinvio alla letteratura indicata nei seguenti miei lavori: sull'autore della costituzione Meiji, rimasta in vigore sino alla fine della Seconda guerra mondiale: Hermann RoesLer (1984), Berichte aus Japan; sull'unico consigliere giuridico italiano: Mario G. Losano (2016a), Alle origini della filosofia del diritto; sulla presenza iberica: Mario G. Losano (2016c), Lo spagnolo Enrique Dupuy; Mario G. Losano (2016b), Il portoghese Wenceslau de Moraes. Cfr. anche infra, note 122, p. 43.

2 Annibale Zambarbieri (2004), Una doppia fedeltà. 
cioè ai Quaccheri, e autore di un libro fortunato sul Bushido), affermava che alcuni valori tradizionali giapponesi affermati nel Bushido si inserivano e trovavano continuità nella dottrina cristiana. ${ }^{3}$

Nel 1905 un inviato del Papa visitò l'imperatore anche per "mettere in luce come i cattolici fossero in grado di staccarsi dalle tendenze occidentali" perché quella religione "vantava un capo indipendente dalle potenze straniere, privo di esercito e quindi essenzialmente pacifico". I cattolici potevano dunque riconoscere nell'Imperatore "la continuità di una dinastia e di un radicamento dai connotati sacri". ${ }^{4}$ Essi, pur essendo cattolici, potevano anche frequentare i templi giapponesi perché "secondo la legge nazionale i templi non dovevano ritenersi organi religiosi”. In realtà, questa "neutralità religiosa del culto nazionale esercitato negli appositi templi" risultava non solo "incomprensibile agli occidentali", ma "anche fra i giapponesi v'erano quelli che confondevano templi e religione". 5

\section{a) I punti di contatto fra i tre Stati del Patto Tripartito}

Il contatto tra Giappone, Italia e Germania si approfondì nel Novecento con l'avvento delle dittature europee e del militarismo giapponese. Negli anni tra il 1930 e il 1945 questi tre Stati rafforzarono i propri legami e a questo fine, tanto nella politica quanto nei mezzi di comunicazione, si impegnarono a fondo nel sottolineare gli elementi comuni, reali o immaginari che fossero. Poiché molti studi hanno analizzato in tutto o in parte queste convergenze rivelatesi poi fatali, ${ }^{6}$ basti qui richiamare alcuni dei principali parallelismi.

3 Annibale Zambarbieri (2004), Una doppia fedeltà, 168-169, note 38 e 39.

4 Annibale Zambarbieri (2004), Una doppia fedeltà, 171.

5 Annibale Zambarbieri (2004), Una doppia fedeltà, 173.

6 Per approfondire gli spunti appena indicati nel testo: Walter A. SKYA (2009), Japan's Holy War; Kunichika Yagyu (2003), Der Yasukuni-Schrein; Francesco Gatti (1997), Il fascismo giapponese; Richard Bessel (ed.) (1996), Fascist Italy and Nazi Germany; Gerhard Krebs (ed.) (1994), Formierung und Fall; Friedrich-Karl von Plehwe (1980), Als die Achse zerbrach; Yasushi Yamaguchi (1976), Faschismus als Herrschaftssystem. 
Dal punto di vista geografico, nel 1935 i tre Stati potevano essere definiti come "medie potenze": il Giappone era lo Stato più popoloso dei tre, la Germania la più estesa, l'Italia la minore per popolazione e superficie. ${ }^{7}$

Mentre il Giappone e l'Italia avevano ed hanno una configurazione geograficamente tutto sommato ben delimitata, la Germania è sempre stata in difficoltà nel trovare una sua identità territoriale. Al momento della sua unificazione ottocentesca, alla "Soluzione Grande-tedesca" (che comprendeva anche le monarchie meridionali della Baviera e dell'Austria-Ungheria, cattoliche e conservatrici) si preferì la "Soluzione Piccolo-tedesca" (che comprendeva gli Stati del Nord, sotto l'egemonia della Prussia, protestante e progressista): secondo quest'ultima soluzione si configurò l'Impero tedesco sorto nel 1871. Rimaneva però aperta la questione degli "altri" territori tedeschi e si apriva così la complessa storia dell'unione di tutti questi territori tedeschi, che si può riassumere nella storia dell'annessione dell'Austria, cioè dell'“Anschluss": termine ambiguo, perché dopo il 1918 indicava un'unione tra Stati socialisti, quale la Repubblica di Weimar e la Repubblica austriaca, mentre nel 1938 l'Anschluss nazionalsocialista dell'Austria austro-fascista rappresentò l'unione fra due Stati autoritari. Quest'unione non era solo un problema interno dei tedeschi, ma un problema internazionale. Nel 1920 D'Annunzio propose di sostituire la vituperata Società delle Nazioni con la Lega di Fiume per "raggruppare in un fascio compatto tutti gli oppressi della terra" e incluse fra i popoli oppressi anche l'"Austria tedesca" accanto agli altri "irredenti tedeschi" e a tutte le colonie del globo. ${ }^{8}$

Quando la fine della Prima guerra mondiale segnò il crollo degli imperi tanto tedesco quanto austro-ungarico, la Costituzione repubblicana di Weimar del 1919 prefigurò una futura unificazione con l'articolo 2: "Il territorio del Reich si compone dei territori dei Länder tedeschi. Altri territori potranno essere riuniti al Reich, con legge di questo, se la loro popolazione ne esprima il desiderio, in virtù del diritto di autodecisione". Dopo il crollo del Terzo Reich e la divisione della Germania, con l'articolo 23 la Legge fondamentale del 1949 della Repubblica Federale di Germania forniva lo strumento giuridico per l'unificazione tedesca, avvenuta quarant'anni dopo: "La

7 Il Giappone aveva 70 milioni di abitanti su quasi $400.000 \mathrm{kmq}$; il Deutsches Reich, 66 milioni di abitanti su $470.000 \mathrm{kmq}$; l'Italia, 43 milioni di abitanti su $310.000 \mathrm{kmq}$. I dati sono desunti dall'edizione del 1936-1942 del Meyers Lexikon, alle corrispondenti voci.

8 Giordano Bruno Guerri (2019), Disobbedisco, 201. 
presente Legge fondamentale ha vigore immediato nel territorio dei Länder” della Germania federale. "[...] Nelle altre parti della Germania entrerà in vigore dopo la loro accessione".' E così avvenne infatti nel 1989. Dell'attuale Germania non fanno però parte i territori tedesco-orientali ceduti all'Unione Sovietica e alla Polonia.

Dal punto di vista storico, i tre Stati avevano in comune il fatto di essersi presentati da pochi anni sullo scenario mondiale: l'Italia nel 1861, il Giappone nel 1868, la Germania nel 1871. Erano "newcomers" che non avevano un passato coloniale, ma che aspiravano a un futuro coloniale.

Dal punto di vista istituzionale, due dei tre Stati erano retti da monarchie, l'altro lo era stato fino al 1919, cioè fino alla Repubblica di Weimar. In Italia per quasi un ventennio la monarchia si sdoppiò in una diarchia a causa dell'incontenibile protagonismo di Mussolini. In Giappone la radicatissima figura dell'imperatore di origine divina costituì un grave problema nell'indirizzare la transizione postbellica verso un regime parlamentare. Uno studio comparativo del 2004 offre "un mosaico, formato da dodici grandi tessere, nel quale risulta ben leggibile [...] l'oggetto indagato: le modalità di configurazione e di consolidamento dei tre più importanti Stati detti late comers: l'Italia, la Germania e il Giappone. L'epoca di riferimento è la stessa, gli anni Sessanta-Ottanta del XIX secolo (anche se qui si prendono le mosse dai primi decenni dell'Ottocento e si giunge fino al secondo dopoguerra), e uguale è anche il punto di osservazione, la monarchia. Il re in Italia, l'imperatore in Germania, il Tenno in Giappone sono stati il perno istituzionale decisivo dei rispettivi processi di unificazione e di trasformazione e perciò fungono da filo conduttore del racconto". Al tema della monarchia "sono intimamente connessi: la 'modernizzazione dall'alto', la 'nazionalizzazione delle masse', l'autorappresentazione comunitaria, il rapporto fra storia e memoria, il legame fra tradizione e identità nazionale": ${ }^{\mathbf{1 0}}$ grandi temi complessivi di

9 Corsivo mio (costituzione di Weimar: http://dircost.di.unito.it/cs/pdf/19190811_germania Weimar_ita.pdf; Legge fondamentale: http://dircost.di.unito.it/cs/pdf/19490523_germania LeggeFondamentale_ita.pdf). Sul problema dell'Anschluss nei testi di Hans Kelsen, cfr. Hans Kelsen (2020), L'annessione dell'Austria al Reich tedesco.

10 Sugli sviluppi di queste coincidenze cfr. Marina Tesoro (2004), Introduzione, 2. 
cui incontreremo riflessi frammentari negli specifici eventi oggetto del presente volume.

Tutti e tre gli Stati erano dual States, cioè Stati in cui alla struttura ufficiale dello Stato si affiancava una struttura ufficiosa ma egualmente potente. ${ }^{11}$ I liberali giapponesi degli anni Venti vedevano nelle Camicie Nere italiane dei "dual political organs" ${ }^{12}$ In Germania e in Italia il partito nazionalsocialista e quello fascista si affiancavano alla struttura statale, mentre in Giappone la tradizione conduceva allo stesso risultato attraverso una via diversa. Poiché in Giappone il Tenno era di origine divina, e quindi sopra le parti, ${ }^{\mathbf{1 3}}$ non esisteva una struttura di partito parallela a quella dello Stato, bensì un forte potere militare parallelo a quello civile. Dal 1928 l'imperatore venne tenuto fuori (almeno formalmente) dalle decisioni prese dal governo civile e sempre più dai militari, divisi a loro volta dalla rivalità tra esercito e marina. Diversamente dai civili, i militari avevano diretto accesso all'imperatore: "This situation gave some of the generals the opportunity to pretend they were speaking in the name of the emperor and impose they will on the government. Their policies, however, were largely dependent on imperial approval". ${ }^{14}$ Di fatto, le loro decisioni erano concordate con l'imperatore, ma non pubblicamente, come sarebbe avvenuto in un iter parlamentare. Questa peculiarità consentì al Giappone, dopo la sconfitta, di tenere l'imperatore fuori dal dibattito (e dai processi) per i crimini di guerra. Tenendo presente questa peculiarità giapponese, per una certa fase della loro storia tutti e tre gli Stati sono accomunati dall'essere "dual States".

11 Il termine si richiama al titolo The Dual State dell'opera di Fraenkel, pubblicata in inglese nel 1941 durante il suo esilio negli Stati Uniti, ma scritta originariamente in tedesco (Der Doppelstaat): Ernst Fraenkel (1983), Il doppio Stato.

12 Reto Hofmann (2015), The Fascist Effect, 9. È uno dei libri più accuratamente documentati sulla recezione del fascismo in Giappone. "My aim is to rethink the history of Japan as a part of a wider, interconnected, history of fascism, arguing that Japanese politics and ideology in the first half of the twentieth century were enmeshed in a dialogue with the European fascism" (2). Bibliografia, 177-193.

13 Cfr. Marina Tesoro (ed.) (2004), Monarchia, tradizione, identità nazionale, 36, nota 4, che cita Кiчoкo Takeda (1988), The Dual-Image, 8-10.

14 Arthur Stam (2003), The Diplomacy of the "New Order", 9. 
Dal punto di vista della propaganda, le dittature dei tre Stati controllavano i mezzi di comunicazione in modo totale. "Mentre in Germania i media devono essere direttamente 'incorporati' dal regime, in Giappone sono delle organizzazioni burocratiche intermedie a regolare i mezzi di comunicazione, per lo più di proprietà privata". ${ }^{15}$

In Italia, "l'ingerenza e il controllo sulla stampa non giunse mai a forme uguali o anche solo approssimativamente simili a quelle tedesche; le direttive per la stampa non furono mai così sistematicamente organizzate e vennero seguite e perseguite con ben minore disciplina". ${ }^{16}$ Il controllo sulle redazioni era comunque stretto. Lo documenta il catalogo d'una mostra della Biblioteca Statale di Cremona, che mette in luce il funzionamento del regime fascista in quella città incentrandolo sulla figura del fascista intransigente Roberto Farinacci e del suo giornale, "Il Regime Fascista", un foglio inizialmente locale divenuto quotidiano a diffusione nazionale sotto la sua direzione. Perennemente in viaggio, Farinacci determinava l'indirizzo del giornale con note scritte sui "punti intorno a cui sviluppare gli articoli che avrebbe poi firmato, esordienti con 'Fare un articolo di fondo', che ha ispirato il titolo della mostra. ${ }^{17}$ L'indicazione risultava duplicemente vincolante perché Farinacci fu segretario nazionale del partito fascista, oltre che proprietario e direttore del giornale.

Erano invece di natura esclusivamente politica le "veline", che - secondo le disposizioni impartite nel 1931 da Gaetano Polverelli, responsabile dell'“Ufficio Stampa del Capo del Governo", cioè di Mussolini - dovevano improntare i giornali a "ottimismo, fiducia e sicurezza nell'avvenire", esigendo perciò di "eliminare le notizie allarmistiche, pessimistiche, catastrofiche e deprimenti”. ${ }^{18}$

15 Julia Siep (2011), Nationalisierte Mütterlichkeit, 108 (sulla politica mediale dei tre regimi, 97-108; bibliografia, 279-307); cfr. anche Gregory J. Kasza (1988), The State of the Mass Media, in particolare 288. Inoltre: Gianni Spulcioni (2014), L'organizzazione del consenso; Clemens Zimmermann (2007), Medien im Nazionalsozialismus (analisi non solo della stampa, ma anche della radio e del cinema, con tre bibliografie su Germania, Spagna e Italia, 269-301); Mauro Forno (2005), La stampa nel Ventennio.

16 Andrea Hoffend (1998), Zwischen Kultur-Achse und Kulturkampf, 104.

17 Stefano Campagnolo (ed.) (2018), Si faccia un articolo di fondo, 21. Sulle direttive alla redazione: Francesco Cignoni (2018), Le "veline".

18 Sulle "veline": Riccardo Cassero (2004), Le veline del Duce; in generale, sul linguaggio da usare, Fabio Foresti (ed.) (2003), Credere, obbedire, combattere; Gabriella B. Klein (1986), Politica linguistica del fascismo. 
Enzo Biagi ricorda una di queste veline giunta al "Resto del Carlino" nel settembre 1940: "Il comunicato del Consiglio dei Ministri va dato su otto colonne. È vietato ogni strillonaggio. Il telegramma del Führer al Duce va pubblicato in 'palchetto' (cioè incorniciato). Non abboccare a notizie tendenziose di fonte straniera. Infine, astenersi da ogni commento". ${ }^{19}$ Dall'efficacia di questa direttiva "si misura la capacità di penetrazione del fascismo, che non intendeva lasciare neutri nemmeno i ristretti spazi dei pronomi, dei complementi e delle operazioni di calcolo": ${ }^{20}$ atteggiamento che ricorre in ogni dittatura. "Complessivamente si possono riconoscere molti parallelismi nella politica mediale, in particolare nella politica della stampa, tra Giappone, Italia e Germania".21

Un'autrice tedesca ha esaminato nella stampa femminile degli anni Trenta il modello di donna imposto dai regimi dittatoriali. Ne risulta una comune visione della donna come madre vincolata alla casa: nibon no haha (la madre giapponese), la Volksmutter (la madre germanica), l'angelo del focolare (la madre italiana). Le tre dittature presentano connotati fortemente maschilisti, benché il Giappone - discusso ancora oggi per certi suoi clichés - sia stato fondato non da un Padreterno, ma da una Madreterna: la dea Amaterasu, da cui si fa discendere la dinastia imperiale.

In realtà i tre regimi, in modo ovviamente diverso, tentavano di acquisire il consenso delle donne, pur mantenendole in una posizione subalterna, come documenta un'ampia ricerca su Donne e fascismo. ${ }^{22}$ Nei 600 giorni della Repubblica di Salò "si assiste infatti ad un prepotente coinvolgimento della donna nella mobilitazione bellica in una fase di estrema emergenza che impone il suo inserimento nella macchina produttiva militare fino al suo arruolamento diretto nelle file dell'esercito repubblicano. Ciò pone le condizioni per una sua più pronunciata emancipazione, contrassegnata dall'indipendenza economica conquistata e dalla più alta autocoscienza derivatane.

19 Enzo Biagi (2008), Io c'ero, 9.

20 Davide Montino (2005), Le parole educate, 9.

21 Julia Siep (2011), Nationalisierte Mütterlichkeit, 108.

22 Donne e fascismo. Immagini e modelli educativi (2010); dei nove saggi, si vedano in particolare l'introduzione di Inge BotTeri (2010), La donna tra modernità e fascismo; Rossella Coarelli (2010a), Da "Bertoldo" a "Settebello"; Rossella CoArelli (2010b), Riviste femminili (elenco di "246 riviste nate in Italia durante il fascismo rivolte ad un vario pubblico femminile", 105). 
È un processo che tendenzialmente stride fino a contraddire il paradigma della donna-angelo del focolare, che, pur tra alcune contraddizioni e sfilacciamenti, aveva retto per tutto il Ventennio".23

Oltre che alla tradizionale stampa, le dittature rivolsero particolare attenzione ai mezzi allora più moderni, la radio e la cinematografia. ${ }^{24}$ In Italia, nel 1931 una legge protezionistica limitò l'importazione di pellicole straniere per favorire la produzione nazionale, che nel 1936 si installò negli studi di Cinecittà, a Roma. A partire dallo stesso anno venne costruito a Venezia il Palazzo del Cinema, per ospitare la Mostra Internazionale d'Arte Cinematografica. I contenuti della produzione cinematografica erano curati dal 1924 dell'Istituto LUCE (L'Unione Cinematografica Educativa). Con Cinecittà il fascismo assicurava la produzione nazionale dei film, con la mostra di Venezia la sua risonanza internazionale e con l'Istituto LUCE la conformità dei contenuti alla linea del regime: il "Film Luce", in particolare, era una breve rassegna settimanale proiettata obbligatoriamente in ogni sala prima della pellicola principale (cfr. p. 149). La diffusione capillare era garantita non solo dalle numerose sale cinematografiche, ma anche dal "Cinemobile": un camioncino blu Fiat 618 dotato di proiettore, schermo e altoparlanti, che portava le immagini del regime anche nelle località prive di sala cinematografica.

In Germania l'importazione dei film era contingentata già dall'epoca di Weimar. L'avvento del nazionalsocialismo nel 1933 regolò la produzione filmica con istituzioni pubbliche: il Ministero della Propaganda e la Camera per la Cinematografia, entrambe sotto la guida di Joseph Goebbels. ${ }^{25}$ Anche il Giappone, sin dalla guerra contro la Cina del 1937, sviluppò una filmografia di propaganda che si ispirava ai modelli tedeschi. ${ }^{26}$ In essa il Giappone veniva presentato anche come il liberatore dei popoli asiatici dalla soggezione coloniale: per superare le barriere linguistiche, la propaganda dedicò una particolare attenzione alla grafica dei manifesti.

23 Elena Pala (2010), Le "donne di Salò" 87.

24 Guido Aristarco (1996), Il cinema fascista.

25 Francis Courtade, Pierre Cadars (1975), Geschichte des Films im Dritten Reich; David Welch (2001), Propaganda and the German cinema.

26 Janine Hansen (1997), Arnold Fancks "Die Tochter des Samurai". 
La politica estera italiana in Asia Orientale ebbe minore rilevanza di quella tedesca, anche perché nell'epoca fascista l'Italia si interessò in un primo tempo alla Cina (soprattutto dopo l'avvento dei nazionalisti nel 1928), per poi concentrare il suo interesse sul Giappone a partire dalla Seconda guerra sino-giapponese (1937). ${ }^{27}$ I documenti mostrano come, dal 1940, la politica estera italiana si avvicinasse sempre più a quella tedesca per la Guerra nel Pacifico: infatti il sistema "Magic" permise agli Stati Uniti e alla Gran Bretagna di decifrare le comunicazioni militari italiane. ${ }^{28}$ D'altra parte, come potenza minore, l'Italia non era al centro degli interessi giapponesi: quando nel 1921 il principe ereditario Hirohito visitò l'Europa, l'Italia fu la sua ultima tappa. ${ }^{29}$ L'affermarsi delle dittature in Europa e del militarismo in Giappone portarono poi a rapporti più stretti.

I bombardamenti degli Alleati misero a dura prova tutti e tre i paesi dell'Asse, anche se in misura diversa. I militari statunitensi, in un rapporto dell'11 settembre 1941, dubitavano che si potesse condurre una guerra contemporaneamente contro la Germania e contro il Giappone e, ritenendo improbabile che il popolo tedesco rovesciasse il regime nazista, chiedevano di concentrare gli sforzi anzitutto verso una "sconfitta totale" della Germania. Franz Neumann, giurista socialdemocratico riconducibile alla Scuola di Francoforte, aveva appena pubblicato un'analisi del nazionalsocialismo in cui esprimeva la speranza che almeno una parte dei tedeschi si opponesse a quel regime. ${ }^{30}$ Nel suo libro indicava in che modo condurre la guerra per giungere a questo risultato, insistendo sulla necessità di evitare $\mathrm{i}$ bombardamenti a tappeto, che avrebbero allontanato tutti i tedeschi dalla causa alleata. Questa tesi non ebbe seguito pratico, purtroppo, e il libro ebbe fortuna solo dopo la fine della guerra: "Ouvrage fondamental de Franz Neumann, généralement considéré, avec Les origines du totalitarisme de Hannah

27 Valdo Ferretti (1983), Il Giappone e la politica estera italiana; Alessandro Vagnini (2015), L'Italia e l'imperialismo giapponese.

28 Peter Herde (1983), Italien, Deutschland, con il materiale inedito (intercettato e decifrato dal sistema "Magic" degli Stati Uniti e dell'Inghilterra) sulla comune politica italo-tedesca in Asia Orientale.

29 Lia Beretta (2013), Hirohito in Italia.

30 Franz L. Neumann (1942), Behemoth. 
Arendt, comme une des contributions pionnières à l'intelligence du phénomène nazi”. ${ }^{31}$

La teoria del bombardamento a tappeto è riconducibile all'italiano Giulio Douhet ${ }^{32}$ e venne discussa e applicata dagli statunitensi. "L'ammiraglio Chester Nimitz era dell'idea che bisognava insistere con i bombardamenti diur$\mathrm{ni}$, da grandi altezze", per distruggere a terra le forze aeree giapponesi e le industrie belliche. Al contrario, il generale Curtis LeMay proponeva "voli notturni a bassa quota, lasciando cadere non bombe dirompenti, ma bombe piccole, numerose e cariche di sostanza incendiarie" sulle città giapponesi fatte di case soprattutto in legno: e, bisogna aggiungere, abitate dalla popolazione civile. Prevalse la tesi di LeMay e, commenta Fosco Maraini, "la ferocia della guerra del Pacifico fece un notevolissimo balzo in avanti, preparando la via agli orrori di Okinawa, di Hiroshima e Nagasaki”.

È ancora Fosco Maraini - con una descrizione che può valere anche per l'analogo dramma di Dresda nel febbraio 1945 - a testimoniare le conseguenze di questi bombardamenti a tappeto su Tokyo del marzo 1945: "Nella notte ardente si calcola fossero morte dalle ottantamila alle centomila persone, quasi tutte civili; le case distrutte furono due milioni e mezzo, lasciando un milione di cittadini privi di dimora. Il furore del fuoco fu tale che si riprodussero [...] dei vortici di fiamme (tatsumaki) che risucchiavano tutto l'ossigeno dell'aria, così che migliaia di persone morirono per asfissia. Altre migliaia morirono affogate, quando cercarono di tuffarsi nelle acque dei numerosi canali e fiumi della Tokyo bassa. Fu insomma un disastro apocalittico"33

Meno apocalittici, ma comunque gravi, furono i bombardamenti sulle città italiane, soprattutto su quelle del triangolo industriale Torino-GenovaMilano, dove si concentrava l'industria bellica. ${ }^{34} \mathrm{~A}$ causa dell'inversione delle alleanze italiane dopo l'8 settembre 1943, esse furono bombardate

31 Questo giudizio è contenuto nella presentazione del già citato dossier Allemagne, Italie, Japon: les fascismes (1982), 104. Una rassegna delle idee e della fortuna del libro di Franz Neumann è in Pierre Ayçoberry (1982), Franz Neumann.

32 Cfr. il paragrafo 4.9. Un protagonista della geopolitica: Giulio Doubet e il "dominio dei cieli", in: Mario G. Losano (2011), La geopolitica del Novecento, 162-179.

33 Fosco Maraini (2001), Case, amori, universi, 599-600 (entrambe le citazioni).

34 Achille Rastelli (2000), Bombe sulla città, con ampia documentazione anche fotografica su Milano. 
dapprima dagli anglo-americani, poi dai tedeschi. Le città disposte sulle lunghissime coste italiane vennero cannoneggiate anche dal mare. Nell'immediato dopoguerra, però, l'Italia poté porre riparo alle distruzioni causate dai bombardamenti perché disponeva di più mano d'opera rispetto alla Germania, dove molti uomini mancavano all'appello perché caduti in battaglia o ancora internati nei campi di prigionia. Intervenendo come nell'industria bellica durante la guerra, ancora una volta furono le donne tedesche a dare inizio alla ricostruzione ricuperando dai cumuli delle macerie i vecchi mattoni, materia prima per la nuova edilizia: le "Trümmerfrauen" divennero un mito (anche se non indiscusso) e in varie città tedesche un monumento ricorda ancora oggi le "donne delle macerie"."35

b) L'Italia in Giappone e il Giappone in Italia: D'Annunzio e il "fratello samurai" Shimoi Harukichi

L'immagine che i giapponesi avevano dell'Italia è colta da Reto Hofmann con queste parole: "Nella cosmologia giapponese dell''Occidente', l'Italia non occupava una posizione privilegiata. L'Italia rimaneva la destinazione di artisti, di viaggiatori e d'un pugno di artisti, nei quali la letteratura inglese aveva destato l'interesse per quell'esotica penisola, patria dell'antica Roma. Invece i politici, gli economisti e gli intellettuali giapponesi si ispiravano ai loro omologhi tedeschi, britannici e francesi". ${ }^{36}$ Invertendo la prospettiva, una descrizione sintetica ma globale della visione italiana del Giappone negli anni del fascismo è contenuta in un articolo che elenca numerosi volumi sul tema e descrive le principali riviste pubblicate in Italia, tra cui "Yamato" ${ }^{37}$

35 I giorni del dopoguerra erano anche i giorni della fame: Franz Severin Berger, Christiane Holler (1994), Trümmerfrauen.

36 Reto Hofmann (2015), The Fascist Effect, 10.

37 Sergio Raimondo et al. (eds.) (2017), Bushido as allied: "Questa ricerca si propone appunto di approfondire quali fossero le espressioni culturali e di costume che risvegliavano l'interesse reciproco almeno tra intellettuali, politici e alti ufficiali delle due nazioni, considerando la limitata conoscenza che i ceti popolari potevano avere di contesti così lontani e diversi"; "La ricerca si sofferma soprattutto sulla produzione culturale in materia apparsa in Italia durante gli anni di cobelligeranza dei due paesi” (trad. it.: Il bushido alleato. Il Giappone guerriero nell'Italia fascista (1940-1943), da https:/www.giornaledistoria.net/ rubriche/frammenti-di-storia/bushido-1940-1943/). 
In Giappone il fascismo venne considerato con attenzione non solo dalle forze di destra, ma anche da "alcuni esponenti degli ideali liberali e democratici", la cui reazione al fascismo "fu spesso ingenua, se non accomodante o addirittura favorevole". Il volume di Hofmann dimostra che non solo i conservatori, ma anche i "liberals" giapponesi "mostravano il più vivo interesse per le strategie mussoliniane del potere, cercando di rafforzare il liberalismo con le nozioni fasciste di capo, di virilità e di morale".38

L'attore principale della ricezione del fascismo in Giappone fu lo scrittore e, poi, politico Shimoi Harukichi (1883-1954), che visse in Italia dal 1915 al 1933. ${ }^{39}$ D'Annunzio sosteneva che quel nome dovesse essere trascritto "Shimoi", per enfatizzarne l'esotismo; Mussolini invece voleva romanizzarne la grafia in "Scimoi": col risultato che le pubblicazioni di Shimoi figurano sotto l'una o l'altra delle due traslitterazioni. Dapprima promosse la letteratura giapponese in Italia come lettore di giapponese al Regio Istituto Orientale di Napoli. In seguito partecipò alla Prima guerra mondiale: ${ }^{40}$ ma su questa partecipazione circolano voci fantasiose che è bene chiarire. Andò al fronte non come volontario negli Arditi italiani, ma come corrispondente di vari giornali giapponesi e anche italiani ("Il Mattino" e "Il Mezzogiorno" di Napoli). Nelle giornate di Caporetto Shimoi incontrò D’Annunzio, che attraverso il comando degli Arditi "gli fece ottenere la carica di Ardito onorario e il diritto di indossare la divisa del corpo": con essa compare in varie fotografie e la indossò "anche in Giappone nelle occasioni ufficiali" 41 Agli Arditi insegnò il judo: introdusse così quest'arte marziale in Italia e nel 1934 accompagnò come interprete il suo fondatore, Jidoro Kano, nel suo viaggio in Italia.

38 Reto Hofmann (2015), The Fascist Effect, 5.

39 Harukichi Shimoi (2019), Un samurai a Fiume. La Prefazione - Harukichi Shimoi, Samurai a Fiume e profeta del fascismo in Giappone - è, credo, la prima vera e propria monografia italiana su Shimoi, 13-131. Seguono Documenti (testi di e su Shimoi, 135-283), Fotografie (287-291), Bibliografia di Harukichi Shimoi (293-300). I documenti vengono riportati con l'indicazione della fonte da cui sono tratti e l'uno di seguito all'altro, senza distinzioni tipografiche.

40 Shimoi pubblicò i suoi ricordi della Grande Guerra: Harukici Scimoi (1919), La guerra italiana. Ora anche in Harukichi Shimor (2019), Un samurai a Fiume, 140-184.

41 Notizie più dettagliate si trovano nel paragrafo: La guerra italiana vista da un giapponese, nella Prefazione di Pautasso in Haruкichi Shimor (2019), Un samurai a Fiume, 43-44. 
Le vicende personali di Shimoi si intrecciando con la complessa "questione fiumana" che travagliò l'Italia alla fine della Prima guerra mondiale. Il destino postbellico dell'area di Fiume era però poco chiaro sin dall'inizio del conflitto, quando il Patto di Londra del 1915 fissò le frontiere che l'Italia avrebbe ottenuto alla fine vittoriosa del conflitto, lasciando però aperta la questione dell'area adriatica negli articoli 4 e $5 .{ }^{42}$ Su questo punto la futura attribuzione di Fiume all'Italia venne compromessa dall'atteggiamento rinunciatario dell'Italia nelle trattative, tanto che il Presidente del consiglio Antonio Salandra scrive: "Sonnino ed io non possiamo esimerci dalla responsabilità dell'abbandono di Fiume", con particolare riferimento alla nota che avrebbe dovuto precisare nell'articolo 5 la posizione di Fiume: "Nel testo dell'accordo [di Londra] la dicitura della nota stessa, non saprei dire perché, [risultò] alquanto variata". Di qui prese inizio la complessa "questione fiumana" ${ }^{43}$

Indro Montanelli, che incontrò Shimoi in Giappone dopo la fine della Seconda guerra mondiale, lo ricorda come "un giapponese che dopo anni d'insegnamento all'Istituto Orientale di Napoli s'era talmente italianizzato che, nel 1915, s'era arruolato volontario nel nostro esercito, aveva seguito D'Annunzio a Fiume, dove aveva tenuto i contatti tra il Vate assediato e Mussolini a Milano, e poi aveva preso parte alla marcia su Roma”. Infatti Shimoi portava a Mussolini, col quale D'Annunzio era ai ferri corti, le lettere del Vate che si aprivano con le parole "T'invio, camerata assente e frigido, questo fratello samurai" ${ }^{44}$ Shimoi poteva uscire da Fiume assediata perché "lui solo, con quella faccia, poteva aggirare i posti di guardia", oppure grazie al suo passaporto, ma soprattutto per il tacito consenso del Regio Esercito: infatti D’Annunzio “se ne serviva per i suoi collegamenti con Mussolini approfittando del fatto che [il generale Enrico] Caviglia ordinava di rilasciare

42 Ferdinando Gerra (1974), L'impresa di Fiume, vol. 1: Fiume d'Italia, 13-22, con una dettagliata ricostruzione sulla formulazione del Patto di Londra e la riproduzione degli articoli sopra citati. Sull'intera "questione fiumana" si vedano i due volumi di Gerra, nell'edizione rivista del 1974.

43 Antonio Salandra (1930), L'intervento, 194-195, corsivo mio.

44 Indro Montanelli (2002), Soltanto un giornalista, 155: l'arruolamento volontario di Shimoi menzionato da Montanelli fa parte della leggenda su questo personaggio, come si è detto poco sopra. Contiene un caustico reportage sull'"ultimo Giappone giapponese che si scontrava con i primi effetti della democratizzazione voluta da Mac Arthur”, 153-156. 
il suo piccolo amico giapponese ogni volta che i carabinieri lo sorprendevano ad attraversare il confine". ${ }^{45}$

Due fotografie illustrano l'evoluzione di Shimoi: una prima lo mostra in kimono, giovane intellettuale appena arrivato a Napoli, l'altra - farsescamente inquietante - lo ritrae in divisa di Ardito, con il pugnale alla cintura e accanto a un grande ritratto di Mussolini. A questo mediatore politicoculturale Reto Hofmann dedica uno dei capitoli più incuriosenti del suo volume. ${ }^{46}$

Contagiato dalla passione di D'Annunzio per l'aviazione, nel 1919 Shimoi cercò di organizzare per il vate-soldato un volo fino a Tokyo, che però non poté essere realizzato a causa del coinvolgimento di entrambi nell'avventura di Fiume. "Fra gli stranieri che numerosi vennero a visitare Fiume in quel periodo, è da ricordare in modo particolare il poeta giapponese Haru Kici Scimoi, noto in Italia anche perché nell'estate 1919 avrebbe dovuto partecipare al progettato raid aereo Roma-Tokio con d'Annunzio e Arturo Ferrarin" ${ }^{47}$ Questo progetto si intersecò con la pubblicazione di "una prima raccolta dei discorsi pronunziati alla ringhiera romana e delle invettive stampate in un foglio di parte nazionale, tra la primavera e l'estate del 1919, quando il Comandante della Squadra di San Marco preparava il lungo volo verso l'Estremo Oriente e la rivendicazione armata di Fiume 'porta d'Oriente', fedele al suo motto guerresco di aviatore e di fante e di marinaio: donec ad metam". ${ }^{48}$

45 Antonio Spinosa (2005), D’Annunzio, 173.

46 Si veda l'intero primo capitolo: Mediator of Fascism: Shimoi Harukichi, 1915-1928, in: Reto Hofmann (2015), The Fascist Effect, 8-37. Le due fotografie di Shimoi sono in corrispondenza alla p. 1 (in divisa da Ardito) e alla p. 16 (in kimono); la prima fotografia è anche in Harukichi Shimor (2019), Un samurai a Fiume, n. 8, 291. A p. 27 è una foto del 1926 di Shimoi con Mussolini (anche in Harukichi Shimor (2019), Un samurai a Fiume, n. 9, 291). A p. 35 si trova la foto della colonna romana che Mussolini donò nel 1928 al Giappone grazie alla mediazione di Shimoi.

47 Ferdinando Gerra (1974), L'impresa di Fiume, vol. 1: Fiume d'Italia, 247: nelle pagine su Shimoi, Gerra riprende il titolo Saluto all'ospite d'Oriente con cui il discorso di D'Annunzio venne pubblicato nel 1949 (esso è riprodotto per intero in Appendice II,1,b). Anche la grafia italianizzata del nome di Shimoi è desunta dal testo di D'Annunzio.

48 Gabriele D’Annunzio (1932a), La penultima ventura, Libro primo, VII: "Con il titolo Contro uno e contro tutti fu già impressa" un'edizione, che però "apparve incompiuta ed ebbe per dure necessità un numero ristretto di esemplari". Sulle edizioni di quest'opera cfr. Appendice II.1, nota 77, p. 282. 
L'idea venne ripresa l'anno dopo dall'asso dell'aviazione Arturo Ferrarin, che a Parigi incontrò un ufficiale italiano "venuto a Parigi a comperare carte geografiche, che dovevano servire per un raid Roma-Tokio vagheggiato da Gabriele D'Annunzio e dal poeta giapponese Shimoi. Era la prima volta che sentivo parlare di questo progetto, nuovo e audace a quei tempi" ${ }^{49}$ Progetto di cui Ferrarin divenne protagonista.

Le condizioni dell'impresa sembrano oggi proibitive. Ferrarin sottrae le carte a quell'ufficiale, ma deve restituirle; ruba allora l'atlante Stieler del Ministero, ma vengono i carabinieri a ricuperarlo. Mentre un ufficiale distrae i carabinieri, Ferrarin strappa le pagine utili per il volo: è la cartografia di base per l'impresa. Iniziano così le tappe di un volo di 18.000 chilometri con un biplano monomotore (residuato della Grande Guerra) che volava in contrade dove, come "a Foochow, come a Canton, non avevano mai visto aeroplani". Quindi si atterrava dove si poteva: "A Foochow dovetti fare una sosta forzata di quattro giorni perché a Shangai si correvano le corse dei cavalli e il campo non era a disposizione per atterrare". Il primo raid RomaTokyo si concluse felicemente: l'aereo di Ferrarin, partito da Centocelle il 14 febbraio 1920, giunse a Tokyo il 30 maggio, dove "la più bella nota d'italianità era data dalle librerie, ove così spesso apparivano le opere di D'Annunzio tradotte in giapponese da farci convinti che il Comandante fosse l'autore prediletto fra le classi intellettuali del paese". ${ }^{\mathbf{5 0}}$ Con l'analogo volo in Brasile del 1928 Ferrarin concludeva l'epoca delle imprese aviatorie individuali, perché il potente Italo Balbo preferiva "le grandi crociere di massa", ben più efficaci dal punto di vista propagandistico.

Un altro volo a Tokyo ebbe luogo un anno dopo la morte di Ferrarin, nel 1942. "Un capitolo ignoto della storia della Seconda guerra mondiale" è "la pianificazione tedesca e italiana per istituire un collegamento aereo tra le potenze europee dell'Asse in Europa e l'alleato giapponese in Estremo Oriente", anche perché l'invasione dell'Unione Sovietica aveva bloccato la via siberiana al Giappone. L'unico volo da Roma a Tokyo, e ritorno, ebbe

49 Arturo Ferrarin (1929), Voli per il mondo, pubblicato dopo il volo in Brasile e ristampato nel 1942. La citazione è a p. 18. L'autore aveva già pubblicato Arturo Ferrarin (1921), Il mio volo Roma-Tokio. Una descrizione anche tecnica delle sue imprese (con bibliografia) è alla voce Ferrarin, Arturo di Giuseppe Sircana (1996), nel Dizionario Biografico degli Italiani.

50 Arturo Ferrarin (1929), Voli per il mondo, 66, 67 e 94. 
luogo dal 29 giugno al 20 luglio 1942 e fu quello italiano, perché il volo tedesco venne solo pianificato, mentre l'aereo giapponese precipitò in volo. ${ }^{51}$

Se Shimoi condivise la passione aviatoria di D'Annunzio, simmetricamente D'Annunzio, nella sua ricerca di esotismo, fu contagiato dalla poesia giapponese prima ancora di conoscere Shimoi: erano infatti gli anni in cui il Japonisme influenzava anche i pittori impressionisti. Negli articoli dedicati a temi giapponesi D’Annunzio rivela il suo debito verso le traduzioni francesi (a partire dalla trascrizione dei vocaboli: "outa" sta infatti per il giapponese "uta", o haiku) e, in particolare, verso l'opera di Edmond de Goncourt che descrive minutamente le giapponeserie di cui è colma la propria casa. ${ }^{52}$ Già fra il 1885 e il 1890 D'Annunzio aveva scritto la "Outa occidentale" secondo la metrica giapponese, seguita dai versi nipponizzanti pubblicati in occasione delle nozze Belmonte-Torlonia. ${ }^{53}$ Nel 1923 D’Annunzio sognava: "Nel Giappone nei dintorni di Kyoto abiterò un vecchio tempio di legno fra i ciliegi lievi e gli stagni coperti dai fiori del loto e i sorrisi discreti dei bonzi” ${ }^{54}$

Nella dionisiaca atmosfera di Fiume, la teatralità e il desiderio di stupire l'uditorio, tipici di D'Annunzio, favorivano gli exploits del Vate. Dopo una cena a Fiume, D'Annunzio sussurrò a Shimoi: "Alzati in piedi e, senza preoccuparti di nulla, pronuncia con voce chiara e sonora un gruppo di una decina o una ventina di parole a casaccio come konnitciwa, sayonara,

51 Peter Herde (2000), Der Japanflug, 9. In appendice, pagine in italiano del diario del generale Publio Magini, secondo pilota nel volo Roma-Tokyo-Roma (265-273).

52 Un preciso percorso del (superficiale) nipponismo dannunziano - nella favola mondana Mandarina, nella Outa occidentale, in alcune pagine del romanzo Il piacere e in varie poesie - è delineato in Mariko Muramatsu (1996), Il buon suddito del Mikado. La fonte cui D'Annunzio attinge è EdMOND DE Goncourt (1881), La maison d'un artiste.

53 Teresa Ciapparoni la Rocca (2005), Gli scrittori italiani; a p. 106 ricorda che D'Annunzio pubblicò quei versi ( 9 strofe, tutte di 5 versi col ritmo 5-7-5-7-7) in Cronaca bizantina il 14 giugno 1885 ("stessa data della Outa occidentale sul 'Fanfulla della domenica") insieme con la recensione delle traduzioni di poesie giapponesi pubblicate da Judith Gautier (figlia di Théophile, "magicien ès lettres françaises"): Judith Gautier (1884), Poëmes de la libellule.

54 D'Annunzio ricorre alla metrica giapponese (tanka di 31 more: 5-7-5-7-7) nello haiku "Outa Occidentale", in cui introduce la rima: "Guarda la Luna | tra li alberi fioriti; | e par che inviti | ad amar sotto i miti | incanti ch'ella aduna". Inoltre: "Veggo da i lidi | selvagge gru passare | con lunghi gridi | in vol triangolare | su 'l grande occhio lunare" (citazioni tratte da https://haikusparsi.wordpress.com/2015/12/13/haiku-g-dannunzio/). Cfr. anche: Mariko Muramatsu (2011), Outa occidentale di Gabriele D’Annunzio; Giulia Agostini (2015), Intrecci letterari tra Italia e Giappone. 
arigatou. Facciamo sbalordire tutti". A voce alta annunciò: "Adesso il signor Shimoi reciterà una poesia. Ascoltatela e poi io ve la tradurrò". Shimoi lo seguì in questo gioco, pronunciando parole giapponesi a caso, e "non appena io prendevo fiato facendo un'interruzione, D'Annunzio cominciava a 'tradurre' trovando sempre le parole giuste" che evocassero "la tristezza al rumore degli insetti o la nostalgia del corso d'acqua limpido in cui si specchia la luna, o la lode delle nuvole passeggere". Ammirazione generale: "Da quel momento in poi, voci sul fatto che D'Annunzio conoscesse bene il giapponese si diffusero fra quelli del governo provvisorio nonché fra i soldati dell'assedio di Fiume", anche perché D’Annunzio "ripeteva spesso il gioco di prestigio delle traduzioni giapponesi". 55

Se il Vate era seriamente sedotto dalla tradizione letteraria giapponese, il D'Annunzio nazionalista e uomo d'azione ammirava l'affermarsi del rinnovato Stato giapponese e additava quella "resurrezione asiatica" come alternativa alla subalternità italiana nella politica internazionale, come si legge nel saluto rivolto a Shimoi riportato nell'Appendice II.1, $b$. È un atteggiamento analogo a quello del bengalese Rabindranath Tagore, che incontreremo fra poco, e di alcuni intellettuali dei due Stati della penisola iberica di fronte al nuovo Giappone: per superare la decadenza si consigliava la "japonización" della Spagna e la "japonização" del Portogallo. ${ }^{56}$

In Giappone gli scritti di D’Annunzio conobbero una grande fortuna, anche se le "opere di letteratura italiana cominciarono ad apparire più tardi [dopo il 1868], in traduzioni parziali, su riviste letterarie, per andare via via aumentando all'inizio del Novecento". Tuttavia "l'autore italiano su cui si concentra la maggiore attenzione e il maggior numero di letterati giapponesi è Gabriele D'Annunzio che in breve tempo diventa un autore straniero di moda nell'ambiente letterario giapponese del primo Novecento. [... D'Annunzio,] dopo Dante e Boccaccio, fu lo scrittore italiano più letto e tradotto e l'unico autore moderno generalmente conosciuto". ${ }^{57}$ Grazie anche

55 Harukichi Shimoi (2019), Un samurai a Fiume, 247, tradotto dal testo di Harukichi Sнimor (1938), D'Annunzio no yokogao, pubblicato in commemorazione della morte di D'Annunzio avvenuta in quell'anno. Le tre parole in giapponese significano buongiorno, arrivederci e grazie.

56 Mario G. Losano (2016b), Il portoghese Wenceslau de Moraes, 142 (e la voce "japonización” nell'indice analitico); Mario G. Losano (2016c), Lo spagnolo Enrique Dupuy, 129-133 (e la voce "japonização" nell'indice analitico).

57 Marisa Di Russo (2005), Influenze e suggestioni letterarie, 176-177. Cfr. anche Maria Mimita Lamberti (1985), Giapponeserie dannunziane, 295-297. 
alle traduzioni di Shimoi, ancora oggi D'Annunzio è un autore noto in Giappone, come hanno dimostrato nel 2013 le celebrazioni a Tokyo e Kyoto per il centocinquantenario della sua nascita. ${ }^{58}$

Infine "il fratello samurai", che aveva fatto da tramite fra D'Annunzio e Mussolini ai tempi dell'impresa di Fiume, fu a fianco di Mussolini nei contatti al massimo livello con il Giappone, divenendo così il riconosciuto propagandista del fascismo nel suo paese. Un resoconto dei contatti fra Shimoi e Mussolini riferisce di una lettera di Mussolini per i bambini giapponesi:

"Oh Shimoi, [dice Mussolini] ho già cominciato il messaggio ai bambini del Giappone. Ho scritto così ...” E legge la prima facciata ancora bagnata. Poi continua a scrivere, intingendo la penna in un grosso calamaio della sua camera nel palazzo Chigi. "Shimoi, che cosa significa la parola Banzai?" - "Letteralmente diecimila anni ed è lo evviva giapponese, che augura una lunga vita. Ma ai giapponesi sarà più gradito Alalà!, che coincide meravigliosamente col grido di vittoria che lanciavano gli antichi guerrieri della Terra del Sole, battendo l'asta delle lance a terra". "Veramente?" - "Giappone e Italia sono, in tutti i punti, perfettamente fratelli". - "Ecco fatto. Il Presidente legge da capo: Ai bimbi del lontano Giappone, sia recato il saluto dei piccoli Balilla italiani che portano oggi la Camicia Nera. Malgrado la distanza, i cuori dei fanciulli virtuosi e amanti della bellezza e della Patria, si incontrano e si amano. Alalà!" -

F. to Mussolini, Roma, luglio $1923 .^{59}$

Mussolini apprese da Shimoi anche la vicenda del Byakkotai, una tragica storia di fedeltà e di morte. Nel 1868-69 la guerra Boshin oppose le forze tradizionaliste di Satsuma all'imperatore aperto all'Occidente. L'unità di riserva "Tigre Bianca", Byakkotai, del clan tradizionalista di Aizu era composta dai figli sui sedici anni dei più importanti samurai. Nel corso di una battaglia, credendo per errore che il loro signore fosse morto, venti giovanetti che si erano ritirati sulla collina di Limori si suicidarono ritualmente. Tutto il Giappone ammirava questa fedeltà estrema, anche se andava ascritta alla parte avversa all'imperatore.

Nel 1928 un matrimonio suggellò nel nuovo Giappone la riconciliazione delle due fazioni: il principe Chichibu, fratello minore dell'imperatore Hirohito, sposò la principessa Setsuko Matsudaira, nipote del signore tradizio-

58 Sulla fortuna di D’Annunzio in Giappone: MARISA Di Russo (2005), Influenze e suggestioni letterarie.

59 Harukichi Shimoi (2019), Un samurai a Fiume, 267-268, con l'indicazione della seguente fonte: "Le parole di Harukichi Shimoi sono riportate in Luigi Vicentini, Mussolini veduto dall'estero, Barion Editore, Milano 1924, pp. 115-121”. 
nalista sconfitto nella battaglia di Aizu. In Italia, Shimoi diffuse la vicenda del Byakkotai presentandola come degna dell'antica Roma. Questa vicenda impressionò Mussolini che nel 1928 inviò a Tokyo una colonna romana sormontata da un'aquila, affinché venisse collocata sulla collina di Limori come monumento per celebrare gli eroi del Byakkotai. Sullo zoccolo di marmo di Carrara si legge: "S.P.Q.R. | nel segno del littorio | Roma | madre di civiltà | con la millenaria colonna | testimone di eterna grandezza | tributa onore imperituro | alla memoria degli eroi di Biacco-tai | Anno MCMXXVIII-VI era fascista”.

Su quella collina la colonna su erge ancora oggi. Poco lontano, una semplice lapide grigia è dedicata da "Un tedesco ai giovani cavalieri di Aizu - 1935". Nulla di ufficiale, ma la croce di ferro al centro, la "Eisernes Kreuz" dei militari tedeschi, ha indotto a pensare che quella lapide sia stata posta da un addetto militare tedesco a Tokyo, ${ }^{\mathbf{6 0}}$ come tributo personale a quell'idea di fedeltà così centrale nell'ideologia nazionalsocialista (cfr. p. 27). Il mito della fedeltà è al centro delle vicende più note anche in Italia, dai "47 ronin" a Madama Butterfly.

Shimoi Harukichi predicava per il Giappone la recezione di un modello politico di tipo fascista, però adattato alle caratteristiche del Giappone, cioè fondato "sul sentimento della terra ancestrale unificata dalla monarchia". Quindi il suo fascismo non aveva bisogno di un capo carismatico, perché ogni riforma politica doveva far capo all'imperatore, "che era, e deve essere per sempre, il centro della solidarietà nazionale". ${ }^{\mathbf{1}}$ L'idea di adattare il fascismo italiano alle esigenze di altre nazioni era diffusa: per esempio, il fondatore dell'“Integralismo" brasiliano, Plínio Salgado, scriveva dall'Italia: "Ho studiato molto il fascismo. Non è esattamente il regime di cui abbiamo bisogno qui [in Brasile], ma è qualcosa di simile".62

Fedele alla sua visione di un fascismo imperiale radicatamente nipponico, Shimoi si allontanò progressivamente dalla politica quando i rapporti fra $\mathrm{i}$ tre paesi del Patto Tripartito si fecero più stretti. Anche dopo l'avventura di Fiume la vita di Shimoi fu sempre dedicata all'Italia e in essa si riflettono le complesse vicende di quegli anni. Lasciata Fiume prima del "Natale di san-

60 "Ein Deutscher den jungen Rittern von Aizu - 1935" (https://www.flickr.com/photos/ bagelmouse/9042885160/in/photostream/).

61 Reto Hofmann (2015), The Fascist Effect, 9 e 32.

62 Hélgio Trindade (1974), Integralismo, 83. 
gue", ritornò ad insegnare all'Orientale di Napoli e poi, in Giappone, all'Università di Tokyo. Scrisse una fortunata biografia di Mussolini, fondò la Società Dantesca in Giappone e tradusse D'Annunzio. Dopo l'8 settembre 1943, quando l'Italia ruppe l'alleanza con la Germania, fu avversato dagli stessi giapponesi come nemico filo-italiano. Poi, con l'occupazione americana, venne epurato, ma infine reintegrato nell'Università di Tokyo. Continuò così la sua attività di italianista fino alla morte, nel $1954 .^{63}$

\section{c) La Germania e il mito dei samurai}

Negli anni Trenta numerosi scritti mirano a promuovere in Germania la conoscenza e l'amicizia con l'alleato orientale del Terzo Reich. I punti di contatto sono naturalmente additati nell'ideologia del nazionalsocialismo e in quella del militarismo. In un manuale del 1936 sulla storia e sulla politica del Giappone, incentrato sui rapporti tra Germania e Giappone, il coautore tedesco (già ufficiale della marina) scrive che "la Germania ha particolare motivo di cercare l'amicizia del Giappone, perché i sentimenti dei due popoli sono per molti versi simili. [...] I giapponesi sentono già ora questa parentela spirituale con noi, ma i tedeschi ne sono ancora lontani". ${ }^{64}$ Il coautore giapponese, nella sua prefazione, descrive come la Germania (vista come caotica e sull'orlo del bolscevismo dopo la Prima guerra mondiale) sia stata salvata dall'"eroico ardimento" dei nazionalsocialisti "nel liberare il popolo tedesco dalla servitù straniera". Inoltre, continua, "come giapponese mi colma della massima soddisfazione il fatto che la Germania debba il suo sorprendente ringiovanimento alle stesse virtù che hanno contribuito alla gloriosa ascesa del Giappone: ardente amore di patria, illimitata capacità di sacrificio e disciplina". ${ }^{55}$

63 Sull'attività di Shimoi dopo Fiume sino alla fine della Seconda guerra mondiale: Harukichi Shimoi (2019), Un samurai a Fiume, 94-131 (nei paragrafi della Prefazione: Shimoi e la Yoga; Shimoi abbandona Fiume; Shimoi e Mussolini; Il ricordo di Indro Montanelli) e la letteratura ivi citata. A Napoli fondò la rivista Sakura; i suoi scritti su questa rivista e sul giornale "Il Mezzogiorno" sono raccolti in HaRuкichi Sнimor (2015), Buio sotto il faro.

64 Eduard von Pustau, Moriguchi Okanouye-Kurota (1936), Japan und Deutschland. La citazione è nella prefazione di Pustau, VII.

65 Paul Ostwald (1941), Deutschland und Japan, IX (entrambe le citazioni). Il volume esamina i rapporti culturali, politici ed economici tra Giappone e Germania. L'appendice è dedicata alla concezione giapponese dello Stato e dell'imperatore, 147-155. 
In quegli anni il punto di contatto tra i due popoli viene ricercato specialmente nel militarismo. ${ }^{66} \mathrm{Nel} 1943$ un autore tedesco rappresenta così il carattere del popolo giapponese: "Dal punto di vista nazionalsocialista è immediato il confronto dell'elemento militare [soldatisches Element] nel popolo tedesco e in quello giapponese". Infatti sono affini tanto "lo spirito di corpo degli ufficiali prussiani e dei samurai dell'epoca Tokugawa", quanto "il soldato politico del nazionalsocialismo e l'atteggiamento eroicamente militare del popolo giapponese del presente". ${ }^{67}$ Questa concezione permetteva di costruire un parallelismo tra i samurai e le SS, chiaramente appoggiato dal regime nazionalsocialista.

In Germania si moltiplicavano i libri sui samurai ${ }^{68}$ e Himmler promuoveva l'immagine del samurai come modello per le SS, di cui era il Reichsführer. "In questa semplice logica, - commenta un odierno germanista statunitense, - Himmler simultaneamente legittima se stesso e il suo gruppo elitario come storicamente necessari e ne accresce il prestigio e la fama collegandolo ai samurai, tradizionalmente ammirati o, almeno, temuti". ${ }^{69}$ A questo fine Himmler fece pubblicare della casa editrice del partito nazionalsocialista una serie di articoli, raccolti poi in un volumetto diffuso in centomila copie, che già nel titolo sancisce questo stretto parallelismo - I Samurai. Cavalieri dell'Impero con onore e fedeltà: il motto delle SS era infatti "Il mio onore si chiama fedeltà", "Meine Ehre heißt Treue" - e vi premise questa prefazione:

Non è un caso che "Das Schwarze Korps", il giornale delle Schutzstaffel di Adolf Hitler, abbia pubblicato la serie di articoli "I Samurai, Cavalieri d'Onore e Fedeltà".

66 Walter Donat (1943a), Deutschland und Japan. Sempre in Walter Donat et al. (1943), Das Reich und Japan cfr. anche: Отто MossDorf (1943), Der soldatische Charakter; WalteR Donat (1943b), Der deutsche und japanische Reichsgedanke; Albrecht von Urach (1943), Die Neuordungsprobleme Japans und Deutschlands.

67 Walter Donat (1943a), Deutschland und Japan, 9-10. "Il soldatisches Element costituisce un collegamento particolarmente forte nell'atteggiamento spirituale del Giappone e della Germania”, Отto Koellreutter (1940), Das politische Gesicht Japans, 23.

68 Thomas Pekar (2008), Held und Samurai (riporta una lista di autori dell'epoca nazionalsocialista, 438, nota 7); SePp Linhart (2005), Das heroische Japan (non tutti gli autori citati erano nazisti, però erano da loro citati o usati). Cfr. infine il monumentale Hans-JoAchim von Bieber (2014), SS und Samurai, che analizza i rapporti culturali nippo-tedeschi dal 1914 (bibliografia, 1173-1249).

69 Bill Maltarich (2005), Samurai und Supermen, 156. 
Le leggi secondo le quali i popoli divengono grandi valgono per tutti, così come le leggi secondo le quali i popoli decadono. È bene per noi prendere sempre in considerazione, per imparare, la vita di altri popoli.

Con questa breve storia dei Samurai vogliamo richiamare alla memoria qualcosa da lungo tempo dimenticata: che già in tempi antichi questo popolo dell'Estremo Oriente aveva le stesse leggi dell'onore come avevano i nostri padri in un passato troppo presto distrutto e che, per lo più, sono minoranze del massimo valore quelle che attribuiscono a un popolo una vita eterna secondo una concezione terrena. Possa essere questo il significato della presente pubblicazione, e in questo spirito possano leggerlo in molti, in particolare gli uomini delle SS.

H. Himmler - Reichsführer $S^{70}$

Questo astratto parallelismo fra élites è però al servizio d'una concreta geopolitica di potenza: "Il sorprendente parallelismo della storia dei nostri due popoli ora alleati sfocia in un futuro con obiettivi e compiti assai simili: la pacificazione e la guida di vasti spazi vitali popolati da una pluralità di genti. Anche i futuri problemi dell'ordine nuovo in Germania e in Giappone saranno all'insegna della grandezza e della responsabilità dei nostri popoli e delle nostre ideologie". ${ }^{71}$ È un accenno alla geopolitica tedesca e alla "Sfera di Co-prosperità della Grande Asia Orientale”, cioè al colonialismo giapponese sul continente asiatico, su cui si tornerà tra poco.

Uno dei più affermati (e nefasti) giuristi nazisti, Otto Koellreutter, pubblicò le sue impressioni di un anno trascorso in Giappone (il 1939) come professore visitante. ${ }^{72}$ Tra le sue fonti indica i volumi Japan und die Japaner, del fondatore della geopolitica Karl Haushofer, e Yamato della svizzera Lily Abegg (ideologicamente neutra). ${ }^{73}$ Il libro di Koellreutter comprende cinque capitoli, ${ }^{74}$ di cui viene qui considerato soltanto il quinto, Japan und

70 Heinz Corazza (1944), Die Samurai.

71 Walter Donat (1943a), Deutschland und Japan, 14.

72 Otto Koellreutter (1940), Das politische Gesicht Japans. Le citazioni sono tratte dalla prima edizione. La Einleitung (7-9) della prima edizione non è più presente nella seconda del 1943. Non ho potuto trovare: Otтo Koellreutter (1939), National Socialism and Japan.

73 Отto Koellreutter (1940), Das politische Gesicht Japans, 7 avverte che può "indicare come realmente informativi soltanto" i seguenti due volumi: Karl Haushofer (1923), Japan und die Japaner; Lily Abegg (1936), Yamato. Su questa autrice, cfr. Mario G. Losano (2016c), Lo spagnolo Enrique Dupuy, 115, nota 159. Su Haushofer, cfr. infra, nota 79 e 103.

74 I capitoli si intitolano Il paese, Il popolo, Lo Stato, L'Impero, Il Giappone e la Germania: questi temi sono trattati dal punto di vista politico. 
Deutschland. Anche per Koellreutter "un forte elemento naturale d'attrazione fra i due popoli è l'elemento militare che vive in loro". ${ }^{75}$ Invece il mondo economico del Giappone subisce "una forte influenza americana" ed è ancora legato "al vecchio pensiero liberale", benché stia ormai andando verso "un'economia controllata dallo Stato" ${ }^{76} \mathrm{Ma}$ i punti di contatto e il progressivo avvicinamento non devono trarre in inganno: "I circoli ufficiali rifiutano di includere il Giappone nell'ambito degli Stati fascisti" ${ }^{77}$ Questa differenziazione era condivisa da Shimoi, come si è visto.

Anche il Giappone aveva conosciuto una crisi dei partiti, frutto del modello americano recepito dai tempi dell'epoca Meiji. In seguito, allo scioglimento dei partiti si era accompagnata l'istituzione del Consiglio Nazionale del Presidente del Consiglio Konoe. Quest'ultimo il 28 agosto 1940 aveva dichiarato che "il Giappone procede nel suo compito di creare un nuovo ordine in Asia Orientale," 78 cioè nel creare il "Grande Spazio" teorizzato da Haushofer. ${ }^{79} \mathrm{Nel}$ prendere le decisioni e nell'appianare le divergenze "secondo il principio di base della politica giapponese c'è solo l'obbedienza incondizionata nell'adempimento degli ordini dell'Imperatore". ${ }^{80}$ Come i tedeschi, anche i giapponesi si sentono un "Volk ohne Raum", un "popolo senza spazio", tema ricorrente della geopolitica espansionistica. ${ }^{\mathbf{8 1}}$

75 Otтo Koellreutter (1940), Das politische Gesicht Japans, 61: "das lebendige soldatische Element”.

76 Отto Koellreutter (1940), Das politische Gesicht Japans, 62.

77 Отto Koellreutter (1940), Das politische Gesicht Japans, 62.

78 Citato da Отto Koellreutter (1941), Der heutige Staatsaufbau Japans, 17. Questo opuscolo venne pubblicato nella collana "Schriften für Politik und Auslandskunde" (della casa editrice Junker und Dünnhaupt), collana che può oggi essere considerata una summa delle posizioni nazionalsocialiste sui più vari temi interni e internazionali.

79 Christian W. Spang (2013), Karl Haushofer und Japan; in particolare: Der deutsche Einfluss auf die Entstehung der Geopolitik in Japan, 480-546, il capitolo Der Einfluss der Geopolitik auf Theorie und Praxis der japanischen Expansion, 547-711 e la vasta bibliografia di testi anche in giapponese: Quellen- und Literaturverzeichnis, 747-937. Inoltre: CHRISTIAN W. SPANG (2000), Karl Haushofer und die Geopolitik in Japan.

80 Отto Koellreutter (1941), Der heutige Staatsaufbau Japans, 19.

81 Otto Koellneutter (1941), Der heutige Staatsaufbau Japans, 21-22: Koellreutter ricorda che l'esercito giapponese è composto prevalentemente da persone di origine contadina, che "conoscono per esperienza diretta la miseranda condizione del contadino giapponese dovuta al problema di un popolo senza spazio (Volk obne Raum)". Nella Germania nazionalsocialista il tema del "popolo senza spazio" era così sentito da divenire anche il titolo di un ponderoso romanzo di quegli anni (con una tiratura di 215.000 esemplari): 
Per questo essi ora mirano alla "creazione di un ordine nuovo in Asia Orientale" in cui Giappone, Cina e Manciukuò divengano "un unico spazio vitale ed economico sotto lo guida del Giappone, che fonda questa sua pretesa sulla sua posizione di finora unico Stato d'ordine (Ordnungsstaat) in Asia Orientale"; infatti "la conclusione del Patto Tripartito con la Germania e l'Italia dà al Giappone libertà d'azione in Asia Orientale e gli rende possibile continuare non soltanto l'espansione sull'area continentale cinese, ma anche verso Sud".82

In questa espansione il Giappone si presenta come forza anticolonialista: ${ }^{83}$ avversaria cioè del colonialismo bianco o, meglio, anglo-francese. Gli alleati del Patto Tripartito appoggiavano questa posizione, che Albrecht von Urach riassume così: "Il governo giapponese sottolinea sempre che, nel Nuovo Ordine dell'Asia orientale, la nuova struttura politica non deve ricorrere ai metodi di sfruttamento coloniale delle potenze occidentali. I popoli dell'Asia orientale devono godere delle loro ricchezze, dei loro tesori minerari e dei frutti del loro lavoro. Il ricavato del lavoro e delle risorse dei popoli dell'Asia orientale non deve più finire nelle mani di pochi plutocrati stranieri nella City di Londra o a Wall Street" ${ }^{\mathbf{4}}$

La speranza con cui anche l'India guardava al Giappone moderno è sintetizzata in un discorso del bengalese Rabindranath Tagore (1861-1941), premio Nobel per la letteratura del 1913. Per lui, il Giappone moderno ha fatto comprendere all'Asia "che abbiamo vita ed energia dentro di noi: per raggiungerle dobbiamo solo eliminare la crosta morta che le ricopre”. Il Giappone moderno costituisce non solo il modello per ridestare l'Asia dal suo letargo secolare, ma anche il correttivo della materialistica civiltà occidentale "che vuole invadere il mondo con l'esclusività con cui prolifica la gramigna”, mentre invece l'Asia orientale ha elaborato una civiltà "non predatrice né meccanicamente efficiente, bensì spirituale e fondata su variopinte

Hans Grimm (1932), Volk obne Raum, dove naturalmente il popolo senza spazio è quello tedesco.

82 Otto Koellreutter (1941), Der heutige Staatsaufbau Japans, 26.

83 Cfr. nel $\mathbb{\$} 9$ le pagine dedicate ai libri di testo giapponesi, infra, p. 135.

84 Albrecht von Urach (1943), Die Neuordungsprobleme Japans und Deutschlands, 199. Il principe Albrecht von Urach (1903-1969) soggiornò in Giappone e scrisse due libri di grande successo: Albrecht von Urach (1940), Ostasien (con 35 fotografie delle zone di guerra); Albrecht von Urach (1942), Das Geheimnis japanischer Kraft (tiratura di 50.000 esemplari). Nazista convinto, dopo la guerra fu arrestato ma non condannato. 
relazioni". "Il Giappone moderno è stata la prima nazione dell'Asia che si è posta il compito di infrangere le barriere e di affrontare il mondo, infondendo la speranza nei cuori di tutta l'Asia. [...] Ringraziamo il Paese del Sol Levante e ricordiamogli solennemente che deve compiere la missione dell'Oriente: infondere la linfa dell'umanità più completa nel cuore della civiltà moderna”. Infatti “il Giappone ha importato dall'Occidente il nutrimento, ma non la propria natura e, quindi, non deve perdersi in una fusione con l'armamentario scientificista acquisito dall'Occidente, convertendosi in una macchina estranea a sé stesso". Tagore ritiene che il Giappone abbia intrapreso questa via: "Non v'è modo migliore per valutare l'immensa eterogeneità dell'era moderna, il cui unico elemento unificatore è l'utilità, che ponendola a confronto con la dignità e con l'occulto potere della reticente bellezza del Giappone”. Da questo confronto, durante la Prima guerra mondiale, nascevano le speranze sul futuro del Giappone: "Sono appena tornato dal Giappone, dove ho esortato questa giovane nazione a schierarsi con gli ideali superiori dell'umanità e a non seguire mai le orme dell'Occidente adottando come religione quell'egoismo organizzato che è il Nazionalismo". ${ }^{85}$ Purtroppo il Giappone, negli anni in cui fece parte del Patto Tripartito, non seguì la via indicata da Tagore e adottò anche verso l'Asia una politica aggressiva, creando la "Sfera di Co-prosperità della Grande Asia Orientale”.

La colonizzazione giapponese assumeva anche forme estreme che invadevano persino l'ambito privato. In Corea, ad esempio, si decretò la "nipponizzazione dei nomi di famiglia, per imporre ai coreani il sistema famigliare giapponese; l'obbligo dell'uso del giapponese non solo in pubblico, ma anche in famiglia; l'obbligo della visita ai templi, delle preghiere giornaliere in direzione del palazzo imperiale giapponese, della recita del giuramento di sudditanza rivolto al Tenno nelle cerimonie scolastiche e d'altro genere, in cui poliziotti o funzionari obbligavano i coreani a recitare a memoria il giuramento; la mobilitazione per il lavoro nelle miniere ecc., cui i coreani

85 Cito e traduco da: Rabindranath Tagore (2012a), Nacionalismo, che non indica su quale edizione dell'originale si fonda; le citazioni nel testo provengono da Rabindranath TAgore (2012b), El nacionalismo en Japón. Non ho potuto consultare né Rabindranath TAgore (1917), Nationalism, né l'edizione italiana: Rabindranath Tagore (1923), Nazionalismo. Il volume contiene tre discorsi letterari sul nazionalismo rispettivamente in Giappone, in Occidente e in India. 
erano in generale trascinati contro la loro volontà; la mobilitazione come soldati giapponesi [...]; infine, la consegna di riso e grano, imposta anche obbligando a consegnare le provviste destinate al proprio consumo" ${ }^{86}$

Koellreutter ritorna sulla costituzione giapponese del 1889 e sulla figura dell'imperatore, concludendo che "la particolare struttura della costituzione del 1889 spiega come sia stato possibile realizzare senza ostacoli e in poco tempo il profondo cambiamento che essa ha conosciuto ai nostri giorni". ${ }^{87}$ Benché quella costituzione avesse preso forma sotto l'influsso del consigliere tedesco Hermann Roesler, con gli anni le concezioni giuspubblicistiche dei due Stati erano divenute inconciliabili: per esempio, il dibattito tedesco "sulla validità o sulla decadenza della costituzione di Weimar dopo la presa di potere di Hitler nel 1933 non può neppure sorgere nel pensiero pubblicistico giapponese" e, a sostegno della sua tesi, Koellreutter cita il costituzionalista Shinichi Fujii, secondo cui "la fede del popolo giapponese si fonda sulla convinzione dell'intramontabilità del suo Stato". Come ogni fede, la si può accettare o no: ma, continua Fujii, "se un europeo rifiuta come irrilevante questa fede, lo fa soltanto perché non ha mai creduto all'intramontabilità del proprio Stato". 88

A grandi linee, questi erano gli elementi comuni addotti per spiegare il legame tra gli Stati del Patto Tripartito. Nel 1942 esso veniva così presentato secondo la prospettiva dei suoi ideatori: "Il Patto Tripartito che ci lega al Giappone non è soltanto una costruzione politica come le passate alleanze, che perseguivano un unico fine e poi si scioglievano. Berlino-Roma-Tokyo è lo spirituale programma mondiale dei giovani popoli d'ordine che mostra al mondo la via verso un futuro migliore, andando oltre il sistema mondiale delle alleanze finalizzate di modello anglosassone, andando oltre la strutturazione imperialistico-occidentale dell'economia mondiale secondo il mo-

86 Tonomura Masaru (2015), Die Erinnerung an die Kolonien, 11. Traduzione di una conferenza che sintetizza il ricordo dell'epoca coloniale in Giappone dalla fine della guerra fino ad oggi e i contrapposti sentimenti che questa memoria suscita ancora oggi in Giappone.

87 Отto Koellreutter (1944), Die politische Entwicklung des heutigen Japans, 48. Il titolo di copertina sopra citato differisce da quello del frontespizio: Die politische Entwicklung Japans.

88 Otto Koellreutter (1944), Die politische Entwicklung des heutigen Japans, 35. Sul parallelo (solo parzialmente possibile) con la Germania nazionalsocialista si veda il capitolo Entstehung und Wesen der japanischen Verfassung, 32-48. 
dello anglosassone, e superando anche lo straripante internazionalismo della follìa bolscevica mirante alla conquista del mondo" ${ }^{89}$

Non sono molti gli studi che paragonino tutti e tre gli Stati del Patto Tripartito, anche a causa delle difficoltà linguistiche che presenta la documentazione giapponese per gli studiosi degli altri due Stati, solo in parte superabile con le numerose pubblicazioni in inglese. La documentazione tedesca è la più dettagliata, anche perché la presenza della Germania in Giappone, sin dall'apertura all'Occidente, fu ben maggiore di quella italiana. La documentazione italiana è più frammentaria e puntuale. Una sintesi equilibrata fra i tre Stati è contenuta in un volume collettivo sulle "culture del ricordo", cioè sul modo in cui i tre Stati hanno elaborato gli anni delle dittature. ${ }^{90}$ Personalmente, forse a causa dei miei percorsi di lettura, ho l'impressione che i rapporti nippotedeschi siano legati al sentimento di comunità, mentre quelli nippo-italiani sono soprattutto rapporti fra individui.

La convergenza dei tre Stati del Patto Tripartito e le sue conseguenze postbelliche sono oggetto di vari studi. Si è già visto l'interesse della Germania nazionalsocialista per il Giappone militarista in pagine condizionate da quel preciso taglio ideologico. Da un'angolatura democratica lo stesso tema è stato affrontato anche nel dopoguerra. Con il titolo Allemagne, Italie, Japon: les fascismes, la rivista "Le débat" ha pubblicato nel 1982 un dossier di quattro articoli, ${ }^{91}$ in cui prevalgono però gli aspetti specifici all'interno di ciascuno dei tre Stati più che un confronto tra $\mathrm{i}$ fenomeni in essi paralleli.

Infine un elemento comune ai tre Stati dell'Asse è individuato da Paul Brooker nel "fraternalismo". Egli esamina il "tentativo da parte dei titolari del potere statale di instillare in una società industrializzata una forma pura di quel tipo di solidarietà che normalmente si incontra in società con una

89 Albrecht von Urach (1942), Das Geheimnis japanischer Kraft, 94. La copertina spiega il "mistero della forza giapponese" (che è il titolo del libro): dietro la testa di un moderno soldato giapponese con elmetto e baionetta si staglia la sagoma del samurai classico.

90 Christoph Cornelissen et al. (eds.) (2003), Erinnerungskulturen. Il termine 'Erinnerungskulturen' (culture del ricordo) è divenuto in tedesco un sinonimo di 'Vergangenheitsbewältigung' (superamento del passato).

91 Allemagne, Italie, Japon: les fascismes (1982), 105-177: Masao Maruyama (1982), Théorie et psychologie; Saul Friedländer (1982), De l'antisémitisme à l'extermination; Tim Mason (1982), Banalisation du nazisme?; Jean-Luc Pouthier (1982), National-syndicalisme et totalitarisme. 
divisione del lavoro inesistente o primitiva".92 Il modello cui Brooker si richiama esplicitamente è la "solidarietà meccanica" di Émile Durkheim e la "conscience collective" che ne scaturisce. Questa solidarietà meccanica viene raggiunta attraverso un indottrinamento capillare, esaminato a fondo per ciascuno dei tre Stati. Brooker offre, nell'Appendice IV della sua opera, una Check-List of Identifying Fraternalist Organizations, confrontando per esempio i non fraternalisti boy scouts con la fraternalistica Hitlerjugend. ${ }^{93}$

\section{L'internamento degli "alien enemies" durante la guerra}

Dopo lo scoppio della guerra, un problema che si pose in termini diversi per ognuno degli Stati belligeranti fu quello dell'internamento dei cittadini di uno Stato divenuto nemico, anche se essi non avevano commesso alcun specifico reato. Gli Stati Uniti - uno Stato di immigrati - allo scoppio della guerra dovette prendere misure contro i giapponesi, gli italiani e i tedeschi che si trovavano nel suo territorio. Essi erano infatti divenuti "stranieri nemici" in base all'Alien Enemies Act del 1798. Secondo valutazioni spesso discordanti, gli stranieri nemici dovevano essere circa 92.000 giapponesi, 315.000 tedeschi, e 695.000 italiani. Benché quello degli italiani fosse il gruppo più numeroso, ne vennero internati poco più di 200 , mentre la maggioranza degli internati era composta da giapponesi e tedeschi. ${ }^{94}$

Nel 1942 gli Stati Uniti istituirono la War Relocation Authority per raccogliere in campi di internamento gli stranieri nemici. In questi campi la situazione era difficile, ma umana anche se spartana: non si trattava né di Gulag né di Lager. Questa Authority cessò la sua attività nel 1946, sei mesi dopo la chiusura dell'ultimo centro di raccolta, a Tule Lake. ${ }^{95}$

Vennero internati circa 100.000 giapponesi. Nel 1976, sotto la pressione del "Redress Movement" dei nippo-statunitensi, il presidente Gerald Ford

92 Paul Brooker (1991), The Faces of Fraternalism, 2.

93 Paul Brooker (1991), The Faces of Fraternalism, 333.

94 Le fonti indicano cifre discordanti. Secondo il Dipartimento di Giustizia gli stranieri nemici erano in tutto 1.100.000; questa e le cifre riportate nel testo vennero pubblicate dal "New York Times" del 31 dicembre 1941.

95 Per ulteriori informazioni si vedano, nella Kodansha Encyclopedia of Japan (1983) le voci (di Roger Daniels): War Relocation Authority; Japanese American, Wartime Relocation of; Wartime Relocation Centers; War Victims Relief Law, 1942. 
riconobbe l'ingiustizia dell'internamento e nel 1988 il Congresso approvò un indennizzo per ogni sopravvissuto all'internamento, cui seguirono altri provvedimenti analoghi. ${ }^{96}$ Infatti i giapponesi negli USA, se da un lato erano considerati Alien Enemies, dall'altro erano anche stimati per la loro lealtà: "I nippo-americani, compresi quelli delle Hawaii, furono considerati tra fra i più coraggiosi soldati americani al fronte in Italia durante la Seconda guerra mondiale", nonostante il fatto che molte famiglia nippo-americane, diversamente da quelle tedesco-americane e italo-americane, "soffrissero di un trattamento discriminatorio dovuto al trasferimento nei campi di concentramento".97

Più complessa la situazione degli italiani allo scoppio della guerra contro gli Stati Uniti, nel 1941. Vennero internati non solo i cittadini italiani che si trovavano momentaneamente in territorio americano, ma anche gli italoamericani immigrati residenti da tempo o nati negli Stati Uniti, però ancora senza cittadinanza americana. ${ }^{98}$ Infine, la comunità tedesca negli Stati Uniti aveva già conosciuto l'internamento durante la Prima guerra mondiale. Con la nuova guerra, l'internamento colpì anche circa 4500 tedeschi provenienti da quindici paesi dell'America Latina e sospettati di attività antiamericane: tutti vennero internati negli Stati Uniti. ${ }^{99}$

In Europa, per gli italiani la situazione si rivelò particolarmente complicata dopo l'8 settembre 1943, con la divisione dell'Italia in due parti, l'una ancora alleata con la Germania nazionalsocialista e l'altra passata dalla parte degli anglo-americani. Divenuti così nemici degli ex alleati, nelle zone controllate dai tedeschi molti italiani divennero prigionieri di guerra, mentre altri furono dichiarati "Internati Militari Italiani" (IMI), una figura ibrida non protetta dalle norme internazionali sui prigionieri di guerra, sulla quale si tornerà a p. 196 ss.

Anche il Giappone creò dei campi di concentramento per gli italiani non collaborazionisti: su di essi il nipponista italiano Fosco Maraini, internato a

96 Cherstin M. Lyon (2012), Prisons and Patriots; Greg Robinson (2009), A Tragedy of Democracy.

97 Keirchi Takeuchi (2004), La politica dell'identità nazionale, 222, nota 17.

98 Lawrence Di Stasi (2004), Una Storia Segreta; U.S. Department of Justice (2001, novembre), Report to the Congress of the United States.

99 Тімотну J. Holian (1996), The German-Americans and World War II e il monumentale Don Heinrich Tolzmann (ed.) (1995-1998), German-Americans in the World Wars. 
Nagoya con la moglie e le tre figlie dal 1943 al $1945,{ }^{100}$ ci ha lasciato una testimonianza di intenso fascino letterario e di spigliata vivacità documentaria: "Immaginarsi venticinque latini sprovveduti, procrastinatori, nuvolai, buttati come giocattoli nelle mani della polizia più burocratica dell'universo!" (p. 555 s.); e poi: "Senza notizie, senza posta, soli con i loro pensieri, con la loro fame appena un poco sopita, il gruppetto viveva fuori dal mondo, in una sorta di strano limbo bianco" (p. 590). Infatti "distanze smisurate, invalicabili, li separavano da qualsiasi congrega d'amici. Fughe? Sarebbe stata assoluta follia pensare a movimenti non autorizzati in un Paese dove un occidentale si distingue a mezzo miglio di distanza, in un paese compattamente unito, simile a un blocco tetragono di cemento. Era un po' come sentirsi sepolti da una frana dentro una miniera" (p. 566).

Dacia Maraini, che aveva sette anni al momento dell'internamento, ricorda quest'esperienza in vari suoi scritti e l'ha così riassunta in un'intervista:

Nel 1943 il Giappone fece un patto con la Germania e con l'Italia. Stando alle regole del patto, la polizia giapponese chiese a tutti gli italiani che stavano allora in Giappone di firmare un'adesione alla Repubblica di Salò. Aggiungendo che chi non avesse firmato sarebbe stato considerato un traditore e un nemico. Mio padre e mia madre, interrogati separatamente, rifiutarono di firmare. Ci considerarono subito nemici e prigionieri di guerra. Prima costretti in casa e poi trasportati in un campo a Nagoya, chiamato Tempaku, dove rimanemmo due anni, fino alla fine della guerra. Non era un campo di sterminio ma le condizioni erano durissime: ci davano talmente poco da mangiare che in capo a pochi mesi eravamo tutti malati di beri-beri, di scorbuto, di anemia perniciosa. Eravamo infestati dai parassiti. E poi c'erano le bombe ogni giorno e i terremoti. Insomma, è stata un'esperienza durissima, da cui pensavo di non uscire viva. Per fortuna è finita la guerra, altrimenti non so quanto avremmo resistito. ${ }^{101}$

100 Fosco Maraini (2001), Case, amori, universi. Le citazioni nel testo sono tratte da quest'ultima edizione; il volume era stato però pubblicato nel 1999. L'epoca e le vicende dell'internamento sono descritte nelle pp. 529-634. In generale: W. Puck Brecher (2017), Honored and dishonored guests (con un paragrafo sulla questione ebraica).

101 La citazione è tratta da Silvia Gigli (2017, aprile), Dacia Maraini. Cfr. anche Dacia Maraini (2001), La nave per Kobe; Dacia Maraini (1993), Bagheria; Dacia Maraini (1978), Mangiami pure; Dacia Maraini (1973), E tu chi eri?. 


\section{Dalla geopolitica dei "Grandi Spazi" al "Patto Tripartito"}

Il 27 settembre 1940 Germania, Italia e Giappone sottoscrissero a Berlino l'aggressivo "Patto Tripartito", ${ }^{\mathbf{1 0 2}}$ che consacrava l'espansione territoriale dei tre Stati secondo la concezione dei "Grandi Spazi” fondata dal geopolitico Karl Haushofer. ${ }^{103}$ In una delle sei note segrete scambiate fra il governo tedesco e quello giapponese in occasione della firma di quel trattato, l'ambasciatore tedesco a Tokyo, Eugen Ott, precisava che "si sta aprendo una fase nuova e decisiva della storia mondiale", in cui "gli Stati firmatari assumevano la guida nella creazione di un ordine nuovo nella Grande Asia Orientale e in Europa”. In particolare, la Germania assicurava l'assistenza al Giappone in caso di aggressione "da parte di uno Stato finora non coinvolto nella guerra europea o nel conflitto con la Cina" e manifestava la sua convinzione "che l'Italia, naturalmente d'accordo con la Germania e il Giappone, presterà il suo aiuto e la sua collaborazione riguardo ai punti enunciati". ${ }^{104}$

Tuttavia l'Italia venne tenuta in disparte perché, "quando [il Ministro degli Esteri Yosuke] Matsuoka decise di trasformare il protocollo segreto in uno scambio di note, decise anche di scambiare le note solo bilateralmente, cioè tra Germania e Giappone. Entrambe le potenze diffidavano infatti della riservatezza degli italiani. Si nota qui un parallelismo con il Patto Anticomintern [del 1936], ai cui protocolli segreti l'Italia non partecipò". 105

102 Questo trattato riconosceva ai tre Stati firmatari il diritto di realizzare i "Grandi Spazi" (Großräume) teorizzati dalla geopolitica delle dittature: "Art. 1. Il Giappone riconosce e rispetta il compito direttivo dell'Italia e della Germania per lo stabilimento di un nuovo ordine in Europa. - Art. 2. L'Italia e la Germania riconoscono e rispettano il compito direttivo del Giappone nello stabilimento di un nuovo ordine nella più grande Asia orientale" (https://team557.wordpress.com/tag/testo-patto-tripartito/).

103 Su questo tema cfr. i capitoli: Geopolitica e diritto: $i$ "Grandi Spazi" di Haushofer e gli "imperi" di Schmitt (50-74), e I Grandi spazi dall'Europa delle dittature al Giappone del militarismo (75-113), in: Mario G. Losano (2011), La geopolitica del Novecento.

104 I sei memorandum segreti scambiati fra Germania e Giappone sono pubblicati in [Johanna M. Menzel] (1957), Der geheime deutsch-japanische Notenaustausch. Il passo citato è contenuto nel Documento 1 (Tokyo, 27 settembre 1940), Deutsche Botschafter Eugen Otto a Yosuke Matsuoka, Ministro degli Esteri del Giappone, 190-191.

105 [Johanna M. Menzel] (1957), Der geheime deutsch-japanische Notenaustausch, 191, nota 34. 
Il Patto Tripartito corrispondeva però al desiderio italiano di affermarsi nella politica mondiale. In Italia era divenuto quasi un luogo comune personificare quel patto con le iniziali delle tre capitali - Roma, Berlino e Tokyo - ed esclamare di fronte alle difficoltà belliche: "Ci pensa Ro-Ber-To" ${ }^{106} \mathrm{Ma}$ "Ro-Ber-To" uscì dalla guerra con le ossa rotte: i regimi dittatoriali italiano e tedesco scomparvero, mentre il regime militarista giapponese subì una drastica trasformazione. Questi mutamenti istituzionali trovarono espressione nelle nuove costituzioni con cui i tre Stati dell'ex "Patto Tripartito" affrontarono il dopoguerra: per volere dei vincitori, come essi erano stati uniti nel provocare la guerra, così dovevano essere uniti anche nell'impegnarsi a conservare la pace postbellica.

Poiché la Seconda guerra mondiale era nata dalla politica bellicistica dei tre Stati di quel Patto, le potenze vincitrici vollero che ciascuna delle costituzioni postbelliche dei tre Stati contenesse un articolo che rifiutasse esplicitamente la guerra come strumento per la risoluzione dei conflitti internazionali. Questa disposizione non era una novità assoluta, perché era presente già nel Patto Briand-Kellogg del 1929: "Articolo I: Le alte parti contraenti dichiarano solennemente in nome dei loro popoli rispettivi di condannare il ricorso alla guerra per la risoluzione delle divergenze internazionali e di rinunziare a usarne come strumento di politica nazionale nelle loro relazioni reciproche. - Articolo II: Le alte parti contraenti riconoscono che il regolamento o la risoluzione di tutte le divergenze o conflitti di qualunque natura o di qualunque origine possano essere, che avessero a nascere tra di loro, non dovrà mai essere cercato se non con mezzi pacifici”. Anche la costituzione della Repubblica spagnola del 1931 stabiliva nell'articolo 6: "España renuncia a la guerra como instrumento de política nacional". Il rifiuto della guerra venne ripreso da numerose altre costituzioni. ${ }^{107}$

106 "Ro-Ber-To", benché sconfitto, non è stato dimenticato e se ne trova menzione anche in opere recenti; per esempio: "Alla vigilia della Seconda guerra mondiale [Ettore] Conti è nominato ambasciatore straordinario e ministro plenipotenziario" per il Giappone e il Manciukuò. "La sua è una missione prettamente commerciale, volta ad aprire una triangolazione tra lo Stato fantoccio del Manciukuò, il Giappone e l'Italia. L'asse Ro-Ber-To, che si formalizzerà con il patto tripartito del 27 settembre 1940 tra Italia, Germania e Giappone, era alle prove generali”, Stefano Righi (2014), La città illuminata, 110.

107 Il testo commentato del Patto Briand-Kellogg è in http://www.studiperlapace.it/view news_html?news_id=briandkellog. Il testo della costituzione repubblicana spagnola è in: http://www1.icsi.berkeley.edu/ chema/republica/constitucion.html. Un elenco di altre co- 
È però curioso notare come, già durante la sanguinosa Prima guerra mondiale, il sentimento di stanchezza per la guerra si facesse strada persino negli stessi Futuristi, il cui manifesto del 1918 proclamava "la guerra, sola igiene del mondo", ma il cui fondatore Filippo Tommaso Marinetti, prima ancora della fine di quel terribile conflitto, proponeva di "mantenere l'esercito e la marina in efficienza fino allo smembramento dell'impero austroungarico. Poi, diminuire gli effettivi al minimo, preparando invece numerosissimi quadri di ufficiali con rapide istruzioni"; in breve, "preparazione di una completa mobilitazione industriale (armi e munizioni) da realizzarsi in caso di guerra contemporaneamente alla mobilitazione militare. Tutti pronti, con la minore spesa, per una eventuale guerra o una eventuale rivoluzione.". 108

D’Annunzio statista, con il sindacalista rivoluzionario Alceste de Ambris, preparò nel 1920 la Carta del Carnaro, una costituzione destinata a reggere la Libera Città di Fiume: "il nostro Statuto novissimo, documento di armonia latina", nel quale le forti utopie sociali di De Ambris si univano al lirismo visionario dannunziano: "Il cardo bolscèvico fioriva in rosa italiana" ${ }^{109}$ Nonostante le divergenze tra i Futuristi e il movimento fiumano, la Carta del Carnaro prevede un depotenziamento militare analogo a quello prefigurato da Marinetti: "In tempo di pace e di sicurezza, la reggenza non mantiene l'esercito armato; ma tutta la nazione resta armata, nei modi prescritti dall'apposita legge, e allena con sagace sobrietà le sue forze di terra e di mare. Lo stretto servizio è limitato ai periodi d'istruzione e ai casi di guerra guerreggiata o di pericolo prossimo". ${ }^{110}$ Tanto Marinetti quanto D'Annunzio sembrano aver presente soltanto la guerra difensiva, e non quella d'aggressione.

stituzioni con clausole pacifiste è in Giuseppe De Vergottini (2004), Guerra e Costituzione, 28-29.

108 Filippo Tommaso Marinetti, Manifesto del partito futurista italiano, 1918, art. 7 (http:// www.irre.toscana.it/futurismo/opere/manifmarinetti.htm con tutti i manifesti di Marinetti); anche in appendice a Giordano Bruno Guerri (2009), Filippo Tommaso Marinetti.

109 Gabriele D’Annunzio (1949c), Al legionario Alceste De Ambris, 215: "Era quella che chiamavamo 'la quinta stagione del mondo'. Il cardo bolscèvico fioriva in rosa italiana. Noi ponevamo nella rivendicazione dell'anima il fondamento dell'ordine nuovo. Nasceva in quel giorno il nostro Statuto novissimo, documento di armonia latina, pegno d'amore all'amore" (Dal Garda, 11 maggio 1921).

110 Sul testo predisposto da de Ambris e riveduto da D'Annunzio (http://www.dircost.unito.it/ cs/docs/carnaro1920.htm): Gabriele D'Annunzio (2009), La Carta del Carnaro; Gugliemo Negri, Silvano Simoni (1990), Le Costituzioni inattuate. 
Questa loro utopia prefigura l'ordinamento d'una ventina di piccoli Stati indipendenti che hanno del tutto abolito le forze armate, ovvero che non hanno più eserciti permanenti: valga per tutti l'esempio della Costa Rica, che dal $1^{\circ}$ dicembre 1948 non ha più un esercito "come istituzione permanente". 111

Abbandonando queste utopiche e seducenti anticipazioni, le pagine seguenti si concentreranno sugli articoli "pacifisti" delle costituzioni dei tre Stati dell'ex Patto Tripartito. Naturalmente il solo diritto non basta a regolare la pace e la guerra. Il vasto dibattito sul pacifismo giuridico cerca di individuare il modo migliore in cui il diritto potrebbe impedire o limitare la guerra; tuttavia l'attuazione delle sue proposte giuridico-filosofiche si scontra troppo spesso con la realtà politica ed economica. Le pagine seguenti si occuperanno soprattutto del conflitto tra realtà e diritto, cioè della difficoltà di tradurre in pratica le teorie del pacifismo giuridico. ${ }^{\mathbf{1 1 2}}$

Quanto il costituzionalista De Vergottini scrive per l'Italia vale anche per la Germania e per il Giappone, e non soltanto per l'immediato dopoguerra: ciascuno di questi tre Stati è una media potenza che "si trova inclusa in un sistema di alleanze egemonizzato da una sola grande potenza ed è per definizione confinata in una situazione di sovranità limitata che trae origine dagli esiti disastrosi del secondo conflitto mondiale, da cui è uscita come Stato debellato che si è visto imporre un trattato di pace intercorso tra vincitori e quindi non discutibile"; quindi gli articoli pacifisti "hanno alle spalle una congenita limitazione di sovranità e non certo libere scelte degli organi costituzionali”. ${ }^{113}$

La Costituzione giapponese è la prima delle tre costituzioni degli Stati dell'ex Patto Tripartito ad essere approvata nel 1947 e a contenere il rifiuto della guerra. L'articolo 9 è cos̀ formulato: "Capitolo II - Rinuncia alla guerra.

111 Costituzione di Costa Rica del 7 novembre 1949, art. 12: "Se proscribe el Ejército como institución permanente. Para la vigilancia y conservación del orden público, habrá las fuerzas de policía necesarias. Sólo por convenio continental o para la defensa nacional podrán organizarse fuerzas militares; unas y otras estarán siempre subordinadas al poder civil; no podrán deliberar, ni hacer manifestaciones o declaraciones en forma individual o colectiva".

112 Una sintesi di queste teorie è in José Antonio García SaÉz (2019), Pacifismo Jurídico; José Antonio García SaÉz, (2016), Kelsen versus Morgenthau, così come altri scritti su Hans Joachim Morgenthau.

113 Giuseppe De Vergottini (2004), Guerra e Costituzione, 9-10. 
Articolo 9. [I] Nella sincera aspirazione alla pace internazionale, basata sulla giustizia e l'ordine, il Popolo Giapponese rinuncia per sempre alla Guerra quale sovrano diritto della nazione e alla minaccia o all'uso della forza come mezzo per la risoluzione delle dispute internazionali. - [II] Allo scopo di raggiungere l'obiettivo di cui al precedente paragrafo, le forze di terra, di mare ed aeree, così come le altre potenzialità belliche, non saranno mai mantenute. Non sarà riconosciuto il diritto dello Stato alla guerra." ${ }^{114}$ Questo articolo verrà analizzato nel Cap. II.

Nella Costituzione italiana del 1948 il rifiuto (anzi, il "ripudio") della guerra è contenuto nell'articolo 11 , che non ha titolo ma è uno dei dodici "princìpi fondamentali” della Repubblica: “Art. 11. L'Italia ripudia la guerra come strumento di offesa alla libertà degli altri popoli e come mezzo di risoluzione delle controversie internazionali; consente, in condizioni di parità con gli altri Stati, alle limitazioni di sovranità necessarie ad un ordinamento che assicuri la pace e la giustizia fra le Nazioni; promuove e favorisce le organizzazioni internazionali rivolte a tale scopo." ${ }^{115}$ Questo articolo verrà analizzato nel Cap. III.

Nella Legge Fondamentale della Germania Federale del 1949 (estesa nel 1990 alla Germania unificata ${ }^{116}$ ) questo principio è contenuto nell'artico-

114 Il testo in italiano della costituzione giapponese del 1947 si trova nel sito: http:// www.filodiritto.com/articoli/2006/08/costituzione-del-giappone/ - Il testo in inglese della Costituzione giapponese si trova nel sito del Primo Ministro del Giappone: http:// japan.kantei.go.jp/constitution_and_government_of_japan/constitution_e.html - Il testo in francese della Costituzione giapponese si trova nel sito: http://mjp.univ-perp.fr/constit/ jp1946.htm.

115 Il testo della Costituzione italiana si trova nel sito della Camera dei Deputati, oltre che in italiano, anche nelle traduzioni in francese e inglese: http://leg16.camera.it/38?conoscerelacamera=28. Le costituzioni storiche italiane sono reperibili in: http://dircost.di.unito.it/ index.shtml.

116 Il 3 ottobre 1990 i cinque Länder che erano confluiti nella DDR entrarono far parte della Repubblica Federale Tedesca, cosicché l'unificazione della Germania fu in realtà l'inclusione della DDR nella RFT, con la conseguente estensione della Legge Fondamentale di quest'ultima ai suoi nuovi territori. Il testo in tedesco della Legge Fondamentale, anche nelle traduzioni in inglese e in francese, si trova nel sito del Parlamento tedesco: https:// www.bundestag.de/grundgesetz. Il testo in tedesco della Legge Fondamentale e delle costituzioni storiche tedesche si trova nel sito: www.verfassungen.de (che contiene anche, in tedesco e spesso in inglese, le costituzioni vigenti di molti Stati del mondo). Il testo in italiano della Legge Fondamentale si trova nel sito: http://dircost.di.unito.it/cs/paesi/ger mania.shtml. Infine, il testo tedesco con a fronte la traduzione italiana è anche in http:// www.consiglioveneto.it/crvportal/BancheDati/costituzioni/de/zGermania_sin.pdf. 
lo 26: “(1) Atti che siano idonei e posti in essere con l'intento di turbare la pacifica convivenza dei popoli, e specificamente di preparare una guerra d'aggressione, sono anticostituzionali. Essi devono essere colpiti da pena. (2) Armi destinate alla condotta di una guerra possono essere fabbricate, trasportate e messe in circolazione solo con l'autorizzazione del governo federale. Una legge federale regola i particolari". ${ }^{117}$ Questo articolo verrà analizzato nel Cap. IV.

Un così radicale rifiuto della guerra non dipendeva soltanto dall'imposizione dei vincitori, ma era condiviso dalla quasi totalità della popolazione. Chi si lasciava alle spalle il peggior conflitto della storia mondiale - soprattutto fra gli europei - viveva nel terrore d'una nuova guerra, alimentato tanto dalla crescente tensione fra i due blocchi (cioè della Guerra fredda che già nell'immediato dopoguerra contrapponeva gli ex-alleati), quanto dalla possibilità che una nuova guerra si trasformasse in un olocausto atomico: il recente ricordo di Hiroshima e Nagasaki era vivo e presente nella mente di tutti, e non solo in Giappone.

Questo diffuso stato d'animo si riflette nel passo di Natalia Ginzburg che, nel descrivere la Torino del dopoguerra, constata la stanchezza di vivere dello scrittore Cesare Pavese: egli "continuò ad aver paura della guerra, anche dopo che la guerra era da gran tempo finita: come, del resto, noi tutti. Perché questo ci accadde, che appena finita la guerra ricominciammo subito ad aver paura di una nuova guerra, e a pensarci sempre". ${ }^{118}$ E nel 1951 una forte donna della Resistenza, ormai prossima a morire, si accomiatava così dalla figlia: "In quest'epoca di bomba atomica è fortunato ancora, si può dire, chi ha la possibilità di scrivere una lettera prima di morire". ${ }^{119}$ In questa prospettiva da incubo, ogni garanzia di pace era quindi ben accetta.

117 "Art. 9. (1) Handlungen, die geeignet sind und in der Absicht vorgenommen werden, das friedliche Zusammenleben der Völker zu stören, insbesondere die Führung eines Angriffskrieges vorzubereiten, sind verfassungswidrig. Sie sind unter Strafe zu stellen. - (2) Zur Kriegsführung bestimmte Waffen dürfen nur mit Genehmigung der Bundesregierung hergestellt, befördert und in Verkehr gebracht werden. Das Nähere regelt ein Bundesgesetz".

118 Natalia Ginzburg (1963), Lessico famigliare, 205; Natalia Ginzburg (2016), Le piccole virtù, 299.

119 Marina Sereni (1955), I giorni della nostra vita, 225; lettera alla figlia del 2 novembre 1951. Cfr. infra, nota 86, p. 287. 
Decenni dopo, le grandi guerre classiche si frammentarono in conflitti dai nomi eufemisticamente allarmanti ${ }^{\mathbf{1 2 0}}$ e le norme costituzionali pacifiste postbelliche dovettero essere interpretate alla luce delle missioni dette "di pace” che si andarono moltiplicando dagli anni Novanta. Un punto cruciale fu senza dubbio la guerra del Kosovo del 1996-99, perché gli Stati della Nato intervennero senza la previa autorizzazione della Nazioni Unite. Il dibattito suscitato da quell'intervento rivela esemplarmente le difficoltà di coordinare il dettato costituzionale pacifista con gli interventi militari definiti "umanitari” o simili. Un volume raccoglie su questo tema i pareri di vari membri dell'Associazione Italiana dei Costituzionalisti (riunitisi nel 1999 su richiesta dell'Associazione dei Costituzionalisti Iugoslavi) e quindici documenti internazionali riferentisi alla guerra del Kosovo. ${ }^{121}$ Si potrebbe organizzare un volume equivalente per ciascuno degli interventi armati successivi, come in parte è stato fatto in un crescente numero di libri e di articoli, e come purtroppo si dovrà continuare a fare nel prossimo futuro.

E così queste pagine - che si sono aperte descrivendo quali discussioni avessero accompagnato oltre mezzo secolo fa la genesi delle norme costituzionali pacifiste - prendono forma in un tempo in cui fervono le discussioni su come aggirare, modificare o forse abrogare quelle stesse norme costituzionali pacifiste.

\section{Il Giappone nel dopoguerra}

Dopo più di due secoli di chiusura ai rapporti esterni, nel 1868 - data di inizio dell'era Meiji - il Giappone recepì la cultura occidentale e, in particolare, un sistema giuridico di tipo europeo continentale. Nel 1889 approvò una costituzione ispirata al modello tedesco (nota come "Costituzione Meiji”), ${ }^{\mathbf{1 2 2}}$ divenendo una monarchia costituzionale sui generis, perché al suo vertice si trovava un imperatore di origine divina.

120 Mario G. Losano (2017b), Guerre ibride, omicidi mirati, droni.

121 Mario Dogliani, Stefano Sicardi (eds.) (1999), Diritti umani e uso della forza. Il volume si riferisce alla crisi del Kosovo del 1998-1999 (con una sintesi degli eventi, 95-96 in nota) e contiene 15 saggi di costituzionalisti italiani, con un'appendice di 15 documenti internazionali (139-287), in buona parte in inglese e francese.

122 Sul suo autore, Roesler, cfr. supra, nota 1, p. 7 e Mario G. Losano (1984), La recezione dei modelli giuridici europei. Sulla recezione del sistema giuridico europeo-continentale: MARIO G. Losano (1973), Tre consiglieri giuridici europei; e la letteratura citata supra, nota 1, p. 7. 
Lo storico Akio Nakai riassume in tre elementi le principali differenze del Giappone rispetto agli altri due Stati dell'ex Patto Tripartito. Anzitutto, il suolo del Giappone non era mai stato occupato da truppe straniere né prima né durante la guerra. Inoltre andava sottolineata la "natura essenzialmente diversa del nazionalsocialismo e del militarismo giapponese": "I militaristi e nazionalisti giapponesi non erano un partito politico e non era sottoposti ad un'unica guida dittatoriale". Infine, "Hitler era un dittatore in un sistema totalitario senza limiti al suo potere. L'imperatore invece [...] doveva muoversi nell'ambito di una monarchia costituzionale. La responsabilità governativa ricadeva per intero sul Consiglio dei Ministri"; quindi "l'imperatore doveva accettare senza riserva le decisioni governative. Ciò rese possibile, nel processo di Tokyo contro i crimini di guerra, che i generali e i politici sotto accusa si assumessero la totale responsabilità, salvando così l'istituzione imperiale". 123

Dopo la sconfitta del Giappone nella Seconda guerra mondiale, con la "Potsdam Declaration" del 1945 (cfr. p. 235) gli Usa, l'Inghilterra e la Cina nazionalista dettarono le condizioni per la sua resa incondizionata, imponendogli anche di democratizzare la propria struttura politica e quindi di approvare una costituzione democratica. Questa radicale modificazione socio-politica avvenne durante l'occupazione militare del Giappone da parte degli Stati Uniti: la prima occupazione straniera nella storia millenaria dell'Impero del Sol Levante.

In un primo tempo gli Alleati avevano pensato a una divisione del Giappone analoga a quella, poi effettivamente realizzata, che si progettava per la Germania. Alla Russia sarebbe stata attribuita - oltre alla Corea del Nord, l'isola di Sakhalin e le isole Kurili - anche l'isola di Hokkaido, la più nordica delle quattro maggiori isole dell'arcipelago giapponese. Tuttavia la crescente diffidenza americana verso i sovietici portò invece a un'occupazione solo americana del Giappone, con un limitato apporto inglese.

Alla fine della guerra, ognuno dei tre Stati dell'ex Patto Tripartito si trovava in una situazione diversa e, di conseguenza, gli Stati Uniti come principale potenza vincitrice adottarono politiche diverse nei riguardi di ciascuno di essi.

L'Italia era divisa in due dalla guerra civile: il "Regno del Sud" firmò la resa di Cassibile l'8 settembre 1943, mentre la "Repubblica Sociale Italiana”,

123 Акıо NakaI (1988), Die "Entmilitarisierung” Japans, 19. 
al Centro-Nord, firmò la resa di Caserta il 29 aprile 1945. La Germania capitolò l'8 maggio 1945, dopo che i combattimenti su tutto il suo territorio avevano distrutto la quasi totalità delle infrastrutture. Venne occupata per intero dagli Alleati che ne assunsero il governo diretto e, della sua precedente struttura statale, rimase operativa solo l'amministrazione locale, che però rispondeva direttamente agli occupanti. Il Giappone capitolò il 14 agosto 1945 (data della resa del Giappone e della fine della Seconda guerra mondiale) ma, nonostante il bombardamento atomico, la sua struttura statale era intatta e il suo territorio non era stato invaso dai vincitori nel corso della guerra. Nell'occupazione seguita alla capitolazione, gli occupanti statunitensi decisero pertanto di affiancare l'amministrazione preesistente per avviarla alla democrazia parlamentare. ${ }^{\mathbf{1 2 4}}$ Inoltre le difficoltà incontrate in Germania nel coordinare fra loro gli Alleati vincitori contribuirono alla decisione degli Stati Uniti di governare da soli il Giappone occupato.

L'occupazione americana del Giappone durò dal 14 agosto 1945 fino al 28 aprile 1952, quando entrò in vigore il trattato di pace di San Francisco. ${ }^{\mathbf{1 2 5}}$ Inizialmente regnava la massima diffidenza reciproca, anche perché la propaganda di entrambe le parti aveva sparso voci terrificanti sulla ferocia del nemico. "Gli americani si facevano vedere soltanto in piccoli gruppi, armatissimi e sospettosi all'estremo", mentre "la maggior parte dei giapponesi si teneva chiusa in casa, almeno dove le case erano rimaste in piedi, in attesa delle temute violenze". Poi venne "un momento magico", quando entrambe le parti "capirono l'inutilità grottesca di tanto terrore fondato sul nulla, su pure voci, sul sentito dire". "Ebbe così inizio una delle occupazioni più calme e di maggior successo di tutta la storia moderna. In altri paesi dei dissidenti si sono subito buttati alla macchia, hanno cercato di continuare la guerra con la guerriglia. In Giappone nulla. Il Tenno aveva parlato. Ipse dixit. Bastava”. ${ }^{\mathbf{1 2 6}}$ Fosco Maraini fu testimone di questo clima, quando a Tokyo, dinanzi all'edificio del quartier generale alleato, "vide il generale MacArthur uscire dal portone a mezzogiorno, da solo, senza scorta, per recarsi a colazione, tra due

124 "La scelta americana di assumere un ruolo dominante in Giappone, sin dall'inizio, mirava a prevenire la competizione tra Alleati ed era il risultato dell'esperienza fatta in precedenza in Italia”, Ilaria Poggiolini (2004), Resa incondizionata e istituto monarchico, 44.

125 Michael Schaller (1985), The American Occupation of Japan; Eiji Takemae (2002), The Allied Occupation of Japan; Yoneyuki Sugita (2003), Pitfall or Panacea. Questi volumi sono stati ristampati più volte.

126 Fosco Maraini (2001), Case, amori, universi, 629-630. 
file di giapponesi d'ogni età e sembianza, che gli battevano fragorosamente le mani. Dicevano: 'È il nuovo shogun dagli occhi azzurri (aoi-me no shogun). Evviva!'” 127 In seguito non mancarono contrasti tra la potenza occupante e il governo giapponese, ma senza violenze individuali. Per comprendere questo atteggiamento "bisogna scrutare l'animo giapponese nei suoi più intimi recessi", avverte Fosco Maraini in un'altra sua opera; per esso "non esistono schema ideale da una parte e bruta materia dall'altra. Dunque la vita non ha mai torto. MacArthur vincendo aveva dimostrato coi fatti la superiorità d'un certo ordine, e questo di conseguenza aboliva il precedente"; insomma "si combatte come demoni per vincere, ma poi si onora il campione chiunque esso sia”. ${ }^{128}$ Questa attitudine permise nel dopoguerra la convivenza di due concezioni della vita profondamente differenti.

Nel 1947 il Giappone doveva passare dal militarismo prebellico a un regime democratico di tipo occidentale. Il modello di costituzione - più imposto che proposto dagli americani - venne accettato quasi integralmente e la sua struttura può essere così sintetizzata: il principio americano dei "checks and balances" si amalgama con quello inglese della "supremacy of the legislature"; i diritti fondamentali sono garantiti nella costituzione; il parlamento detiene costituzionalmente il controllo sul governo; è sancita la separazione dei tre poteri; la Camera Alta della nobiltà viene trasformata in un senato elettivo. In particolare, i princìpi costituzionali più innovativi rispetto alla tradizione giapponese portano la firma di MacArthur: la posizione dell'imperatore come simbolo dello Stato senza potere politico e il celebre articolo 9, che abolisce il diritto alla guerra e al riarmo.". ${ }^{129}$

Due temi vanno sottolineati: nell'intera storia del Giappone, l'occupazione americana è l'unico caso di potere straniero insediatosi sul territorio giapponese (la presenza britannica postbellica fu un'appendice di quella americana, ebbe scarsa rilevanza e durò poco ${ }^{\mathbf{1 3 0}}$ ); l'Unione Sovietica rimase

127 Fosco Maraini (2001), Case, amori, universi, 621. Questa sensazione di sicurezza è confermata dalle osservazioni del giudice statunitense Oppler, cfr. infra, p. 175.

128 Fosco Maraini (1988), Ore giapponesi, 105.

129 Акіо Nakai (1988), Die "Entmilitarisierung" Japans, 272-273.

130 "The official British Commonwealth Occupation Force (BCOF), composed of Australian, British, Indian and New Zealand personnel, was deployed in Japan on February 21, 1946. While U.S. forces were responsible for overall military government, BCOF was responsible for supervising demilitarization and the disposal of Japan's war industries. BCOF was 
esclusa dall'occupazione del Giappone, a differenza di quanto avvenne in Germania. ${ }^{131}$ Proprio in funzione antisovietica furono gli stessi Stati Uniti a spingere il Giappone a iniziare un limitato riarmo, preoccupati per la situazione cinese (che portò nel 1949 alla fondazione della Repubblica Popolare Cinese) e per la guerra di Corea che, scoppiata nel 1950, obbligò gli Stati Uniti a ritirare molti militari dal Giappone per impiegarli in Corea.

Sull'occupazione americana in Giappone è disponibile un'ampia documentazione originale, che va però valutata con cautela: quei "documenti sono un'istantanea scattata dalla prospettiva della burocrazia occupante, perciò si presentano ovviamente come notizie di successi e vanno quindi valutate di conseguenza. Il loro valore consiste nella descrizione di fatti e dettagli che invitano a un confronto con quelli sull'occupazione della Germania, poiché rispecchiano l'autostima dei funzionari e lo spirito missionario della potenza occupante". ${ }^{132}$

Oggettivamente l'occupazione americana in Giappone (e quella alleata in Germania) possono essere considerate "un esperimento eccezionalmente riuscito di educazione d'una nazione alla democrazia." ${ }^{133}$ Per il Giappone va ricordato che già una volta, nell'epoca Meiji (1868-1912), esso aveva recepito con successo modelli sociali e giuridici più imposti che importati dall'Occidente.

È particolarmente significativo che il $1^{\circ}$ gennaio 1946 l'imperatore Hirohito abbia rivolto alla nazione un appello che si richiamava esplicitamente al rinnovamento ottocentesco dell'epoca Meiji, ricollegando quel lontano messaggio al rinnovamento che in quei giorni attendeva il Giappone sconfitto:

also responsible for occupation of several western prefectures and had its headquarters at Kure. At its peak, the force numbered about 40,000 personnel. During 1947, BCOF began to decrease its activities in Japan, and it was officially wound up in 1951" (Occupation of Japan, New World Encyclopedia).

131 Un confronto tra l'occupazione statunitense in Giappone e in Germania è in Robert Wolfe (ed.) (1984), Americans as Proconsuls.

132 Wolfgang Benz (1978), Amerikanische Besatzungsherrschaft, 270. L'introduzione ai documenti (265-279) è in tedesco, mentre tutti i documenti sono riportati nell'originale inglese.

133 Wolfgang Benz (1978), Amerikanische Besatzungsherrschaft, 268. 
Oggi entriamo nel nuovo anno. In passato, all'inizio dell'era Meiji, l'Imperatore Meiji emanò un proclama in cinque articoli:

1. Si devono indire riunioni nella maggior misura possibile, e tutti gli affari di Stato devono essere decisi in accordo con l'opinione pubblica. 2. Tutti, in posizione elevata o inferiore, di comune accordo devono fare del loro meglio in tutti gli affari di Stato. 3. È necessario che a tutti, dai funzionari civili e militari fino al semplice popolo, venga data la possibilità di realizzare i propri obiettivi, in modo che non siano insoddisfatti. 4. Devono essere abbandonate tutte le cattive abitudini e tutto deve fondarsi sui princìpi giusti e al di sopra delle parti del Cielo e della Terra. 5. Si devono acquisire conoscenze da ogni parte del mondo, per favorire così lo Stato imperiale dalle sue fondamenta.

Queste parole dell'imperatore sono giuste e al di sopra delle parti: che mai si potrebbe aggiungere ad esse? Noi vogliamo quindi prestare nuovamente questo giuramento, nell'intento di aprire una nuova via al bene del paese. Seguendo i suoi princìi dobbiamo dismettere le vecchie cattive abitudini e far valere l'opinione del popolo; funzionari e popolo devono votarsi unanimemente a un atteggiamento pacifico, costruire una cultura della ricca abbondanza e così elevare le condizioni di vita del popolo e costruire un nuovo Giappone.

Le distruzioni che le nostre città hanno sofferto durante la guerra, le sofferenze di chi ha subito la guerra, la paralisi del commercio e delle imprese, la mancanza di cibo e il crescente numero dei disoccupati sono effettivamente disperanti. Se il nostro popolo prende in considerazione la situazione attuale e insieme è saldamente deciso a muoversi pacificamente verso la civiltà, senza dubbio si deve aprire un sereno futuro non solo per il nostro popolo, ma per l'intera umanità.

L'amore per la famiglia e l'amore per la patria sono caratteristiche particolarmente rilevanti nel nostro paese. Ora è necessario sviluppare appieno questo atteggiamento, mettendolo con spirito di sacrificio al servizio dell'amore dell'umanità. ${ }^{134}$

Questo autorevole (e condiviso) richiamo alla tradizione contribuisce a spiegare perché i tentativi di modificare in senso radicalmente democratico la Costituzione Meiji si siano scontrati con l'opposizione dei politici giapponesi, obbligando il generale Douglas MacArthur - comandante in capo delle forze di occupazione - ad affidare a una commissione di americani la stesura di un progetto costituzionale conforme alla "Potsdam Declaration". Forse il proconsole americano non chiedeva di meglio. Forse anche l'articolo sulla rinuncia alla guerra era stato suggerito dal primo ministro Kijuro Shidehara: ma questa informazione è controversa. Comunque, richiamandosi all'art. 73 della Costituzione Meiji, la nuova costituzione venne approvata come emen-

134 Mia traduzione dal testo in tedesco: Нiroнito (1964), Neujahrsbotschaft des Tenno, 23. Anche l'art. 9 viene ricollegato alla tradizione giapponese: cfr. infra, Appendice I,1,4. 
damento della precedente ${ }^{135}$ dalle due camere elettive nell'ottobre 1946, ed entrò in vigore il 3 maggio 1947. Di fatto essa rappresenta però "una grande frattura piuttosto che una continuità tra la vecchia e la nuova costituzione", perché il potere sovrano è passato da un monarca teocratico al popolo. ${ }^{\mathbf{1 3 6}}$

In Giappone funzionò il modello di governo militare sperimentato anche in Italia (cfr. p. 56). MacArthur stesso afferma che - mentre in Germania alla fine della guerra l'amministrazione si dissolse o fu necessario procedere alle epurazioni ("all government agencies disintegrated, or had to be purged") in Giappone le strutture autoctone continuarono a funzionare, sotto la supervisione del "Supreme Command of the Allied Powers" (SCAP). ${ }^{\mathbf{1 3 7}}$

Wilhelm Röhl riassume così il percorso legislativo sul limitato riarmo giapponese, cioè l'iter di quella che definisce "la normativa più violentemente combattuta della costituzione giapponese":

Luglio 1950: MacArthur chiede l'istituzione di una "Police-Reserve";

Agosto 1950: Il decreto governativo n. 260 istituisce la Police Reserve (composta dapprima da 75.000 uomini, poi da 110.000);

Settembre 1951: Trattato nippo-americano sulla sicurezza;

Febbraio 1952: Accordo amministrativo nippo-americano sul trattato per la sicurezza;

Luglio 1952: Legge sull'Ente per la Sicurezza; scioglimento della Police Reserve; istituzione di un corpo militare e di un corpo di polizia;

Luglio 1953: Inizio delle trattative nippo-americane sull'aiuto MSA (Mutual Security Act);

Marzo 1954: Trattato MSA;

Giugno 1954: Leggi sull'istituzione di un Ufficio per la Difesa e per le Forze di Autodifesa. ${ }^{138}$

135 The Constitution of the Empire of Japan (1889): "Article 73. When it has become necessary in future to amend the provisions of the present Constitution, a project to the effect shall be submitted to the Imperial Diet by Imperial Order. (2) In the above case, neither House can open the debate, unless not less than two-thirds of the whole number of Members are present, and no amendment can be passed, unless a majority of not less than two-thirds of the Members present is obtained", Нiroвumi Iто (1889), Commentaries on the Constitution.

136 La rivista Aus Politik und Zeitgeschichte ha dedicato un fascicolo alla costituzione giapponese: Nobushige Ugai, Herbert Zachert (eds.) (1964), Die japanische Verfassung. Esso contiene: Nobushige Ugai, Herbert Zachert (1964), Ideal und Realität. Il testo giapponese, tradotto da un nipponista tedesco, contiene un'introduzione (3-7; da cui è tratta la citazione, p. 3) ai testi della costituzione Meiji del 1889 (8-12) e della costituzione del 1946 (13-23), oltre al già citato messaggio imperiale del 1946 (cfr. supra, nota 134).

137 Douglas MacArthur (1966), Reports of General MacArthur, 193-194.

138 Questa sintesi dell'analisi della costituzione giapponese si trova in WiLhelm Röhl (1963), Die japanische Verfassung, 57. Il Mutual Security Act (MSA) del 1951-61 (titolo ufficiale: 
La Germania seguiva con diretto interesse le vicende iniziali del riarmo giapponese perché, "osservando il riarmo del Giappone, ormai in pieno sviluppo, si riscontrano molti parallelismi con il previsto riarmo della Germania: li si nota nelle difficoltà che là si sono già manifestate e che qui sono da attendersi" ${ }^{139}$ Per mantenere l'ordine interno - fermo restando il divieto del riarmo per il Giappone (e per la Germania) - venne creato un corpo di polizia, la National Police Reserve, che divenne il nucleo delle future forze armate giapponesi. ${ }^{140}$ La descrizione delle caratteristiche di quelle unità di polizia, consolidatesi nei primi due anni della loro esistenza, portano l'autore a concludere: "Tanto nella sua organizzazione e struttura, quanto nel suo armamento e istruzione, questo corpo rivela un carattere simile a quello di un esercito" ${ }^{141}$ Infatti "esperti giapponesi ritengono che la potenza di fuoco di una divisione della National Police Reserve sia oggi nove volte maggiore di quella di una divisione del precedente esercito giapponese." ${ }^{\mathbf{1 4 2}}$

Il crescente timore anticomunista, tipico della situazione postbellica, emerge nella descrizione delle difficoltà di reclutamento dei ranghi inferiori di quel corpo di polizia: i giovani giapponesi erano o pacifisti (atteggiamento che "può essere ricondotto soprattutto alla 'rieducazione' americana" dopo il 1945) o conservatori (mentre "il liberalismo non ha fatto presa nelle scuole superiori"). "La stragrande maggioranza degli studenti giapponesi appartiene a gruppi socialisti o comunisti”, o comunque influenzati dal marxismo: quindi in Giappone "l'introduzione della leva obbligatoria in questi anni critici sarebbe la via più certa per costruire un'Armata Rossa giapponese." ${ }^{\mathbf{1 4 3}}$ Insomma, il blocco del riarmo previsto dell'art. 9 poteva anche svolgere un'utile funzione anticomunista.

Per rendere il riarmo compatibile con l'art. 9, la "National Police Reserve" venne trasformata nel 1954 in "Japan Self-Defense Force", in considerazione

"An Act to maintain the security and promote the foreign policy and provide for the general welfare of the United States by furnishing assistance to friendly nations in the interest of international peace and security") sostituì il Piano Marshall (o, meglio, "European Recovery Progam”, ERP, del 1946). Entrambe le leggi miravano al contenimento del comunismo.

139 Nota redazionale che precede, a p.41, l'articolo di Karl Ferdinand Reichel (1953), Japans "Nationale Polizei-Reserve".

140 La stessa evoluzione si verificò in entrambe le Germanie, cfr. nota 30, p. 181.

141 Karl Ferdinand Reichel (1953), Japans "Nationale Polizei-Reserve" (1), 41.

142 Karl Ferdinand Reichel (1953), Japans "Nationale Polizei-Reserve" (1), 42.

143 Karl Ferdinand Reichel (1953), Japans "Nationale Polizei-Reserve" (2), 32. 
del fatto che l'art. 9 proibisce la guerra d'aggressione, ma non quella di difesa. Da allora le Forze di Auto-difesa non hanno cambiato nome, ma sono andate costantemente ampliandosi. Poiché nel frattempo il Giappone è giunto ad essere una delle prime dieci potenze mondiali per le spese militari, importanti forze politiche spingono oggi per una revisione dell'art. 9, anche in considerazione della mutata situazione internazionale e della crescente partecipazione del Giappone alle missioni internazionali.

Il 2018 registra in Giappone un intensificarsi di misure militari: "Il rafforzamento delle Forze d'Autodifesa, le forze armate de facto del Giappone, mira in primo luogo a proteggere le isole periferiche come le Senkaku (oggetto di un contenzioso territoriale con la Cina, che le chiama Diaoyu). Nell'aprile [2018] le Forze d'Autodifesa avevano presentato la loro prima brigata anfibia d'intervento rapido: un'unità di 2100 militari addestrata da consiglieri statunitensi secondo il modello dei marines e destinata alla riconquista di isole occupate" ${ }^{\mathbf{1 4 4}}$

La Cina è infatti un vicino preoccupante: "Il tonnellaggio di navi militari varate dalla Cina dal 2014 supera quello di Francia, Germania, Italia, Spagna, India, Corea del Sud e Taiwan messe insieme. Non solo, Pechino sta sviluppando la sua capacità di affondare navi nemiche: e i missili antinave, da terra, aria e mare sono più avanzati di quelli americani. Inoltre, la Cina sta rafforzando la presenza esattamente lungo la 'Sealine', la tratta marittima globale: Senkaku, le isole Spratly al largo delle Filippine, Sri Lanka, dove ha preso in leasing un porto per cento anni, Gibuti, dove costruisce la sua prima base militare africana. Infrastrutture per lo più civili, per ora". L'attivismo cinese lungo la "Sealine" (la via dell'approvvigionamento petrolifero tra Stati arabi e Giappone) ha provocato un incidente: "Qui tutto è cambiato nel 2010, quando un grosso peschereccio cinese speronò la Guardia costiera giapponese vicino alle isole Senkaku. Una provocazione, una collisione 'volontaria' che provocò arresti, uno spettacolare processo al capitano cinese a Tokyo, tensione alle stelle”. E anche a una contromisura militare del Giappone: nell'isola di Ishigaki ("il lembo più meridionale del Giappone") "si sta costruendo una base giapponese 'di autodifesa' aerea, e saranno dislocati 500-600 soldati”, perché "Ishigaki controlla le Senka$\mathrm{ku}$, cinque scogli disabitati, terra di albatros" ${ }^{\mathbf{1 4 5}}$

144 Philippe Mesmer (2018, 18 dicembre), Le Japon muscle les capacités offensives.

145 Reportage dell'inviata sul posto Mara Gergolet (2019, 7 gennaio), Tokyo prepara l'isolatrincea. Questo attivismo è parte del piano geopolitico cinese delle "nuove via della seta" 
È in quest'atmosfera che il 6 agosto 2018 è stata presentata a Yokohama la nave "Izumo", l'ammiraglia della flotta giapponese entrata in servizio nel 2015 come portaelicotteri per il servizio antisommergibili e di controllo costiero, ma ora convertita in portaerei, anche se ufficialmente non può essere dichiarata tale in omaggio all'art. 9 della costituzione. La Cina (che possiede già una portaerei simile a quelle della classe Izumo e ne sta costruendo altre due) ha protestato contro la costruzione di queste "portaerei travestite". Infatti una portaerei è una tipica arma di attacco, anche se viene presentata come "portaerei difensiva".

"La decisione di riconvertire le unità della classe Izumo avviene in un contesto di crescente vulnerabilità delle basi aeree giapponesi nei confronti dei missili da crociera e balistici di Cina e Corea del Nord. Tokyo ha sempre pubblicamente negato la vera natura delle Izumo a causa delle questioni relative alla violazione costituzionale del sistema d'arma di proiezione. In base all'articolo 9 della Costituzione giapponese, il governo proibisce la costruzione di armi considerate principalmente di natura offensiva come le portaerei. Tuttavia il Giappone possiede una straordinaria capacità marittima latente". Inoltre, come indispensabile complemento a questa trasformazione, "in base alle nuove linee guida del Programma Nazionale per la Difesa, entro il 2023 il Giappone riceverà i 42 F-35A acquistati nel dicembre del 2011". ${ }^{146}$

Il Giappone non è isolato in questa trasformazione navale: "The vessel is similar in size to the French and Italian carriers, the Charles de Gaulle and the Cavour" ${ }^{\mathbf{1 4 7}}$ anche se l'Italia trova nell'articolo 11 della sua costituzione lo stesso principio pacifista che vincola il Giappone. Una rivista specializzata conferma che "queste navi [della classe Izumo] presentano caratteristiche analoghe alla portaerei leggera italiana Cavour; senza contare che, contraria-

(Belt and Road Initiative), che non può essere qui affrontato ma la cui cartografia documenta anche i suoi approdi in Europa e l'interesse per i porti italiani di Trieste e Genova (http://www.limesonline.com/tag/nuove-vie-della-seta), con i relativi allarmi ("La Repubblica”, 20 marzo 2019: https:/genova.repubblica.it/cronaca/2019/03/20/news/via_della_seta_ toti_genova_non_fara_la_fine_del_pireo_-222089511/).

146 Reportage dell'analista accreditato con il Ministero della Difesa e con la NATO Franco IACCH (2018, 28 novembre), Il Giappone sta per annunciare: "Le portaelicotteri saranno riconvertite in portaerei difensive così da aggirare la Costituzione del Giappone che vieta vettori d'attacco. Acquistati altri cento F-35".

147 Wide-mouthed frog (2013, 20 agosto). 
mente agli altri DDH giapponesi, l'armamento imbarcato è strettamente difensivo e, al pari della Cavour, sono presenti pure capacità anfibie con spazi per accogliere fino a 400 soldati e 50 veicoli leggeri”. ${ }^{\mathbf{1 4 8}}$ Analoghe caratteristiche presenta la portaelicotteri "Trieste", la maggior nave italiana costruita nel dopoguerra e varata nel maggio 2019.

Infine il già citato "Le Monde" ricorda che "martedì 18 dicembre 2018 il governo ha annunciato le nuove direttive per pianificare gli acquisti di materiale per $\mathrm{i}$ prossimi cinque anni, definendo le priorità strategiche per $\mathrm{i}$ dieci anni a venire. Di fronte alle minacce della Corea del Nord e della Cina, ma anche della Russia, il Giappone vuole rafforzare le sue capacità di attacco e di cyberwar".

Nei settant'anni trascorsi fra l'entrata in vigore della costituzione giapponese e le attuali tensioni internazionali, le forze armate giapponesi sono andate costantemente crescendo, mentre l'articolo 9 della costituzione è rimasto invariato: perciò il crescente divario fra il precetto costituzionale e le reali esigenze militari sta giungendo al punto di rottura, che si manifesta nel dibattito sulla modifica o abrogazione dell'articolo 9. La finzione che si adotta per le "portaerei difensive" è soltanto un aspetto della generale finzione sulle "Forze di Autodifesa".

Il già citato "The Economist" (richiamandosi al "duck test": "If it looks like a duck, swims like a duck, and quacks like a duck, then it probably is a duck") risolve con pragmatismo britannico questo farisaico dibattito sulle Forze di "Autodifesa" e sulla natura "difensiva" della portaerei Izumo: "If it's as big as an aircraft-carrier and looks like an aircraft-carrier, chances are that it is an aircraft-carrier - or can readily be turned into one”.

Attualmente il Giappone è diviso tra chi ricorda che, grazie all'art. 9, il Giappone ha goduto di settant'anni di pace e chi, invece, vede l'art. 9 come un'imposizione postbellica degli USA che impedisce al Giappone di poter far fronte alle minacce provenienti dalla Cina e dalla Corea del Nord. Una documentazione su questo dibattito tuttora in corso è contenuta nell'Appendice $I$.

148 Giuliano Da Frè (2018, 2 gennaio), Portaerei per il Giappone?, con ulteriori dati tecnici e confronti con la nave Cavour. 


\section{L'Italia nel dopoguerra}

Lo Stato italiano moderno nasce formalmente nel 1861, quando il Regno di Sardegna, dopo aver unificato con tre guerre d'indipendenza i vari Stati della Penisola, si trasforma in Regno d'Italia. A quest'ultimo venne estesa la costituzione del Regno di Sardegna, cioè lo Statuto Albertino del 1848, che rimase in vigore sino alla fine della Seconda guerra mondiale. Quella costituzione flessibile permise nell'Ottocento di trasformare la monarchia costituzionale in monarchia parlamentare, ma poi - con l'avvento del fascismo consentì anche di abolire il parlamento elettivo e di sostituirlo con la "Camera dei Fasci e delle Corporazioni”.

Infatti nel 1922 era avvenuto il colpo di Stato fascista con la "Marcia su Roma" e il re aveva nominato Benito Mussolini primo ministro di un governo che progressivamente svuotò lo Statuto Albertino dei suoi contenuti liberali, fino a vietare nel 1925 i partiti politici, ad eccezione del Partito Nazionale Fascista. Iniziava così il ventennio della dittatura fascista, durato fino al 1945. In quel ventennio lo Statuto Albertino rimase formalmente in vigore, ma privo ormai di rilevanza, così come avveniva nella Germania hitleriana con la Costituzione di Weimar del 1919 e nel Giappone militarista con la costituzione Meiji del 1889.

Di quegli anni tragici possono essere qui ricordate soltanto alcune date cruciali. Nel 1935 l'Italia invase l'Etiopia e la Società delle Nazioni la colpì con le sanzioni, definite "inique" dal fascismo. Il 9 maggio 1936 Mussolini dichiarava: "L'Italia ha finalmente il suo impero". Nel 1936 Mussolini intervenne con Hitler nella Guerra civile spagnola. Nel 1938 il "Manifesto della razza" aprì la via alla legislazione razziale in Italia. Nel 1939 l'Italia invase l'Albania e il 22 maggio 1939 sottoscrisse il "Patto d'Acciaio" con la Germania. Il $1^{\circ}$ settembre 1939 la Germania invase la Polonia e diede inizio alla Seconda guerra mondiale, mentre l'Italia entrò in guerra solo il 10 giugno 1940. Il 27 settembre 1940 l'Italia firmò il Patto Tripartito con la Germania e il Giappone.

Dopo le rovinose vicende belliche, il 25 luglio 1943 il Gran Consiglio del Fascismo dichiarò decaduto Mussolini che, arrestato, venne liberato dai tedeschi e portato prima a Monaco di Baviera e poi nell'Italia settentrionale, dove fondò la "Repubblica Sociale Italiana" o "Repubblica di Salò". ${ }^{149}$ Intanto l'8

149 Le strutture ministeriali, politiche e militari della RSI erano sparse in varie località sul lago di Garda. Nella cittadina di Salò avevano sede il Ministero degli Esteri e il Ministero 
settembre 1943 il re e il primo ministro Pietro Badoglio erano fuggiti da Roma e avevano organizzato nell'Italia meridionale un esercito che combatteva a fianco delle truppe alleate che risalivano la penisola.

L'Italia venne così a trovarsi in una situazione di tragica complessità: i soldati italiani ancora sparsi in tutto il teatro di guerra dalla Russia all'Africa, dalla Francia all'Egeo, si trovarono da un giorno all'altro a dover decidere - senza informazioni e senza ordini dal re in fuga - se combattere a fianco dei tedeschi o degli anglo-americani. Essi divenivano così nemici dei precedenti alleati e alleati dei precedenti nemici, in un clima di reciproche ostilità e diffidenza dalle conseguenze spesso drammatiche: esecuzioni sommarie, prigionia, deportazioni.

Per ritardare il più possibile la risalita degli Alleati nella penisola, i tedeschi costruirono la "Linea Gotica", un sistema difensivo che attraversava la Toscana e l'Emilia (all'incirca da Livorno a Firenze fino a Pesaro) e tagliava l'Italia in due parti fra loro nemiche. Con le truppe regie nell'Italia meridionale schierate a fianco degli Alleati e contrapposte ai tedeschi attestati sulla Linea Gotica, per due anni (dal 14 novembre 1943 al 25 aprile 1945) l'Italia così divisa visse una situazione di guerra civile: nel Centro-nord gli italiani della fascista Repubblica Sociale Italiana combattevano insieme con i tedeschi contro gli anglo-americani e contro i partigiani (che intanto formavano un crescente movimento: la Resistenza); nell'Italia meridionale - man mano che risalivano la penisola - altri italiani combattevano, insieme con gli angloamericani e con i partigiani, contro i tedeschi e contro gli italiani della Repubblica Sociale. Furono i due anni più tragici della recente storia italiana. Poiché nel momento finale della guerra crollavano i governi ufficiali degli Stati sconfitti, nel 1943 gli Alleati crearono ad Algeri l'“Allied Military Government of Occupied Territories” (AMGOT) con ufficiali appositamente preparati ad amministrare i territori progressivamente occupati. ${ }^{150}$ Con que-

della Cultura Popolare di quel governo, le cui comunicazioni (diffuse dall'Agenzia Stefani, anch'essa a Salò) venivano spesso riportate con le parole "Salò afferma", "Salò informa".

150 Su questo periodo: Claudio Li Gotti (2008), Gli americani a Licata; Giovanni Di Capua (2005), Il biennio cruciale. Charles Poletti, politico statunitense, giunse in Italia col grado di tenente colonnello e partecipò alle attività dell'AMGOT in varie città. A Palermo ebbe come interprete il mafioso Vito Genovese, luogotenente di Lucky Luciano. Sugli inizi di questo inquietante connubio: Ezio Costanzo (2006), Mafia e Alleati; nonché il classico Michele Pantaleone (1978), Mafia e politica. Inoltre Davide Caracciolo (2008), La ignominiosa alleanza. 
sta struttura gli organismi locali originari restavano in funzione, ma sotto la supervisione degli Alleati. Il primo esperimento di questo "governo militare" iniziò in Sicilia nel luglio 1943, subito dopo lo sbarco alleato. Con altra denominazione, l'AMGOT continuò ad operare in Italia fino al 31 dicembre 1945. Questo modello di gestione venne poi esteso ai paesi occupati dagli Alleati, in particolare alla Germania e al Giappone, ${ }^{\mathbf{1 5 1}}$ ma l'occupazione militare in Italia fu più breve rispetto agli altri due paesi dell'ex Patto Tripartito. ${ }^{152}$

L'estendersi dell'occupazione alleata dalla Sicilia verso l'Italia settentrionale è simbolicamente segnato della circolazione delle AM-Lire (Allied Military Currency), che dal luglio 1943 sostituirono la valuta italiana, poi dal 1946 la affiancarono, fino ad essere del tutto ritirate dal 1950: a questa valuta (equiparata al dollaro) si imputa la forte inflazione postbellica in Italia.

L'Italia ritornò a una situazione di progressiva normalità con la fine della guerra e con il voto del 2 giugno 1946, con cui gli italiani - e le italiane, che votavano per la prima volta in una consultazione a livello nazionale - passarono dalla monarchia alla repubblica e istituirono un'Assemblea Costituente, incaricata di preparare la carta che avrebbe sostituito lo Statuto Albertino. Da quell'Assemblea scaturì la costituzione attuale, che entrò in vigore $1^{\circ}$ gennaio 1948. ${ }^{153}$

Essa contiene l'articolo 11 sul ripudio della guerra, completato da altre norme che evitano le incertezze che tuttora travagliano il Giappone. In particolare, lo stesso articolo 11 prevede una limitazione della sovranità nazionale a favore degli organismi internazionali per il mantenimento della pace. Completano le regole costituzionali in caso di guerra l'art. 27 ("Non è ammessa la pena di morte, se non nei casi previsti dalle leggi militari di guerra"; modificato con la legge costituzionale del 2001: "Non è ammessa la pena di morte"); l'art. 60 ("La durata di ciascuna Camera non può essere

151 L'AMGOT non operò mai sul territorio francese per la netta opposizione del generale De Gaulle, il quale anzi dichiarò che la valuta alleata equivalente alle AM-Lire era denaro falso. In Francia gli americani finirono per rinunciare al progetto dell'AMGOT e riconobbero il "Gouvernement Provisoire de la République Française", voluto da De Gaulle.

152 Nella Zona A (il "Territorio Libero di Trieste" tolto alla Jugoslavia) l'AMGOT operò fino al 26 ottobre 1954, quando la Zona A venne assegnata all'Italia.

153 Nel sito della Camera dei Deputati, il testo della Costituzione italiana è presente anche nelle traduzioni in francese e inglese: http://leg16.camera.it/38? conoscerelacamera $=28$. 
prorogata se non per legge e soltanto in caso di guerra"); l'art. 78 ("Le Camere deliberano lo stato di guerra e conferiscono al Governo i poteri necessari"); l'art. 87 (il Presidente della Repubblica "ha il comando delle Forze armate, presiede il Consiglio supremo di difesa costituito secondo la legge, dichiara lo stato di guerra deliberato dalle Camere"); l'art. 103 ("I Tribunali militari in tempo di guerra hanno la giurisdizione stabilita dalla legge. In tempo di pace hanno giurisdizione soltanto per i reati militari commessi da appartenenti alle Forze armate."); l'art. 111 ("Contro le sentenze e contro i provvedimenti sulla libertà personale, pronunciati dagli organi giurisdizionali ordinari o speciali, è sempre ammesso ricorso in Cassazione per violazione di legge. Si può derogare a tale norma soltanto per le sentenze dei tribunali militari in tempo di guerra"; questo articolo è rimasto invariato anche dopo la legge costituzionale del 1999).

\section{La Germania nel dopoguerra}

Alla fine della guerra, i tre Stati del Patto Tripartito si avviarono verso la democrazia seguendo direttrici diverse. Nel calcolare le perdite territoriali dovute alla sconfitta è opportuno partire dalla situazione prebellica, perché l'inclusione dei "Grandi Spazi" acquisiti in tempo di guerra renderebbe poco realistico il confronto. Ad esempio, al Giappone era internazionalmente riconosciuto il dominio su Formosa (dal 1885), sulla Corea e su parte dell'isola di Sakhalin (1905): la loro perdita ridusse il territorio giapponese al $45 \%$ di quell'estensione. Però se si includesse nel calcolo anche la Manciuria conquistata nel 1931, alla fine della guerra il Giappone sarebbe stato ridotto al 19\% della sua superficie prebellica. ${ }^{154}$ La sua riduzione risulterebbe ancora più drammatica se si considerassero i territori conquistati durante la guerra, su cui si tornerà tra poco.

Tutti e tre gli Stati persero le colonie - i loro "Grandi Spazi” dell'era dittatoriale - mentre diversa fu la sorte dei loro classici territori nazionali. Il Giappone e l'Italia ne persero alcune parti marginali (geograficamente, anche se non emotivamente), ma la loro struttura territoriale prebellica rimase sostanzialmente invariata. L'Italia cedette alla Jugoslavia una parte della Venezia Giulia, alla Grecia il Dodecaneso e alla Francia i territori alpini

154 Wolfgang Benz (1978), Amerikanische Besatzungsherrschaft, 265, nota 2. 
di Briga e Tenda. Il Giappone perse le isole occupate nel Pacifico (Wake, Salomone, Guam e altre) e le aree del Sud-est asiatico che erano state incluse nell'area coloniale eufemisticamente detta "Sfera di Co-prosperità della Grande Asia Orientale”: Indonesia, Singapore, Malesia, Birmania, Filippine, Manciuria e parti della Cina, Taiwan, Corea, Papua Nuova Guinea e altre zone ancora.

Invece la Germania imperiale venne divisa fra le quattro potenze vincitrici in "Zone di occupazione": le tre zone occidentali, occupate da Stati Uniti, Gran Bretagna e Francia, divennero in seguito la Repubblica Federale Tedesca, mentre la Zona di Occupazione Sovietica divenne la Repubblica Democratica Tedesca (DDR). Al termine di questo processo, il territorio del dissolto Impero tedesco risultava suddiviso in cinque parti: mentre dalle quattro zone di occupazione alleata nacquero i due Stati tedeschi, i territori più orientali vennero assegnati all'URSS, mentre alla Polonia andarono i territori sino ai fiumi Oder e Neisse; infine la ex capitale Berlino venne retta da uno speciale statuto quadripartito, che ne faceva un tertium rispetto ai due nuovi Stati tedeschi.

Questa frammentazione si rifletteva anche nel linguaggio della Guerra fredda, che evitava di menzionare la DDR, non riconosciuta internazionalmente: la si chiamava "Mitteldeutschland", Germania centrale, perché i territori ceduti alla Polonia e alla Russia erano "Ostdeutschland", Germania Orientale (nome che invece in Italia, insieme con "Germania Est", veniva spesso usato in sostituzione della denominazione ufficiale "Repubblica Democratica Tedesca", Stato ufficialmente non riconosciuto).

Questi spostamenti di confine provocarono un forte flusso di profughi tedeschi soprattutto verso le tre Zone d'Occupazione occidentali, in parte per scelta personale, in parte per espulsioni in massa, per esempio dai Sudeti. ${ }^{155}$

Le nuove costituzioni, con il loro articolo pacifista, si applicavano quindi all'intero territorio postbellico del Giappone e dell'Italia, ma alla sola Repubblica Federale Tedesca. La costituzione della Repubblica Democratica Tedesca del 7 ottobre 1949, nata nell'area di influenza sovietica, contiene analoghe disposizioni, ma formulate secondo la prospettiva di internazionalismo pro-

155 Manfred Kittel, Horst Möller (2006), Die Beně̌-Dekrete. Nell'Europa orientale vennero cacciati tutti i tedeschi, mentre in quella occidentale si investigarono soltanto le colpe individuali, e con un certo lassismo: sui processi postbellici, sulla denazificazione tedesca e sull'epurazione italiana si tornerà in seguito. 
letario. L'art. 5 stabilisce: “(1) Le norme generalmente riconosciute del diritto internazionale impegnano i pubblici poteri nonché tutti i cittadini. (2) È dovere dei poteri pubblici vegliare al mantenimento e alla difesa delle relazioni amichevoli con tutti i popoli. (3) Nessun cittadino potrà partecipare ad azioni di guerra tendenti ad opprimere un popolo".

L'art. 6 stabilisce nel primo comma che "tutti i cittadini godono di uguaglianza di diritti dinanzi alla legge”, e prosegue: “(2) Qualsiasi propaganda diffamatoria o di boicottaggio contro istituzioni e organismi democratici, qualsiasi incitamento all'assassinio di uomini politici democratici, qualsiasi manifestazione di odio religioso, razziale e contro altri popoli, qualsiasi propaganda militarista e bellicista e tutte le altre azioni dirette contro l'eguaglianza dei diritti costituiscono dei delitti ai sensi del codice penale. L'esercizio dei diritti democratici nello spirito della Costituzione non costituisce una propaganda diffamatoria. (3) Le persone condannate per siffatti reati non possono esercitare funzioni né negli uffici pubblici né nei posti direttivi dell'attività economica e culturale. Esse perdono qualsiasi diritto elettorale attivo e passivo". ${ }^{156}$ Queste norme costituzionali erano state integrate da una Legge a tutela della pace del 15 dicembre 1950. ${ }^{157}$

I testi legislativi della DDR, dopo la caduta del Muro di Berlino, hanno un valore puramente storico: su di essi ritornerà l'Appendice III,2 e 3, mentre la controversia sulla visione comunista della pace, cui si ispirano anche questi testi della DDR, verrà documentata nell'Appendice II,2 dal dibattito sui "Partigiani della Pace".

In conclusione, i vincitori occuparono militarmente in maniera diversa i territori così riconfigurati: il Giappone conobbe un'iniziale forte presenza statunitense, in Germania l'occupazione quadripartita fu accompagnata da una simbolica presenza militare belga e olandese nelle corrispondenti zone di frontiera, ${ }^{158}$ in Italia la presenza alleata (soprattutto statuni-

156 Die Verfassung der Deutschen Demokratischen Republik vom 7. Oktober 1949, in: HansUlrich Hochвaum (ed.) (1958), Staats- und verwaltungsrechtliche Gesetze, 29-30. La traduzione degli art. 5 e 6 è tratta da http://www.dircost.unito.it/cs/pdf/19491007_germania RepubblicaDemocratica_ita.pdf.

157 Questa legge - interamente tradotta nell'Appendice III,3 - si compone di un preambolo e di 15 articoli: Gesetz zum Schutze des Friedens vom 15. Dezember 1950, in: Hans-Ulrich Носнваuм (ed.) (1958), Staats- und verwaltungsrechtliche Gesetze, 168-172.

158 Belgio, Olanda e Lussemburgo, alla fine della guerra, aspiravano a occupare una parte dei territori tedeschi confinanti come riparazione per i danni subìti durante la guerra. 
tense) si ridusse ben presto a numerose basi militari extraterritoriali, tuttora in funzione.

Oggi, in generale, la storiografia e la politica non mettono in discussione l'aspetto positivo del processo postbellico di democratizzazione. Non bisogna però dimenticare che nell'immediato dopoguerra quell'intervento dei vincitori venne vissuto anche come un'imposizione, come una violazione della sovranità nazionale: questo atteggiamento emerge anche nei dibattiti sugli articoli "pacifisti" delle tre costituzioni. Quest'avversione continuò nei gruppi minoritari di estrema destra e ancora oggi trova espressione in una storiografia minoritaria, i cui prodotti vengono collocati nell'“Armadio dei veleni" delle biblioteche, dove sono conservati i libri cui si accede con una speciale autorizzazione.

Uno di questi libri proibiti distingue una "denazificazione con la corda", cioè con l'esecuzione capitale, da una "senza corda", quest'ultima ascritta alla Zona di occupazione americana. La prima viene presentata come un annullamento del diritto internazionale ricondotto al parere del "professore russoebreo A. N. Trainine”, a Parigi. La seconda denazificazione viene attribuita alla "Psychological Warfare Division" del generale R. A. McClure, che per la Germania prevedeva "tre anni di fame, tre anni di rieducazione e tre anni di ricostruzione"; in particolare "l'istituzione centrale per la creazione della colpa collettiva fu, per la guerra psicologica degli Alleati, la Scuola di Francoforte", il cui "prodotto principale" del dopoguerra fu "la Nuova Sinistra". 159

Nel febbraio 1945, nel comunicato sulla conferenza di Yalta, venne annunciato il progetto di cancellare le organizzazioni e le leggi naziste, nonché di punire i maggiori colpevoli. Queste misure suscitarono infiniti dibattiti e resistenze, anche perché, nel crepuscolo postbellico, era pressoché impossibile distinguere la purga politica (cioè la sostituzione dell'élite sconfitta con l'élite vittoriosa) dalla condanna penale (cioè la punizione dei reati). ${ }^{\mathbf{1 6 0}}$

159 Georg Franz-Willing (1991), Umerziehung, 131 e 149. Si può discordare sul contenuto di questo volume, ma la forma è quella di una classica trattazione accademica tedesca.

160 Clemens Vollnhals (ed.) (1991), Entnazifizierung. Politische Säuberung, con un'appendice di documenti, statistiche e immagini (65-346) e bibliografia (354-360). A p. 331 è riprodotta la vignetta della "macchina denazificatrice": cfr. nota seguente. Cfr. anche Clemens Vollnhals (1989), Entnazifizierung und Selbstreinigung. 
Già nel 1946 una vignetta della rivista satirica della zona americana, "Der Simpl", ironizzava sulla facilità con cui le commissioni attestavano il candore delle pecore nere naziste: una fila di pecore nere su un nastro trasportatore finisce dall'alto dentro una macchina "denazificatrice" e, dal basso, ne esce una fila di pecore bianche con un giglio in mano, il tutto sovrastato dalla scritta: "Il nero diventa bianco, ossia la denazificazione meccanica" ${ }^{\mathbf{1 6 1}}$ E per i troppo accondiscendenti certificati di assoluzione la vox populi aveva coniato il termine "Persilscheine", attestati sbiancati col Persil, dal nome di un noto detersivo.

Le costituzioni storiche tedesche hanno riservato al sovrano il diritto di dichiarare guerra (ius ad bellum) e hanno regolato in varia misura il diritto nel corso della guerra (ius in bello). ${ }^{\mathbf{1 6 2}}$ Solo dopo la Prima guerra mondiale il diritto internazionale ha cominciato a porre dei limiti al diritto sovrano di dichiarare guerra, dapprima nello statuto della Lega delle Nazioni, poi soprattutto con il Patto Briand-Kellogg del 1929: il suo primo articolo stabilisce che le parti contraenti rinunciano alla guerra come strumento per la soluzione dei conflitti internazionali e, nel secondo articolo, che esse si impegnano a risolvere le tensioni mediante negoziati. Questo principio venne recepito in vari trattati e ritorna nella carta delle Nazioni Unite del 1945 (art. 2, c. 4, e art. 33): unica eccezione sono le misure militari collettive prese dalle Nazioni Unite (art. 42 s.) e l'autodifesa individuale e collettiva (art. 51). Sono queste le radici dell'articolo 26 dell'attuale costituzione tedesca federale (Grundgesetz).

"Nella sua prima stesura la Legge Fondamentale non conteneva un'articolata regolamentazione della sovranità militare (Wehrhoheit)" a causa "della particolare situazione della Germania come paese occupato. Gli obiettivi politici degli occupanti miravano anche al disarmo e alla smilitarizzazione della Germania, come si vede in numerose decisioni emanate per distruggere il potenziale bellico tedesco e per impedire una rinascita del militarismo

161 Max Radler (1946), Schwarz wird weiss. Già nel nome "Der Simpl" si richiamava a "Der Simplicissimus", la classica rivista satirica tedesca pubblicata dal 1896 al 1944.

162 Bruno Rieder (1984), Die Entscheidung über Krieg und Frieden. Il volume esamina il ius belli et pacis nel Sacro Romano Impero della Nazione Tedesca, nonché nelle costituzioni dei singoli Stati tedeschi (1815-1886), della Paulskirche del 1848, dell'impero bismarckiano del 1871, di Weimar del 1919 e dell'attuale Grundgesetz. 
tedesco" ${ }^{163}$ È questa la ragione per cui i costituenti tedeschi nel 1948 rinunciarono a includere tra le competenze del Bund una "Wehrverfassung" o una "Wehrfrage", cioè una regolamentazione del diritto alla guerra.

Anche la partecipazione della Germania federale alla NATO fu caratterizzata da una grande prudenza terminologica. Ad esempio, dal 1952 al 1957, in piena Guerra fredda, la NATO elaborò una strategia per far fronte a un eventuale attacco da parte dei paesi dell'Est europeo, la "Forward Strategy", che i tedeschi tradussero con "strategia in avanti" (Vorwärtsstrategie). Questo termine venne ben presto sostituito da "difesa in avanti" (Vorwärtsverteidigung, cioè difesa preventiva) per sottolineare che le azioni militari previste erano soltanto difensive e che, quindi, rispettavano l'articolo 26 della costituzione. Il linguaggio ufficiale compiva strane contorsioni per la limitarsi alla sola guerra difensiva: "Se per ogni altro paese la Nato si riarmava (aufrüsten), in Germania la dizione ufficiale era Nachrüstung: il termine sottintendeva che l'Occidente si riarmava sì, ma dopo (nach) e quindi $d i$ conseguenza all'ammodernamento dei missili nucleari sovietici. Dire che la Nato si riarmava per i tedeschi sarebbe stato già dar ragione ai pacifisti". ${ }^{164}$ Negli anni Ottanta, il dibattito sullo stazionamento dei missili sovietici provocò in Germania ( $\mathrm{ma}$ anche in Italia ${ }^{\mathbf{1 6 5}}$ ) un'ondata pacifista paragonabile soltanto a quella che, nel 1949, aveva accompagnato il dibattito sulla ricostituzione delle forze armate tedesche. Nel vivace concorso sulla "parola dell'anno" annualmente indetto dalla "Società per la lingua tedesca", nel 1979 al terzo posto figurava Nachrüstung.

Negli anni successivi questo aspetto della Legge Fondamentale venne progressivamente integrato da nuove norme, ma sempre alla luce dell'art. 26. Negli anni della Guerra fredda il rischio di un conflitto armato era incombente e, per questo, sembrò opportuno includere una norma costituzionale che regolasse la dichiarazione di guerra: ma di "guerra" non si doveva parlare. Così, quando nel 1956 una modifica costituzionale regolò l'intervento

163 Bruno Rieder (1984), Die Entscheidung über Krieg und Frieden, 289. Per questi documenti alleati cfr. Dieter S. Lutz (1982), Krieg und Frieden als Rechtsfrage, $21 \mathrm{s.}$

164 Vanna Vannuccini, Francesca Predazzi (2019), Piccolo viaggio nell'anima tedesca, 128 (sulla "parola dell'anno": https://gfds.de/aktionen/wort-des-jahres/). Le due corrispondenti dalla Germania analizzano l'“anima tedesca” attraverso 16 parole, spesso intraducibili: tra di esse, a p. 60-72, 'Vergangenheitsbewältigung' (superamento del passato), usata anche nel presente libro (cfr. nota 90, p. 33).

165 Centro Militare di Studi Strategici (1990), I movimenti pacifisti. 
delle forze armate tedesche, intanto ricostituite, il nuovo art. 59a GG, usò per tre volte non la tradizionale espressione "dichiarazione di guerra", ma l'inconsueta perifrasi "constatazione che si è verificata la necessità di difendersi" (Verteidigungsfall), proprio per indicare che non si trattava di una guerra di aggressione e che quindi si rispettava l'art. 26 GG: "La constatazione che si è verificata la necessità di difendersi spetta al Parlamento" (comma 1); in casi estremi il Presidente può "dichiarare e annunciare questa constatazione" (comma 2); il Presidente può rilasciare dichiarazioni di rilevanza internazionale sull'"esistenza della constatazione che si è verificata la necessità di difendersi” (comma 3). ${ }^{\mathbf{1 6 6}}$

\section{Il superamento del passato: $i$ grandi processi}

La transizione dalla guerra alla pace e dalle dittature alle democrazie parlamentari esigeva una serie di misure che verranno qui di seguito sintetizzate in quattro grandi temi, che però non esauriscono certo l'argomento: sul piano internazionale, $i$ grandi processi contro i criminali di guerra (esaminati nel presente paragrafo); sul piano interno, le epurazioni delle persone più compromesse con le dittature $(\mathbb{8} 8$; nell'economia, il tentativo di riparare i danni materiali prodotti dalla guerra o dalle occupazioni con l'obbligo per gli sconfitti di pagare delle riparazioni belliche ai vincitori $(\mathbb{9} 9)$; infine, mentre tutte queste misure si riferiscono al passato e alle persone che in quel passato erano vissute, i libri scolastici del dopoguerra cercavano di offrire alle nuove generazioni postbelliche (cioè ai futuri cittadini ed elettori) una rivisitazione del passato, una descrizione della transizione e una rappresentazione positiva della giovane democrazia $(\$ 10)$. Tra tutte queste misure, le più spettacolari furono i grandi processi contro i crimini di guerra, e ad essi - nei singoli territori nazionali - si accompagnarono numerosi processi egualmente traumatici e divisivi sul piano locale.

I vincitori esigevano che i tre Stati sconfitti facessero i conti con il proprio passato e, a questo fine, presero tre misure per facilitare il passaggio dai regimi autocratici alla democrazia parlamentare. Subito, con la fine delle

166 L'articolo 59a venne abrogato nel 1968. Sulle varie modifiche costituzionali che introducono una sovranità militare (Wehrverfassung) nella Legge Fondamentale (art. 59 e art. 115 GG): Bruno Rieder (1984), Die Entscheidung über Krieg und Frieden, 290-318. 
ostilità, iniziava l'occupazione militare dei tre Stati e l'elaborazione di una costituzione democratica. Poi, in tempi brevi, all'interno dei tre Stati dovevano essere processate e punite le persone che avevano contribuito a scatenare la guerra o che nel suo corso si erano macchiate di delitti gravissimi. Infine, nel medio periodo, andavano allontanate dalle più rilevanti cariche pubbliche o socio-economiche le persone più compromesse con i regimi dell'epoca bellica, per evitare che ostacolassero il processo di democratizzazione appena iniziato e per rendere giustizia a chi aveva subìto i soprusi delle dittature. L'insieme di queste misure, insieme con gli articoli pacifisti inclusi nelle nuove costituzioni, avrebbero dovuto evitare future guerre di aggressione.

I due processi principali che si svolsero a Norimberga e a Tokyo vennero accompagnati da altri processi intentati dagli Alleati o dagli Stati coinvolti soprattutto nell'espansionismo tedesco e giapponese. In Europa, oltre ai tribunali militari statunitensi, inglesi e francesi, operarono anche quelli cecoslovacchi, polacchi e sovietici, mentre il processo contro Adolf Eichmann si svolse in Israele nel 1961. ${ }^{167}$ Ancora più vasta fu la cerchia degli Stati coinvolti nei processi contro i crimini di guerra giapponesi.

I due grandi processi si conclusero abbastanza rapidamente perché il numero degli imputati era limitato. Invece le epurazioni (delle quali si occuperà il prossimo paragrafo) coinvolgevano un numero ben maggiore di persone, provocavano resistenze più diffuse e richiedevano quindi tempi più lunghi. ${ }^{168}$ Tuttavia il rigore con cui avrebbero dovuto essere condotte andò attenuandosi con il consolidarsi della Guerra fredda e della sua ideologia anticomunista: quale miglior anticomunista di un ex fascista o di un ex nazista, che per di più deve farsi perdonare quel suo passato?

Questa attenuazione non poteva però valere per i principali responsabili della guerra e delle atrocità belliche, che dovevano essere puniti anche come ammonimento per il futuro. In tutti e tre gli Stati sconfitti, gli Alleati e chi si

167 Una sinossi dei processi e delle condanne (con bibliografia) è alla voce Kriegsverbrecherprozesse della Brockhaus Enzyklopädie (2005). Sugli analoghi dati per il Giappone cfr. infra, nota 188 , p. 71.

168 Sulle diversità della denazificazione nelle due Germanie, cfr. Gerhard Werte (2001), Die Bestrafung von NS-Unrecht in Westdeutschland e KLaus MarXen (2001), Die Bestrafung von NS-Unrecht in Ostdeutschland, nella seconda parte di Der Umgang mit den nationalsozialistischen Verbrechen in Deutschland del volume di Klaus Marxen et al. (eds.) (2001), Der Umgang mit Kriegs- und Besatzungsunrecht. 
identificava con la loro posizione politica chiedevano una punizione esemplare dei militaristi, dei fascisti e dei nazionalsocialisti. Però, secondo le regole politiche della democrazia, questa resa dei conti non doveva essere una vendetta, ma una punizione da adottare secondo le regole del diritto. Si ponevano così i problemi tipici di ogni giustizia di transizione: ${ }^{169}$ poiché l'appartenenza a un partito o l'obbedienza agli ordini non era un delitto al momento dell'adesione o dell'azione, bisognava emanare nuove norme che retroattivamente definissero dei crimini. Inoltre bisognava stabilire in che modo qualificare come colpevole, in base alle norme di oggi, chi ieri aveva tenuto un comportamento che non era stato considerato delittuoso. Gli Stati democratici vincitori si trovarono così a dover fronteggiare una contraddizione: in base ai propri princìi non potevano punire i criminali di guerra senza un dovuto processo fondato su norme penali, però proprio quei princìpi non ammettevano norme penali retroattive. Dal punto di vista strettamente giuridico, ogni soluzione poteva quindi essere messa in discussione: $\mathrm{e}$ di fatto lo fu.

Simboli della giustizia sono la bilancia e la spada: se gli articoli pacifisti nelle costituzioni rappresentano la bilancia, i due spettacolari processi di Norimberga e di Tokyo rappresentano la spada d'una giustizia transizionale, accusata anche di essere la giustizia del vincitore. ${ }^{\mathbf{1 7 0}}$

a) Il processo di Norimberga - cui si affiancarono altri dodici processi - ebbe luogo davanti a un Tribunale militare internazionale ("International Military Tribunal”). Esso era composto da giudici militari delle quattro potenze

169 David Cohen (s.d.), Transitional Justice in Divided Germany; Sanya Romeike (2016), Transitional Justice in Germany; bibliografia, 68-79. Più in generale: Anja MinR (2018), Regime Consolidation.

170 Sul Giappone è importante il libro di Richard H. Minear (1971), Victors' Justice, ristampato più volte fino al 2015. Sulla Germania Gerhard E. Gründler, Arnim von Manikowsky (1967), Das Gericht der Sieger. Un confronto sul perseguimento dei crimini commessi durante la guerra e le occupazioni è in Kuaus Marxen et al. (eds.) (2001), Der Umgang mit Kriegs- und Besatzungsunrecht, citato nella precedente nota 168, p. 64. Inoltre: Arnold C. Brackman (1989), The Other Nuremberg. Brackman assistette al processo di Tokyo come giornalista e ricostruisce cronologicamente gli eventi, con osservazioni personali; sulla posizione critica del giudice francese Henri Bernard nel processo di Tokyo, 407-408 e 436-437 (cfr. infra, p. 77). 
alleate e giudicava i principali responsabili dello Stato hitleriano. ${ }^{\mathbf{1 7 1}} \mathrm{Negli}$ altri dodici processi, invece, il tribunale era composto da soli giudici statunitensi e giudicava i crimini commessi da funzionari pubblici d'alto livello, da industriali, da medici, da giudici e da altre personalità civili. ${ }^{172}$ Complessivamente l'insieme dei processi di Norimberga durò dal 20 novembre 1945 all'aprile $1949 .{ }^{173}$

Al processo principale di Norimberga si stabilì quali organizzazioni dovevano essere ritenute criminali. Nelle zone occupate dagli Alleati occidentali vennero emanate delle leggi per sottoporre a giudizio chi aveva fatto parte di quelle organizzazioni, graduandone in cinque categorie le colpe, e quindi le punizioni: colpevoli principali (cioè criminali di guerra); corresponsabili (cioè attivisti, militaristi e approfittatori); gregari (cioè in sospeso, da valutare); assolti (cioè non colpevoli). Anche nel processo di Tokyo e nei singoli processi nazionali, come si vedrà, ritorna un'analoga griglia per graduare le colpe e, quindi, le pene.

In sintesi, nella zona americana, dal 20 novembre 1945 al $1^{\circ}$ ottobre 1946 il tribunale di Norimberga emanò 12 condanne a morte, di cui 10 eseguite. A questo processo si aggiunsero i processi indetti autonomamente dalle potenze vincitrici. Nelle tre Zone di occupazione occidentali si ebbero oltre 5000 condanne, con 806 condanne a morte, di cui 481 seguite. Nel processo di Tokyo, dal 31 luglio 1946 al 12 novembre 1948, vennero comminate 7 condanne a morte. Nella Zona di Occupazione Sovietica si valutano circa 45.000 condanne, mentre non è accertato il numero di esecuzioni capitali. Altri processi si svolsero negli Stati che erano stati occupati dai tedeschi e dagli italiani. Nel 1955 con il Deutschlandsvertrag le potenze vincitrici trasferirono alla Germania federale la competenza per il perseguimento dei cri-

171 Office of the United States Chief of Counsel For Prosecution of Axis Criminality (1946-1948), Nazi Conspiracy and Aggression, cui seguono Supplements (2 voll.), Opinion and Judgement (1 vol.).

172 La documentazione di questi processi è raccolta in 330.000 pagine, una parte delle quali è stata pubblicata in inglese: Trials of War Criminals Before the Nuernberg Military Tribunals under Control Council Law no. 10 (1949-1953). I primi 14 volumi documentano singoli procedimenti, mentre il vol. 15 (Procedure, Practice, and Administration) raccoglie le regole seguite in questi processi.

173 Gli atti ufficiali del processo di Norimberga sono: Lawrence D. Egbert, Paul A. Joosten (eds.) (1947-1949), Der Prozess gegen die Hauptkriegsverbrecher. 
mini nazisti e dal 1958 fino ad oggi funziona a Ludwigsburg un apposito ufficio per il perseguimento dei crimini nazisti. ${ }^{174}$

b) Il processo di Tokyo. Già durante la guerra gli Stati Uniti avevano ammonito il Giappone sulle conseguenze che sarebbero scaturite dai suoi comportamenti illegali. Con l'occupazione del Giappone la realizzazione di quei duri propositi si concretizzò nell'istituzione di un tribunale militare contro i crimini di guerra giapponesi; tuttavia il mutare della situazione internazionale impose una revisione dell'atteggiamento degli Stati Uniti. "Nel settembre 1945, per esempio, il generale MacArthur promise che il Giappone non sarebbe mai più stato una potenza mondiale e che la sua punizione, 'appena iniziata, sarebbe stata lunga e amara'. Ma dopo un anno e mezzo, MacArthur constatava i notevoli risultati raggiunti con l'occupazione e dichiarava che il Giappone era già pronto per un trattato di pace". ${ }^{175}$ Il mutamento dell'atmosfera internazionale non influenzò tuttavia lo svolgimento anche in Asia dei processi contro i criminali di guerra. Il più importante di essi si svolse a Tokyo dal 3 maggio 1946 al 12 novembre 1948 e fu accompagnato da altri processi cui si accennerà tra poco.

Sulla base della "Potsdam Declaration" (riportata a p. 235) venne istituito un "International Military Tribunal for the Far East" che a Tokyo condusse un processo analogo a quello di Norimberga. Fra i due processi si notano parallelismi e discordanze, ma è condivisibile la conclusione secondo cui il processo di Tokyo "seguì gli schemi generali del documento di Norimberga". ${ }^{176}$

Un primo orientamento sul processo di Tokyo può essere fornito da un volume del $1979^{\mathbf{1 7 7}}$ (la cui bibliografia consente un ulteriore accesso agli innumerevoli scritti su questo tema) e dagli atti del processo stesso. ${ }^{\mathbf{1 7 8}}$

174 “Zentrale Stelle der Landesjustizverwaltung zur Aufklärung nationalsozialistischer Verbrechen": non proprio iperattiva, a giudicare dalle critiche dell'olandese Christiaan F. Rueter nelle sue numerose opere in tedesco dedicate al perseguimento giudiziario dei crimini nazisti.

175 Philip R. Piccigallo (1979), The Japanese on Trial, 46 (vedi anche nota 188, p. 71); sulla fonte delle due asserzioni di MacArthur, cfr. Philip R. Piccigallo (1979), The Japanese on Trial, 227, nota 45.

176 Solis Horwitz (1950), The Tokyo Trial, 475.

177 Philip R. Piccigallo (1979), The Japanese on Trial; Bibliography, 267-280.

178 R. John Pritchard, Sonia M. Zaide (eds.) (1987), The Tokyo War Crimes Trial; Bernard V. A. Röling, ChristiaAn F. Rüter (eds.) (1977), The Tokyo Judgement. "This publication is 
Poiché la "Potsdam Declaration" esigeva fra l'altro l'epurazione di "chi aveva ingannato e traviato il popolo giapponese", la "4th January [1946] Directive" del Supreme Commander of Allied Powers (SCAP), cioè del generale MacArthur, individuava i crimini e i comportamenti su cui fondare i processi e le epurazioni: questa lista, analoga a quella su cui si fondava il processo di Norimberga, è quindi il fondamento tanto del processo di Tokyo quanto delle epurazioni in tutto il Giappone. Al tribunale internazionale di Tokyo vennero sottoposti i crimini maggiori (individuati dalle lettere A, B, C, della lista che segue), mentre gli altri furono oggetto dei processi di epurazione.

Purge Categories.

La direttiva di base ordinava al Governo Giapponese di rimuovere dai pubblici uffici e di escludere dai servizi governativi esponenti del nazionalismo militante e delle aggressioni, ricadenti nelle seguenti categorie:

Categoria A. Criminali di guerra.

Categoria $B$. Militari di carriera e personale della marina; polizia speciale e personale dei ministeri della guerra.

Categoria C. Membri influenti di associazioni patriottiche ultranazionaliste, terroristiche o segrete.

Categoria D. Persone influenti nelle attività della Imperial Rule Assistance Association, Imperial Rule Assistance Political Society e nella Political Association of Great Japan.

Categoria E. Personale delle organizzazioni finanziarie e di sviluppo coinvolte nell'espansione giapponese.

Categoria F. Governatori dei territori occupati.

Categoria G. Altri militaristi e ultranazionalisti.

Ciascuna di queste categorie è accompagnata da una specificazione sui vari livelli o qualifiche delle persone da epurare ma, nonostante ciò, la Categoria $G$ può essere interpretata come un'estensione elastica delle persone da perseguire. ${ }^{179}$

Una parte dei giapponesi, sia pur minoritaria, condivideva la punizione dei crimini di guerra. Tra di essi era un intellettuale che incontreremo nell'esame dei libri scolastici giapponesi: Saburo "Ienaga was one of the very few scho-

based on the stenciled copies of the judgement of the International Military Tribunal for the Far East (majority judgment) and on the opinion of the five judges who gave evidence of a different opinion", vol. I, V.

179 Wolfgang Benz (1978), Amerikanische Besatzungsherrschaft. Le "Purge Categories" sono in tedesco (273-274, nelle pagine successive vengono riportati i dati quantitativi sulle epurazioni) e in inglese (299-300). In questo scritto Benz ha raccolto - in inglese - i documenti ufficiali "che forniscono un panorama complessivo della politica di occupazione americana in Giappone" (265). 
lars who recognized in the 1960s two aspects of the ordinary Japanese war experience - that is, that of offenders (externally) and that of victims (internally). He unequivocally argued the need to pursue, by the Japanese themselves through their own judiciary, the issue of war crimes and responsibility. His uniqueness was that he was not just arguing the position, but was himself pursuing the question of war responsibility in his own way, along with his scholarly work" ${ }^{\mathbf{1 8 0}}$

Nel processo di Tokyo, che durò dal 1946 al 1948, vennero sottoposti a giudizio i crimini commessi durante l'invasione della Manciuria nel 1931, durante la Seconda guerra sino-giapponese del 1937 (con il concomitante massacro di Nanchino) e durante la Seconda guerra mondiale. ${ }^{\mathbf{1 8 1}}$

L'“International Military Tribunal for the Far East" aveva però una struttura diversa da quello di Norimberga. Istituito dal generale MacArthur, era composto da undici giudici da lui scelti tra quelli indicati dagli undici Stati firmatari della capitolazione giapponese, mentre la pubblica accusa era affidata agli Stati Uniti.

La famiglia imperiale venne esclusa dal processo, evitando così l'abdicazione dell'imperatore Hirohito. In realtà, il coinvolgimento diretto dell'imperatore a favore della guerra è oggetto di forti controversie. Durante e immediatamente dopo la guerra, l'opinione pubblica americana ne era convinta, anche sulla base di pubblicazioni allora molto diffuse e oggi ritenute scientificamente poco fondate. ${ }^{182}$ I materiali addotti nel processo e le interviste dei giudici si prestano a interpretazioni divergenti, perché dipendono dalla prospettiva in base alla quale si considera la posizione dell'imperatore. Secondo la tradizione giapponese l'imperatore è un'istanza metapolitica e religiosa; invece, secondo l'interpretazione giuridica occidentale, è un monarca costituzionale i cui poteri sono limitati dalla costituzione. D'altro lato, l'imperatore di discendenza divina era il comandante supremo delle forze armate e senza il suo assenso sarebbe stata impossibile una guerra d'aggressione. In conclusione, "la chiave per comprendere questa polemica dipende

180 Yoshiкo Nozaki (2008), War Memory, 153.

181 Il volume di Giovanni Contini et al. (eds.) (2010), Memoria e rimozione contiene saggi di autori giapponesi e italiani. Verranno richiamati singoli saggi su specifici temi. Su Nanchino: Toikushi Kasahara (2010), Il massacro di Nanchino; Guido Samarani (2010), Il massacro di Nanchino; Harumi Watanabe (2010), Come condividere.

182 Per esempio, David Bergamini (1971), Japan's Imperial Conspiracy, oggi superato. 
da come le parti definiscono certi concetti. Esse sono divise in due gruppi: la prima giustifica l'imperatore sulla base sia di certe peculiarità della cultura giapponese, sia della politica interna; la seconda lo ritiene colpevole applicando certi princìi occidentali, che ritiene 'universali'". ${ }^{\mathbf{1 8 3}}$ MacArthur accettò la tesi dell'estraneità dell'imperatore alla politica aggressiva e questa decisione plasmò la storia del Giappone postbellico.

Passando dalle alte sfere ai comuni mortali, qualche studioso si è chiesto quale involuzione avessero subìto i soldati giapponesi. Infatti un'anomalia difficile da spiegare è la differenza di giudizio sul comportamento dei soldati giapponesi prima e dopo il 1937: nella Prima guerra sino-giapponese "erano definiti dagli osservatori occidentali come disciplinati e valorosi”; nella Guerra russo-giapponese del 1904-05 gli osservatori rilevarono "la valorosa condotta tenuta dai soldati giapponesi"; sembrerebbe infine che fino al 1937 "i prigionieri di guerra (compresi i cinesi, divenuti in seguito bersaglio delle più brutali azioni) fossero trattati rispettando le norme internazionali dell'epoca". ${ }^{\mathbf{1 8 4}}$ A questa involuzione può aver contribuito, fra le altre cause, la propaganda ultranazionalista e il suo contesto sociale. ${ }^{\mathbf{1 8 5}}$

Di certo, comunque, il comportamento dei militari giapponesi durante la Seconda guerra mondiale fu spesso inumano. Poiché l'espansione coloniale giapponese nella vasta area asiatica della "Co-Prosperità" aveva coinvolto molti Stati e colonie del Sud-Est asiatico, i processi per crimini di guerra si svolsero non solo in Giappone e furono promossi non solo dagli Stati Uniti.

Il 27 ottobre 1945 gli Stati Uniti iniziarono nelle Filippine il processo contro il generale Tomoyuki Yamashita, che nella conquista di Singapore era stato definito la "Tigre della Malesia" e che venne impiccato il 23 febbraio 1946. Questo processo suscitò forti critiche, perché alcuni degli stessi giudici statunitensi sostennero che fossero state violate le regole minime per un

183 Peter Wetzler (1989), Kaiser Hirohito, 615. L'articolo passa in rassegna la letteratura anche giapponese dall'epoca Meiji in poi, e conclude: non ci sono le prove che l'imperatore fosse l'artefice della congiura, ma neppure che egli fosse soltanto "un simbolo dello Stato": "Il suo ruolo si colloca in qualche modo fra questi due estremi" (642).

184 Rosa Caroli (2010), Storia e storiografia in Giappone, 101, con varie fonti.

185 Masao Maruyama (1990), Le radici dell'espansionismo, specialmente 3-25: Teoria e psicologia dell'ultranazionalismo. 
giusto processo e che quindi si trattasse di un "unfair trial". ${ }^{186}$ "Il caso Yamashita è una pietra miliare tanto del diritto internazionale quanto del diritto costituzionale americano": infatti "una generazione dopo, il caso Yamashita si dimostrò rilevante nei dibattiti sulla responsabilità del Comando americano nelle atrocità commesse nel Vietnam" ${ }^{\mathbf{1 8 7}}$

Gli Stati Uniti iniziarono analoghi processi in Cina il 21 gennaio 1946, mentre nel 1945 iniziarono quelli delle isole del Pacifico, che erano affidati alle autorità della marina militare statunitense e seguivano regole diverse dagli altri analoghi processi gestiti dall'esercito. A Yokohama ebbero luogo più di 300 processi che coinvolsero più nazioni. ${ }^{\mathbf{1 8 8}}$

Altri processi vennero intrapresi dalla Gran Bretagna, secondo procedimenti propri ma concordati con gli Stati Uniti, però a loro volta diversi da quelli dell'Olanda, della Francia e della Cina, che avevano aperto anch'esse analoghi processi. Il primo processo inglese iniziò a Singapore il 21 gennaio 1946, mentre altri si svolsero nei vari territori asiatici controllati dalla Gran Bretagna. A questi si aggiunsero i processi intentati dagli Stati del Commonwealth come il Canada e la Nuova Zelanda.

L'inizio della Guerra fredda, infine, portò a discrepanze tra l'Unione Sovietica e gli Stati Uniti anche nel perseguimento dei crimini di guerra giapponesi: per i sovietici l'atteggiamento degli Stati Uniti era troppo conci-

186 A. Frank Reel (1949), The Case of General Yamashita. Una sintesi del discusso processo è in Philip R. Piccigallo (1979), The Japanese on Trial, 49-62. Le critiche erano condivise dalle dissenting opinions dei giudici Frank Murphy e Wiley Rutledge, statunitensi, e da Gregorio Perfecto, della Suprema Corte filippina (57). George F. Guy (1981), The Defence of General Yamashita; l'importante giurista del Wyoming George F. Guy (1904-1980) fece parte del collegio di difesa del Generale Yamashita. Su questa sua esperienza pubblicò un articolo nel "Wyoming Law Journal" del 1950, poi riprodotto nell'edizione citata. Durante il processo, Guy "came to respect General Yamashita and to believe in his innocence” (52, nella presentazione di Jeffrey B. Morris). La Kodansha Encyclopedia of Japan (1983, s.v. War Crimes Trials) dedica al caso Yamashita l'intera sottovoce The "Minor" Trials, cita un lungo passo del giudice Murphy e considera questi processi "drumhead justice". Questa voce filo-nipponica è scritta dall'importante nipponista americano Richard $\mathrm{H}$. Minear (Richard H. Minear (1971), Victors' Justice).

187 Telford Taylor (1970), Nuremberg and Vietnam (trad. it. 1971: Norimberga e Vietnam: una tragedia americana, Milano: Garzanti, 211 pp.); JaY W. BAIRD (ed.) (1972), From Nuremberg to My Lai: uno dei vari contributi è dedicato al caso Yamashita.

188 Per quanto riguarda i processi degli USA in Asia contro i crimini di guerra giapponesi, una statistica sul numero dei casi, degli accusati, delle condanne, delle assoluzioni e delle sentenze capitali è in Philip R. Piccigallo (1979), The Japanese on Trial, 95. 
liante e, quindi, sorsero contrasti sulle persone da convocare come imputati, sulla formazione dei collegi giudicanti e sugli avvocati della difesa. Il punto culminante di questo disaccordo è rappresentato dall'accusa come criminali di guerra che l'URSS rivolse a 300.000 prigionieri di guerra giapponesi. ${ }^{189}$

La Cina era il paese che più aveva sofferto sotto l'attacco del Giappone, le cui truppe rimasero sul territorio cinese sino alla fine del 1946. La guerra civile cinese degli anni postbellici si concluse nel 1949, con la formazione di due Cine e con la firma di due trattati di pace: tra il Giappone e Taiwan, il 28 aprile 1952, e tra il Giappone e la Repubblica Popolare Cinese, il 12 agosto 1978. Per questo il perseguimento dei crimini giapponesi seguiva in Asia una legislazione diversificata e una prassi spesso tortuosa. ${ }^{190}$

Due Stati europei avevano colonie asiatiche coinvolte nell'area della "CoProsperità" giapponese: l'Olanda con le Indie Olandesi e la Francia con l'Indocina. A Batavia già nel dicembre 1945 le autorità olandesi avevano iniziato i processi contro più di 200 criminali di guerra giapponesi: processi che risultarono più duri dei corrispondenti processi olandesi contro i criminali di guerra tedeschi. ${ }^{191}$ In Asia la Francia perseguiva i crimini commessi nella sua colonia indocinese, che però era stata oggetto dal 1941 al 1945 di un accordo franco-giapponese tra Vichy e Tokyo. L'attenzione della Francia liberata, tuttavia, si concentrava sui crimini europei dei tedeschi e dei collaborazionisti della Repubblica di Vichy. Si spiega così, in Asia, il basso numero di processi e di condanne francesi rispetto a quelli degli altri Stati. ${ }^{192}$

In conclusione, i processi contro i criminali di guerra giapponesi non sono omogenei: si riscontrano diversità di procedure tra gli Stati Uniti e altri Stati e, all'interno degli USA stessi, tra marina ed esercito; i territori in cui vennero commessi i crimini presentavano uno status giuridico differente (Stati indipendenti o colonie europee, per esempio); l'azione processuale era condizionata dal fatto che, per esempio, le Filippine fino al 1946 furono territorio degli USA, i quali potevano così agire direttamente, mentre invece

189 Philip R. Piccigallo (1979), The Japanese on Trial, 250, nota 36.

190 I dati statistici complessivi sui processi cinesi sono in Philip R. Piccigallo (1979), The Japanese on Trial, 173 , con fonte: 256, nota 88.

191 I dati statistici sulle condanne olandesi e le comparazioni statistiche con altre condanne sono in Philip R. Piccigallo (1979), The Japanese on Trial, 184; fonti: 258, note 46-49.

192 I dati statistici sulla Francia sono in Philip R. Piccigallo (1979), The Japanese on Trial, 208 ; 262, nota 27.

72 Capitolo I 
la Cina, essendo uno Stato indipendente, esigeva un accordo previo con gli USA sui processi. Per i processi asiatici contro i crimini di guerra giapponesi, quindi, non è possibile tracciare un quadro unitario delle procedure seguite né comparare i dati dei singoli processi.

Il processo di Tokyo fu accompagnato da una minore attenzione internazionale rispetto al processo di Norimberga. Complessivamente, i giapponesi non si sentirono coinvolti in questo processo per una serie di ragioni che andavano dall'atteggiamento verso la classe dirigente (cui si rimproverava più la sconfitta che la criminalità di guerra) alla durata ritenuta eccessiva dei processi, che distolse l'attenzione della gente alle prese con le gravi difficoltà materiali del dopoguerra.

Rispetto agli altri due Stati del Patto Tripartito, il Giappone sembra essere quello che più tende a respingere la rielaborazione del passato, anche con atteggiamenti di netto negazionismo. Oltre al massacro di Nanchino, tre argomenti continuano ad essere fortemente dibattuti: gli esperimenti di guerra batteriologica compiuti su prigionieri cinesi (almeno tremila, senza alcun sopravvissuto) e la mirata diffusione della peste in alcune aree cinesi; i lavori forzati obbligatori; la prostituzione coatta di donne dei territori occupati. ${ }^{193}$

Un'accurata ricostruzione degli aspetti giuridici del processo di Tokyo si sofferma anche sulla reazione dei giuristi giapponesi di fronte a quella sentenza. Dopo di essa pochi penalisti se ne occuparono, non solo per le carenze del processo, ma anche per esprimere il diniego "ad occuparsi del proprio ruolo nello Stato oggetto del Tribunale di Tokyo. Questa reazione è da ricondurre soprattutto alla continuità personale dei principali penalisti prima e dopo la guerra". ${ }^{194}$

Anche gli internazionalisti giapponesi seguirono una linea analoga: dopo un primo periodo di prese di posizione quasi sempre favorevoli a processare $\mathrm{i}$

193 Takao Matsumura (2010), L'Unità 731; Hisashi Yano (2010), I lavoratori forzati delle colonie giapponesi (a mio giudizio poco convincente); Aiкo Kurasawa (2010), Romusha; sul sempre più dibattuto caso delle "comfort women", cfr. infra, nota 211, p. 78.

194 Sulla natura giuridica del processo di Tokyo, cfr. Philipp Osten (2003), Der Tokioter Kriegsverbrecherprozeß (bibliografia, 187-208). La citazione è a p. 145 . Oltre alle reazioni dei giuristi giapponesi (130-161) vengono esaminate le posizioni del politologo Masao Maruyama (1914-1996) e dello storico Ienaga Saburo (cfr. p. 138). 
criminali di guerra, "il cambio nella politica di occupazione degli Stati Uniti, la grazia e la riabilitazione concessa a molti criminali di guerra discreditò - agli occhi di molti internazionalisti, ma anche nell'opinione pubblica - le condanne e gli obiettivi del processo di Tokyo. [...] Questo mutamento politico condusse a una fase di disinteresse per il processo durata due decenni (dalla metà degli anni Cinquanta alla metà dei Settanta).". ${ }^{195}$

Si può così giungere a una conclusione generale: "Il tentativo di fare del processo di Tokyo ai criminali di guerra il punto iniziale di un processo di chiarificazione etico-morale e un nuovo inizio nella società giapponese non è riuscito. [...] Da parte dei tribunali giapponesi non ha avuto luogo un'ulteriore elaborazione giudiziaria, accanto ai processi condotti dagli Alleati, dei reati commessi da parte dei giapponesi nel corso della guerra e dell'occupazione. Il merito consolidato del Tribunale di Tokyo è il lavoro di chiarimento storico, cioè l'accertamento di quanto è accaduto". ${ }^{196}$

c) Nessun grande processo in Italia. L'Italia non conobbe processi analoghi a quelli di Norimberga e di Tokyo a causa degli eventi che accompagnarono la fine della guerra e che videro la penisola italiana divisa in due, con il Sud a fianco degli Alleati e il Nord a fianco dei nazionalsocialisti. ${ }^{197}$ In Italia, dunque, "nessuna aula di tribunale si è pronunciata fino adesso sulle accuse di crimini di guerra mosse a connazionali, né i manuali scolastici hanno dedicato attenzione a questi aspetti poco edificanti della storia nazionale". ${ }^{\mathbf{1 9 8}}$

Il colonialismo e il neocolonialismo sono uno dei temi di grande rilevanza attuale non solo per i paesi che ne hanno sofferto in passato ma anche, oggi, per l'Europa che ne sta vivendo le conseguenze attraverso le migrazioni di massa. Sul modo in cui il tema del colonialismo è affrontato nei manuali di storia della scuola media italiana è stata condotta un'analisi su 13 manuali di storia per le scuole medie inferiori e 22 per le scuole medie superiori. ${ }^{199}$ Ogni manuale è stato analizzato secondo una griglia precostituita, giungendo così a un'analisi articolata, di cui non è qui possibile occuparci. Anche i manuali scolastici di geografia e di storia in Germania e Giappone sono stati

195 Philipp Osten (2003), Der Tokioter Kriegsverbrecherprozeß, 161.

196 Philipp Osten (2003), Der Tokioter Kriegsverbrecherprozeß, 184.

197 Filippo Focardi (2010), Criminali a piede libero.

198 Giovanni Contini et al. (eds.) (2010), Memoria e rimozione; così nell'Introduzione, 9.

199 Michele Brondino, Alfonso Di Giovine (eds.) (1987), Colonialismo e neocolonialismo. 
analizzati con un metodo analogo, per verificare quale immagine comunicano dell'altro Stato.

Nonostante il mito degli "Italiani, brava gente" sono documentati crimini commessi dagli italiani sia durante le guerre per la conquista delle colonie africane (Libia, Etiopia, Eritrea, Somalia italiana), sia durante la Seconda guerra mondiale, nelle colonie italiane e negli Stati invasi dall'esercito italiano (Jugoslavia, Albania, Grecia, Unione Sovietica).

Le responsabilità italiane risalgono ad anni anteriori rispetto a quelle attribuite ai tedeschi e ai giapponesi, perché iniziano con le imprese coloniali in Libia e in Abissinia, cioè a partire dal 1911. Di "riparazioni" italiane si è parlato molto tardi, quando anzi il passato coloniale serviva a giustificare interventi politici più recenti: ad esempio, il trattato italo-libico, concluso il 30 agosto 2008 tra Silvio Berlusconi e Muhammar Gheddafi, contiene vari riferimenti al passato coloniale italiano che "ha cagionato delle ferite ancora ricordate da molti libici”. "Il Governo italiano, - precisa inoltre il trattato, esprime il proprio rammarico per le sofferenze arrecate al popolo libico a seguito della colonizzazione italiana e si adopererà per rimuoverne per quanto possibile gli effetti". I ricordi vanno dalla devastazione di Tripoli dopo la disfatta di Sciara Sciatt nel 1911 alle repressioni in Abissinia (1936-1941). ${ }^{200}$

In particolare, sui diciotto mesi di occupazione italiana in Jugoslavia, si assiste a una rimozione collettiva. Indubbiamente i soldati italiani potevano essere "brava gente" nelle pause fra le attività belliche, ma nel corso queste ultime "la documentazione dimostra che i comportamenti bellici dei soldati italiani, sia nelle colonie africane che nei Balcani occupati, sono stati simili a quelli di tutti gli eserciti aggressori della storia, cioè improntati all'estrema violenza”. ${ }^{201}$ Questi crimini sono rimasti impuniti perché i colpevoli "non sono mai stati consegnati agli Stati sul cui territorio avevano commesso i crimini, né processati e condannati in contumacia dagli stessi Stati, né sono mai stati giudicati da corti italiane". 202

Gli Stati contro cui l'Italia aveva combattuto - l'Unione Sovietica, la Gran Bretagna, la Jugoslavia, la Grecia e l'Albania, ma anche l'Etiopia - dal 1945 presentarono all'Italia due liste distinte: una di coloro che avevano maltrat-

200 Nicola Labanca (2010), Compensazioni, che elenca i crimini italiani dal 1911 al 1941 (148-149); LAURA Magi (2010), Fino a che punto l'Italia ha risarcito $i$ danni alle vittime.

201 Alessandra Kersevan (2008), Lager italiani, 10.

202 Laura Magi (2010), Fino a che punto l'Italia ha risarcito $i$ danni alle vittime, 203. 
tato i prigionieri di guerra (richiesta proveniente soprattutto dagli angloamericani) e un'altra di veri e propri criminali di guerra per violenze sulla popolazione civile dei paesi invasi. "Si parla di 729 criminali di guerra richiesti dalla Jugoslavia, 11 dalla Grecia, 9 dalla Francia, 833 dagli anglo-americani, 12 dall'Unione Sovietica, 3 dall'Albania". 203

Contro l'Italia, un vero processo internazionale per crimini di guerra venne proposto dall'Etiopia, ma senza successo. Nel 1943 l'Etiopia aveva chiesto di partecipare alla "Commissione delle Nazioni Unite per i Crimini di Guerra”, ma la sua richiesta era stata respinta perché la Commissione doveva occuparsi esclusivamente dei crimini commessi durante la Seconda guerra mondiale, mentre l'Etiopia era stata invasa dall'Italia nel 1935-36. Nel 1947 l'Etiopia intendeva processare per crimini di guerra e per crimini contro l'umanità i generali Pietro Badoglio e Rodolfo Graziani. Poiché intanto era entrato in vigore il trattato di pace italo-etiopico, la Commissione sopra ricordata riconobbe come fondata la richiesta dell'Etiopia e preparò una lista di dieci criminali di guerra italiani. L'anno dopo l'Etiopia comunicò di voler far processare solo i generali Badoglio e Graziani, che avevano usato gas velenosi anche contro la popolazione civile etiope, "da un tribunale internazionale formato da una maggioranza di giudici non etiopi”, "secondo i principi legislativi e la procedura seguiti dal tribunale militare internazionale di Norimberga". Il sorgere di alcune difficoltà diplomatiche e la netta opposizione inglese alle richieste etiopi, considerate "estremamente inopportune" dal Foreign Office, misero fine a questa vicenda. ${ }^{204}$

Il Trattato di Pace di Parigi del 1947 prevedeva che l'Italia pagasse "riparazioni" per i danni di guerra, ma sin dall'inizio non fu chiaro se, con queste riparazioni, fossero esauriti gli effetti delle violenze commesse dall'Italia con le guerre di aggressione. "In conclusione risulta molto difficile accertare quali condotte illecite tenute dalle forze armate italiane si intendesse riparare attraverso il pagamento delle riparazioni previste dal trattato di pace con l'Italia" ${ }^{205}$ Infine prevaleva la tesi secondo cui la riparazione era dovuta soltanto allo Stato di appartenenza della vittima, e non alla vittima stessa.

Richiamandosi a quest'ultima tesi i tribunali giapponesi negarono il diritto a un risarcimento individuale alle donne costrette alla prostituzione (le

203 Massimo Recchioni (2013), Francesco Moranino, 72.

204 Massimo Recchioni (2013), Francesco Moranino, 73-74.

205 Laura Magi (2010), Fino a che punto l'Italia ha risarcito $i$ danni alle vittime, 206. 
"comfort women" soprattutto filippine e coreane) e alle vittime o di esperimenti medici oppure dell'uso di armi chimiche. Sussisteva soltanto la possibilità che lo Stato beneficiario del risarcimento decidesse di trasferire una parte dell'indennità alle vittime individuali. Ma l'India e la Cina nazionalista rinunciarono al risarcimento sottoscrivendo un trattato bilaterale con il Giappone, mentre il Laos e la Cambogia rinunciarono unilateralmente alla riparazione. $^{206}$

Dopo il 1945 non ci sono più stati processi simili a quelli di Norimberga e di Tokyo contro le guerre d'aggressione, ma sono stati istituiti tribunali contro i crimini di guerra: per esempio, contro quelli commessi in Jugoslavia, nel 1993; in Ruanda, nel 1994; in Cambogia, nel 1997; in Sierra Leone, nel 2004.

Le difficoltà di questi processi si fondano non soltanto su resistenze di tipo politico, ma anche sull'incompatibilità (vera o presunta) di sistemi giuridici diversi: sono difficoltà sorte anche nei grandi processi del dopoguerra.

Nel processo di Norimberga, il giudice francese Henri Donnedieu de Vabres dichiarava in un suo memorandum che la nozione di 'complotto' introduceva una categoria "ignota al diritto francese" ${ }^{207}$ e per questa ragione non votò nessuna delle condanne per il reato di "complotto". Il suo atteggiamento contribuì a far escludere il reato di complotto dall'elenco dei crimini giudicati a Tokyo. Egualmente controverse fra gli stessi giudici furono le condanne a morte, ma soprattutto le assoluzioni; e il giudice Donnedieu criticò questo atteggiamento "contrario alle consuetudini francesi", secondo le quali "i giudici sono solidali". 208

Al termine del processo di Tokyo, il giudice francese Henri Bernard - che era stato un giudice coloniale democratico e aveva partecipato alla Resistenza - criticò la sentenza finale e ritenne "suo dovere" "ristabilire la verità", pur trovandosi in posizione sfavorevole perché conosceva poco la situazione asiatica durante la guerra e "non conosceva le due lingue ufficiali del processo, l'inglese e il giapponese". ${ }^{\mathbf{2 0 9}}$ Ciononostante inviò vari memoranda ai

206 Laura Magi (2010), Fino a che punto l'Italia ha risarcito $i$ danni alle vittime, 209.

207 Ann-Sophie Schöpfel (2013), La voix des juges français, 107.

208 Ann-Sophie Schöpfel (2013), La voix des juges français, 107.

209 Ann-Sophie Schöpfel (2013), La voix des juges français, 109. 
colleghi giudici: riteneva "difettosa" la procedura del processo, ingiusta l'assenza dell'imperatore Hirohito ${ }^{\mathbf{2 1 0}}$ e incompleto l'elenco dei crimini giapponesi. Infatti la corte non discusse né l'Unità 731, incaricata della guerra batteriologica, né le "comfort women", ${ }^{211}$ le donne asiatiche costrette alla prostituzione dagli occupanti giapponesi. Sulle ricerche batteriologiche c'era stato un accordo segreto con gli Stati Uniti che, desiderando appropriarsi dei risultati, fecero ritirare il dossier. Infine, gli avvocati della difesa non avevano avuto accesso ai documenti accusatori, cosa che comprometteva l'imparzialità del giudizio finale.

Quest'ultimo, di 1200 pagine, era stato redatto da un comitato estensore presieduto dall'americano Cramer, nel quale non erano stati inclusi quattro giudici dissenzienti, tra cui il già ricordato Henri Bernard, che presentò una memoria critica "per lealtà verso gli accusati" e "per senso del dovere".212

I giudici francesi "eredi di una tradizione, espressero il loro dissenso criticando il cuore stesso dei processi. Speravano di migliorare la procedura, di arricchire la qualità delle decisioni e di esprimere la loro concezione d'una giustizia universale". ${ }^{\mathbf{2 1 3}}$ "Per il giudice francese [Henri Donnedieu], i princìpi di Norimberga erano dei princìi immutabili, scoperti dalla Ragione. A Norimberga sosteneva il Diritto naturale, diritto scaturito dalla natura umana, dunque proprio di ogni persona, che prenderebbe in considerazione la natura dell'uomo e la sua finalità nel mondo. Però l'idea di stabilire l'universalità di questi princìpi non era condivisa da alcuni degli attori presenti a Norimberga. Al diritto naturale preferivano il positivismo giuridico che stabilisce che il diritto positivo (leggi, giurisprudenza ecc.) ha un suo

210 Bernard asseriva che il principale autore dei delitti era l'imperatore Hirohito, del quale i convenuti dovevano essere ritenuti soltanto complici; e criticava l'assenza di un incontro preliminare fra i giudici prima della sentenza. Infine, "the French member's dissent was written in a convoluted prose", "in obscurantist language", mentre la dissenting opinion (di oltre 1000 pagine) del giudice indiano Pal "was virtually unreadable", Arnold C. Brackman (1989), The Other Nuremberg, 436. Cfr. Radhabinod PaL (1953), International Military Tribunal.

211 Naoko Kumagai, David Noble (2016), The Comfort Women; Chunchee Sarah Soh (2008), The Comfort Women; Maria Clara Maffei (2002), Tratta, prostituzione forzata (recensione: Mario G. Losano (2003), Recensione a Tratta, prostituzione forzata).

212 Dissenting Judgment of the Member from France, 481-496, in: Bernard V.A. Röling, ChristiaAn F. Rüter (eds.) (1977), The Tokyo Judgement, 112.

213 Ann-Sophie Schöpfel (2013), La voix des juges français, 102. 
valore. Queste differenze di concezione erano al cuore dei dibattiti a Norimberga".214

I grandi processi di Norimberga e di Tokyo suscitarono e continuano a suscitare controversie, ma da questi processi e dai dibattiti che ne seguirono nacque "l'idea di una giurisdizione penale internazionale autonoma; bisognava ora metterla in pratica superando le reticenze degli Stati in un contesto internazionale sfavorevole: Guerra fredda, guerra di Corea." ${ }^{215}$ Nonostante l'avverso clima politico, quei grandi e discussi processi influirono di certo sulla concezione del futuro Tribunale Penale Internazionale istituito all'Aia nel 2003. Inoltre i loro servizi di traduzione simultanea divennero un modello per le strutture sovranazionali che si andarono moltiplicando nella seconda metà del secolo XX.

D'altra parte, quei grandi processi europei ed extraeuropei contro i crimini di guerra presentano due aspetti contraddittori. L'aspetto positivo consiste nel fatto che in essi si manifesta per l'ultima volta lo spirito di fratellanza, e la conseguente attività concorde, degli Stati che erano state potenze alleate durante la Seconda guerra mondiale. L'aspetto negativo emerge dalle critiche rivolte a quei processi, spesso intesi come giustizia dei vincitori. Lo si è visto nei pareri contrastanti del giudici francesi e lo si ritrova nell'opinione di Frank Murphy, giudice della Corte Suprema degli Stati Uniti: il desiderio di punire non deve "giustificare l'abbandono della nostra devozione per la giustizia; - ha sostenuto nel già ricordato caso Yamashita, - diversamente ammetteremmo che il nemico ha perduto la battaglia ma ha distrutto i nostri ideali". 216

\section{Il superamento del passato: le epurazioni}

La fine violenta di un ciclo storico esige la sostituzione dell'élite sconfitta con quella vincitrice. Tuttavia non è possibile una sostituzione meccanica e radicale di un ceto con un altro, perché negli Stati industrializzati del XX secolo esistono competenze gestionali e burocratiche che non possono essere improvvisate. Inoltre il crollo dei tre Stati del Patto Anticomintern era stato

214 Ann-Sophie Schöpfel (2013), La voix des juges français, 105.

215 Ann-Sophie Schöpfel (2013), La voix des juges français, 114.

216 Philip R. Piccigallo (1979), The Japanese on Trial, 215. Fonte, 265, nota 13: U.S. Supreme Court, In re Yamashita, 327 U.S. I (1946). Sul caso Yamashita, cfr. supra, p. 70. 
progressivamente annunciato dalle irrimediabili sconfitte militari, dai conseguenti conflitti interni del ceto al potere e da operazioni di avvicinamento a quella che si prevedeva la futura parte vincitrice, sia interna sia esterna. Di conseguenza, alla fine del conflitto la sostituzione dell'élite sconfitta si presentava come un progetto politico di difficile esecuzione.

Le potenze vincitrici si proponevano di demilitarizzare il Giappone, di defascistizzare l'Italia e di denazificare la Germania. In questo progetto di vasto respiro si possono individuare tre fasi: la volontà politica di rieducare in senso democratico lo Stato sconfitto; l'emanazione di norme giuridiche in base alle quali il processo di epurazione avesse luogo secondo i princìi dello Stato di diritto; infine, l'applicazione di queste norme ai casi singoli e l'esecuzione delle pene comminate.

Queste tre fasi erano presenti nei tre Stati sconfitti, ma con modalità e accentuazioni diverse, anche perché il mondo postbellico non era più quello degli anni di guerra: una delle quattro potenze alleate, l'Unione Sovietica, si era trasformata nel principale nemico delle altre tre potenze; all'interno della Germania e soprattutto dell'Italia esistevano partiti di sinistra che creavano tensioni nella compagine dei governi postbellici; nei tre Stati sconfitti bisognava ricostruire dalle fondamenta l'economia e rinnovare la struttura statale. Per questo totale rinnovamento i vincitori non potevano però contare su un numero sufficiente di persone qualificate: per conservare e migliorare il funzionamento dell'apparato statale ed economico, in tutti e tre gli Stati era quindi pressoché impossibile realizzare una radicale epurazione delle persone compromesse con il precedente regime dittatoriale.

In questa situazione, Konrad Adenauer - a chi, nel 1949, gli rimproverava l'amnistia delle persone compromesse col nazismo e, quindi, una loro eccessiva presenza nella società e nell'apparato statale - rispondeva: finché non si dispone di acqua pulita, bisogna usare l'acqua sporca. Questa metafora vale per tutti e tre gli Stati dell'ex Patto Tripartito. Per l'Italia, è corretto dire che il "difetto della neonata Repubblica non sia stato quello di cadere nelle mani di antifascisti comunisti, quanto, se mai, di non avere avviato un serio e profondo processo di defascistizzazione e di educazione civile democratica".217

217 Davide Montino (2005), Le parole educate, 9. L'affermazione citata si riferisce alla polemica sulla cultura egemonizzata dalla sinistra. 
Alla fine della guerra gli Stati Uniti affrontarono la "rieducazione" del Giappone secondo gli stessi principi che li avevano guidati in Germania, sintetizzati nelle "quattro D": Demilitarization, Denazification, Democratization, Decentralization. Quest'ultimo principio venne applicato solo alla Germania, che divenne uno Stato federale, mentre non ebbe luogo la temuta divisione del Giappone in quattro zone di occupazione. ${ }^{218}$ Però questi princìpi venivano applicati a società con un diverso passato, e quindi con una diversa memoria sociale. In particolare, i crimini di guerra venivano distinti in "comuni crimini di guerra" ("gewöhnliche Kriegsvebrechen", cioè violazioni di norme già in vigore al momento della loro commissione) e "crimini nazionalsocialistici” ("nazionalsozialistische Gewaltverbrechen”, ciò̀ crimini fondati sull'ideologia e sulla prassi dello Stato nazionalsocialista).

Gli Stati Uniti affrontarono il dopoguerra giapponese presupponendo che giapponesi e tedeschi avessero commesso gli stessi crimini contro l'umanità: i tedeschi contro gli ebrei, i giapponesi contro i cinesi. "Però questa equiparazione dei crimini del militarismo giapponese con i crimini del nazionalsocialismo non è priva di problemi”, osserva lo storico Akio Nakai in un articolo dedicato proprio al confronto tra la "smilitarizzazione" del Giappone e la "denazificazione" della Germania. ${ }^{219}$ Nel 1940 un convinto nazionalsocialista constatava che "la questione razziale presenta in Giappone un aspetto completamente diverso rispetto all'Europa, ed è errato affrontarla secondo opinioni europee e nazionalsocialiste. Praticamente non esiste un problema ebraico in Giappone".220

Per Nakai l'antisemitismo, l'annientamento dei minorati fisici e psichici, cioè "i crimini nazionalsocialistici, vanno considerati come una ribellione contro la civiltà europea umanistica" (p. 8). Invece il Giappone aveva conosciuto nell'epoca Meiji una tradizione di rispetto delle norme internazionali, poi venuta meno; aveva concepito quell'ascesa come un modello per il riscatto dei popoli non bianchi e cos̀ aveva presentato la sua espansione nel continente asiatico come la realizzazione del classico principio di ricon-

218 Si temeva che gli Stati Uniti avrebbero occupato l'isola di Honshu, la Gran Bretagna quella di Shikoku, la Cina quella di Kyushu e l'URSS quella di Hokkaido.

219 Акıо Nakal (1988), Die "Entmilitarisierung" Japans. Le citazioni nel testo sono tratte da questo articolo, seguite dal rinvio alla pagina. Cfr. anche Bernd Martin (1970), Restauration.

220 Отto Koellreutter (1940), Das politische Gesicht Japans, 19. 
durre "gli otto angoli del mondo" sotto "un unito tetto": il Giappone. Però nei tempi più recenti "era cresciuto anche nei giapponesi il disprezzo per gli altri popoli asiatici; in particolare $\mathrm{i}$ giapponesi considerarono inferiori $\mathrm{i}$ coreani e i cinesi" (p. 8). Di qui gli eventi come il massacro di Nanchino: Nakai riconduce al 1937, cioè allo scoppio della Seconda guerra sino-giapponese, "l'inizio del mutamento", e infine si chiede: "Le guerre in Corea, Vietnam, Afghanistan e così via non possono in certo modo essere intese anche come una continuazione di tutti i conflitti razziali?" (p. 10).

Quando iniziarono anche in Giappone i processi contro i crimini di guerra (gruppo A), il numero di accusati fu minore che in Germania, anche perché non pochi personaggi in vista si erano tolti la vita: ma non per espiare i crimini (che non avvertivano come colpe, essendo atti compiuti per fedeltà all'imperatore), bensì perché l'etica lo esigeva dal guerriero che era stato sconfitto. I processi contro i criminali di guerra del Gruppo B si svolsero in varie capitali asiatiche; invece "in Giappone non si sono mai svolti processi per crimini contro l'umanità, adducendo la motivazione che non era possibile l'accertamento dei fatti per mancanza di documentazione", mentre le vittime tra i civili vennero ritenute "un male inevitabile in una guerra totale" (p. 12).

Dopo la capitolazione del 1945 il comando americano (SCAP) iniziò a preparare il grande processo di Tokyo. Il governo giapponese fu sorpreso da quanto estesa fosse la concezione statunitense dei crimini di guerra e ne chiese invano la revisione. Infine, "l'imperatore era dell'opinione che i processi non dovessero essere svolti da tribunali giapponesi, perché dei cittadini giapponesi non potevano essere puniti in nome dell'imperatore per comportamenti che in guerra avevano tenuto per fedeltà verso di lui. Il Ministro degli esteri Shigemitsu presentò una richiesta di revisione alle potenze vincitrici, che però venne respinta" (p. 12).

In parallelo con i grandi processi, iniziò anche in Giappone l'epurazione dei quadri medio-altri compromessi con i crimini bellici, secondo una procedura analoga a quella applicata in Germania: gli inquisiti compilavano un questionario, che veniva poi sottoposto alla valutazione di un organismo giapponese, i cui risultati venivano controllati dal Comando Supremo statunitense (SCAP).

Queste disposizioni provocarono nel 1946 una crisi di governo, perché portarono alle dimissioni di tre ministri e di vari alti funzionari. Nel 1947 il mondo politico giapponese veniva rivoluzionato: dei 3384 candidati alla 
Camera Bassa ne vennero esclusi 252. La prima fase del 1946 epurò i politici, mentre la seconda nel 1947 colpì il mondo dell'economia e dei funzionari pubblici. Intanto erano già stati epurati circa 5000 insegnanti e 6000 appartenenti alla polizia segreta. Nel 1948 il Tribunale Militare di Tokyo condannò a morte 7 imputati di crimini di guerra (cat. A), e all'ergastolo altri 16. Seguirono condanne per le altre categorie di criminali. ${ }^{221}$

Il giornalista Kazuo Kawai attesta lo scarso interesse dei giapponesi per queste condanne: ai loro occhi, la storia aveva già punito quei leaders con la sconfitta; inoltre quei processi erano durati troppo a lungo, e il pubblico aveva rivolto al sua attenzione ai pressanti problemi del dopoguerra. ${ }^{222}$ Questo atteggiamento valeva in realtà per tutto il processo epurativo: la decapitazione della politica, della burocrazia e dell'economia venne sentita come una ritorsione. Infatti gli stessi statunitensi contribuirono alla revoca di molti provvedimenti e, infine, la pace di San Francisco portò alla revoca di tutte le leggi sull'epurazione.

In Giappone quei processi vennero sentiti come ingiusti. Ancora negli anni Ottanta una delle principali enciclopedie giapponesi si richiama a un nipponista statunitense per descrivere "una serie di tribunali militari semilegali durante e dopo la Seconda guerra mondiale, di fronte ai quali militari e civili giapponesi e tedeschi vennero giudicati dagli Alleati vittoriosi per presunti crimini di guerra". In particolare, pesava ai giapponesi che venissero passate sotto silenzio le azioni degli Stati Uniti (in particolare, l'uso della bomba atomica) e dell'Unione Sovietica (che attaccò il Giappone nell'agosto del 1945, quando la sua resa era già stata annunciata). La situazione di chi aveva perso la guerra, e quindi era l'unico ad essere perseguito, veniva così riassunta da un pubblico accusatore statunitense nel processo di Tokyo: "La ricetta per lo stufato di coniglio esige che anzitutto si catturi il coniglio" ${ }^{223}$

221 Dati più dettagliati in Wolfgang Benz (1978), Amerikanische Besatzungsherrschaft, 274-275. Con una formulazione originale delle "Purge categories", 299-300. L'insieme delle misure statunitensi sull'epurazione è nell'Appendix II, The Purge, 298-312.

222 L'articolo di Kazuo Kawai, Japan's American Interlude, "Nippon Times" (Tokyo), 23, è citato da Wolfgang Benz (1978), Amerikanische Besatzungsherrschaft, 275, nota 30.

223 Kodansha Encyclopedia of Japan (1983), s.v. War Crimes Trial: con un elenco-tabella degli accusati (224), tratto da Richard H. Minear (1971), Victors' Justice. Ecco il testo originale (223) dei passi riportati nel testo: in Giappone operò "a series of quasi legal military tribunals during and after World War II in which Japanese and German military and civilians were tried by the victorious Allies for alleged war crimes": inoltre "the bulk of 
I criteri in base ai quali effettuare le epurazioni erano contenuti in una direttiva del Quartier Generale degli Alleati emanata il 4 gennaio 1947, che individuava sette gruppi da sottoporre all'epurazione. Come in Germania, anche in questa casistica la categoria residuale degli "altri militaristi e nazionalisti” (Gruppo G) fu all'origine di molti casi controversi, contro cui si poteva fare ricorso. "Però la maggior parte dei giapponesi ritenne infruttuoso il ricorso contro l'autorità assoluta delle potenze occupanti e assunse un atteggiamento di rassegnazione" (p. 14).

Nakai spiega l'accettazione della nuova costituzione e delle radicali riforme agrarie da un lato con questa rassegnazione verso i detentori del potere e, dall'altro, con il ricordo "della democrazia dell'epoca Taisho dopo la Prima guerra mondiale, al cui sistema parlamentare e partitico ci si poteva ora richiamare" (p. 14), anche se proprio il fallimento dei partiti aveva aperto la porta al militarismo e alla guerra.

Nakai ha una visione decisamente critica del successo rieducativo degli Alleati. Col ritorno della sovranità al Giappone, "gli epurati e i licenziati vennero tutti riabilitati; anche i criminali di guerra lasciarono le prigioni e ricomparvero sulla scena della politica e dell'economia. In non pochi casi ripresero semplicemente la loro precedente posizione, loro restituita da chi al momento la deteneva. Come notava ironicamente un giornale giapponese, la qualifica di 'ex-epurato' divenne una specie di titolo onorifico per personaggi in vista” (p. 15). Nonostante l'art. 9 della costituzione si ricostituirono le forze armate, sia pure dapprima come National Police Reserve (cfr. p. 49). Ma soprattutto si ricostituirono dei raggruppamenti di estrema destra con le medesime persone dell'epoca militaristica.

Kishi Nobusuke è un caso paradigmatico. Incarcerato come criminale di guerra alla fine del conflitto, nelle sue memorie scrive: "Durante la mia permanenza nella prigione di Sugamo non avevo l'impressione di essere considerato, con i miei colleghi, come un criminale. Ero consapevole sol-

the prosecution at Tokyo Trial rested on charges not clearly considered illegal". In generale, questi tribunali "considered only Japanese acts, and not acts committed by the Allies", come le bombe atomiche ("at the Tokyo Trial evidence concerning the two bombs was declared inadmissible”). Lo stesso vale per l'attacco russo al Giappone dell'8 agosto 1945: "Asked after the Tokyo Trial about various Russian acts, the American prosecutor at Tokyo commented: The recipe for rabbit stew is first to catch the rabbit"; e quindi "The logic applies equally to American acts and American actors; and it explains why only Japanese acts and Japanese actors were prosecuted". 
tanto del fatto che eravamo stati vinti. Ma con la sconfitta totale, di cui ero colpevole anch'io, sul popolo giapponese si era abbattuta un'intollerabile onta, un'indicibile sventura. Non potrei sottrarmi a questa responsabilità neppure se potessi espiarla morendo più volte. Ho ininterrottamente riflettuto su come tentare di porvi riparo. Come risposta ho deciso di mettere nuovamente tutta la mia energia, dopo la scarcerazione, al servizio dello Stato e della nazione. Sono giunto alla conclusione che proprio ora la politica e la gestione dello Stato rivestono la massima importanza per apprendere dagli errori passati e per agire per il bene del Giappone e del suo popolo" ${ }^{224}$ E così Kishi Nobusuke, che dal 1941 al 1944 era stato membro del ministero Hideki Tojo (condannato a morte dal Tribunale internazionale di Tokyo), tornò ad essere presidente del Consiglio dei ministri dal 1957 al 1960.

In Italia la guerra terminava lasciando una situazione confusa, con la già ricordata rimozione di Mussolini il 25 luglio 1943, con l'occupazione alleata nel Sud e quella tedesca nel Centro-Nord dell'Italia, con la formazione della Repubblica Sociale Italiana, con la guerra partigiana e con la fine del conflitto mondiale il 25 aprile 1945.

L'Italia repubblicana conobbe, come la Germania Federale, un tiepido processo di epurazione dei fascisti, che, come nel caso tedesco, si andò rapidamente attenuando con l'inasprirsi della Guerra fredda. Anche in Giappone l'esitante epurazione avvenne con le caratteristiche ed i problemi derivanti dalla peculiare struttura di quella società, che continuava ad essere retta dall'imperatore sotto il quale aveva potuto affermarsi il militarismo.

Il governo di transizione di Ivanoe Bonomi (1944-45) fu poco incisivo nell'epurazione (poiché colpì soprattutto in basso), mentre il successivo governo di Ferruccio Parri cadde proprio per i contrasti sull'epurazione.

La divisione della penisola aveva dei riflessi anche in questo campo: "Nel Sud della penisola e nelle isole il fascismo era finito per sempre nel luglio del 1943 e non aveva avuto, a differenza del Nord, alcuna 'resurrezione'. Erano pertanto mancate non per scelta soggettiva, ma per circostanze oggettive, le occasioni di comportamenti di connivenza (con i fascisti repubblicani e i

224 Riportato in Aкio NakaI (1988), Die "Entmilitarisierung" Japans, 15-16; tratto da Nobusuke Kishi (1983), Kishi Nobusuke Kaikoroku, 19-20. 
loro padroni tedeschi)". 225 In sostanza, nell'Italia meridionale c'era meno materia prima per l'epurazione.

Riprendendo i tre criteri dell'epurazione già illustrati, la volontà politica esisteva, ma aveva difficoltà ad esprimersi perché le diverse anime della Resistenza, unite durante la lotta contro il nazi-fascismo, con l'avvento della pace assumevano posizioni diverse all'interno del nuovo governo democratico. ${ }^{226}$ I socialisti erano più radicali dei comunisti. Pietro Nenni scriveva articoli preconizzando la fine del capitalismo, l'avvento della società socialista e "la socializzazione dei grandi mezzi di produzione e di scambio" ${ }^{227}$ I comunisti volevano l'epurazione degli industriali arricchitisi col fascismo, ma escludevano una nazionalizzazione delle imprese, perché miravano a una politica di unità nazionale in cui anche i capitalisti contribuissero alla ricostruzione del paese. Democristiani e liberali erano contrari a ogni epurazione classista e chiedevano l'allontanamento soltanto di chi si era compromesso nel modo più grave col fascismo. Infine il partito dell'“Uomo qualunque", rapidamente scomparso ma allora non privo di seguito, si opponeva a ogni forma di epurazione, ritenendola una forma di dittatura dell'antifascismo.

Questa discordanza di opinioni contribuì nel novembre 1945 all'uscita dei liberali dal governo Bonomi e alla formazione del primo governo del democristiano Alcide De Gasperi, con il quale comincia l'atrofia e, di fatto, la fine dell'epurazione. ${ }^{228}$

Le contrastanti posizioni politiche portarono a emanare una legge $d i$ compromesso sull'epurazione, sulla quale si sarebbero fondate le future azioni giudiziarie. I due gradi di giudizio legalitariamente previsti e il gran numero di epurandi ostacolarono sin dall'inizio l'applicazione di questa normativa. Il Decreto Legislativo Luogotenenziale del 27 luglio 1944, n. 159, - la "Magna

225 Romano Canosa (1999), Storia dell'epurazione in Italia, 380. Sull'epurazione nel Regno del Sud: Lamberto Mercuri (1988), L'epurazione in Italia, 21-49.

226 Sandro Setta (1993), Profughi di lusso. Una prima parte analizza la collusione tra grandi imprenditori e fascismo e, nella fase finale della dittatura, il loro appoggio anche alla Resistenza (1-66); una seconda parte documenta come questa ambiguità abbia contribuito a vanificare l'epurazione dei vertici imprenditoriali italiani (67-123). La documentazione proviene soprattutto dal "liberale Archivio federale di Berna" (9), perché gli archivi italiani non erano allora accessibili all'autore.

227 Pietro Nenni (1978), Vento del Nord, 290-292, con gli articoli Vento del Nord ("Avanti!", 7 febbraio 1945) e La resa (“Avanti!", 29 aprile 1945).

228 Sandro Setta (2005), L'Uomo Qualunque, 22. 
Charta" dell'epurazione - indicava quali reati dovevano essere puniti come delitti fascisti:

Art. 3. Coloro che hanno organizzato squadre fasciste, le quali hanno compiuto atti di violenza o di devastazione, e coloro che hanno promosso o diretto l'insurrezione del 28 ottobre 1922 [Marcia su Roma] sono puniti secondo l'art. 1120 del Codice penale del 1889. Coloro che hanno promosso o diretto il colpo di Stato del 3 gennaio 1925 e coloro che hanno in seguito contribuito con atti rilevanti a mantenere in vigore il regime fascista sono puniti secondo l'art. 118 del Codice stesso. Chiunque ha commesso altri delitti per motivi fascisti o valendosi della situazione politica creata dal fascismo è punito secondo le leggi del tempo. [...]

Art. 5. Chiunque, posteriormente all' 8 settembre 1943, abbia commesso o commetta delitti contro la fedeltà e la difesa militare dello Stato, con qualunque forma di intelligenza o corrispondenza o collaborazione col tedesco invasore, di aiuto o di assistenza ad esso prestata, è punito a norma delle disposizioni del Codice penale militare di guerra. Le pene stabilite per i militari sono applicate anche ai non militari. I militari saranno giudicati dai Tribunali militari, i non militari dai giudici ordinari. ${ }^{229}$

L'art. 8 prevedeva l'interdizione temporanea dai pubblici uffici o la privazione dei diritti politici per non più di dieci anni (nonché la decadenza dalle Assemblee elettive) nei confronti di chi, "per motivi fascisti o avvalendosi della situazione politica creata dal fascismo, abbia compiuto fatti di particolare gravità che, pur non integrando gli estremi di reato, siano contrari a norme di rettitudine e di probità politica”.

In realtà, l'apparente severità di queste norme poteva essere aggirata grazie all'art. 7 dello stesso decreto-legge del 27 luglio 1944: "Per i reati previsti nel presente titolo, la pena può essere ridotta fino ad un quarto, e alla pena di morte o dell'ergastolo può essere sostituita la reclusione non inferiore a cinque anni: a) se il colpevole, prima dell'inizio della presente guerra, ha preso posizione ostile al fascismo; b) se ha partecipato attivamente alla lotta contro i tedeschi. Se ricorrono le circostanze attenuanti generiche, previste dal Codice penale del 1889, alla pena di morte o all'ergastolo è sostituita la reclusione per trent'anni e le altre pene sono diminuite di un sesto. Il

229 Il testo legislativo completo è in http://www.straginazifasciste.it/wp-content/uploads/2015/ 02/Decreto-Legislativo-Luogotenenziale-27-luglio-1944-n.-159.pdf. Cfr. Hans Woller (1996), Die Abrechnung mit dem Faschismus, 193; Tommaso Fortunio (1946), La legislazione definitiva, 45; e, dello stesso autore, Tommaso Fortunio (1948), Revisione e revoca dell'epurazione. 
colpevole potrà essere dichiarato non punibile, se nella lotta contro i tedeschi si sia particolarmente distinto con atti di valore".

Questo decreto-legge, pietra angolare delle sanzioni contro i fascisti, entrava in vigore nel luglio 1944: a quella data, la guerra era ancora in corso e alcune sue norme erano un invito a collaborare con la Resistenza, invito che ritorna anche in vari documenti del Comitato di Liberazione Nazionale per l'Alta Italia. Inoltre era pressoché impossibile dare attuazione a quel decreto in un paese diviso, con fluide strutture statali in difficoltà nell'applicare ovunque le nuove norme.

L'Alto Commissariato per le Sanzioni contro il Fascismo" operava secondo tre ulteriori decreti luogotenenziali. ${ }^{230}$ Due successivi decreti-leggi del 4 agosto e del 9 novembre 1945 prevedevano il sequestro dei beni e dei profitti di regime, nonché l'allontanamento dalle alte cariche delle aziende private. Però anche la "Legge Nenni", cioè il decreto-legge 9 novembre 1945, n. 702, prevedeva all'art. 17 di non applicare quelle sanzioni a coloro che "posseggano una comprovata capacità tecnica e amministrativa e ricorra per essi una delle circostanze seguenti: si siano distinti nella lotta contro i tedeschi ovvero, prima dell'inizio della presente guerra, abbiano preso posizione ostile al fascismo".

Le eccezioni previste aprivano la via a scappatoie che avevano successo data la stretta clandestinità del movimento partigiano e quella ancor più rigorosa in cui aveva dovuto agire la fitta rete dei suoi fiancheggiatori nella società civile: infatti gli antifascisti attivi o i partigiani combattenti non si conoscevano fra di loro, e questo facilitò il sorgere di pseudo-partigiani, che aggiravano così le norme sull'epurazione. Basti un esempio: con gli Alleati della V Armata era entrato in Roma anche uno sconosciuto battaglione partigiano dalle camicie verdi, chiamato perciò "Battaglione verde", comandato da Gabriellino D’Annunzio (figlio del poeta). Oggi salta agli occhi quanto quella "camicia verde" fosse incongrua per dei partigiani: essa era infatti l'equivalente della camicia nera fascista per gli Integralisti in Brasile, ${ }^{231}$ per i nazisti ungheresi delle Croci Frecciate, per la Guardia di Ferro

230 Decreti luogotenenziali del 3 settembre 1944, n. 198 (contro i maggiori criminali fascisti); del 3 ottobre 1944, n. 238, ("Ordinamento dell'Alto Commissariato per le Sanzioni contro il Fascismo"); del 23 ottobre 1944, n. 285, (regole procedurali per l'Alto Commissariato ed estensione delle epurazioni fuori Roma).

231 Cfr. supra, p. 25. 
all'epoca del maresciallo Ion Antonescu (le "cămășile verzi” del conducător, il duce della Romania) ${ }^{\mathbf{2 3 2}}$ e anche per l'attuale Lega Nord italiana che, inconsapevole di queste assonanze, aveva adottato la camicia verde per la sua Guardia Padana, oggi tramontata. ${ }^{233}$

Il comandante del "Battaglione verde" "distribuì, nei giorni immediatamente successivi [all'entrata in Roma], numerose attestazioni di partecipazione alla guerra partigiana. A chi? Naturalmente a fascisti e collaborazionisti che avevano bisogno di salvare la pelle. Attestati in tutto e per tutto veri, reperiti chissà dove". La cattura di un messaggio dimostrò "il legame tra i repubblichini e [Gabriellino] D'Annunzio, il quale venne subito arrestato con l'accusa di tradimento. Ma pochi mesi dopo, assolutamente libero e senza essere stato sottoposto a processo alcuno, il capo di quelle 'Camicie verdi' si era rifugiato in una chiesa gesuita". ${ }^{234}$ La rete dell'epurazione era dunque tesa, ma le sue maglie erano molto larghe.

Può considerarsi emblematico per l'intero processo di epurazione e di condanne in Italia quanto avvenne con i militari italiani accusati dagli inglesi di crimini contro i prigionieri di guerra. Essi vennero giudicati e condannati da tribunali inglesi sul territorio italiano. Nell'ottobre del 1946 le truppe inglesi si ritirarono dall'Italia, concordando con le autorità italiane che i condannati scontassero in Italia il resto della detenzione.

Furono presto esercitate pressioni per ottenere misure di riduzione della pena. Un interessamento in questo senso fu manifestato anche dalla Segreteria di Stato del Vaticano. Nel luglio 1949 il governo inglese venne incontro alle esigenze italiane riconoscendo la possibilità della riduzione di un terzo della pena per buona condotta e la possibilità dell'immediata scarcerazione in caso di grave malattia contratta in carcere, tale da 'accorciare le normali aspettative di vita'. Le autorità italiane sfruttarono immediatamente la situazione: chiesta la riduzione di un terzo della pena per buona condotta, applicarono poi di propria iniziativa il beneficio della libertà provvisoria, previsto dall'ordinamento italiano dopo che il condannato avesse scontato almeno i due terzi della pena. In questo modo si procedette nel 1950 a un'ondata di scarcerazioni. Nel novembre dello stesso anno [1950] solo tre persone erano ancora in carcere. ${ }^{235}$

232 Nicholas M. Nagy-Talavera (2001), The Green Shirts and the Others.

233 Gianluca Passarelli, Dario Tuorto (2012), Lega e Padania.

234 Franco Felice Napoli (1996), Villa Wolkonsky, citato in Massimo Recchioni (2013), Francesco Moranino, 76-77.

235 Massimo Recchioni (2013), Francesco Moranino, 74. 
Le elezioni del 1948 avevano segnato la sconfitta dei partiti di sinistra e l'affermazione delle forze cattoliche e conservatrici: in questo clima politico si svolgevano i processi per le epurazioni e i giudici dovevano decidere sulla base delle ambigue norme appena esaminate. Gli accusati proponevano in generale una documentazione accuratamente precostituita a propria discolpa, mentre troppo spesso le accuse presentate da parte dei rappresentanti dei Comitati Nazionali di Liberazione erano generiche. Già prima della fine della guerra molti industriali e politici si erano premurati di precostituirsi benemerenze antifasciste giocando spregiudicatamente su più tavoli. ${ }^{236}$

I dati su queste epurazioni sono incerti, anche perché si riferiscono a territori che man mano venivano liberati e quindi vivevano precarie situazioni organizzative. Dai dati disponibili si può concludere che poche delle persone indagate vennero incriminate e ancor meno rimosse. ${ }^{237}$ Le condanne davanti all'Alta Corte istituita dal Decreto Luogotenenziale 159 furono poche e circondate, da un lato, da crescenti critiche per la lentezza dei procedimenti e, dall'altro, da una progressiva incertezza sulla loro opportunità.

In quell'epoca di transizione i soprusi subìti ad opera dei fascisti bruciavano ancora, le armi della Resistenza erano ancora in mano a molti partigiani e l'impunità di certi fascisti suscitava perplessità o sdegno, ma talora anche desiderio di vendetta. Alcuni settori della sinistra iniziarono a dubitare dell'effettiva applicazione delle sanzioni antifasciste e decisero di farsi giustizia da sé. Su quei mesi e anni dopo la fine della guerra si scrivono ancora oggi pagine aspre e contraddittorie. Da un lato, la democrazia vincitrice e, in particolare, il movimento partigiano ricordavano le atrocità nazifasciste e tendevano a giustificare ogni reazione. Dall'altro, i vinti avvertivano come un sopruso il silenzio calato sulle violenze da loro subìte anche dopo la fine ufficiale delle ostilità: un silenzio che venne rotto nel 2003 da Giampaolo Pansa, che con il libro Il sangue dei vinti iniziò a sollevare sistematicamente il velo sulle violenze dei vincitori, il che gli valse l'accusa di fascista. E infatti quasi vent'anni dopo, nel 2019, tracciò il bilancio di quella sua lunga campagna in uno scritto autobiografico il cui titolo riecheggia proprio l'accusa che gli rivolgevano le sinistre, e non solo loro: Quel fascista di Pansa (anche se

236 Alcuni esempi sono riportati nel mio libro Mario G. Losano (2018), Norberto Bobbio, 99-100; cfr. anche Sandro Setta (1993), Profughi di lusso, 35-36.

237 Roy Palmer Domenico (1991), Italian Fascists on Trial. Con una sintesi dei dati sulle epurazioni, 89; e una descrizione degli eventi processuali, 90-108. 
Pansa parte dal giusto principio che esporre le ragioni dei vinti non significa condividerle). ${ }^{238}$

Con il titolo Da partigiani a briganti, un altro grande giornalista, Enzo Biagi, racconta una vicenda della quale nel 1945 parlarono tutti i giornali e di cui furono protagonisti due suoi compagni nella lotta partigiana: una storia stralunata e disperata, esemplare anche se incomprensibile fuori da quel contesto senza più regole. Quei due non erano dirigenti comunisti importanti come Francesco Moranino, ma pesci piccoli e feroci, che di fronte alle esitazioni dell'epurazione ufficiale erano passati all'“idea di una giustizia fatta alla svelta" in un "mondo diviso in due", i buoni e i cattivi. Quel "mondo diviso in due", che prima della vittoria era ancora pieno di speranza, come nei versi Oltre il ponte Italo Calvino spiegava alla generazione venuta dopo di lui: “Avevamo vent'anni e oltre il ponte | oltre il ponte ch'è in mano nemica | vedevam l'altra riva, la vita | tutto il male avevamo di fronte. | Tutto il bene avevamo nel cuore | a vent'anni la vita è oltre il ponte". Il due compagni di Biagi, invece, in quel "mondo diviso in due" vedevano con disperazione la vittoria sbiadirsi in sconfitta: e allora "sparano e strangolano, e fanno 'l'epurazione sul serio:"239 una quarantina di morti nella sola Castelfranco.

A questo clima si possono ricondurre sia l'uccisione il 17 luglio $1945 \mathrm{di}$ cinquantacinque fascisti detenuti nella prigione di Schio, sia i casi di giustizia sommaria della "Volante Rossa", che continuò a colpire fino al 1949. ${ }^{240}$

238 Giampaolo Pansa (2003), Il sangue dei vinti. Ovviamente il libro di Pansa non è il primo ad affrontare questo tema, ma va segnalato perché sollevò un dibattito nazionale, che l'autore alimentò al ritmo di pressoché un volume all'anno sul medesimo tema: (2002), I figli dell'Aquila; (2003), Il sangue dei vinti; (2005), Sconosciuto 1945; (2006), La grande bugia; (2007), I gendarmi della memoria. Tuttavia la sua ricostruzione non si arresta con questi volumi: per esempio, (2012), La guerra sporca; ed egli la ripercorre nella sua autobiografia: (2019), Quel fascista di Pansa.

239 Enzo Biagi (2008), Io c'ero, 17-21; la citazione è a p. 19. Biagi fu partigiano con Carlo Azeglio Ciampi, in seguito Presidente della Repubblica: "Siamo tutti e due vecchi ragazzi classe 1920 e l'8 settembre 1943 decidemmo di entrare nei partigiani di Giustizia e Libertà e poi per tutta la vita ci siamo portati dietro quello che abbiamo imparato sui monti, lui della Toscana, io dell'Emilia, il rispetto per gli altri, qualunque fosse la loro opinione, e la giustizia sociale" (484); "I quattrodici mesi in cui ho fatto il partigiano sono il periodo che ricordo con più orgoglio ma anche con tanti rimpianti” (505).

240 Francesco Trento (2016), La guerra non era finita. Il volume ricostruisce le vicende dell'organizzazione (ripudiata dal Partito Comunista Italiano) e i processi che, nel 1951 e nel 
Queste innegabili violenze oscurarono gli aspetti positivi della Resistenza, e un grande giornalista come Indro Montanelli (che aveva fatto "la Resistenza in divisa" come monarchico, ma che riteneva l'antifascismo "peggiore del fascismo") sostenne che "la strage di Schio [...] si differenzia da quella delle Fosse Ardeatine solo per il fatto che nessun Kappler e nessun Priebke la pagarono". ${ }^{241}$ Un giudizio politicamente inaccettabile e storicamente insostenibile.

L'incerto confine tra atti di guerra e vendette postbelliche fu al centro delle vicende del comunista Francesco Moranino (1920-1971): comandante partigiano accusato di omicidio per l'uccisione di sette presunte spie, si rifugiò a Praga; rieletto deputato nel PCI, ritornò in Italia e venne amnistiato. $^{242}$ Il suo processo doveva decidere se le esecuzioni contestate erano state delitti comuni ovvero atti di guerra; ma, al tempo stesso, quel processo rivelava esemplarmente il cambio di clima politico negli anni Cinquanta, diretto a sminuire la rilevanza politica e militare della Resistenza. L'onda lunga di quel processo del 1955 non si era ancora spenta nel 2019, quando il Parlamento doveva votare l'autorizzazione a procedere contro Matteo Salvini per il blocco dei migranti sulla nave "Diciotti". In un forzato parallelismo con il caso Salvini, la destra riesumò il caso Moranino: la sua condanna all'ergastolo venne commutata in dieci anni di detenzione, poi nel 1965 Giuseppe Saragat, "il Presidente della Repubblica, piemontese come Moranino, cancellò, con un colpo di spugna, la pena" e "l'Italia uscì così, definitivamente, dal clima avvelenato della guerra civile, ma il prezzo pagato allo Stato di diritto fu altissimo".243

1953, portarono alla condanna di vari suoi componenti, alcuni dei quali, condannati all'ergastolo, vennero poi graziati, mentre altri avevano trovato rifugio soprattutto in Cecoslovacchia. Cfr. anche Massimo Recchioni (2011), Il tenente Alvaro (autore anche di (2013), Francesco Moranino); Gianfranco Stella (1993), Rifugiati a Praga.

241 Indro Montanelli (2002), Soltanto un giornalista, 115. Montanelli entrò nella Resistenza attraverso gli esponenti del futuro Partito d'Azione, venne arrestato e condannato a morte dai tedeschi, ma si salvò evadendo dal carcere di San Vittore alla fine dell'estate del 1944.

242 Massimo Recchioni (2013), Francesco Moranino. Una sintesi del caso Moranino è nell'intervento appassionatamente di parte del socialista Guido Bernardi (1923-1995), relatore di minoranza alla Camera dei Deputati, 120-132.

243 Pietro Mancino (2019, 21 febbraio), Il Colle graziò il comunista assassino. Nel sottotitolo: "Saragat salvò il partigiano Moranino, colpevole di 7 omicidi. Altro che i 'reati' di Salvini”. 
In questo modo all'agiografia della Resistenza si accompagna fino ad oggi la leyenda negra descritta da Pansa, e sui singoli fatti è sempre angosciante cercare un equilibrio fra le due opposte narrazioni. ${ }^{244}$

Uno degli ultimi atti del governo Bonomi fu l'istituzione delle Corti Straordinarie d'Assise e di una Sezione Speciale della Corte di Cassazione a Milano, per tentare di far fronte all'alto numero di processi per le epurazioni nell'amministrazione e nell'economia. Con l'estate del 1945, le sanzioni contro il fascismo erano in seria difficoltà e, "consolidata nel 1948", l'egemonia democristiana "garantiva che i pochi strumenti epurativi rimasti sarebbero stati applicati con prudenza, fino alla chiusura delle attività".245

I numerosi tentativi di valutare nel suo insieme l'intricata e contraddittoria epurazione italiana sono giunti quasi tutti, anche se con sfumature diverse, a questa conclusione: "È giudizio unanime di tutti coloro che si sono occupati degli avvenimenti del periodo $1943-46$ che in Italia le sanzioni penali contro gli ex fascisti e i collaborazionisti e l'epurazione nelle amministrazioni pubbliche, nelle aziende private ecc. si sono risolte dopo un anno o due in un nulla di fatto".246

Il magistrato Domenico Peretti-Griva aveva diretto il "Commissariato per l'epurazione" ma, pur riconoscendo che agli inizi qualche serio tentativo di epurazione era stato fatto, si dimise dalla carica suggellando quell'esperienza con un articolo dal titolo tombale: Il fallimento dell'epurazione. L'equilibrata prosa del magistrato individua la deludente transizione dalla Resistenza alla desistenza:

Ormai troppo vasta e spregiudicata era stata l'attuazione dell'epurazione secondo le norme precedenti: troppe le sperequazioni, troppe le attenuazioni in rapporto alle non molte eccessività operatesi nei primi tempi a causa del pur spiegabile spirito di ritorsione, o, talvolta, a causa di indesiderabile faziosità. L'applicazione delle disposizioni sopravvenute non poteva più deviare il corso della prassi precedente che si era nettamente, mano a mano, orientata verso la desistenza. Può anzi darsi che la desistenza sia stata da quelle disposizioni incoraggiata, ancorché si fosse inteso con esse indulgenze ai più, a patto di una maggior severità verso i maggiori colpevoli, specialmente altolocati. Sui poco meno di 400 alti gradi proposti per il congedo

244 Cfr. il discusso libro di Gianfranco Stella (2018), Compagno mitra; sull'argomento Stella è autore di numerose opere sorrette da un'ideologia di destra.

245 Roy Palmer Domenico (1991), Italian Fascists on Trial, 190. Con una sintesi dei dati sulle epurazioni, 89.

246 Romano Canosa (1978), Le sanzioni contro il fascismo, 7. 
dal Commissario per l'epurazione [cioè dello stesso Peretti-Griva], solo poche decine furono collocati a riposo dal Consiglio dei ministri. E codesto esiguo numero si ridusse ulteriormente in misura assai sensibile attraverso le successive revoche. ${ }^{247}$

Questa era ormai la rassegnata atmosfera del tempo. "Il Ponte" intitolava l'intero ultimo fascicolo del 1947 con le parole La crisi della Resistenza. In esso, Il fallimento dell'epurazione appena citato era accompagnato da titoli altrettanto deprimenti: rileggerli oggi permette di ripercorrere, attraverso le parole dei protagonisti, l'inizio della restaurazione clandestina che Piero Calamandrei vedeva calare sull'Italia. ${ }^{\mathbf{2 4 8}}$

Conviene arrestare qui l'esame dell'epurazione in Italia, la cui storia si va scrivendo man mano che è possibile accedere a nuovi documenti. Su questa "legalità incerta" 249 si iniziò a costruire la nuova Italia.

In Germania l'adesione al regime nazionalsocialista era stata vasta; la Germania non aveva conosciuto un movimento di riscatto come quello partigiano in Francia, Italia o Jugoslavia; la rovinosa sconfitta si era conclusa con la divisione del paese, la cui l'occupazione militare gravava sui rapporti dei tedeschi con gli Alleati: insomma, la chiusura dei conti con quel passato si rivelava ardua.

Nel gennaio 1946 una direttiva della Commissione Alleata di Controllo di Berlino fissava per la Germania occupata i criteri della denazificazione, che venne però realizzata in modo diverso nelle varie zone d'occupazione: nella Zona d'Occupazione Sovietica era usata come uno degli strumenti per edificare una società socialista; nelle zone occidentali (accanto ad altre misure) si

247 Domenico Riccardo Peretti-Griva (1947), Il fallimento dell'epurazione, 1077-1078.

248 La crisi della Resistenza (1947). Gaetano Salvemini concludeva l'articolo di apertura dal volenteroso titolo Ottimismo con le parole: "Ecco il pessimismo di sempre che mi riprende. Punto e basta" (Gaetano Salvemini, 1947); seguivano poi Piero Calamandrei (1947), Restaurazione clandestina; Arturo Carlo Jemolo (1947), La battaglia che non fu data; Vittorio Foa (1947), La crisi della Resistenza; Riccardo LeVI (1947), L'azione economica; Roberto Battagla (1947), Il riconoscimento dei partigiani; Alberto Predieri (1947), Garibaldini e partigiani; Dante Livio Bianco (1947), Partigiani e C.L.N.; Carlo Galante Garrone (1947), Guerra di liberazione; PaOlo Barile (1947), La magistratura si ribella alle leggi?; Giovanni Ravagli (1947), La mancata avocazione; Mario BraCCI (1947), Come nacque l'amnistia; Luigi Bianchi d’Espinosa (1947), Il "caso Pilotti”; Mario Vinciguerra (1947), Un equivoco.

249 Achille Battaglia (1955), Giustizia e politica, 335.

$94 \quad$ Capitolo I 
ridusse a un'epurazione del personale che non toccò l'economia, a differenza di quanto avvenne nella zona sovietica. "La denazificazione rappresentò per il governo militare americano uno dei pilastri fondamentali della sua politica di occupazione, mentre non rivestì questa importanza nella zona britannica e soprattutto in quella francese". 250

Con la "Legge della liberazione" del 5 marzo 1946 la denazificazione venne affidata alle autorità tedesche, ${ }^{251}$ che istituirono degli appositi organi a questo fine: le "Spruchkammern". Questi organi dovettero fronteggiare circa 13 milioni di casi: una quantità ingestibile, che produsse ritardi nelle decisioni anche a causa delle procedure adottate. Anzitutto si cominciò dai casi meno gravi, cosicché - nel progressivo alleggerimento della denazificazione - $\mathrm{i}$ casi più gravi vennero giudicati tardi, o non giudicati del tutto. Un'altra causa della mitezza o dell'assenza delle condanne fu l'inversione dell'onere della prova: poiché erano gli imputati stessi che dovevano dimostrare di essere innocenti, gli organi giudicanti furono inondati da scritti di parenti o amici che si attestavano la non colpevolezza. Sono i già ricordati "Persilscheine" (cfr. p. 61), pratica cui non furono estranee neppure le Chiese tedesche. ${ }^{252}$ In conclusione, "il procedimento delle 'Spruchkammern' produsse continui conflitti con il governo militare americano" ed è uno degli esempi dell'insuccesso della denazificazione. ${ }^{253}$

Inoltre, come già si è visto per il Giappone, gli inquisiti dovevano compilare un questionario con circa un centinaio di domande: anche questo

250 Clemens Vollnhals (ed.) (1991), Entnazifizierung. Politische Säuberung, 9, con un'appendice di documenti, statistiche, immagini e bibliografia (354-360).

251 "Gesetz Nr. 104 zur Befreiung von Nationalsozialismus und Militarismus vom 5. März 1946, emanata nella zona americana ed estesa poi nel 1946 alle altre zone occidentali: regolava la procedura con cui ogni Spruchkammer valutava il formulario compilato obbligatoriamente da ogni tedesco maggiorenne e, se non risultava un passato nazionalsocialista a suo carico, gli rilasciava un attestato liberatorio (Entlastungs-Spruch), indispensabile per occupare posizioni pubbliche o semi-pubbliche. Dal 1948 gli Stati Uniti ristabilirono le strutture statali con le vecchie élites. Nella DDR la legge dell'11 novembre 1949 prevedeva che tutti quelli che non erano stati condannati a una pena detentiva superiore a un anno tornavano a godere dei diritti politici e potevano svolgere ogni attività, meno che nella giustzia e nell'amministrazione pubblica.

252 ERnst KLeE (1991), Persilscheine und falsche Pässe (trad. it: Chiesa e nazismo (1993), Torino: Einaudi, 230 pp.).

253 Dennis Meyer (2009), Entnazifizierung, 18-19. 
"strumento d'indagine fu oggetto di molte satire, la più celebre della quali fu probabilmente il romanzo scritto nel 1951 dal conservatore Ernst von Salomon e intitolato appunto Il questionario, che fino al 2003 aveva conosciuto ben 17 edizioni. ${ }^{254}$

L'applicazione delle norme sulla denazificazione venne affidata alle "Deutsche Spruchkammern", uffici che agivano secondo le regole giudiziarie, ma che formalmente non erano dei tribunali. Nella Zona d'Occupazione Sovietica operavano, secondo schemi simili, le "Commissioni per la denazificazione”. Queste ultime cessarono la loro attività nel 1948, e da quella data anche l'attività delle "Spruchkammern" occidentali andò estinguendosi. Complessivamente, "il perseguimento - iniziato tardi e in sostanza insoddisfacente - dei reati nazionalsocialisti da parte degli organi giudiziari tedeschi" conseguì risultati modesti. ${ }^{255}$ Benché questi tribunali fossero stati affidati a un'élite di tedeschi non coinvolti nel passato regime, le condanne furono lievi. Poiché nel determinare la durata delle pene detentive si teneva conto anche della durata dell'internamento nei campi di prigionia alleati alla fine della guerra, "questi tribunali si trasformarono in fabbriche di assoluzioni". ${ }^{256}$ La denazificazione fu tutto sommato un'operazione fallita, che costituì comunque un passo importante verso la democratizzazione della Germania occidentale.

La denazificazione nelle tre zone controllate dagli Alleati occidentali procedette dunque a rilento. Al tempo stesso, con la fine della guerra erano tornati in servizio molti giuristi dell'epoca nazionalsocialista, nettamente (e comprensibilmente) avversi a ogni forma di denazificazione. Esemplare in proposito è uno scritto che Otto Koellreutter - uno dei "terribili giuristi" dell'epoca nazionalsocialista - pubblicava negli anni Cinquanta, intitolan-

254 Ernst von Salomon (1951), Der Fragebogen (trad. franc.: Le questionnaire (1953), Paris: Gallimard, 648 pp.). Questa satira della denazificazione è uno dei tanti "scritti giustificativi” che inondarono la Germania federale nel dopoguerra, creando quasi un genere letterario: le "Rechtfertigungsschriften". Su questo autore: ERnSt von SALOmon (2018), Un destino tedesco.

255 Clemens Vollnhals (ed.) (1991), Entnazifizierung. Politische Säuberung, 8. Venne individuato anche un criterio temporale: "Gli imputati erano accusati di essere rimasti fedeli a quelle organizzazioni [criminose] anche quando, dopo il 1939, le loro finalità criminose erano chiare a tutti".

256 Petra Weber (2000), Justiz und Diktatur, 112. Cfr. anche Ernst Sontag (1950-51), Die Deutschen Spruchgerichte. 
dolo La denazificazione, un peccato contro il diritto e l'onore e presentando la denazificazione come "la guerra fredda civile messa in scena nel 1945". 257

Il primo passo di Koellreutter consiste nel delegittimare gli organi chiamati a realizzare la denazificazione, cioè le Spruchkammern. Per questo egli nega anzitutto che "le Spruchkammern siano 'tribunali' e le loro decisioni siano 'sentenze' secondo il $\$ 839$, c. 2 del BGB” (p. 3); e in questo, come si è visto, ha formalmente ragione. Anche nel suo Deutsches Staatsrecht aveva scritto che "il carattere puramente politico della denazificazione" fa dei "membri delle Spruchkammern dei funzionari politici". Per lui la "Legge per la liberazione dal nazionalsocialismo e dal militarismo"258 è "una legge puramente politica che non può essere valutata dal punto di vista dello Stato di diritto" (p. 4 s.). La soluzione da lui proposta è una legge federale che superi le decisioni delle Spruchkammern (decisioni che risultano diverse da regione e regione) e che colmi "la spaccatura politica provocata nel popolo tedesco dalla denazificazione. Solo così si rafforza la 'democrazia tedesca' e lo 'Stato cristiano di diritto' contro il comunismo".259

Sull'accidentato processo della denazificazione esiste una letteratura tanto vasta quanto discrepante che non è qui possibile esaminare, anche se se ne è appena visto un esempio nel giudizio di Koellreutter. Una guida lineare per seguire quel complesso percorso in Germania è offerta dalla legislazione sulla denazificazione esaminata qui di seguito, con cui gli Alleati dapprima e le autorità tedesche poi hanno cercato di realizzare quel progetto.

Ormai si dà per acquisito che la denazificazione "si debba considerare fallita, ${ }^{260}$ mentre resta aperto un quesito: alla de-nazificazione è seguita una ri-nazificazione? Chi sostiene che la rinazificazione non abbia avuto luogo invita a considerare globalmente gli eventi del dopoguerra: la politica alleata di rieducazione alla democrazia ha convinto buona parte dei giudici

257 Otto Koellreutter [1954], Die Entnazifizierung. "Politici, giuristi e parte del corpo giudicante tedesco hanno evitato finora una chiara indagine dei problemi della denazificazione e hanno così protratto fino ad oggi la guerra fredda civile messa in scena nel 1945" (2 n.n.).

258 Cfr. nota 251.

259 Otto Koellreutter (s.d.), Das Wesen der Spruchkammern, 32. Pubblicato dopo il 1954, perché afferma di non aver avuto risposta allo scritto Die Entnazifizierung (KoellReutter, [1954]), citato nella nota 257. Sulla copertina: "Professore ordinario emerito di diritto pubblico dell'Università di Monaco".

260 Klaus-Dettlev Godau-Schüttke (2001), Von der Entnazifizierung, cpv. 1. 
adattatisi per opportunismo al nazionalsocialismo ad apprezzare i valori democratici e, quindi, a contribuire alla rinascita democratica della Germania. Chi al contrario ritiene che i giudici ritornati in servizio dopo la guerra non avessero accettato del tutto i valori democratici imposti dalle potenze vincitrici asserisce che la rinazificazione della giustizia tedesca ha avuto luogo e che essa contribuisce a spiegare il fallimento dell'intera denazificazione.

Per esperienza personale so che, nel dopoguerra, i giovani già cresciuti nella democrazia (e legati alla cultura tedesca) guardavano con preoccupazione a questo troppo lento distacco dal passato. I miei primi articoli, scritti quando avevo vent'anni, erano dedicati proprio alla "giustizia impunita" della Germania federale e a sentenze retrograde, che sembravano tradire le speranze di chi sperava in una Germania "altra" rispetto al passato appena lasciato alle spalle. ${ }^{261}$ Per questo guardavamo con interesse agli inizi dell'“altra" Germania, anch'essa "risorta dalle rovine": si sperava che imboccasse una direzione diversa, ma venne bloccata dallo stalinismo. Lasciando le esperienze personali, è utile passare dal particolare al generale e concentrarsi sulla Germania federale.

Seguendo la legislazione tedesco-federale sulla denazificazione nel quinquennio dalla fine del 1945 all'inizio del 1951, si nota come nella Germania federale questa normativa voluta dagli Alleati abbia progressivamente perduto vigore e, simmetricamente, come grazie a questo indebolimento molti funzionari pubblici compromessi con varia intensità col nazionalismo siano tornati ai loro posti, portando con sé la vecchia mentalità.

Alla radice di questa normativa è una precisa disposizione dell'Accordo di Potsdam del 2 agosto 1945, le cui formulazioni ritornano spesso alla lettera nella successiva legislazione alleata e tedesca sulla denazificazione: "Tutti i membri del partito nazista in modo non soltanto nominale e tutti i soggetti ostili agli obiettivi perseguiti dagli Alleati vanno allontanati da tutte le posizioni pubbliche e semipubbliche e dai posti di responsabilità in importanti

261 Mario G. Losano (1960, 15 marzo), Giustizia impunita (nel sottotitolo: "Nella Germania di Adenauer sono ancora in servizio centinaia di giudici e procuratori dello Stato che si sono macchiati di gravi delitti durante il regime nazista"). Qualche anno dopo: Mario G. Losano (1963), L'aeroporto non c'entra (su una sentenza del Tribunale del lavoro di Monaco di Baviera); Mario G. Losano (1964), La Germania spiegata agli studenti; Mario G. Losano (1965), Il diritto alla sberla (sul mancato perseguimento penale di atteggiamenti fascisti). 
imprese private. Questi soggetti devono essere sostituiti da persone che per le loro qualità politiche e morali sembrino in grado di collaborare allo sviluppo di istituzioni veramente democratiche in Germania".62

La data del passaggio dell'attività di denazificazione dagli Alleati ai tribunali tedeschi non è sempre indicata un maniera unitaria, anche a causa della frammentazione delle norme in proposito: una data comunemente accettata è quella del $1^{\circ}$ ottobre 1947 , coincidente con l'Ordinanza 110 che verrà esaminata tra poco. $^{263}$ Per chiarezza questa fase di transizione può essere riassunta in sei punti.

[I] Le quattro potenze alleate intrapresero congiuntamente la riforma della giustizia nella Germania sconfitta con il "Proclama n. 3" del 20 ottobre 1945. ${ }^{264}$ In esso sancirono l'indipendenza dei giudici, senza porsi ancora il problema dell'atteggiamento da tenere nei confronti dei giudici in servizio durante il regime nazionalsocialista.

[II] Questo problema venne affrontato pochi giorni dopo dalla legge n. 4 della Commissione Alleata di Controllo (Kontrollrat) sulla Trasformazione del sistema giudiziario tedesco, la quale all'Art. IV stabiliva che "tutti gli ex membri del partito nazionalsocialista che si sono impegnati attivamente per la sua attività, e tutti gli altri soggetti direttamente coinvolti nei metodi punitivi del regime hitleriano, vengono sollevati dai loro uffici di giudici o di pubblici ministeri e non possono essere ammessi a ricoprire questi posti". ${ }^{265}$

In particolare, gli inglesi progettavano per i tribunali tedeschi un azzeramento che ricordava quello previsto per l'intera Germania dal Piano Mor-

262 Potsdamer Abkommen, Cap. III, Sez. A, $\$$ 6: “Alle Mitglieder der nazistischen Partei, welche mehr als nominell an ihrer Tätigkeit teilgenommen haben, und alle anderen Personen, die den alliierten Zielen feindlich gegenüberstehen, sind aus den öffentlichen oder halböffentlichen Ämtern und von den verantwortlichen Posten in wichtigen Privatunternehmungen zu entfernen. Diese Personen müssen durch Personen ersetzt werden, welche nach ihren politischen und moralischen Eigenschaften fähig erscheinen, an der Entwicklung wahrhaft demokratischer Einrichtungen in Deutschland mitzuwirken" (https://www.fdj.de/infoportal/artikel/pdf/potsdamer_abkommen.pdf).

263 Un esame delle varie posizioni è in Bärbel EICKHOFf et al. (1988), Restauration im Recht, 111-112.

264 Proklamation Nr. 3 vom 20. Oktober 1945, Grundsätze für die Gestaltung der Rechtspflege, "Amtsblatt des Kontrollrats für Deutschland", Nr. 2.

265 Kontrollratsgesetz Nr. 4 vom 30. Oktober 1946, Umgestaltung des deutschen Gerichtswesens, "Amtsblatt des Kontrollrats für Deutschland", Nr. 26. 
genthau (cfr. p. 117): da un lato, i tribunali tedeschi avrebbero dovuto essere chiusi per dieci anni, durante i quali sarebbero stati sostituiti con una giustizia di tipo coloniale; dall'altro, si sarebbe usato quel decennio per preparare in senso democratico una nuova leva di giudici. Nella zona sovietica venne realizzato un piano con questo genere di azzeramento della situazione pregressa. Invece nelle tre zone occidentali si decise di escludere dai tribunali soltanto i giudici con un attivo passato nazionalsocialista. Ma nel regime nazionalsocialista tutti avevano dovuto compromettersi, anche se in varia misura: bisognava quindi stabilire sino a che punto doveva giungere il coinvolgimento con il passato regime per giustificare l'esclusione dalla posizione di giudice.

[III] Per precisare queste misure le quattro potenze alleate emanarono la Direttiva n. 24 del 12 gennaio 1946, Per l'allontanamento dagli uffici e dalle posizioni di responsabilità dei nazionalsocialisti e delle persone ostili alle misure degli Alleati. Nell'art. 1 vengono elencati gli uffici e le attività da cui dovevano essere epurati "tutti gli appartenenti al NSDAP in modo non soltanto nominale e tutti i soggetti ostili agli obiettivi perseguiti dagli Alleati". Al loro posto dovevano andare "soggetti che per il loro atteggiamento politico e morale vengono ritenuti capaci di favorire lo sviluppo di vere istituzioni democratiche in Germania" ${ }^{\mathbf{2 6 6}}$ Ecco in che cosa doveva consistere la denazificazione.

[IV] Il sistema previsto dalla Direttiva 24 generò crescenti resistenze fra i tedeschi sia per la difficoltà di organizzare i procedimenti di epurazione nella situazione ancora caotica del dopoguerra, sia per l'eterogeneità dei casi singoli e quindi per le conseguenti arbitrarietà nelle decisioni. Gli Alleati organizzarono in modo più preciso i procedimenti di epurazione con la Direttiva n. 38 del 12 ottobre 1946, Arresto e punizione dei criminali di guerra, dei nazionalsocialisti e dei militaristi, e l'internamento, il controllo e la sorveglianza dei tedeschi potenzialmente pericolosi. ${ }^{267}$ Con questa legge gli Alleati introdus-

266 Kontrollratsdirektive Nr. 24 vom 12. Januar 1946, Entfernung von Nationalsozialisten und Personen, die den Bestrebungen der Alliierten feindlich gegenüberstehen, aus Ämtern und verantwortlichen Stellugen (testo che non ho potuto vedere).

267 Kontrollratsdirektive Nr. 38 vom 12. Oktober 1946, "Verhaftung und Bestrafung von Kriegsverbrechern, Nationalsozialisten und Militaristen und die Internierung, Kontrolle und Überwachung von möglicherweise gefährlichen Deutschen" (testo che non ho potuto vedere). Questa legge si richiama al Potsdamer Abkommen, Cap. III, Sez. A, $\mathbb{} 6$. 
sero una griglia di valutazione articolata in cinque categorie, analoga a quelle già esaminate per i processi di Norimberga e Tokyo: "I. Colpevoli principali; II. Corresponsabili; III. Poco coinvolti; IV. Gregari; V. Assolti” (Hauptschuldige; Belastete; Minderbelastete; Mitläufer; Entlastete).

Nella Zona di Occupazione Sovietica (cfr. Appendice III,3) questa direttiva venne applicata in tutta la sua durezza e, insieme con l'art. 6 della costituzione della DDR, ${ }^{\mathbf{2 6 8}}$ ispirò il diritto penale dell'altro Stato tedesco. In particolare qui affiora il tema della guerra e della pace - oggetto centrale del presente lavoro - perché in base a questa direttiva veniva punito chi, con la diffusione "di notizie tendenziose, ha messo in pericolo e potenzialmente mette ancora in pericolo la pace del popolo tedesco e la pace del mondo".

L'incontro dei quattro Ministri degli esteri a Mosca, nella primavera del 1947, raccomandò al Kontrollrat "di trasferire alle competenti autorità tedesche, con un atto legislativo dell'organo legislativo tedesco, la responsabilità dell'applicazione delle Direttive n. 24 e n. 38", facendo in modo che "con questo provvedimento legislativo si consegua un trattamento unitario di tutti gli ex nazionalsocialisti e militaristi in base alla loro responsabilità. La scelta dei metodi per assolvere questo compito è affidata alla valutazione delle autorità tedesche". Questa misura era resa possibile dal fatto che, nel frattempo, erano stati istituiti i Länder e quindi l'organizzazione della società tedesca stava tornando alla normalità. Però l'unità degli Alleati stava già volgendo al termine e quindi l'applicazione delle norme sulla denazificazione non avvenne in modo omogeneo in tutti i Länder.

[V] Seguendo la direttiva dei Ministri degli esteri, i Länder della zona americana, nel sud della Germania, emanarono il 5 marzo 1946 l' "Ordinanza n. 104 sulla liberazione dal nazionalsocialismo e dal militarismo". Invece i

268 Art. 6, Cost. DDR 1949: "1) Tutti i cittadini godono di uguaglianza di diritti dinanzi alla legge. 2) Qualsiasi propaganda diffamatoria o di boicottaggio contro istituzioni e organismi democratici, qualsiasi incitamento all'assassinio di uomini politici democratici, qualsiasi manifestazione di odio religioso, razziale e contro altri popoli, qualsiasi propaganda militarista e bellicista e tutte le altre azioni dirette contro l'eguaglianza dei diritti costituiscono dei delitti ai sensi del codice penale. L'esercizio dei diritti democratici nello spirito della Costituzione non costituisce una propaganda diffamatoria. 3) Le persone condannate per siffatti reati non possono occupare funzioni né nei servizi pubblici né nei posti direttivi dell'attività economica e culturale. Esse perdono qualsiasi diritto elettorale attivo e passivo" (http://www.dircost.unito.it/cs/pdf/19491007_germaniaRepubblica Democratica_ita.pdf). 
francesi conservarono di fatto il controllo sull'epurazione nei Länder di loro competenza. Nella zona sovietica l'epurazione era stata realizzata anche nell'ambito delle imprese private. Gli inglesi tentarono di emanare una regolamentazione unitaria per tutta la Germania, ma dovettero ripiegare sull'applicazione del Decreto $110,{ }^{269}$ incontrando però una certa resistenza nei Länder di loro competenza e riuscendo infine ad approvare, nel Land dello Schleswig-Holstein, la "Legge per la continuazione e la conclusione della denazificazione" del 10 febbraio 1948.

[VI] La legge dello Schleswig-Holstein del 10 febbraio 1948 fu la prima legge tedesca che decideva come procedere alla denazificazione e, di conseguenza, divenne il modello delle analoghe leggi di altri Länder. ${ }^{270}$ Considerando l'obbligatorietà dell'iscrizione al Partito Nazionalsocialista per tutti i giudici e pubblici ministeri, la legge prevede che l'appartenenza a quel partito non costituisca da sé sola una prova della responsabilità personale, la quale però non è esclusa neppure dalla non appartenenza a quel partito: $\mathrm{i}$ casi andavano valutati singolarmente.

Anche questa legge recepiva la griglia di cinque categorie di valutandi, ma introduceva alcune attenuazioni: chi ricadeva nelle due categorie intermedie (III. Poco coinvolti; IV. Gregari) poteva chiedere la revisione dell'epurazione un anno dopo la sua pronuncia $(\mathbb{1 2}, \mathrm{c} .1)$. Per questa via si poteva porre riparo a rigori eccessivi, ma anche vanificare progressivamente il processo epurativo. A questo svuotamento contribuì anche il divieto di riaprire un procedimento una volta concesso l'attestato liberatorio $(\$ 11)$. Questa ragionevole misura ebbe un effetto negativo nello specifico contesto del dopoguerra tedesco perché, nell'immediato dopoguerra, gli Alleati avevano ammesso nella categoria degli "Assolti" (la quinta e ultima categoria) i giudici e i pubblici ministeri necessari affinché la vita giudiziaria potesse riprendere con un minimo di normalità. Successivamente risultò che non pochi di questi magistrati avevano operato in tribunali speciali o erano comunque gravemente compromessi con il regime: però avevano ricevuto l'attestato

269 Verordnung Nr. 110 zur Übertragung der Entnazifizierungsaufgaben auf die Regierungen der Länder vom 1. Oktober 1947, "Amtsblatt der Militärregierung Deutschland, Britisches Kontrollgebiet”, Nr. 41, pp. 608 s.

270 Gesetz zur Fortführung und zum Abschluß der Entnazifizierung, 10. Februar 1948, "Gesetzund Verordnungsblatt für Schleswig-Holstein”, 1948, pp. 33 s. 
liberatorio e quindi - non essendo più possibile epurarli grazie al $₫ 11$ restarono al loro posto nonostante le norme sulla denazificazione.

Non sempre era possibile reintegrare le persone epurate nella posizione originaria o equivalente. Il regolamento di attuazione prevedeva però che il funzionario potesse essere reintegrato in una posizione inferiore oppure che, in assenza anche di quest'ultima, dovesse ricevere un'indennità mentre era in attesa della collocazione definitiva. Questo imponeva però costi quasi insostenibili per un piccolo Land ancora alle prese con le difficoltà postbelliche. $^{271}$

Allo smantellamento della denazificazione nella magistratura contribuì anche una strana regola propria della sola zona britannica e dovuta alle pressioni dei massimi livelli della magistratura stessa: nei tribunali, a ogni assunzione di un assolto in base alla legge sull'epurazione doveva corrispondere l'assunzione di un colpevole riconosciuto come tale dalla stessa legge.

Di fronte a queste diversità tra i vari Länder, il parlamento federale approvò il 15 ottobre 1950 una direttiva secondo la quale i Länder dovevano concludere l'epurazione. Dei cinque livelli previsti dalla griglia di valutazione, i tre livelli inferiori non sarebbero più stati perseguibili dopo il $1^{\circ}$ gennaio 1951 e di conseguenza si sarebbero estinti i processi ancora pendenti. Gli accusati dei due comportamenti più gravi (I. Colpevoli principali; II. Coinvolti) potevano chiedere entro il 3 marzo 1951 di essere inclusi in una categoria di livello inferiore, cioè in una delle categorie i cui procedimenti venivano azzerati.

È comprensibile che un'intera nazione non volesse vivere in un perenne stato d'accusa; e infatti i parlamenti dei Länder si affrettarono a recepire la normativa del parlamento federale ed emanarono le leggi di conclusione del processo di denazificazione. Nello Schleswig-Holstein la denazificazione venne conclusa nel modo più radicale, cioè rendendola di fatto impossibile: infatti due decreti del 1952 prescrivevano di distruggere i documenti ovvero di cancellare (nei documenti che non era possibile distruggere) le frasi che potessero servire di base per future accuse di coinvolgimento nel nazionalsocialismo. ${ }^{272}$

271 CaRmen Smiatacz (2015), Ein gesetzlicher Schlussstrich?, 297.

272 Erlass vom 23. September 1952, “Amtsblatt für Schleswig-Holstein”, 1952, 396; Erlass vom 18. Dezember 1952, “Amtsblatt für Schleswig-Holstein”, 1953, 2. 
Intanto il 23 maggio 1949 era entrata in vigore la costituzione della Germania federale e nell'agosto dello stesso anno era stato eletto per la prima volta il parlamento federale, il Bundestag.

L'articolo 131 della nuova costituzione prevedeva un'ulteriore possibilità di svuotamento della denazificazione. ${ }^{273}$ In base a questa norma, i funzionari pubblici e i magistrati allontanati dalle loro cariche perché compromessi con il nazionalsocialismo potevano chiedere di essere reintegrati nei posti occupati sotto quel regime. Il Bundestag approvò nel 1951 una legge applicativa dell'articolo 131 della costituzione che permetteva la riammissione in servizio degli ex nazionalsocialisti che non ricadessero nelle prime due categorie (I. Colpevoli principali; II. Coinvolti). ${ }^{274}$ L'esito della votazione dimostra l'insofferenza per la denazificazione non solo nella popolazione, ma anche nella stessa classe politica tedesca: la legge venne approvata con due sole astensioni.

Negli anni successivi, però, la Guerra fredda e le continue schermaglie tra i due Stati tedeschi portarono alla luce molti inaccettabili comportamenti dell'epoca nazionalsocialista da parte di giudici ritornati in carica nella Germania federale. Nel 1965 la DDR pubblicò un Braunbuch $^{275}$ sulle presenza di 1800 ex nazisti nell'apparato statale della Germania federale. La documentazione era nella quasi totalità autentica e corretta, anche se presentata con toni troppo propagandistici. Le polemiche che ne scaturirono obbligarono i politici della Germania federale ad approvare una modifica della legge sui

273 Art. 131 GG: [Stato giuridico dei dipendenti pubblici.] "La situazione giuridica delle persone, compresi i rifugiati e gli espulsi, che si trovavano 1'8 maggio 1945 in pubblico servizio, dimesse per motivi differenti da quelli previsti dal diritto che regola il pubblico impiego o i contratti collettivi e che finora non sono impiegati, o, se lo sono, si trovano collocati in una posizione diversa da quella precedente, dev'essere regolata con legge federale. Ciò vale anche per le persone, compresi i rifugiati e gli espulsi, che 1'8 maggio 1945 avevano diritto all'assistenza e che non ricevono, per motivi differenti da quelli di servizio o contrattuali, alcuna assistenza, o la ricevono, ma non in corrispondenza del loro ufficio. Fino all'entrata in vigore della legge federale, salvo che il legislatore del Land non provveda diversamente, non possono far valere diritti” (http://www.consiglioveneto.it/ crvportal/BancheDati/costituzioni/de/zGermania_sin.pdf).

274 Gesetz zur Regelung der Rechtsverhältnisse der unter Artikel 131 des Grundgesetzes fallenden Personen, vom 11. Mai 1951, BGBl. 1951, 307-308.

275 Braunbuch. Kriegs- und Naziverbrecher in der Bundesrepublik und in Westberlin. Staat, Wirtschaft, Verwaltung, Armee, Justiz (1965). Una valutazione di queste polemiche fra i due Stati tedeschi è in Klaus Bästlein (2009), "Nazi-Blutrichter als Stützen des Adenauer-Regimes". 
giudici, che prevedeva: "Un giudice o un pubblico ministero attivo nella giustizia penale dal $1^{\circ}$ settembre 1939 al 9 maggio 1945 può essere messo a riposo su sua richiesta. ${ }^{276}$ Si permetteva così una specie di denazificazione volontaria e retribuita, che però ebbe poco successo: ne fecero uso 149 magistrati su $15.000 .^{277}$

Col 1951 si può quindi ritenere che nella Germania federale sia terminato il processo di denazificazione, senza dubbio il più complesso fra quelli più tentati che realizzati nei tre Stati dell'ex Asse.

Tuttavia non si può chiudere il discorso sulla rieducazione e sulle epurazioni senza almeno accennare alla rinascita nel secolo XXI dei movimenti di estrema destra che stanno conquistando importanti posizioni istituzionali in tutto il mondo. Le misure postbelliche avevano momentaneamente attenuato le pulsioni autoritarie sempre presenti in ogni contesto sociale, ma la loro presenza minoritaria e sotterranea si rafforza e si manifesta ciclicamente in forme storiche diverse.

Theodor Adorno, richiamandosi una sua conferenza del 1959, già nel 1967 aveva parlato all'Unione degli Studenti Socialisti Austriaci degli Aspetti del nuovo radicalismo di destra ed aveva confrontato la situazione postbellica della Germania con quella dell'Italia. In Germania "nel 1945 il vero panico, l'effettiva presa di distanza dal regime e dalla sua disciplina non aveva avuto luogo, come in Italia, ma si era rimasti coerenti sino alla fine. In Germania l'identificazione con il sistema [nazionalsocialista] non è mai stata distrutta in modo realmente radicale e ciò offre naturalmente ai gruppi cui mi riferisco una delle possibilità di richiamarsi ad essa." ${ }^{278}$ Adorno metteva dunque in guardia contro il nazismo ancora latente: ed ecco che "un livre d'hier devient le miroir d'aujourd'hui", ${ }^{279}$ perché allora si andava affermando il partito neonazista "Nationaldemokratische Partei Deutschlands" (NPD), mentre oggi è in ascesa il partito di estrema destra "Alternative für Deutschland" (AfD).

276 Deutsches Richtergesetz vom 1. Juli 1962, $\$ 116$ (https://www.gesetze-im-internet.de/drig/ index.html).

277 Hans Wrobel (1989), Verurteilt zur Demokratie, 151, nota 85.

278 Theodor W. Adorno (2019), Aspekte des neuen Radikalismus, 17. Questa conferenza verrà inclusa nel volume Vorträge 1949-1968, in stampa presso la casa editrice Suhrkamp.

279 Johanna Luyssen (2019, 16 ottobre), Adorno, icône de la lutte, 13. 
Il tema della rinascita delle destre ritorna in una conferenza che Umberto Eco tenne nel 1995 agli studenti della Columbia University per ricordare che il fascismo mussoliniano o il nazismo hitleriano non possono ritornare oggi nelle forme di allora, ma possono manifestarsi in nuove forme autocratiche. Sono le pulsioni autoritarie sempre presenti in ogni società, cui Eco dà il nome di "fascismo eterno" o "Ur-fascismo". L'epurazione, come ogni altra misura ad essa equivalente, tenta di cancellare le tracce di un fascismo storico, ma non può cancellare il "fascismo eterno", cioè l'eterna pulsione autoritaria presente in ogni società: "L'Ur-Fascismo può ancora tornare sotto le spoglie più innocenti. Il nostro dovere è di smascherarlo". 280

\section{Il superamento del passato: le riparazioni belliche}

Le guerre sono eventi distruttivi e i vincitori tendono a rifarsi sui vinti per le distruzioni e le perdite subìte. In Europa, una svolta nella concezione delle riparazioni belliche si ebbe a partire dalla Prima guerra mondiale, quando si cominciò a commisurare l'entità delle riparazioni non solo in base a un fatto - il dato incontrovertibile della sconfitta - ma anche in base a un valore: la colpa di aver provocato la guerra. Quindi il vinto non doveva soltanto ripagare, ma anche riparare: doveva indennizzare non perché aveva perduto la guerra, ma perché l'aveva provocata.

Questa "moralizzazione del concetto" ${ }^{281}$ provocò forti risentimenti nella Germania dopo il Trattato di Versailles e non arrecò vantaggi materiali ai vincitori, che cercarono di limitarla nelle misure riparatorie previste alla fine della Seconda guerra mondiale. Il Giappone aveva invece una diversa tradizione culturale e religiosa alle spalle. Lo storico Akio Nakai, di fronte alla concezione occidentale insita nel caso specifico delle riparazioni belliche, si chiede: "Ma deve rimanere un eterno e inestinguibile complesso di colpa, rievocabile in ogni momento?", e spiega che la concezione giapponese - espressa nei vocaboli "tsumi" e "kegare" - consente a chi espiato la propria colpa "di riprendere legalmente e senza gravami la precedente attività in campo economico o sociale". Questo modo di concepire il mondo "spiega

280 Umberto Eco (2018), Il fascismo eterno, 50.

281 JöRg FISCH (1992), Reparationen und Entschädigungen; cfr. il paragrafo Von den Kriegsentschädigungen zu den Reparationen: die Moralisierung des Begriffes, 19-25, con l'analisi della terminologia usata nei trattati. Vasta bibliografia, 327-343 (con 29 tabelle). 
molto dell'atteggiamento giapponese dopo la guerra e dopo il trattato di pace di San Francisco".282

Alla fine della guerra gli Stati sconfitti dovettero restituire ai soggetti internazionali, originariamente proprietari, tanto i territori occupati quanto i beni trafugati. La negativa esperienza della Prima guerra mondiale gravava sulla conferenza di Potsdam del luglio 1945, nella quale le potenze vincitrici stabilirono i criteri delle riparazioni belliche a carico degli Stati sconfitti. Perciò esse rinunciarono a stabilire per la Germania (e anche per il Giappone) l'obbligo di pagare le riparazioni in scadenze annue e in denaro, da ottenere attraverso l'eccedenza della produzione industriale. Questa misura aveva infatti portato a un rafforzamento dell'industria tedesca, grazie al quale la Germania aveva potuto ritornare in breve tempo al livello di grande potenza, scatenando la Seconda guerra mondiale.

Dopo quest'ultima, dunque, i vincitori imposero alla Germania di pagare le riparazioni in natura e mediante il trasferimento di impianti industriali, in primo luogo quelli dell'industria bellica: è la Demontage ${ }^{\mathbf{2 8 3}}$ di cui si parlerà nelle pagine dedicate alla Germania. Venne in sostanza stabilito il principio, applicato anche al Giappone, di fondare le riparazioni sul trasferimento sia dei beni situati all'estero, sia dei prodotti e delle industrie non necessarie al mantenimento dell'economia di pace. In particolare, le industrie belliche non convertibili a una produzione civile andavano o smantellate, o smontate e trasferite ai vincitori nell'ambito delle riparazioni belliche.

Invece gli Alleati ritornarono ai princìi di Versailles nei riguardi dell'Italia (e degli altri Stati alleati dell'Asse: Romania, Bulgaria, Ungheria, Finlandia). Essi stabilirono cioè che l'Italia dovesse versare in denaro l'ammontare delle riparazioni, attraverso l'eccedenza della produzione. Inoltre vennero confiscati i beni italiani presenti nel territorio degli Stati vincitori. Ancora una volta, questo trattamento di relativo favore nasceva dal fatto che era sconfitta l'Italia repubblicana e fascista del Nord, mentre era a fianco degli Alleati vincitori l'Italia monarchica e parlamentare del Sud.

Lo stesso principio doveva essere applicato ai singoli danneggiati durante le occupazioni o la guerra: le potenze alleate prima, i singoli Stati sconfitti

282 Aкio Nakai (1988), Die "Entmilitarisierung” Japans, 19-20.

283 In questo contesto delle riparazioni uso il termine corrente in tedesco, cioè il sostantivo femminile Demontage, e non l'originale francese, il sostantivo maschile démontage che ne è all'origine. 
poi, si impegnarono verso i privati a restituire loro i beni sottratti ovvero a indennizzarli per i danni materiali e immateriali sofferti. Si trattava, per esempio, di indennizzare chi aveva subito pratiche di lavoro coatto, chi era stato allontanato dal servizio pubblico per motivi razziali o politici, ovvero di ristabilire i rapporti di proprietà sui beni espropriati forzosamente agli ebrei. Ovviamente nei casi specifici la situazione era diversa nei tre Stati: per esempio, la pratica del lavoro coatto di massa in Germania e in Giappone "non ha corrispettivo nel caso italiano, anche se non sono mancati esempi di sfruttamento di manodopera da parte italiana, ad esempio in Libia" ${ }^{284}$

Sul complesso tema delle riparazioni è possibile procedere qui soltanto per accenni relativi ai tre Stati dell'ex Patto Tripartito, rinviando ai testi citati in nota per un primo approfondimento.

Il Giappone - in base all'art. 11 della Dichiarazione di Potsdam - poteva conservare le industrie necessarie per la propria economia di pace e per il pagamento in natura delle riparazioni, ma non le industrie belliche. Il problema delle riparazioni si presentò più semplice in Giappone che in Europa, perché in Giappone il controllo postbellico era detenuto di fatto dai soli Stati Uniti e quindi non potevano sorgere contrasti tra le varie potenze vincitrici, come in Europa. Il governo militare degli Stati Uniti era affiancato soltanto dalla Far Eastern Commission, con poteri limitati. ${ }^{285}$ Anche per questa ragione non fu necessario progettare una divisione del Giappone in zone di occupazione, come in Germania.

Nell'aprile 1946 venne reso pubblico il piano di Edwin W. Pauley, ambasciatore di Truman. ${ }^{286}$ Esso prevedeva di trasferire ai paesi asiatici danneggiati dalla guerra gli impianti industriali giapponesi non necessari al Giappone per il mantenimento del livello di vita prebellico: qualcosa di analogo, ma in forma più attenuata, rispetto a quanto si vedrà per la Germania. Però il piano di Pauley venne ritenuto troppo duro e suscitò le proteste non solo dei giapponesi, ma anche dello stesso MacArthur, perché avrebbe impedito la

284 Giovanni Contini et al. (eds.) (2010), Memoria e rimozione, 10 (Introduzione).

285 La Far Eastern Commission era composta da USA, Unione Sovietica, Cina, Gran Bretagna (Stati con diritto di veto) e, inoltre, da Australia, Francia, Indonesia, Canada, Nuova Zelanda, Olanda, Filippine. Cfr. Hubertus SeIfer (1971), Die Reparationen Japans; sulla Far Eastern Commission, 67-71.

286 Kodansha Encyclopedia of Japan (1983), s.v. Reparations for Southeast Asia. 
ricostruzione industriale del Giappone. Si cominciava infatti a comprendere che il Giappone sarebbe stato il principale alleato degli USA in Oriente. Perciò dal 1946 al 1949 il piano Pauley venne progressivamente disapplicato e poi abbandonato, anche per le difficoltà di stabilire sia l'ammontare delle riparazioni, sia la loro distribuzione fra i singoli Stati asiatici coinvolti nella guerra.

Accanto allo smontaggio (limitato) delle industrie belliche, le riparazioni giapponesi comprendevano altri cespiti, alcuni dei quali anche importanti: la confisca delle proprietà dello Stato giapponese all'estero, il pagamento dei costi delle truppe d'occupazione, la cessione dei territori all'estero (come il Manciukuò) e altre voci ancora. Inoltre gli Stati Uniti concessero al Giappone numerosi crediti. I dati economici di questi pagamenti sono riassunti in numerosi dati e tabelle, il cui autore ricorda però la discussa attendibilità dei dati. ${ }^{287}$

Le opposizioni statunitensi al piano Pauley e i contrasti interni alla Far Eastern Commission ritardarono sino al febbraio 1947 l'inizio dello smontaggio delle industrie giapponesi, che terminò nel maggio 1950: esso si rivelava di dimensioni notevolmente inferiori rispetto a quello avvenuto in Germania. Intanto la vittoria dei comunisti in Cina nel 1949 e l'inizio della guerra di Corea nel 1950 avevano trasformato l'atteggiamento degli Alleati verso il Giappone, divenuto ora il loro principale alleato in quello scacchiere: ogni indebolimento dell'economia giapponese sarebbe stato in contrasto con le nuove esigenze strategiche. Per questo il trattato di pace di San Francisco del 1951, all'art. 15, stabilì per il Giappone soltanto un generico obbligo di riparazione, fondato sulla sua colpa nel provocare la guerra. Ma le sanzioni economiche si rivelarono meno onerose del prevedibile e il numero degli Stati aventi diritto alle riparazioni venne limitato a quelli che erano stati occupati dal Giappone: le due Cine non presentarono richieste, e così anche la Cambogia, il Laos, l'India e l'Unione Sovietica.

L'insistenza soprattutto delle Filippine portò a includere nel trattato di pace di San Francisco l'obbligazione per il Giappone di negoziare le richieste di riparazioni con gli Stati che ne facessero richiesta: le Filippine, Burma, l'Indonesia e (dopo il 1954) il Vietnam del Sud. Dopo lunghe trattative, e

287 Le prestazioni del Giappone sono riassunte in JöRG FISCH (1992), Reparationen und Entschädigungen, 233-239, con tabelle riassuntive. 
anche accuse di collusioni, si giunse a concludere accordi con tutti e quattro i paesi. I pagamenti vennero fatti fornendo beni, le cui materie prime erano fornite dal paese richiedente. Questo era anche un modo per riaprire al Giappone l'accesso alle materie prime dell'Asia, nel corso di una progressiva normalizzazione postbellica.

Le trattative bilaterali del Giappone con i paesi sopra indicati collocarono il Giappone in una posizione di forza, che gli permise di allungare i tempi delle trattative fino al 1958 e di ottenere una riduzione delle richieste. Alla fine, "le riparazioni andavano onorate con beni e servizi, non in denaro. Erano valori estremamente bassi rispetto alle capacità produttive del Giappone, soprattutto tanto tempo dopo la fine della guerra. Era poco anche in confronto con le riparazioni della Germania federale dopo il 1952. Gli americani si erano imposti completamente. [...] Il Giappone riconobbe ed apprezzò appieno questo trattamento di riguardo, senza polemizzare sulle regole, ma riconoscendo come grande e fino ad allora unico progresso nella storia il fatto che per la prima volta, di fronte alle riparazioni belliche, c'era stata una libera trattativa tra debitori e creditori".288

Anche il Giappone aveva praticato su vasta scala il lavoro forzato nei territori occupati, adottando nei riguardi dei cinesi, considerati nemici, una politica diversa da quella diretta ai coreani, per i quali si mirava all'assimilazione. Comunque i giapponesi si avvalsero di mano d'opera coatta in tutta l'area della "Co-Prosperità". ${ }^{289}$

Un problema particolarmente controverso è quello delle donne asiatiche costrette a prostituirsi nel corso della conquista giapponese sul continente asiatico. La polemica per il riconoscimento dovuto alle "comfort women" per le sofferenze subìte si è aperta tardi, nel 1991, ma riaffiora costantemente, come si è accennato più volte.

In Occidente si è tornati a discuterne nel 2018, grazie all'esordiente scrittrice coreano-americana Mary Lynn Bracht, ${ }^{290}$ che con il suo romanzo Figlie del Mare "vuole riportare l'attenzione dell'Occidente su di loro, unendosi a

288 Jörg FISCH (1992), Reparationen und Entschädigungen, 141-142.

289 Mark SPOerer (2007), Zwangsarbeitsregimes im Vergleich (la prima pubblicazione di alcuni saggi di SEIDEL (ed.) (2007), Zwangsarbeit, è in Geschichte und Gesellschaft, 2005, 1, Göttingen: Vandenhoek \& Ruprecht, 144 pp.).

290 Mary Lynn Bracht (2018), Figlie del Mare; il titolo originale è White Chrysanthemum. Sulle "comfort women", cfr. nota 211, p. 78. 
una protesta cominciata nel 1991, quando una delle vittime, Kim Hak-sun, denunciò gli orrori che fu costretta a subire"; infatti "la loro tragedia è ancora ingiustamente sconosciuta ai più, è un capitolo mancante nei libri di storia". ${ }^{291}$ La giovane scrittrice racconta così la genesi del suo romanzo: "Sono figlia di una donna coreana e di un soldato americano. Sono nata in Germania e cresciuta nel Texas. Fino al college pensavo di diventare una pilota di fighter come mio padre, poi ho preso un'altra strada. Ho studiato storia e antropologia, e ho scoperto questo 'segreto non segreto'. Le donne coreane, infatti, sanno dell'esistenza delle 'comfort women', ma non ne parlano. È stata la testimonianza di una donna olandese che viveva in Indonesia, anche lei ridotta in schiavitù negli anni della guerra, a smuovere l'opinione pubblica negli anni '90. Da noi il senso di vergogna, e la paura di parlare, hanno tenuto nascosto il segreto per anni". ${ }^{292}$ Ancora oggi negli Stati asiatici si ritorna su questo tema solo se costretti (e più per sopire che per risarcire), mentre vanno scomparendo le donne che soffrirono quella violenza.

In conclusione, nel caso del Giappone le riparazioni belliche furono contenute, nonostante gli iniziali annunci minacciosi, perché erano mutati i rapporti di forza nel quadro geopolitico mondiale e gli Stati Uniti avevano bisogno dell'appoggio dei loro ex nemici. Fisch traccia questo quadro complessivo:

"La politica delle riparazioni porta la firma degli Stati Uniti. I fini già accertati per l'Italia e per la Germania occidentale emergono qui [per il Giappone] ancora più chiari. Dopo i progetti iniziali di distruggere il potenziale industriale dominò il principio del non gravare la mano, con l'eccezione dei beni all'estero e del knowhow. Solo i costi dell'occupazione avevano la precedenza. Le prestazioni per le riparazioni vennero tenute basse e la ricostruzione dell'industria venne favorita con la concessione di crediti. Solo dopo la creazione di una solida base vennero richieste ulteriori prestazioni. Il Giappone ne uscì in misura decisamente più vantaggiosa che la Germania occidentale e anche in modo non più sfavorevole dell'Italia".293

291 Nathascia Severgnini (2018, 4 maggio), Schiave dei soldati, 46.

292 Intervista a Mary Lynn Bracht di Michela Ravalico: Mary Lynn Bracht (2018, 4 maggio), "Svelo il Giappone delle ipocrisie ...".

293 Jörg FISCH (1992), Reparationen und Entschädigungen, 237-238. 
La posizione dell'Italia nelle trattative di pace si rivelò particolarmente ambigua perché, da un lato, era stata un paese alleato della Germania nazista e quindi colpevole della guerra di aggressione, ma, dall'altro, dopo l'8 settembre 1943 era stata anche alleata degli anglo-americani ed aveva quindi contribuito alla vittoria sul nazismo. Per questa ragione gli Alleati applicarono all'Italia condizioni meno gravose che per la Germania e il Giappone. $\mathrm{Da}$ un lato gli anglo-americani tendevano a ridurre al minimo le pretese verso un'Italia in gravi difficoltà economiche, dall'altro gli Stati che avevano sofferto un'occupazione italiana chiedevano delle riparazioni (in primo luogo l'Unione Sovietica).

Secondo la ricostruzione di Fisch, le richieste di Unione Sovietica, Jugoslavia, Albania, Etiopia, Egitto e Grecia ammontavano a 15 miliardi di dollari, a fronte dei quali l'Italia offrì 300 milioni di dollari, sostenuta in ciò dagli Alleati occidentali. ${ }^{294}$ Nei lunghi dibattiti, "per i sovietici era importante non tanto la prestazione materiale, quanto il fatto che si procedesse secondo gli stessi principi applicati agli altri alleati di Hitler. Ed era proprio quello che gli americani volevano a tutti i costi impedire: essi volevano realizzare le loro idee, che erano centrali per la Germania, ma che dovevano valere anche per l'Italia, l'Austria e il Giappone, consistenti nel realizzare una politica economica nella quale, in fondo, non c'era posto per alcuna riparazione." ${ }^{295}$ Infine le riparazioni a carico dell'Italia vennero fissate nel 1947 a condizioni molto favorevoli: "In particolare, per le riparazioni provenienti dalla produzione corrente vennero incluse tante clausole a tutela dell'Italia, che sorprende che ci sia stata ancora una qualche prestazione".296

In Italia, inoltre, i vincitori si preoccuparono anche della salvaguardia del patrimonio artistico danneggiato dalla guerra. I danni subìti dal patrimonio artistico italiano erano ingenti e bisognava di impedire che venissero aggravati ulteriormente dall'incuria o da misure non appropriate. ${ }^{297}$ Nella fase finale della guerra, tra il 1943 e il 1945, gli Alleati anglo-americani crearono una divisione incaricata di prendersi cura dei monumenti danneggiati dai tedeschi in ritirata o dai bombardamenti degli stessi Alleati. Questi "Monu-

294 Jörg FISCH (1992), Reparationen und Entschädigungen, 89. Con ricostruzione dell'aspetto economico di queste trattative, 86-92.

295 JöRG FISCH (1992), Reparationen und Entschädigungen, 88.

296 Jörg FISCH (1992), Reparationen und Entschädigungen, 172-173.

297 Carlotta Coccoli (2017), Monumenti violati. 
ments Officers" erano architetti, direttori di musei, archeologi e altri esperti incaricati di provvedere ai "first aid and repairs", in stretta collaborazione con i sovrintendenti artistici italiani. Nel dopoguerra le loro imprese divennero oggetto di un romanzo, da cui venne tratto il film Monuments Men. ${ }^{298}$ In un grande affresco dei furti d'arte da Napoleone a Hitler compaiono anche le "Monuments Women" italiane: la romana Palma Bucarelli, la milanese Fernanda Wittgens, la torinese Noemi Gabrielli. ${ }^{299}$

Il cambiamento di fronte dell'8 settembre 1943 trasformò gli italiani da alleati in nemici non solo negli Stati Uniti, ma anche in Giappone: "Le relazioni tra il Giappone imperiale e l'Italia degenerarono. Poco dopo l'8 settembre le autorità giapponesi entrarono nell'Ambasciata italiana a Tokyo e arrestarono gli italiani presenti. Ad eccezione di Mirko Ardemagni e di un pugno di fascisti irriducibili che giurarono fedeltà alla Repubblica Sociale Italiana, tutti gli altri si rifiutarono di farlo e vennero inviati in vari campi di internamento sparsi per il Giappone, dove vennero trattati duramente”. 300 Per questi internamenti il governo italiano richiese le scuse del governo giapponese e un indennizzo, richiesta che venne lasciata cadere con il ristabilimento delle normali relazioni nel 1952. ${ }^{301}$ Simmetricamente, a Pistoia i partigiani uccisero l'addetto navale giapponese e, infine, l'Italia dichiarò guerra al Giappone il 14 luglio 1945 (quando già la Germania si era arresa): una dichiarazione puramente simbolica, poiché l'Italia non disponeva di mezzi militari per raggiungere quell'area, ma non senza conseguenze sui cittadini italiani residenti in Giappone, divenuti d'un tratto sudditi di una potenza nemica. In proposito è già stata ricordata l'esperienza di Fosco Maraini e della sua famiglia.

Mentre per la Germania si esamineranno le riparazioni nell'ambito dell'industria e delle infrastrutture, è interessante, per l'Italia, soffermarsi sui risarcimenti nei riguardi delle persone danneggiate dalle politiche del fascismo.

298 Robert M. Edsel (2009), Monuments Men (trad. it.: Monuments Men. Eroi alleati, ladri nazisti e la più grande caccia al tesoro della storia, Sperling \& Kupfer 2013, XXV-430 pp.). Da questo libro è tratto il film del 2014 The Monuments Men, diretto e interpretato da George Clooney.

299 Alessandro Marzo Magno (2017), Missione grande bellezza.

300 Reto Hofmann (2015), The Fascist Effect, 158.

301 Reto Hofmann (2015), The Fascist Effect, 173-174, nota 4. 
Al momento dello scoppio della Seconda guerra mondiale l'Italia aveva già una legge sui danni di guerra risalente al 1919, che prevedeva un vero e proprio diritto soggettivo al risarcimento del danno bellico: per i beni mobili, il risarcimento doveva corrispondere al loro valore per l'acquisto e, per i beni immobili, al valore occorrente per la loro riparazione o ricostruzione, in base ai costi del momento in cui essa avveniva. La sua applicazione si rivelò macchinosa, perché i soggetti danneggiati preferivano non accettare l'offerta di liquidazione, ma adire $\mathrm{i}$ tribunali per conseguire un risarcimento più vantaggioso. L'attuazione della legge del 1919 ebbe quindi costi alti e tempi lunghi, tanto che si concluse circa 18 anni dopo la sua emanazione. Questa esperienza indusse il legislatore, al momento dello scoppio della guerra, ad emanare una nuova legge che presentava l'indennizzo di guerra come una concessione dello Stato, e non più come un diritto del danneggiato. ${ }^{302}$

La legge del 1940 è meno favorevole al danneggiato: i beni mobili vengono valutati in base al loro valore commerciale al momento del danno, mentre $\mathrm{i}$ beni immobili vengono risarciti in base al loro valore nel mese anteriore allo scoppio della guerra, detraendo però il valore dell'eventuale parte residua. Inoltre la nuova legge non prendeva più in considerazione $\mathrm{i}$ danni alle persone, mentre invece la legge precedente prevedeva una pensione privilegiata di guerra in caso di invalidità $o$, per i superstiti, in caso di morte per causa di guerra. Questa normativa, emanata all'inizio della guerra, non poteva prevedere l'estensione e la gravità dei danni che sarebbero seguiti e si rivelò di impossibile attuazione su un territorio nazionale frammentato: infatti dal 1943 venne applicata soltanto nelle zone occupate dai tedeschi. Una serie di provvedimenti frammentari emanati dal 1944 aumentò la confusione nell'applicazione delle norme, generando gravi sperequazioni negli indennizzi.

Le norme del 1940 vennero progressivamente estese alle colonie italiane in Africa e, dopo la fine del conflitto italo-greco, all'Albania. Con la fine della guerra si moltiplicarono le norme settoriali per la ricostruzione dell'edilizia, dell'agricoltura e delle imprese civili indispensabili per la ripresa economica. Intanto anche in Italia dal 1948 si poneva il problema dei profughi dalla Dalmazia, dalla Venezia Giulia e dall'Egeo. Infine, poiché biso-

302 I due testi fondamentali sono il Testo Unico del 27 marzo 1919, n. 426, e la Legge del 26 ottobre 1940, n. 1543; Luciano Boccini (1940), Il problema dei danni di guerra (con bibliografia); Antonio Visco (1943), La riparazione dei danni di guerra. 
gnava mettere ordine anche nella contabilità della lotta partigiana, col Decreto legislativo del 19 aprile 1948, n. 517, lo Stato assumeva le obbligazioni contratte dalle formazioni partigiane sia in denaro, sia in beni o servizi, purché risultasse che si trattava di prestazioni ricevute ai fini della guerra di liberazione. Però questa ragionevole prescrizione si rivelò di difficile applicazione, perché chi agiva nella clandestinità o combatteva in montagna non sempre poteva rilasciare ricevute, e qualcuno aveva certo approfittato dell'eccezionalità della situazione: di qui la difficoltà di tracciare un confine processuale tra comportamenti leciti e quelli illeciti. Ma, in generale, molti casi individuali erano difficili da indennizzare per la fluidità in cui viveva l'intera nazione negli anni di guerra.

Tra le persone che avevano subito danni materiali o morali in seguito alle condanne fasciste, un gruppo significativo era quello degli ebrei allontanati dalle professioni liberali e dalle università. ${ }^{303}$ Un esemplare case study per spiegare quanto fosse complessa ogni forma di riparazione è il defatigante percorso burocratico di Renato Treves, perseguitato politico e razziale, per essere riammesso alla cattedra da cui era stato allontanato nel 1938: percorso ricostruito da Carlo Nitsch con grande precisione. ${ }^{304}$

Nel caso di Treves, l'iter burocratico si dipanava per posta tra il consolato italiano di Córdoba in Argentina e l'Italia, con l'invio della documentazione "non appena le comunicazioni lo permettano", scriveva Treves (p. 70). Inoltre le cattedre universitarie di chi era stato allontanato erano intanto occupate da altri docenti. Adolfo Ravà, amico e collega di Treves, gli scriveva: "Con l'abolizione delle leggi razziali rientrano in servizio, oltre me, anche Donati e Levi di filosofia del diritto: ciò disturba quelli che sono ai nostri posti, ed io [mi] rammarico di dover disturbare Bobbio": il giovane Norberto Bobbio, infatti, era stato chiamato alla cattedra da cui Ravà era stato rimosso perché ebreo; in realtà, Bobbio "era stato richiamato a Torino, ma non c'è posto, essendo rientrati due professori ebrei”. Ravà cita poi altre analoghe situazioni, e conclude: "Tutto ciò determina un ambiente poco simpatico; perché,

303 Cfr. per esempio AnNalisa CAPristo (2002), L'espulsione degli ebrei. Questo studio si limita al periodo dell'espulsione, non al ripristino postbellico della situazione, che è invece trattato in Mario Toscano (ed.) (1988), L'abrogazione delle leggi razziali in Italia.

304 Carlo Nitsch (2014), Renato Treves esule in Argentina; in particolare il dettagliato Cap. IV, "Nostos": la revisione del concorso del 1938 e il rientro in Italia, 65-98. Le citazioni tratte da questo volume sono indicate nel testo con il numero della pagina tra parentesi. 
come non fu gradevole che siano stati occupati i nostri posti, così non è bello andare al posto dei professori ora epurati. $\mathrm{E}$ io non sono sicuro che il nostro ritorno sia gradito a tutti, perché sposta notevoli interessi" ${ }^{305}$ Le difficoltà di reinserimento dei docenti esiliati sembravano essere state ancora più forti in Germania, come si vedrà tra poco.

Ai problemi posti dalla lenta ripresa della vita normale e al ripristino delle situazioni alterate dalle leggi razziali si aggiungeva la lentezza burocratica, anche perché, "commentava sconsolato Ravà, 'quasi tutti i bocciati dei concorsi dicono ora che ciò è avvenuto per ragioni politiche"” (p. 74). A questo si aggiungevano i grandi mutamenti politici legati anche al primo governo De Gasperi del 1946: "Da mesi ormai non si riuniva più il Consiglio Superiore" della Pubblica Istruzione (cioè l'organo che doveva decidere i numerosi casi ancora pendenti) e inoltre "il Ministero era intenzionato a modificare le norme per la revisione attualmente in vigore, che avrebbero evidenziato, nella loro applicazione pratica, una serie di criticità" (p. 78). Insomma, la riparazione per i torti inflitti dal fascismo ebbe luogo, ma con lentezza ed esitazioni.

La sopravvenuta Guerra fredda, in realtà, portò ben presto anche a un'inversione di tendenza tanto nelle riparazioni quanto nelle epurazioni, provocando casi, per così dire, di indennizzi al contrario: valga per tutti l'esempio di Nicola Pende, il firmatario del "Manifesto della razza" su cui in Italia si fondò la discriminazione razziale del 1938, della quale era stato vittima, come tanti altri, anche Renato Treves. "Sospeso dall'insegnamento fin dal gennaio 1946 per la sua partecipazione attiva alla politica fascista in quanto senatore del Regno, con una serie di ben congegnati ricorsi, proprio mentre si esaurisce la spinta propulsiva del vento del Nord dopo la Liberazione del 25 aprile [1945], quando sono passati quasi tre mesi dalla vittoria democristiana del 18 aprile, l'8 luglio del 1948, con una pronuncia definitiva della consulta, il professor Nicola Pende può tornare a insegnare alla facoltà medica di Roma". ${ }^{306}$ Riabilitazioni analoghe furono frequenti anche in Germania. ${ }^{307}$

305 Adolfo Ravà a Renato Treves, Roma, 11 settembre 1945, in: Carlo Nitsch (2014), Renato Treves esule in Argentina, 138-139; inoltre, 72.

306 Pasquale Chiesa (2012), L'algoritmo del perdono, 10. Esemplare anche la storia di Gaetano Azzariti, dal Tribunale dalla razza a Presidente della Corte Costituzionale della Repubblica (sempre in Barbara RaGgi (2012), Baroni di razza, 190-191), insieme a non pochi altri.

307 Norbert Frei (ed.) (2003), Carriere, i cui singoli capitoli esaminano le carriere nel dopoguerra di imprenditori, giornalisti, ufficiali, medici e giuristi. 
L'intricata storia della Germania postbellica ha imposto un percorso complesso anche alle riparazioni per i danni e per le ingiustizie causate dal nazionalsocialismo. Gli Alleati occidentali realizzarono la divisione e il controllo della Germania secondo un progetto duro (ma non quanto il piano di Henry Morgenthau, che di fatto distruggeva la Germania ${ }^{308}$ ); l'URSS impose alla sua zona di occupazione misure complessivamente più gravose.

La divisione in quattro zone di occupazione e la cessione dei territori orientali dell'ex impero tedesco disgregarono un'economia che, prima della guerra, era unitaria e integrata. I tentativi di "autarchizzare" le quattro zone di occupazione; la fusione delle zone statunitense e britannica (Bizone), cui si aggiunse quella francese (Trizone); la contrapposizione fra queste ultime e la Zona di Occupazione Sovietica (SBZ), poi DDR: tutte queste misure disarticolarono le tradizionali linee di approvvigionamento delle materie prime e i rapporti tra le imprese e il loro indotto. Alle ingenti distruzioni belliche si aggiunsero i saccheggi incontrollati nei mesi finali della guerra (la "caccia ai trofei”), accompagnati da violenze sui civili. Quando i singoli governi militari ristabilirono un minimo di ordine, le riparazioni iniziarono con la Demontage, cioè con lo smontaggio e il trasferimento di quanto restava di alcune industrie strategiche tedesche.

Alcuni anni dopo la guerra, uno degli ufficiali statunitensi incaricati di scegliere le imprese da smantellare ha pubblicato i suoi ricordi, che attestano uno dei maggiori prelievi di prodotti dell'ingegno e di materiali industriali mai avvenuti. ${ }^{309}$ Prelievo difficile da documentare: "Sembra chiaro che di questi trasferimenti si sarebbe dovuto tener conto nel valutare le riparazioni versate agli Stati Uniti, ma non è facile valutare i beni immateriali. [...] Inoltre il programma FIAT [Field Informations Agency, Technical] divenne sempre più rapace, e ogni tentativo di ricorrere alla contabilità per rendere più chiaro, o anche più dignitoso, quanto stava avvenendo risultò sempre

308 Il Piano Morgenthau prevedeva di dividere la Germania in più Stati (non quindi di formare uno Stato federale) e, di fatto, di azzerare tutte le industrie tedesche, con la conseguente trasformazione della Germania in un territorio soltanto agrario: Henry Morgenthau (1945), Germany is our Problem; una sintesi è in Rainer Karlsch (1993), Allein bezahlt?; la citazione è alle pp. 47-53. Il timore verso i tedeschi generava atteggiamenti quasi razzistici non solo in Morgenthau: il sottosegretario di Stato inglese, Lord Robert Vansittart, attribuiva loro una "patologica aggressività". John Gimbel (1990), Science, Technology, and Reparations. 
meno praticabile". ${ }^{\mathbf{3 1 0}}$ Sembra che le conoscenze tecnologiche così acquisite siano state cedute anche alle imprese private statunitensi e che i trasferimenti riconducibili a questo programma si siano aggirati sui 10 miliardi di dollari, fino al 1947, quando il programma venne chiuso.

La Demontage era importante soprattutto per l'Unione Sovietica, perché aveva subìto ingenti distruzioni cui poteva porre un riparo almeno parziale trasferendo le infrastrutture industriali tedesche, spesso di livello tecnico superiore a quello russo. Invece gli Alleati occidentali, che si muovevano a un livello industriale più avanzato, erano interessati a specifiche industrie e, soprattutto, ai loro piani di ricerca e sviluppo; spesso, anzi, agli scienziati che promuovevano quelle ricerche. Nella Zona di Occupazione Sovietica, poi DDR, la Demontage ostacolò molto il possibile sviluppo industriale di quell'area che nell'Impero tedesco, con la Sassonia, era stata fra le più industrializzate.

La Demontage avvenne spesso in modo disorganizzato. Mosca inviò degli specialisti delle sue varie industrie per scegliere gli obiettivi da smontare, ma questi inviati spesso agirono senza coordinarsi con il governo militare sovietico, anche perché i due gruppi perseguivano fini diversi. Si smontarono più impianti di quanti si potessero trasportare, e poi fu necessario creare dei magazzini dove ricoverare questi impianti, che alla fine vennero restituiti come rottame alla DDR.

Un simbolo della distruttività della Demontage fu la sua applicazione alle ferrovie: "Fino al marzo 1947 [i sovietici] asportarono rotaie per un totale di 11.800 chilometri. La rete ferroviaria venne così ridotta del $48 \%$ rispetto al 1938. Il danno più grave per il trasporto ferroviario venne dallo smontaggio del secondo binario, per un totale di 6300 chilometri di rotaia. [...] Lo smontaggio del secondo binario divenne addirittura il simbolo della Demontage nella SBZ [Zona di Occupazione Sovietica]. Nessun'altra azione di smontaggio ha lasciato tracce così durature e profonde nella memoria delle persone, come lo smontaggio del secondo binario". ${ }^{311}$ Effettivamente io stes-

310 Recensione al volume di John Gimbel (citato supra, nota 309) di Stuart Macdonald (1991), Science, Technology, and Reparations.

311 Rainer Karlsch (1993), Allein bezablt?, 81-82. Il volume descrive l'intero svolgimento delle riparazioni nella SBZ/DDR, in particolare la formazione di società miste tedescosovietiche, l'esportazione dell'uranio, il trasferimento in URSS di scienziati tedeschi e i flussi finanziari spesso mascherati. Cfr. anche Hermann-Josef Brodesser et al. (2000), 
so ricordo ancora i viaggi in treno nella DDR degli anni Sessanta, sull'unico binario che correva parallelo all'altro terrapieno vuoto: per ogni viaggiatore e in ogni viaggio, un memento per le rotaie che erano state portate via.

Nelle singole zone di occupazione si sono susseguite, e in parte sovrapposte, situazioni non coordinate: le varie misure delle potenze vincitrici; gli indirizzi non coincidenti tra gli Alleati occidentali e l'Unione Sovietica; poi, le singole e divergenti decisioni di ciascuno dei due Stati tedeschi; infine, le specifiche difficoltà interne della DDR. A proposito di queste ultime, va ricordato che anche in questo territorio i nazisti avevano espropriato dei beni (per esempio degli immobili) di ebrei e di avversari politici; con l'occupazione sovietica e con l'avvento della DDR, molti di questi immobili vennero statalizzati; con la fine della DDR nel 1990, quei beni statali vennero privatizzati, ma il potenziale acquirente privato si trovò talora di fronte gli eredi della famiglia ebrea originariamente espropriata dai nazisti.

Qui di seguito verranno indicate soltanto le linee generali delle principali misure di riparazione, rinviando ogni approfondimento alla voluminosa documentazione che si è andata accumulando sul tema. Entrambi gli Stati tedeschi nati dall'occupazione alleata pagarono le riparazioni di guerra, ma in misura ben diversa. Da un lato, la Repubblica Federale Tedesca fruì del Piano Marshall e del condono dei debiti di guerra; dall'altro, la Repubblica Democratica Tedesca pagò a lungo ingenti riparazioni all'Unione Sovietica, tanto che un autore occidentale si chiede se la DDR sia stata "l'unica a pagare", e pone il quesito come titolo al suo libro. ${ }^{312}$

Infatti la Repubblica Democratica Tedesca, occupata da mezzo milione di militari sovietici, dovette far fronte a obbligazioni ben più gravose rispetto a quelle della Germania federale. Tuttavia "il segreto sui documenti relativi alle riparazioni venne mantenuto sino alla fine della DDR", ${ }^{313}$ cioè fino al 1990, ostacolando così il dibattito scientifico. Anzi, ciascuno dei due Stati tedeschi riteneva di aver pagato una parte delle riparazioni dovute dall'altro Stato e gli richiedeva quindi la restituzione di ingenti somme. Un dato emerge comunque da questa polemica: "Fino al 1953 la Zona di Occupazione Sovie-

Wiedergutmachung und Kriegsfolgenliquidation (dettagliata ricostruzione a partire dall'occupazione alleata della Germania); KARL Doenring et al. (2001), Jahrhundertschuld, Jahrhundertsübne.

312 Si tratta del volume Rainer Karlsch (1993), Allein bezahlt?.

313 Rainer Karlsch (1993), Allein bezablt?, 10. 
tica/DDR ha sopportato la maggiore quota di riparazioni": ${ }^{314}$ Ripetutamente la DDR aveva richiesto il riconoscimento di questa situazione e, quindi, un conguaglio da parte della Germania federale. Se ne parlò nel 1970 nell'incontro tra Willy Brandt e Willi Stoph, a Erfurt, e di nuovo in occasione della riunificazione nel 1989-90: solo in quel contesto, nell'imminenza della fusione dei due Stati, si concluse la discussione senza alcun conguaglio.

Il territorio della DDR era privo di materie prime, e doveva quindi importarle soprattutto dall'Unione Sovietica. Il trattato commerciale del 3 dicembre 1965 (in vigore fino al 1970) imponeva alla DDR di importare dall'Unione Sovietica materie prime a prezzi superiori a quelli di mercato, e di esportare in Unione Sovietica i prodotti della propria industria a prezzi di favore. Con questo pagamento indiretto e permanente dei danni di guerra iniziava così un rapporto sbilanciato che avrebbe condizionato la DDR per tutti i quarant'anni della sua esistenza. Infatti per la DDR sarebbe stato preferibile produrre beni industriali esportabili in Occidente, ricevendone il pagamento in valute convertibili, la cui carenza fu sempre una spina nel fianco degli Stati comunisti, tutti operanti con valute non convertibili.

Un esempio attesta la gravità di questo contrasto nell'indirizzo economico. Tra gli scienziati ritornati nella DDR dopo la guerra c'era anche Brunolf Baade, ${ }^{315}$ che a Dresda continuava per la DDR la tradizione aeronautica della Germania prebellica. In quel contesto venne progettato il primo aereo a reazione tedesco, il "Baade 152", di cui l'URSS aveva prenotato non pochi esemplari. Poi il 2 febbraio 1961 il Politbüro della DDR dovette interrompere quel promettente programma perché l'Unione Sovietica aveva deciso di costruire essa stessa quel tipo di aereo e, quindi, aveva stornato il contratto con la DDR. Quest'ultima dovette così cancellare un progetto "che era già costato 2 miliardi di marchi ed era alla soglia della produzione in serie". 316

314 Rainer Karlsch (1993), Allein bezablt?, 12.

315 Brunholf Baade è considerato il padre dell'industria aeronautica della DDR: ReInHARD Müller (2010), Brunolf Baade (in particolare: Die Sowjetunion und der DDR-Flugzeugbau, 351 s.).

316 Gunnar Decker (2015), 1965, der kurze Sommer der DDR, 49. Documentato volume di un giornalista che descrive gli eventi intorno al 1965, "punto drammaticamente culminante degli anni Sessanta, tra la costruzione del Muro di Berlino e la Primavera di Praga”: nel 1965 fallisce il tentativo di riforma della DDR e inizia la tensione interna che porterà all'implosione di quello Stato. 
Questa linea si consolidò con Brežnev: "L'Unione Sovietica impone indirizzi quasi irrealizzabili su che cosa, quando e dove si deve produrre, e dove si deve vendere. La DDR deve soprattutto restare un fornitore a buon mercato dell'URSS, non della Germania occidentale"; a questo fine "deve essere frenato il ritiro della DDR dall'industria pesante e si deve impedire che essa indirizzi la sua economia verso i mercati occidentali" ${ }^{317}$ Priva di materia prime, la DDR puntava a un'industria "intelligence intensive", mentre all'URSS interessava un'industria pesante di trasformazione delle sue materie prime.

Proprio su questi contrastanti indirizzi verteva, tra il 1963 e il 1965, l'aspro dibattito sul trattato economico tra URSS e DDR, firmato nel 1965. Furono giorni drammatici: alla vigilia della firma di quel trattato, Erich Apel - responsabile dell'economia nel Politbüro della DDR e oppositore di quell'accordo troppo filosovietico - venne trovato morto nel suo ufficio. Non è mai stato chiarito se si sia trattato di suicidio o di omicidio politico. $^{318}$

Gli esempi di questo conflitto non solo economico potrebbero moltiplicarsi, ma hanno ormai un interesse soltanto storico perché dal 1990 la DDR non esiste più. Passando alle riparazioni da parte della Germania occidentale, la situazione si capovolge: alla spogliazione della DDR fa riscontro il piano Marshall per gli Stati europei sconfitti, anche se gli anni postbellici non furono certo rose e fiori.

Alla fine della Seconda guerra mondiale la Germania non aveva ancora estinto i suoi debiti derivanti dalla Prima guerra mondiale e regolati dal trattato di Versailles. Anche questi "debiti imperiali” interni ed esteri confluirono del trattato di Londra del 1953, che stabiliva il tipo e l'ammontare delle riparazioni che la Germania doveva pagare a vari Stati, per esempio sotto forma di cessioni di parte della flotta mercantile, di beni situati all'estero o di "Demontage" di industrie tedesche occidentali. Nel 1954 il trattato di Parigi mise fin alle riparazioni per la Repubblica Federale Tedesca.

317 Gunnar Decker (2015), 1965, der kurze Sommer der DDR, 35.

318 Jörg Roesler (2010), Zwischenfall unterwegs oder Vorbote vom Ende?; RaINER Karlsch, Agnes TANdler (2001), Ein verzweifelter Wirtschaftsfunktionär? Il "Neues Ökonomisches System" (NÖS) del 1963 era in progetto di riforma dell'economia pianificata voluto da Erich Apel e azzerato dal trattato del 1965 con l'URSS. 
La costituzione del 1949 della Germania federale attribuisce all'Unione (Bund) il potere concorrente di legiferare sui danni di guerra e sulle riparazioni (“die Kriegsschäden und die Wiedergutmachung", art. 74, nr. 9). ${ }^{319}$ I "danni di guerra" sono "i danni materiali direttamente prodotti dagli eventi bellici", mentre per "riparazione" "si deve intendere la riparazione per i danni provocati dalle misure persecutorie del regime nazista. Ricadono in questa categoria anche la restituzione di beni materiali che sono stati asportati da un territorio occupato ad opera delle truppe tedesche di occupazione". 320

Un caso specifico ma importante riguarda l'espropriazione di opere d'arte nella stessa Germania (di cui furono vittime soprattutto gli ebrei, ma anche gli oppositori) e nei paesi occupati (Raubgut, Raubkunst). Dalla fine della guerra ad oggi si svolgono pratiche per la restituzione di questi beni e dal 2003 è stata istituita a questo fine una "Commissione consultiva per la restituzione di beni culturali sottratti in seguito alle persecuzioni nazionalsocialiste, in particolare a proprietari ebrei”. Nel 2013 la stampa tedesca valutava che circa 20.000 oggetti artistici si trovavano ancora nei magazzini federali, perché non si riusciva a restituirli. ${ }^{321}$ All'inizio del 2019 il direttore della Galleria degli Uffizi, il tedesco Eike Schmidt, chiedeva a una collezione privata tedesca la restituzione di un quadro di Jan van Huysum, rubato nel 1940 da un soldato della Wehrmacht. ${ }^{322}$ Il Kunstmuseum di Berna (che ha già in corso indagini su centinaia di opere d'arte donate da "Cornelius Gurlitt, morto nel 2014, il cui padre, Hilderbrand, fu incaricato dai nazisti di vendere opere d'arte rubate o confiscate dai nazisti”) è ora alle prese con

319 Il termine generale è "Wiedergutmachung", cioè riparazione: questo termine implicitamente indica che è stato compiuto un sopruso, cui si mette riparo. Nella legislazione ricorre il termine più neutro di "Rückerstattung", cioè di restituzione o rimborso. Entrambi i termini indicano la volontà di indennizzare chi aveva subìto un'ingiustizia nell'epoca nazionalsocialista.

320 Theodor Maunz, Günter Dürig (1984), Grundgesetz - Kommentar, Art. 74, Nr. 9, Rdnr. 122-125 (vol. V, Lfg. 23, Oktober 1984).

321 Si veda per esempio Alfred Flechtheim (2015), Raubkunst und Restitution.

322 Ne hanno parlato, per esempio, la televisione tedesca il 2 gennaio 2019 (https://www.tages schau.de/ausland/uffizien-101.html) e il settimanale "Der Spiegel", che ha pubblicato la lettera del 17 luglio 1940 - con cui un caporalmaggiore annunciava alla moglie l'invio "di un meraviglioso quadro ad olio" - con il sottotitolo: "Giallo intorno a un'opera d'arte: un soldato la ruba, un museo italiano la vuole indietro, gli avvocati chiedono soldi, le autorità non aiutano. E nessun vuol dire dov'è" (Norbert F. Pötzl [2019, 5 gennaio], Räuberlobn). 
un'analoga donazione problematica del mercante d'arte franco-svizzero brasiliano Georges Keller. E storie di questo genere si ripetono in tutti gli Stati in cui è passata l'occupazione nazionalsocialista. ${ }^{323}$ Insomma, quella dell'arte rubata, della Raubkunst, è una storia infinita.

Nei riguardi degli individui, il diritto alla restituzione e il regime delle riparazioni vennero regolati da una serie di leggi, mentre due accordi internazionali regolarono nel 1952 le riparazioni dovute a Israele (Luxemburger Abkommen) e agli ebrei che non vivevano in Israele (Haager Protokolle). ${ }^{\mathbf{2 2 4}}$ Seguirono poi altri accordi per indennizzare tutti gli Stati che avevano subìto danni dall'occupazione tedesca. Un rapido sguardo a queste obbligazioni internazionali di indennizzo documenta quanto fosse complesso e gravoso il compito che ricadeva su una Repubblica Federale di Germania distrutta dalla guerra.

Da un lato, le vittime si trovano in tutti gli Stati occupati o coinvolti durante la guerra, dalla Grecia alla Norvegia, dalla Francia alla Polonia, con la situazione particolare degli Stati dell'Europa orientale entrati nel blocco comunista: Jugoslavia, Polonia, Ungheria, Cecoslovacchia. Esistevano poi situazioni particolari, come per esempio la richiesta di riparazioni da parte dei prigionieri inglesi detenuti nella Germania nazista, ovvero la speciale situazione di Israele. Un panorama di questa complessa situazione è contenuto in un volume collettivo che offre anche un'antologia degli accordi internazionali per l'indennizzo degli stranieri perseguitati dal nazismo. ${ }^{325}$

Nello stabilire i criteri per accedere all'indennizzo fu inevitabile tracciare linee di demarcazione che potevano rivelarsi arbitrarie o ingiuste: da un lato, era fondato il timore che le spese per le riparazioni aumentassero in modo incontrollabile, mettendo a repentaglio il bilancio della Repubblica Federale di Germania; dall'altro, le norme si rifacevano a una specifica definizione di

323 FABrice Coffrini (2019, 7 gennaio), Obras roubadas por nazistas.

324 Nana SAgi (1981), Die Wiedergutmachung für Israel; Constantin Goschler (1992), Wiedergutmachung, 257-285 (Das Luxemburger Abkommen mit Israel und der Claims Conference); Benjamin B. Ferencz (1986), Lohn des Grauens.

325 Il seguente volume è complementare a quello di Walter Schwarz (ed.) (1974-1987): Die Wiedergutmachung nationalsozialistischen Unrechts: Hans GüNTER Hockerts et al. (eds.) (2006), Grenzen der Wiedergutmachung. Gli Stati esaminati sono, verso l'Europa occidentale, Olanda, Lussemburgo, Francia, Norvegia, Danimarca, Svezia, Grecia, Italia, nonché Svizzera e Gran Bretagna; verso l'Europa orientale, Jugoslavia, Polonia, Ungheria e Cecoslovacchia. 
"persecuzione" e, inoltre, escludevano chi non accettava il sistema politico della Repubblica Federale. Come si vedrà alla fine del presente paragrafo, questo principio portò ad escludere i comunisti dalle riparazioni, finché questa discriminazione venne cancellata da una sentenza del Tribunale Costituzionale tedesco.

La DDR aveva approvato delle misure di risarcimento, senza però procedere "a una restituzione di beni patrimoniali o a indennizzi per ingiustizie sofferte, che fossero anche solo approssimativamente paragonabili alle riparazioni per le ingiustizie nazionalsocialiste decise dalla Repubblica Federale" ${ }^{\mathbf{3 2 6}}$ Infatti la DDR non si considerava erede del Reich, e inoltre, come si è detto, alcuni beni espropriati dai nazionalsocialisti (e passati quindi a proprietari ariani, più o meno in buona fede) erano poi stati riespropriati dalle autorità sovietiche di occupazione e dallo Stato loro succeduto, nell'ambito della nazionalizzazione d'imprese e d'immobili e della collettivizzazione della terra.

Dal punto di vita tedesco-occidentale, la DDR seguiva "un principio politicamente plasmato sullo Stato sociale", in quanto la sua concezione delle riparazioni "si subordinava del tutto al modello di trasformazione sociale cui mirava la SED [il partito comunista della DDR]. Le prestazioni per i perseguitati dal regime nazista assunsero le forme di una cura paternalistica e privilegiata, che mirava alla loro integrazione nella società proletaria tedesco-orientale". ${ }^{327}$ Ovviamente esisteva anche una visione ufficiale della DDR diametralmente opposta, ma non è qui possibile soffermarci su queste divergenze di interpretazione.

Le ingiustizie del nazionalsocialismo riguardavano tanto i beni quanto le persone. La legislazione della Germania federale ha regolato la restituzione dei beni materiali ai loro proprietari originari con un corpus legislativo di grande complessità. ${ }^{328}$

326 Josef Isensee, Paul Kirchiof (eds.) (1997), Handbuch des Staatsrechts, 530.

327 Constantin Goschler (2005), Schuld und Schulden, 407; in particolare il capitolo Wiedergutmachung in der DDR, 361-411.

328 Il Ministro federale delle finanze e lo studioso Walter Schwarz (insieme con numerosi altri) hanno curato sei volumi di documentazione: Walter Schwarz (ed.) (1974-1987), Die Wiedergutmachung nationalsozialistischen Unrechts, i cui sei volumi di documentazione sono: vol. 1: Rückerstattung nach den Gesetzen der Allierten Mächte, 1974; vol. 2: Das Bundesrückerstattungsgesetz, 1981; vol. 3: Der Werdegang des Entschädigungsrechts unter national- und völkerrechtlichem und politologischem Aspekt, 1985; vol. 4: Das Bundesentschädigungsgesetz. Erster Teil (SS 1 bis 50 BEG), 1981; vol. 5: Das Bundesentschädigungsgesetz. Zweiter Teil 
L'applicazione di questa legislazione si scontrava con un'ulteriore difficoltà, che ci riporta ad altri problemi già esaminati a proposito dell'epurazione: i giudici chiamati ad applicare questa norme riparatorie erano spesso giudici dell'epoca nazionalsocialista, le cui sentenze avevano creato le situazioni cui ora dovevano porre riparo. Da questa situazione nascevano critiche politiche, disagi delle parti in causa e lentezze procedurali che non agevolarono le riparazioni.

Nella Germania nazionalsocialista le leggi razziali colpirono ancora più duramente che in Italia. Gli ebrei, oltre che avviati ai campi di sterminio, vennero espulsi dalle loro professioni e quelli che riuscirono a ritornare conobbero le difficoltà di reinserimento illustrate poco sopra per Renato Treves. L'esilio di due giuristi ebrei illustra la diaspora dei docenti universitari tedeschi: Theodor Sternberg ${ }^{329}$ ed Ernst Hirsch. Valgano come esempio le vicende di quest'ultimo, emigrato in Turchia e rientrato in Germania come sociologo; esse presentano analogie con quelle di Treves, anche se il rientro di Hirsch in patria fu più traumatico.

Quando nell'estate del 1948 gli giunse l'invito a tenere alcune conferenze in quella parte della Germania che allora si chiamava Zona di Occupazione Americana, [Ernst Hirsch] partì con un passaporto diplomatico turco e con l'incarico di riallacciare gli scambi culturali tra le università tedesche e quelle turche. La prima tappa a Monaco fu caratterizzata da un'accoglienza della Facoltà di Giurisprudenza così agghiacciante, da fargli interrompere il soggiorno dopo la prima conferenza, 'profondamente colpito e ferito dall'accoglienza di questo collega tedesco in una Germania apparentemente da tre anni liberata dal nazismo ${ }^{[330]}$. E l'avverbio 'apparentemente' spiega a sufficienza l'atmosfera incontrata. Riinvitato a Monaco con le scuse del rettore, nel viaggio di ritorno tenne ancora una conferenza e il professore di diritto commerciale, Alfred Hueck, gli chiese se sarebbe stato disposto ad accettare una chiamata a quella facoltà: 'Ma una chiamata a quella facoltà non l'ho ricevuta né allora né poi'. Gli altri contatti universitari in Germania furono migliori, ma comunque non bastarono a convincerlo a tornare, ${ }^{331}$ infatti solo nel 1950 Ernst Hirsch si trasferì alla Freie Universität di Berlino.

(SS 51 bis 171 BEG), 1983; vol. 6: Entschädigungsverfahren und sondergesetzliche Entschädigungsregelungen, 1987. Questa normativa interna è complementare agli accordi internazionali sui risarcimenti: cfr. nota 325, p. 123. Per un commentario giuridico alla legge federale sugli indennizzi (BEG), cfr. Erich BLEssin (1967-1969), Bundesentschädigungsschlußgesetz.

329 Mario G. Losano (2001), Il diritto libero di Theodor Sternberg.

330 Ernst Hirsch (1982), Aus Kaisers Zeiten, 346 e 349 di questa autobiografia di Hirsch.

331 Sull'intera vicenda di Hirsch: Mario G. Losano (2009a), Ernst Hirsch ..., Parte I. La formazione e l'esilio di Ernst Hirsch; Mario G. Losano (2009b), Ernst Hirsch ..., Parte II. Ernst Hirsch sociologo del diritto; la citazione nel testo è tratta da quest'ultimo articolo, 362. 
Senza scendere nei dettagli di questa situazione che si prolungò per vari anni, alcuni dati riassumono i risultati delle complesse misure politiche, legislative e giudiziarie fin qui descritte. Alla fine del 1948, cioè 14 mesi dopo l'emanazione delle norme alleate sugli indennizzi, nella sola Zona di Occupazione Americana erano state presentate 220.000 richieste di restituzione, per un totale valutato sui 3 miliardi di marchi dell'epoca: una somma ingestibile. Le richieste vennero poi ripartite fra i vari Länder intanto costituiti (e competenti per le decisioni sugli indennizzi). In Baviera nel 1963 il 93\% delle richieste era stata decisa: questa percentuale era leggermente superiore a quella degli altri Länder. ${ }^{332}$ Una parte di queste riparazioni - sotto forma di indennizzi puntuali o di pensioni - veniva pagata all'estero, soprattutto in Israele, poiché molti dei danneggiati erano ebrei emigrati in quello Stato.

Ancora alla fine del 1987 il Bundestag stanziava una forte somma per "gruppi finora dimenticati o trascurati, come i Sinti e i Rom, gli omosessuali, le vittime delle sterilizzazioni forzate, degli esperimenti medici, delle azioni di eutanasia e dei lavori forzati". 333

Infine, il caso dei comunisti nella Germania federale è particolarmente rivelatore della complicata situazione politica di quegli anni, tanto che un testo ne parla come di "vittime squalificate": "Allora era fuori discussione che, accanto agli ebrei, i comunisti erano stati uno dei principali obiettivi del terrore nazionalsocialista. Quindi rientravano indiscutibilmente tra i fruitori delle prime misure di risarcimento. Però, dall'inizio degli anni Cinquanta, $\mathrm{i}$ comunisti attivi vennero esclusi dal pagamento dei risarcimenti. Quest'esclusione, che è un prodotto della Guerra fredda, venne corretta - sia pure informalmente - nella seconda metà degli anni Sessanta" ${ }^{334}$ Il fondamento giuridico per questa discriminazione si trova nella legge del 1953 che completava la precedente normativa sulle riparazioni (Bundesergänzungsgesetz, $\mathrm{BEG})$.

332 Tobias Winstel (2006), Verhandelte Gerechtigkeit, 103-120. Le statistiche sopra citate si riferiscono a tutte le richieste (e non solo a quelle degli ebrei, come suggerisce il titolo del volume), perché negli atti giudiziari tutte le richieste erano trattate congiuntamente.

333 GotThard Jasper (1989), Die disqualifizierten Opfer, 361-362; segue una precisa ricostruzione del clima in cui nacque quella modifica legislativa e la sua applicazione.

334 Gotthard Jasper (1989), Die disqualifizierten Opfer, 362. 
La nuova formulazione del primo articolo prevedeva che venisse escluso dagli indennizzi "chi combatte l'ordinamento liberal-democratico": una formulazione ambigua che si prestò a una successiva applicazione estensiva, ma non nel senso voluto dal legislatore, che si riferiva a chi aveva fatto parte del sistema nazionalsocialista. Infatti nei primi anni del dopoguerra prevaleva ancora il "consenso antifascista", ma con l'intensificarsi della Guerra fredda andò prendendo corpo la convinzione che il "combattere l'ordine liberaldemocratico" potesse riferirsi anche alla lealtà democratica verso la Germania federale. Così negli anni Cinquanta la magistratura cominciò a escludere dalle riparazioni i comunisti, anche se nel contempo il Partito Comunista Tedesco (KPD, nella Germania federale) era rappresentato in alcuni parlamenti di Länder e si candidava al parlamento federale. Questo clima portò nel 1956 la Corte Costituzionale a vietare il partito comunista tedesco ma, successivamente, a precisare che solo un'attività a favore del partito comunista dopo la sentenza di incostituzionalità costituiva un attacco all'ordinamento liberal-democratico. Quindi l'attività comunista anteriore al 1956 non precludeva l'accesso agli indennizzi.

Questo tortuoso percorso può essere così sintetizzato: "L'effetto della clausola politica di esclusione [dagli indennizzi] veniva così ancora una volta limitato. Mentre la legge del 1956 (BEG) aveva precisato che soltanto una lotta contro l'ordinamento liberal-democratico dopo il maggio 1949 annullava il diritto all'indennizzo, ora il Tribunale Costituzionale fissava l'agosto 1956 come nuova data, abolendo ogni effetto retroattivo. Dal punto di vista della politica del diritto ciò portava alla problematica conseguenza che $\mathrm{i}$ vecchi comunisti rimasti inattivi dopo il 1956 potevano ottenere la loro pensione di perseguitati. L'indennizzo era divenuto un premio per il buon comportamento politico". Si poneva però un problema: "Chi cercava di mantenere i rapporti con il partito, anche in forme relativamente neutrali, cadeva nelle mani della giustizia e metteva in pericolo la decisione sul suo caso. Un'attività estremamente limitata bastava alla magistratura per negare o ritirare l'indennizzo, in conformità con le sue precedenti decisioni. Ancora oggi [1959] il Tribunale di Cassazione si attiene alla sua problematica posizione, secondo cui si può negare l'indennizzo anche in assenza di una sentenza penale per una violazione delle norme penali sulla politica". ${ }^{335}$

335 Gotthard Jasper (1989), Die disqualifizierten Opfer, 376. 
Tra i profondi sconvolgimenti fin qui esaminati, i grandi processi ai criminali di guerra colpivano indirettamente la maggioranza della popolazione tedesca, mentre essa era direttamente coinvolta dalle epurazioni, che spesso creavano risentimento e sconcerto in vasti ambiti famigliari, e dalle riparazioni, che rendevano precarie le condizioni della vita quotidiana, segnata dalla penuria dei beni (dal cibo alle case) e dai servizi pubblici ancora ridotti all'osso dalle distruzioni. A questi gravami si aggiungevano le morti dei famigliari nella guerra, l'incertezza per il futuro lavorativo e mille altre angustie. Questa situazione rendeva invincibile il desiderio di pace e, nei politici, facilitò l'accettazione della norma costituzionale che vietava la guerra, imposta dalle potenze vincitrici. Però alle nuove generazioni andava spiegato tanto il passato (che aveva prodotto la catastrofe mondiale di cui anche i bambini vivevano quotidianamente le conseguenze) quanto il futuro (che sarebbe stato retto da regole politiche nuove): la secolare storia tedesca, infatti, aveva conosciuto la democrazia soltanto nella decina d'anni della travagliata Repubblica di Weimar, dal 1919 al 1933. Bisognava dunque superare il passato per preparare il futuro: questo compito era affidato alla scuola e, in particolare, ai libri scolastici.

\section{Il superamento del passato: i libri scolastici}

La fine di ogni sistema di potere esige ripensamenti e revisioni che si riflettono anche nei manuali scolastici: di recente ciò è avvenuto con la fine dell'Unione Sovietica, ma nel corso del tempo si è verificato anche nell'ex Jugoslavia, in India, in Medio Oriente, in Gran Bretagna e negli Stati Uniti. ${ }^{336}$ Quindi anche in Giappone, Italia e Germania la cesura politica della fine della guerra si riflette nei libri scolastici. Un confronto tra i manuali adottati prima e dopo la guerra rivela una contrapposizione tanto forte, quanto forte era la diversità dei regimi politici nelle due fasi storiche dello stesso paese.

Durante il suo esilio americano, Erika Mann rivolse contro l'indottrinamento nazionalsocialista nelle scuole tedesche una critica che può essere estesa alla politica scolastica degli altri due Stati dell'ex Patto Tripartito. ${ }^{337}$

336 Un quadro complessivo è in Giuliano Procacci (2003), La memoria controversa.

337 Erika Mann (1938), School for Barbarians. L'edizione in tedesco venne stampata ad Amsterdam nel 1938, presso il Querido Verlag (una delle maggiori case editrici dell'esilio 
Dopo aver esaminato come l'ideologia nazionalsocialista avesse permeato ogni momento della vita sociale tedesca (e l'ossessivo saluto "Heil Hiler!", ripetuto a ogni incontro, ne diviene il simbolo) e non avesse risparmiato neppure la vita famigliare (inquinata dal timore della delazione), Erika Mann dedica il nucleo del suo libro alla scuola e, citando testi scolastici e documenti ufficiali, analizza la trasformazione in senso nazionalsocialista d'ogni materia: dalla matematica alla storia, dalla religione al disegno, dalla chimica alla geopolitica.

Sono tuttora coinvolgenti le pagine in difesa della libertà nella scuola che Thomas Mann premise al libro della figlia:

"Il tema fondamentale del libro, l'insegnamento in Germania, si rivela un punto di partenza estremamente fruttuoso per esporre l'intera concezione nazionalsocialista. Che sia una donna ad averlo scelto non è strano, mentre invece è sorprendente constatare come questa voluta limitazione a un singolo tema fornisca un'immagine esauriente e ben informata dell'intero Stato totalitario. Il quadro è così completo che uno straniero desideroso di penetrare in questo mondo inquietante può dire di conoscerlo dopo aver letto questo libro. Tutto il tetro concentrarsi degli attuali leader tedeschi sulla sola idea del potere dello Stato; tutta la loro disperata determinazione nel subordinare a questa idea l'intera vita intellettuale e spirituale della nazione, senza una sola riserva umana: tutto ciò viene alla luce con sorprendente chiarezza in questa descrizione e analisi, accompagnata da un'abbondanza di dettagli fin troppo convincenti sul programma educativo del nazionalsocialismo.

Parlo di 'programma' perché esso si rivolge al futuro. È un primo inesorabile abbozzo di quello che sarà il tedesco del futuro. Nulla vi si sottrae. Con ferrea e implacabile coerenza, fanaticamente, deliberatamente, meticolosamente, i nazionalsocialisti si sono impegnati nel mettere in pratica quest'unica idea, applicandola a ogni singolo settore e fase dell'istruzione. Il risultato è che l'istruzione non viene presa in considerazione di per se stessa; il suo contenuto non è mai indirizzato alla preparazione, alla cultura, alla conoscenza, alla promozione del progresso umano mediante l'educazione. Invece di tutto ciò l'istruzione ha un unico riferimento, spesso connotato dalla violenza: l'idea fissa della supremazia nazionale e della preparazione militare". 338

I grandi processi e le epurazioni cercavano di regolare i conti con il passato. Ma come descrivere quel passato - così inappellabilmente condannato dalle appassionate parole di Thomas Mann - alle generazioni del Giappone, dell'Italia e della Germania che non l'avevano vissuto? È soprattutto attraverso i

tedesco) ed è ora ristampata in Erika ManN (2011), Zehn Millionen Kinder (trad. it. 1997: La scuola dei barbari. L'educazione della gioventu nel Terzo Reich. Introduzione di Thomas Mann, Firenze: Giuntina, 207 pp.); Alessandra Minerbi (1999), La scuola dei barbari.

338 Thomas Mann (1938), Introduction, la citazione è a p. 6 ed è stata tradotta da me. 
libri scolastici che il passato viene presentato alle generazioni future: ma spesso quei libri narrano il passato non come è stato, ma come si vorrebbe fosse stato; e su certi temi spesso non narrano, ma tacciono. I grandi processi e le epurazioni sono stati al centro di vaste discussioni nel momento in cui ebbero luogo: ma come sono stati presentati alle generazioni successive? E come sono stati presentati i regimi che hanno indotto ai comportamenti puniti poi con i grandi processi e con le epurazioni? Per rispondere a queste domande è utile sfogliare i libri scolastici: nel dopoguerra essi erano lo strumento più capillare per raggiungere le nuove generazioni, anche se oggi non è più così.

Per il costituzionalista Di Giovine - che è "affascinato e turbato dal fenomeno dei mass-media, dal ruolo sempre più totalizzante che essi giocano nel mondo contemporaneo" - il "primo motivo d'interesse" per compiere una ricerca sui manuali di storia per la scuola media "è stato quello di accostarmi al meno noto fra i messaggi che raggiungono il grande pubblico". Tuttavia, afferma il coautore della ricerca, "nell'era dell'informatica e dell'immagine, il testo scolastico rimane pur sempre uno strumento di conoscenza essenziale nei 'curricula' educativi della nostra scuola secondaria.".399

Tutti e tre gli Stati affrontavano con strategie diverse alcuni grandi problemi comuni. Nell'ambito di una generale riforma scolastica, bisognava riscrivere in poco tempo i libri nati sotto i regimi sconfitti. Però le difficoltà organizzative ed economiche del dopoguerra imponevano di ricuperare il ricuperabile, non solo tra $\mathrm{i}$ testi, ma anche tra i loro autori, per quanto compromessi con il passato regime. Quindi la riforma scolastica intersecava le procedure di epurazione, caratterizzate da risultati limitati anche in questo settore. Inoltre alle distruzioni belliche delle tipografie si aggiungeva la scarsezza delle materie prime: la carta era contingentata e di cattiva qualità, il che compromette ancora oggi la conservazione di quei manuali scolastici, che d'altra parte erano (e spesso sono ancora) considerati materiale bibliografico non destinato alla lunga conservazione.

Limitando l'esame alla trasformazione dei libri scolastici, certi aspetti esteriori della grafica facevano parte di professionalità acquisite da tempo, e sotto un regime diverso: le si poteva indirizzare diversamente, ma non soppiantarle del tutto. Lo stesso vale per la scrittura: il contenuto innovato

339 Michele Brondino, Alfonso Di Giovine (eds.) (1987), Colonialismo e neocolonialismo; le citazioni sono rispettivamente a p. 125 e a p. 238. 
veniva spesso formulato con uno stile espositivo che risentiva della retorica dei decenni precedenti. Nella descrizione dei regimi caduti, infine, le storie personali degli autori, i contesti sociali ancora incerti, il desiderio di pacificazione sociale dopo una lunga guerra ( $\mathrm{ma}$ anche l'opportunismo o la nostalgia per il passato regime) portavano a limitare o ad omettere l'esame degli eventi degli ultimi due decenni.

Le incertezze della transizione andarono attenuandosi dopo i primi anni e, a partire dai primi anni Cinquanta, si consolidò sempre più l'indirizzo democratico dell'editoria scolastica come riflesso del consolidamento dello Stato democratico, dell'economia liberale e dell'avvicendamento anche generazionale tra gli autori di libri scolastici. ${ }^{340}$ All'interno di questi grandi problemi comuni ai tre Stati che uscivano da una dittatura per entrare in una democrazia parlamentare, la riforma scolastica e i testi su cui essa poggiava assumeva alcuni aspetti specifici sintetizzati qui di seguito.

In Giappone l'istruzione è stata una struttura sociale rilevante sin dai tempi antichi e se ne può seguire l'evoluzione in un'attendibile opera collettiva. ${ }^{341}$ L'epoca Meiji (1868-1912) aveva quindi ereditato un articolato sistema di istruzione, che venne innovato secondo il modello prussiano. Il ministro dell'istruzione Arinori Mori (1847-1889) "nella sua politica scolastica seguì il modello tanto prussiano quanto americano. Il conte Ito [Hirobumi], suo importante e rilevante collega politico, si impegnò energicamente per la recezione del sistema statuale prussiano. Ito e Mori avevano incontrato a Vienna Lorenz von Stein, che tenne loro delle conferenze, donò loro i suoi apprezzati libri sulla dottrina amministrativa e, in seguito, intrattenne con entrambi un fitta corrispondenza."342

Poiché, seguendo il modello tedesco, Mori mirava a rafforzare l'identificazione dello scolaro con lo Stato, sin dall'epoca Meiji anche la geografia

340 I diversi modi di presentare l'altro Stato e alcuni pregiudizi o steretotipi tuttora persistenti nei libri scolastici tedeschi e giapponesi sono esposti in KarL-Ernst Jeismann (ed.) (1982), Deutschland und Japan.

341 Günther HaAsch (ed.) (2000), Bildung und Erziehung in Japan. Un terzo del volume ripercorre l'evoluzione dell'educazione giapponese dalle origini alla fine del 1600 (21-36); nell'epoca Tokugawa (37-65); nella trasformazione dell'epoca Meiji (66-92); nelle epoche Taisho e Showa (93-103) e nell'epoca della Seconda guerra mondiale (103-125), particolarmente rilevante per la presente esposizione.

342 Günther HaAsch (ed.) (2000), Bildung und Erziehung in Japan, 67-68. 
veniva presentata come un elemento unificante e come espressione della politica governativa. Per questo un'analisi giapponese dei testi scolastici è appunto dedicata ai manuali di geografia, anche se i manuali sono oggi in parte superati dalle nuove tecnologie. "Prima dell'avvento relativamente recente della televisione, - scriveva un autore nel 2005, - per la maggior parte dei giapponesi i manuali scolastici costituivano la fonte principale delle immagini del mondo esteriore e quindi i manuali di geografia erano una fonte di ispirazione per il popolo, cui indicavano il compito da svolgere nelle date circostanze mondiali. L'istituzione dell'ordinamento scolastico del 1872 era animata dall'ideale di una modernizzazione che imitasse i modelli dei paesi occidentali” ${ }^{343}$

Il Giappone ha una storia antica di interventi pubblici sui manuali scolastici. "Nel 1886, sotto il Ministro Mori Arinori furono pubblicati i decreti che modificarono fondamentalmente il modello scolastico istituito nel 1872", cambiando l'impostazione dei testi: "Si ebbe una reazione contro l'occidentalizzazione sotto forma di enfasi per il carattere della storia e della cultura propri del Giappone, come, per esempio, la divinità della famiglia imperiale" (p. 218). Inoltre "veniva dedicato sempre più spazio alle descrizioni e alle illustrazioni dei santuari shintoisti” (ivi). I temi erano trattati in modo da assecondare le politiche governative: ad esempio, un manuale attribuiva la prosperità della Gran Bretagna ai suoi giacimenti minerari e alle sue colonie, offrendo una descrizione che "poteva solo servire a giustificare una politica di espansionismo coloniale” del Giappone (p. 219).

A causa dell'“introduzione del sistema di approvazione ministeriale" (p. 219), si generò una situazione in cui i testi ministeriali esistevano accanto ad altri testi, in un clima di concorrenza che culminò nel 1902 con lo "scandalo dei libri di testo", dovuto alle "tangenti pagate da alcuni editori alle autorità prefetturali e scolastiche per influenzare la scelta dei libri di testo delle scuole primarie". Questo scandalo offrì al Governo il pretesto "per escludere l'uso di testi che non fossero scritti e pubblicati dal Ministero della pubblica istruzione" (p. 220).

In quei manuali, dal 1903 al 1930, i paesi stranieri venivano presentati in modo costante e stereotipo. La crisi economica del 1929 colpì anche il Giappone e, come reazione all'impoverimento soprattutto dei contadini,

343 Keirchi Takeuchi (2004), La politica dell'identità nazionale, 216. Gli altri rinvii contenuti nel testo si riferiscono al saggio qui citato.

132 Capitolo I 
nelle scuole e nelle università si affermarono le idee socialiste. Come reazione a questo indirizzo, dal 1930 aumentò l'ultranazionalismo e, dopo la guerra contro la Cina, anche l'isolamento del Giappone. Questi eventi provocarono una revisione dei manuali, nei quali la Cina veniva presentata "come l'origine di tutti i mali del Giappone" (p. 220).

Un simbolo di questa trasformazione sono le dimissioni del giuspubblicista e deputato Minobe Tatsukichi, reo di aver sostenuto che l'imperatore era un organo dello Stato. Gli si contrappose una visione radicale del kokutai, cioè dell'essenza della nipponicità. Dalla concezione dell'ininterrotta linea dinastica di origine divina "il significato del kokutai si trasformò in un'essenza nazionale dal fondamento mitico, in un'indiscutibile fonte di superiorità nazionale. I principali responsabili di questa trasformazione furono le società segrete dei militari e i funzionari di estrema destra dei ministeri degli interni e dell'istruzione, che miravano a rafforzare lo spirito nazionale come reazione all'agitazione socialista nelle università e tra gli intellettuali”. Nel ministero dell'istruzione prese così forma un ufficio che propagandava lo spirito nazionale (kokumin seishin), secondo il quale il Giappone, attraverso la dinastia imperiale, era un paese di origine divina. ${ }^{344}$

La "manipolazione dei libri scolastici da parte dello Stato culminò con l'edizione del 1943 dei testi di storia per la quinta e sesta classe e con le edizioni del 1943 e 1944 dei testi di geografia" per le stesse classi. "Di questo, - scrive Keiichi Takeuchi, - ho una conoscenza diretta, perché proprio in quegli anni avevo frequentato la quinta e la sesta classe della scuola primaria. Nel settembre 1945, a seguito degli ordini delle forze alleate dell'occupazione, gli insegnanti chiesero agli scolari di cancellare con la penna buona parte dei libri di testo: quindi appartengo alla sola classe che ha studiato su quei libri che rappresentano il culmine dell'ultranazionalismo e del militarismo giapponese". ${ }^{345}$

Alla fine della guerra, la rieducazione del Giappone perseguita dall'occupazione americana aveva per oggetto immediato le generazioni che avevano

344 Günther Haasch (ed.) (2000), Bildung und Erziehung in Japan, 108. Cfr. anche Klaus J. Antoni (1991), Der himmlische Herrscher; Wolfgang SchwentKer (2003), Die Grenzen der Entzauberung.

345 Keirchi TaKeuchi (2004), La politica dell'identità nazionale, 221. Egli riferisce inoltre la sua esperienza diretta nel 1944 e nel 1945 a Tokyo, quando i suoi studi erano di continuo "interrotti dai bombardamenti aerei e navali". 
già subìto un'educazione autoritaria, e che da essa andavano allontanate. Le generazioni successive, invece, andavano educate secondo i nuovi princìi democratici, e questo doveva avvenire soprattutto nella scuola. I libri di testo costituirono quindi lo strumento principale per formare le nuove generazio$\mathrm{ni}$, soprattutto in un contesto educativo rigoroso come quello giapponese, che privilegiava l'apprendimento mnemonico e non incoraggiava il dibattito critico.

Nonostante il crescente peso di altri mezzi d'informazione per i giovani, i libri scolastici sono ancora oggi oggetto di particolare attenzione in Giappone: proprio per questo le descrizioni degli eventi della storia contemporanea che essi propongono sono state - e continuano ad essere - oggetto di controversie. In generale, i libri scolastici giapponesi sono stati accusati, soprattutto dall'estero, di essere reticenti sul passato. Negli anni Ottanta una forte polemica sulla rappresentazione dell'espansionismo del Giappone nei suoi libri scolastici venne suscitata da alcuni paesi che erano stati invasi durante la guerra. ${ }^{346} \mathrm{Da}$ un lato, quei paesi lamentavano il silenzio o la sottovalutazione di eventi gravi; dall'altro lato, però, certi ambienti giapponesi contro-criticavano quelle rappresentazioni come "antigiapponesi" o "masochistiche". Queste polemiche durano dalla fine della guerra ad oggi, con alterne vicende.

Le potenze occupanti proibirono l'uso dei testi scolastici dell'epoca militarista, che in un primo tempo circolarono con pagine tagliate e frasi censurate. Nel 1947 venne abolito il testo unico di Stato per le scuole primarie e secondarie, e cominciarono a diffondersi testi di ispirazione democratica, fra cui quello di Saburo Ienaga (1913-2002), approvato nel 1953: la storia di questo testo è esemplare per l'evoluzione-involuzione giapponese, e quindi su di esso si tornerà più in dettaglio. Con la Guerra fredda, però, le aperture democratiche vennero attenuate con norme restrittive e dal 1953 i testi scolastici vennero nuovamente sottoposti a una revisione ministeriale che, nel corso degli anni, si fece sempre più rigida. ${ }^{347}$

346 Si veda la discussione sul passato nel Giappone degli anni Ottanta in Tаміко Kuroda (1994), Aufarbeitung oder Restauration?

347 Una precisa sintesi delle polemiche sui libri di testo giapponesi è contenuta in Givliano Procacci (2003), La memoria controversa, nel capitolo Il caso giapponese: un passato che ritorna $(63-75)$. 
Una critica generale può valere a grandi linee sino a oggi, con i dovuti adattamenti caso per caso: "I testi scolastici delle medie non menzionano le 'comfort women' e pochi parlano delle colonie; inoltre vi sono anche libri scolastici in cui si tenta di negare i reati del Giappone". Spesso "si odono pareri che non solo abbelliscono il dominio coloniale giapponese e negano il suo carattere oppressivo, ma che addirittura sottolineano che esso è avvenuto nell'interesse dei popoli dominati". Questi argomenti sono diffusi nella stampa non soltanto di destra e "solo di rado si odono critiche a questa prospettiva" 348

Alcuni autori - anteriormente di sinistra - assunsero posizioni vicine a quelle del governo, come Fujioka Nobukatsu con la sua "visione liberale della storia”, su cui si tornerà tra poco, o Fusao Hayashi, che a metà degli anni Sessanta reinterpretava in chiave anticolonialista l'espansione giapponese.

Un tema che ha portato a frequenti contrasti con i governi della Cina e della Corea è quello dell'invasione giapponese del continente asiatico, descritta come l'espansione "per una più grande Asia Orientale": l'autore di riferimento su questo tema è Fusao Hayashi. L'applicazione della dottrina dei "grandi spazi" di Karl Haushofer ${ }^{349}$ venne presentata dai militaristi giapponesi come un contributo alla liberazione dei popoli asiatici dal colonialismo europeo e statunitense: ed effettivamente alcuni leader indipendentisti asiatici si allearono dapprima con i giapponesi, per poi distaccarsene. ${ }^{350}$ Secondo Fusao Hayashi, dalla Seconda guerra mondiale il Giappone sarebbe uscito sconfitto solo a metà: persa la guerra contro gli Stati democratici, avrebbe però vinto quella sul fronte della decolonizzazione. "Si aprirebbe così il varco a una considerazione separata e autosufficiente della storia giapponese non solo in relazione alla Seconda guerra mondiale, ma anche rispetto alla stessa storia contemporanea e si giustificherebbe una sorta di isolazionismo storiografico". ${ }^{351}$

348 Tonomura Masaru (2015), Die Erinnerung an die Kolonien, 5-6; cfr. supra, nota 86, p. 32.

349 KeIICHI TAKeuchi (2004), La politica dell'identità nazionale, 222, nota 17: cfr. Christian W. Spang (2000), Karl Haushofer und die Geopolitik in Japan.

350 Il leader indipendentista indiano Subhas Chandra Bose, favorevole a un liberazione armata dell'India (e quindi avversario del non violento Gandhi), con gli aiuti dell'Asse e con gli indiani prigionieri di guerra o lavoratori nelle Aree di Co-Prosperità creò l'Indian National Army e, a Singapore, fondò nel 1943 un governo indiano in esilio: Mario G. Losano (2011), La geopolitica del Novecento, 103-104.

351 Giuliano Procacci (2003), La memoria controversa, 71. 
Questa rivalutazione dell'espansione continentale giapponese (unita alla revisione del massacro di Nanchino, delle "comfort women" e di altri eventi) ha provocato non solo una revisione dei testi scolastici, ma anche scontri diplomatici con la Cina e la Corea del Sud: quest'ultimo Stato, in uno specifico caso, giunse a richiamare il proprio ambasciatore. Infatti il manuale di Kanji Nishio venne approvato dal ministero giapponese con una serie di correzioni che però, secondo l'autore, non modificavano la linea direttrice del lavoro. Sulla correzione della formula "grande Asia Orientale" la controversia giunse in parlamento e il premier Junichiro Koizumi dichiarò che la formulazione era appropriata e che quindi l'autorizzazione per il volume non veniva ritirata. "Una siffatta dichiarazione, - ricorda Procacci,- aveva importanti implicazioni politiche: essa suonava infatti come un preannuncio della modifica dell'articolo 9 della costituzione" giapponese attuale, ${ }^{352}$ cioè dell'articolo con il quale "the Japanese people forever renounce war". Come si vede, la possibile revisione dell'articolo pacifista della costituzione affiora spesso, per quanto sottotraccia, nel dibattito politico giapponese.

Steffi Richter individua tre dispute sui libri di testo giapponesi. A metà degli anni Cinquanta, le forze conservatrici intervennero sui libri di testo dell'immediato dopoguerra, ritenuti strumenti per la diffusione di idee comuniste: "Ciò significò una crescente revisione della politica educativa, democratizzata nel dopoguerra, a favore di un'interpretazione ufficiale e statale della storia, il cui controllo è garantito dall'introduzione di un procedimento di autorizzazione centralizzato attraverso il Ministero dell'Istruzione (1953)". Una seconda disputa si sviluppò negli anni Ottanta, quando le descrizioni edulcorate del comportamento delle forze armate giapponesi, soprattutto nei territori occupati, provocò le proteste degli Stati vicini e alcune crisi diplomatiche. Per far fronte a queste critiche, il Ministero dell'Educazione "emanò una 'Clausola sugli Stati vicini' come criterio di valutazione dei libri di testo, invitando a evitare ogni formulazione che potesse offendere gli Stati vicini". ${ }^{353}$ La terza "Historikerstreit" giapponese scoppiò a

352 Giuliano Procacci (2003), La memoria controversa, 75.

353 Steffi Richter (2003), Zurichtung der Vergangenheit, 7, nota 7. Il volume Steffi Richter, Wolfgang Höpken (eds.) (2003), Vergangenheit im Gesellschaftskonflikt contiene inoltre: JaCQueline Berndt (2003), Eine zeitlos schöne Nation (su Kobayashi Yoshinori, cfr. infra, p. 144); Yoshiaki Yoshimi (2003), Das Problem der "Trostfrauen"; Ryuichi Narita (2003), 
metà degli anni Novanta, in concomitanza con il cinquantenario della capitolazione del Giappone. La si può far iniziare con la fondazione dell'“Associazione per la produzione di nuovi libri di storia” (promossa dal germanista Nishio Kanji e dal pedagogista Fujioka Nobukatsu, su cui si ritornerà) da parte dei promotori dello "studio liberale della storia" (dove 'liberale' significa come minimo 'conservatore') e dei nazionalisti che richiedono la revisione non solo dei libri scolastici, ma anche della "costituzione pacifista".

La traduzione tedesca del capitolo sul periodo dal 1931 al 2003 di un libro scolastico per le scuole elementari giapponesi permette di farsi un'idea in concreto della struttura e del messaggio trasmesso da questi manuali. Gli argomenti e le illustrazioni riguardano soprattutto i bambini coinvolti nella guerra. Il tema della rinuncia alla guerra viene desunto dal testo scolastico Sulla nuova costituzione, di cui si riproduce una pagina con le parole: "Affinché il Giappone non inizi una seconda volta una guerra, rinunciamo ai soldati, alle navi da guerra, agli aerei e a tutti gli altri strumenti di guerra. Ma non scoraggiatevi! Il Giappone sta solo facendo la cosa giusta, e più in fretta di altri paesi. Al mondo nessuno è più forte di chi è nel giusto!" ${ }^{354} \mathrm{La}$ nuova costituzione, aggiunge il manuale nel capitolo Non facciamo più nessuna guerra!, "persegue l'alto ideale di un mondo che viva in pace" e si fonda su tre principi: democrazia, pace, diritti umani. Sulla pace in particolare viene spiegato: "Si è riflettuto su questa guerra durata quindici anni e si è dichiarato al paese e all'estero: 'Noi rinunciamo per sempre alla guerra'. [...] L'educazione perseguiva ora, come suo elemento centrale, lo scopo di educare sulla base della democrazia un popolo che costruisce uno Stato pacifico e una società pacifica. In questo contesto è stata introdotta la materia 'educazione civica': in essa i bambini stessi studiano la struttura della società e la vita delle persone". ${ }^{355}$

Il capitolo Il Giappone in Asia ricorda agli scolari la lotta contro la base americana di Okinawa, un tema particolarmente sensibile in Giappone: "Per proteggere la nostra vita e sperando in un mondo pacifico, ci ostinammo

Geschichte schreiben in Schulbüchern; Aтsuкo Onuki (2003), Instrumentalisierung der Geschichte und Nationaldiskurse.

354 Gesellschaft: Ein japanisches Geschichtslehrbuch (2006), 38; con traduzione tedesca, 39.

355 Gesellschaft: Ein japanisches Geschichtslehrbuch (2006), pp. 39-43: traduzione in tedesco con la riproduzione del manuale giapponese a fronte. 
affinché Okinawa potesse tornare alla terra dei suoi antenati" (cosa che avvenne nel 1971). Resta invece aperta la questione dei "territori del Nord", cioè delle isole Kurili, "che sono un territorio tradizionalmente giapponese [...] e che non sono ancora state restituite". 356

Okinawa è uno dei temi proibiti nella storiografia del Giappone postbellico, libri scolastici inclusi: ultima delle isole Ryukyu e parte integrante del Giappone, fu conquistata dagli americani in una dura battaglia in cui l'esercito giapponese ordinò il suicidio della popolazione civile (o forse lo eseguì, almeno in parte). Il "suicidio di massa" (traduzione di jiketsu, suicidio per evitare la resa) è uno dei temi controversi nel dibattito storico, e non solo su Okinawa: anche l'isola di Saipan, conquistata dagli statunitensi il 15 giugno 1942, visse un analogo dramma. ${ }^{357}$

Nel dopoguerra Okinawa era un territorio a sé, occupato dalla base militare americana (per andare e venire dal Giappone occorreva il passaporto), e di lì partivano gran parte degli attacchi aerei contro il Vietnam: per i movimenti giapponesi anti-establishment, un ulteriore argomento per chiedere il ritorno di Okinawa al Giappone.

La lunga polemica anche giudiziaria sui libri di testo di Ienaga Saburo ha un valore esemplare: "Ienaga Saburo (1913-2002) was one of the twentieth-century Japan's outstanding historians. He not only wrote history; he also made history. He did so by suing the Japanese government over its censorship of his text-books. Ienaga's three suits became the focus of a major and sustained anti-establishment movement in the late twentieth-century Japan" ${ }^{358} \mathrm{Su}$

356 Gesellschaft: Ein japanisches Geschichtslehrbuch (2006), 51 e 53.

357 "Quando gli americani riuscirono a mettere piede sull'isola [di Saipan], grande circa metà dell'Elba, restarono letteralmente sconvolti dal fatto che migliaia di civili giapponesi, comprese donne d'ogni età e perfino bambini, preferirono suicidarsi, facendosi esplodere addosso bombe a mano fornite dai militari, o lanciandosi in folli voli mortali dalle altissime scogliere"; questo comportamento era dovuto anche al "fatto che la propaganda dei militari e dei militaristi politici aveva tracciato un quadro così orripilante degli americani, che qualsiasi fato sembrava preferibile a quello di cadere nelle loro mani”, Fosco Maraini (2001), Case, amori, universi, 592.

358 Richard H. Minear (2008), Foreword, X: cfr. nota seguente. Cfr. Saburō Ienaga (1978), The Pacific War, dove spiega la scelta del titolo (VII) non accettato dagli storici conservatori. Poco prima di morire Ienaga pubblicò la sua biografia: SABurō Ienaga (2001), Japan's Past, Japan's Future. 
questa polemica è opportuno spendere qualche parola, perché essa va oltre ai problemi didattici da cui ha preso origine. ${ }^{359}$

L'autorizzazione ministeriale dei libri di testo, non solo nel caso giapponese, può trasformarsi in censura. Per questo l'analisi dei processi di Ienaga è stata interpretata in Germania anche come "un tentativo - mostrando nel caso giapponese la funzione e le possibili implicazioni politiche dell'autorizzazione statale dei libri di testo - di fornire un contributo alla riflessione sulla prassi dell'autorizzazione dei libri scolastici nella Repubblica Federale Tedesca". ${ }^{360}$ Il libro di Ienaga era stato pubblicato nel 1975 e il parallelismo si riferisce quindi alla Germania di quegli anni. Un analogo parallelo con gli Stati Uniti è tracciato dal nipponista Richard Minear, secondo il quale negli USA "national authorities play little role", mentre "commercial considerations rule": però "a few major States - notably, Texas - play an outsized role, with right-wing, 'Christian' vigilantes guarding the ideological gates as tenaciously as the Japanese right-wingers with whom Ienaga and his movement contended and contend" ${ }^{361}$

Nel 1952 Ienaga presentò al Ministero il manoscritto del suo libro Shin nihonshi (Nuova storia del Giappone), destinato alla scuola media. Il ministero respinse il manoscritto, che però, ripresentato nello stesso anno a una commissione diversa, venne approvato per l'adozione a partire dal 1953. Nel 1955 l'editore chiese a Ienaga una revisione del volume, che era stato respinto dal Ministero con 216 indicazioni di passi da correggere. Ienaga corresse il testo, che però venne ri-respinto con 37 richieste di correzione. Infine il testo corretto da Ienaga venne approvato nel 1955. Però nel 1956 la riforma scolastica della scuola media impose una nuova revisione del volume, respinta ancora una volta dal ministero con questa motivazione: "A seguito dell'eccessiva passione nell'invitare alla riflessione attraverso fatti storici, sussiste l'impressione che non vengano raggiunti gli obiettivi dell'insegnamento della materia 'Storia giapponese', attraverso la quale devono essere riconos-

359 L'analisi dei tre processi e del loro contesto è in Yoshiko Nozaki (2008), War Memory; l'autrice è una storica che ha partecipato al movimento internazionale a sostegno di Saburo. Una puntuale ricostruzione dei processi, con la traduzione dei passi contestati, delle osservazioni ministeriali e delle risposte di Ienaga è in Gesine Foljanty-Jost (1979), Schulbuchgestaltung (bibliografia, 151-157).

360 Gesine Foljanty-Jost (1979), Schulbuchgestaltung, 8: l'analisi di Gesine Foljanty-Jost nasce da una dissertazione difesa nel 1975.

361 Richard H. Minear (2008), Foreword, X. 
ciuti i meriti degli antenati, deve essere approfondita la propria coscienza di giapponesi e deve essere suscitato un forte amore per la nazione". ${ }^{362}$ Dopo uno scambio di memorie tra editore, autore e ministero, il testo venne di nuovo respinto ma, infine, accettato dopo un'ulteriore revisione di Ienaga. Il testo così approvato venne adottato dal 1959 al 1962 e una quarta versione aggiornata venne adottata dal 1962 al 1964.

La riforma scolastica del 1960 impose una nuova revisione del libro. La quinta stesura venne sottoposta al ministero nel 1962 e respinta nel 1963. Ienaga eseguì le 323 correzioni richieste e il libro venne autorizzato per il 1964, però a condizione che fossero modificati 293 passi. La versione corretta da Ienaga venne di nuovo respinta, con la richiesta di modificare dei passi che prima non erano stati indicati. Dopo uno scambio di osservazioni con il ministero, Ienaga apportò le modifiche affinché il libro potesse essere adottato nel 1964.

Per Ienaga, però, il dover apportare più di 300 modifiche sotto la pressione della scadenza ravvicinata e senza poterne discutere con il ministero costituì un attacco alla sua libertà scientifica e un limite alla sua libertà di espressione: per questo citò in giudizio il Ministero per violazione della libertà di espressione (art. 21 delle Costituzione giapponese), della libertà scientifica (art. 23), del diritto all'educazione (art. 26) e per eccessivo controllo sull'educazione, in violazione dell'art. 10 della legge sull'istruzione.

Contro la decisione di respingere il libro, Ienaga ricorse in giudizio affinché il ministero ritirasse il divieto di adozione. Questa richiesta venne respinta il 17 luglio 1970. Contro questa sentenza Ienaga presentò ricorso al Tribunale Supremo, che il 20 dicembre 1975 riconobbe che il Ministero non aveva rispettato le proprie norme per l'approvazione dei libri di testo, ma respinse la richiesta più importante di Ienaga: quella che richiedeva di dichiarare incostituzionale l'approvazione ministeriale dei libri di testo.

Mentre erano in corso questi processi, un'ulteriore revisione del manuale portò Ienaga a un nuovo scontro con il ministero e ad un terzo processo per la violazione della sua libertà scientifica. In un suo articolo, Ienaga precisava che "what I have been seeking from the court is only a decision concerning the violation of the constitution and due process of law by the textbook certification. I am not seeking a decision as on whether the argument of the author or of the Ministry is right with respect to the content of the text-

362 Gesine Foljanty-Jost (1979), Schulbuchgestaltung, 40. 
book". ${ }^{363}$ Ienaga chiedeva insomma al governo di non sostituirsi agli storici, ma di governare applicando la costituzione del 1946.

Il terzo processo di Ienaga si concluse nel 1997: “On August 27, Ienaga's thirty-two-years challenge to the Japanese government's textbook censorship came to an end, as Japan's Supreme Court, consisting of five judges, handed down his decision on his third lawsuit. As expected, the court avoided entering arguments concerning the constitutionality of the state textbooks screening - a central issue Ienaga had fought for more than three decades but it ruled in his favor on several points, including those regarding the descriptions of Japan's wartime conduct". 364

La vittoria di Ienaga spianava la strada ad altri storici, perché "the MOE [Ministry of Education] had no choice but to relent from its hard-line approach to textbook screening policies. In the 1972-1973 screening, for example, a high school Japanese history textbook containing a reference to the Japanese occupation of Nanjing Massacre was approved". ${ }^{365}$ Si cominciò anche a parlare del "suicidio di massa" a Okinawa e della criticabile condotta della guerra.

La nipponista Foljanty-Jost ha ricostruito e tradotto le vicende processuali e lo scambio di osservazioni col ministero. Come esempio della discussione basti qui citare uno dei punti criticati dal Ministero (le cui argomentazioni erano soltanto orali, e sono quindi state ricostruite sulla base dei documenti processuali o editoriali). L'esempio si riferisce alla Seconda guerra mondiale:

Ienaga: "Questa guerra "irresponsabile".

Ministero: Non è ingiusto, rispetto agli altri paesi, attribuire la responsabilità al solo Giappone? Per motivi pedagogici "irresponsabile" dovrebbe essere cancellato.

Ienaga: Poiché è indiscusso che si tratta di una guerra irresponsabile, la cancellazione non è necessaria.

Ministero: "Irresponsabile" va assolutamente cancellato".366

363 Gesine Foljanty-Jost (1979), Schulbuchgestaltung, 42; il passo è tratto da Saburō Ienaga (1970), The Historical Significance.

364 Yoshiko Nozaki (2008), War Memory, 130. La lunga descrizione di questo processo (72-135) va ricondotta al fatto che nel suo corso vennero ridiscussi e definiti singoli eventi bellici, in particolare il massacro di Nanchino, il "suicidio di massa" di Okinawa e gli esperimenti di guerra batteriologica dell'Unità 731 (su quest'ultima, cfr. nota 193, p. 73).

365 Yoshiko Nozaki (2008), War Memory, 63. Infatti quello che il Ministero "attempted to erase entirely from the textbooks was the representation of the Nanjing Massacre" (72).

366 Gesine Foljanty-Jost (1979), Schulbuchgestaltung, 103. 
Una revisione involutiva dei testi scolastici venne indirettamente provocata nel 1991 dalla Guerra del Golfo e dalle discussioni su una possibile partecipazione del Giappone, nonostante l'articolo 9 della Costituzione. Una figura rilevante in queste polemiche fu il pedagogista Nobukatsu Fujioka: per lui "l'atteggiamento del Giappone era vergognoso, poiché il Giappone accettò solo le richieste di appoggio economico degli altri Stati, senza poter rappresentare alcun proprio interesse. Un simile comportamento era per lui inaccettabile. Quest'esperienza lo portò a considerare criticamente la posizione pacifista come il risultato dell'educazione seguita alla Seconda guerra mondiale". ${ }^{367}$

Mosso da questo ripensamento, nel 1995 Fujioka fondò il "Gruppo di ricerca per un'immagine liberale della storia" che criticava l'insegnamento della storia recente nei libri didattici giapponesi. Per lui, la storia recente giapponese era deformata da due interpretazioni. Una proveniva dal Tribunale di Tokyo che, conformemente alla visione delle potenze vincitrici, presentava il Giappone come il principale responsabile della Seconda guerra mondiale: ritorna qui la difficoltà di accettare la "moralizzazione del concetto di colpa" e la differente percezione della nozione di colpa in una certa parte della cultura giapponese (cfr. supra, p. 106). L'altra interpretazione proveniva dal Komintern che, partendo dalla concezione comunista, propagandava un Giappone liberato dalla struttura imperiale. Entrambi le visioni, per Fujioka, erano influenzate da interessi stranieri e trasmettevano ai giovani giapponesi un'immagine negativa del proprio paese. Invece i nazionalisti presentavano un'immagine del Giappone bellico coincidente con quella del "Sfera di Co-prosperità della Grande Asia Orientale": il Giappone avrebbe aiutato gli altri paesi asiatici a liberarsi dal giogo coloniale e ne avrebbe iniziato l'industrializzazione. Anche negli scritti tedeschi nazionalsocialisti l'espansione coloniale giapponese è presentata come "liberazione dei giovani popoli" ${ }^{638}$ (ma sulla "gioventù" di civiltà antiche come quelle non solo dell'India si potrebbe discutere).

367 Hiromi Igari (2000), Die Schulbuchkontroverse, 272.

368 Albrecht von Urach (1942), Das Geheimnis japanischer Kraft, 94: "Entrando far parte del sistema d'ordine delle potenze dell'Asse e affermandosi con decisione nella lotta di liberazione dei giovani popoli, il Giappone getta sulla bilancia della storia mondiale l'intera sua forza non solo per la costruzione di una sfera comune di benessere con i popoli dell'Asia orientale, ma anche per la costruzione di un nuovo ordine mondiale". 
Per Fujioka la verità si collocava fra questi due estremi: questa era la sua "immagine liberale della storia", in base alla quale avrebbero dovuto essere riequilibrati i libri scolastici giapponesi, per lui troppo negativi. I suoi critici ritengono però che il risultato finale della sua dottrina, nonostante il nome, sia una parziale riabilitazione del Giappone militarista, vicina più ai nostalgici della "Sfera di Co-prosperità della Grande Asia Orientale" che agli storici liberali nel senso proprio di quest'ultimo termine.

Fujioka prese posizione anche contro il Ministero dell'educazione, quando esso nel giugno 1996 approvò i libri di testo che sarebbero stati usati nell'anno scolastico successivo. In essi, per la prima volta, si parlava delle "comfort women" e della prostituzione cui esse erano state costrette durante la guerra. ${ }^{369} \mathrm{Il}$ gruppo di lavoro appositamente formato presentò una serie di osservazioni sostanzialmente negazioniste del fenomeno, osservando inoltre che quel tema era inadatto per libri destinati alle scuole medie. Queste posizioni vennero condivise da giornali conservatori e anche da alcuni deputati del Partito Liberal-Democratico.

Un'analoga revisione viene richiesta anche per il massacro di Nanchino, di cui si metteva in dubbio non solo l'entità, ma anche l'efferatezza. Effettivamente i dati sulle vittime non sono univoci: si va dalle 300.000 vittime indicate dai cinesi alle 200.000 del Tribunale di Tokyo, a cifre minori indicate in vari libri scolastici, sino ai 40.000 che alcuni - tra cui Fujioka ritengono l'unica cifra scientificamente provata. Anzi, Fujioka e altri sostengono che il massacro di Nanchino fu opera dei cinesi stessi, ma che la notizia venne fraintesa dal giornalista del "New York Times": e da lì prese inizio l'accusa contro i giapponesi. Oggi in Giappone questo negazionismo è proibito, così come in Germania il negazionismo dell'Olocausto è punito dall'art. 194 del codice penale.

Il revisionismo giapponese colpisce "per l'audacia e per i toni passionali, talvolta persino spettacolari, con cui si propone al grande pubblico, servendosi di programmi televisivi, di produzioni cinematografiche e di una letteratura per lo più popolare e rivolta in primo luogo ai giovani”. ${ }^{370}$ Il successo dei manga (fumetti) di Kobayashi Yashinori deve far riflettere sul fatto che

369 Nel 2000 questi “crimini contro l'umanità" vennero giudicati dal Tribunale internazionale delle donne: Rosa Caroli (2010), Storia e storiografia in Giappone, 106-107. La polemica pubblica sulle "comfort women" aveva preso inizio nel 1991: cfr. supra, nota 292, p. 111. Rosa Caroli (2010), Storia e storiografia in Giappone, 97. 
questi mezzi di comunicazione hanno sui giovani una presa maggiore che $\mathrm{i}$ libri scolastici.

Kobayashi "si appella ai lettori affinché condannino le ricerche su crimini di guerra e le scuse ufficiali rivolte alle popolazioni asiatiche. Nei suoi fumetti il tema delle cosiddette 'comfort women' [...] viene liquidato asserendo che la loro reclusione da parte dei soldati giapponesi fu motivata dalla volontà di difenderle dalla violenza che regnava nelle zone di guerra". ${ }^{371}$ Queste opinioni non sono minoritarie: in Giappone, il manga di Kobayashi intitolato Sensoron (Dibattito sulla guerra) venne considerato l'evento editoriale del 1998 e l'importante rivista "Seikai" pubblicò un numero speciale sul "fenomeno Kobayashi". In Germania, il settimanale "Die Zeit" criticò aspramente quel manga, e fu a sua volta attaccato dal disegnatore. ${ }^{372}$

In sintesi, secondo il "Gruppo di ricerca per un'immagine liberale della storia" (ma 'liberale' nel senso conservatore già visto) la storia giapponese dovrebbe dedicare molto spazio all'epoca Meiji, che gettò le fondamenta del Giappone moderno. La Prima guerra sino-giapponese (1894-1895) e quella contro la Russia (1904-1905) sarebbero state guerre di difesa (e non di invasione, come invece scrivono i libri scolastici) e la colonizzazione della Corea sarebbe stata soltanto un'imitazione di quanto gli europei facevano su vasta scala. La revisione della storia giapponese proposta dal "Gruppo di ricerca per un'immagine liberale della storia” venne dapprima trascurata come inattendibile, però il suo progressivo diffondersi suscitò una crescente serie di opposizioni, che ne contestavano il negazionismo sulla base di dati storici.

Il nuovo manuale di storia dell'editrice Fushoka si differenzia da tutti gli altri perché punta sull'arte giapponese, per illustrare la storia non tanto descrivendo fatti, quanto suscitando sentimenti.

A Kobayashi vengono affidati i temi più sensibili - la narrazione dei miti, la guerra nell'Asia orientale e nel Pacifico, gli anni dell'occupazione e del processo di Tokyo - che egli affronta secondo il suo piglio consolidato: "Dal

371 Rosa Caroli (2010), Storia e storiografia in Giappone, 97-98 (l'autrice indica anche gli autori che si sono opposti a questo revisionismo, 99-100); cfr. anche Harumi Watanabe (2010), Come condividere.

372 Henrik Bork (1999, 11 marzo), Geschichtsklitterung in Sprechblasen; cfr. anche Gallige Sprechblasen (1999); Berndt WeILER (1999, 7 agosto), Kobayashi - und wie er die Welt sieht. Cfr. anche Neo-Gömanism Manifesto Special - on War, in: Wikipedia, L'enciclopedia libera. 
suo debutto nel 1976 Kobayashi ha satireggiato la società giapponese contemporanea, contrapponendo infine ai punti deboli della sua cultura l'asserzione di valori nazionalistici, riferiti al recente passato". ${ }^{373}$ Dal 1992 Kobayashi pubblica la serie dei suoi manga atipici con il titolo Manifesto dell'Arrogantismo (Gomanizumu sengen), in aperta polemica con lo spirito "masochistico" di cui vengono accusati i democratici postbellici in Giappone. Il manga di Kobayashi non segue i canoni del genere, però non soltanto è riuscito a evitare il rifiuto da parte dei giovani lettori, ma anzi è divenuto uno degli eventi editoriali di quegli anni.

In Italia, dagli anni Venti agli anni Quaranta la politica scolastica subì due radicali cambi di indirizzo politico: da quello monarchico-costituzionale a quello fascista e, poi, dall'indirizzo fascista a quello repubblicano-democratico. ${ }^{374}$

Con l'avvento del fascismo il Ministero dell'educazione nazionale venne affidato al filosofo Giovanni Gentile, che a sua volta nel 1923 nominò il pedagogista Giuseppe Lombardo-Radice direttore generale dell'istruzione elementare e responsabile della commissione che avrebbe dovuto esaminare o respingere i libri di testo destinati alle scuole elementari. In questa fase $\mathrm{i}$ manuali esprimevano più una fedeltà dinastico-costituzionale che un'adesione al fascismo. L'indirizzo mutò nel 1929, con la fine dell'attività ministeriale di Lombardo-Radice e con l'introduzione del libro unico di Stato.

Un saggio che ripercorre vicende e contenuti dell'editoria scolastica nell'epoca fascista giunge alla conclusione che "gli anni '30, in sostanza, avevano fascistizzato la scuola e instaurato un conformismo culturale e scolastico (di programmi e di parole d'ordine) di regime, ma non avevano prodotto modelli formativi che non fossero quello totalitario e ormai extra-scolastico (o super-scolastico) della Gioventù Italiana del Littorio

373 Jacqueline Berndt (2003), Eine zeitlos schöne Nation, 210.

374 Un panorama sintetico di questa evoluzione è contenuto in due libri-catalogo: Rossella Coarelli (ed.) (2001), Dalla scuola all'Impero; Rossella Coarelli (ed.) (2004), Istruiti e laboriosi. Entrambi i volumi, composti di vari saggi, contengono anche un'importante iconografia e le schede bibliografiche dei libri esposti nelle due mostre presso la Biblioteca Braidense di Milano, nonché gli indici degli editori e degli autori scolastici di quegli anni. Cfr. anche Luigi Cajani (2003), Italien und der Zweite Weltkrieg. 
(GIL)", ${ }^{375}$ che dipendeva non dal Ministero dell'educazione nazionale, ma direttamente dal partito fascista.

Si è già visto che il regime, con le sue "veline", imponeva alla stampa non solo i temi da trattare, ma anche il tono con cui trattarli. Questo vincolo esisteva anche per i testi scolastici, tenuti a "seguire i suggerimenti delle circolari ministeriali inserendo nei libri di lettura la parafrasi o la spiegazione del pensiero ufficiale dei ministri”, ${ }^{376}$

Poiché in Italia il fascismo non finì ovunque nello stesso momento, la riforma scolastica e l'intervento sui libri di scuola ebbero luogo in modi e tempi diversi, ${ }^{377}$ e con grandi difficoltà. Infatti alla fine della guerra anche l'Italia scolastica era spezzata in due. Nel centro-nord la Repubblica Sociale Italiana confermava il testo unico dettato dal suo fascismo repubblicano. Nelle limitate zone del nord liberate dai partigiani - le "repubbliche partigiane” delle Langhe, dell'Alto Monferrato, dell'Ossola e della Carnia, e altre ancora $^{378}$ - gli embrioni di amministrazioni locali democratiche tentarono anche una riforma dei testi scolastici. In particolare, nella Repubblica dell'Ossola (durata soltanto dal 10 settembre al 23 ottobre 1944) la giunta provvisoria preparò una carta programmatica di riforma dell'intero insegnamento, in cui "si raccomanda l'esaltazione dei valori umani insiti nella cultura classica, il ripristino dello studio delle lingue straniere, l'eliminazione delle forme militaresche della ginnastica; per i libri di testo, si sollecita la correzione di quelli esistenti e, nel frattempo, si chiede l'invio di quelli adottati nelle scuole della Svizzera di lingua italiana", confinante con le valli dell'Ossola. ${ }^{379}$

Invece nell'Italia centro-meridionale - con il fronte in continuo benché lento movimento verso Nord - nei primi tempi non si riusciva material-

375 Fulvio De Giorgi (2004), Nel "cantiere scolastico" della Repubblica, 26, saggio che costituisce l'ampia Presentazione al volume di Rossella Coarelli (ed.) (2004), Istruiti e laboriosi.

376 Marcella Bacigalupi, Piero Fossati (1986), Da plebe a popolo, 171. Si vedano in particolare: La pedagogia delle circolari, 171-176; Bibliografia, 267-282.

377 Rossella Coarelli (2004), Il secondo dopoguerra. Questo saggio contiene anche vari esempi del modo in cui i testi scolastici dell'immediato dopoguerra descrivevano la fine del regime fascista (144-149).

378 Nunzia Augeri (2014), L'estate della libertà; Giongio Bocca (2005), Una repubblica partigiana; Massimo Legnani [1968], Politica e amministrazione.

379 Gianni Oliva (2018), La grande storia della Resistenza, 364 (che rinvia a Quinto Casadio (1967), Gli ideali pedagogici della Resistenza). Sulla Repubblica dell’Ossola, pp. 155 s. 
mente a scrivere e a stampare nuovi libri scolastici, quindi si usavano i testi fascisti strappando le pagine più "di regime": qualcosa di simile, quindi, a quanto già si è visto per il Giappone. Nel 1944 il Ministero dell'Educazione Nazionale prese il nuovo nome di Ministero della Pubblica Istruzione ed emanò i nuovi programmi scolastici, influenzati anche dal pedagogista statunitense Carleton W. Washburne, che faceva parte del Sottocomitato per l'Istruzione del Governo Alleato in Italia. I testi scolastici, che erano stati rivisti con la riforma Bottai nel 1940, dovettero essere nuovamente modificati nell'autunno del 1945, in tempo per l'inizio del nuovo anno scolastico. Nel 1946 divenne ministro della pubblica istruzione il democristiano Guido Gonella, che organizzò un'inchiesta in vista della riforma dell'insegnamento ma, soprattutto, "con l'obiettivo di creare le condizioni per una collaborazione convinta da parte della classe magistrale che usciva dal fascismo con atteggiamenti di passività o di diffidenza verso la nuova concezione democratica della scuola". 380

Nell'Italia del Nord, dopo la caduta della Repubblica Sociale Italiana e la resa dell'esercito tedesco, il Governo militare alleato si occupò anche della scuola promuovendo sia l'epurazione del personale compromesso col fascismo, sia nuovi programmi con i corrispondenti libri di testo. Nel 1945 viene istituita una commissione per defascistizzare i manuali scolastici, la quale operò sino all'autunno del 1945, incontrando con regolarità autori ed editori. La sua relazione finale contiene chiare linee direttive e stabilisce le sanzioni per i contravventori:

Non potranno essere venduti, conservati in magazzino, o ristampati, libri scolastici ad eccezione di quelli approvati dal Governo militare alleato. [...] I libri che contengono propaganda fascista vanno sequestrati, e saranno fatti pervenire al Sindaco che li terrà a disposizione del Provveditore agli Studi. Con l'approvazione dell'Ufficiale Regionale dell'Educazione i libri sequestrati devono essere inventariati dai librai ed editori e riposti in luogo sicuro, per essere venduti alle cartiere come carta da macero. [...] Quei libri che posseggono soltanto poche pagine di propaganda fascista, potranno essere venduti ed usati quando dette pagine siano state asportate. Le pagine dovranno essere tolte dai volumi prima che questi siano esposti alla vendita. ${ }^{381}$

380 Rossella Coarelli (2004), Il secondo dopoguerra, 134. Cfr. anche l'intero paragrafo: La defascistizzazione dei testi scolastici, 134-149.

381 Commissione Alleata, Sottocommissione dell'Educazione (ed.) (1945), Defascistizzazione dei libri di testo, in: Archivio INSMLI, fondo CLN, busta 79, fasc. 271, cit. in Rossella CoArelli (2004), Il secondo dopoguerra, 139-140. Sull' INSMLI: “La rete degli Istituti per 
Le valutazioni dei testi da adottare avvenivano su scala regionale, con la conseguente discordanza di giudizi fra le diverse regioni: giudizi che dovevano infine essere armonizzati da una Commissione suppletiva. Un atteggiamento di base accomunava però questi documenti dall'indirizzo spesso eterogeneo: la lotta all'analfabetismo non solo "strumentale", che era ancora una vera piaga sociale, ma anche "spirituale", cioè la lotta all'atteggiamento mentale che ostacolava la diffusione della democrazia; l'“analfabetismo spirituale" equivaleva a "immaturità civile, impreparazione alla vita morale e politica e insensibilità ai problemi che essa presenta. ${ }^{382}$

Per le scuole elementari, come si è visto, potevano essere venduti o usati solo i testi approvati dal ministero dell'istruzione. Però, accanto al problema dei contenuti fascisti, i librai e gli editori sollevavano anche i problemi pratici della fornitura di carta, ${ }^{383}$ della distribuzione in un contesto dalle comunicazioni ancora precarie a causa dei danni bellici, e - per ragioni economiche - anche il ricupero almeno parziale di testi stampati entro il 1944. L'Italia riviveva così il fenomeno inverso rispetto a quello vissuto al momento dell'avvento del fascismo: "spesso si riconfermò la modalità, già attuata per fascistizzare $\mathrm{i}$ testi in uso nelle scuole prima dell'avvento del regime", cioè la tendenza ad adattare $\mathrm{i}$ vecchi testi alla nuova situazione con i minimi ritocchi possibili: "anche adesso si potevano individuare interventi sui libri con i quali gli autori di fatto andavano a camuffare i vecchi contenuti attraverso aggiustamenti e mimetizzazioni". ${ }^{384}$

Quanto ai contenuti politicamente più spinosi, nei testi tanto per le scuole elementari quanto per le secondarie sembra che "gran parte degli autori preferisse sorvolare del tutto sul ventennio appena trascorso". ${ }^{385} \mathrm{Si}$

la storia della Resistenza e dell'età contemporanea in Italia è un'associazione coordinata dall'Istituto Nazionale Ferruccio Parri (ex INSMLI-Istituto Nazionale per la Storia del Movimento di Liberazione in Italia), ente fondato per l'appunto da Ferruccio Parri nel 1949 con lo scopo di raccogliere, conservare e studiare il patrimonio documentario del Corpo Volontari della Libertà e del Comitato di Liberazione Nazionale Alta Italia" (http://www.italia-resistenza.it/chi-siamo/).

382 Fulvio De Giongi (2004), Nel "cantiere scolastico" della Repubblica, 15.

383 Nell'immediato dopoguerra la carta era razionata e di cattiva qualità (carta acida); ciò rende problematica la conservazione di quasi tutte le pubblicazioni dell'epoca, compresi i testi scolastici: Franca Alloatti (2004), La carta di guerra.

384 Rossella Coarelli (2004), Il secondo dopoguerra, 143.

385 Rossella Coarelli (2004), Il secondo dopoguerra, 143. 
resero così necessarie due circolari ministeriali per obbligare i manuali a includere nella trattazione anche il periodo fino alla costituzione repubblicana, cioè a occuparsi tanto del Fascismo quanto della Resistenza. Su questo tema era cautissimo anche il testo scolastico del noto romanista Antonio Guarino, che negli anni Settanta sarebbe stato eletto senatore nelle liste del Partito Comunista Italiano. ${ }^{386}$ Altri temi riproducevano stereotipi ereditati dal passato: "infelicissima era poi spesso la parte dei manuali relativa alla geografia umana in cui si possono notare residui, forse pure inconsapevoli, di razzismo".387

La lentezza della defascistizzazione dei testi è a volte legata alle apparenze, allo stile, cioè alla consuetudine acquisita nel ventennio: diviene a volte difficile distinguere l'inerzia intellettuale dalla resistenza passiva verso la nuova democrazia. Benché dopo il 1946 i testi fossero divenuti gradualmente più estesi ed espliciti, spesso la loro formulazione linguistica risentiva ancora della retorica tipica del fascismo. Del resto, lo stesso avveniva nei commenti parlati che accompagnavano la "Settimana Incom", il breve "cinegiornale" sull'attualità settimanale proiettato prima dei film dal $1946 \mathrm{al}$ 1965: una voce stentorea enunciava frasi imbottite di aggettivi altisonanti e lontane dalla lingua parlata.

Poiché era inevitabile riutilizzare, sia pur riveduti, i "libri di materie letterarie e scientifiche provenienti dall'editoria fascista", il passato regime tendeva a sopravvivere non tanto nei contenuti, quanto nelle vecchie forme sotto forma di "decaloghi, massime o illustrazioni o le stesse copertine, requisiti che rendevano le pubblicazioni maggiormente gradite al regime, e dalla cui valutazione dipendeva la possibilità per gli insegnanti-autori di ottenere il 'merito distinto' e l'iscrizione al 'ruolo d'onore' e, quindi, anticipati aumenti di stipendi e avanzamenti di grado" ${ }^{388}$ La fretta dell'immediato dopoguerra fece sì che molti di questi stilemi non venissero eliminati dalle edizioni postbelliche. Si spiega così perché le commissioni di defascistizzazione censurassero la forma anche nei testi di materie il cui contenuto si prestava poco ad essere fascistizzato, come l'economia domestica, la stenografia o l'igiene.

386 Rossella Coarelli (2004), Il secondo dopoguerra, 144.

387 Fulvio De Giorgi (2004), Nel "cantiere scolastico" della Repubblica, 53.

388 Rossella Coarelli (2004), Il secondo dopoguerra, 151-152. 
Dal punto di vista estetico-editoriale, negli anni dal 1946 al 1953 le pubblicazioni scolastiche sono "piuttosto povere nell'impostazione grafica o meglio meno ricche, rispetto alla produzione degli anni Trenta e dei primi anni Quaranta". ${ }^{389}$ Un esempio della defascistizzazione iconografica può essere desunto da due manuali di matematica. Un manuale del 1941 spiega la moltiplicazione con l'immagine a colori di 3 Balilla in uniforme allineati in 7 file. Nel 1950, invece, le operazioni matematiche vengono illustrate soltanto con i numeri allineati su una lavagna nera a quadretti. ${ }^{390}$ Con l'avvento della repubblica cambiano le persone di riferimento anche nei manuali di matematica: "Per aiutare un compagno di lavoro fra i 9 operai di un laboratorio si sono raccolte L. 1129. Avendo versato tutti la stessa quota, quanto ha versato ogni operaio?"? ${ }^{391}$

La repubblica italiana doveva affrontare anche le questioni ereditate dal colonialismo. L'accordo italo-libico del 2 ottobre 1956 tentava di risolvere i problemi pratici post-coloniali ancora aperti dopo l'indipendenza della Libia nel 1951. Anni dopo, con il comunicato congiunto del 4 luglio 1998, si affrontarono anche i problemi culturali, uno dei quali fu la creazione di un "Libyan Studies Centre" presso l'Istituto Italiano per l'Africa e l'Oriente, a Roma. Uno dei frutti di questa cooperazione è l'analisi del modo in cui i manuali scolastici dell'Italia dapprima liberale, poi fascista e infine repubblicana hanno affrontato il problema della colonizzazione in Libia. ${ }^{392}$

Uno stereotipo sbrigativamente italocentrico dominava i manuali dall'inizio della conquista libica fin quasi ai nostri giorni, e bastano pochi esempi a dimostrarlo. Nel 1914 i bambini della terza elementare imparavano che "l'Italia per far rispettare i sudditi italiani che lavoravano in Libia, nel 1911 dichiarò guerra alla Turchia. Il 5 ottobre i nostri marinai entrarono vittoriosi

389 Guido Mura (2004b), Oltre l'immagine, 111 (Documentazione iconografica, 161-188); Guido Mura (2004a), Esempi di grafica del ventennio, (Documentazione iconografica, 101-146). Entrambi i saggi analizzano la forma dell'illustrazione o della grafica (riprodotta a colori), ma non il messaggio che esse trasmettono.

390 L'illustrazione con i Balilla costituisce la copertina del volume di Francesco Morra (1941), Numeri e figure (riprodotta in: Rossella Coarelli (2001), Dalla scuola all'Impero, 101). Il sobrio esempio numerico è contenuto Giorgio Cammelli (1950), Aritmetica, illustrazione non numerata dopo p. 161.

391 Fulvio De Giongi (2004), Nel "cantiere scolastico" della Repubblica, 14.

392 Nicola Labanca (ed.) (2003), La Libia nei manuali (bibliografia, 179-186; riproduzioni di scritti e documenti, 189-235). Vengono esaminati 43 libri di testo. 
a Tripoli, cacciando nel deserto i Turchi e gli Arabi” ${ }^{393}$ Il tono è invariato anche nel 1927: "La Libia, che comprende la Tripolitania e la Cirenaica, apparteneva alla Turchia. L'Italia, avendo i suoi interessi, chiese ai Turchi, che governavano pessimamente, di poterli difendere. Ebbe un rifiuto e dichiarò guerra il 27 settembre del 1911" ${ }^{394}$ In seguito, nel 1936, la colonizzazione fascista è presentata come la continuazione di quella romana: "Gli italiani vivevano laggiù come nel loro proprio paese. Del resto non era stato paese loro anche quello, una volta? Dappertutto ritrovarono i segni dei loro padri. A Tripoli, un bell'arco romano di trionfo. [...] E il fante poté mettere i suoi accampamenti, dove aveva accampato il legionario romano."395

L'avvento della repubblica democratica e antifascista in Italia non modifica la prospettiva della narrazione e nel 1947 si legge: dopo l'occupazione di Massaua, "tale possedimento venne ampliato lungo la costa e nell'interno e si formò la Colonia Eritrea. Ma l'Italia dovette allora combattere contro gli Abissini che ostacolavano la nostra pacifica penetrazione in quelle regioni”. Si noti: "pacifica", anche se i generali Badoglio e Graziani vennero accusati di crimini di guerra per aver usato i gas asfissianti in quella campagna. ${ }^{396}$

Considerando soltanto la fase storica che vide il passaggio dal fascismo alla repubblica, le soluzioni più adottate nei testi scolastici erano o il silenzio (infatti la quasi totalità dei manuali terminava con la Prima guerra mondiale) o la ripubblicazione dei testi anteriori. Per spiegare le guerre africane, sino agli anni Sessanta veniva proposta una giustificazione demografica o l'attenuante della buona colonizzazione: il mito degli "Italiani brava gente". In generale ritornavano appena rivisti i testi degli anni Venti, anche se non mancavano positive eccezioni, rappresentate da testi innovativi.

Come l'autore giapponese già citato, posso ricordare come esperienza autobiografica un testo dei miei anni liceali. L'ottimo manuale di storia di

393 AeC (1914), Brevi cenni, 27, riprodotto in Nicola Labanca (ed.) (2003), La Libia nei manuali, 189.

394 Ettore Gliozzi (1927), Il risorgimento italiano, 43, riprodotto in Nicola Labanca (ed.) (2003), La Libia nei manuali, 200.

395 Gioncchino Volpe (1936), La storia d'Italia raccontata ai ragazzi delle scuole di avviamento professionale, 144, riprodotto in Nicola Labanca (ed.) (2003), La Libia nei manuali, 210.

396 Mario Conti Pertanini (1947), Corso di storia e geografia per la classe V, 51, riprodotto in Nicola Labanca (ed.) (2003), La Libia nei manuali, 217; Simone Belladonna (2015), Gas in Etiopia. 
Armando Saitta apre la terza edizione del 1957 annunciando "l'aggiunta del periodo fascista e della Seconda guerra mondiale”. Un'aggiunta forse tardiva, ma consistente perché, sulle 943 pagine del manuale, 74 sono dedicate a temi allora attualissimi: infatti il volume si chiude ricordando che "l'Italia fa parte del sistema atlantico e sul finire del 1955 è stata ammessa all'ONU". 397 Tuttavia questa attenzione alla contemporaneità e all'oggettività era, ancora alla fine degli anni Cinquanta, più l'eccezione che la regola.

L'inadeguatezza dei testi scolastici era stata segnalata dagli storici, ma senza successo. ${ }^{398}$ Anzi, negli anni Novanta, cioè "nel decennio seguito alla fine del bipolarismo e nel quadro di un'ascesa al governo in Italia di maggioranze di centrodestra, quella critica storiografica è stata volta in polemica politica" ${ }^{399}$ Nell'ambito di una campagna contro la cultura gramscianamente egemonizzata dalla sinistra si giunse a chiedere anche in parlamento che si istituissero commissioni per la censura dei libri di testo "ideologici". La proposta non venne accolta e la polemica andò dissolvendosi nel tempo. In conclusione, nell'Italia recente si è svolta una polemica sui libri di testo di indirizzo opposto a quella esaminata in Giappone a proposito di Ienaga Saburo: mentre in Giappone la polemica mirava a portare verso sinistra i libri di destra, in Italia essa mirava a portare verso destra i libri di sinistra.

D'altra parte, con le nuove fedeltà politiche del dopoguerra nascevano anche i nuovi conformismi: è stato notato che, "dopo il breve sussulto washburiano" già ricordato, "cala sulla scuola elementare il modello del nuovo cittadino repubblicano che nel frattempo si è scoperto filoatlantico, centrista e cattolico". ${ }^{400}$ Ma il tema del nuovo conformismo postbellico non può qui essere affrontato.

Una circolare ministeriale del $2012^{401}$ ha reso obbligatoria per i manuali scolastici la forma ibrida, cioè digitale e cartacea, che ha accresciuto il mate-

397 Armando Saitta (1957), Il cammino umano; le citazioni sono nell'Avvertenza iniziale e a p. 942.

398 Per esempio, Giongio Rochat (1970), Inchiesta sui testi per l'insegnamento.

399 Nicola Labanca (ed.) (2003), La Libia nei manuali, 55 e l'intero paragrafo: La recente polemica italiana sui libri di testo.

400 Marcella Bacigalupi, Piero Fossati (1986), Da plebe a popolo, 259.

401 Circolare Ministeriale n. 18 del 9 febbraio 2012, Adozione dei libri di testo nelle scuole statali di ogni ordine e grado per l'anno scolastico 2012-2013: indicazioni operative. La soluzione adottata dal Ministero di abolire il libro scolastico nella sola forma stampata ha suscitato critiche: cfr. p. es. Libri scolastici, digitalizzazione all'italiana (2012, febbraio 12). 
riale a disposizione degli studenti ma non ha sinora influenzato la stesura dei manuali.

Infine, nel 2014 è stata presentata una vasta ricerca sul tema che si ricollega direttamente ai temi di fondo dei tre articoli costituzionali pacifisti: Guerra e pace nei manuali scolastici italiani. ${ }^{\mathbf{4 0 2}}$ Le conclusioni della ricerca sono positive: "I manuali italiani sono generalmente aggiornati rispetto alla ricerca storiografica. La presentazione di argomenti come la guerra e la pace è approfondita e dettagliata, condotta sempre a partire dal contesto internazionale e non sono omessi aspetti contraddittori o che sono oggetto di discussione o testimoniano memorie opposte. [...] La formazione di una coscienza critica di fronte alle guerre e alle loro cause o alla difficoltà di costruire e mantenere la pace è favorita dal tono obiettivo dell'esposizione e dalla presenza di dossier di documenti a partire dai quali l'insegnante può costruire percorsi di apprendimento atti a suscitare riflessione e discussione".

I manuali giungono sino ai conflitti più recenti e analizzano la funzione delle Nazioni Unite: "Questo argomento è affrontato a più riprese: nelle sezioni in cui si parla del nuovo ordine del mondo dopo la Seconda guerra mondiale e nelle pagine dedicate ai nuovi conflitti dopo la fine della Guerra fredda quando si mettono in evidenza i nuovi problemi che l'Onu deve affrontare (guerre civili, terrorismo internazionale, moltiplicazione dei rifugiati, problemi di finanziamento e di rappresentatività delle sue istanze). [...] Se passiamo dalla pace al pacifismo è infine interessante notare come tutti i manuali dedichino qualche paragrafo e qualche documento all'opera di Gandhi e di Martin Luther King”. I manuali, conclude la ricerca, mirano quindi a fornire "non soltanto conoscenze, ma anche vere competenze di cittadinanza": anche oggi essi sono quindi uno strumento efficace contro quell'“analfabetismo spirituale" che abbiamo visto minacciare la nostra democrazia al suo sorgere, e che continua a minacciarla nella sua maturità.

402 Francesco Monducci (2015, 27 luglio), Guerra e pace nei manuali scolastici italiani, da cui provengono le citazioni nel testo. La ricerca - presentata a Fribourg (Svizzera) in occasione della Deuxième Conferénce de l'Association Internationale de Recherche en Didactique de l'Histoire et des Sciences Sociales (AIRDHSS), Guerres et paix: enjeux éducatifs (11-13 settembre 2014), - è stata svolta da ricercatori degli istituti storici della rete INSMLI (Istituto Nazionale per la Storia del Movimento di Liberazione in Italia: cfr. nota 381, p. 147. 
La Germania del secolo XX subì cambi di regime tanto numerosi quanto radicali e, ad ogni cambio di regime, corrispose anche una rivoluzione nei libri scolastici. Con la Repubblica di Weimar la Germania passò dal regime monarchico a quello repubblicano, che durò dal 1919 al 1933; alla Repubblica di Weimar subentrò la dittatura nazionalsocialista dal 1933 al 1945; dopo la fine della guerra, i due Stati tedeschi dovettero fare i conti con la dittatura nazionalsocialista partendo da due impostazioni differenti: secondo la democrazia parlamentare la Repubblica Federale (come si vedrà nelle prossime pagine) e secondo la dittatura del proletariato la $\mathrm{DDR} ;{ }^{403}$ perciò in entrambi gli Stati i libri scolastici vennero radicalmente rinnovati, ma seguendo indirizzi antitetici. Infine, dopo il 1990, i manuali della ex DDR dovettero essere sostituiti dai manuali dell'altro Stato tedesco, o da nuovi testi armonizzati con la nuova situazione politica. ${ }^{404} \mathrm{La}$ Germania unificata doveva ora fare i conti anche con la "seconda dittatura tedesca", 405 cioè con un doppio superamento del passato.

Nell'area tedesca questi cambiamenti furono così numerosi e radicali che qui sarà necessario limitarsi ad alcuni cenni generali sugli anni dopo il 1945 nella Germania federale e, soprattutto, all'insegnamento della storia contemporanea, la materia più direttamente chiamata a descrivere questi avvicendamenti politici e, quindi, a rispecchiare i corrispondenti mutamenti di narrazione nel tentativo di indirizzare le nuove generazioni verso un modello democratico di società. Più che i libri di testo verranno prese in considerazione le misure dello Stato sul contenuto dei libri di testo, e non i libri stessi, troppo numerosi: si rivelerà così lo stretto intreccio fra eventi politico-sociali, reazioni governative e indirizzo didattico.

Due intellettuali autorevoli tennero vivo il pensiero critico sul nazionalsocialismo. Thomas Mann parlò ai tedeschi dalla BBC, per tutta la durata della guerra fino a pochi giorni dopo la capitolazione: il suo ultimo discorso

403 Annette Leo (1993), Die Auseinandersetzung mit der nationalsozialistischen Vergangenheit.

404 Una descrizione per ciascuna delle fasi sin qui illustrate si trova in Hans-Werner Kunn et al. (1993), Politische Bildung in Deutschland.

405 Carola S. Rudnik (2009), Doppelte Vergangenheitsbewältigung; William John Niven (2002), Facing the Nazi Past; Christoph Kleßmann et al. (eds.) (1999), Deutsche Vergangenheit - eine gemeinsame Herausforderung; Ludger KüHnhardt et al. (eds.) (1996), Die doppelte deutsche Diktaturerfahrung; KLAus SüHL (ed.) (1994), Vergangenheitsbewältigung 1945 und 1989; Ludwig Elm (1991), Nach Hitler, nach Honecker. 
dell'8 maggio 1945 - sui campi di concentramento tedeschi, Die deutschen $K Z$ - metteva i suoi concittadini di fronte alle loro responsabilità: "Non era un piccolo numero di delinquenti, erano centinaia di migliaia di una cosiddetta élite tedesca, uomini, giovani e donne disumanizzate, che sotto l'influenza di folli maestri, in preda a un piacere malsano, hanno commesso questi delitti".06

Sulla responsabilità politica dei tedeschi si era soffermato in uno scritto del 1946 anche Karl Jaspers, ${ }^{407}$ finalmente liberato dal totale isolamento che si era autoimposto perché avversato dal regime nazista per il suo matrimonio con una donna ebrea, dalla quale si rifiutò di divorziare venendo per questo allontanato dall'insegnamento. Mentre i processi per la denazificazione passavano al vaglio le colpe dei nazionalsocialisti secondo griglie giuridiche (cfr. supra, p. 101), Jaspers reagì alla "piattezza del discorso sulla colpa" ("Flachheit des Schuldgeredes") proponendo una griglia etica in cui distingue quattro dimensioni della colpa: la dimensione criminale, politica, morale e metafisica. La colpa "morale" è quella più difficile da far accettare ai tedeschi, che contribuirono al nazionalsocialismo con "l'autoillusione, la collaborazione o l'esecuzione di ordini disumani". Poiché questa colpa morale è un problema esclusivamente individuale, Jaspers considera ingerenze indebite ("Einmischung") la rieducazione e la denazificazione volute dagli Alleati. Infine c'è la colpa "metafisica", che solo Dio può giudicare: chi non ha incondizionatamente messo in gioco la propria vita per impedire l'uccisione di altri ha questa colpa metafisica, "che non è comprensibile giuridicamente, politicamente e moralmente. Il fatto che io viva ancora, dopo che sono avvenute cose simili, grava su di me come una colpa inestinguibile". L'ambiente tedesco non era ricettivo per questo pensiero e già nel 1948 Jaspers prese la via

406 Citato in Torben Fischer (2009), Exildebatte, 48. Durante la guerra Thomas Mann svolse un'intensa attività con la BBC (http://ifb.bsz-bw.de/bsz434791377rez-1.pdf); Тномаs ManN (1997), Die deutschen KZ.

407 Karl Jaspers (1946), Die Schuldfrage; cfr. Anne-Kathrin Herrmann (2009), Karl Jaspers: Die Schuldfrage, 44-45 (analisi del testo di Jaspers e letteratura sul tema). Lo separava dalla Germania federale anche la convinzione dell'impossibilità della riunificazione tedesca: Karl Jaspers (1990), Freiheit und Wiedervereinigung. Cfr. Julia Weis (2009), Karl Jaspers: Freibeit und Wiedervereinigung, 156-157 (analisi e letteratura). La proposta di Jaspers di rinunciare all'unificazione tedesca in cambio di un mutamento democratico della DDR provocò anche la reazione del presidente della DDR, Walter Ulbricht, che alla visione di Jaspers (da lui visto come ideologo della BRD) contrappose la SED, il partito comunista della DDR, come unica sostenitrice dell'unità tedesca. 
dell'esilio - quell'esilio che non erano riusciti a imporgli i nazisti - e si trasferì a Basilea, dove restò sino alla fine dei suoi giorni. In generale la società tedesca, pur sollecitata da alcuni dei suoi migliori intellettuali, mostrava una scarsa propensione a riflettere sulle sue colpe collettive.

Nell'immediato dopoguerra, in alcune aree gli Alleati bloccarono l'insegnamento della storia, mentre in altre lo permisero purché si escludesse il periodo dal 1933 al 1945. I manuali di storia dell'epoca nazionalsocialista vennero requisiti e, parallelamente, l'epurazione allontanò gli insegnanti più compromessi con il passato regime. I nuovi manuali dovevano ricevere l'approvazione degli Alleati, che vedevano in essi uno strumento importante per l'educazione dei giovani tedeschi alla democrazia.

Con il 1947 l'insegnamento della storia era ripreso in tutto il territorio tedesco e anche i manuali scolastici riflettevano il dibattito fra gli storici, che cercavano di spiegare in quale modo come un'intera nazione avesse potuto essere coinvolta nel nazionalsocialismo. Il libro di Erich Weniger è esemplare per questa atmosfera di rinnovamento, in cui si affrontava il tema del nazionalsocialismo e della sua politica del terrore. Per lui, l'insegnamento della storia è "l'organon attraverso cui lo Stato e il popolo si accertano della propria esistenza e dei propri compiti, chiariscono la propria responsabilità davanti alla storia e introducono ogni nuova generazione alla sua responsabilità storica". ${ }^{408}$ Però questo approccio critico - benché esteso ed approfondito anche dal libro di storia più diffuso negli anni ' $50^{\mathbf{4 0 9}}$ - costituiva più l'eccezione che la regola.

Con la Guerra fredda degli anni '50, nella Germania federale l'analisi storica del nazionalsocialismo subì un mutamento di indirizzo perché su di essa si innestò un radicale anticomunismo, alimentato anche dalla costante polemica con l'“altro" Stato tedesco. Nel dibattito storico e politico si affermò la "teoria del totalitarismo", che accomunava nella trattazione il nazionalsocialismo e il comunismo come forme specifiche di una medesima concezione politica da rigettare in blocco. In particolare, si evitava il problema di coinvolgere nel nazionalsocialismo una vasta parte della popola-

408 Erich Weniger (1949), Neue Wege im Geschichtsunterricht, 4; la prima edizione è del 1946 e raccoglie alcuni saggi pubblicati nel 1945-46 nella rivista Die Sammlung. Un'analisi del modello didattico proposto da Weniger è contenuta nel saggio di Horst Kuss (2008), Neue Wege - alte Ziele?

409 Fritz Wuessing et al. (1951), Wege der Völker. 
zione, attribuendo ogni eccesso al controllo totalitario del partito nazionalsocialista sulla società e riconducendo le persecuzioni razziali alla personalità di Hitler. Al duplice totalitarismo veniva contrapposto il regime democratico della Germania federale. Però negli anni Cinquanta anche questo indirizzo didattico fu contestato e sempre più spesso si preferì far terminare l'insegnamento della storia con la Prima guerra mondiale.

Con il rinsaldarsi della struttura federale, l'organizzazione dell'insegnamento era affidata ai Länder, coordinati a livello nazionale dalla Conferenza dei Ministri dell'Istruzione. ${ }^{410}$ Questa Conferenza emana decisioni (ne vedremo tra poco alcuni esempi) recepite nei singoli Länder come programmi didattici che indirizzano i singoli docenti, ovviamente nel rispetto delle loro libertà fondamentali. I politici erano consapevoli dell'importanza politica dell'insegnamento della storia e per questo intervennero ripetutamente per indirizzarlo in una direzione ritenuta più favorevole alla democrazia $\mathrm{o}$, almeno, alle esigenze politiche del momento.

Bisognava affrontare un passato che riaffiorava in varie forme. Per esempio, nelle elezioni del 1950 il "Partito Imperiale Socialista della Germania" $(\mathrm{SRP})$ si presentò come erede del NSDAP e per questo venne vietato nel 1952 dal Tribunale costituzionale federale. Sulla scia di questa sentenza, nel 1953 le linee direttive per l'insegnamento della storia prevedevano il tema "Dittature e la Seconda guerra mondiale", seguendo l'indirizzo di includere il nazionalsocialismo e il comunismo nella comune categoria del totalitarismo. Però ancora nel 1956 in alcuni Länder non si affrontava il tema della persecuzione degli ebrei. L'atmosfera cominciò a cambiare dalla metà degli anni Cinquanta, con il subentrare di una generazione che non aveva avuto un'esperienza diretta del nazionalsocialismo e quindi affrontava quella parte del passato tedesco senza esserne stata personalmente coinvolta.

Il processo di approfondimento didattico dell'epoca nazionalsocialista venne accelerato come reazione alle manifestazioni di antisemitismo degli anni 1959-1960. La serie di attacchi alle sinagoghe e ai cimiteri ebraici nel 1959 era la continuazione dell'atteggiamento antisemita esploso con il nazionalsocialismo. Ancora nel 1949 un'inchiesta accertò che un quarto della

410 Kultusministerkonferenz, Grundsätze für den Geschichtsunterricht, Beschluß vom 17.12.1953, "Sammlung der Beschlüsse der Ständigen Konferenz der Kultusminister in der Bundesrepublik Deutschland”. 
popolazione tedesca si dichiarava antisemita. Questa percentuale crebbe ancora nel 1952. A questo ritorno dell'antisemitismo contribuì anche, nel dibattito sulle riparazioni, la strana accusa rivolta agli ebrei di arricchirsi per mezzo dell'Olocausto, esemplarmente illustrata dal "Caso Auerbach". Il responsabile dell'ufficio bavarese per gli indennizzi, Philipp Auerbach, venne accusato insieme con altri politici di essersi appropriato d'una parte dei fondi destinati ai risarcimenti. Auerbach venne assolto, ma si suicidò poco dopo.

Tra la fine del 1959 e l'inizio del 1960 il Ministero degli interni accertò più di 600 casi di azioni antisemite. Il caso più emblematico fu l'attacco alla sinagoga di Colonia, inaugurata a marzo e imbrattata il 25 dicembre 1959. In questo contesto la reazione del governo federale fu netta, ma risentiva del clima della Guerra fredda. Infatti il governo federale pubblicò un libro bianco per condannare quegli eventi, però nella sezione Influenza delle forze anticostituzionali accentuò la partecipazione dei (pochi) estremisti di sinistra, giungendo alla conclusione che "le concezioni del DRP [Deutsche Reichspartei] avevano contribuito a provocare quelle azioni, ma che delle 'forze comuniste' avevano utilizzato quegli eventi per screditare il governo federale".411

Nei dibattiti suscitati da questi eventi si rimproverò all'insegnamento scolastico di non aver attribuito la giusta rilevanza al passato nazionalsocialista. Per questo, nel febbraio del 1960, la Conferenza dei Ministri dell'Istruzione raccomandò la Trattazione del recente passato nell'insegnamento della storia e dell'educazione civica. ${ }^{\mathbf{4 1 2}} \mathrm{Vi}$ si raccomandava l'analisi del nazionalsocialismo, ma non si menzionava specificamente l'Olocausto, tema particolarmente spinoso che gli insegnanti affrontavano con esitazione. Due anni dopo, la stessa Conferenza indicava le linee direttrici secondo cui preparare i libri per l'insegnamento della storia, invitando a mettere in luce la connessione tra conoscenza storica e agire politico. ${ }^{413}$ Quasi contemporaneamente

411 Maria Munzert (2009), Neue Antisemitismuswelle, 86.

412 Kultusministerkonferenz, Behandlung der jüngsten Vergangenheit im Geschichts- und Gesellschaftskunde-Unterricht, Beschluß vom 11.-12. Februar 1960 (fonte: cfr. nota 410, p. 157). Con questa decisione la KMK reagì alle attività antisemite del 1959.

413 Kultusministerkonferenz, Gestaltung der Lehrbücher für den Unterricht in neuester Geschichte und Zeitgeschichte, Beschluß vom 2. Juli 1962 (fonte: cfr. nota 410, p. 157). Per un quadro complessivo sulla didattica dell'Olocausto: Falk Pingel (1994), Nationalsozialismus und Holocaust. 
un'altra raccomandazione indicava le Linee direttrici per la trattazione della teoria del totalitarismo nell'insegnamento. ${ }^{\mathbf{4 1 4}}$

Gli avvenimenti all'inizio degli anni Sessanta esigevano un continuo confronto della società tedesca con il suo passato. Nell'indifferenza dei più, l'8 maggio 1960 si sarebbero prescritti i delitti nazisti: il dibattito sul da farsi durò dal 1960 al 1979, e alla fine vinse la non prescrittibilità degli omicidi. Nel 1961 lo Stato di Israele processò Adolf Eichmann, figura centrale nell'Olocausto. L'opinione pubblica tedesca venne scossa dal processo contro il personale del campo di concentramento di Auschwitz, celebrato a Francoforte dal dicembre 1963 al 1965.

Intanto maturava il 1968, con il movimento studentesco e con le frange armate come la Rote Armee Fraktion. In particolare, gli studenti appartenevano ormai a una generazione cresciuta fuori dal nazionalsocialismo e assumevano una posizione critica rispetto ai propri genitori. Attraverso il movimento degli studenti e la sua ripercussione nella società, la "teoria critica della società" - che ha le sue radici nell'"Istituto per la Ricerca Sociale” fondato nel 1922 a Francoforte - si fece strada nell'insegnamento della storia, e anche l'educazione civica venne presentata come uno strumento per il mutamento sociale.

Era inevitabile che in quegli anni si intensificasse il dibattito sociale sull'insufficienza della preparazione democratica dei cittadini. Sempre nel 1960 venne perciò istituita la nuova disciplina "Gemeinschaftskunde", ${ }^{15}$ equivalente all'italiana "Educazione civica". Però anni dopo, nel 1964, un'indagine constatava che nei giovani la conoscenza sull'epoca nazionalsocialista si riduceva per lo più alla figura di Hitler. D’altra parte, anche la maggior parte dei libri di testo tendeva a far coincidere il nazionalsocialismo con la nefasta figura di Hitler, riducendo al minimo la trattazione del coinvolgimento della società tedesca e degli orrori di quella dittatura. Non pochi manuali, infine, si cavavano d'impaccio chiudendo col 1933 la trattazione della storia del secolo XX.

414 Kultusministerkonferenz, Richtlinien zur Behandlung des Totalitarismus im Unterricht, Beschluß vom 5. Juli 1962 (fonte: cfr. nota 410).

415 Saarbrücker Rahmenvereinbarungen zur Einrichtung des Faches Gemeinschaftskunde, 1960. "Queste direttive erano una risposta politica all'ondata di indignazione che accompagnò gli imbrattamenti delle sinagoghe nel dicembre 1959": Hannah Zimmermann (2012), Geschichte ohne Zeitgeschichte, 39. L'evoluzione di questa materia è nel capitolo 3.1.2.1. Exkurs: Geschichte des Faches Gemeinschaftskunde, 38 s. 
A questa situazione si richiama il testo radiofonico del 1966 di Theodor W. Adorno, Educazione dopo Auschwitz, ${ }^{\mathbf{4 1 6}}$ "le [cui] frasi iniziali divennero il Credo di un'intera generazione di insegnanti: 'L'esigenza che Auschwitz non avvenga una seconda volta è il primo principio dell'educazione. Essa precede in tale misura tutte le altre esigenze, che non credo di doverla in alcun modo giustificare." $4 \mathbf{4 1 7}$

Per verificare quanto gli alunni avessero recepito dall'insegnamento sul nazionalsocialismo, Dieter Boßmann assegnò nel 1976-1977 il tema "Was ich über Hitler gehört habe" (Quello che ho sentito dire su Hitler) e raccolse in un volume i punti salienti di oltre 3000 componimenti di allievi tra i 10 e i 23 anni. ${ }^{418}$ Il risultato dimostrò che $\mathrm{i}$ tentativi didattici precedenti non avevano dato il frutto sperato: tra il contenuto dei testi scolastici e le conoscenze degli alunni c'era un tale divario, che si parlò di un "BoßmannSchock", di un "trauma" con il quale si riaprì il dibattito sul modo più efficace per insegnare la storia recente. In questo dibattito prese posizione anche un organismo federale, organizzando un convegno nazionale sul Nazionalsocialismo come problema didattico. ${ }^{\mathbf{4 1 9}}$

Dalla fine degli anni '70 si manifestarono correnti della destra estrema, cui una parte degli insegnanti reagì con insegnamenti antifascisti. Però non mancarono le critiche: il credere che una precisa conoscenza del nazionalsocialismo fosse un argine contro l'estremismo di destra, si sosteneva, si stava rivelando un errore perché sembrava che, di fronte alle spiegazioni dei docenti, non pochi gli allievi avessero assunto atteggiamenti di rifiuto.

Intanto a livello internazionale tornavano a manifestarsi le tendenze che sin dal dopoguerra avevano negato l'esistenza delle camere a gas e dell'Olocausto, per giungere così a cancellare queste colpe ascritte al popolo tedesco nella sua totalità. ${ }^{\mathbf{4 2 0}}$ In coincidenza con il $40^{\circ}$ anniversario della "Notte dei Cristalli" del novembre 1938, la Conferenza dei Ministri dell'Istruzione

416 Theodor W. Adorno (1970), Erziehung nach Auschwitz. Alcuni estratti vennero pubblicati nel 1993 anche dal settimanale Die Zeit, perché “il tema 'educazione' sembra oggi più attuale che mai" (https://www.zeit.de/1993/01/erziehung-nach-auschwitz).

417 Andrea Höft (2009), Nationalsozialismus im Schulunterricht, 174. Il passo menzionato da Höft è in Theodor W. Adorno (1970), Erziehung nach Auschwitz, 92.

418 Dieter Boßmann (1982), "Was ich über Adolf Hitler gehört habe ...."

419 Dieter Schmidt-Sinns (1980), Der Nationalsozialismus als didaktisches Problem.

420 Brigitte Bailer-Galanda (ed.) (1996), Die Auschwitzleugner; Claudio Vercelli (2013), Il negazionismo. 
- nella raccomandazione dell'aprile 1978 sulla Trattazione del nazionalsocialismo nell'insegnamento ${ }^{\mathbf{4 2 1}}$ - indicava come reagire al diffondersi delle teorie negazioniste per condurre gli scolari a un'autonoma capacità di giudizio politico.

Dalla fine degli Settanta l'epoca nazionalsocialista è oggetto di romanzi, film e serie televisive che presentarono quella fase storica in una forma più avvincente di quanto possano fare i libri scolastici. In Germania, nel gennaio 1979 la televisione diffuse la serie statunitense Olocaust, che ebbe una grande popolarità. Ma non mancarono le critiche, perché il linguaggio televisivo impone ritmi e semplificazioni non sempre consone a un tema così tragico: per Elie Wiesel, premio Nobel per la pace e reduce dai campi di concentramento, quella serie televisiva aveva trasformato l'Olocausto in una soapopera.

Infine la raccomandazione del 1980 della Conferenza dei ministri dell'Istruzione affronta il difficile discorso sulla resistenza al nazionalsocialismo, indirizzandone le ricerche anche nell'ambito della storia locale: Trattazione nell'insegnamento della resistenza nell'epoca del nazionalsocialismo.

In base a questa direttiva la scuola deve cercare di collocare la resistenza nel contesto complessivo del dominio e della politica del nazionalsocialismo. Vanno anche presi in considerazione i movimenti di resistenza fuori dalla Germania e le attività degli esiliati. Accanto alla descrizione di figure rilevanti della resistenza è particolarmente rilevante l'ambito individuato dalla storia locale e regionale. Va mostrato che la capitolazione di fronte alla dittatura è spesso iniziata non con crolli spettacolari, ma con le piccole viltà quotidiane; e che proprio nella quotidianità si trova anche una silente resistenza, senza la quale una descrizione della vita nel Terzo Reich non sarebbe completa. Tuttavia va anche illustrato come abbiano potuto sorgere la paura e l'adattamento, togliendo a molti il coraggio di rendersi conto dell'ingiustizia, o anche di reagire ad essa. ${ }^{422}$

Gli insegnanti tedeschi si confrontarono anche con l'estero sulla didattica della storia recente e del nazionalsocialismo. In particolare, nella conferenza tedesco-israeliana del 1979 sui libri scolastici si manifestò la critica secondo la

421 Kultusministerkonferenz (1978), Behandlung des Nationalsozialismus im Unterricht (che non potuto vedere; citato in Andrea Höft (2009), Nationalsozialismus im Schulunterricht, 175).

422 Kultusministerkonferenz (1980), Empfehlung zur Behandlung des Widerstandes in der NSZeit im Unterricht (Beschluss vom 4. Dezember 1980), fonte: cfr. nota 410, p. 157. Il testo è tratto dalla "Gazzetta Ufficiale" bavarese (http://www.gesetze-bayern.de/Content/Docu ment/BayVV_2230_1_1_1_1_3_UK_184/true). 
quale i testi tedeschi descrivono gli eventi, ma non li collocano nel contesto politico che li ha provocati, favorendo così la visione secondo cui il nazionalsocialismo va ricondotto alla personalità malata di Hitler. Per contribuire alla formazione degli insegnanti sulla didattica dell'Olocausto e seguendo il modello americano di valutazione di quell'insegnamento, nel 1995 venne fondato l'“Istituto Fritz Bauer". Esso è intitolato a un giudice perseguitato dal nazionalsocialismo, emigrato e poi reintegrato nella carriera con il compiti di giudicare i crimini nazisti. Fritz Bauer è internazionalmente noto per aver contribuito in modo determinante a due eventi-chiave: la cattura di Eichmann nel 1961 e l'organizzazione dell'“Auschwitz-Prozess" nel 1963.

Con la diffusione dei mezzi di comunicazione e di Internet, veniva sempre più alla luce che gli allievi acquisivano le notizie sul recente passato da una pluralità di fonti, e che quindi l'apporto della didattica non aveva più il monopolio della formazione della loro visione del mondo passato. Non solo le notizie fluivano da Internet e dai mezzi di comunicazione, ma esisteva anche una comunicazione all'interno del gruppo famigliare, per ricuperare ricordi ormai lontani ma legati al proprio ambito esistenziale. La didattica della storia recente, del nazionalsocialismo e dell'Olocausto dovette quindi sperimentare nuove vie: "Si iniziò a discutere sul distogliere l'attenzione da una comunicazione fortemente razionale a favore di una sul piano emozionale. Quello che negli anni Settanta era ancora discusso, negli anni Ottanta divenne, almeno in forma attenuata, una parte ovvia delle biografie. Il nazionalsocialismo e l'Olocausto divennero temi non più soltanto dell'insegnamento della storia e delle materie legate all'educazione civica, ma anche dell'insegnamento della religione, dell'etica e del tedesco". ${ }^{\mathbf{2 3} 3}$

Con l'unificazione della Germania lo studio del passato recente e il superamento della dittatura nazionalsocialista si estese all'intero territorio tedesco, che dal 1989 dovette anche affrontare la "seconda dittatura tedesca": quella che aveva plasmato durante un quarantennio la parte orientale della Germania. ${ }^{424}$

I grandi processi di Norimberga e Tokyo, le epurazioni negli Stati sconfitti e le riparazioni belliche sono tre tentativi di concludere la tragedia della guerra

423 Andrea Höft (2009), Nationalsozialismus im Schulunterricht, 185.

424 Katja Schweizer (1999), Täter und Opfer in der DDR. 
con misure che per il futuro evitino un evento simile, nella convinzione che esso sarebbe infintamente più catastrofico a causa della minaccia nucleare. Alla scuola è stato affidato il compito di rendere chiare le cause e le conseguenze di quella tragedia affinché le generazioni future ne siano consapevoli e non commettano gli stessi errori. Le pagine precedenti si sono soffermate su questi quattro temi per spiegare l'atmosfera sociale del dopoguerra e il bisogno di pace che pervadeva tutti i ceti sociali. Gli articoli costituzionali con cui i tre Stati dell'ex Asse rifiutavano la guerra si presentavano come la garanzia d'una futura politica di pace. Quello che però i padri costituenti non potevano prevedere era la radicale trasformazione della guerra stessa nel mezzo secolo successivo: trasformazione che ha progressivamente ridotto l'efficacia di quegli articoli pacifisti e, con essa, la garanzia di pace che sembrava acquisita alla fine della Seconda guerra mondiale.

È quindi opportuno esaminare in breve come si è trasformata la guerra e come la sua trasformazione stia rendendo obsoleti quegli articoli costituzionali, di cui con crescente frequenza si propone la modifica o l'abrogazione.

\section{Oltre mezzo secolo dopo la guerra: gli articoli costituzionali pacifisti e i nuovi conflitti}

Dopo la guerra, il ricordo ancora vivo delle catastrofi e la presenza schiacciante delle truppe d'occupazione alleate rese non solo politicamente accettabili, ma anche praticabili gli articoli pacifisti delle tre costituzioni, benché non mancassero prese di posizione che criticavano la limitazione di sovranità che essi imponevano. Tuttavia il contrasto tra quelle norme pacifiste e la realtà risultò sempre più evidente a partire dalla crisi e, poi, dal crollo del blocco sovietico, perché le guerre locali andarono coinvolgendo sempre più gli Stati della Nato, della quale erano membri tanto l'Italia quanto la Germania.

Numerose trasformazioni rafforzarono il dibattito sugli articoli pacifisti. Nel 1949, l'Italia fu tra gli Stati fondatori della Nato, cui nel 1955 si aggiunse la Germania Federale. Poiché, in base all'art. 10 del trattato, della Nato possono far parte solo Stati europei, ${ }^{425}$ il Giappone non è né membro né

425 “Art. 10. Le parti possono, con accordo unanime, invitare ad aderire a questo Trattato ogni altro Stato europeo in grado di favorire lo sviluppo dei princìi del presente Trattato e di 
partner di questa alleanza, ma dagli anni '90 collabora con la Nato ${ }^{\mathbf{4 2 6}}$ che lo considera il più antico fra i suoi "partners across the globe", in quanto partecipante non solo a operazioni di "peace-support", ma anche a "military activities". $\mathbf{4 2 7}$

La cautela con cui il Giappone si muoveva sullo scenario internazionale dopo la fine della Guerra fredda venne superata, sia pur con prudenza, a partire dagli anni Novanta. Per il Giappone esistono però più limitazioni che per altri attori internazionali: "Queste limitazioni risultano anzitutto dai vincoli imposti dal passato militarismo giapponese. Da esso si possono dedurre, sul piano interno, le disposizioni della Costituzione, una regola sociale del pacifismo e una cultura politica dell'antimilitarismo, nonché, sul piano esterno, il modo negativo in cui i suoi vicini asiatici percepiscono il Giappone". 228

Questi elementi condizionano le reazioni giapponesi di fronte alle trasformazioni degli anni Novanta e hanno generato una sua politica della sicurezza che rivede l'alleanza con gli Stati Uniti e la cooperazione multila-

contribuire alla sicurezza della regione dell'Atlantico settentrionale. Ogni Stato così invitato può divenire parte del Trattato depositando il proprio strumento di adesione presso il governo degli Stati Uniti d'America. Il governo degli Stati Uniti d'America informerà ciascuna delle parti del deposito di ogni strumento di adesione" (https:/www.nato.int/cps /fr/natohq/official_texts_17120.htm?selectedLocale=it).

426 Japan: NATO's longest-standing partner in the Asia-Pacific (2013, 13-16 aprile), http:// www.nato.int/cps/en/natohq/news_99788.htm?selectedLocale=en: "Building on initial contacts that were made in the early 1990s, Nato and Japan have developed political dialogue and practical cooperation in a wide range of areas of mutual interest over the past two decades." Un esempio di attività congiunta: NATO and Japan conduct first ever joint counter-piracy drill (2014, 25 settembre), http://www.nato.int/cps/en/natohq/news_113373. htm?selectedLocale=en.

427 Relations with Japan (2016, 7 aprile), NATO, http://www.nato.int/cps/en/natohq/topics_50336.htm: “Japan is the longest-standing of Nato's 'partners across the globe'. Building on initial contacts in the early 1990s, dialogue on common security interests has become more regular and structured. Practical cooperation has been developed in a wide range of areas, including peace-support and crisis-management activities, humanitarian assistance and disaster relief, cyber defence, defence against terrorism, non-proliferation, as well as participation in military activities. Stabilising Afghanistan has been a key focus of cooperation over the past decade. Nato and Japan signalled their commitment to strengthen cooperation in a joint political declaration signed in April 2013, during the visit of Nato's Secretary General to Japan."

428 Carsten Otтo (1999), Japans bewaffneter Pazifismus, 8. Questo articolo sintetizza la politica internazionale del Giappone a partire dagli anni Novanta. 
terale soprattutto nell'area asiatica. La diplomazia giapponese mira a rispettare la norma pacifista della costituzione, a evitare interventi militari (richiesti dal suo principale alleato, gli Stati Uniti, ma visti con sospetto dai vicini asiatici) e ad esercitare un'influenza pacifista nelle organizzazioni internazionali. Al Giappone e all'Olanda si deve nel 1991 l'istituzione del registro sul commercio di armi presso le Nazioni Unite, al fine di evitare la destabilizzazione dell'area asiatica nel corso delle attuali tensioni.

Dagli anni Ottanta le guerre medio-orientali ripropongono ciclicamente il dibattito sulla liceità dell'intervento in esse di uno dei tre Stati costituzionalmente pacifisti. Alla Guerra del Golfo fra Iran e Iraq (1980-88, chiusa con risoluzione ONU n. 598) l'Italia partecipò soltanto con forniture di armi, in parte bloccate in parlamento dai partiti di sinistra. La situazione si radicalizzò con la guerra del Kosovo (1996-99): la Nato vi intervenne dal 1999 senza la preliminare autorizzazione delle Nazioni Unite, e quella fu la prima partecipazione tedesca nel dopoguerra a un'attività bellica, mentre l'Italia fornì un rilevante supporto aereo-navale che coinvolgeva le basi statunitensi in Italia. Nella guerra russo-afghana (1979-89) gli USA appoggiarono la guerriglia antisovietica, poi, dal 2001 a oggi, l'intervento della Nato a favore del governo afghano capovolse quelle alleanze. Italia e Germania partecipano ancora oggi a questa non-guerra, ${ }^{429}$ mentre il Giappone si è limitato a un contributo economico e ad attività umanitarie in senso lato. ${ }^{\mathbf{4 3 0}}$

Nella criticata invasione dell'Iraq del 2003, tuttora in corso, l'Italia fornì un suo apporto crescente, mentre la Germania si rifiutò di partecipare dopo intense polemiche interne. Dal febbraio 2004 al luglio 2006 alla coalizione internazionale si aggregò un contingente del Giappone, le cui forze armate compivano così la loro prima azione militare dopo la Seconda guerra mondiale. Ma la dichiarazione del primo ministro Koizumi Junichiro rivela quali acrobazie verbali imponesse il rispetto almeno formale dell'articolo 9 della costituzione giapponese (il corsivo è mio): “Voglio esprimere il mio profondo rispetto e la mia gratitudine alle Forze di Autodifesa che hanno operato in condizioni difficili con la forte consapevolezza che stavano conducendo le

429 Sulla partecipazione tedesca in Afghanistan: Philıpp Münch (2015), Die Bundeswehr in Afghanistan; sulla partecipazione italiana: Fabrizio Сотіссніa (2014), La guerra che non c'era.

430 Una domentazione ufficiale è in Ministry of Foreign Affairs, Japan (2007), Japan's Contribution to Afghanistan. 
loro attività in Iraq per tutto il popolo giapponese', ha detto [il premier Junichiro] Koizumi in una conferenza stampa in cui ha precisato che Tokyo continuerà a sostenere la ricostruzione dell'Iraq". ${ }^{431}$

Il 24 aprile 2018 gli Stati Uniti (che la settimana prima avevano dichiarato di volersi ritirare dall'Iraq in 48 ore), la Gran Bretagna e la Francia bombardarono l'Iraq come punizione per l'uso di armi chimiche contro la popolazione. In una preoccupante situazione di non-guerra, per la prima volta dopo la crisi di Cuba del 1962 si rischiava uno scontro diretto fra militari statunitensi e russi, entrambi presenti in Iraq in schieramenti opposti. Per questo gli Stati Uniti avevano avvertito la Russia dell'attacco. Anche turchi, iraniani e quant'altri erano stati invitati in qualche modo a scansarsi perché di lì a poco sarebbero stati lanciati i missili. Una settimana dopo questo bombardamento quasi amichevole giungeva in Iraq la commissione dell'Organization for the Prohibition of Chemical Weapons che doveva accertare se il regime siriano avesse effettivamente usato quelle armi chimiche che erano all'origine del bombardamento punitivo.

A questa bizzarra operazione mancava l'assenso delle Nazioni Unite. Perciò l'Italia e la Germania non vi parteciparono, limitandosi a vaghe frasi di approvazione. La posizione tedesca venne sintetizzata nella formula "dafür aber nicht dabei”: a favore, ma senza esserci. In realtà, una loro partecipazione avrebbe violato l'articolo pacifista presente nelle loro costituzioni, anche se esso non venne evocato nei comunicati ufficiali. In Italia un quotidiano ha richiamato esplicitamente l'art. 11 della Costituzione per affermare ancora una volta l'incostituzionalità di un intervento anche indiretto, per esempio consentendo l'uso delle basi statunitensi in territorio italiano per lo svolgimento di bombardamenti fuori dall'area Nato. ${ }^{\mathbf{4 3 2}}$

431 Il Giappone ritira le sue truppe dall'Iraq (2006, 20 giugno), in: Corriere della Sera.

432 "Qui bisogna domandarsi se l'appartenenza all'Alleanza atlantica può in qualche modo mettere in secondo piano il dettato costituzionale, aggrappandosi, come è accaduto in passato, a quella seconda parte [dell'art. 11] che consente le 'limitazioni di sovranità'. [... Esso] non contiene commi e nemmeno un punto: la prima e la seconda parte sono separate da un punto e virgola (originariamente solo una virgola), a dimostrazione dell'unità logica del testo. La Corte costituzionale ha chiarito poi il carattere tassativo ed essenziale degli scopi cui tende la limitazione di sovranità (sentenza 304/1984): 'Le condizioni e le 'finalità' cui sono subordinate le 'limitazioni di sovranità', sono quelle stabilite nell'art. 11 della Costituzione [...]. È il trattato che, quando porta limitazioni alla sovranità, non può ricevere esecuzione nel Paese se non corrisponde alle condizioni e alle finalità 
Le guerre al "terrorismo islamico" sono oggi estese a più continenti, anche se questi interventi militari non vengono definiti "guerre". In Giappone, in Italia e in Germania gli interventi militari in questi conflitti sono sempre stati accompagnati da opposizioni politiche forti ma minoritarie che, richiamandosi agli articoli pacifisti delle rispettive costituzioni, affermavano - invano - l'incostituzionalità dei vari interventi militari.

dettate dall'art. 11 della Costituzione', Silvia Truzzi (2018, 15 aprile), L'articolo 11 e $i$ limiti alle guerre "travestite", che rinvia anche al testo della costituzionalista Lorenza Carlassare (2013, 11 febbraio), L'art. 11 della Cost. nella visione dei Costituenti. 



\section{Capitolo II Il rifiuto della guerra nella Costituzione giapponese del 1947}

Costituzione giapponese - Capitolo II. Rinuncia alla Guerra Articolo 9.

Nella sincera aspirazione alla pace internazionale, basata sulla giustizia e l'ordine, il Popolo Giapponese rinuncia per sempre alla Guerra quale sovrano diritto della nazione e alla minaccia o all'uso della forza come mezzo per la risoluzione delle dispute internazionali.

Allo scopo di raggiungere l'obiettivo di cui al precedente paragrafo, le forze di terra, di mare ed aeree, così come le altre potenzialità belliche, non saranno mai mantenute. Non sarà riconosciuto il diritto dello stato alla guerra.

Nell'agosto del 1945 - con l'invasione sovietica della Manciuria e con la tragedia nucleare di Hiroshima e Nagasaki - si concluse per il Giappone la Seconda guerra mondiale, cui seguì l'occupazione statunitense. Rispetto agli altri due Stati del Patto Tripartito, la pressione statunitense per l'emanazione di una nuova costituzione si manifestò con particolare energia e incontrò una resistenza parimenti forte, perché l'omogenea società giapponese tende a non accettare mutamenti radicali e repentini, soprattutto se provenienti dall'esterno.

Dalla fine del Cinquecento al 1868 quella società era rimasta pressoché chiusa agli influssi occidentali; col 1868 e con l'apertura dell'era Meiji aveva innovato molto, ma rispettando il più possibile le tradizioni. In particolare, il militarismo giapponese affondava le sue radici nella secolare tradizione dei samurai. ${ }^{1}$ L'occupazione statunitense si trovava così a dover innovare in senso democratico una società ancora legata alle sue concezioni tradizionali

1 In Germania la culla del militarismo era stata la Prussia e per questa ragione, durante l'alleanza col Giappone, era stato messo in luce il parallelismo tra samurai e Junker (e, più tardi, tra samurai e SS, cfr. p. 27: cfr. Gerhard Krebs (ed.) (2002), Japan und Preußen, in particolare: Junko Ando (2002), Japan und die Preußische Verfassung. 
di obbedienza a un imperatore di origine divina, celebrato in una religione ufficiale che aveva preso il nome di "Shinto di Stato" e che aveva appoggiato il militarismo giapponese. ${ }^{2}$ In questo contesto gli Stati Uniti volevano introdurre in Giappone la democrazia parlamentare e cancellare il militarismo che aveva caratterizzato la politica giapponese dagli anni pre-bellici fino alla sconfitta. $^{3}$

I suicidi che seguirono la dichiarazione di resa annunciata per radio dall'imperatore sono il simbolo della forza delle tradizioni antiche nella società giapponese moderna. "Ricordo ancora le fotografie dei molti giapponesi che si suicidarono in quei giorni, - scrive Fosco Maraini, - sempre nel medesimo luogo fatale, in vista del Niju-Bashi, del Doppio Ponte" (cioè il ponte d'ingresso al Palazzo Imperiale, dove ci si recava "per rendere omaggio alla sacra persona del Tenno, il Re del Cielo"): "i loro corpi stavano ordinatamente in fila, accucciati per terra, abbattuti in avanti, come deve scrupolosamente curare avvenga al proprio cadavere chi sceglie quest'ultimo e tremendo sacrificio" 4

La nuova costituzione venne dunque imposta dagli occupanti, ma la forza della tradizione giapponese indusse a presentarla come una continuazione della Costituzione Meiji del 1889, benché quest'ultima prevedesse una monarchia assoluta di origine divina, che poteva sciogliere in ogni momento il parlamento. ${ }^{5}$ La costituzione giapponese del 1947, "drafted and imposed by the occupying United States and the so-called 'Supreme Commander of the Allied Powers' General Douglas MacArthur, proclaimed the sovereignty of the people, abolished Japan's feudal system, established the separation of powers as well as the separation of State and religion and, most importantly, introduced the 'pacifist' Article 9 to Japan's constitution". I decenni finora trascorsi portano a valutare così questo radicale intervento istituzionale: "The most important heritage of seven years of US occupation is without a doubt

2 Sulla revisione postbellica dello Shinto: Herbert Zachert (1950), Shinto und Staatsführung im neuen Japan.

3 Sugli inizi della costituzione giapponese: Dan F. Henderson (ed.) (1968), The Constitution of Japan; John M. MaKi (ed.) (1980), Japan's Commission on the Constitution; SногcH Koseki (1998), The Birth of Japan's Postwar Constitution. Cfr. inoltre RoBert E. Ward, Frank Joseph Shulman (1974), The Allied Occupation of Japan 1945-1952.

4 Fosco Maraini (1988), Ore giapponesi, 104.

5 Un confronto fra le due costituzioni è in Куоко Inoue (1991), MacArthur's Japanese Constitution. 
Japan's post war constitution and its 'pacifist' or 'war-renouncing' Article 9 which does not allow the country to maintain armed forces other than for the defense of Japanese territory" ${ }^{6}$

Nei dibattiti che accompagnarono le nuove costituzioni nei tre Stati dell'ex Patto Tripartito, i conservatori protestarono (invano) contro questa lesione della sovranità nazionale; tuttavia gli effetti di questa opposizione sono particolarmente visibili tanto nel dibattito costituente giapponese quanto, dopo l'approvazione dell'articolo 9, negli sforzi di interpretarlo restrittivamente, praticando (col tacito appoggio degli Stati Uniti, come si vedrà) quello che è stato chiamato "pacifismo verbale". ${ }^{7}$ D'altra parte, in Giappone preesisteva un movimento pacifista, attivo ma minoritario, che appoggiava invece l'approvazione e, poi, il rigoroso rispetto dell'articolo 9. ${ }^{8}$ Al centro di queste tensioni e sottoposto a interpretazioni di segno opposto, l'articolo 9 visse una "unintended life" che dura tuttora. ${ }^{9}$

Il 10 agosto 1945 i giapponesi iniziarono le trattative per la resa, partendo dalla "Potsdam Declaration" che prevedeva la resa incondizionata, ma sottolineando che essa lasciava impregiudicate le prerogative dell'imperatore. Contemporaneamente continuarono per alcuni giorni le ultime ostilità in Manciuria contro i sovietici e nel Pacifico contro gli statunitensi. Il 15 agosto l'imperatore lesse alla radio la dichiarazione di resa: per la prima volta nella millenaria storia giapponese l'imperatore, ancora divino, si rivolgeva direttamente ai suoi sudditi, che per la prima volta ne udivano la voce. Lo udivano ma non lo capivano: "Verso mezzogiorno l'imperatore pronunciò il suo discorso, nel quale effettivamente annunciava la resa, ma nessuno dei giapponesi del Kosai-ji e dintorni riuscì a capirne il senso. Il testo era infatti

6 Axel Berkofsky (2010), Japan's Post-War Constitution, 6 e 5. Per approfondire: Axel Berkofsky (2012), A Pacifist Constitution for an Armed Empire (in particolare: Chap. 6, Codifying Japanese Pacifism: Art. 9, 101-116); e la sua tesi dottorale: Axel Berkofsky (2005), Die neuen Amerikanisch-Japanischen Leitlinien.

7 Peter Getreuer (1986), Der verbale Pazifismus; James Llewelyn et al. (2009), A Pacifist State in a Hostile Region.

8 Cyril H. Powles (1999), Pacifism in Japan, 1918-1945. Sul dissenso giapponese rispetto al potere attraverso i secoli: Maria Chiara Migliore et al. (eds.) (2016), Il dissenso in Giappone e, in particolare, Noemi Lanna (2016), Il duplice dissenso di Oda Makoto (sullo scrittore pacifista Oda Makoto, 1932-2007).

9 Kenneth L. Porth (2010), Transcending Law. Cfr. anche Federico Lorenzo Ramaioli (2016a), Addio alle armi: l'articolo 9 della Costituzione giapponese; Federico Lorenzo Ramaioli (2016b), Disarmo e riarmo nella costituzione giapponese. 
composto in un linguaggio di corte talmente lontano da quello ordinario, che bisognava essere dei veri filologi per afferrarne il senso"; fu necessario attendere "fino alla sera, quando giunsero i giornali con notizie chiare e spiegazioni”. ${ }^{10}$

Nei giorni seguenti, e fino all'ottobre del 1945, vennero perfezionati gli accordi formali per la resa, per la liberazione dei prigionieri di guerra e per l'occupazione militare del Giappone. Negli USA venne istituita un'agenzia per stabilire il da farsi in Giappone, e l'esecuzione delle sue decisioni fu affidata al generale Douglas MacArthur, che divenne di fatto il governatore del Giappone postbellico, lo "shogun dagli occhi azzurri" (aoi-me no shogun; blue-eyed shogun). Il dibattito costituente si svolse dunque fra quell'agenzia negli USA, il "Supreme Commander" e il governo giapponese; ma la forte volontà di MacArthur lo portò spesso ad agire indipendentemente dalle indicazioni dell'agenzia della madrepatria. ${ }^{12}$

D'altra parte quel governo militare, benché straniero, non era estraneo alle tradizioni del Giappone, che fino all'epoca Meiji era stato guidato da un governo militare (il Bakufu), e poi di nuovo dai militari negli anni dal 1930 al 1945. "MacArthur aveva capito straordinariamente bene le esigenze emotive del popolo giapponese ed incarnava nell'aspetto, nei modi, nei gesti portentosi di signore rinascimentale, l'erede dei grandi capitani di quattro secoli or sono". ${ }^{13}$ Poi la gestione quotidiana dell'occupazione prese il sopravvento:

"Una dietro l'altra uscivano dal suo ufficio le direttive ai governi, prima di Shidehara, poi di Yoshida, intese a riformare dalle radici una società con almeno quindici secoli di tradizioni civili: smantellamento dei monopoli (zaibatsu, 'cricca capitalista'), riforma della scuola, nuova costituzione, emancipazione della donna, processo ai criminali di guerra ... Era evidente che un gigantesco schema astratto, preso di

10 Fosco Maraini (2001), Case, amori, universi, 619. Gli internati italiani erano stati trasferiti presso un "antico e incantevole tempio buddista, il Kosai-ji (il Santuario della Vasta Salvezza)", 604.

11 Cfr. p. 46, e per es.: Maurizio Brunori (1993), Il Giappone. Storia e civiltà del Sol Levante, 273.

12 Douglas MacArthur (1964), Reminiscences. Sull'azione di MacArthur in Giappone: Ray A. Moore, Donald L. Robinson (2002), Partners for Democracy; Ray A. Moore, Donald L. Robinson (1998), The Constitution of Japan, 800 pp. di documenti su CD. Inoltre: Shoichi Koseki (1998), The Birth of Japan's Postwar Constitution.

13 Fosco Maraini (1988), Ore giapponesi, 104. Le pp. 104-108 sono dedicate agli inizi dell'occupazione alleata, con folgoranti squarci sulle differenze di mentalità fra i due popoli. 
peso da una civiltà e posto come tante ordinate ed ascisse d'acciaio sulla materia vivente di un'altra, fosse destinato a fallire, o per lo meno a generare ogni sorta di confusioni. Poi (10 aprile 1951) MacArthur venne esonerato dai suoi incarichi da Truman ed i malintesi si moltiplicarono; ebbe così modo di consolidarsi il sordo risentimento di tutto un popolo di vinti verso un popolo vincitore che aveva agito con magnanimità e larghezza davvero uniche nella storia delle guerre". ${ }^{\mathbf{1 4}}$

Nonostante questi sussulti d'assestamento, il Giappone conserva di MacArthur un ricordo complessivamente positivo, mentre in Occidente la sua immagine venne offuscata dalla sua decadenza post-nipponica segnata dalla sconfitta in Corea, dal contrasto con Truman, dalla sua destituzione e dalla sua negativa campagna presidenziale. I suoi critici ricordano inoltre che "the entire occupation was under his egocentric shadow" e che "his overwhelming personal vanity demanded fealty from his staff and a public image of himself as the personal creator of Japans 'spiritual revolution", ${ }^{15}$

Le misure di MacArthur furono di certo decisive nell'avviare il Giappone verso una democrazia di tipo occidentale. Il Giappone militarista aveva represso i diritti umani e uno dei primi atti di MacArthur, già il 4 ottobre 1945, fu l'emanazione della "Human Rights Directive" (più esattamente "Removal of Restrictions on Political, Civil, and Religious Liberties"), che avviava anche l'epurazione dei funzionari compromessi col regime militarista, la liberazione immediata dei prigionieri politici e l'abrogazione di quasi una ventina di normative illiberali.

A distanza di pochi giorni seguirono le "Five Fundamental Reforms Directives", e cioè: "1. Enfranchisement of women; 2. Encouragement of labor unions; 3. Introduction of liberal principles into Japan's education; 4. Reform of the judicial system; 5. Democratization of the economic institutions" ${ }^{\mathbf{1 6}}$ La riforma della costituzione non è inclusa nei cinque punti, ma è ricordata nel loro preambolo: "The achievement of the Potsdam Declaration will unquestionably involve a liberalization of the constitution". Questa

14 Fosco Maraini (1988), Ore giapponesi, 105-106.

15 Joyce e Gabriel Kolko (1972), The Limits of Power, 305. Questi autori sono fortemente critici verso l'intera politica statunitense in Asia.

16 Quest'ultima misura - che aboliva le grandi concentrazioni industriali, zaibatsu - coincideva con l'abolizione dei Konzerne tedeschi e delle corporazioni italiane: Тномаs A. Bisson (1954), Zaibatsu Dissolution in Japan; Karsten Heinz Schönbach (2015), Die deutschen Konzerne und der Nationalsozialismus, 1926-1943. Sulla rilevanza politica degli zaibatsu: Federico Lorenzo Ramaioli (2017), Il nazional-capitalismo degli zaibatsu. 
curiosa tournure (equivalente nel gioco degli scacchi all'indiretta "mossa del cavallo") era dovuta al fatto che MacArthur non aveva ricevuto lo specifico incarico di riformare la costituzione Meiji. ${ }^{17}$ In realtà, dagli Stati Uniti giungevano istruzioni di fare in modo che fossero i giapponesi stessi a procedere alla revisione costituzionale.

Seguendo questa direttiva, MacArthur assegnò la riforma costituzionale al principe Konoe Fumimaro, che entrò nel governo Higashikuni con l'incarico di rivedere la costituzione Meiji. Però Konoe Fumimaro era un politico della vecchia guardia ed era stato due volte Primo ministro tra il 1936 e il 1941. Sotto la sua guida il Giappone aveva realizzato la propria quota nella spartizione del mondo prevista dal patto trilaterale ed aveva dichiarato la "war of annihilation" contro la Cina. In breve: la riforma democratica della costituzione giapponese era stata affidata a un criminale di guerra. ${ }^{18}$ Ciononostante nel novembre 1945 Konoe presentò all'imperatore una proposta di revisione della Costituzione Meiji, ma “on December 6, 1945, Konoe's name first appeared on an official list of Japanese A-Class criminals of war and a few days later Konoe committed suicide". ${ }^{19}$

Nonostante questo incidente, MacArthur insistette affinché la costituzione riveduta contenesse un articolo di rinuncia alla guerra (war-renouncing article) e il governo di Shidehara Kijuro affidò il compito al ministro Matsumoto Joji, sul quale si tornerà tra poco. Nel dopoguerra si discusse molto se l'articolo pacifista fosse stato voluto da MacArthur o dal primo ministro Shidehara, come affermò nel 1951 MacArthur davanti al "Committee on Armed Service” del Senato. Dal canto suo, Shidehara confermò questa versione, asserendo anche di essersi convinto della necessità del pacifismo quando, nel dopoguerra, aveva ascoltato su un tram un giovane che raccontava agli altri passeggeri la sua disperazione per le devastazioni della guerra e del

17 "MacArthur never received the orders and authorization from Washington to assign the task of constitutional revision to himself and to the so-called 'Government Section' (GS)", Axel Berkofsky (2010), Japan's Post-War Constitution, 11; e ancora: "Washington did neither instruct MacArthur to draft a revised Japanese constitution nor to dissolve Japan's armed forces"; le successive mosse costituzionali di MacArthur sono illustrate sotto il significativo titolo: Ignoring Washington, 11-12.

18 Questa qualifica venne usata nell'articolo di Nathaniel Pfeffer già il 26 ottobre 1945, sul New York Times.

19 Axel Berkofsky (2010), Japan's Post-War Constitution, 14. I crimini di classe A erano quelli "contro la pace". 
militarismo. Meno melodrammaticamente, MacArthur non poteva imporre in prima persona l'articolo pacifista perché la Far Eastern Commission (che insieme con l'Allied Council for Japan sovrintendeva alle attività alleate in Giappone) ne esigeva l'accettazione unanime da parte delle autorità giapponesi. Inoltre MacArthur era andato oltre al suo mandato e quindi, attribuendo a Shidehara la paternità dell'articolo 9 , evitava a se stesso possibili dissapori politici in patria. Resta comunque il fatto che " $\mathrm{i}$ principi della costituzione che più saltano all'occhio sono scritti di pugno da MacArthur: la posizione dell'imperatore come simbolo dello Stato senza potere politico e il celebre articolo 9, in cui si abolisce il diritto alla guerra e al riarmo".20

La travagliata genesi dell'articolo 9 si colloca nel contesto della globale trasformazione giuridica del Giappone postbellico. Le memorie del giudice Alfred Oppler ne offrono un quadro complessivo, in cui i giudizi personali di un protagonista si associano alla descrizione sistematica dell'intero complesso di riforme legislative realizzate in Giappone alla fine della guerra. ${ }^{21}$ Alfred Oppler, nato nel 1893 in Alsazia-Lorena, aveva percorso la carriera giudiziaria in Germania fino all'avvento di Hitler. Le leggi razziali lo avevano obbligato a emigrare negli Stati Uniti, dove riprese la carriera giudiziaria che lo portò in Giappone. La sua descrizione della trasformazione giuridica del Giappone postbellico associa quindi la testimonianza diretta di un protagonista a un'impostazione tecnico-giuridica vicina alla concezione sistematica dei giuristi europei continentali, perché tale era la sua formazione originaria. Nella sua opera - accanto alle esperienze personali di lavoro legislativo in un contesto anomalo come quello dell'occupazione militare in Giappone - si susseguono le notizie sulla riforma giudiziaria, sulle riforme dei codici civile e penale, nonché su leggi specifiche, da quella sui giovani a quella sull'amnistia.

Due capitoli meritano una speciale attenzione per comprendere la norma pacifista della nuova costituzione giapponese: quello dedicato a The New Constitution e quello sulla National Security versus Pacifism. Incaricato di esa-

20 Wolfgang Benz (1978), Amerikanische Besatzungsherrschaft, 273, che si richiama al documento Political Reorientation, I, 102 (cioè: SCAP, Government Section, ed., Political Reorientation of Japan, Sept. 1945 to Sept. 1948, Washington 1949, 2 voll.; anche: Westport, Ct, 1970), e ritiene "poco verisimile" la paternità attribuita a Shidehara.

21 Alfred C. Oppler (1976), Legal Reform in Occupied Japan. Si vedano in particolare: The New Constitution (43-64) e National Security vs Pacifism (208-213). A p. 5 è la biografia prussiana di questo giudice teuto-statunitense. 
minare i problemi connessi con la proposta di presentare la nuova costituzione come una riforma della costituzione Meiji del 1889, Oppler conclude: "While the draft Constitution actually represented a revolutionary substitution for the old Constitution, the desire of SCAP [Supreme Command of the Allied Powers] and of the Japanese cabinet to retain the imperial institution resulted in the policy of sustaining the fiction of legal continuity" (p. 50). Nella nuova costituzione "unusal stress is laid upon the peaceful cooperation of the people with other nations, the horror of war, and on pacifism. These features are evident in the preamble and in the unique Renunciation of War clause" (p. 60).

A quest'ultima disposizione Oppler dedica un apposito capitolo. Le sue radici tedesche lo portano a confrontare la nuova costituzione giapponese con la costituzione di Weimar, che presentava a "similarly noble language", che era nata in un contesto di "defeat and surrender", ma che proprio per questa ragione non poteva divenire "the guiding emblem for a lasting democracy”. Il pragmatismo giapponese dissolveva però i suoi timori su un possibile parallelismo tra le due costituzioni: in particolare non vedeva in Giappone il rifiuto della sconfitta, la "leggenda della pugnalata alle spalle" (Dolchstosslegende) della Germania. ${ }^{22}$

Quando la guerra di Corea suggellò l'impossibilità di una convivenza pacifica col mondo comunista, gli Stati Uniti dovettero trasferire dal Giappone alla Corea una parte delle truppe originariamente stazionate in difesa del disarmato Giappone, e così "we who had disarmed the defeated enemy now desired to see the potential ally rearmed” (p. 208). In questa situazione, il partito comunista giapponese - che aveva conosciuto un successo elettorale nel 1949 e che si era sempre opposto alla costituzione voluta dal vincitore si schierò a difesa dell'applicazione integrale dell'articolo 9. Oppler descrive questa contraddittoria battaglia interna accompagnata da dimostrazioni antiamericane anche violente, giungendo però alla conclusione che, "during all our years in Japan my wife and I felt perfectly safe in the streets of Tokyo, even by night, something I could never say of New York City” (p. 213).

22 "I found little Dolchstosslegende among the Japanese", Alfred C. Oppler (1976), Legal Reform in Occupied Japan, 62. In Germania, alla fine della Prima guerra mondiale, si era affermato il mito di un esercito invitto sul campo, ma "pugnalato alle spalle" dal fronte interno politicamente ostile perché composto di democratici, comunisti ed ebrei. 
Nei tre Stati dell'ex Patto Tripartito gli Alleati dovevano creare un nuovo ordinamento con personale autoctono proveniente dalle strutture burocratiche dei vecchi ordinamenti sconfitti. Nel caso del Giappone, uno studioso russo ha di recente attribuito l'articolo 9 all'influenza di un altro giapponese: "On December 10, 1945, Shiratori Toshio [1887-1949], former Japanese ambassador to Italy and now a Class A war criminal, finished writing a lengthy letter addressed to Foreign Minister Yoshida Shigeru as he awaited trial in Sugamo Prison": in questa lettera - scritta in inglese nell'intento di diffonderla - Shiratori proponeva di includere la rinuncia alla guerra nella costituzione giapponese allora in preparazione. Scrive l'autore russo: "I presented this theory at the public dispute on my doctoral dissertation, Shiratori Toshio and Japanese Foreign Policy (1931-1941), at the University of Tokyo in 2002. Many participants listened with interest but also with skepticism. They seemed to find too bold the idea that a 'war criminal' with a reputation as a militarist ideologue could have proposed the renunciation of war as a basic tenet of the constitution. I later discussed the theory in greater detail in my 2006 book The Era of Struggle: Toshio Shiratori (1887-1949), Diplomat, Politician, Thinker, which I wrote in Russian. To this day, it is the only booklength biography of Shiratori. The full text of the letter to Yoshida is included in Russian-language collection of Shiratori's selected writings The Re-awakening of Japan (2008)". ${ }^{23}$ Questi esempi attestano quanto complessa e opaca sia stata la battaglia politica intorno alla revisione costituzionale nel Giappone postbellico.

In realtà, le vicissitudini del testo costituzionale dimostrano che l'establishment politico giapponese non era propenso ad accettare le radicali proposte americane e riteneva invece che poche modifiche del testo della Costituzione Meiji potessero soddisfare le esigenze degli occupanti. A sostegno di questa convinzione i politici giapponesi adducevano il silenzio della "Potsdam Declaration" su una riforma costituzionale e, anzi, sostenevano che il promuoverla avrebbe addirittura violato quella dichiarazione. Nel 1946 sembrava ormai quasi certo che il testo costituzionale non avrebbe contenuto il war-renouncing article voluto da MacArthur.

23 Vassili Molodiakov (2017, 7 settembre), Who Is the Author of Article 9 of the Constitution of Japan?. Su Shiratori Toshio, cfr. Philipp Osten (2003), Der Tokioter Kriegsverbrecherpro$z e \beta, 50$ (bibliografia, 187-208). 
Un'interessante spiegazione di queste contrastanti vedute sulla riforma costituzionale venne proposta dallo studioso Tanaka Hideo, che le riconduceva non al classico conflitto di mentalità tra "Est" e "Ovest", ma al conflitto tra due sistemi giuridici: i giapponesi si erano formati sui testi tedeschi (o di ispirazione tedesca) di diritto costituzionale e amministrativo, e tendevano quindi a un'interpretazione giuspositivistica - cioè letterale - della "Potsdam Declaration", mentre invece gli americani, nell'interpretarla alla luce del Common Law, si rifacevano ai concetti di democrazia, di sovranità popolare e di diritti umani, estranei alla tradizione culturale giapponese. ${ }^{24}$

All'inizio del 1946 si diffusero le prime notizie su una proposta di revisione costituzionale, chiamata "Matsumoto Draft" dal nome del ministro responsabile del "Committee to Study Constitutional Problems", il già ricordato Matsumoto Joji. ${ }^{25}$ Essa fu però ritenuta troppo conservatrice per poter essere accettata dagli americani, soprattutto per la mancanza dell'articolo pacifista. MacArthur a questo punto avocò a sé la riforma costituzionale, senza informarne specificamente né Washington né Tokyo. Su sua indicazione, la Government Section (GS) del Supreme Command of Allied Powers divenne l'istanza costituente del Giappone, sotto la guida del maggior generale Courtney Whitney ${ }^{\mathbf{2 6}}$ e del colonnello Charles Kades. Quest'ultimo ha pubblicato il documento-guida per la revisione costituzionale preparato dallo stesso MacArthur per indirizzare la Government Section:

The Emperor is the head of the State. His succession is dynastic. His duties and powers will be exercised in accordance with the Constitution and responsible for the basic will of the people as proved therein.

War as a sovereign right of the nation is abolished. Japan renounces it as an instrumentality for settling its disputes and even for preserving its own security. It relies upon the higher ideals which are now stirring the world for its defense and its protection. No Japanese Army, Navy, or Air Force will ever be authorized and nor rights of belligerency will ever be conferred upon any Japanese forces.

The feudal system will cease. No rights of peerage except those of the Imperial family will extend beyond the lives of those now existent. No patent of nobility will come from this time forth embody within itself any National or Civic power of Government. Pattern budget after British system. ${ }^{27}$

24 Hideo Tanaka (1987), The Conflict between Two Legal Traditions, specialmente pp. $107 \mathrm{~s}$.

25 Il testo originale con commenti in inglese è in: 2-8 Joji Matsumoto, "Draft of Tentative Revision of the Constitution Draft", http://www.ndl.go.jp/constitution/e/shiryo/02/058cshoshi.html.

26 Courtney Whitney (1956), MacArthur, his Rendez-Vous with History.

27 Charles Kades (1989), The American Role in Revising Japan's Imperial Constitution, 223-224, corsivo mio. 
Le disposizioni di questo documento sono eccezionalmente dure, perché prevedono non solo la rinuncia tanto al diritto sovrano del ius ad bellum quanto alla guerra come strumento di soluzione dei conflitti, ma persino la rinuncia alla guerra "per preservare la propria sicurezza", cioè alla guerra difensiva. Questa formulazione troppo drastica dovette essere attenuata nella stesura finale. La costituzione riformata venne approntata in soli sei giorni, il che sollevò numerosi dubbi sulla sua accuratezza ed applicabilità, anche se probabilmente gli americani avevano già alle loro spalle importanti lavori preparatori.

Due punti - "non negoziabili" per gli americani - colpirono l'opinione pubblica giapponese: l'imperatore non solo non era più divino, ma perdeva $\mathrm{i}$ suoi poteri divenendo soltanto il "symbol of the State"; l'articolo "pacifista", finalmente incluso, disarmava di fatto il Giappone. Questo testo, sottoposto al parlamento il 5 marzo 1946, venne approvato nell'agosto del 1946: era la costituzione imposta dal vincitore, anche se gli americani tendono a respingere questo giudizio, diffuso invece in Giappone non solo negli ambienti conservatori. ${ }^{28}$

L'esame del travagliato percorso costituente del Giappone postbellico spiega perché l'articolo 9 abbia avuto (ed abbia) per il Giappone una rilevanza politica maggiore dei corrispondenti articoli tedeschi e italiani. MacArthur voleva un articolo che escludesse per sempre l'esistenza di forze armate giapponesi, anche se destinate all'autodifesa dell'arcipelago, la cui protezione sarebbe stata garantita dalle forze statunitensi. La formulazione dell'articolo sottoposto all'approvazione parlamentare conserva una chiara traccia di questa visione nelle parole "the Japanese people forever renounce war as a sovereign right of the nation" (art 9, c. 1, corsivo mio); inoltre la costituzione giapponese è priva di due disposizioni equivalenti agli articoli 52 e 78 della Costituzione italiana, che sembrano permettere il ricorso alle forze armate per l'autodifesa e le operazioni di peace keeping. In sostanza, gli americani volevano il disarmo totale del Giappone e quindi anche la rinuncia all'autodifesa, impegnandosi come contropartita alla difesa del Giappone in caso di aggressione armata: il documento-guida sopra citato vieta espressamente al Giappone il diritto di dichiarare guerra "even for preserving its own security".

Nel dibattito parlamentare, Hitoshi Ashida - responsabile dell'attività costituente e futuro premier - propose l'“Ashida Emendment”, che consisteva

Glenn D. Hook, Gavan McCormack (2001), Japan's Contested Constitution. 
nell'aggiungere all'inizio del secondo comma le parole "for the above purpose", sostituite nella redazione finale con i termini equivalenti "in order to accomplish the aim of the preceding paragraph" (cioè, per realizzare l'obiettivo indicato con le parole: "Aspiring sincerely to an international peace based on justice and order"). L'articolo 9 conteneva così la rinuncia alla guerra "forever" e al riarmo ("war potential will never be maintained") per realizzare l'obiettivo della "pace internazionale". In questo modo si escludeva che il Giappone potesse avere delle forze armate per sovvertire la pace internazionale con una guerra d'aggressione; ma non si escludeva che il Giappone potesse avere delle forze armate per difendersi da una guerra d'aggressione. Lungo questo tortuoso sentiero si mosse da allora l'interpretazione dell'articolo 9 e la successiva creazione delle forze armate del Giappone postbellico, che sino ad oggi si chiamano "Forze di Autodifesa".

La discussione in Giappone si spostò quindi sulla definizione di "guerra di autodifesa", dal momento che era indiscutibile il fatto che l'art. 9 escludesse la guerra di aggressione. Secondo una teoria, la guerra di autodifesa era un mezzo per comporre i conflitti internazionali, e quindi ricadeva nel divieto dell'art. 9, cosicché il Giappone non avrebbe dovuto avere alcun apparato militare. Un'altra teoria sosteneva che era ammessa la guerra difensiva, anche se il secondo comma dell'art. 9 non riconosce allo Stato giapponese il diritto a "condurre una guerra".

Parallelamente si discuteva sulla possibilità di ricostituire le forze armate giapponesi. Anche in questo caso una teoria sosteneva il divieto di qualsiasi tipo di forza armata, mentre un'altra riteneva che fosse vietata soltanto una forza armata atta a condurre una guerra moderna, mentre sarebbe stata lecita la formazione di una forza di polizia o di forze armate per l'autodifesa. Il governo giapponese seguì questa seconda via e istituì una "National Police Reserve", che più tardi sarebbe confluita nelle Forze di Autodifesa.

I pacifisti giapponesi ricorsero alla giustizia per far valere il divieto di ogni forma di forza armata. Il ricorso contro la "National Police Reserve" venne respinto l'8 ottobre 1946 dal Tribunale Supremo giapponese. Il 16 dicembre 1959 lo stesso tribunale respinse il ricorso contro lo stazionamento di truppe americane sul territorio giapponese, affermando che le truppe straniere non ricadevano nel divieto dell'articolo 9 e che anzi esse garantivano la sopravvivenza stessa del Giappone come Stato sovrano. ${ }^{29}$ 
Con l'occupazione alleata e con l'entrata in vigore della nuova costituzione, in base all'articolo 9 il Giappone non aveva più un esercito, né un Ministero della Guerra o della Difesa, né un'industria bellica, che nel frattempo era stata smantellata dagli Alleati. Però lo scoppio della guerra di Corea nel 1950 provocò il trasferimento dal Giappone alla Corea di un forte contingente delle truppe americane di occupazione, e le tensioni interne dovute ai partiti di sinistra offrirono l'occasione per creare nel 1950 un corpo di polizia, la già ricordata "National Police Reserve", che costituì il primo nucleo del futuro esercito giapponese. Questo primo passo verso il riarmo è analogo a quello della Germania Federale e della DDR. ${ }^{30}$

I contrasti interni sull'articolo 9 hanno dato luogo a varie azioni giudiziarie sorte dal basso, cioè dall'opposizione di gruppi di cittadini giapponesi. È quindi interessante ricordare queste sentenze, che non hanno parallelo in Italia e Germania anche perché il dibattito sull'incostituzionalità delle norme segue in Giappone una procedura diversa da quella prevista nelle altre due costituzioni pacifiste. Per brevità viene qui riassunto il tenore delle singole sentenze, che sono state oggetto di numerose trattazioni cui si rinvia per ogni approfondimento. ${ }^{31}$

Suzuki Decision. - Questa sentenza dell'8 ottobre 1952 è la prima pronuncia della Corte Suprema giapponese sull'art. 9. Nel luglio 1950 il leader socialista Suzuki Mosaburo aveva presentato un ricorso contro l'istituzione della National Police Reserve, sostenendo che così si violava l'art. 9. Si poneva anzitutto un problema procedurale legato ai poteri della Corte Suprema: da un lato, Suzuki si rifaceva all'art. 81 (che attribuisce alla Corte Suprema il potere di stabilire la costituzionalità delle leggi), mentre la Corte stessa

30 Nel 1946 venne sciolta la Wehrmacht. Nella RFT, la polizia di frontiera (Bundesgrenzschutz) del 1951 (oggi Bundespolizei) si trasformò poi in Bundeswehr nel 1955. Nella DDR, la Kasernierte Volkspolizei, fondata nel 1948 dagli occupanti sovietici, si trasformò nel 1956 in Nationale Volksarmee der DDR. Tanto nelle due Germanie quanto in Giappone questi corpi di polizia erano equipaggiati come reggimenti di fanteria leggera.

31 In generale: Lawrence W. Beer, Hiroshi Itoh (eds.) (1996), The Constitutional Case Law in Japan, 122; John MaKi (1964), Court and Constitution in Japan. Sui singoli casi: Alfred C. Oppler (1961), Sunagawa Case; Robert L. Seymour (1974-1975), Japan's Self-Defense; Carl F. Goodman (2008), The Rule of Law in Japan, cfr. chapter 8, Art 9. Renunciation of War. Military Power and Responsibility, 211-240. Riporta cinque casi: The Sunakawa Case (225-226); The Naganuma Case (226-227); The Hyakuri Air Base Case (227-228); The Okinawa Mandamus Case (228-229); nonché Recent Lower Courts Cases, 229 s. 
dichiarava invece di avere poteri soltanto sulle sentenze, cioè nell'ambito del potere giudiziario, e non anche in quello legislativo. Questa decisione chiarisce così i poteri della Corte Suprema (e per questo viene paragonata al caso Marbury v. Madison negli USA, 1803), ma lascia aperta la questione sulla costituzionalità della Police Reserve e delle Forze di Autodifesa, che dal 1954 sostituiscono la Police Reserve.

Sunagawa Case. - Intorno al 1957 il Giappone stava rinegoziando con gli USA il trattato sulla sicurezza, al cui rinnovo si opponevano le forze politiche di sinistra appoggiate internazionalmente dall'URSS e dalla Cina. La vicenda giudiziaria inizia nel luglio 1957, quando gli USA chiedono di estendere una strada d'accesso alla loro base aerea di Tachikawa passando per un vicino villaggio. Mentre si stanno effettuando i sopralluoghi, un migliaio di giapponesi si riuniscono per protestare e sette di loro vengono arrestati. Sulla base di accordi speciali tra gli Stati Uniti e il Giappone, nei loro riguardi sono previste pene più severe che per una normale occupazione di un edificio o di un'area. Nel marzo 1959 la District Court di Tokyo assolveva i sette imputati, sostenendo nella sentenza che la presenza delle forze armate americane violava l'art. 9 della Costituzione, che l'autodifesa era possibile solo attraverso una decisione dell'ONU e che l'art. 9 - fondato sulla volontà popolare - prescriveva la pace: quest'ultima avrebbe potuto essere messa in pericolo perché gli USA avrebbero potuto compiere attività belliche anche fuori dal Giappone, coinvolgendo così il Giappone stesso. Inoltre anche la difesa del Giappone da parte degli USA avrebbe coinvolto il Giappone come Stato belligerante. Il tribunale concludeva quindi che il trattato sullo stazionamento di truppe statunitensi in Giappone (US-Japan Security Treaty) violava la Costituzione e pertanto, se quello stazionamento era incostituzionale, anche il trattamento privilegiato per l'occupazione eventuale di una base era nullo, in base al "due process of law" previsto dall'art. 32 della Costituzione.

L'assoluzione degli imputati venne celebrata come una vittoria del movimento contrario al trattato e divenne quindi politicamente rilevante anche per il governo giapponese. Il Pubblico Ministero si appellò direttamente alla Corte Suprema, senza passare attraverso il livello giudiziario intermedio della High Court. Nella sentenza del 16 dicembre 1959 del Grand Bench della Corte Suprema la sentenza di prima istanza venne annullata (con decisione unanime, integrata però da dieci pareri supplementari) e rinviata alla District Court di Tokyo per un nuovo giudizio. Il tribunale supremo riconosceva l'esigenza di mantenere la pace generale attraverso l'autodifesa, 
però non soltanto attraverso l'ONU, come era stato sostenuto in primo grado.

Per risolvere il problema della legittimità dello stazionamento dei militari statunitensi, la Corte Suprema si riferì non più al Trattato USA-Giappone sulla sicurezza, bensì al trattato di San Francisco del 1952, sottoscritto da quaranta Stati dell'ONU: esso consentiva lo stazionamento di truppe straniere sul suolo giapponese. La Corte affermò che, nel sottoscrivere i trattati, il governo giapponese aveva agito in modo conforme alla costituzione, però - richiamandosi anche in questo caso alla divisione dei poteri - affermò pure che, come potere giudiziario, non poteva pronunciarsi sulla costituzionalità dei trattati. La Corte Suprema si limitava a constatare che il trattato USAGiappone sulla sicurezza "deve certamente essere in armonia con l'art. 9 e con l'art. 92, c. 3 (che richiede una fedele osservanza dei trattati e del diritto delle nazioni) e non si può assolutamente ammettere che esso sia in violazione di quella norma o che sia chiaramente ovvio che esso è incostituzionale e non valido"32 Quel trattato non viola quindi la costituzione. D'altra parte, le forze statunitensi non sono potenziale bellico giapponese (sono infatti sotto il totale controllo statunitense) e servono al mantenimento della pace in Giappone e in Asia, data l'inadeguatezza militare del Giappone postbellico. Il caso venne rinviato al tribunale distrettuale, che - vincolato ad accettare la costituzionalità dell'accordo tra Stati Uniti e Giappone - doveva limitarsi a comminare una corretta sanzione. Conclusione: una multa per i sette imputati di occupazione dell'area statunitense.

Anche se questa decisione toglieva un'arma agli oppositori del trattato, il contrasto politico non si attenuò e, anzi, culminò con l'uccisione del premier Kishi Nobusuke (accusato di appoggiare il trattato in Parlamento) e con l'annullamento della visita a Tokyo del Presidente Eisenhower. Dal punto di vista giuridico, questa sentenza è importante perché per la prima volta il Tribunale Supremo si pronunciava sulla costituzionalità della rinuncia alla guerra; tuttavia non si pronunciava sulla costituzionalità delle Forze di Auto-

32 Alfred C. Oppler (1983), Sunagawa Case, 275: "The main purpose of the security treaty", cioè lo stazionamento delle truppe statunitensi, "must certainly be in accord with the intent of the article 9 and of article 98, paragraph 2, (which requires faithful observation of treaties and established laws of nations) and it absolutely cannot be admitted that it is in violation of the said provisions or that it is clearly obvious that it is unconstitutional or invalid". 
difesa. Quest'ultimo tema venne deciso anni dopo, nel 1969, dalla Sapporo High Court, che si richiamò anche al Sunagawa Case.

Naganuma Case. - Nel 1969 le Forze di Autodifesa avevano iniziato a costruire una base per missili antiaerei Nike sulla cima di una montagna che era stata dichiarata riserva naturale. Circa trecento abitanti (fra cui molti agricoltori preoccupati per l'alterazione delle falde acquifere dovuta alla deforestazione) diedero inizio a un processo in cui si sosteneva l'incostituzionalità delle Forze di Autodifesa. Nel settembre 1973 la District Court di Sapporo dichiarò incostituzionali le Forze di Autodifesa e bloccò la costruzione della base missilistica. Però nell'agosto 1976 la Sapporo High Court annullò la precedente sentenza, sostenendo che la questione sulla costituzionalità delle Forze di Autodifesa era una "political question" e che quindi non era di competenza del potere giudiziario. Il caso venne portato alla Corte Suprema, la quale il 9 settembre 1982 confermò l'annullamento della sentenza di primo grado perché i ricorrenti non avevano titolo per stare in giudizio: infatti non avevano un interesse diretto nel dichiarare "riserva naturale" quella foresta e non potevano dimostrare di essere danneggiati dalla cancellazione della riserva. In particolare, l'alterazione della forestazione e delle falde acquifere era successiva al ritiro della qualifica di riserva naturale. Anche in questo caso la Corte non affrontava il problema della costituzionalità delle Forze di Autodifesa. È stato osservato che la sua posizione può essere sintetizzata nell'affermazione che il fatto in esame non costituiva un problema giuridico, ma "was merely a political issue".33

Il trattato di mutua assistenza con gli Stati Uniti tutelava il Giappone dagli attacchi esterni, mentre il Giappone avrebbe dovuto provvedere con forze proprie alla sicurezza interna e alla protezione civile. Nel contesto della dottrina statunitense del containment dell'URSS, nel 1952 la "National Police Reserve" si trasformò in "Nation Safety Forces" (forte di oltre 100.000 effettivi); nel 1954 il "National Security Board" si trasformò in "Defense Agency" e gli effettivi vennero riorganizzati nella "National Self-Defense Force", suddivisa in forze di autodifesa di terra, di mare e di cielo. Nel 2006 la "Defense Agency" divenne "Ministry of Defense". Intanto gli effettivi delle tre armi e il loro equipaggiamento erano andati espandendosi, tanto che nel 2017 il bilancio delle forze armate giapponesi si collocava tra i dieci maggiori del mondo.

33 James E. Auer (1990), Article 9 of Japan's Constitution. 
In presenza di questa progressione è sempre più difficile sostenere la denominazione postbellica di "forze di autodifesa" per le forze armate giapponesi, mentre il crescente numero di missioni all'estero urta contro la lettera dell'articolo 9 (come del resto avveniva e avviene anche in Germania e in Italia con riferimento agli equivalenti articoli costituzionali).

Intanto è passato più di mezzo secolo da quando la nuova costituzione giapponese è entrata in vigore e la geopolitica mondiale è radicalmente mutata. Per il Giappone il pericolo sembra essersi spostato dalla Russia alla Cina, mentre l'imprevedibile Corea del Nord impone al Giappone una difesa missilistica difficilmente conciliabile con l'articolo 9. Per questo il Partito Liberal-Democratico (LPD) oggi al potere istituì nel 1956 una Commissione per la Costituzione, che nei sette anni successivi propose varie riforme, ma non quella dell'art. 9. Dagli anni Novanta le proposte di modifiche costituzionali si sono susseguite ${ }^{34}$ e dal 1997 la potente lobby conservatrice "Nippon Kaigi" (Conferenza del Giappone) si batte per la modifica dell'articolo $9,{ }^{35}$ ritenuto un limite incompatibile con la sovranità nazionale: solo con la sua abolizione il Giappone tornerebbe ad essere "un paese normale" ${ }^{\mathbf{3 6}}$ In particolare, l'attuale premier Abe Shinzo (nel cui governo sono presenti alcuni membri di questa lobby) ha più volte proposto di modificare l'articolo 9, incontrando non poche difficoltà. Già nel 2006 il suo programma politico prevedeva esplicitamente la riforma dell'articolo 9: "Per me il conservatorismo non è un'ideologia, ma pensare al Giappone e ai Giapponesi. È naturale pensare al presente e al futuro, ma abbiamo delle responsabilità anche verso coloro che sono vissuti in passato. In altre parole, lo spirito del conservatorismo è il mantenimento di una prudente consapevolezza di come siano state conservate le tradizioni attraverso i secoli della lunga storia del Giappone. Proprio per questo motivo credo fermamente che occorra

34 Christian G. Winkler (2011), The Quest for Japan's New Constitution; in particulare: Four Visions for a New National Security Policy, 36 s. RaINer WAHL (ed.) (2008), Verfassungsänderung, Verfassungswandel, Verfassungsinterpretation; in particolare: Ausländische Einwirkungen auf die Entstehung der Verfassung Japans und Deutschlands im Vergleich, $107 \mathrm{s.}$

35 Sul Nippon Kaigi (Nippon Conference) cfr. Glenn D. Hook, Gavan McCormack (2001), Japan's Contested Constitution.

36 William C. Middlebrooks (2008), Beyond Pacifism. 
rivedere l'articolo 9 della Costituzione per meglio adattarlo allo spirito e alle tradizioni del popolo Giapponese". ${ }^{37}$

Questa revisione è una costante nel programma del Partito LiberalDemocratico, che nel suo congresso del 2018 ha proposto non di abolire l'articolo 9, ma di attenuarlo con un articolo 9 bis. Alcuni documenti di questo dibattito sono riportati nell'Appendice I,5.

Le prese di posizione nella politica interna erano accompagnate dai mutamenti della situazione internazionale, che coinvolgevano direttamente il Giappone. Nel 1990 gli Stati Uniti chiesero che il Giappone partecipasse alla Guerra del Golfo, il che provocò un'intensa polemica fra i due Stati. Dal 1992 una legge permette ai militari giapponesi di partecipare alle missioni internazionali umanitarie in cooperazione con le Nazioni Unite. Da allora forze non combattenti giapponesi sono state presenti in Cambogia (1991) e in Mozambico (1993), ma è soprattutto con il coinvolgimento in Iraq (2004-2006) che l'opinione pubblica giapponese è tornata a schierarsi contro il riarmo. ${ }^{38}$

Un attento osservatore ritiene che l'inversione di tendenza rispetto al pacifismo costituzionale si possa far risalire al febbraio 2004, quando il governo giapponese inviò 150 soldati in Iraq. Con quella decisione "il processo compiuto nei decenni precedenti per una nuova definizione della politica giapponese della sicurezza ha trovato la sua conclusione provvisoria. L'impegno in Iraq è stato ed è estremamente controverso nello stesso Giappone e anche il fondamento legislativo per l'uso delle forze armate in caso di conflitto non va oltre il principio dell''autodifesa individuale' del singolo soldato: una circostanza che senza dubbio limita la capacità operativa del contingente giapponese". ${ }^{39}$

Una conclusione meno provvisoria, anche se non meno controversa, venne con le "Security Laws", approvate il 19 settembre 2015 ed entrate in vigore il 29 marzo 2016. Esse concludono formalmente una serie di interpretazioni estensive dell'articolo 9, tese ad ammettere sia l'appoggio militare

37 Shinzō Abe (2013), Utsukushii kuni e [Verso un paese meraviglioso]. Ringrazio Emil Mazzoleni per la segnalazione e per la traduzione del passo citato.

38 A queste missioni si aggiunsero quelle in Indonesia (2005), in Nepal (2007), sulle alture del Golan (2005-2012), in Somalia contro la pirateria (2009) e a Haiti dopo il terremoto del 2012.

39 Axel Berkofsky (2004, 26 aprile), Japanisches Militär in Irak. 
a favore di un alleato aggredito, sia la guerra di autodifesa in caso di attacco esterno. Non solo i movimenti pacifisti, ma anche molti politici e cittadini ritengono però che l'articolo 9 consenta l'uso della forza solo per la difesa individuale - cioè, il soldato giapponese in missione di pace può rispondere al fuoco solo se attaccato - mentre invece non ne preveda l'uso in un intervento di prevenzione collettiva.

Inoltre queste leggi ordinarie sono ritenute incostituzionali perché non è stato rispettato l'art. 96 che regola le modifiche costituzionali: esso richiede infatti una maggioranza qualificata di due terzi e un referendum confermativo. Invece il Governo Abe ha realizzato questa modifica con una legge ordinaria, presentandola come una reinterpretazione dell'articolo 9: reinterpretazione che, a livello internazionale, è stata approvata dagli Stati Uniti, ma che ha suscitato le proteste della Cina e della Corea del Sud. Un'autorevole sintesi delle critiche rivolte a queste leggi è stata formulata dalla "Japan Association of Constitutional Law". In essa Professor Yasuo Hasebe della Waseda University ricostruisce l'origine e i limiti costituzionali dell'interpretazione estensiva dell'articolo 9:

The government's statement submitted to the National Diet on 14 October 1972 held that: "the current constitution, which is based on pacifist principle, cannot be understood to tolerate unlimited exercise of the right of self-defence. The constitution recognises the use of the right only in cases it is essential to protect Japanese people's rights to life, liberty, and pursuit of happiness, as these rights are jeopardised by foreign military attacks". Since the right of collective self-defence is to be invoked when foreign states are under military attack and request support from Japan, such use of force is beyond the constitutional limit. In concrete terms, the government may use force only when

(1) Japan itself is under on-going or imminent, unlawful armed attack emanating from abroad;

(2) to terminate the attack, use of force is necessary; and

(3) the extent of the use of force is proportionate to the end to be achieved. These three conditions should be co-existent.

In accordance with this line of reasoning, the government has, again and again, stated that among the right of self-defence recognised by Article 51 of the UN Charter, ${ }^{[40]}$ the constitution recognises only the right of individual self-defence; in

40 [Art. 51: Nothing in the present Charter shall impair the inherent right of individual or collective self-defence if an armed attack occurs against a Member of the United Nations, until the Security Council has taken measures necessary to maintain international peace and security. Measures taken by Members in the exercise of this right of self-defence shall be immediately reported to the Security Council and shall not in any way affect the 
other words, the use of the right of collective self-defence is unconstitutional. Various government spokespersons, including successive chiefs of the cabinet legislation bureau who are primarily in charge of providing legal advices to the government, have stated that in order for the government to exercise the right of collective self-defence, amendment of Article 9 is essential. ${ }^{\mathbf{4 1}}$

Secondo questa ricostruzione era opinione diffusa anche nell'ambito parlamentare che l'autodifesa collettiva fosse incostituzionale, finché nel 2014 una mirata sostituzione del direttore dell'Ufficio Legislativo del Premier portò quell'ufficio a formulare un parere che andava in direzione opposta. Rispetto ai tre punti sopra citati, al punto 1 ("Japan itself is under on-going or imminent, unlawful armed attack emanating from abroad"), la nuova interpretazione aggiungeva un Punto 1a: "Use of force will be also permitted when (1a) Japanese people's rights to life, liberty, and pursuit of happiness are jeopardised because of military attacks against foreign countries, which are in close relationship with Japan”. Solo un'interpretazione politica può attribuire un contenuto alla vaghissima formula della messa in pericolo di "life, liberty, and pursuit of happiness" dei giapponesi.

In realtà, il Professor Yasuo Hasebe sostiene che i casi concreti riconducibili al Punto 1a sono sempre controversi: ad esempio, si chiede, si potrebbe decidere che la chiusura dello Stretto di Hormuz provocherebbe una crisi petrolifera e quindi un peggioramento delle condizioni di vita dei giapponesi, consentendo così l'intervento delle forze armate giapponesi? Il primo ministro Abe ritiene di sì, il partito Komeito suo alleato ritiene di no.

Ma questa - che sembrava un'astratta ipotesi geopolitica - ha rischiato di tradursi in realtà proprio mentre il premier Abe si trovava in visita a Teheran: il 13 giugno 2019 sono state attaccate due navi cisterna proprio nell'area di Hormuz e le immagini delle navi in fiamme sono state diffuse in Internet. Non è chiaro da chi sia venuto l'attacco, ma gli Stati Uniti hanno subito accusato i pasdaran iraniani e sembrano così ripetere il copione che portò alla guerra in Iraq. "A prescindere da chi sia il responsabile - l'Iran, una

authority and responsibility of the Security Council under the present Charter to take at any time such action as it deems necessary in order to maintain or restore international peace and security.]

41 Yasuo Hasebe (2015, 24 dicembre), The End of Constitutional Pacifism in Japan?: "In response to the Japanese Diet enacting 'Security Laws' on 19 September 2015, the Japan Association of Constitutional Law has decided to post the following entry written by Professor Yasuo Hasebe at Waseda University, former vice-president of IACL”. 
provocazione o un terzo attore malintenzionato - la cosa da fare al più presto è ridurre la tensione prima che un incidente di troppo scateni un conflitto catastrofico per il mondo intero" ${ }^{\mathbf{2}}$ Pochi giorni dopo, i Royal Marines britannici hanno sequestrato a Gibilterra la petroliera "Grace I", battente bandiera panamense, appartenente a una società di Singapore e accusata di trasportare petrolio iraniano in Siria. Se la guerra delle petroliere dovesse aggravarsi, il Giappone si riterrà o no vincolato dall'articolo pacifista della sua costituzione?

La risposta al quesito sarà politica, come è stata politica finora l'interpretazione dell'articolo 9, favorita dalla trasformazione mirata dell'Ufficio Legislativo: "If the bureau [cioè il Cabinet Legislation Bureau, l'Ufficio Legislativo del Premier] has come to say that some government action is constitutional because the prime minister wants it to be, there remains scarce respect for its opinions".

Le "Security Laws", benché al centro di queste critiche, sono in vigore dal 2016 e regolano quindi gli interventi delle forze armate giapponesi - che continuano a chiamarsi "Forze di Autodifesa" - negli interventi internazionali di peace keeping.

Verso la metà del 2016 due nuovi avvenimenti sembravano facilitare la revisione del pacifismo giapponese. Nella politica interna, le elezioni politiche del luglio 2016 avevano portato il Partito Liberal-Democratico dell'attuale primo ministro assai vicino alla maggioranza dei due terzi della Camera Alta, con la quale esso potrebbe approvare la modifica dell'art. 9, per sottoporla poi a referendum. Nella politica estera, il nazionalismo di Shinzo Abe veniva rafforzato dagli esperimenti atomici e missilistici della Corea del Nord e dalle rivendicazioni della Cina sul 90\% del Mar Cinese meridionale, che coinvolgevano anche alcune isolette collocate sulla rotta degli approvvigionamenti energetici per il Giappone. ${ }^{43}$ Una di queste è Ishigaki, isoletta vicina alla costa cinese: "La recente decisione di armare la prima portaerei [giapponese] è vista da alcuni come una chiara violazione dell'articolo 9. Per ora, il $60 \%$ dei giapponesi è contrario a modifi-

42 Pierre Haski (2019, 14 giugno), Chi vuole scatenare una guerra nello stretto di Hormuz, in: Internazionale.

43 Limitandomi al principale quotidiano italiano ( $m a$ articoli simili sono presenti ovunque): Guido Santevecchi (2016, 11 luglio), Giappone, Abe più forte; Guido Santevecchi (2016, 10 luglio), Un rischio il Giappone che archivia il pacifismo. 
care la Costituzione, come vorrebbe il primo ministro Shinzo Abe. Ma cresce anche la percezione della minaccia esterna. Le conseguenze di questo attivismo si vedono. A Ishigaki si sta costruendo una base giapponese 'di autodifesa' aerea, dove saranno dislocati 500-600 soldati”. ${ }^{44}$

In realtà le rivendicazioni cinesi hanno messo in allarme, oltre al Giappone, anche i governi di Filippine, Malesia, Brunei e Taiwan. Nel 2013 le Filippine avevano fatto ricorso alla Corte Permanente di Arbitrato sulla Legge del Mare contro l'occupazione cinese degli scogli o secche di Scarborough Shoal, dove nel 2012 la marina militare della Filippine aveva catturato otto pescherecci della Cina continentale. Però quelli che sono "scogli" per le Filippine sono "isole" per la Cina, il che modifica il controllo delle acque territoriali circostanti: 12 miglia per uno scoglio, 200 per un'isola. Le colate di cemento della Cina stanno trasformando vari scogli (in genere disabitati) in "isole", sulle quali vengono insediati cittadini cinesi. Queste tensioni hanno richiamato in quelle acque forze navali tanto cinesi quanto statunitensi.

La corte dell'Aia - sulla base della "United Nations Convention of the Law of the Sea" (UNCLOS), sottoscritta anche dalla Cina nel 1996 - ha deciso che "la Cina non ha diritti storici di sovranità sul Mar Cinese meridionale e molte sue azioni hanno violato la legalità internazionale". ${ }^{45} \mathrm{La}$ Cina però ha rifiutato di riconoscere l'autorità della Corte dell'Aia, affermando: "È deplorevole che il Tribunale arbitrale [...] abbia forzato la propria giurisdizione processuale ed esecutiva, violando gravemente sia la UNCLOS che il diritto della Cina. ${ }^{46}$ Il nuovo, pragmatico e discusso presidente delle Filippine, Rodrigo Duterte, sta tuttavia cercando una soluzione di compromesso con la Cina, nella quale si dia meno spazio ai problemi di sovranità territoriale e più attenzione a un comune diritto di pesca nelle acque contese, in cambio di tecnologie cinesi nell'acquacoltura. ${ }^{47}$

44 Mara Gergolet (2019, 7 gennaio), Tokyo prepara l'isola-trincea.

45 Citato in Guido Santevecchi (2016, 13 luglio), "La Cina non ha diritti sulle isole contese”, con una carta dettagliata dell'area. Le attuali pretese cinesi si fondano sui "nove tratti di penna” con cui Chiang Kai-shek, dopo la Seconda guerra mondiale, delimitò l'area di controllo cinese sul Mar Cinese meridionale. I cinesi hanno installato missili antiaerei sull'isola di Woody, nelle isole Paracelso, e stanno trasformando in isole almeno 7 atolli delle isole Spratly, antistanti le Filippine e il Brunei, e contese fra sei Stati rivieraschi.

46 Li Ruiyu (2016, 13 luglio) (ambasciatore della Repubblica Popolare Cinese in Italia), Vi spiego perché non accettiamo questo verdetto.

47 Brice Pedroletti (2016, 16 agosto), Pékin et Manille cherchent l'apaisement. Le trattative con i cinesi sono affidate all'ex presidente Fidel Ramos, che "a dit regretter l'époque où 
Le pretese cinesi coinvolgono anche le isole giapponesi Senkaku (Diaoyu per i cinesi): esse sono l'ultima propaggine meridionale del Giappone di cui fa parte l'isola di Ishigaki, citata poco sopra, e sono vicine alle basi statunitensi di Okinawa. Perciò l'espansionismo cinese - unito ai test atomici e missilistici della Corea del Nord - contribuisce a ravvivare il nazionalismo giapponese, favorendo così il partito del premier Abe e la revisione radicale dell'art. 9.

'pêcheurs philippins, chinois, et même vietnamiens évoluaient librement autour de l'atolle de Scarborough'” Parallelamente, però, la Cina continua "une propagande rageuse à l'encontre des Philippines et des États-Unis" e non riconosce la sentenza della UNCLOS. 



\section{Capitolo III \\ Il rifiuto della guerra nella Costituzione italiana del 1948}

Costituzione italiana - Princìpi fondamentali - Articolo 11.

L'Italia ripudia la guerra come strumento di offesa alla libertà degli altri popoli e come mezzo di risoluzione delle controversie internazionali; consente, in condizioni di parità con gli altri Stati, alle limitazioni di sovranità necessarie ad un ordinamento che assicuri la pace e la giustizia fra le Nazioni; promuove e favorisce le organizzazioni internazionali rivolte a tale scopo.

Per l'Europa, gli ultimi anni della guerra e i primi anni del dopoguerra furono "anni dell'anarchia", che la trasformarono in un "continente selvaggio". In Italia ci sono "ponti saltati, case senza servizi, disoccupazione dilagante, inflazione alle stelle, reduci che faticano a inserirsi nella società, borsa nera, prostituzione e sciuscià disposti a tutto". Ma proprio pochi anni dopo quel disastro "nascono i prototipi della Vespa e della Ferrari, sperimentati sulle strade devastate dai bombardamenti, i gelati Algida nati da un residuato bellico americano, la prima lavatrice Candy messa a punto grazie agli schizzi inviati da un prigioniero degli USA, il primo volo dell'Alitalia". ${ }^{2}$ Per seguire questa parabola in Italia bisogna rifarsi all'estate del 1943.

In poche settimane, nella penisola si susseguirono lo sbarco degli angloamericani in Sicilia il 10 luglio 1943, la destituzione di Benito Mussolini il 25 luglio 1943, la fuga del re e del governo da Roma a Pescara (e, di lì, all'Italia del Sud per ricongiungersi con i nuovi alleati), la divisione dell'Italia in due Stati contrapposti (la Repubblica Sociale Italiana al Centro-nord, che combatteva a fianco dei tedeschi, e il Regno d'Italia a Sud, che combat-

1 KeIth Lowe, Der wilde Kontinent; Ian Nish, The Japanese in War and Peace, 1942-1948 (documenti raccolti da un membro delle forze britanniche di occupazione in Giappone); Mario Avagliano, Marco Palmieri, Dopoguerra.

2 Commento al libro di Avagliano e Palmieri citato nella nota precedente: L'Italia postguerra tra distruzione e voglia di ripartire, in: Libero, 27 settembre 2019, p. 26. 
teva a fianco degli Alleati), il forte movimento partigiano soprattutto nel Nord e la situazione di guerra civile generarono in Italia una situazione diversa da quella degli altri due Stati dell'ex Patto Tripartito. Infatti in Italia Mussolini era stato esautorato e il potere era tornato al re; in quegli stessi mesi, invece, in Germania il potere di Hitler e in Giappone quello dell'imperatore continuavano sostanzialmente invariati.

Il biennio 1943-45 è fondamentale per la storia dell'Italia contemporanea, ma qui - riprendendo i temi accennati nel $\$ 5$, p. 54 - se ne possono indicare solo i punti salienti, e in modo sommario. ${ }^{3}$ La sconfitta in Africa settentrionale e in Russia, i bombardamenti alleati sulle principali città italiane e le difficoltà quotidiane avevano creato un profondo malcontento popolare. I vertici dello Stato italiano pensavano ormai a uno sganciamento dalla Germania e ad una pace separata con gli angloamericani. Il 25 luglio 1943 il Gran Consiglio Fascista approvò un ordine del giorno che rimuoveva Mussolini dalle sue funzioni. Nello stesso giorno Mussolini incontrò il re, che - dopo avergli annunciato di averlo sostituito come primo ministro con il generale Badoglio - lo fece arrestare all'uscita dall'incontro.

Dopo la detenzione in vari luoghi, Mussolini venne liberato dai nazisti il 12 settembre 1943, trasportato a Monaco di Baviera e poi, di lì, nell'Italia settentrionale, dove in quello stesso mese fondò la Repubblica Sociale Italiana. Essa si estendeva sino a sud di Roma, dove fronteggiava il Regno d'Italia. Si fronteggiavano anche due eserciti italiani: quello repubblicano al centro-nord, alleato con i tedeschi, e quello monarchico nel sud, alleato con gli anglo-americani.

Il 25 luglio 1943 segnò la fine del governo fascista, ma non della guerra. Con quella data iniziarono i "quarantacinque giorni" del governo Badoglio, che cominciò a smantellare le istituzioni fasciste e raccolse intorno alla monarchia il precedente apparato statale depurato solo nelle sue frange più estreme, cercando nel contempo di coinvolgere i più moderati tra i partiti antifascisti del Comitato di Liberazione Nazionale. ${ }^{4}$

3 Per un piano resoconto degli eventi: Gianni Oliva (2018), La grande storia della Resistenza. Per approfondire: Piero Pieri, Giorgio Rochat (2002), Badoglio, Maresciallo d'Italia.

4 Il Comitato di Liberazione Nazionale (CLN) comprendeva a sinistra il Partito Comunista Italiano (PCI), il Partito Socialista Italiano di Unità Proletaria (PSIUP) e il Partito d'Azione (PdA) e, al centro, la Democrazia Cristiana (DC), il Partito Liberale Italiano (PLI) e il Partito Democratico del Lavoro (PDL); inevitabili i contrasti politici fra i due schieramenti. 
L'8 settembre 1943 il generale Badoglio annunciò alla radio l'armistizio tra il Regno d'Italia e gli Alleati, che avrebbero dovuto sbarcare sulla penisola entro pochi giorni (anche se la data era incerta). L'armistizio rinsaldava la posizione della monarchia rispetto agli angloamericani, ma esponeva i vertici del regno italiano alle rappresaglie dei tedeschi. Per questo il governo Badoglio continuò il doppio gioco con i tedeschi, ma nel contempo preparò una fuga da Roma, perché non si sapeva quando i nuovi Alleati anglo-americani sarebbero giunti a Roma. Alla periferia di Roma, invece, presero posizione le truppe tedesche. Di fronte a ciò, il 9 settembre il re, Badoglio, le personalità della Corte e i responsabili militari - preoccupati per la propria sorte di classe dirigente più che per quella del paese - attraversarono l'Appennino, raggiunsero Pescara e di lì si imbarcarono sulla corvetta "Baionetta" alla volta di Brindisi. In questa fuga senza dignità i vertici della monarchia non diedero alcun ordine alle forze armate italiane, esponendole alle rappresaglie tedesche, che si verificarono puntualmente.

La logica dei quarantacinque giorni giunge così al suo epilogo drammatico eppure conseguente: la monarchia, Badoglio, Ambrosio salvano se stessi e la continuità dello Stato che incarnano [...]. La conservazione dello Stato come istituzione (che i protagonisti rivendicheranno come proprio merito) si trasforma in abbandono del paese reale, comportando un costo umano e materiale altissimo: nessuna indicazione chiara ai militari esposti alla rappresaglia tedesca, nessuna indicazione ai civili, nessun appello, nessuna prospettiva. Il 10 settembre, nel momento in cui i fuggiaschi sbarcano in Puglia, il bilancio è politicamente in attivo, ma moralmente fallimentare. $^{5}$

È difficile oggi immaginare il dramma dell'Italia tra l'8 settembre 1943 e la Liberazione del 25 aprile 1945. Nel Nord, solo una limitata percentuale accettò di arruolarsi con i tedeschi e anche i volontari dell'esercito di Salò furono pochi. Centinaia di migliaia vennero fatti prigionieri e inviati in Germania. Molti (gli "sbandati") indossarono panni civili e cercarono di ritornare a casa. ${ }^{6}$ Altri - talora interi gruppi di militari - stretti fra la morsa hitleriana e la coscrizione di Salò, formarono bande partigiane o si unirono a quelle già esistenti, apportando alla Resistenza armi ed esperienza militare. Nel Sud l'unione della monarchia con gli anglo-americani rese meno drammatico quel biennio.

5 Gianni Oliva (2018), La grande storia della Resistenza, 90-91.

6 Gabriella Gribaudi (2016), Combattenti, sbandati, prigionieri. 
Invece per le truppe italiane in Grecia e nei Balcani (lontane da casa e in territorio estraneo, quando non ostile) era difficile "sbandarsi" perché la casa cui tornare era di là dal mare: molti si arresero ai tedeschi, altri resistettero e vennero uccisi (come avvenne a Cefalonia e a $\operatorname{Kos}^{7}$ ), alcuni si unirono ai partigiani locali. L'atmosfera di questa dissoluzione si respira nel film del 1960 di Luigi Comencini dal titolo emblematico, Tutti a casa: era quello il desiderio di tutti, che però non per tutti si trasformò in realtà. Le tragedie di quegli anni spiegano come il rifiuto della guerra fosse per la stragrande maggioranza degli italiani un'aspirazione radicata nel più profondo dell'anima.

Per il governo Badoglio il problema centrale era l'uscita dalla guerra. Dopo un confuso periodo tra Roma e Pescara di contatti con gli angloamericani, di reticenze interne e di doppio gioco con i tedeschi, il 13 ottobre 1943 il Regno d'Italia dichiarò guerra alla Germania e le truppe dell'Italia monarchica affiancarono quelle anglo-americane nella lenta riconquista della penisola. Al Nord, invece, tedeschi diffidavano dell'efficienza militare dell'esercito fascista-repubblicano e andarono perciò rafforzando le loro posizioni accanto a quelle delle forze armate di Salò, approntando anzi un piano per l'occupazione dell'Italia: il piano "Achse" (Asse).

Firmando la propria resa incondizionata agli Alleati l'8 settembre 1943, il Regno d'Italia fu il primo dei tre Stati dell'ex Patto Tripartito a uscire formalmente dalla guerra, anche se le truppe del Regno d'Italia continuavano a fianco degli anglo-americani la guerra contro gli ex alleati tedeschi, a fianco dei quali combattevano invece le truppe della Repubblica di Salò: una situazione disperata e complicatissima. Infatti il re e i vertici politici e militari erano fuggiti da Roma senza dare disposizioni alle truppe italiane sparse dalla Provenza all'Egeo e ancora integrate con i militari tedeschi, che avevano invece ordini precisi nei riguardi degli italiani: o incorporarli nelle proprie truppe, o internarli o, in caso di resistenza, passarli per le armi.

Chi rifiutava questa incorporazione poteva anche essere internato non come prigioniero di guerra, ma come lavoratore per il Reich. Nascevano cos̀ gli Internati Militari Italiani (IMI), che non erano prigionieri di guerra

7 Elena Aga-Rossi (2016), Cefalonia; a Cefalonia vennero fucilati circa 400 ufficiali italiani e uccisi tra 5000 e 9000 militari; il generale Hubert Lanz, responsabile dell'eccidio, venne condannato a dodici anni di reclusione dal Tribunale di Norimberga. Isabella InSOLVIBILE (2010), Kos 1943-1948; a Kos (Coo) vennero fucilati oltre un centinaio di ufficiali italiani. 
e ai quali non si applicavano quindi le Convenzioni di Ginevra: circa 600.000-800.000 persone quasi dimenticate dalle istituzioni italiane anche dopo la fine della guerra. "Nei lager tedeschi morirono 17 generali italiani: silenzio! Morirono alcune decine di migliaia di soldati e ufficiali italiani: silenzio!"” È una riprovevole dimenticanza cui tuttora non si è saputo porre riparo, anche se si trattava di soldati italiani che avevano assunto una gravosa posizione piena di dignità: né con i fascisti di Salò, né con la Wehrmacht dei nazisti.

Tra gli Internati Militari Italiani ritroviamo i personaggi più diversi della futura Italia repubblicana, dallo scrittore Giovanni Guareschi - l'irresistibile anticomunista alla cui penna dobbiamo Don Camillo e Peppone, nonché il motto lanciato nelle elezioni del 1948: "Nel segreto della cabina Dio ti vede, Stalin no" - al politico Alessandro Natta, il neolaureato fatto prigioniero a Rodi, poi deputato comunista per dieci legislature e infine successore di Enrico Berlinguer come segretario generale del Partito Comunista Italiano. Alessandro Natta ha dedicato un libro di memorie al suo internamento, da cui si possono ricavare precise notizie sulla vita in quei campi di lavoro. ${ }^{9}$

Anzitutto le condizioni degli IMI erano meno disperate di quelle dei lager di sterminio, anche perché Natta si trovava in un campo di prigionia per ufficiali: "Dopo la Liberazione avemmo modo di misurare la nostra condizione sul metro di Mauthausen", ma durante l'internamento il confronto non c'era. Tuttavia "se l'intensità della persecuzione non fu uguale, eguale fu il sentimento di esecrazione e di condanna dei nazisti” (p. 93). "Noi abbiamo avuto una sorte diversa da quella dei prigionieri nei campi di sterminio, dei lager politici, anche se occorre dire che non vi fu una differenza nella sostanza ma solo nel grado di intensità della persecuzione” (p. 137).

Questa differenza si nota, per esempio, in un minor rigore rispetto alla religione e alla cultura. "Numerosi erano nei lager i cappellani militari, che svolsero un'intensa, e dai tedeschi tollerata, attività di assistenza spirituale” (p. 73). "Non dimenticherò mai, ad esempio, i pastori valdesi che, nel campo

8 Così Alessandro Natta (1997), L'altra Resistenza, 124.

9 Alessandro Natta (1997), L'altra Resistenza. Nel 1991 (non c'è più il PCI e Natta ha lasciato la politica attiva) partecipa a un convegno sugli IMI con una relazione: Alessandro Natta (1992), Reducismo o silenzio? Lì ricorda il suo manoscritto del 1954 e il fatto che gli Editori Riuniti rifiutarono il volume, che rimase inedito fino al 1997. 
di Sandbostel, tenevano conferenze all'aperto, tra una baracca e l'altra, e avevano sempre un uditorio numeroso, e non formato certo dai soli correligionari. È vero che le loro parole esercitavano un fascino particolare, soprattutto per quanti, e non erano pochi, non avevano mai avuto occasione di ascoltare sacerdoti nei quali il senso religioso fosse tanto vivo e tanto fortemente si unisse al gusto della libertà" (p. 56).

Tra gli ufficiali internati non mancavano i libri: "Parve una singolare ironia della sorte riuscire a leggere nel campo di concentramento tedesco ciò che era stato proibito in Italia. Lo stesso accadde con il Manifesto di Marx e per altre pubblicazioni di cui molti ebbero allora per la prima volta notizia. I tedeschi non sequestravano, in genere, i libri e tanto erano sospettosi e diffidenti nei riguardi della carta scritta a mano, tanto erano accomodanti verso la carta stampata" (p. 75 s.).

La Liberazione del 1945 concluse positivamente questa "altra" resistenza: "L'insurrezione di aprile [1945] conferiva un più preciso senso e valore alla nostra lotta e concludeva, insieme alla guerra di liberazione, la resistenza degli internati nei lager. Quella era la sanzione della giustezza e della necessità del sacrificio umile e doloroso che per quasi venti mesi aveva impegnato le energie migliori degli internati contro i fascisti e i tedeschi e contro le molte insidie e debolezze del movimento di resistenza". "Tra i reticolati tedeschi eravamo diventati uomini liberi” (p. 133 s.).

Nel 1992 un autore tedesco constatava: "Considerata la straordinaria importanza assunta dopo il 1945 dalla Resistenza per la consapevolezza e la coscienza politica della nazione italiana, non ci si rende conto del perché si siano voluti ignorare per tanto tempo gli internati militari nel contesto di tutte le manifestazioni intese a superare il passato. Un fatto ancor più incomprensibile se si tiene conto del fatto che i militari rinchiusi nei campi di prigionia nazionalsocialisti, nel rifiutare ogni forma di collaborazione con la Repubblica Sociale e con il Terzo Reich - decisione che significava rinuncia a trattamenti privilegiati per continuare a subire quanto di peggio potessero offrire i Lager - attuarono in pratica anche loro, sia pure senza l'uso delle armi, una forma di resistenza". ${ }^{\mathbf{1 0}}$ Negli anni successivi vennero pubblicate

10 Gerhard Schreiber (1992), I militari italiani internati, 15-16. Il volume è l'edizione italiana "riveduta e ampliata" di Gerhard Schreiber (1990), Die italienischen Militärinternierten, edito dal Militärgeschichtliches Forschungsamt, Freiburg i. B.; Mario Avagliano, Marco Palmieri (2020), I militari italiani nei lager nazisti. 
alcune approfondite ricerche storiche ${ }^{\mathbf{1 1}} \mathrm{e}$, di recente, sono riaffiorati i ricordi dell'uccisione di oltre un centinaio di internati che lavoravano in una fabbrica munizioni di Treuenbrietzen, nel Brandeburgo. ${ }^{\mathbf{1 2}}$

Anche la Repubblica Sociale Italiana costituì le proprie forze armate e gli abitanti del suo territorio vennero obbligati al servizio militare, altrimenti sarebbero stati puniti come renitenti alla leva. ${ }^{13}$ Molte persone non disposte a collaborare con i tedeschi o a militare con i fascisti - e non finite negli Internati Militari Italiani - si unirono alla Resistenza. I militari delle forze armate di Salò fatti prigionieri vennero internati dagli Alleati soprattutto negli Stati Uniti. Nella primavera del 1945 iniziò l'offensiva alleata contro la Repubblica Sociale Italiana, che capitolò il 29 aprile. Le sue forze armate vennero dissolte. Bisognava ora iniziare la ricostruzione di un paese devastato.

Dopo la fine della guerra e con l'avvento della repubblica italiana, la rifondazione di un nuovo esercito italiano - proprio per la sua specifica storia che lo vide combattere a fianco degli anglo-americani - presentò meno ostacoli che il riarmo del Giappone e della Germania: già alla fine del 1946 l'"Esercito di Transizione" italiano era di fatto completato. La sua genesi non incontrò l'ostacolo dell'articolo pacifista nella costituzione, come negli altri due Stati dell'ex Asse, perché in Italia quell'articolo prese forma soltanto nel 1948, cioè quasi due anni dopo la formazione dell'"Esercito di Transizione". La "transizione" viene così descritta dal Ministero della Difesa italiano:

11 Mario Avagliano, Marco Palmieri (2009), Gli internati militari italiani. La più vasta ricerca italiana è probabilmente quella di Nicola Labanca (ed.) (1992), Fra sterminio e sfruttamento; Nicola LABANCA (ed.) (2000), La memoria del ritorno.

12 Nella sabbia del Brandeburgo (in tedesco Im märkischen Sand), documentario "web doc" prodotto da Out of Focus Filmproduktion: "Un progetto crossmediale in tre lingue (tedesco, italiano e inglese) che si dipana in ventiquattro episodi tra passato, presente e futuro di Treuenbrietzen. Perché se la memoria di Antonio Ceseri [uno dei quattro sopravvissuti] 'ha iniziato soltanto da poco a sciogliersi', come racconta la figlia in uno degli approfondimenti sul sito del documentario, anche quella della cittadina tedesca [Treuenbrietzen] ha dovuto 'aspettare di essere matura' [...] per poter mettere insieme i pezzi di una vicenda dalle letture contrastanti. Per non parlare dell'Italia, che da pochi anni affronta il tema”, Alessia Grossi (2017, 4 novembre), La sabbia che ricopri le vergogne della Storia.

13 Sulla consistenza delle forze armate repubblicane e dei corpi paralleli: http://www.storiaxxisecolo.it/rsi/rsiesercito.htm. 
Al termine della $2^{\mathrm{a}}$ Guerra Mondiale l'Esercito Italiano [quello cioè del Regno d'Italia] era rappresentato dai cinque Gruppi di Combattimento che avevano combattuto nell'ambito della $5^{\mathrm{a}}$ Armata americana e dell' $8^{\mathrm{a}}$ britannica, e da altre unità, anch'esse direttamente dipendenti dai comandi alleati. Il 14 novembre 1945 la Missione Militare Alleata emanò una direttiva fondamentale che indicava l'ordinamento dell'Esercito detto di Transizione.

All'Esercito Italiano fu imposta una struttura che doveva rimanere in vigore fino alla conclusione del trattato di pace [...]. La direttiva, dopo la definizione degli organici particolareggiati, diede luogo alla prima normativa organica del dopoguerra, diramata dalla Stato Maggiore dell'Esercito nel marzo 1946. [...]

Alcune unità restarono ancora alle dipendenze degli Alleati: una divisione ausiliaria e 6 raggruppamenti e 2 gruppi battaglioni. Nel corso dello stesso anno 1946, le tre divisioni per la sicurezza interna furono trasformate in altrettante brigate [...]; l'Arma di Cavalleria, ancora ufficialmente esclusa dalla ricostruzione dell'Esercito, riprese vita con l'assegnazione ad ogni divisione di fanteria di un gruppo squadroni esplorante, montato su cingolette. Nel febbraio 1947, allorché fu firmato il trattato di pace di Parigi, l'Esercito di Transizione era ormai completato. ${ }^{\mathbf{1 4}}$

Il 2 giugno 1946 gli italiani parteciparono alle prime libere elezioni dal 1924. In esse si decise il passaggio al regime repubblicano e l'istituzione di un'Assemblea costituente, che in quella travagliata transizione doveva non solo predisporre la nuova costituzione, ma anche votare la fiducia al nuovo governo, ratificare i trattati internazionali e approvare il bilancio nazionale. Al suo interno operava la Commissione per la Costituzione, o "Commissione dei 75", suddivisa in tre Sottocommissioni e in un Comitato per la Redazione, detto anche "Comitato dei 18", che curava la stesura vera e propria del progetto di costituzione. ${ }^{15} \mathrm{E}$ in questo contesto che venne discusso anche il principio pacifista che avrebbe dovuto essere incluso nella futura costituzione.

Una peculiarità della costituente italiana fu la forte presenza delle sinistre, che rappresentavano circa il 40\% dei voti. ${ }^{16}$ Questo determinò nella nuova costituzione una forte attenzione ai temi sociali e, nell'ambito che qui interessa, al tema della pace, che era allora quasi un monopolio delle sinistre.

14 Il testo citato, con maggiori dettagli, è tratto da sito del Ministero della Difesa: http://www.esercito.difesa.it/storia/Pagine/La-Storia-1946-1947.aspx.

15 La commissione terminò i suoi lavori il 12 gennaio 1947 e la nuova Costituzione venne pubblicata sulla "Gazzetta Ufficiale" il 27 dicembre 1947, entrando in vigore il $1^{\circ}$ gennaio 1978.

16 Dei tre partiti che nei decenni successivi avrebbero determinato la politica italiana, la Democrazia Cristiana ottenne il 35,2\% dei voti (207 seggi); il Partito Socialista il 20,7\% dei voti (115 seggi); il Partito Comunista il 18,9\% (104 seggi). 
Un passo sintetizza tanto le direttrici politiche del Movimento dei Partigiani della Pace, quanto anche le divisioni del mondo postbellico:

"Nell'aprile 1949 a Parigi nasce ufficialmente il Movimento dei Partigiani della Pace. La denominazione discende dall'esperienza della resistenza europea e asiatica e raccoglieva il messaggio della politica antimperialista e della cultura antifascista. La lotta sarà indirizzata all'interdizione dell'arma atomica e all'incontro delle cinque grandi potenze per un patto di pace con la consapevolezza di poter evitare una nuova disastrosa guerra. Le parole d'ordine sono: la difesa della pace è il compito di tutti $i$ popoli e l'unità per la difesa della pace è il più sacro dei doveri.

La partecipazione al primo Congresso mondiale per la pace è straordinaria e si intreccia con le manifestazioni contrarie al Patto Atlantico. Giungono a Parigi 2287 delegati di 72 paesi, ma molti sono gli assenti: gli USA impediscono la partenza ai delegati nipponici, mentre la Francia nega i visti di ingresso a Cina, Mongolia, Corea, Unione Sovietica (tra i cui delegati è presente Shostakovich), Bulgaria, Polonia, Ungheria, tutto il blocco dell'est resterà bloccato a Praga. $\mathrm{Ci}$ si domanderà: "Chi erige barriere di incomunicabilità tra $\mathrm{i}$ popoli?”. Tra gli aderenti: Frédéric Joliot-Curie (premio per la fisica a cui spetterà la presidenza e la relazione introduttiva), Picasso (che dipingerà il manifesto del congresso: la famosa "Colomba della pace"), Aragon, Farge, Amado, Matisse, Ehrenburg, Neruda, Einstein. Nella delegazione italiana guidata da Nenni: Vittorini, Guttuso, Quasimodo, N. Ginzburg, G. Levi, G. Einaudi. Il movimento non è però limitato agli intellettuali, anzi vuole rivolgersi alle masse." ${ }^{17}$

Lo scrittore sovietico Ilya Ehrenburg, rievocando l'entusiasmo che circondò Picasso quando nel 1953 soggiornò a Roma in occasione per la sua mostra, sottolinea l'importanza di quella colomba come simbolo dell'intero movimento: con essa, Picasso

"ha inventato l'immagine che ha unito i popoli. Ho visto le sue colombe nelle diverse città della Cina. Ho visto la colomba di Picasso nell'aula di una scuola a Leningrado e sul petto di una coreana. Le colombe di Picasso hanno volato sul Congresso dei Popoli a Vienna”. [Infatti al centro della pagina campeggia la grande immagine del manifesto "Congrès des Peuples pour la Paix, Vienne 12-18 décembre 1952", con la scritta sormontata da una colomba.] "Ricordo un comizio dei Partigiani della Pace a Roma nell'autunno del 1949. Una grande piazza, le colombe, le fiaccole. Dopo il comizio, insieme a Picasso andai in una piccola osteria. Gli operai accorsero verso Picasso, cominciarono ad abbracciarlo. Egli era molto commosso, e gli operai erano fieri e felici. Meraviglioso quadro, indimenticabile. Esso contiene un senso profondo e tutti commuove, anche rievocandolo con semplici parole: gli operai romani, Pablo Picasso, la colomba. In quell'istante anch'io fui fiero: per l'arte". ${ }^{\mathbf{1 8}}$

17 Ruggero Giacomini (1984), I partigiani della pace.

18 Ilya Ehrenburg (1953), Partigiano della Pace. L'articolo è contenuto nel numero speciale a 16 pagine dedicato per intero alla Mostra di Picasso, Roma 1953, ed è datato "Mosca, aprile 1953 ". 
Come si vede dai nomi dei partecipanti più illustri al "Congresso mondiale per la pace", i Partigiani della Pace erano un movimento di sinistra, egemonizzato dall'Unione Sovietica e dai partiti comunisti dei singoli Stati europei. Il suo pacifismo antiamericano suscitava dubbi e critiche non solo tra $\mathrm{i}$ conservatori: nell'Appendice II,2,a il testo di Stalin sintetizza la posizione ufficiale dei comunisti sui Partigiani della Pace, mentre l'articolo di Norberto Bobbio critica con argomenti razionali e con tono pacato questo pacifismo unilaterale; infine, lo scambio di lettere tra Norberto Bobbio e il pittore Gabriele Mucchi attesta come anche all'interno della sinistra italiana la discussione su quel movimento pacifista fosse viva e ricca di spunti critici.

In questo clima andava prendendo forma la futura costituzione della Repubblica Italiana. La relazione con cui Meuccio Ruini ne presentò il progetto definitivo esprime con chiarezza la comune matrice antifascista che animava i costituenti nella stesura dell'articolo pacifista di quella costituzione e ribadisce lo stretto collegamento tra il ripudio della guerra e il diritto internazionale:

"La costituzione, dopo aver affermato il concetto della sovranità nazionale, intende inquadrare nel campo internazionale la posizione dell'Italia: che dispone il proprio ordinamento giuridico in modo da adattarsi automaticamente alle norme del diritto internazionale generalmente riconosciute. Rinnegando recisamente la sciagurata parentesi fascista, l'Italia rinuncia alla guerra come strumento di conquista e di offesa alla libertà di altri popoli. Stato indipendente e libero, l'Italia non consente, in linea di principio, altre limitazioni alla sua sovranità, ma si dichiara pronta, in condizioni di reciprocità e di eguaglianza, a quelle necessarie per organizzare la solidarietà e la giusta pace fra i popoli. Contro ogni minaccia di rinascente nazionalismo, la nostra costituzione si riallaccia a ciò che rappresenta non soltanto le più pure tradizioni ma anche lo storico e concreto interesse dell'Italia: il rispetto dei valori internazionali" ${ }^{19}$

Il dibattitto della Commissione sull'attuale articolo 11 prese in considerazione due norme pacifiste già esistenti. Il patto Briand-Kellogg del 1928 affermava all'art. 1: "Le alte parti contraenti dichiarano solennemente in nome dei loro popoli rispettivi di condannare il ricorso alla guerra per la

19 Assemblea Costituente, Commissione per la costituzione. Progetto di costituzione della Repubblica Italiana. Relazione del Presidente della Commissione presentata alla Presidenza dell'Assemblea Costituente il 6 febbraio 1947, Tipografia della Camera dei Deputati, Roma 1947, p. 5 (http://legislature.camera.it/_dati/costituente/lavori/ddl/00Anc.pdf). 
risoluzione delle divergenze internazionali e di rinunziare ad usarne come strumento di politica nazionale nelle loro relazioni reciproche". La costituzione della Repubblica spagnola del 1931 dichiarava all'art. 6: "La Spagna rinuncia alla guerra come strumento di politica nazionale". La Commissione ritenne che il concetto di "condanna" contenuto nel patto Briand-Kellogg non fosse sufficientemente univoco, mentre la "rinuncia" (contenuta nella costituzione spagnola) esprimeva in modo chiaro e diretto il "rifiuto", anzi - nella formulazione finale italiana - il "ripudio" della guerra. Con il termine "ripudio" i costituenti intendevano anche condannare ogni propaganda bellicista e ogni dottrina che predicasse la guerra: l'Italia era appena uscita dal Fascismo che per vent'anni aveva esaltato la guerra.

Poiché nel 1946 le donne avevano avuto per la prima volta l'elettorato attivo a livello nazionale, alla costituente partecipavano anche 21 deputate (su oltre cinquecento colleghi). La più giovane di loro era Teresa Mattei, ${ }^{20}$ comunista e partigiana Comandante di Compagnia (cioè capitano), ma anche pacifista: compiva le incursioni - per sequestrare armi in casa di fascisti, o prosciutti per sfamare i partigiani - impugnando una grossa stilografica nera come se fosse un revolver. La sua intervista nel quarantennale della Costituzione è un esempio della tensione morale che dominava i lavori della Costituente:

Teresa Mattei il 2 giugno del 2006 rilasciava un'intervista alla trasmissione Radio 3 Mondo nella quale raccontò, tra le altre cose, questo episodio accaduto durante la votazione dei principi fondamentali della Costituzione: "Al momento della votazione per l'art. 11, cioè quello contro la guerra - 'L'Italia ripudia la guerra', è stato scelto il termine più deciso e forte - tutte le donne che erano lì, ventuno, siamo scese nell'emiciclo e ci siamo strette le mani tutte insieme, eravamo una catena, e gli uomini hanno applaudito". E poi continuava: "Per questo, quando ora vedo tutti questi mezzucci per giustificare i nostri interventi italiani nelle varie guerre che aborriamo, io mi sento sconvolta perché penso a quel momento, penso a quelle parole e penso che se non sono le donne che difendono la pace prima di tutto non ci sarà un avvenire per il nostro paese e per tutti i paesi del mondo".

20 Nel dopoguerra, Teresa Mattei (1921-2013) fu parlamentare comunista e si impegnò a favore delle donne, facendo per esempio abolire divieti assurdi, come quello che proibiva alle infermiere di sposarsi, essendo quella professione concepita quasi come un sacerdozio (142). Venne radiata dal partito nel 1955 perché il suo antistalinismo la mise in urto con Togliatti, ma continuò a dedicarsi alle donne e all'infanzia: Patrizia Pacini (2009), Teresa Mattei, una donna nella storia; PAtrizia Pacini (2011), La Costituente: storia di Teresa Mattei, per una recensione: Mauro De VincentiIs (2011, 25 settembre).

21 Pasquale Pugliese (2013, 15 marzo), Ispiratevi a Teresa Mattei, cancellate gli F35. 
La recente pubblicazione di due attente analisi dei due articoli costituzionali sull'ordinamento internazionale e sul ripudio della guerra ${ }^{22}$ consente di limitarci qui a riassumerne la genesi. Del termine "ripudio" è stato sottolineato l'"accento energico" anche da parte del presidente Ruini: "La Commissione ha ritenuto che, mentre 'condanna' ha un valore etico più che politico-giuridico, e 'rinunzia', presuppone, in certo modo, la rinunzia a un bene, ad un diritto, il diritto alla guerra (che vogliamo appunto contestare), la parola 'ripudia', se può apparire per alcuni richiami non pienamente felice, ha un significato intermedio, ha un accento energico ed implica così la condanna come la rinuncia alla guerra". ${ }^{23}$

Mentre la costituzione tedesca esprime il rifiuto della guerra con un vocabolo inconsueto ("Kriegsächtung", cfr. p. 223), il testo italiano ricorre al verbo "ripudiare" da secoli presente nella lingua italiana letteraria, legato per lo più al "rifiutare la propria moglie" ovvero - sempre esprimendo "un'iniziativa unilaterale" - a molte altre forme di rifiuto netto, anche in campo giuridico: già il legislatore dell'inizio dell'Ottocento regolava il "ripudiare un'eredità". ${ }^{24}$

È possibile seguire le varie stesure dell'art. 11 che hanno preceduto la sua formulazione definitiva. Il variare della sua posizione nel testo costituzionale - da articolo 4 ad articolo 11 - attesta come i costituenti stessero elaborando a fondo non solo questo articolo, ma l'intero sistema costituzionale. Ecco dunque, in sintesi, l'evoluzione dell'articolo 11 nel corso del dibattito costituente:

22 "L'ordinamento giuridico italiano si conforma alle norme del diritto internazionale generalmente riconosciute" (art. 10, c. 1); "L'Italia ripudia la guerra come strumento di offesa alla libertà degli altri popoli e come mezzo di risoluzione delle controversie internazionali” (art. 11): Luigi Bonanate (2018), Art. 11; Pietro Costa (2018), Art. 10. I singoli volumetti di questa collana analizzano i 12 articoli che costituiscono i "Principi fondamentali" della Costituzione italiana.

23 Luigi Bonanate (2018), Art. 11, 27, tratto da Atti dell'Assemblea Costituente, 24 marzo 1947, 2432.

24 Un panorama completo dell'uso di "ripudiare" e derivati è in Salvatore Battaglia (1992), Grande dizionario della lingua italiana, s.v. Ripudiare, nella quale si cita il Codice di Napoleone il Grande per il Granducato Lucchese, Lucca 1806, art. 461: "Il tutore non potrà né accettare né ripudiare un'eredità devoluta al minore, se non previa l'autorizzazione del consiglio di famiglia"; inoltre, al punto 4 s.v. Ripudio, viene riportato l'art. 11 della Costituzione Italiana del 1948. 
Il 3 dicembre 1946 la prima Sottocommissione della Commissione per la Costituzione approva il seguente articolo: "La Repubblica rinunzia alla guerra come strumento di conquista o di offesa alla libertà degli altri popoli e consente, a condizioni di reciprocità, le limitazioni di sovranità necessarie alla difesa e alla organizzazione della pace”. Il 24 gennaio 1947 la Commissione per la Costituzione in seduta plenaria, non approvando gli emendamenti proposti, approva implicitamente il seguente articolo nel testo formulato dal Comitato di redazione: "L'Italia rinunzia alla guerra come strumento di conquista e di offesa alla libertà degli altri popoli e consente, a condizione di reciprocità e di eguaglianza, le limitazioni di sovranità necessarie ad un'organizzazione internazionale che assicuri la pace e la giustizia per i popoli”. Testo definitivo del Progetto di Costituzione elaborato dalla Commissione: Art. 4: L'Italia rinunzia alla guerra come strumento di conquista e di offesa alla libertà degli altri popoli e consente, a condizione di reciprocità e di eguaglianza, le limitazioni di sovranità necessarie ad una organizzazione internazionale che assicuri la pace e la giustizia tra i popoli. - Il 24 marzo 1947, nella seduta pomeridiana, l'Assemblea Costituente approva il seguente articolo: "L'Italia ripudia la guerra come strumento di offesa alla libertà degli altri popoli e come mezzo di risoluzione delle controversie internazionali, e consente, in condizioni di parità con gli altri Stati, alle limitazioni di sovranità necessarie ad un ordinamento internazionale, che assicuri la pace e la giustizia fra le Nazioni”. - Testo coordinato dal Comitato di redazione prima della votazione finale in Assemblea e distribuito ai Deputati il 20 dicembre 1947: Art. 8. L'Italia ripudia la guerra come strumento di offesa alla libertà degli altri popoli e come mezzo di risoluzione delle controversie internazionali; consente, in condizioni di parità con gli altri Stati, alle limitazioni di sovranità necessarie ad un ordinamento che assicuri la pace e la giustizia fra le Nazioni; promuove e favorisce le organizzazioni internazionali rivolte a tale scopo. - Il 22 dicembre 1947, nella seduta antimeridiana, l'onorevole Ruini, a nome del Comitato di coordinamento, comunica che l'articolo viene spostato ed assume quindi il numero 10. - Testo definitivo dell'articolo: Art. 11. L'Italia ripudia la guerra come strumento di offesa alla libertà degli altri popoli e come mezzo di risoluzione delle controversie internazionali; consente, in condizioni di parità con gli altri Stati, alle limitazioni di sovranità necessarie ad un ordinamento che assicuri la pace e la giustizia fra le Nazioni; promuove e favorisce le organizzazioni internazionali rivolte a tale scopo. ${ }^{25}$

La comune matrice antifascista dei partiti rappresentati nella Costituente spiega perché l'articolo pacifista sia stato approvato quasi all'unanimità, cioè con due soli voti contrari. Mentre i grandi partiti votarono congiuntamente, dimostrando che erano ancora uniti dal forte spirito antifascista che aveva animato la Resistenza, i due voti contrari provennero dai rappresentanti di due formazioni minori. Poiché l'Italia era ancora occupata dagli Alleati e il

25 Questo sito consente di seguire non solo l'evolversi delle formulazioni dei singoli articoli della Costituzione italiana, ma riporta anche gli interventi dei vari costituenti (http://www.nascitacostituzione.it/01 principi/011/index.htm?art011-999.htm2). 
suo esercito non era ancora stato ricostituito, il costituente Guido Russo Perez $^{\mathbf{2 6}}$ riteneva ridicolo che si dichiarasse pacifista uno Stato disarmato; inoltre era impossibile distinguere tra guerre giuste e ingiuste, visto che a dichiararlo è sempre il vincitore (argomento che, più tardi, ritornerà anche in Bobbio). Per il costituente Francesco Saverio Nitti, ${ }^{27}$ invece, non aveva senso che quella norma venisse emanata da un paese sconfitto e distrutto, che non aveva ancora ricuperato la sua piena sovranità e che quindi non era in grado né di dichiarare né di condurre una guerra. Ma nella votazione queste posizioni erano meno che minoritarie: erano voci isolate.

Nella Seconda guerra mondiale le grandi potenze avevano arruolato anche persone provenienti dalle varie etnie delle proprie colonie, dove già da tempo erano presenti movimenti indipendentisti. Non pochi di questi militari ritornarono nelle colonie con una nuova o rafforzata consapevolezza e con nuove cognizioni, anche militari. Poiché gli Stati Uniti e l'Unione Sovietica erano contrari al mantenimento delle colonie, con la fine della guerra si intensificò e si avviò a conclusione il processo di decolonizzazione. $^{28}$

La simpatia delle sinistre per le guerre di liberazione nazionale ebbe un fugace riflesso anche nella costituente italiana, nel corso del dibattito sulla condanna della guerra offensiva. Tutti erano concordi nel respingerla, "con peraltro un iniziale distinguo dei comunisti, i quali - pur non insistendo poi a che ciò fosse menzionato nella disposizione finale che si stava discutendo - 'si differenziarono in certa misura dalla concezione degli altri partiti, perché, pur essendo contrari alle guerre di aggressione o di conquista, dichiararono di approvare le 'guerre giuste', e cioè sia le guerre di legittima difesa sia le guerre di liberazione nazionale, ossia quelle miranti a liberare i popoli da regimi oppressivi, in particolare dal giogo colo-

26 L'ex fascista Russo Perez nella costituente rappresentava il partito "L'Uomo Qualunque", che oggi si definirebbe "populista". Il motto di quel movimento era: "Il popolo è come l'asino: utile, paziente e bastonato". Sulla sua effimera storia: SANDro SETTA (2005), L'Uomo Qualunque.

27 Importante politico meridionalista costretto all'esilio perché antifascista, l'ormai settantasettenne Francesco Saverio Nitti rappresentava nella costituente l'“Unione Democratica Nazionale", una scomparsa coalizione di partiti che oggi si definirebbe di centro-destra.

28 L'intero arco della decolonizzazione va dall'indipendenza dell'India nel 1947 alla restituzione della portoghese Macao alla Cina, nel 1999. 
niale.”29 Il riferimento alle guerre di liberazione nazionale non venne poi incluso nel testo definitivo. Tuttavia non mancano autori che tendono a ritenere compatibile "la guerra in difesa della libertà dei popoli" con il "ripudio della guerra" dell'art. 11, quando "una delle parti, o con la minaccia dell'uso della forza, o con blocchi [...] volti a sovvertire le istituzioni della parte avversa" miri a "ottenere risultati offensivi della sua libertà."30

I costituenti democristiani precisarono che ritenevano illegittima la guerra perché immorale, mentre quelli comunisti e socialisti si dichiaravano favorevoli a un disarmo unilaterale per garantire all'Italia un futuro di pace, come aveva dichiarato anche il segretario generale del Partito comunista, Palmiro Togliatti, in un discorso dell'11 aprile 1946.

Il rifiuto della guerra non si spingeva però sino alla dichiarazione di neutralità, perché la neutralità deve durare nel tempo, mentre il ripudio della guerra ammette l'accettazione preventiva della partecipazione a specifici conflitti, specie nell'ambito di organizzazioni per la sicurezza collettiva.

Si era così giunti al testo definitivo dell'articolo 11, cardine del principio pacifista nella nuova costituzione italiana. I costituenti lo inserirono in un'architettura più articolata rispetto alle altre costituzioni del dopoguerra, già ricordata nel quadro generale delle vicende italiane (cfr. p. 56). Così, l'art. 78 della costituzione italiana stabilisce che le Camere decidono lo stato di guerra e l'art. 87 che il Presidente della Repubblica lo proclama; inoltre, in presenza dello stato di guerra, l'art. 27 prevedeva la pena di morte in base al codice penale militare di guerra (ora trasformata in ergastolo); l'art. 60, la proroga della vigenza delle Camere; l'art. 103, la competenza dei tribunali militari in tempo di guerra e l'art. 111 la non ricorribilità in Cassazione delle sentenze dei tribunali militari di guerra. L'art. 52 non escludeva la guerra difensiva, stabilendo anzi il servizio militare generale e obbligatorio, oggi sospeso: "La difesa della Patria è sacro dovere del cittadino. - Il servizio militare è obbligatorio nei limiti e modi stabiliti dalla legge. Il suo adempimento non pregiudica la posizione di lavoro del cittadino, né l'esercizio dei diritti politici. - L'ordinamento delle Forze armate si informa allo spirito democratico della Repubblica”.

29 Stefano Sicardi (1999), I mille volti della guerra, 98; il passo citato da Sicardi viene da Antonio Cassese (1975), Commento all'art. 11 della Costituzione. Sul volume di Mario Dogliani, Stefano Sicardi (eds.) (1999), Diritti umani e uso della forza cfr. nota 121, p. 43.

30 Manlio Mazziotti di Celso (1993), Lezioni di diritto costituzionale, 158. 
Nella seconda parte dell'articolo 11 l'Italia dichiarava il proprio desiderio di partecipare alla comunità internazionale, accettando le limitazioni di sovranità che ciò comportava: i legami con gli Stati Uniti erano stati confermati dal viaggio di De Gasperi negli USA nel 1947; l'Italia era poi entrata nella Nato nel 1949 e si preparava a entrare nell'ONU nel 1955.

La presenza dell'Italia nelle organizzazioni internazionali comportò una progressiva attenuazione del divieto contenuto nell'art. 11, perché l'Italia prese parte a operazioni internazionali che, per la loro natura militare, suscitarono aspre controversie: nel 1991 partecipò alla Prima guerra del Golfo, poi alle missioni in Serbia nel 1999, in Afghanistan nel 2001, in Iraq nel 2003 e così via. ${ }^{31}$ Nell'opinione pubblica e nel Parlamento sorgevano queste domande: fin dove può giungere "la difesa della Patria"? Una "missione di pace" in che cosa si distingue da una guerra quando usa materiale bellico e annienta vite umane, spesso di civili? È costituzionalmente ammissibile che militari italiani muoiano all'estero combattendo in "missioni di pace"? Che ne è del dovere di non ingerenza negli affari interni di uno Stato terzo? Sono gli interrogativi che, di fronte a quelle missioni, sono riecheggiati anche in Giappone e in Germania.

Le articolate risposte negative a questi interrogativi possono essere riassunte dalla posizione netta e radicale di una costituzionalista italiana: "Le ragioni per ritenere l'intervento della Nato nel Kosovo contrario alle norme interne e alle norme internazionali mi sembrano insuperabili: è mancata ogni autorizzazione da parte del Consiglio di Sicurezza delle Nazioni Unite, come richiesto dall'art. 53 della Carta; solo un'aggressione subìta da uno degli Stati aderenti all'Alleanza Atlantica avrebbe potuto giustificare una reazione armata della Nato (art. 5 del Patto) e niente di simile era in atto e nemmeno era minacciato. L'intervento armato, dunque, altro non è stato che una guerra di offesa, certamente vietata dall'art. 11 della Costituzione".32

31 In base al Decreto-legge 16 maggio 2016, n. 67, l'Italia era impegnata in 26 missioni in 18 Stati. Un elenco delle missioni compiute e in corso si trova nel sito del Ministero della Difesa (http://www.difesa.it/OperazioniMilitari/Pagine/RiepilogoMissioni.aspx).

32 Rosanna Tosi (1999), La guerra: il dovere di ripudiarla, 113. Anche Lorenza Carlassare (1999), Costituzione italiana e guerra "umanitaria", 23 sostiene: "Dunque è stata una guerra. I nomi diversi con cui la si è dapprima qualificata sono subito apparsi un inutile velo per una fin troppo visibile realtà. L'art. 11 della Costituzione $[\ldots]$ innegabilmente risulta infranto". 
Le risposte affermative sull'ammissibilità degli interventi designabili con l'ossimoro "guerra pacifica" si caratterizzano per le distinzioni linguistiche (come si definisce la guerra? Come la definivano i costituenti? ecc.), che in italiano, quando c'è da mascherare qualche conseguenza sgradevole, si ammantano spesso di terminologia in inglese: peace keeping, peace restoring, humanitarian warfare, intervento "out of area" ecc.

L'argomentazione giuridica più diffusa è che il rinvio "alle norme del diritto internazionale generalmente riconosciute" dell'art. 10 della Costituzione italiana ${ }^{33}$ sta subendo una modificazione consuetudinaria, che consente interventi come quello in Kosovo e simili. Però anche i sostenitori di questa giustificazione devono ammettere che questa consuetudine è ancora in formazione, e che quindi non esiste. ${ }^{34} \mathrm{E}$, se anche esistesse, nel caso specifico dell'Italia la Corte costituzionale ha più volte ribadito che non possono essere modificati dalla consuetudine i "princìpi fondamentali" della Costituzione italiana, dei quali fanno parte gli articoli 10 e 11. Insomma, l'intervento "umanitario" nel Kosovo (senza mandato ONU e senza l'approvazione dello Stato interessato) non aveva precedenti e ha aperto una serie di quesiti insoluti; esso ha una giustificazione solo politico-fattuale, ma non giuridica.

La crisi del Kosovo è soltanto uno dei fallimenti dell'Unione Europea. Sul generale richiamo alla tutela dei diritti umani cui servono questi interventi umanitario-militari si deve concludere con Mario Dogliani: "La verità dei diritti dell'uomo, se vuole essere universale, ha senso solo all'interno di un'organizzazione universale che li riconosca e li sanzioni: solo, dunque, se si risolve il problema dell'interprete (e cioè, oggi, solo se gli Stati Uniti e la Nato smetteranno di volerne prendere loro, in quanto parti, il posto)".35

L'art. 11 della Costituzione italiana bilancia le due esigenze del ripudio della guerra e della partecipazione attiva alla politica internazionale: da un lato, vieta la guerra di aggressione, e dall'altro inserisce l'Italia nell'ordinamento internazionale (con la limitazione della propria sovranità in condi-

33 Art. 10, comma 1: "L'ordinamento giuridico italiano si conforma alle norme del diritto internazionale generalmente riconosciute."

34 Filippo VARI (1999), La "vecchia" Costituzione e la "nuova" guerra, in particolare il $\$ 3$ : L'art. 10 e la presunta formazione della consuetudine innovativa, 125-128.

35 Mario Dogliani (1999), Diritti dell'uomo, 47. 
zioni di reciprocità). Da questo bilanciamento derivano anche specifiche norme costituzionali e infracostituzionali.

Il diritto costituzionale italiano non ha elaborato una definizione di guerra, ma l'ha delegata agli organismi internazionali di cui l'Italia fa parte. È dunque l'ONU, cioè il diritto internazionale, a definire la "guerra" e la "minaccia alla pace" che consentono un intervento armato. ${ }^{36}$ In questo quadro, "il Consiglio di sicurezza ha progressivamente stabilito un collegamento diretto fra crisi umanitarie e minacce alla pace, una delle tre ipotesi che giustificano l'azione del Consiglio". ${ }^{37}$ Si è così ampliato l'ambito delle azioni di forza, ammettendo anche la ratifica successiva del Consiglio alle azioni di guerra, come nel caso dell'intervento in Kosovo.

Le tre costituzioni di Giappone, Italia e Germania sono state scritte partendo da una concezione di guerra che prendeva a modello il conflitto mondiale appena terminato. In particolare, nel mondo bipolare del dopoguerra che aveva ben presente Hiroshima e Nagasaki, il rischio nucleare rendeva teorico lo scoppio di una guerra, perché la guerra sarebbe stata nucleare e avrebbe comportato la possibile distruzione dell'intera umanità. Esemplari per quello stato d'animo postbellico (ricordato supra, p. 42) furono gli scritti di Günther Anders, al cui volume sulla guerra atomica Norberto Bobbio scrisse la prefazione nel $1961 .^{38}$ Bobbio ricorda Anders anche in un altro volume sulla pace e sulla guerra del 1989: "Dedico idealmente il libro a Günther Anders, da cui ho tratto la prima ispirazione a occuparmi del problema della guerra nell'era atomica." ${ }^{39}$ Oggi il problema nucleare sembra derubricato a puro problema tecnologico, anche se le testate nucleari si sono moltiplicate in numero e potenza, e talora si trovano nelle mani di

36 Alla "minaccia alla pace" si riferisce l'art. 39 della Carta delle Nazioni Unite: "Il Consiglio di Sicurezza accerta l'esistenza di una minaccia alla pace, di una violazione della pace, o di un atto di aggressione, e fa raccomandazione o decide quali misure debbano essere prese in conformità agli articoli 41 e 42 per mantenere o ristabilire la pace e la sicurezza internazionale".

37 Antonio Cassese (2001), International Law, 297.

38 Günther Anders (1961), Essere o non essere.

39 Norberto Воввіо (1989), Il Terzo assente, 11. La prefazione a Günther Anders del 1961 vi è riprodotta alle pp. 15-22 col titolo: Pace o libertà?. Bobbio ritornò sull'argomento nel capitolo Pace e guerra della sua autobiografia (Norberto Boвbio (1997), Autobiografia, 217-246) e nel volume Norberto Boвbio (1979), Il problema della guerra. 
politici non affidabili. È allarmante, ad esempio, la leggerezza con cui Kim Jong-un e Donald Trump minacciano il ricorso all'arma atomica. ${ }^{40} \mathrm{Ed}$ è comprensibile l'apprensione con cui il Giappone segue queste minacce che coinvolgono direttamente la sua area geopolitica: di qui il rafforzamento delle proposte di revisione dell'articolo pacifista della sua costituzione, già esaminate.

Intanto l'evoluzione successiva alla caduta del muro di Berlino è stata caratterizzata dal frammentarsi delle guerre in conflitti locali. In caso di guerra difensiva, il sistema delle alleanze si rimette alla decisione di organizzazioni internazionali o regionali (o della potenza che si sostituisce a quelle organizzazioni) rispetto allo Stato nazionale. Di qui il progressivo depotenziamento dell'intero sistema normativo legato al diritto bellico italiano.

Il dovere della difesa della patria, sancito dall'art. 52 ("La difesa della Patria è sacro dovere del cittadino"), venne limitato nel 1972 dalla legge sull'obiezione di coscienza e nel 2000 dall'abolizione (o, meglio, dalla sospensione) della coscrizione obbligatoria. ${ }^{41}$ Anche in Germania il servizio militare obbligatorio, istituito nel 1956, venne sospeso dal 2011, limitandolo soltanto ai casi di guerra di difesa o di tensioni internazionali. In Giappone la coscrizione obbligatoria è stata abolita dalla Costituzione del 1947.

Dal 1990 i conflitti locali hanno portato anche l'Italia ad attenuare il ripudio della guerra. Oggi "la guerra è dissimulata sotto altre vesti, per cui si tratta di decidere l'invio di unità militari finalizzato al mantenimento o imposizione della pace, l'intervento umanitario, la partecipazione ad operazioni di polizia internazionale, la resistenza al terrorismo internazionale". ${ }^{\mathbf{2}}$ Con il terrorismo internazionale, la guerra non ha più limiti né nello spazio

40 Il 'cinguettìo' di Trump rivolto a Kim Jong-un è così riportato dalla CNN: “Will someone from his depleted and food starved regime please inform him that I too have a Nuclear Button, but it is a much bigger \& more powerful one than his, and my Button works!' Trump tweeted"; e la CNN si chiede "whether the President has thought deeply about the awesome destructive power at his command" (https:/edition.cnn.com/2018/01/03/politics/ donald-trump-nuclear-button-north-korea/index.html).

41 Legge 15 dicembre 1972, n. 772 (e successive modifiche); legge 14 novembre 2000, n. 331 (che prevede il ritorno alla coscrizione obbligatoria in caso di guerra o di grave crisi internazionale).

42 Giuseppe De Vergottini (2004), Guerra e Costituzione, 10. Questo volume di 350 pp. offre un panorama dei temi controversi legati all'interpretazione evolutiva dell'art. 11. Cfr. anche Paolo Carnevale (ed.) (2004), Guerra e Costituzione. 
né nel tempo; non c’è più un teatro della guerra, né un inizio con la dichiarazione di guerra, né la conclusione con un trattato di pace; conflitto e pace spesso convivono sullo stesso territorio e nello stesso tempo. Si genera così una situazione ibrida come ibride sono le guerre che si combattono: al fattuale stato di guerra verso l'esterno non fanno riscontro le conseguenze interne previste dalla legge.

Questa incertezza si riflette anche nel diritto interno italiano, per il quale le missioni di pace non sono guerre, mentre però ai soldati che vi partecipano si applica il diritto penale militare. ${ }^{43}$ Per esempio, la Legge 14 novembre 2000, n. 331, equipara alla guerra anche una "grave crisi internazionale nella quale l'Italia sia coinvolta direttamente o in ragione della sua appartenenza a un'organizzazione internazionale". ${ }^{44} \mathrm{Di}$ conseguenza, "ai fini della legge penale militare di guerra, per conflitto armato si intende il conflitto in cui almeno una delle parti fa uso militarmente organizzato e prolungato delle armi nei confronti di un'altra per lo svolgimento di azioni belliche" (legge 27 febbraio 2002, n. 15, art. 2). In conclusione, nei testi legislativi i concetti di "grave crisi internazionale", "conflitto armato" o simili tendono a identificarsi con il concetto di "guerra", col risultato che chi va in missione di pace è soggetto al codice militare penale di guerra.

I nuovi conflitti pongono il legislatore ordinario di fronte al problema di estendere i compiti delle forze armate anche alle missioni all'estero, evitando però l'annullamento per incostituzionalità delle norme al riguardo. In precedenza le leggi facevano sempre riferimento alla "difesa della Patria, ${ }^{45}$ richiamandosi così indirettamente al "sacro dovere del cittadino" dell'art. 52 della Costituzione. Una peculiarità della legge italiana istitutiva del servizio

43 In un primo momento si era rifiutato di applicare il codice penale militare alle missioni di pace all'estero (ANDrea De Guttry (1997), Le missioni delle forze armate italiane fuori area, 118 s.), poi intervennero la legge 31 gennaio 2002, n. 6, e la legge 27 febbraio 2002, che lo applicano (Giulio Bartolini (2002), Le modifiche al codice penale militare di guerra).

44 Legge 14 novembre 2000, Norme per l'istituzione del servizio militare professionale, n. 331, art. 2, comma 2, f (http://www.ngnu.org/leggi/331.html), seguita dal Decreto legislativo n. 215 del 2001 e dal Decreto legislativo n. 236 del 2003.

45 Norme di principio sulla disciplina militare e istituzione della Rappresentanza Militare, Legge 382/1978, art. 1: "Le Forze Armate sono al servizio della Repubblica; il loro ordinamento e la loro attività si informano ai princìpi costituzionali. Compito dell'Esercito, della Marina e dell'Aeronautica è assicurare, in conformità con il giuramento prestato e in obbedienza agli ordini ricevuti, la difesa della Patria e concorrere alla salvaguardia delle libere istituzioni e al bene della collettività nazionale nei casi di pubbliche calamità”. 
militare professionale è il suo art. 1 , che richiama direttamente due precetti costituzionali: "L'ordinamento e l'attività delle forze armate sono conformi agli articoli 11 e 52 della Costituzione”.

Una prima conseguenza di questa inclusione dell'art. 11 in una legge ordinaria è la differenza fra le regole d'ingaggio dei militari italiani in Iraq rispetto a quelle degli statunitensi. In Italia, il Regolamento di disciplina militare (DPR 545/1986) ${ }^{46}$ prevede il "dovere di disobbedienza" agli ordini che violano una legge. Il riferimento diretto all'art. 11 in una legge ordinaria permette così di fondare il rifiuto di obbedienza con un richiamo altrettanto diretto alla costituzione, mentre in precedenza bisognava richiamarsi al giuramento prestato dal militare (che obbliga a obbedire alla Costituzione), giungendo così indirettamente alla costituzione stessa.

L'attività legislativa e le crisi nel Mediterraneo sono andate aumentando e condizionandosi reciprocamente. Nel 2011 un primo attacco francese, nel contesto di una coalizione internazionale approvata dall'ONU, dava inizio alla guerra in Libia che doveva portare alla caduta di Gheddafi e a una destabilizzazione dell'area che dura tuttora. Non è possibile descrivere qui il frammentarsi dello Stato libico, né il contrapporsi degli interessi petroliferi della Francia (che con l'Egitto, gli Emirati Arabi e la Russia sostiene il governo libico di Tobruk, non riconosciuto internazionalmente) e quelli dell'Italia (che, con gli altri alleati, sostiene il Governo libico di Tripoli riconosciuto dall'ONU), né l'inserimento dell'Isis in questa guerra fino alla "somalizzazione" attuale della Libia: ci si soffermerà soltanto sul duplice coinvolgimento dell'Italia in questa non-guerra. Coinvolgimento indiretto perché - avendo autorizzato l'uso delle basi statunitensi in Sicilia per le incursioni aeree in Libia - si è scoperto che dal 2011 i droni statunitensi "hanno lanciato almeno 550 attacchi sul suolo libico" dalla base di Sigonella e, nei sei mesi di attacco alla Libia, secondo il comandante statunitense " $\mathrm{i}$ nostri [droni] Predator hanno lanciato 243 missili Hellfire: un quinto di tutti quelli usati nei quattrodici anni d'impiego di quest'arma". ${ }^{47} \mathrm{Ma}$ anche coinvolgimento diretto, perché l'Italia ha dovuto inviare truppe di terra in Libia. In entrambi i casi l'Italia si espone a ritorsioni terroristiche.

46 Regolamento di disciplina militare: http://www.militari.org/Legge_disciplina_dpr_545.htm.

47 Gianluca Di Feo (2018, 21 giugno), Libia, la guerra segreta dei droni. Cfr. Mario G. Losano (2017b), Guerre ibride, omicidi mirati, droni. 
Soffermiamoci sull'invio di truppe italiane "out of area": la base normativa per aggirare non solo l'art. 11, ma anche l'approvazione parlamentare di interventi armati all'estero era stata creata nel 2015, con un complicato sistema legislativo atto a mascherare l'esclusione del parlamento.

Anzitutto era stato emanato un decreto governativo sulla "Proroga delle missioni internazionali delle Forze armate e di polizia", ${ }^{48}$ presentato poi al parlamento per la sua conversione in legge. ${ }^{49}$ Però, nella conversione del decreto, venne inserito un lungo articolo 7 bis ("bis" perché il decreto da convertire conteneva solo sette articoli) dalla complicatissima formulazione, che conviene qui citare per esteso: ${ }^{\mathbf{5 0}}$

Art. 7 bis, Disposizioni in materia di intelligence.

1. Il Presidente del Consiglio dei ministri, acquisito il parere del Comitato parlamentare per la sicurezza della Repubblica, emana, ai sensi dell'articolo 1, comma 3, della legge 3 agosto 2007, n. 124, disposizioni per l'adozione di misure di intelligence di contrasto, in situazioni di crisi o di emergenza all'estero che coinvolgano aspetti di sicurezza nazionale o per la protezione di cittadini italiani all'estero, con la cooperazione di forze speciali della Difesa con i conseguenti assetti di supporto della Difesa stessa.

48 Decreto-Legge 30 ottobre 2015, n. 174: Proroga delle missioni internazionali delle Forze armate e di polizia, iniziative di cooperazione allo sviluppo e sostegno ai processi di ricostruzione e partecipazione alle iniziative delle organizzazioni internazionali per il consolidamento dei processi di pace e di stabilizzazione (15G00189) ("Gazzetta Ufficiale", Serie Generale n. 253 del 30.10.2015 - in http://www.gazzettaufficiale.it/eli/id/2015/10/ 30/15G00189/sg\%20).

49 Legge 11 dicembre 2015, n. 198: Conversione in legge, con modificazioni, del decretolegge 30 ottobre 2015, n. 174, recante proroga delle missioni internazionali delle Forze armate e di polizia, iniziative di cooperazione allo sviluppo e sostegno ai processi di ricostruzione e partecipazione alle iniziative delle organizzazioni internazionali per il consolidamento dei processi di pace e di stabilizzazione (15G00212) ("Gazzetta Ufficiale", Serie Generale n. 292 del 16.12.2015 - in http://www.gazzettaufficiale.it/eli/id/2015/12/16/ $15 \mathrm{G} 00212 / \mathrm{sg}$ ).

50 Testo del decreto-legge 30 ottobre 2015, n. 174 (in: Gazzetta Ufficiale, Serie Generale, n. 253 del 30 ottobre 2015), coordinato con la legge di conversione 11 dicembre 2015, n. 198 (in questa stessa Gazzetta Ufficiale, alla pag. 24), recante: "Proroga delle missioni internazionali delle Forze armate e di polizia, iniziative di cooperazione allo sviluppo e sostegno ai processi di ricostruzione e partecipazione alle iniziative delle organizzazioni internazionali per il consolidamento dei processi di pace e di stabilizzazione" (15A09336) ("Gazzetta Ufficiale", Serie Generale n. 292 del 16.12.2015 - in http://www.gazzettaufficiale. it/eli/id/2015/12/16/15A09336/sg). 
2. Il Presidente del Consiglio dei ministri informa il Comitato parlamentare per la sicurezza della Repubblica, con le modalità indicate nell'articolo 33, comma 4, della legge 3 agosto 2007, n. 124, delle misure di intelligence di cui al comma 1 del presente articolo.

3. Al personale delle Forze armate impiegato nell'attuazione delle attività di cui al comma 1 del presente articolo si applicano le disposizioni dell'articolo 5 del decreto-legge 30 dicembre 2008, n. 209, convertito, con modificazioni, dalla legge 24 febbraio 2009, n. 12, e successive modificazioni, dell'articolo 4, commi 1-sexies e 1-septies, del decreto-legge 4 novembre 2009, n. 152, convertito, con modificazioni, dalla legge 29 dicembre 2009, n. 197, e, ove ne ricorrano i presupposti, dell'articolo 17, comma 7, della legge 3 agosto 2007, n. 124.

4. Il comma 3 del presente articolo non si applica in nessun caso ai crimini previsti dagli articoli 5 e seguenti dello statuto istitutivo della Corte penale internazionale, adottato a Roma il 17 luglio 1998, ratificato ai sensi della legge 12 luglio 1999, n. 232.

5. Il Comitato interministeriale per la sicurezza della Repubblica di cui all'articolo 5 della legge 3 agosto 2007, n. 124, e successive modificazioni, può essere convocato dal Presidente del Consiglio dei ministri, con funzioni di consulenza, proposta e deliberazione, in caso di situazioni di crisi che coinvolgano aspetti di sicurezza nazionale, secondo modalità stabilite con apposito regolamento ai sensi dell'articolo 43 della legge 3 agosto 2007, n. 124.

6. Il Comitato parlamentare per la sicurezza della Repubblica, trascorsi ventiquattro mesi dalla data di entrata in vigore della legge di conversione del presente decreto, trasmette alle Camere una relazione sull'efficacia delle norme contenute nel presente articolo.

In sintesi, gli interventi "in situazioni di crisi o di emergenza all'estero" divengono "misure di intelligence" "con la cooperazione di forze speciali della Difesa” (comma 1), forze alle quali vengono applicate le regole vigenti per gli interventi dei servizi segreti (è questa la sostanza dell'illeggibile comma 3), cioè l'impunibilità, salvo i gravissimi crimini di competenza della Corte penale internazionale. ${ }^{51}$ Il "Comitato parlamentare per la sicurezza della Repubblica” (Copasir) viene informato delle decisioni governative e, a sua volta, informa il Parlamento: quindi il Parlamento è escluso dal processo decisionale e, al massimo, riceve "una relazione sull'efficacia delle norme contenute nel presente articolo" (comma 6).

51 Il testo dell'articolo 5 dello statuto istitutivo della Corte penale internazionale è il seguente: "Art. 5. Crimini di competenza della Corte. 1. La competenza della Corte è limitata ai crimini più gravi, motivo di allarme per l'intera comunità internazionale. La Corte ha competenza, in forza del presente Statuto, per i crimini seguenti: a) crimine di genocidio; b) crimini contro l'umanità; c) crimini di guerra; d) crimine di aggressione". 
Nel luglio del 2016 i mezzi di comunicazione italiani hanno cominciato a dare notizie di interventi di truppe italiane in Iraq e in Libia, dapprima smentite dal Governo, poi confermate dall'organo parlamentare di controllo sui servizi segreti, il Comitato Parlamentare per la Sicurezza della Repubblica (Copasir). Per le truppe italiane in Iraq vengono specificate anche le unità in azione. Per la Libia, 'l' 'Huffington Post' specifica che si tratta di operazioni effettuate in applicazione della normativa approvata lo scorso novembre dal Parlamento, che consente al Presidente del Consiglio di autorizzare missioni all'estero di militari dei nostri corpi d'élite ponendoli sotto la catena di comando dei servizi segreti con tutte le garanzie connesse. Immunità compresa". 52 Queste notizie vennero riprese dal "Corriere della Sera" ("Per la prima volta da quando è autorizzata a farlo, ovvero dal febbraio scorso, la Presidenza del consiglio ha messo nero su bianco la presenza, più volte ufficiosamente trapelata negli ultimi mesi, di piccoli nuclei di reparti militari speciali sul territorio libico" ${ }^{53}$ ), che le pubblica accanto a un'intervista del Ministro degli esteri italiano, il quale le smentisce: "Adesso militari in Libia ci sono? Non abbiamo missioni militari in Libia. Se le avremo saranno autorizzate dal parlamento. Per le operazioni a copertura dei servizi segreti non vi basterebbe informare il Comitato per la Sicurezza della Repubblica? Non commento per definizione operazioni di natura riservata." ${ }^{54}$ L'imbarazzo del governo è risultato chiaro anche pochi giorni dopo, quando i due ministri della Difesa e degli Esteri hanno preferito farsi rappresentare dai sottosegretari alla riunione delle corrispondenti due commissioni parlamentari, provocando le critiche non solo delle opposizioni e l'abbandono della seduta da parte di vari deputati.

In presenza di questa situazione normativa si può quindi concludere: "Che l'Italia sia o meno già in guerra è una questione di sottigliezze terminologiche, adesso che anche gli ultimi dubbi sono stati dissipati sul fatto che

52 Reparti italiani nella guerra all'Isis. Ma il parlamento non ne sa nulla (2016, 30 luglio), in prima pagina; ripreso in: Libia e Iraq, forze speciali italiane sul terreno: lo scoop del Fatto confermato dal documento trasmesso al Copasir (2016, 10 agosto).

53 Marco Galluzzo (2016, 11 agosto), Reparti speciali a difesa degli 007 già sul terreno.

54 Intervista al Ministro degli Esteri, Paolo Gentiloni: Maurizio Caprara (2016, 11 agosto), Presto riapriremo l'ambasciata a Tripoli. 
le nostre forze speciali - oltre a quelle statunitensi, britanniche e francesi siano in Libia al fianco delle forze fedeli al governo Al Sarraj”. 55

Quanto detto finora riguardava la legislazione vigente nel 2016; tuttavia in Italia nello stesso anno era in corso una discussa riforma costituzionale che, abbinata a una nuova legge elettorale, rischiava di sconvolgere varie parti della costituzione del 1948, di cui modificava 47 articoli su 139. In particolare, nel sistema bicamerale venivano radicalmente modificate le competenze del Senato e, di conseguenza, l'attuale art. 78 ("Le Camere deliberano lo stato di guerra e conferiscono al Governo i poteri necessari”) veniva così trasformato: "La Camera dei deputati delibera a maggioranza assoluta lo stato di guerra e conferisce al Governo i poteri necessari." Se il referendum costituzionale del 4 dicembre 2016 avesse approvato la riforma in discussione, si sarebbe verificata la grave situazione messa in luce da un sito pacifista:

La modifica [costituzionale] in discussione attualmente prevede invece che tale dichiarazione di guerra sia in capo a un solo ramo del Parlamento: una situazione che, combinata con la nuova legge elettorale che prevede un alto premio di maggioranza, configura la possibilità che un singolo partito - in minoranza nel Paese e nell'elettorato ma avente la maggioranza in Parlamento grazie alla legge elettorale possa prendere tale decisione. Le associazioni delle nostre Reti per la pace e il disarmo esprimono perciò una chiara preoccupazione per questa possibilità e, soprattutto, per la leggerezza con cui si sta intervenendo su un tema alquanto delicato. Riteniamo pericoloso e poco responsabile che si intervenga sull'articolo 78 della Costituzione, che rappresenta come detto una deroga eccezionale a princìi ben più fondanti delle nostre istituzioni repubblicane, solo come conseguenza automatica e quasi "tecnica" di una decisione sull'assetto parlamentare. ${ }^{56}$

La riforma costituzionale proposta dal referendum venne però respinta da quasi il 60\% dei votanti e quindi la costituzione non ha subìto modifiche. Il dibattito sull'intervento dei militari italiani all'estero continua quindi con gli stessi riferimenti legislativi secondo cui si è svolto finora.

55 Libia e Iraq, forze speciali italiane sul terreno: lo scoop del Fatto confermato dal documento trasmesso al Copasir (2016, 10 agosto).

56 L'Italia ripudia la guerra: non rendiamo più facile dichiararla $(2015,27$ gennaio). 



\section{Capitolo IV \\ Il rifiuto della guerra nella Legge Fondamentale tedesca del 1949}

Costituzione tedesca (Grundgesetz) - Il Bund e i Länder - Articolo 26. (1) Le azioni che possono turbare la pacifica convivenza dei popoli e intraprese con tale intento, in particolare al fine di preparare una guerra offensiva, sono incostituzionali. Tali azioni devono essere perseguite penalmente.

(2) Le armi da guerra possono essere prodotte, trasportate e messe in commercio soltanto con l'autorizzazione del Governo federale. I particolari sono stabiliti da una legge federale.

Dopo la fine della Seconda guerra mondiale, dopo l'occupazione militare e dopo la divisione del territorio tedesco, nel 1949 era entrata in vigore la nuova costituzione della Repubblica Federale di Germania contenente un precetto pacifista: l'art. 26, oggetto della presente analisi.

La società tedesca del XIX e della prima metà del XX secolo era pervasa da un forte sentimento militarista e la diffusione del movimento pacifista aveva quindi incontrato molte difficoltà. Infatti la Germania è stata accusata di aver provocato entrambe le guerre mondiali e, al loro termine, la discussione sulla colpevolezza della Germania aveva diviso non solo la società tedesca, ma anche gli stessi pacifisti tedeschi. Nonostante queste difficoltà, il pacifismo germanico può vantare due Premi Nobel per la pace: entrambi però non tedeschi, ma austriaci. ${ }^{\mathbf{}}$

La Germania, sconfitta in entrambe le guerre mondiali, ha dovuto affrontare due volte il dibattito sul proprio riarmo: esigenza fondamentale per uno Stato sovrano, da un lato, e fonte di timori non infondati per le nazioni vicine, dall'altro. ${ }^{2}$ Tanto in Giappone e in Italia quanto in Germania, inoltre,

1 Sono gli austro-ungarici Bertha von Suttner (1843-1914) e Alfred Hermann Fried (1864-1921). In generale: ROger CHICKerIng (1975), Imperial Germany and a World without War.

2 Sul dibattito dopo la Prima guerra mondiale: Wolfder (1934), Il riarmo della Germania e le moderne forze militari; Celestino Coppellotti (1936), Evoluzione della Germania hitle- 
il dibattito sul riarmo, sul disarmo e sulla pace assumeva una particolare polarizzazione a causa della Guerra fredda. I due blocchi si erano organizzati in patti militari contrapposti: la Nato, fondata nel 1949, e il Patto di Varsavia, fondato nel 1955. Le due alleanze militari venivano così a fronteggiarsi direttamente sul territorio della Germania divisa. Questa stessa tensione, benché in misura minore, si verificava al confine nord-orientale dell'Italia: in quest'ultima era però attivo il più forte partito comunista dell'Occidente, che faceva della pace, cioè del disarmo e della non-aggressione agli Stati comunisti, il tema di un'intensa campagna politica. ${ }^{3}$ Questo movimento pacifista politicamente orientato - noto col nome di "Movimento dei Partigiani della Pace” - aveva una diffusione mondiale (cfr. p. 201 con nota 17; e Appendice II,2).

Il dibattito sul riarmo risultava particolarmente aspro in entrambi gli Stati tedeschi. ${ }^{4}$ Le vicende dei partiti comunisti delle due Germanie riflettono in modo estremo questa contrapposizione. Nella DDR il partito comunista $\left(\mathrm{SED}^{5}\right.$ ) era il partito-guida (anche se esistevano altri partiti, comunque da esso egemonizzati). Nella Germania federale, invece, il partito comunista (KPD, proibito al momento della presa di potere hitleriana) era stato approvato dagli Alleati e nel 1949 era entrato nel parlamento tedesco con circa il $5 \%$ dei voti, però nel 1951 era stato dichiarato illegale. ${ }^{6}$ Il dibattitto sul rifiuto della guerra previsto dall'articolo 26 si inseriva dunque in queste complesse tensioni interne alla Germania federale.

riana. Sul dibattito dopo la Seconda guerra mondiale: Mario SiLvestri (2002), Dal riarmo della Germania alla catastrofe; Maria Venturini (1953), Per non tornare indietro.

3 Con riferimento alla Germania: I lavoratori tedeschi contro il riarmo della Germania (1951); Gisella Floreanini (1955), Si impedisca il riarmo della Germania di Bonn!

4 Angelika Klein (ed.) (1987), Der Friedensgedanke.

5 La "Sozialistische Einheitspartei Deutschlands" (SED) nacque nella DDR e a Berlino Est dalla fusione dei tradizionali partiti socialdemocratico (SPD, Sozialdemokratische Partei) e comunista (KPD, Kommunistische Partei Deutschlands). Dopo l'unificazione della Germania la SED passò a chiamarsi PDS (Partei des Demokratischen Sozialismus), che esistette fino al 2007.

6 Patrick Major (1998), The Death of the KPD; Angelika Lehndorff-Felsko (1981), Der KPD-Verbotsprozeß 1954 bis 1956. Il divieto del KPD era stato preceduto nel 1952 dal divieto di un partito neonazista. Dal 1972 l'appartenenza a un partito non democratico (come il KPD) comportava il divieto di essere funzionario dell'amministrazione pubblica (Berufsverbot, come conseguenza del Radikalenerlaß del 1972, che era stato preceduto dall'Adenauererlaß del 1950). 
Subito dopo l'occupazione alleata del territorio tedesco, i governatori militari delle tre zone di occupazione occidentali invitarono i rappresentanti locali a progettare una futura costituzione. Ne nacquero una proposta federalista, ispirata in particolare dalla Baviera e dai partiti cristiano-sociali, e una proposta unitaria, avanzata da altri Länder e dal partito socialdemocratico. Va sottolineato quindi che, nella Germania occidentale, le costituzioni dei Länder hanno preceduto la "Legge Fondamentale" della Federazione (Bund): per questo nel dibattito costituente federale sono frequenti i richiami alle preesistenti costituzioni dei Länder.

Il tema della costituzione tedesca venne ripreso ufficialmente nell'aprilegiugno 1948 nella Conferenza delle Sei Potenze, a Londra. Oltre a Stati Uniti, Gran Bretagna e Francia (ostile al risorgere di uno Stato tedesco) vi parteciparono Belgio, Olanda e Lussemburgo, ma non l'Unione Sovietica, benché potenza vincitrice: la Guerra fredda era già iniziata. Il documento finale - le decisioni londinesi, i "Londoner Beschlüße" - optava per una struttura federale del futuro Stato tedesco e venne approvato senza entusiasmo anche dall'Assemblée Nationale francese. Ma l'atteggiamento francese era ben sintetizzato dal bon mot secondo cui i francesi amavano tanto la Germania da volerne addirittura due.

Poiché i tedeschi accolsero con freddezza questi accordi di Londra, gli Alleati cercarono di migliorare il clima con i tre successivi "Documenti di Francoforte" ("Frankfurter Dokumente") del luglio 1948. Il più importante di essi, il primo, conteneva gli statuti che regolavano il regime di occupazione e, al tempo stesso, invitavano i tedeschi a eleggere un'Assemblea costituente che predisponesse una costituzione caratterizzata dal federalismo e dalla garanzia dei diritti fondamentali. Anche in Germania, come in Giappone, gli Alleati volevano evitare che la costituzione fosse sentita come un documento imposto dalle potenze occupanti.

Il contrasto fra gli organi tedeschi e quelli alleati venne alla luce quando, nel luglio 1948, i rappresentanti dei governi e dei parlamenti dei soli Länder occidentali si riunirono a Coblenza e accettarono il contenuto dei "Frankfurter Dokumente", respingendo però gli statuti sul regime di occupazione e, in particolare, rifiutando la creazione di uno Stato tedesco-occidentale, che avrebbe ratificato da divisione definitiva della Germania. Le tensioni con i governatori militari durarono per tutto il 1948 e si conclusero con la decisione che la costituzione sarebbe stata ratificata dai parlamenti dei Länder, e non da un referendum popolare, come avrebbero invece voluto i governatori 
militari. A partire da questo momento, in ogni documento o attività i tedeschi e i loro alleati - tanto a ovest quanto a est - ricorrevano a termini o a circonlocuzioni che evitassero di avallare la divisione della Germania postbellica, al fine di non compromettere in alcun modo la possibile riunificazione futura delle terre tedesche.

Le discrepanze tra le autorità alleate e quelle tedesco-occidentali accompagnarono anche l'attività dell'assemblea costituente (chiamata "convenzione", "Verfassungskonvent", proprio per non evocare la divisione della Germania), che si riunì dal 10 al 23 agosto 1948 nel castello di Herrenchiemsee, su un'isola dell'omonimo lago della Baviera. Il testo che ne risultò venne da alcuni criticato perché redatto da "privati" (anche se questi "privati" erano i premier dei Länder occidentali), ma i suoi lavori preparatori influirono non poco sulla redazione della futura costituzione federale.

L'attività costituente venne affidata a 95 rappresentanti dei Länder occidentali (riuniti in un "Consiglio Parlamentare", Parlamentarischer Rat, non dunque "Assemblea Costituente"), che avrebbero approvato per la parte occidentale della Germania una "Legge Fondamentale" ("Grundgesetz", non dunque una "Costituzione": altro escamotage linguistico per non compromettere una futura unità tedesca). Infatti il Preambolo della "Legge Fondamentale" afferma: "Nell'intento di dare alla vita statuale un nuovo ordinamento, per un periodo transitorio, in virtù del suo potere costituente, [il Consiglio Parlamentare] ha deliberato la presente Legge fondamentale della Repubblica Federale Germanica". L'articolo 146 è ancora più esplicito: "La presente Legge fondamentale cesserà di aver vigore il giorno in cui subentrerà una Costituzione approvata con libera deliberazione dal popolo tedesco." ${ }^{7}$ In attesa di questa lontana unificazione, la nuova Germania occidentale nasceva all'insegna della provvisorietà: "Ist die Bundesrepublik ein Provisorium?” è stata la domanda che per decenni circolò nella pubblicistica tedesca. Tuttavia questa provvisorietà serviva a mantenere viva la speranza di un'unificazione futura, come è ribadito nel Preambolo e nell'articolo 23 (nel testo originale) della Grundgesetz.

Nel 1949 la Grundgesetz venne approvata anzitutto dal Parlamentarischer Rat (ma non all'unanimità); poi dai governatori militari delle potenze alleate; infine dai parlamenti dei Länder: ma la Baviera dapprima la respinse

7 Corsivo mio (http://dircost.di.unito.it/cs/pdf/19490523_germaniaLeggeFondamentale_ita.pdf). 
e poi la approvò obtorto collo. La tormentata storia di questa non-costituzione si concludeva il 23 maggio 1949 con la sua entrata in vigore.

Come le costituzioni giapponese e italiana, anche la Legge Fondamentale tedesco-occidentale contiene un articolo pacifista, il 26. Esso è preceduto da due articoli sulle relazioni internazionali della Germania federale, concepiti in funzione del mantenimento della pace nel mondo. Ecco il loro testo integrale:

Art. 24 - 1) La Federazione può trasferire mediante una legge diritti di sovranità ad organizzazioni interstatali. 1a) ${ }^{[\mathbf{8}]}$ Nella misura in cui i Länder sono competenti per l'esercizio di funzioni statali e per il compimento di compiti statali, con l'approvazione del Governo federale possono trasferire diritti sovrani a istituzioni confinanti. 2) La Federazione può, per la tutela della pace, inserirsi in un sistema di reciproca sicurezza collettiva; essa pertanto consentirà a quelle limitazioni dei suoi diritti sovrani che realizzino ed assicurino un ordinamento pacifico e duraturo in Europa e tra i popoli del mondo. 3) Per la risoluzione delle controversie interstatali la Federazione aderirà a convenzioni relative ad una giurisdizione arbitrale internazionale, generale, ampia ed obbligatoria.

Art. 25 - Le regole generali del diritto internazionale costituiscono parte integrante del diritto federale. Esse sono anteposte alle leggi e creano immediatamente diritti e doveri per gli abitanti del territorio federale.

In questo contesto, l'articolo 26 regola il pacifismo e il riarmo all'interno della Germania federale, ma esprime il rifiuto della guerra con la circonlocuzione "turbare la pacifica convivenza dei popoli". Nel dibattito costituente e nei testi successivi, anche per esigenze di sinteticità, ricorre il termine tedesco "Kriegsächtung", più raro e più recente dell'italiano "ripudio" (cfr. supra, p. 204 e nota 24). Assente nei dizionari classici (come l'ottocentesca opera fondata dai Fratelli Grimm), il termine tedesco sembra risalire agli anni dopo la Prima guerra mondiale, con particolare riferimento al Patto Briand-Kellogg. ${ }^{9}$ A partire da quelle fonti il termine è poi stato recepito nelle successive pubblicazioni sulla pace e sul diritto internazionale e costituzionale, fino alla sua inclusione nell'art. 26 della Grundgesetz. ${ }^{\mathbf{1 0}}$

8 Il capoverso 1a venne introdotto nella Legge Fondamentale con la legge del 21 dicembre 1992 ed entrò in vigore il 25 dicembre dello stesso anno.

9 Die Idee der Kriegsächtung in Amerika (1925), traduzione dall'inglese di un articolo su "Unity"; Helene Stöcker (1926), Das Problem der Kriegsächtung; Auswärtiges Аmt (ed.) (1928), Materialien zum Kriegsächtungspakt.

10 Per esempio il termine è stato ripreso nell'ottantesimo anniversario di quel patto: ERHARD Своме (2008, agosto), Kriegsächtung - aktuell und drängend. 
L'articolo 26 si compone di due commi che è opportuno esaminare separatamente. Il primo comma è esplicitamente pacifista: "1) Atti che siano idonei e posti in essere con l'intento di turbare la pacifica convivenza dei popoli, e specificamente di preparare una guerra d'aggressione, sono anticostituzionali. Essi devono essere colpiti da pena". ${ }^{11}$

La pena prescritta dalla costituzione è precisata nell'art. 80 del codice penale tedesco con un esplicito richiamo alla norma costituzionale: "Preparazione d'una guerra d'aggressione. Chi prepara una guerra d'aggressione (art. 26, c. 1 della Legge Fondamentale), esponendo così la Repubblica Federale Tedesca al pericolo di una guerra, viene punito con la reclusione a vita $o$ con una pena detentiva non inferiore ai dieci anni”.

Attualmente è aperta la discussione sui rapporti tra l'art. 26 GG e il nuovo $\$ 80$ a del codice penale tedesco ("Incitamento al reato di aggressione"), che si richiama al $₫ 13$ della legge penale tedesca del 30 giugno 2002 sul perseguimento dei reati internazionali ("Völkerstrafgesetzbuch", codice penale per i crimini conto la legge internazionale). I pacifisti sostengono che così è stato svuotato l'articolo della costituzione, mentre i fautori della modifica sostengono che ciò non è avvenuto. In questa sede conviene esaminare anzitutto il contenuto del nuovo $\mathbb{8} 80$ a del codice penale tedesco: "Chi, nell'ambito di validità di questa legge, incita pubblicamente in una riunione o con la diffusione di scritti (art. 11, c. 3) al reato di aggressione (art. 13 del "Völkerstrafgesetzbuch") è punito con una pena detentiva da tre mesi a cinque anni”. Il $\$ 13$ del "Völkerstrafgesetzbuch" si richiama a sua volta alla Carta delle Nazioni Unite:

1. Chi conduce una guerra d'aggressione o compie qualsiasi altra azione aggressiva che per le sue caratteristiche, la sua gravità e la sua ampiezza rappresenta una chiara violazione della Carta delle Nazioni Unite, viene punito con l'ergastolo.

2. Chi pianifica, prepara o intraprende una guerra d'aggressione oppure qualsiasi altra azione aggressiva nel senso del comma 1, è punito con l'ergastolo o con la detenzione non inferiore a dieci anni. L'azione prevista dal comma 1 è punibile soltanto se (I) la guerra d'aggressione è avvenuta ovvero se un'altra azione aggressiva è stata compiuta; (II) se per mezzo loro si fa sorgere il pericolo di una guerra d'aggressione o di un'altra azione aggressiva contro la Repubblica Federale Tedesca.

11 La traduzione italiana dell'intera costituzione tedesca del 1949 si trova in: http://www.dircost.unito.it/cs/pdf/19490523_germaniaLeggeFondamentale_ita.pdf; il testo tedesco con a fronte la traduzione italiana è in: http://www.consiglioveneto.it/crvportal/BancheDati/ costituzioni/de/zGermania_sin.pdf. 
3. È aggressiva l'azione rivolta contro la sovranità, l'integrità territoriale o l'indipendenza politica di uno Stato, ovvero l'uso da parte di uno Stato della forza armata in modo inconciliabile con la Carta delle Nazioni Unite. ${ }^{\mathbf{1 2}}$

Si sostiene che il nuovo art. 80a sia in contrasto con l'art. 26, c. 1, GG e con l'abrogato $₫ 80$ StGB perché consente un'interpretazione estensiva della nozione di intervento armato (azione aggressiva, "Angriffshandlung”), dal momento che la Carta delle Nazioni Unite non ha impedito "interventi umanitari" o "autodifese preventive", ad esempio nel caso del Kosovo. Questa modifica legislativa si risolverebbe quindi in una legittimazione degli interventi "fuori area" nonostante l'art. 26 GG. A riprova di questo indirizzo viene citato il discorso del Presidente federale Frank-Walter Steinmeier, che ha affermato: "Proprio perché siamo tra i pochi Stati al mondo che sono politicamente e democraticamente stabili, si attende da noi una partecipazione più forte che dieci o vent'anni fa nell'eliminazione dei conflitti", in particolare "da quando il problema della pace e della guerra (che noi ritenevamo risolto sul suolo europeo) si è ripresentato con l'annessione della Crimea da parte della Russia in violazione del diritto internazionale". ${ }^{\mathbf{1 3}}$

Altri interpretano invece questa nuova normativa come una precisazione, e non come una sostituzione o abrogazione, del precedente $\mathbb{} 80$ StGB.

L'ambito di applicazione delle due versioni del $₫ 80$ è limitato dal fatto che questo delitto può essere commesso soltanto da chi occupa una posizione di vertice nella struttura statale. Un'ulteriore difficoltà deriva dalla mancanza di una definizione costituzionale del concetto centrale di "guerra d'aggressione": definizione che va dunque ricercata nel diritto internazionale. Poiché anche il diritto internazionale non ne fornisce una nozione univoca, è stato sostenuto che questa norma penale sarebbe inapplicabile a causa dell'indeterminatezza del contenuto.

12 Tradotto dal testo ufficiale tedesco (http://www.gesetze-im-internet.de/vstgb/index.html), di cui esiste una traduzione non ufficiale in inglese (http:/www.iuscomp.org/gla/statutes/ VoeStGB.pdf).

13 Steinmeier fordert stärkeres Engagement der Bundeswehr (2017, 16 giugno). La critica al nuovo $\mathbb{8} 8$ a è contenuta in Wolfgang Bittner (2017, 7 aprile), Verbot der Vorbereitung eines Angriffskrieges. Una serie di precisazioni sulla responsabilità dei vertici politici e sull'applicazione all'estero di queste norme è contenuta in una decisione della Procura generale federale sull'intervento della Bundeswehr in Siria; cf. Florian Rötzer (2017, 7 gennaio), $\$ 80$ StGB "Vorbereitung eines Angriffskriegs". 
Anche il documento che nel 1990 preparò l'unificazione delle due Germanie contiene un esplicito riferimento all'art. 26 della Grundgesetz: "The Governments of the Federal Republic of Germany and the German Democratic Republic reaffirm their declarations that only peace will emanate from German soil. According to the constitution of the united Germany, acts tending to and undertaken with the intent to disturb the peaceful relations between nations, especially to prepare for aggressive war, are unconstitutional and a punishable offence. The Governments of the Federal Republic of Germany and the German Democratic Republic declare that the united Germany will never employ any of its weapons except in accordance with its constitution and the Charter of the United Nations." ${ }^{14}$

Si noti che, in questo documento, il richiamo al valore pacifista della costituzione è integrato con il richiamo al diritto internazionale, cioè agli art. 42 e 53 della Carta delle Nazioni Unite: richiamo che nel 1990 era indispensabile per giustificare gli interventi militari all'estero sotto l'egida della Nazioni Unite. Intanto, con l'unificazione della Germania, la Grundgesetz si è estesa anche all'ex DDR e quindi l'art. 26 si applica all'intero Stato tedesco attuale, mentre il richiamo agli articoli della Carta delle Nazioni Unite aiuta a precisare il significato della vaga formulazione sull'uso delle forze armate.

Il secondo comma dell'articolo 26 si riferisce alla temuta industria bellica tedesca, sulla quale la Francia - durante il dibattito pre-costituzionale avrebbe voluto conservare il controllo anche dopo la formazione dell'avversato Stato tedesco. Come in Giappone e in Italia, anche in Germania gli Alleati avevano disciolto i cartelli (Konzerne). Il secondo comma dell'art. 26 non impone alcun divieto, cioè non proibisce tout court la produzione bellica, ma obbliga il parlamento a regolare la produzione militare con una legge ordinaria: "Armi destinate alla condotta di una guerra possono essere fabbricate, trasportate e messe in circolazione solo con l'autorizzazione del governo federale. Una legge federale regola i particolari”.

14 Art. 2 (corsivo mio) del cd. “Zwei-plus-Vier-Vertrag” (“Two Plus Four Agreement”, ma ufficialmente "Treaty on the Final Settlement with Respect to Germany") del 12 settembre 1990: è l'accordo intercorso tra i due Stati tedeschi e le quattro potenze vincitrici che preparò la riunificazione tedesca del 3 ottobre 1990. La traduzione in inglese di questo trattato è in http://usa.usembassy.de/etexts/2plusfour8994e.htm. 
Questo secondo comma dell'art. 26 nacque per dare concretezza al pacifismo espresso nel primo comma, ma nella sua formulazione i costituenti dovettero risolvere numerosi problemi definitori, che risultano con chiarezza dai verbali delle sedute tradotti nell'Appendice III.1. Il divieto di ogni attività che metta in pericolo la pace viene rafforzato dal divieto di produrre e diffondere "armi destinate ad attività bellica" ("Zur Kriegsführung bestimmte Waffen"). La formulazione iniziale parlava di "armi e munizioni" ("Waffen und Munition"), ma venne respinta perché troppo estesa, in quanto poteva includere anche armi e munizioni per la caccia o per lo sport. Vennero respinte tanto la formulazione "attrezzature belliche d'ogni tipo" ("Kriegsgerät jeder Art”, perché avrebbe potuto comprendere anche le materie prime per produrre armi), quanto "armi utilizzabili in guerra" ("Im Kriege verwendbare Waffen”), perché avrebbe potuto comprendere anche le dotazioni destinate alle polizie. Si giunse così alla formulazione ancora oggi vigente "armi destinate ad attività bellica" ("Zur Kriegsführung bestimmte Waffen”).

In essa ritorna però il problema di definire che cosa si intende per guerra. ${ }^{15}$ Infatti nel 1948 non si pensava ancora ai prodotti "dual use", cioè ai beni civili utilizzabili anche a fini bellici, e le "hybrid wars" erano ancora lontane. ${ }^{16}$ L'Appendice III. 1 riporta ampi brani del dibattito costituente in cui si affina la terminologia da adottare in questi articoli.

Si discusse anche se la norma doveva contenere un divieto generale di produrre "armi utilizzabili in guerra", oppure una riserva di legge, e infine si optò per questa seconda soluzione: si intendeva così salvaguardare la libertà di impresa. Vennero perciò emanate due leggi ordinarie che riguardano la produzione bellica.

15 Sul dibattito da cui nacque il secondo comma dell'art. 26: Zum Hintergrund von Art. 26 Abs. 2 GG und der Entstehung des Kriegswaffenkontrollgesetzes bzw. des Außenwirtschaftsgesetzes, Ohne Rüstung Leben, Oktober 2011, 11 pp.: http://www.aufschrei-waffenhandel.de/ fileadmin/dokumente/dateien-or/pdf-dokumente/Brosch\%C3\%BCre_Hintergrund_26_Abs _2_GG_Oktober_2011_.pdf. “Die Kampagne gegen Rüstungsexport bei 'Ohne Rüstung Leben' dokumentiert in dieser Broschüre eine Expertise der Tübinger Juristin Nehle Betz zur Entstehung des Art. 26 Abs. 2 GG”; “Die Kampagne gegen Rüstungsexport bei 'Ohne Rüstung Leben' wird vom Evangelischen Entwicklungsdienst (EED) finanziell unterstützt”, 2.

16 Sui riflessi giuridici dei nuovi tipi di conflitto: Mario G. Losano (2017b), Guerre ibride, omicidi mirati, droni. 
La prima è la "Legge sul controllo delle armi da guerra", entrata in vigore il $1^{\circ}$ giugno 1961 e più volte aggiornata (Kriegswaffenkontrollgesetz, KrWaffKontrG). La sua connessione con l'articolo pacifista della Grundgesetz è esplicito sin dal titolo: "Legge applicativa dell'art. 26, c. 2 della Grundgesetz”. Questa legge si concentra sulle procedure per autorizzare la produzione e l'esportazione di armi da guerra. ${ }^{17}$

La seconda è la più vasta "Legge sul commercio estero", che non si riferisce soltanto al materiale bellico, ma regola in generale l'importazione e l'esportazione di qualsiasi bene e servizio, e quindi riguarda indirettamente anche $\mathrm{i}$ beni di natura bellica. ${ }^{18} \mathrm{Al}$ momento della preparazione della costituzione non pochi costituenti dubitavano che la Germania sarebbe tornata a produrre materiale bellico, dato l'atteggiamento negativo degli Alleati, ma infine si abbandonò l'idea di vietare il commercio di materiale bellico e si optò per un controllo su questo commercio, perché in questo modo si sarebbe evitato, da un lato, che la pace venisse messa in pericolo anche fuori dalla Germania, senza d'altro lato limitare la libertà d'impresa all'interno della Germania.

Mentre la prima legge costituisce l'adempimento del precetto costituzionale di tutelare la pace, la seconda ha il più vasto obiettivo di garantire la libera attività economica dei singoli, senza che questo urti contro gli interessi dello Stato, soprattutto nell'ambito delle relazioni internazionali. Con l'articolo 26 e con queste due leggi di attuazione lo Stato tedesco-federale aveva ottemperato alle indicazioni delle potenze alleate.

Nel 1968 sono stati introdotti due nuovi articoli nella Legge Fondamentale per precisare come possono essere impiegate le forze armate tedesche: il lungo art. 87a, Finalità delle forze armate, riportato qui di seguito, e l'art. 87b, Amministrazione federale della difesa, qui omesso perché riguarda l'amministrazione interna.

Art. 87a.

(1) La Federazione organizza forze armate per la difesa. I loro effettivi e i lineamenti fondamentali della loro organizzazione devono risultare nel bilancio di previsione.

17 JoAchim Steindorf (2007), Waffenrecht.

18 Außenwirtschaftsgesetz, AWG (https:/www.gesetze-im-internet.de/bundesrecht/awg_2013/AWG.pdf). 
(2) Al di fuori della difesa, le forze armate possono essere impegnate soltanto nella misura in cui la presente Legge Fondamentale lo ammette esplicitamente.

(3) Durante lo "stato di difesa" o "di tensione", le forze armate hanno la potestà di proteggere gli obiettivi civili e di assumersi la cura dei compiti inerenti alla regolazione del traffico, nella misura in cui ciò sia necessario per l'espletamento del loro compito difensivo. Oltre a ciò, nel caso di "stato di difesa" o "di tensione", può essere trasferita alle forze armate anche la protezione di obiettivi civili in appoggio ai provvedimenti della polizia; in tale caso le forze armate collaborano con le autorità competenti.

(4) Allo scopo di difendersi da un incombente pericolo per l'esistenza o per l'ordinamento costituzionale liberale e democratico della Federazione o di un Land il Governo federale può, se ricorrono i presupposti dell'articolo 91, secondo comma, e se le forze di polizia, così come la polizia confinaria federale non sono sufficienti, impiegare le forze armate in appoggio alla polizia e alla polizia confinaria federale per proteggere obiettivi civili e per combattere ribelli organizzati e armati militarmente. L'impiego delle forze armate dev'essere sospeso se lo richiedono il Bundestag o il Bundesrat. ${ }^{19}$

Accettato il principio che le forze armate tedesche potevano agire anche fuori dall'area della Nato ("out of area"), restava aperta la questione se questi interventi dovevano essere approvati dal parlamento. Le missioni in Bosnia e in Somalia offrirono ai socialdemocratici e ai liberali l'occasione per sollevare davanti al Tribunale Costituzionale un problema di conflitto di competenze riassumibile nel seguente quesito: il governo, decidendo da solo l'invio dei soldati, aveva leso le prerogative parlamentari sull'uso delle forze armate? Il Tribunale Costituzionale ritenne di no.

Dagli anni Ottanta la situazione internazionale è però cambiata e anche la Germania unificata, come partner di alleanze internazionali, ha dovuto conciliare gli interventi militari all'estero con la norma pacifista della propria costituzione. Si ripeteva così per la Germania la situazione che andavano vivendo anche il Giappone e l'Italia. Nel 1990 le forze armate tedesco-federali (che erano state ricostituite nel 1955, ma che fino ad allora erano state impiegate all'estero solo in missioni umanitarie) per la prima volta vennero dispiegate in armi fuori dall'area della Nato per lo sminamento nel Golfo Persico durante la seconda Guerra del Golfo, poi per la difesa antimissilistica in Turchia nell'operazione Desert Storm, poi per un ospedale da campo in Cambogia, poi ancora nell'Adriatico, nei Balcani, in Somalia, e così via.

19 L'art. 87a è stato introdotto con la legge del 24 giugno 1968 (testo italiano: http:// www.consiglioveneto.it/crvportal/BancheDati/costituzioni/de/zGermania_sin.pdf). 
Tuttavia in Somalia e in Cambogia la Bundeswehr agiva su mandato dell'ONU, ma senza una preventiva autorizzazione del parlamento. Su queste "operazioni di pace" il governo si divise, e soltanto nel 1992 il partito socialdemocratico cessò di opporvisi (anche se con ciò non cessarono le divergenze di opinioni) in un incontro svoltosi nei pressi di Bonn.

Nel 1992 il Partito Socialdemocratico con la "Svolta di Petersberg" ("Petersberger Wende") impresse un nuovo indirizzo programmatico alla politica estera e si dichiarò disponibile a dare il proprio assenso alle missioni della Bundeswehr sotto l'egida dell'ONU. Con questo non si era però conclusa la disputa sull'interpretazione della Grundgesetz. Mentre l'art. $87 \mathrm{a}^{[20]}$ afferma che la Federazione (Bund) appresta le forze armate per la difesa, l'art. 24, c. $2,{ }^{[\mathbf{2 1}]}$ ammette la possibilità che la Federazione faccia parte di un "sistema di reciproca sicurezza collettiva" per il "mantenimento della pace", accettando così "limitazioni alla propria sovranità". Non veniva però chiarito se erano ugualmente ammissibili interventi militari sotto l'egida della Nazioni Unite e della Nato, né se il Parlamento doveva decidere su tali interventi. $^{22}$

Accettato il principio che le forze armate tedesche potevano agire anche fuori dall'area della Nato ("out of area"), restava dunque aperta la questione se questi interventi dovevano essere approvati dal parlamento. Le missioni in Bosnia e in Somalia offrirono ai socialdemocratici e ai liberali l'occasione per sollevare davanti al Tribunale Costituzionale un problema di conflitto di competenze riassumibile nel seguente quesito: il governo, decidendo da solo l'invio dei soldati, aveva leso le prerogative parlamentari sull'uso delle forze armate?

Con la sentenza del 12 luglio 1994 la Corte Costituzionale ribadì la liceità costituzionale dell'invio di militari "out of area", però ritenne incostituzionale il comportamento del governo che non aveva ottenuto preliminarmente l'autorizzazione del parlamento. Nel caso che l'urgenza lo avesse richiesto, continuava la Corte, il governo poteva decidere da solo l'invio dei militari, chiedendo però successivamente la ratifica parlamentare della propria decisione.

Sulla base di questa sentenza la "riserva parlamentare" (Konstitutiver Parlamentsvorbehalt) nasceva però come prassi, cioè senza che una legge

20 Il testo dell'art. $87 \mathrm{a}$ è a p. $228 \mathrm{~s}$.

21 Il testo dell'art. 24, c. 2, è a p. 223.

22 Vor 25 Jahren: Bundesverfassungsgericht billigt Auslandseinsätze (2019, 8 luglio), il testo è desunto dal sito della Bundeszentrale für politische Bildung. 
ordinaria la regolasse. Il moltiplicarsi delle missioni all'estero portò all'emanazione di una legge sulla riserva parlamentare, che si apre con la dichiarazione: "Per l'invio di militari tedeschi armati fuori dall'ambito di validità della Grundgesetz occorre l'approvazione del parlamento".23

L'intervento dell'esercito federale tedesco in Kosovo venne approvato dal Parlamento il 16 ottobre 1998, ma ciononostante il Partito del Socialismo Democratico (PDS) ${ }^{24}$ presentò al Tribunale Costituzionale un ricorso contro il Governo e contro il Ministro Federale della Difesa, ritendo che la decisione di partecipare alle operazioni militari avesse "violato delle competenze costituzionali del Bundestag. Il Bundestag sarebbe stato leso nei suoi diritti e doveri dagli atti impugnati". Secondo i ricorrenti quella decisione implicava una revisione costituzionale dell'articolo "pacifista". Anche in questo caso, però, il Tribunale Costituzionale respinse il ricorso perché "la delibera [del Bundestag del 16 ottobre 1998] copre gli attuali attacchi aerei della Nato" e inoltre "le più recenti delibere del $14^{\circ}$ Bundestag non hanno rimosso né modificato la precedente delibera del 16 ottobre", e quindi "non sono violati i diritti del Bundestag." 25 Secondo un commentatore, "la decisione del Tribunale Costituzionale tedesco di non entrare nel merito è anche un segno di prudenza" perché "il dibattito tra giuristi rispecchia e tocca a questo proposito i dilemmi della costituzione europea in fieri" ${ }^{26}$

L'aver regolato formalmente le missioni all'estero non ha però eliminato le riserve di fondo dell'opposizione e il dibattitto si rinnova ogni volta che il governo chiede l'autorizzazione parlamentare a una di queste mis-

23 "Der Einsatz bewaffneter deutscher Streitkräfte außerhalb des Geltungsbereichs des Grundgesetzes bedarf der Zustimmung des Bundestages" (Parlamentsbeteiligungsgesetz, $\mathbb{S} 1$, c. 2; il titolo ufficiale è Gesetz über die parlamentarische Beteiligung bei der Entscheidung über den Einsatz bewaffneter Streitkräfte im Ausland; in vigore dal 24 marzo 2005). Cfr. Philipp Scherrer (2010), Das Parlament und sein Heer; Florian Schröder (2005), Das parlamentarische Zustimmungsverfahren.

24 La "Partei des Demokratischen Sozialismus" (PDS) fu il partito in certa misura erede della SED (il partito comunista della DDR) e dal 1989 al 2007 agì su posizioni di sinistra soprattutto nei Länder dell'Est ricostituiti dopo l'unificazione.

25 I passi citati sono nella traduzione della sentenza - 15. Bundesverfassungsgericht (Tribunale Costituzionale Federale), Karlsruhe, 2a sezione, ordinanza del 25 marzo 1999; 2 BVE 5/99 - in Mario Dogliani, Stefano Sicardi (eds.) (1999), Diritti umani e uso della forza, 285-287; sul commento di Jörg Luther, cfr. nota seguente.

26 Jörg Luther (1999), Il giudice costituzionale può fermare la guerra?, 89. 
sioni, per esempio ad ogni rinnovo della missione tedesca in Afghanistan. Anche nella società civile continua l'opposizione agli interventi militari presentati come operazioni di pace. ${ }^{27}$

La polemica continuò sino alla sentenza del 1990 del Tribunale Costituzionale tedesco: "L'autorizzazione dell'art. 24, c. 2 GG, permette al Bund non soltanto di entrare a far parte di un sistema di sicurezza collettiva reciproca e di accettare le limitazioni di sovranità a ciò connesse. Essa offre anche il fondamento costituzionale per l'assunzione dei compiti tipicamente connessi con un tale sistema e, quindi, anche per l'uso della Bundeswehr in missioni realizzate nell'ambito e secondo le regole di questo stesso sistema., ${ }^{28}$

Mentre nel parlamento e nella società civile continuano, sia pure in misura minore, le critiche a questi interventi "pacificatori", le forze armate tedesche sono state finora impiegate in circa 130 missioni all'estero, mentre alcune decine sono tuttora in corso. ${ }^{29}$

Giunge così al termine questo rapido esame degli articoli "pacifisti" contenuti nelle costituzioni che reggono i tre Stati dell'ex Patto Tripartito dopo la sconfitta nella Seconda guerra mondiale. Tutti e tre gli articoli sono stati

27 Il sito della "Arbeitsstelle für Frieden und Abrüstung” pubblica le massime della citata sentenza del Tribunale Costituzionale sotto il titolo "Bundesverfassungsgericht ebnet den Weg für weltweite Militäreinsätze der Bundeswehr”, e commenta: "Am 12. Juli 1994 hat das Bundesverfassungsgericht den Weg für weltweite Einsätze der Bundeswehr freigemacht. Diese 'out of area'-Entscheidung markiert das Ende einer jahrelangen Diskussion über die verfassungsrechtlichen Grundlagen für Auslandseinsätze. Es hat juristisch die von der CDU/CSU/FDP-Bundesregierung längst geschaffenen Fakten nachträglich sanktioniert” (http://www.asfrab.de/urteil-bverfg-1271994-2-bve-392.html).

28 Testo della prima massima della sentenza: "Die Ermächtigung des Art. 24 Abs. 2 GG berechtigt den Bund nicht nur zum Eintritt in ein System gegenseitiger kollektiver Sicherheit und zur Einwilligung in damit verbundene Beschränkungen seiner Hoheitsrechte. Sie bietet vielmehr auch die verfassungsrechtliche Grundlage für die Übernahme der mit der Zugehörigkeit zu einem solchen System typischerweise verbundenen Aufgaben und damit auch für eine Verwendung der Bundeswehr zu Einsätzen, die im Rahmen und nach den Regeln dieses Systems stattfinden", sentenza 286 del 1990 (http://www.servat.unibe.ch/ dfr/bv090286.html, https:/www.bundesverfassungsgericht.de/SharedDocs/Entscheidungen/DE/ 2008/05/es20080507_2bve000103.html).

29 Ulteriori dati nel sito: http://www.einsatz.bundeswehr.de/. Sulle missioni di pace all'estero delle forze armate tedesche, cfr. per esempio: Сhristoph Schwegmann (ed.) (2011), Bewährungsproben einer Nation; Bernhard ChIARI (ed.) (2010), Auslandseinsätze der Bundeswehr; Jürgen Schwarz, Armin A. Steinkamm (eds.) (1993), Rechtliche und politische Probleme. Una voce critica: Julian Reichelt (2010), Rubet in Frieden, Soldaten! 
oggetto di controversie, sia al momento della loro approvazione, perché erano sentiti come una limitazione della sovranità nazionale, sia negli anni tra i due millenni, in cui tutti e tre gli Stati presero parte ad attività belliche che, per rispettare la costituzione, non vengono definite 'guerre', anche se ci assomigliano molto. Le crisi internazionali e il terrorismo internazionale hanno rafforzato le critiche sull'inattualità di questi articoli "pacifisti", che nei fatti le decisioni politiche svuotano sempre di più.

Prima o poi si giungerà a modificarli, e il più prossimo a questo destino sembra essere l'articolo 9 della costituzione giapponese, come attestano le posizioni del partito di maggioranza documentate nell'Appendice I,5. In Italia, le polemiche sulla conciliabilità fra l'articolo 11 e gli interventi militari all'estero - dapprima sopite perché il dibattito politico si concentrava sull'ingovernabilità, sulla crisi economica e sui flussi migratori - sono riprese con la guerra civile in Libia della primavera del 2019, nella quale la politica italiana esclude un intervento militare: ma in politica certe negazioni spesso rivelano le intenzioni. ${ }^{30}$

L'art. 26 della Legge Fondamentale tedesca, benché circondato da un ben congegnato insieme di leggi d'esecuzione, si concilia sempre meno con gli interventi militari sempre più frequenti e impegnativi.

Tuttavia le modifiche costituzionali sono difficili in tutti e tre gli ordinamenti, e quindi non si può escludere che i tre articoli "pacifisti" continuino una loro esistenza formale, mentre di caso in caso la politica prenderà decisioni indipendenti dallo spirito pacifista che anima il dettato costituzionale.

30 La Ministra della difesa Elisabetta Trenta ("che non ferma il mercato di bombe per lo Yemen, né l'acquisto degli F35") "fa sapere che non ci sarà nessun intervento militare. Dimenticando anche lei che i soldati italiani, e in pericolo, in Libia già ci stanno", Tommaso Di Francesco (2019, 14 aprile), La nostra Libia dimenticata, con una sintesi delle vicende libiche e del coinvogimento italiano dalla primavera del 2011. 



\section{Appendici}

\section{Giappone: Il disarmo totale in un'area dalle tensioni crescenti}

\section{I.1 Dichiarazione di Potsdam (Potsdam Declaration, 1945) ${ }^{\mathbf{1}}$}

Nella Dichiarazione di Potsdam (Potsdam Declaration or the Proclamation Defining Terms for Japanese Surrender) del 26 luglio 1945 Harry S. Truman, Winston Churchill e Chiang Kai-Shek precisarono i termini della resa del Giappone enunciati nel corso della Conferenza di Potsdam del 17 luglio - 2 agosto 1945. Dopo la Dichiarazione di Potsdam venne firmato l'Accordo di Potsdam (Potsdam Agreement) del 2 agosto 1945 per regolare la ristrutturazione politica e geografica della Germania, il trattamento dei tedeschi prigionieri guerra e le riparazioni belliche. ${ }^{2}$

La Dichiarazione di Potsdam viene riportata per intero qui di seguito perché contiene le dure linee direttrici che le potenze vincitrici intendevano seguire nei confronti del Giappone dopo la sua resa incondizionata. In particolare, il punto 9 prevede il disarmo e la fine delle forze armate giapponesi (ma non parla ancora del divieto di riarmo e del rifiuto della guerra), mentre il punto 10 annuncia la condanna dei criminali di guerra.

Di particolare rilevanza è la formulazione del punto 13. Mentre nei punti precedenti si parla sempre del "Giappone" come di una totalità, nel punto 13 gli Alleati chiedono "al governo del Giappone" di proclamare "immediatamente la resa incondizionata di tutte le forze armate giapponesi”. Gli Alleati erano giunti a questa formulazione perché si erano convinti che il governo giapponese non avrebbe mai accettato un ultimatum che coinvolgesse anche la figura dell'imperatore. Perciò essi chiedevano la resa non del Giappone, ma delle "forze armate

1 Il testo in italiano qui riprodotto è desunto da https:/it.wikipedia.org/wiki/Dichiarazione _di_Potsdam; il testo in inglese è in http://www.ndl.go.jp/constitution/e/etc/c06.html.

2 Testo in tedesco: http://www.documentarchiv.de/in/1945/potsdamer-abkommen.html. Testo in inglese: https:/www.nato.int/ebookshop/video/declassified/doc_files/Potsdam\%20 Agreement.pdf; e anche https://en.wikipedia.org/wiki/Potsdam_Agreement. 
giapponesi". Questo punto 13 è quindi all'origine della persistenza dell'istituzione imperiale attraverso le fasi della capitolazione e dell'occupazione americana, sino alla ripresa della normale vita internazionale dello Stato giapponese, con la nuova costituzione che riconosceva la figura dell'imperatore sancendo cosi una continuità plurisecolare fondamentale per la società del Giappone.

1. Noi - il Presidente degli Stati Uniti d'America, il Presidente del Governo Nazionale della Repubblica di Cina e il Primo Ministro del Regno Unito - in rappresentanza delle centinaia di milioni di nostri compatrioti, ci siamo consultati e conveniamo che al Giappone sia data la possibilità di porre fine a questa guerra.

2. Le enormi forze di terra, mare e aria degli Stati Uniti d'America, dell'Impero britannico e della Cina, ampiamente rinforzate dalle loro armate e flotte aeree provenienti dall'ovest, sono pronte a infliggere i colpi finali al Giappone. Questa potenza militare è sostenuta e ispirata dalla determinazione di tutte le Nazioni Alleate a proseguire la guerra contro il Giappone finché esso non cessi di resistere.

3. Il risultato della vana e insensata resistenza tedesca alla potenza dei popoli liberi della Terra dovrebbe essere un esempio di terribile chiarezza per il popolo del Giappone. Le forze che stanno ora convergendo sul Giappone sono incommensurabilmente maggiori di quelle che, dirette contro la resistenza nazista, hanno necessariamente sconvolto le terre, le industrie e le vite di tutto il popolo tedesco. Il pieno uso della nostra potenza militare, sospinta dalla nostra risolutezza, significherà un'inevitabile e completa distruzione delle forze armate giapponesi e inevitabilmente la totale devastazione della patria giapponese.

4. Per il Giappone è giunto il tempo di decidere se continuare a essere controllato da quei caparbi consiglieri militaristi i cui stupidi calcoli hanno portato l'Impero del Giappone sulla soglia dell'annientamento, o se seguire la via della ragione.

5. Qui di seguito sono le nostre condizioni. Non devieremo da esse. Non ci sono alternative. Non sopporteremo ritardi.

6. Deve essere eliminata per sempre l'autorità e l'influenza di quelli che hanno ingannato e fuorviato il popolo del Giappone conducendolo alla conquista del mondo, poiché siamo certi che non sarà possibile un nuovo ordine di pace, sicurezza e giustizia finché non sarà eliminato dal mondo il militarismo irresponsabile.

7. Finché tale nuovo ordine non sarà stabilito e fino a che non ci sarà la prova che la potenza militare del Giappone è distrutta, saranno occupati dei punti nel territorio giapponese scelti dagli Alleati per assicurare il conseguimento degli obiettivi qui esposti. 
8. Le condizioni della Dichiarazione del Cairo ${ }^{[3]}$ saranno rispettate e la sovranità giapponese sarà limitata alle isole di Honshu, Hokkaido, Kyushu, Shikoku e altre isole minori come da noi determinato.

9. Le forze militari giapponesi, dopo essere state completamente disarmate, saranno libere di tornare alle proprie case con l'opportunità di trascorrere vite pacifiche e laboriose.

10. Non intendiamo che i giapponesi siano asserviti come razza o distrutti come nazione, ma sarà applicata una severa giustizia ai criminali di guerra, inclusi coloro che sono stati crudeli con i nostri prigionieri. Il governo giapponese rimuoverà tutti gli ostacoli al rifiorire e al rafforzarsi delle tendenze democratiche nel popolo giapponese. Dovranno essere assicurati libertà di parola, di religione e di pensiero, così come il rispetto dei diritti fondamentali dell'uomo.

11. Al Giappone sarà permesso di mantenere le industrie atte a sostenere la sua economia e gli sarà permessa la riparazione di quelle dello stesso tipo, ma non di quelle che lo renderebbero in grado di riarmarsi per la guerra. A questo fine gli sarà accordato l'accesso alle materie prime, ma non il loro controllo. Sarà consentita la futura partecipazione giapponese al commercio mondiale.

12. Le forze di occupazione degli Alleati saranno ritirate dal Giappone appena questi obiettivi saranno stati raggiunti e si sarà stabilito in accordo con la volontà liberamente espressa del popolo giapponese un governo pacifico e responsabile.

13. Noi facciamo appello al governo del Giappone affinché proclami immediatamente la resa incondizionata di tutte le forze armate giapponesi e fornisca adeguate garanzie della loro buona fede in tale azione. L'alternativa per il Giappone è la rapida e totale distruzione.

I.2 Dopo la resa: le istruzioni per il Gen. MacArthur e la politica degli Stati Uniti verso il Giappone (1945)

Gli Stati Uniti occuparono il Giappone dopo la resa e attuarono i principi enunciati nella Dichiarazione di Potsdam. Nel primo documento riportato qui di seguito (Le istruzioni per il Generale Douglas MacArthur) si precisano i compiti e i poteri del generale MacArthur. Nel secondo documento (L'iniziale politica

3 La Dichiarazione di Potsdam richiama qui la Conferenza del Cairo del 27 novembre 1943, che sancì la pressione militare sul Giappone sino alla sua resa incondizionata, la restituzione alla Cina dei territori conquistati dai Giapponesi (Manciuria, isole Pescadores e Taiwan) e l'indipendenza della Corea. 
degli Stati Uniti verso il Giappone $e$, in particolare, nella sua Parte III) si stabilisce il disarmo del Giappone (1. Disarmo e smilitarizzazione) e il perseguimento dei criminali di guerra (2. Criminali di guerra.)

a) Le istruzioni per il Generale Douglas MacArthur $(1945)^{4}$

1. L'autorità dell'Imperatore e del governo giapponese nel guidare lo Stato è a Lei subordinata in quanto Comandante Supremo dei Poteri Alleati (SCAP). Lei eserciterà la Sua autorità nel modo che riterrà opportuno per condurre a compimento la sua missione. Il nostro rapporto con il Giappone non si fonda su una base contrattuale, ma su una resa incondizionata. Poiché la Sua autorità è suprema, Lei non avrà alcun rapporto con i giapponesi nel perseguire il Suo fine.

2. Il controllo sul Giappone sarà esercitato attraverso il Governo Giapponese nella misura in cui questa soluzione produrrà risultati soddisfacenti. Questo però non pregiudica il Suo diritto di procedere direttamente, se necessario. Lei può applicare gli ordini da Lei emanati ricorrendo alle misure da Lei ritenute necessarie, compreso l'uso della forza.

3. Alla dichiarazione di intenti contenuta nella Dichiarazione di Potsdam verrà data piena attuazione. Tuttavia non verrà applicata perché ci consideriamo contrattualmente vincolati con il Giappone sulla base del presente questo documento. Esso verrà rispettato ed applicato perché la Dichiarazione di Potsdam fa parte della nostra politica fondata sulla buona fede verso il Giappone e per la pace e la sicurezza nell'Estremo Oriente.

(U.S. Department of State, Foreign Relations of the United States: Diplomatic Papers, U.S. Government Printing Office, 1945, VI, p. 712).

4 Fonte: L'iniziale politica degli Stati Uniti verso il Giappone dopo la resa (6 settembre 1945), in: Nibon kokan shuan bunsho [Raccolta dei principali trattati internazionali del Giappone] (1985), 81-91. 
b) L'iniziale politica degli Stati Uniti verso il Giappone dopo la resa $(1945)^{5}$

Finalità del documento. Questo è un documento sull'iniziale politica generale verso il Giappone dopo la sua resa. È stato approvato dal Presidente e distribuito al Comandante supremo delle Forze Alleate e alle competenti agenzie e dipartimenti degli Stati Uniti per la sua applicazione. Non affronta tutte le materie relative all'occupazione del Giappone e richiedenti decisioni politiche. Queste materie qui non incluse o incluse solo parzialmente sono state trattate (o verranno trattate) separatamente.

\section{Parte I - Obiettivi finali}

Gli obiettivi finali degli Stati Uniti rispetto al Giappone, cui devono adeguarsi le politiche nel periodo iniziale, sono le seguenti:

(a) Garantire che in Giappone non torni a divenire una minaccia per gli Stati Uniti o per la pace e la sicurezza mondiali.

(b) Realizzare la formazione di un governo pacifista e responsabile che rispetti i diritti degli altri Stati e che appoggi gli obiettivi degli Stati Uniti cos̀̀ come sono enunciati nella Carta delle Nazioni Unite. Gli Stati Uniti desiderano che questo governo si attenga quanto più è possibile ai princìpi dell'autogoverno democratico, tuttavia non è una responsabilità che incomba ai Poteri Alleati l'imporre al Giappone qualsiasi forma di governo che non si fondi sulla volontà popolare liberamente espressa.

Questi obiettivi verranno conseguiti seguendo queste direttive principali:

(a) La sovranità del Giappone si limiterà alle isole di Honshu, Hokkaido, Kyushu, Shikoku [81-82] e quelle periferiche isole minori da determinarsi secondo la Dichiarazione del Cairo e secondo altri accordi di cui gli Stati Uniti siano o possano essere parti.

(b) Il Giappone verrà completamente disarmato e smilitarizzato. L'autorità dei militaristi e l'influenza del militarismo verranno del tutto eliminate

5 Fonte: L'iniziale politica degli Stati Uniti verso il Giappone dopo la resa (6 settembre 1945), in: Nibon kokan shuan bunsho [Raccolta dei principali trattati internazionali del Giappone] (1985), 81-91. Nel testo ho segnalato con corsivi miei due passi a mio giudizio significativi per l'atteggiamento degli Stati Uniti verso il Giappone sconfitto. 
dalla sua vita politica, economica e sociale. Le istituzioni che esprimono lo spirito del militarismo e dell'aggressione verranno energicamente soppresse.

(c) Il popolo giapponese dovrà essere incoraggiato a desiderare le libertà individuali e a rispettare i diritti umani fondamentali, in particolare la libertà di religione, di riunione, di opinione e di stampa. Dovrà anche essere incoraggiato a costituire organizzazioni democratiche e rappresentative.

(d) $\mathrm{Al}$ popolo giapponese dovrà essere offerta l'opportunità di sviluppare una sua economia che permetta alla popolazione di conseguire gli obiettivi propri del tempo di pace.

\section{Parte II - L'Autorità alleata}

1. L'occupazione militare. Verrà realizzata l'occupazione militare delle isole giapponesi per realizzare i princìi della resa e, inoltre, per conseguire le finalità sopra indicate. L'occupazione dovrà avere il carattere di un'operazione a carico dei principali Poteri Alleati in guerra col Giappone, che agiranno nell'interesse delle Nazioni Unite. Per questa ragione ci si aspetta e sarà benvenuta la partecipazione di altre forze armate che abbiano avuto una funzione rilevante nella guerra contro il Giappone. Le forze di occupazione saranno sottoposte a un Comandante Supremo designato dagli Stati Uniti.

Anche se, consultando e istituendo organi appropriati, [81-82] si farà ogni sforzo pe stabilire delle politiche di occupazione e di controllo del Giappone che soddisfacciano i principali Poteri Alleati, in caso di divergenze di opinione fra di loro prevalgono le politiche degli Stati Uniti.

2. Rapporti col Governo giapponese. L'autorità dell'Imperatore e del Governo giapponese saranno subordinate al Comandante Supremo, che sarà dotato di tutti i poteri necessari per realizzare i termini della resa e per realizzare le politiche fissate per lo svolgimento dell'occupazione e il controllo del Giappone.

Considerando l'attuale carattere della popolazione giapponese e il desiderio degli Stati Uniti di conseguire i propri obiettivi con il minimo impiego delle sue forze e delle sue risorse, il Comandante Supremo eserciterà la sua autorità attraverso gli organi governativi e le agenzie giapponesi, compreso l'Imperatore, nella misura in cui ciò favorisca in modo soddisfacente il conseguimento degli obiettivi degli Stati Uniti. Si permetterà al Governo giapponese, sotto la sua direzione, di esercitare i normali poteri di governo 
nell'ambito dell'amministrazione interna. Questa politica, tuttavia, sarà soggetta ai diritti e ai doveri del Comandante Supremo di richiedere cambiamenti nella struttura governativa o nel personale, ovvero di agire direttamente nel caso che l'Imperatore $\mathrm{o}$ altre autorità giapponesi non si attengono in modo soddisfacente alle richieste de Comandante Supremo nel realizzare i termini della resa. Inoltre questa politica non vincola il Comandante Supremo ad appoggiare l'Imperatore o ogni altra autorità governativa giapponese che operi in contrasto con i mutamenti tesi a conseguire gli obiettivi degli Stati Uniti. La nostra politica è di usare la forma di Governo esistente in Giappone, non di appoggiarla. ${ }^{6}$ I mutamenti nella forma di Governo iniziati dal popolo o dal Governo giapponese al fine di modificare le sue tendenze feudali e autoritarie devo essere permesse e appoggiate. Nel caso in cui la realizzazione di questi cambiamenti involva l'uso della forza da parte del popolo o del governo giapponese contro le persone che vi si oppongono, il Comandante Supremo dovrà intervenire solo se necessario per garantire la sicurezza delle sue forze e il raggiungimento di tutti gli altri obiettivi perseguiti dall'occupazione.

3. Pubblicità delle politiche. Il popolo giapponese, e il mondo intero, dovrà essere pienamente informato degli obiettivi e delle politiche di occupazione e dei progressi conseguiti nella loro realizzazione.

\section{Parte III - Politiche}

1. Disarmo e smilitarizzazione. Il disarmo e la smilitarizzazione sono i compiti primari dell'occupazione militare e dovranno essere realizzati con rapidità e determinazione. Si dovrà fare ogni sforzo per rendere i giapponesi consapevoli del ruolo svolto dai capi militari e navali e dai loro collaboratori nel cagionare i disagi presenti e futuri della popolazione.

Il Giappone non dovrà avere forze armate di terra, di mare e di cielo, né una polizia segreta, né un'aviazione civile. Le forze militari giapponesi di terra, di mare e di cielo dovranno essere disarmate e disciolte; dovranno essere disciolti anche il Quartier Generale Imperiale, il Comando Supremo e ogni organizzazione della polizia segreta. Il materiale militare e navale, le imbarcazioni militari, le installazioni militari e navali, nonché gli aerei mili-

6 Corsivo mio.

I. Giappone 
tari, navali e civili dovranno essere consegnati e messi a disposizione come richiesto da Comandante Supremo.

Gli alti ufficiali del Quartier Generale Imperiale, il Comando Supremo, altri alti ufficiali militari e navali del Governo [84-85] giapponese, i capi delle organizzazioni ultra-nazionaliste e militaristiche e altri importanti esponenti del militarismo e delle forze aggressive dovranno essere arrestati e tenuti a disposizione. Le persone che siano state esponenti attive del militarismo e nazionalisti militanti verranno rimossi ed esclusi dagli uffici pubblici e da ogni altra posizione di responsabilità pubblica o privata. Verranno disciolte e proibite le associazioni e le istituzioni ultra-nazionalistiche o militaristiche di tipo sociale, politico, professionale e commerciale.

Dal sistema educativo andranno eliminati il militarismo e l'ultra-nazionalismo, tanto nella teoria quanto nella pratica, comprese le attività paramilitari. I militari di carriera dell'esercito e della marina - ufficiali e sottufficiali ${ }^{7}$ - e ogni altro esponente del militarismo e dell'ultra-nazionalismo dovranno essere esclusi da attività di controllo e di insegnamento.

2. Criminali di guerra. Le persone accusate dal Comandante Supremo o dalle apposite agenzie delle Nazioni Unite di essere criminali di guerra, incluse quelle accusate di aver partecipato a crudeltà contro prigionieri delle Nazioni Unite o di altre nazionalità, devono essere arrestati, giudicati e, se condannate, punite. Coloro che sono ricercati da una nazione che non fa parte delle Nazioni Unite per reati contro i suoi cittadini - se non sono ricercati dal Comandante Supremo per essere giudicati o per rendere testimonianza o per altre ragioni - devono essere affidati alla custodia di quelle nazioni.

3. Incentivi al desiderio di libertà individuali e di procedure democratiche. La libertà di culto deve essere proclamata subito dopo l'occupazione. Al tempo stesso va spiegato ai giapponesi che non sarà permesso alle organizzazioni $\mathrm{e}$ ai movimenti ultra-nazionalistici e militaristici di celarsi dietro una copertura religiosa. [85-86]

$\mathrm{Al}$ popolo giapponese si dovrà offrire l'opportunità e l'incoraggiamento a famigliarizzarsi con la storia, la cultura e le istituzioni degli Stati Uniti e delle altre democrazie. L'associazione di personale delle forze di occupa-

7 I termini "ufficiali e sottufficiali" rendono con una certa approssimazione "commissioned or non-commissioned". 
zione con la popolazione giapponese andrà tenuta sotto controllo, ma soltanto nella misura necessaria, per favorire le politiche e gli obiettivi dell'occupazione.

I partiti politici democratici, con il diritto di riunione e di pubblica discussione, andranno incentivati, restando però soggetti alle esigenze per il mantenimento della sicurezza delle forze di occupazione.

Le leggi, i decreti e le normative che stabiliscono discriminazioni di razza, di nazionalità o di opinione politica dovranno essere abrogate; quelle in conflitto con gli obiettivi e le politiche delineate in questo documento dovranno essere revocate, sospese o modificate come richiesto; e gli enti incaricati della loro applicazione dovranno essere aboliti o modificati opportunamente. Le persone ingiustamente recluse dalle autorità giapponesi per ragioni politiche dovranno essere liberate. Il sistema giudiziario, giuridico e poliziesco dovrà essere riformato appena possibile per risultare conforme alle politiche esposte negli articoli 1 e 3 di questa Parte III; successivamente esso andrà progressivamente orientato alla protezione delle libertà individuali e dei diritti civili.

\section{Parte IV - Economia}

1. Smilitarizzazione economica. L'attuale base economica della forza militare giapponese deve essere distrutta, né si deve permettere che risorga.

Verrà di conseguenza realizzato un programma contenente, fra gli altri, $\mathrm{i}$ seguenti elementi: la cessazione immediata della produzione, nonché il divieto di produzione futura, di tutti i beni atti a equipaggiare, a riparare o a essere usati da qualsiasi forza o struttura armata [86-87]; la messa al bando di ogni struttura specializzata nella produzione o riparazione di materiale bellico, incluse le imbarcazioni e ogni tipo di aereo; l'istituzione di un sistema di ispezione e controllo su una selezione di settori dell'attività economica giapponese per prevenire la preparazione occulta o mascherata di materiale bellico; l'eliminazione in Giappone di quelle specifiche industrie o settori produttivi il cui precipuo valore in Giappone consiste nella preparazione della guerra; il divieto di ricerche specializzate e di addestramento aventi per oggetto il potenziale bellico; la limitazione delle dimensioni e della natura delle industrie pesanti giapponesi in vista del loro futuro uso pacifico; infine, la limitazione della marina mercantile giapponese alla dimensione richiesta per conseguire l'obiettivo della demilitarizzazione. 
L'eventuale assetto delle strutture produttive esistenti in Giappone che andranno eliminate in base a questo programma, o che andranno convertite ad altro uso, o trasferite all'estero, o distrutte, verrà determinato dopo un inventario. Nell'attesa, gli impianti facilmente convertibili alla produzione civile non andranno distrutti, salvo situazioni di emergenza.

2. Promozione delle forze democratiche. Nell'ambito del lavoro, dell'industria e dell'agricoltura andrà incoraggiato e favorito lo sviluppo di organizzazioni fondate sui princìi democratici. Si dovranno favorire le politiche che permettano una vasta distribuzione del reddito e della proprietà sui mezzi di produzione e di commercio.

Andranno favorite quelle forme di attività economica, di organizzazione e di leadership che siano ritenute adatte a rafforzare l'atteggiamento pacifico del popolo giapponese, rendendo difficile il controllo o la direzione di attività economiche indirizzabili a fini militari.

Per conseguire questa finalità, la politica [87-88] del Comandante Supremo dovrà:

(a) proibire la continuità o la nomina in posizioni importanti nel campo economico di individui che non indirizzino il futuro sforzo economico giapponese unicamente a fini pacifici;

(b) favorire un programma per lo scioglimento dei grandi conglomerati industriali e bancari che hanno esercitato un controllo su gran parte del commercio e dell'industria giapponese;

3. Ripresa di una pacifica attività economica. Le politiche del Giappone hanno imposto al popolo giapponese grandi danni economici, prospettandogli un futuro di gravi difficoltà e sofferenze economiche. Il dissesto del Giappone è la diretta conseguenza del suo proprio comportamento, e gli Alleati non si faranno carico di porre riparo al danno. ${ }^{8} \mathrm{Vi}$ si potrà porre riparo soltanto se il popolo giapponese abbandona tutte le mire militari e si impegna diligentemente nell'unica prospettiva di costruire una vita pacifica. Sarà necessario che esso intraprenda la ricostruzione materiale, che riformi a fondo la natura e l'indirizzo delle sue attività e istituzioni economiche, occupando gli individui secondo una direttrice diretta alla pace e ad essa consacrata. Gli Alleati non hanno intenzione di imporre condizioni che possano compromettere il compimento di queste imprese nei tempi opportuni.

8 Corsivo mio. 
Dal Giappone ci si attende che produca beni e servizi in grado di rispondere alle esigenze delle forze di occupazione nella misura in cui questo risultato può essere raggiunto senza provocare carestia, diffuso malessere e gravi sofferenze fisiche.

Dalle autorità giapponesi ci si attende (e, se necessario, verrà imposto) di favorire, sviluppare e realizzare i programmi che servono ai seguenti fini:

(a) evitare gravi disfunzioni economiche; [88-89]

(b) garantire la distribuzione giusta e imparziale delle risorse disponibili;

(c) adempiere alle richieste di riparazione concordate con i Governi alleati;

(d) facilitare la ripresa dell'economia giapponese, in modo che vengano soddisfatte le richieste ragionevoli e pacifiche della popolazione;

A questo proposito sarà permesso alle autorità giapponesi, sotto la loro stessa responsabilità, di stabilire e applicare controlli sull'attività economica nazionale, come i servizi pubblici essenziali, la finanza, le banche, la produzione e distribuzione dei beni essenziali, il tutto soggetto all'approvazione e al controllo de Comandante Supremo al fine di garantire la loro conformità con i fini dell'occupazione.

4. Riparazioni belliche e indennizzi.

Le RIPARAZIONI per l'aggressione giapponese consisteranno:

(a) nel trasferimento - come verrà determinato dalle competenti autorità Alleate - delle proprietà giapponesi collocate fuori dai territori assegnati al Giappone;

(b) nel trasferimento dei beni o delle strutture rilevanti e non necessarie all'economia di pace del Giappone, né alle forniture dovute alle forze di occupazione. Le esportazioni diverse da quelle da imbarcarsi come riparazioni o indennizzi possono esser fatte solo verso quei destinatari che accettano di provvedere a corrispondenti importazioni o di pagare tali esportazioni in valuta estera. Non sarà possibile pretendere alcuna forma di riparazione che interferisca o pregiudichi il programma di smilitarizzazione del Giappone.

Un INDENNIZzo completo e rapido verrà richiesto [89-90] per tutte le proprietà identificabili come prede belliche.

5. Politiche fiscali, monetarie e bancarie. Le autorità giapponesi continueranno ad essere responsabili per la gestione e la direzione delle politiche interne fiscali, monetarie e creditizie, sotto l'approvazione e il controllo del Comandante Supremo. 
6. Commercio estero e relazioni finanziarie. Si permetterà al Giappone di riprendere le normali relazioni commerciali con il resto del mondo. Durante l'occupazione e sotto opportuni controlli sarà consentito al Giappone di comperare da paesi terzi materie grezze e altri beni che ritenga necessari per scopi pacifici, esportando beni per pagare le importazioni approvate.

Verrà esercitato un controllo su tutti i beni importati ed esportati, sugli scambi internazionali e sulle transazioni finanziarie. Tanto le politiche seguite nell'esercizio di questi controlli, quanto la loro effettiva amministrazione saranno assoggettate all'approvazione e alla supervisione del Comandante Supremo per garantire che non siano contrarie alle politiche delle autorità occupanti, in particolare che il potere d'acquisto nella disponibilità del Giappone sia utilizzato soltanto per bisogni essenziali.

7. Proprietà giapponesi all'estero. Beni giapponesi all'estero o in territori distaccati dal Giappone in base ai termini di resa, compresi i beni posseduti in tutto o in parte dalla Casa Imperiale e dal Governo, devono essere denunciati alle autorità occupanti e tenuti a disposizione secondo la decisione della autorità Alleate.

8. Pari opportunità per le imprese straniere in Giappone. Le autorità non dovranno dare né permettere ad alcuna organizzazione imprenditoriale giapponese [90-91] di accordare a un'impresa di qualsiasi paese straniero una posizione preferenziale o di esclusiva, né cedere a tale impresa il controllo di un settore importante dell'attività economica.

9. Proprietà della Casa Imperiale. Le proprietà della Casa Imperiale non saranno esentate da alcuna azione necessaria al raggiungimento degli obiettivi dell'occupazione.

(U.S. Department of State, Department of State Bulletin, U.S. Government Printing Office [DSB], N 326, pp. 423-427) 
I.3 Come i giapponesi hanno percepito la costituzione del dopoguerra (1997) ${ }^{9}$

Nel 1997 un volume collettivo ha analizzato l'atteggiamento dei giapponesi di fronte alla costituzione postbellica, cioè in quale modo essi avessero percepito le nuove linee direttive della politica nazionale. L'indice del volume, riportato qui di seguito, mostra nella prima parte quali sono state le reazioni di fronte ai principali temi costituzionali e, nella seconda parte, come queste reazioni si siano configurate nelle varie aree dell'arcipelago giapponese.

Indice del volume: Come i giapponesi hanno percepito la Costituzione: I. La formulazione della costituzione giapponese. Analisi delle modalità effettive di diffusione. 1. Come è stato percepito il processo di creazione della costituzione (di Koseki Shoichi); 2. Lo sviluppo del movimento democratico nel dopoguerra e la promulgazione della costituzione giapponese (di Kinji Umeda); 3. La logica della "difesa nazionale" nella "storia di una nuova costituzione" (di Satoru Ito); 4. La nuova visione alla guida del movimento di diffusione costituzionale (di Nagami Hata); 5. La consapevolezza dei cittadini prima e dopo la promulgazione della nuova costituzione (di Takane Kawashima); 6. Luci e ombre della costituzione secondo la stampa (di Kenji Watanabe); 7. La costituzione ed i residenti cinesi e coreani in Giappone (di Kaoru Komaki); 8. La costituzione del Giappone per Okinawa (di Masaki Anya). - II. I rapporti tra i cittadini e la nuova costituzione all'interno del Giappone. 1. Hokkaido (di Chunichi Saito); 2. Area del Tohoku (di Fujio Ichinohe); 3. Prefettura di Ibaraki (di Hirofumi Takahashi); 4. Prefettura di Yamanashi (di Tamotsu Asakawa); 5. Prefettura di Shizuoka (di Seiichi Tsukamoto); 6. Comune di Kyoto (di Ichiro Ikeda); 6. Prefettura di Okayama (di Hisao Ömori); 7. Prefettura di Aichi (di Naoki Furuya); 8. Prefettura di Fukuoka (di Toyo Shindo).

In particolare, viene qui presentato il nucleo centrale del contributo dello storico contemporaneo Kinji Umeda, Lo sviluppo del movimento democratico nel dopoguerra e la promulgazione della costituzione giapponese: se ne traduce qui di seguito il paragrafo sull'atteggiamento dei giapponesi di fronte alla nuova costituzione e alla democrazia che essa introduce e, inoltre, lo si fa precedere e seguire dal riassunto delle restanti parti del medesimo contributo, in modo da facilitarne l'inquadramento concettuale e storico. ${ }^{\mathbf{1 0}}$

La traduzione dal giapponese è stata curata da Pier Giorgio Girasole, autore anche dei riassunti e delle note fra parentesi quadre, per distinguerle dalle stringate note dell'originale di Kinji Umeda, che vengono riportate senza alcuna parentesi.

9 Fonte: Kinji Umeda (1997a), Sengo minshu-shugi undo no nagare, le parti qui tradotte sono alle pp. 46-61. I riassunti e la traduzione dal giapponese sono di Pier Giorgio Girasole.

10 Di Umeda si veda anche il volume: KInJı UMEDA (1997b), Ima manabitai kingendai rekishi. 


\section{[Riassunto]}

"La costituzione giapponese come punto di arrivo del ventesimo secolo".

"La seconda guerra mondiale verrà ricordata nella storia mondiale come la vittoria delle nazioni unite da ideali liberali contro il fascismo": con queste parole Umeda inizia il proprio saggio, in cui prende in considerazione $i$ movimenti che, dopo le guerre mondiali, hanno sviluppato gli ideali di libertà nel mondo contemporaneo, concentrandosi poi sul caso giapponese e sulla sua costituzione pacifista. La prima parte del Novecento è caratterizzata dal fenomeno coloniale, come ad esempio la guerra di conquista della Corea, intrapresa dal Giappone tra il 1908 ed il 1909 e avvenuta in un clima in cui le principali potenze europee, come la Gran Bretagna e la Francia, perseguivano analoghe politiche espansionistiche. Dopo la seconda conferenza di pace dell'Aja del 1907 sembrava però che le questioni internazionali fossero divenute maggiormente controllabili, in particolare se annunciate da "un chiaro e previo avviso". Tuttavia a distanza di pochi anni scoppiò la Prima Guerra mondiale. In questo conflitto globale caratterizzato da uno scontro tra imperi, scrive Umeda, il Giappone, alleato di Francia e Regno Unito, si presentò in Asia come il liberatore del continente dai vecchi colonizzatori, avvantaggiato dal fatto che essi erano impegnati nelle trincee in Europa. In questi conflitti si inserì anche la Rivoluzione d'Ottobre, che fece della pace uno degli elementi principali della propria lotta contro il potere zarista.

Il Giappone nel 1925 è tra i firmatari del protocollo di Ginevra, cui si aggiunge nel 1928 il patto Briand-Kellogg. "Nel giugno del 1929”, continua Umeda, "in Giappone viene emanata una dichiarazione del governo di cui viene criticata l'espressione 'a nome di ogni libero individuo', essendo il Paese retto dall'Imperatore". Il capitolo prosegue con la descrizione della decade successiva, in cui iniziano a svilupparsi i totalitarismi, come dimostra l'affermarsi di Stalin. In quegli anni i nuovi Stati sorti in Germania e in Italia sembrano essere gli alleati ideali per il Giappone, anche per la sua politica di espansione in Asia. Dopo le dittature in Europa e il secondo conflitto mondiale, nel giugno del 1945 fu approvata la carta delle Nazioni Unite. Dopo la sconfitta del Giappone, gli Stati Uniti vi introdussero una nuova costituzione di modello occidentale. Uno dei suoi punti centrali fu la smilitarizzazione. Quindi la costituzione giapponese, conclude Umeda, rappresenta il punto di arrivo dei movimenti libertari della prima metà del Novecento. 
La nascita dei movimenti democratici in Giappone nel dopoguerra ed il problema della costituzione ${ }^{11}$

Nonostante il Giappone fosse stato sconfitto a Okinawa, gli scontri sulle isole maggiori continuavano. Hiroshima e Nagasaki erano in preda al caos, mentre per il Manciukuò abbandonato dall'armata del Kwantung stava cominciando un periodo difficile. Inoltre (anche se si apprendeva con un certo sollievo che negli altri territori dell'impero giapponese la guerra era finita) ovunque erano state distrutte le abitazioni ed i luoghi di lavoro e, a causa dell'inflazione, mancava il cibo e le famiglie non potevano sostenersi. Persino nelle campagne era difficile trovare del riso tanto che, subito dopo la resa, si svilupparono associazioni volontarie per la vendita di cibo casa per casa. In questa situazione coloro cui era stato proibito esprimersi durante il conflitto iniziarono ad organizzarsi per diffondere le proprie idee, anche se a guerra conclusa la legge sulla sicurezza interna era ancora in vigore. Uno di questi gruppi fu l'Alleanza Culturale del Popolo di Inosuke Nakanishi, che si organizzò verso la fine del settembre 1945. I tre obiettivi di questo movimento erano l'"abolizione della legge sulla sicurezza, il rilascio dei prigionieri politici e la punizione dei responsabili di guerra”. Subito dopo l'abrogazione della legge sulla sicurezza (4 Ottobre 1945), i prigionieri politici liberati inaugurarono l'“assemblea di benvenuto per i combattenti per la libertà rilasciati". ${ }^{12}$ Anche se durante la guerra avevano dovuto mantenere segrete le loro posizioni, subito dopo essi organizzarono numerose assemblee libere in quanto gruppo ormai legale. Infatti, immediatamente dopo il 15 agosto, ${ }^{13}$ già il $1^{\circ}$ ottobre si era costituito il nucleo fondativo del movimento. Di questa organizzazione (il cui slogan era: "Lottiamo per eliminare la persistenza di fascisti testardi e senza scrupoli, insieme ai feudalisti ${ }^{\mathbf{1 4}} \mathrm{del}$ Paese") presero parte anche Tetsu Katayama e Itoshi Ashida, che sarebbero poi diventati primi ministri. ${ }^{15}$ Un'ulteriore caratteristica del dopoguerra fu

11 [Kinji Umeda (1997a), Sengo minshu-shugi undo no nagare, 46-50. Traduzione di Pier Giorgio Girasole.]

12 Fronte popolare, prima pubblicazione, 10 dicembre 1945.

13 [Il 15 agosto 1945 è la data della resa del Giappone.]

14 [In giapponese hoken shugi, termine utilizzato dalla Sinistra dopo la guerra per indicare i conservatori.]

15 Edizione dell'Assemblea del Pensiero Libero (annuario di cultura del Giappone), edizione del 1948. 
la richiesta di rimpatrio da parte dei cittadini coreani e cinesi che erano stati deportati in Giappone per contribuire alla sua difesa. Nella fase finale del conflitto, inoltre, il primo ministro Naruhito Higashikuni, dopo essere stato convocato dall'imperatore Hirohito, continuò a sostenere lo spirito della legge di sicurezza lavorando sul kokutai ${ }^{\mathbf{1 6}}$ In queste circostanze il movimento democratico giapponese incominciò a prendere forma. Anche se erano presenti posizioni diverse e benché non si ponesse ancora il problema della costituzione, si posero le fondamenta per gli sviluppi futuri. Il 4 ottobre 1945 venne abrogata la legge sulla sicurezza e il giorno seguente il primo ministro Higashikuni diede le dimissioni. Il giorno 10 invece vennero rilasciati $\mathrm{i}$ detenuti politici, producendo nuovamente una svolta nel Paese. In seguito, da un lato Kijuro Shidehara, il Primo Ministro nominato successivamente, aveva difeso il kokutai, dall'altro il quartier generale statunitense diede inizio al processo di democratizzazione sulla base della conferenza di Postdam, mentre il partito comunista giapponese arrivò a proporre l'abolizione del sistema imperiale. Uno dei primi segnali tangibili della trasformazione che seguì l'abrogazione della legge sulla sicurezza fu il caso del quotidiano nazionale "Yomiuri Shimbun", che annoverava tra le sue file sia democratici, sia responsabili della guerra, assunti in precedenza. Venne perciò avanzata la richiesta di rinnovare il personale e di indicare quali fossero i responsabili durante la guerra. ${ }^{17} \mathrm{~A}$ dicembre ci furono poi scontri durante la ricostruzione della linea ferroviaria Keisei, accompagnati dalla richiesta di un aumento di salario, di un contratto collettivo regolato e delle dimissioni dell'esecutivo reazionario. ${ }^{18}$ Come prima conseguenza dell'abrogazione della legge sulla sicurezza aumentarono anche i movimenti che chiedevano un mutamento nelle condizioni di lavoro. La seconda conseguenza generata dall'abrogazione della legge sulla sicurezza fu in tentativo di portare la democrazia nelle campagne. Prima della riforma agraria, ${ }^{19}$ infatti, la sottra-

16 [Nella Germania nazionalsocialista si confrontava l'idea del Reich con quella di kokutai. Per i tedeschi, il Reich è "più una fede che un sapere", è "qualcosa di maggiore, migliore e superiore al semplice Stato". "Ancora più esclusiva per i giapponesi è la parola kokutai, che si dovrebbe tradurre con 'idea dell'Impero' o 'essenza dell'Impero': qualcosa di unicamente giapponese e quindi intrasferibile”, Walter Donat (1943b), Der deutsche und der japanische Reichsgedanke, 105-106.]

17 Taro Miyamoto, Ricordo della guerra all'interno dello Yomiuri, edizioni Shin Nippon.

18 Gazzetta dei lavoratori del Giappone, 9 gennaio 1945.

19 [La riforma agraria risale al 1946.] 
zione dei campi ai contadini da parte dei grandi proprietari causò vari scontri; invece all'interno dei villaggi vigevano regole e atteggiamenti totalmente democratici. Ad esempio, nel villaggio di Nakagawa (presso l'odierno comune di Sashima nella prefettura di Tochigi) il 24 novembre venne istituita un'assemblea di agricoltori per opporsi agli espropri delle terre e venne richiesta la sospensione totale tanto dei capi villaggio quanto dei responsabili della guerra. La stessa cosa avvenne nei villaggi limitrofi. ${ }^{20}$ Anche nelle campagne quindi si trovavano fianco a fianco i difensori della democrazia e i responsabili della guerra. Questa situazione richiedeva una revisione del sistema di controllo sulle terre presente durante la guerra. Terza conseguenza fu invece la lotta per i viveri. Divenuti introvabili durante il conflitto, venivano anche rubati o sottratti a quelli destinati all'esercito. Ciò scatenò un'ondata di malcontento tra i cittadini che dovevano difendersi da soli e nel dicembre del 1945 - nei quartieri di Ebara, nel distretto di Meguro (oggi Shinagawa), in quelli di Oji e di Ryunogawa (oggi Kita) ed in quello di Toyoshima) organizzarono assemblee in cui l'accaparramento di riserve di cibo venne considerato un crimine di guerra. Proprio in questo periodo si iniziò a considerare un emendamento costituzionale per modificare le vecchie modalità di compravendita e venne così istituito un gruppo di ricerca e confronto sugli emendamenti costituzionali proposti a suo tempo da Fumimaro Konoe e dall'allora primo ministro Shidehara. Inoltre all'interno dei movimenti democratici si incominciava a considerare il problema della costituzione. Per primi i rappresentanti del partito comunista giapponese l'11 novembre 1945 annunciarono la richiesta di una costituzione che attribuisse il potere al popolo, sottraendolo all'autorità imperiale. Invece il 27 dicembre Iwaburo Takano e Yasuzo Suzuki ${ }^{21}$ organizzarono un incontro costituzionale in cui presentarono come preferibile un modello di monarchia costituzionale piuttosto che un modello del tutto democratico. Tuttavia, poiché l'imperatore non aveva alcuna autorità politica, il giorno seguente Takano propose una costituzione che "superasse il modello imperiale consegnando il potere nelle mani di un presidente della repubblica”. Questo movimento costituzionale divenne, come si vedrà meglio in seguito, il modello per le direttive imposte dal quartier generale americano: è tuttavia doveroso ricor-

20 Gazzetta dei lavoratori del Giappone, 5 dicembre 1945.

21 [Iwaburo Takano, sociologo, e Yasuzo Suzuki, costituzionalista, erano legati al movimento socialista giapponese.] 
dare che questo gruppo si formò il 13 novembre a partire dalla Lega culturale del Giappone. La Lega culturale del Giappone aveva come slogan "Fondare una cultura democratica" e "Far chiarezza sui responsabili del conflitto"; inoltre al suo interno erano presenti studiosi e uomini di cultura. Si può quindi asserire che i movimenti costituzionalistici siano nati da quelli democratici. Inoltre, secondo il parere dello studioso Michio Sagawa, dopo che all'interno dei gruppi dei lavoratori di Nagoya era stato preparato "il progetto di una costituzione per la costruzione di un nuovo Giappone" nel quale "uno dei temi era la rinuncia totale al riarmo, oltre all'abolizione del sistema imperiale da sostituirsi con uno totalmente repubblicano". 22 Sagawa era stato infatti arrestato in quanto membro del Movimento di educazione liberale, ma dopo l'abrogazione della legge sulla sicurezza alla fine della guerra poté finalmente esprimere le sue opinioni sul tema della costituzione. E come lui anche molti altri.

\section{Il Movimento rivoluzionario democratico del dopoguerra e il problema della costituzione ${ }^{23}$}

Il 15 dicembre 1945 il quartier generale delle forze di occupazione americane emanò "l'ordinanza di separazione tra Stato e religione". ${ }^{24}$ Con ciò si dovette eliminare l'ideologia secondo cui "l'Imperatore del Giappone era ritenuto per la sua origine capo religioso oltre che politico" sulla quale si fondava il kokutai. Inoltre l'imperatore Hirohito, il $1^{\circ}$ gennaio 1946, emanò un editto, noto come la "dichiarazione della natura umana dell'imperatore". Sul piano internazionale e soprattutto agli occhi dell'America era infatti necessario eliminare l'idea che il sovrano giapponese fosse colpevole del conflitto in quanto comandante supremo dell'esercito. In quegli anni, a partire dagli scontri per i viveri già ricordati, sorge il Movimento rivoluzionario-democratico. Infatti, mentre pochi anni prima i cittadini di varie parti del Giappone erano impegnati nelle lotte per il cibo, il ministero dell'agricoltura organizzò un gruppo che mirava a rendere democratico il governo e

22 Michio Sagawa, La mia vita come educatore, nuova edizione critica. [Il Movimento di educazione liberale era stato un movimento clandestino.]

23 [Kinji Umeda (1997a), Sengo Minshu-shugi undo no nagare, 50-54. Traduzione di Pier Giorgio Girasole.]

2420 anni di documenti dopo la guerra, $1^{\mathrm{a}}$ edizione critica. 
di porre fine al problema delle risorse alimentari. Il movimento allora chiese di "risolvere il problema del cibo" e di "licenziare i criminali di guerra, i militaristi e i burocrati corrotti". 25 In seguito, il 21 gennaio i vertici industriali del Kanto ${ }^{26}$ si riunirono in un'assemblea democratica per risolvere il problema dei viveri (Asahi Shimbun del 22 gennaio 1946). Inoltre l'11 febbraio 200 gruppi si unirono nell'associazione per le risorse alimentari del Kanto. In essa erano rappresentati " $i$ produttori dei sindacati, i coltivatori di cooperative agricole e i commercianti urbani per dare il migliore esempio di amministrazione popolare contro la mancanza di regolamentazioni sul cibo". ${ }^{27}$ Ovviamente non tutti gli intenti vennero rispettati alla lettera ma, sotto l'influenza dei partiti comunista e socialista, i sindacati si unirono, coinvolgendo agricoltori e commercianti. Il 17 febbraio, dopo che furono prese misure di emergenza per il problema dei viveri, gli scontri nelle campagne si intensificarono in tutto il Paese. A partire dal 4 marzo per due mesi in 18 prefetture ci furono sommosse. Ad esempio, a Ibaraki il 23 marzo si mobilitarono 4000 persone, mentre il 25 marzo circa diecimila si radunarono di fronte al palazzo della prefettura chiedendo le dimissioni del primo ministro Shidehara. ${ }^{28}$ Inoltre il 22 aprile sempre diecimila contadini si organizzarono in corteo di fronte alla sede della prefettura di Akita. ${ }^{29}$ Data l'estrema rilevanza del problema relativo alle risorse alimentari, il 22 aprile fu indetta un'assemblea in opposizione al primo ministro Shidehara, mentre il $1^{\circ}$ maggio fu festeggiato come giornata dei lavoratori, cui seguì la giornata del cibo stabilita il 19 maggio in un'ulteriore riunione. Oltre al problema degli approvvigionamenti si prese posizione "contro una classe politica immobile" a favore di "una realizzazione della democrazia popolare in un vertice composto dai sindacati del partito socialista e del partito comunista, nonché dai gruppi di agricoltori per un governo popolare". ${ }^{30}$ Un'ulteriore caratteristica di questo periodo fu il movimento del Fronte popolare (o fronte unito) che generò il Movimento dei rivoluzionari democratici, che dà il titolo a questo paragrafo. Il movimento era nato durante le lotte per i

2520 anni di documenti dopo la guerra, $1^{\mathrm{a}}$ edizione critica.

26 [Zona pianeggiante in cui sorgono Tokyo e il suo hinterland.]

27 Gazzetta dei lavoratori del Giappone, 9 febbraio 1946.

28 Jo Hamano, Storia dei movimenti popolari di Ibaraki nel dopo guerra, Edizioni Jihi.

29 Gazzetta dei lavoratori del Giappone, 25 aprile 1946.

3020 anni di documenti dopo la guerra, $1^{\text {a }}$ edizione critica. 
viveri e riuniva esponenti delle città e delle campagne che chiedevano di punire i responsabili di guerra. In un secondo momento tutti questi gruppi nel Paese, insieme con quelli di Tokyo, decisero di unirsi in un unico fronte. Questo avvenne a Kyoto, dove il 18 gennaio si formò l'Assemblea di Kyoto del Fronte Popolare, cui presero parte il 21 febbraio il partito socialista, il partito comunista e quello liberale. L'11 febbraio era stata organizzata un'altra assemblea ad Aomori con la presenza dei socialisti, dei comunisti e dei liberali. Nella prefettura di Ishikawa la data scelta fu il 26. Così facendo a maggio nelle 30 prefetture del Giappone fu realizzato un unico fronte ed il 27 settembre furono indette delle elezioni per i vertici che avrebbero avuto luogo il 5 Ottobre, alle quali tuttavia si stabilì di far precedere quelle dei rappresentanti locali. ${ }^{31}$ Ciononostante il fronte popolare, benché appena formato, entrò subito in difficoltà. Il partito comunista aveva annunciato nel novembre del 1945 la "campagna per un fronte popolare", ma i socialisti non avevano aderito. Il 15 Gennaio del 1946 Hitoshi Yamakawa ${ }^{32}$ aveva proposto il Fronte Democratico Popolare, mentre il 26 fu organizzata una riunione di politici esiliati durante la guerra (tra cui Sanso Nosaka), cui parteciparono intellettuali comunisti e liberali. Di conseguenza a marzo Nosaka propose nuovamente la creazione del fronte ma, a differenza dei comunisti, i socialisti non vi presero parte. Mentre non si riusciva a creare un fronte unito, il quartier generale delle forze di occupazione decise di tenere le elezioni per il 10 aprile. Il partito di maggioranza del primo ministro Shidehara venne riconfermato. In questo periodo aumentarono le proteste contro il primo ministro Shidehara ed il 19 aprile i partiti socialista, comunista e liberale si riunirono in una coalizione contro il capo del governo, costringendolo alle dimissioni. Successivamente il $1^{\circ}$ maggio fu celebrata la giornata dei lavoratori e a Tokyo si riunirono cinquecentomila persone con i motti: "Potere alla democrazia popolare", "Costruire un fronte popolare democratico", ma soprattutto "Una costituzione per il popolo dal popolo". Infine, mentre si attendeva di vedere come Shidehara avrebbe organizzato il governo dopo le dimissioni, si celebrò la giornata del cibo (19 maggio). Tuttavia per circa un mese non si riuscì a trovare un nuovo capo di governo e si rimase in una posizione di stallo fino a quando Yoshida Shigeru divenne

3120 anni di documenti dopo la guerra, $1^{\mathrm{a}}$ edizione critica.

32 [Hitoshi Yamakawa (1888-1953) fu tra i fondatori del partito comunista giapponese.] 
primo ministro. Proprio in quegli anni in cui il movimento rivoluzionariodemocratico si stava formando, il comando supremo delle forze di occupazione decise di accelerare i tempi di stesura della costituzione. Il quartier generale americano aveva infatti preso in considerazione il gruppo di ricerca sui problemi costituzionali, sorto durante il mandato di Shidehara, ma sapeva di non essere ancora pronto ad affrontare l'opinione pubblica internazionale e decise quindi di non lasciare che il governo giapponese decidesse da solo sulla questione. Il comandante supremo delle forze di occupazione, MacArthur, il giorno 3 febbraio organizzò il gruppo per la stesura della costituzione e lo rese operativo dalla settimana successiva, ovvero dal giorno 10. Tra i vari gruppi di studio giapponesi il quartier generale scelse il già ricordato Gruppo di ricerca costituzionale. ${ }^{33}$ Gli americani affrettarono la stesura della costituzione a causa dell'imminente prima riunione della Commissione l'Estremo Oriente (Far Eastern Commision) prevista per il 25 febbraio. In questa occasione, facendo riferimento alla conferenza per gli affari esteri di Mosca del precedente dicembre, si discusse sugli elementi di maggior rilievo per la politica giapponese e ci si concentrò sul fatto che nella nuova costituzione il potere dell'imperatore doveva restare immutato. MacArthur volle infatti dimostrare al governo giapponese che la nuova costituzione non avrebbe intaccato il sistema imperiale prima della convocazione della commissione. Stando cos̀̀ le cose, il primo ministro Yoshida, il segretario di Stato Matsumoto e il direttore per gli affari civili Whitney riuscirono nel loro incontro a creare un governo secondo le direttive del quartier generale. La conservazione dell'autorità imperiale, voluta da MacArthur, consentì all'ormai dimesso Shidehara di continuare ad esercitare la sua influenza. ${ }^{34} \mathrm{Da}$ questa situazione prese origine la linea direttrice nella formazione del governo del Giappone.

\section{[Riassunto]}

Shidehara, ultimo premier difensore del kokutai

Umeda analizza il kokutai, ovvero la dottrina che indicava come sacra la figura dell'imperatore giapponese, partendo da Kiichiro Hiranuma, figura molto vicina a Hirohito nelle fasi finali del conflitto. Dopo la Dichiarazione

33 Masui Takayanagi (ed.), Il processo di creazione della costituzione, edizioni Yuhikaku.

34 Shoichi Koseki, La nascita della nuova costituzione, edizioni Chuko. 
di Potsdam, infatti, quel consigliere difese il sovrano sostenendo la necessità di conservare la sua figura al vertice dello Stato, sulla base non delle leggi umane, ma della sua natura divina. Tuttavia, con la dichiarazione della natura umana dell'imperatore del $1^{\circ}$ gennaio 1946 , il sovrano stesso ammise di non essere una divinità, lasciando però aperta l'idea di una discendenza divina. Il primo ministro Shidehara, ricorda Umeda, diede le dimissioni e rimase l'ultimo difensore del kokutai classico. Il suo successore Shigeru Yoshida invece riuscì a mantenere il kokutai adeguandolo alle esigenze del tempo. Infatti (con un'affermazione definita "ambigua" da Umeda) il primo ministro annunciò che "l'Imperatore non era ormai da considerarsi al vertice della società giapponese, ma parte del suo popolo”. Inoltre, iniziati nel $1946 \mathrm{i}$ preparativi per la stesura del nuovo testo costituzionale, Yoshida ribadì di aver informato l'imperatore dei lavori, appellandosi all'articolo 73 della costituzione Meiji del $1889 .{ }^{35}$ Si pose però un problema: la differenza di contenuto tra i documenti in inglese del Quartier generale americano e i testi in giapponese del governo provocò le critiche del partito comunista. Umeda continua la sua analisi sostenendo che il kokutai rimase attivo sotto il motto "imperatore parte del suo popolo", con il quale non si aboliva il suo potere. "Oggi la parola kokutai", conclude l'autore, "è ormai in disuso", anche se risulta necessaria nello studio della genesi della costituzione entrata finalmente in vigore il 3 novembre del 1946.

35 [La costituzione giapponese del 1947 si apre infatti così: "I rejoice that the foundation for the construction of a new Japan has been laid according to the will of the Japanese people, and hereby sanction and promulgate the amendments of the Imperial Japanese Constitution effected following the consultation with the Privy Council and the decision of the Imperial Diet made in accordance with Article 73 of the said Constitution. Signed: HIROITO, Seal of the Emperor". - L'articolo 73 della costituzione Meiji del 1889 invece recita: "Il Gabinetto, oltre ad altre funzioni amministrative generali, svolge le seguenti funzioni: 1. Amministrare fedelmente la legge e condurre gli affari di stato; 2. Gestire gli affari esteri; 3. Concludere i trattati dopo l'approvazione della Dieta; 4. Amministrare il servizio civile, in conformità con le norme stabilite dalla legge; 5 . Preparare il bilancio e presentalo alla Dieta. Tuttavia, non può includere disposizioni penali in tali ordini di gabinetto se non autorizzati da tale legge. 6. Decidere l'amnistia generale, l'amnistia speciale, la commutazione della pena, la tregua e il ripristino dei diritti".] 
I.4 Le radici storiche del pacifismo in Giappone: intervista a Karatani Kojin (2017)

Kojin Karatani (nato nel 1941 e docente alla Hosei University, alla Kinki University e visiting professor alla Columbia University) si laureò in economia all'Università di Tokyo, e dal 1969 è attivo come critico letterario e come studioso di filosofia. È autore anche di un libro sulla pace perpetua in Kant. ${ }^{\mathbf{3 6}}$ Nell'aprile 2016 ha pubblicato il volume L'inconscio e la Costituzione, ${ }^{37}$ la cui ricchezza di contenuti (tra cui il riferimento al Kant appena ricordato) è documentata dall'indice qui riprodotto:

Indice del volume: L'inconscio e la costituzione: I. La coscienza, l'inconscio e la costituzione. 1. Costituzione e inconscio; 2. La Prima guerra mondiale e Freud; 3. Il sistema imperiale e la rinuncia alla guerra 4. Inconscio e sondaggi di opinione. - II. I precedenti della costituzione. 1. Gli articoli 1 e 9; 2. I precedenti del testo costituzionale; 3. Dal consiglio degli anziani all' istituzione imperiale; 4. Alle radici della costituzione del dopoguerra; 5. Il sistema Tokugawa come sistema del dopoguerra. - III. La teoria della pace di Kant. 1. Chomin Nakae ${ }^{38}$ e Tokoku Kitamura; ${ }^{39}$ 2. Le critiche di Hegel alla teoria della pace kantiana; 3. La Storia universale e La Pace perpetua; 4. Kant e Marx; 5. Kant e Freud; 6. La forza del dono. - IV. Il nuovo movimento liberale e la guerra. 1. La revisione della teoria kantiana sulla pace; 2. L'imperialismo visto dal modello del libero scambio; 3. I tre tipi di accumulazione del capitale; 4. La politica economica di uno Stato egemone; 5. La trasformazione di uno Stato egemone; 6. L'ideologia liberale (jiyu shugi) e l'ideologia neoliberale (shin-jiyu shugi); 7. Corsi e ricorsi storici; 8. Le speranze per il futuro. Postfazione.

Un volume fortunato, che era giunto alla quinta edizione già nell'anno successivo alla prima pubblicazione. Per questo uno dei più importanti giornali giapponesi, il "Mainichi Shimbun", intervistò Kojin Karatani sui temi di quel libro e, in particolare, sulle radici dell'articolo 9 della costituzione giapponese che possono essere fatte risalire alla "Pax Tokugawa".0 L'intervista offre una sintesi degli argomenti affrontati da Karatani e viene qui tradotta per intero.

36 Kojin Karatani (2006), Sekai kyowakoku he.

37 Kojin Karatani (2016), Kenpo no muishiki, giunto alla quinta edizione nel 2017. Traduzione di Pier Giorgio Girasole.

38 [Chomin Nakae (1847-1901) introdusse le teorie di Rousseau in Giappone.]

39 [Tokoku Kitamura (1868-1894), scrittore e critico letterario giapponese, fu uno dei fondatori del movimento pacifista.]

40 Fonte: Keita Minami (2017, 27 novembre), Kempo 9-jo no genzai ishiki rutsu wa "Tokugawa no heiwa. La traduzione e le note tra parentesi quadra sono di Pier Giorgio Girasole. 
Intervista a Kojin Karatani sull'articolo 9 e sulla "pax Tokugawa" ("Mainichi Shimbun", 27 novembre 2017).

A ottobre [2017] alla Camera dei Rappresentati il Partito Liberal Democratico di maggioranza, insieme con il Komeito, ${ }^{41}$ il Partito della Speranza e il Restoration Party of Japan, noti come "i riformisti costituzionali", hanno ottenuto i due terzi dei seggi rendendo concreta l'ipotesi di un emendamento [costituzionale] soprattutto per quanto concerne l'articolo 9. Si pensa infatti che il Primo Ministro Shinzo Abe voglia accentuare l'importanza delle Forze di Autodifesa (Jietai).

L'articolo 9 ci ha quindi portato dei benefici o ci ha ostacolato? Abbiamo chiesto al filosofo e pensatore Kojin Karatani, che ha 76 anni, se si possa parlare di coscienza reale dell'articolo 9.

Il Partito Liberal Democratico alla Camera dei Rappresentanti ha promesso di emendare quattro punti della costituzione, tra cui la ridefinizione dell'articolo 9 e delle Forze di Autodifesa, ed ha ottenuto la maggioranza. Ritiene che si possa prevedere una possibile modifica costituzionale per il futuro prossimo?

Fino ad oggi il Partito Liberal Democratico ha parlato di emendamenti che lasciassero immutato l'articolo 9, ridefinendo soltanto le Forze di Autodifesa. Il Primo Ministro Abe è stato il primo ad annunciare alla Camera Bassa di voler modificare l'articolo 9. Tuttavia, è probabile che voglia aggiungere solo una norma sull'inserimento delle Forze di Autodifesa nella Costituzione. $\mathrm{Ma}$ - anche se ottenesse i due terzi dei voti [necessari per una modifica costituzionale] - si andrebbe al voto e comunque la modifica verrebbe ritardata. Certamente il Primo Ministro Abe cercherà di abbreviare i tempi. Sembra che pensi di poter superare il referendum dicendo: "Non si tratta di un emendamento, ma di un aggiornamento della costituzione".

Mi chiedo però se, così facendo, potrà realmente modificare la Costituzione. Finché l'articolo 9 (che esclude il diritto di belligeranza del Paese) resterà tale, qualsiasi "aggiornamento" equivarrà a una modifica costituzio-

41 [Partito della Giustizia, ispirato al buddismo del movimento Sokka Gakkai.] 
nale. L'articolo 9 impedisce alle Forze di Autodifesa di operare in un Paese straniero. Il diritto all'autodifesa collettiva, che è una parafrasi di alleanza militare, non è consentito con l'attuale Costituzione. Il Primo Ministro Abe, se davvero ambisce a modificare la Costituzione, dovrebbe leggerla con molta attenzione. Ma non può farlo, perché rischierebbe di perdere il referendum [necessario per modificare la costituzione].

Perché pensa che un referendum popolare possa respingere un emendamento costituzionale?

Perché sull'articolo 9 non è una questione di coscienza collettiva giapponese, bensì di inconscio. L'inconscio viene spesso equiparato al subconscio, ma non è così. Sono due entità distinte. Il subconscio può venir influenzato dall'educazione o dai media. L'inconscio invece è stato anche chiamato con il nome di superego dallo psicoanalista austriaco Freud (1856-1939). Ed è proprio il superego/inconscio a governare la coscienza. L'articolo 9 proveniva da esperienze di guerra giapponesi, che però non vanno intese come riflessioni coscienti. Pertanto non può essere modificato dall'educazione o dai media. Se l'articolo 9 fosse dovuto a una riflessione consapevole, sarebbe stato abbandonato già da tempo.

\section{Allora perché l'articolo 9 è radicato nell'inconscio giapponese?}

Bisogna tener presente che l'articolo 9 era stato imposto dal Quartier Generale delle forze alleate (GHQ). A quel tempo, MacArthur pensava che, se non si fosse conservato il sistema imperiale, in Giappone si sarebbe verificata una grande ribellione. L'articolo 9 era perciò fondamentale per rendere effettivo l'articolo 1 della Costituzione (che prescriveva il ruolo dell'imperatore solo come simbolo della sovranità nazionale) e poter dimostrare - per esempio all'Unione Sovietica - come "il Giappone fosse cambiato" con gli Alleati.

Tuttavia, non c'è contraddizione tra il fatto che l'articolo 9 sia stato imposto dal GHQ e che i Giapponesi lo abbiano accettato volontariamente. In realtà, sebbene MacArthur abbia imposto la Costituzione, essa è stata controfirmata dal Primo Ministro giapponese di allora, Yoshida [Shigeru]. Venne così dapprima imposto "il rifiuto della guerra" da parte di forze esterne come quelle alleate, cui seguì una coscienza che ha ulteriormente 
cercato "il rifiuto della guerra". In questo senso l'articolo 9 è una scelta volontaria del popolo giapponese. È un aspetto di quello che si chiama "cultura".

È possibile trovare degli eventuali antecedenti dell'articolo 9 nella storia del Giappone?

Dopo il lungo periodo degli Stati Combattenti, ${ }^{42}$ si instaurò il sistema politico dello shogunato Tokugawa che evitava le guerre e che ebbe come conseguenza una pace presente non solo in Giappone, ma in tutta l'Asia orientale. Quest'epoca viene chiamata anche "pax Tokugawa". Il samurai portava sì una spada, ma questa era un simbolo di identità, non un'arma. La cultura di Tokugawa è la "forma embrionale" dello spirito dell'articolo 9. Tuttavia, dopo la Restaurazione Meiji, il Giappone inaugurò il sistema della coscrizione obbligatoria, colonizzò la penisola coreana e invase la Cina. L'articolo 9 affonda le sue radici nel rimpianto inconscio per la "pax Tokugawa" che i Giapponesi provarono dopo la restaurazione Meiji [a partire dal 1868].

Non dimentichiamo come anche l'articolo 1 abbia le sue radici nel periodo dello shogunato Tokugawa. Lo shogun considerò infatti la figura dell'imperatore con attenzione e rispetto. Se lo shogun decise di opporsi all'imperatore fu solo perché dei daimyo rivali avevano deciso di farne la propria bandiera contro lo shogunato. ${ }^{43}$ Lo shogun Tokugawa pose l'imperatore al vertice della struttura dello Stato, isolandolo dalla politica attiva ma inserendolo comunque nel sistema dello shogunato. Non ci sono dubbi che questa fu la prima manifestazione dell'Imperatore inteso come simbolo, come avvenne poi nel secondo dopoguerra e continua tuttora.

42 [L'epoca Sengoku, XIV-XV secolo, è nota come epoca degli "Stati Combattenti" ed è anteriore a quella della pax Tokugawa.]

43 [Riferimento alla guerra Boshin, combattuta tra il 1868 ed il 1869, che vide la sconfitta dello Shogunato e l'ascesa del potere imperiale nel Giappone moderno.] 
Quale connessione vede tra l'articolo 1 e l'articolo 9 dell'attuale Costituzione? ${ }^{44}$

Esiste una relazione di interdipendenza tra l'articolo 1 e l'articolo 9. L'attuale Imperatore e la sua consorte sono i primi garanti dell'articolo 9. Si può dire che [alla fine della guerra] l'Imperatore, assumendo su di sé la responsabilità della guerra condotta dalla nazione giapponese, abbia protetto la propria linea dinastica. In altre parole, proteggere l'articolo 9 significa proteggere anche l'articolo 1.

In passato si diceva: "L'articolo 9 (rifiuto della guerra) garantisce l'articolo 1 (imperatore)", mentre oggi la situazione è rovesciata: "L'articolo 1 garantisce l'articolo 9". ${ }^{45}$

Qual è il ruolo svolto dall'articolo 9 nella comunità internazionale?

Penso che "il rifiuto della guerra" dell'articolo 9 non sia un semplice rifiuto, ma debba essere considerato come un "dono" rivolto alla comunità internazionale. Che uso se ne farà? Ad esempio, se un paese attaccasse o minacciasse il Giappone indifeso, verrebbe condannato dalla comunità internazionale. Avendo infatti garantito un "dono", il Giappone non resterà indifeso. Vincerà l'opinione pubblica internazionale. Il potere del dono supera quello della forza armata e dell'economia.

Data la tensione con la Corea del Nord, sembra che questo modo di pensare sia "troppo idealista".

In realtà, il Giappone, essendo dotato delle Forze di Autodifesa, non "rispetta" alla lettera l'articolo 9, e questo rappresenta una grave minaccia anche per la Corea del Nord. Tuttavia, se si rispettasse alla lettera l'articolo 9, la situazione sarebbe diversa: il Giappone potrebbe dimostrare all'Assemblea

44 Costituzione giapponese del 1946: “Art. 1. - L'Imperatore è il simbolo dello Stato e dell'unità del popolo; egli deriva le sue funzioni dalla volontà del popolo, in cui risiede il potere sovrano".

45 [Il che significa: grazie al rifiuto della guerra (art. 9) l'imperatore non è stato coinvolto nei processi alla fine della guerra; e, simmetricamente, l'esistenza in carica dell'imperatore oggi garantisce il rifiuto della guerra.] 
generale delle Nazioni Unite "la completa attuazione dell'articolo 9" e, per questa via, portare le nazioni vincitrici della Seconda guerra mondiale a modificare il loro ruolo di leadership avuto finora ed a realizzare la "Repubblica mondiale" cui aspirava il filosofo tedesco Kant (1724-1804).

Mi si consenta un'ultima parola come intervistatore. Ci si chiede perché i cittadini giapponesi non abbiano provato a modificare prima l'articolo 9 della Costituzione che oggi è al centro dei dibattiti. Se pensiamo che la ragione che porta a sostenere l'articolo 9 sia un atteggiamento collettivo contro la guerra, oggi questo atteggiamento dovrebbe indebolirsi perché siamo di fronte a numerose generazioni che non la conoscono per nulla. Eppure non è così. Il professor Karatani ne individua la causa nell'inconscio collettivo del popolo giapponese e spiega che "non c'è incoerenza nel fatto che l'articolo 9 sia stato imposto, ma anche accettato volontariamente dai Giapponesi". Quindi sarebbe possibile una psicanalisi dell'inconscio nei confronti dell'articolo 9? È difficile giungere a una conclusione logica, ma le considerazioni del professor Karatani ci offrono del materiale per riflettere sull'articolo 9 .

\section{I.5. Il congresso del Partito Liberal-Democratico (LDP): verso un art. 9 bis (2018)}

La crescente tensione con la Corea del Nord (e quella più taciuta ma non meno grave con la Cina nel Mar Cinese Meridionale) hanno riportato al centro del dibattito politico la revisione dell'articolo 9. Nel marzo 2018 ne ha discusso anche il congresso nazionale del partito di maggioranza - il Partito Liberal-Democratico $(L D P)$ - e devo alla cortesia della costituzionalista Hidemi Suzuki, docente della Keio University, $i$ testi inglesi del "Yomiuri Shimbun" qui di seguito tradotti.

Nel secondo di essi si parla del "caso Moritomo", sul quale va fornito qualche chiarimento: si tratta di una controversia o scandalo (secondo il punto di vista) in cui il premier Shinzo Abe venne indirettamente coinvolto attraverso l'attività della moglie nella "Gakko Hojin Moritomo Gakuen", un'impresa che gestisce alcune scuole private secondo un indirizzo ultranazionalista: ogni giorno vi si leggeva infatti il rescritto imperiale sull'educazione, seguendo un uso praticato dal 1890 al 1945. Per la costruzione di una scuola elementare questa impresa acquistò un terreno governativo a un prezzo di eccessivo favore: prezzo tenuto dapprima segreto, ma rivelato dall"Asabi Shimbun" il 9 febbraio 2017. La vicenda coinvolse la moglie del premier Shinzo Abe (nominata presidente onoraria della scuola, e 
poi dimessasi) e vari ministri del suo governo, anche perché $i$ documenti della transazione vennero manipolati o non vennero più ritrovati. Un alto funzionario del Ministero delle Finanze che aveva già dovuto deporre sul caso Moritomo, Nobushisa Sagawa, venne riconvocato l'anno dopo. L'opposizione chiese le dimissioni del ministro delle finanze e anche dell'intero governo Abe ${ }^{\mathbf{4 6}} \mathrm{A}$ partire dal 2017 lo "scandalo Moritomo" incide sulla sempre vasta popolarità del premier Shinzo Abe. ${ }^{47}$

a) Shinzo Abe deciso a modificare la costituzione ${ }^{48}$

Nel marzo 2018, in occasione del congresso del Partito Liberal-Democratico (LDP), il primo ministro e presidente di quel partito Shinzo Abe comunicò la sua decisione di modificare la costituzione in quattro punti, dando così alle Forze di Autodifesa anche quel fondamento costituzionale che finora era loro mancato.

La revisione costituzionale è "un compito che il partito si è assunto sin dalla sua fondazione, e vogliamo assolverlo nel corso di questa generazione", ha detto Abe sabato [24 marzo 2018] in un incontro presso il quartier generale del Partito Liberale Democratico con i segretari generali delle sezioni locali del partito: "Vogliamo giungere a questo risultato".

Sul fatto che il Ministero delle Finanze abbia modificato i documenti sulla vendita di terreni di Stato all'impresa scolastica Moritomo Gakuen [di cui si è parlato in apertura di questa parte I.5], il primo ministro ha espresso il suo rincrescimento con queste parole: "Sono dolorosamente consapevole della grave responsabilità in quanto a capo del settore amministrativo". E ha aggiunto: "Sono deciso ad esaminare a fondo le ragioni che hanno generato quel problema, a chiarire l'intera situazione e a ricostruire dalle

46 Sagawa to testify in Diet on March 27 over falsified files, in: The Asabi Shimbun (2018, 20 marzo).

47 Di questo scandalo si è occupata anche la stampa italiana nel marzo 2018 (cfr. ad esempio in: Il Sole - 24 Ore, http://argomenti.ilsole24ore.com/moritomo-gakuen.html).

48 Fonte: Abe determined to revise Constitution (2018, March 26). Didascalia della foto: "Il primo ministro Shinzo Abe parla alla convenzione del Liberal Democratic Party (LDP) a Minato Ward, Tokyo, dove ha riaffermato la sua volontà di portare a termine la revisione dell'art. 9 della Costituzione". 
fondamenta l'organizzazione [amministrativa] in modo che questo problema non abbia mai più a ripetersi”.

Prima dell'incontro, alcuni dirigenti del partito - tra cui Hiroyuki Hosoda, in carica come Presidente del Quartier Generale per la Revisione della Costituzione - aveva indetto una riunione dei principali funzionari delle sezioni di partito delle prefetture ed aveva loro presentato un progetto di revisione costituzionale suddiviso in quattro parti.

I dirigenti del partito spiegarono che la loro proposta di includere le Forze di Autodifesa nella Costituzione - inclusione con la quale l'art. 9 viene conservato perché l'esistenza delle Forze di Autodifesa verrà sancita da un art. 9 bis - è una priorità per il partito.

A Hosoda è stato affidato il compito di redigere il progetto di riforma proposto dal partito, ed egli intende fondarne il testo soprattutto su questa proposta.

Durante l'incontro affiorarono anche opinioni diverse presenti nel partito. Una di esse propone di eliminare il divieto di detenere potenziale bellico (contenuto nel secondo comma dell'art. 9) e nel dichiarare [ufficialmente] l'esistenza delle Forze di Autodifesa.

I dirigenti del partito presentarono anche tre proposte legislative su tre temi ulteriori: il miglioramento dell'istruzione; il fronteggiare le situazioni di emergenza; e l'eliminazione delle coalizioni (merged constituencies) nelle elezioni dei House of Councillors (Senato).

Il partito ha approvato queste tre proposte.

Forte reazione dell'opposizione.

I partiti dell'opposizione, compreso il Partito Democratico Costituzionale del Giappone (CDPJ), stanno intensificando la loro opposizione ai tentativi del LDP di modificare la costituzione.

La loro forte opposizione alle modifiche costituzionali sembra dovuta in parte alla crescente sfiducia nei confronti di Abe a causa dello scandalo dovuto all'alterazione di documenti nella vicenda legata a Moritomo Gakuen.

Il leader del CPDJ, Yukio Edano, ha apertamente preso le distanze dalla proposta di modifica costituzionale del LDP che asserisce l'esistenza delle Forze di Autodifesa con l'espressione "prendere le necessarie misure di autodifesa". 
Sabato scorso [24 marzo 2018], a Sendai, Edano ha comunicato alla stampa che "l'LDP ha ripetuto che l'estensione del diritto all'autodifesa non avrà luogo anche se si modifica l'art. 9: però non possiamo fidarci”.

Il maggior partito di opposizione (il CPDJ, la cui politica si fonda sul costituzionalismo) concorda invece con le revisioni legislative come quelle che limiterebbero il potere del Governo di sciogliere il parlamento.

A dicembre [2017] il CPDJ ha reso pubbliche le sue linee-guida per il dibattito sulla revisione costituzionale, che comprendono anche un'estensione del diritto di informazione su questo tema. D'altra parte, questo partito si oppone in questi termini alla revisione dell'art. 9 desiderata da $\mathrm{Abe}$ : “Ci opponiamo a un cambiamento peggiorativo dell'art. 9, che abbandona una politica orientata unicamente all'autodifesa".

In una conferenza stampa di giovedì [22 marzo 2018] il presidente del JCP [Japanese Communist Party], Kazuo Shii, ha criticato l'amministrazione Abe chiedendo: "Un'amministrazione che non si vergogna di infrangere la costituzione, ha il diritto di modificare la costituzione?"

Il Partito della Speranza (Kibo no To), favorevole alla modifica costituzionale al momento della sua fondazione nello scorso anno, ha modificato la sua posizione. Questo partito è andato aumentando il suo atteggiamento critico verso il governo Abe dopo che Yuriko Koike, nella carica di governatore conservatore di Tokyo, è stata sostituita da Yuichiro Tamaki, ex membro del Partito Democratico.

In mezzo alle tensioni politiche generate dallo scandalo Moritomo Gakuen, anche il Partito per la Restaurazione del Giappone (Nippon Ishin no Kai), vicino ad Abe, ha iniziato a prendere le distanze dai tentativi di rivedere la costituzione. "In questo momento il Parlamento non è in grado di prendere decisioni su questo tema con la necessaria freddezza", ha affermato Ichiro Matsui, leader di Nippon Ishin no Kai.

b) Il tentativo di revisione costituzionale del LDP travolto dal "caso Moritomo" - Diminuisce l'influenza di Abe a causa dello scandalo ${ }^{49}$

È sempre meno chiaro se il Partito Liberal-Democratico, oggi al potere, sarà in grado, ancora durante il presente anno [2018], di realizzare il suo obietti-

49 Fonte: Yasumasa Takada, Akinisa Ota [redazione del Yomiuri Shimbun] (2018, March 26), LPD quest to revise top law buffeted by Moritomo scandal. Didascalia della foto: Sabato [24 marzo 2018] a Tokyo, il Primo ministro Shinzo Abe parla all'incontro con i segretari generali delle sezioni locali del LDP. 
vo di far approvare dal Parlamento le modifiche costituzionali, dal momento che il Primo Ministro e Presidente del partito Shinzo Abe sta perdendo peso politico a causa della vicenda della vendita di terreni di Stato all'operatore scolastico Moritomo Gakuen.

Abe ha insistito sulla sua posizione a sostegno degli emendamenti costituzionali durante un incontro con i segretari generali delle sezioni locali dell'LPD, lo scorso sabato [24 marzo 2018]. Infatti la revisione costituzionale è parte del programma di partito dalla sua fondazione.

Nello stesso giorno, in un incontro presso il quartier generale del partito a Tokyo, Hiroyuki Hosoda (Presidente del Quartier Generale per la Revisione della Costituzione) aveva invitato i dirigenti del partito e i principali funzionari delle sezioni di partito delle prefetture a unire i loro sforzi in vista di un referendum nazionale sulla revisione costituzionale. "Vi invito caldamente, ha detto Hosoda, - a predisporre un ambiente favorevole, facendo sforzi ovunque, per conseguire, in un referendum nazionale, la maggioranza non del 51\%, bensì del 60\% o del 70\%\%"

Quell'incontro è stato un'eccellente occasione per informare a fondo i membri del partito dell'intenzione della direzione di iniziare in parlamento le revisioni costituzionali entro la fine del presente anno [2018]. Infatti all'incontro partecipavano rappresentanti delle sezioni locali del partito e membri del partito presenti negli organi elettivi locali.

Dopo aver così consolidato la loro posizione su come procedere alla revisione di quattro temi costituzionali, i dirigenti del Quartier Generale per la Revisione della Costituzione presentarono uno schema secondo il quale avrebbero potuto trarre vantaggio da un forte appello all'opinione pubblica.

\section{Entusiasmo calante nel partito.}

Però, all'interno del partito, sulla revisione costituzionale si registra meno entusiasmo di quanto ci si aspettava, perché il governo sta incontrando difficoltà a causa dell'alterazione di documenti ufficiali nel caso Moritomo Gakuen.

Durante l'incontro sopra ricordato, alcuni partecipanti incoraggiarono i dirigenti del partito ad affrontare la revisione costituzionale: "Portate a compimento questa vicenda senza farvi piegare", ha detto un partecipante. Altri invece manifestarono la loro preoccupazione sul possibile esito del progetto. 
All'ultimo incontro dei segretari generali locali, un membro della Prefettura di Osaka si è espresso così sulla vicenda Moritomo: il partito "deve rendere conto sino in fondo" dello scandalo sull'alterazione dei documenti.

Rivolgendosi ai partecipanti, Abe ha espresso il suo profondo dispiacere sulla vicenda Moritomo, dopo di che ha menzionato solo brevemente le revisioni costituzionali. Si può dire che il partito abbia perso la sua capacità d'impulso nella modifica costituzionale, sotto i colpi delle critiche al primo ministro per la vicenda Moritomo. Alcuni membri di partito si sono spinti sino a temere che le proposte di modifica costituzionale siano percepite soltanto come proposte personali di Abe. Il caso Moritomo getterà probabilmente un'ombra sulle trattative tra il partito di maggioranza e quelli di opposizione.

A partire dal congresso del partito di domenica [25 marzo 2018], il LDP spera di seguire un piano in cui presentare i suoi progetti di modifica costituzionale alle Commissioni affari costituzionali di entrambe le camere del parlamento e di iniziare una serie di colloqui con i partiti di opposizione, in modo da poter presentare ufficialmente al parlamento la proposta di emendamento costituzionale durante la sessione straordinaria d'autunno.

I partiti d'opposizione sono però nettamente contrari all'attuale posizione del governo sul caso Moritomo, mettendo a rischio i progetti di riforma legislativa sul lavoro, cui il governo ha dato priorità.

\section{Dubbi si addensano sul Komeito.}

Il partito Komeito ${ }^{50}$ è il più recente alleato dell'attuale governo di coalizione e non è particolarmente allineato con il LPD, perché è sempre stato tradizionalmente tiepido sul tema della revisione costituzionale. Un dirigente del Komeito ha affermato: "L'LPD ha messo a punto le proposte di emendamento costituzionale solo per giungere in tempo al congresso di partito".

Kazuo Kitagawa, presidente della Commissione di Ricerca per la Costituzione del Komeito, si è mostrato disponibile a riformare la legge nazionale sul referendum, come richiesto dai partiti di opposizione. 
Nel LDP si sospetta che il Komeito voglia dare priorità alla revisione della legge sul referendum nazionale, insieme con i partiti dell'opposizione, mettendo così in secondo piano il dibattito sugli emendamenti costituzionali.

Se dovessero sorgere difficoltà nell'iniziare in parlamento per la fine dell'anno [2018] le revisioni costituzionali, diverrebbe problematico realizzare il calendario proposto dal LDP.

Un punto essenziale nell'elezione della House of Councillors (Senato), nell'estate del prossimo anno [2019], riguarda la possibilità che i due terzi dei seggi continuino ad essere assegnati ai partiti di maggioranza e ai loro membri (quindi favorevoli alle modifiche costituzionali). Il LDP vuole proporre le revisioni costituzionali al più tardi prima delle elezioni della Camera Alta, ma sono già in calendario altri importanti eventi come le elezioni locali su scala nazionale e l'abdicazione dell'Imperatore.

Lo stesso LDP non è unito su questi temi. A settembre [2018] è prevista l'elezione del presidente del LDP ed è probabile che in quell'occasione si manifestino contrasti su come presentare i provvedimenti che nella costituzione dovranno regolare le Forze di Autodifesa.

Il precedente segretario generale del LDP, Shigeru Ishiba, che aveva manifestato la sua intenzione di candidarsi alla presidenza, venerdì scorso nel suo blog ha criticato il primo ministro. Ishiba ha detto: "Provo un forte senso di disagio per il modo in cui il LDP ha deciso di conservare l'art. 9 senza alcuna spiegazione da parte del presidente”.

c) Testo del progetto dell'art. 9 bis.

Proposta di modifica dell'articolo 9 della Costituzione

da parte del Partito Liberal-Democratico ${ }^{51}$

Con questa proposta, il partito di maggioranza non interviene direttamente sul testo dell'originario art. 9, ma vi aggiunge il riconoscimento giuridico dell'esistenza delle Forze di Autodifesa, finora negata dalla lettera dell'art. 9: "land, sea, and air forces, as well as other war potential, will never be maintained. The right of belligerency of the state will not be recognized".

51 Fonte: Sankei Shinbun, 25 marzo 2018, importante quotidiano di destra; il testo è tratto da un'immagine del documento nel sito del Sankei Shimbun: http:/www.sankei.com/politics /news/180325/plt1803250009-n1.html. Traduzione di Pier Giorgio Girasole. 
Articolo 9 bis.

(Comma 1) Ferma restando la normativa dell'articolo precedente che garantisce protezione al nostro Paese e stabilisce la necessità delle Forze di Autodifesa per il nostro Stato e per i nostri cittadini come forza collettiva, nel rispetto della legge chiediamo di mantenere le Forze di Autodifesa sotto la guida del Primo Ministro come comandante supremo.

(Comma 2) Le Forze di Autodifesa serviranno lo Stato secondo le direttive approvate in Parlamento e secondo quanto stabilito dalle leggi.

\section{Italia: Tra guerra e pace: D’Annunzio, Shimoi Harukichi, Stalin e Bobbio}

II.1. D'Annunzio tra l'Occidente "che non ci ama" e l'“esempio inaudito di trasformazione" del Giappone

I rapporti tra Gabriele D'Annunzio e Shimoi Harukichi sono stati illustrati alle pp. 17-26. Essi riflettono, da un lato, quel momento veramente irripetibile che fu l'occupazione di Fiume e lo spirito che ne animò i protagonisti (il "fiumanesimo"), $e$, dall'altro, la visione dannunziana dell'Oriente estremo: un luogo di sogno e, al tempo stesso, un modello di potente affermazione nazionale contro le avversità. Non solo in D'Annunzio, ma anche in tutt'Europa era ancora forte lo stupore per la vittoria dell'esercito giapponese su quello zarista, nella guerra del 1904-1905.

Qui di seguito vengono riportati due discorsi di D'Annunzio. Il primo del 1919 sulla sua visione generale dell'Oriente e, in particolare, sul suo progetto di volo in Estremo Oriente: un testo in cui i richiami ai gloriosi viaggiatori del passato si intrecciano con l'amarezza sul presente dell'Italia appena uscita dalla Grande Guerra con una vittoria mutilata, ma percorso anche da futuristici fremiti d'entusiasmo per le macchine volanti. Il secondo del 1920 in onore del "fratello samurai" Shimoi Harukichi, che per lui incarnava quel suo mondo orientale, verso cui progettavano insieme di volare.

a) Dopo l'inerzia forzata, “Si vola!": e allora "a Tokio, in dieci o dodici tappe"

D’Annunzio tenne il primo discorso nel 1919, quando l'avventura fumana non era ancora iniziata ed egli era incerto tra la marcia su Fiume e il volo a Tokyo progettato con Shimoi. La guerra era terminata e finalmente $i$ comandi avevano revocato la sospensione dei voli: di qui il titolo del discorso, L'ala d'Italia è

II. Italia 
liberata. D'Annunzio ricorda i giorni dell'inazione, "quando per la prima volta, dissipando col gesto il fumo delle sigarette e delle malinconie, io proposi la rotta dell'Estremo Oriente". Allora poteva sembrare quasi un diversivo contro la noia nata dall'inazione dopo la guerra, ma ora quel piano assumeva forme più concrete nelle parole del giovane Natale Palli (1895-1919), il pilota che aveva guidato l'aereo con D'Annunzio nel volo su Vienna il 9 agosto 1918.

Quel piano di volo verso l'Oriente estremo traccia non solo le singole tappe di un'impresa ardimentosa, ma suggerisce anche la via di fuga, almeno momentanea, "dall'Occidente che non ci ama e non ci vuole", perché insiste "in ostinate ingiustizie e in ostinate servitù," come appunto la mancata annessione di Fiume all'Italia dovuta soprattutto al diniego degli Stati Uniti. E qui dalla filigrana dannunziana traspaiono già il linguaggio e l'ideologia del fascismo che andava prendendo forma in quegli anni e per il quale il fiumanesimo costitui sotto più aspetti un'ispirazione: per D'Annunzio, l'Occidente - "lo splendore dello spirito senza tramonto" - si è estinto, ed "è divenuto un'immensa banca giudea in servizio della spietata plutocrazia transatlantica".

Nel discorso di D'Annunzio il volo verso l'Estremo Oriente è a tratti un'impresa futuristica della macchina volante, a tratti una rêverie d'evasione da un mondo che si avverte ostile. In questo primo discorso la figura centrale è Natale Palli, che traccia l'itinerario d'un viaggio ancora onirico, costellato di tappe dagli affascinanti nomi esotici. Però "l'eroe fanciullo" non vedrà $i$ "palmeti che fanno vento alle stelle". Morirà invece nel 1919 - a ventitré anni - schiantandosi sulle Alpi in un raid che era anche una prova in vista dell'impresa verso l'Estremo Oriente: "Natal Palli, comandante della Serenissima, dopo guidate le gloriose aquile d'Italia sul cielo di Vienna e su cento campi nemici, cadde sul Monte Pourry di Savoia in un volo superbo di giovinezza, di ardimento e di fede", come ricorda la lapide sulla sua casa in un quieto viale di Casale Monferrato.

Gabriele D'Annunzio, L'Ala d'Italia è liberata. ${ }^{52}$

Compagni, dopo giorni e giorni d'irosa tristezza, ecco che il mio cuore balza di gioia. Ecco che anche una volta io sono per voi un messaggero di gioia. Vi porto un grande annuncio. L'Ala d'Italia è liberata.

52 Gabriele D'Annunzio (1932d), L'ala d'Italia è liberata, di cui vengono qui riprodotte alcune pagine iniziali e, per intero, le pp. 248-255. 
Mi sembra di tornare al tempo che giungevo d'improvviso su i vostri campi lontani, su i bei campi del Veneto e del Friuli, quando per compiere un'impresa ardita era necessario lottare contro l'inettitudine e il malvolere dei Capi ( $\mathrm{mi}$ ci vollero quasi tre anni di pertinacia per ottenere licenza di volo su Vienna); e, avendo alfine strappato un consenso difficile, gridavo alla vostra ansietà: "Si vola! Si vola!" [...53].

L'annuncio di oggi sotto il cielo di pace è come l'annuncio di allora sotto il cielo di guerra. Un soffio eroico rompe l'aria morta.

Avete veduto su questo campo atterrare apparecchi stranieri in sosta per seguire le rotte di levante. Al paragone i nostri erano come il purosangue di Federico Tesio accanto all'alfana di Mambrino. Non appariva in quelli nessuna cura della linea, della leggerezza, della proporzione. Nei nostri già appare lo stile; il quale non è se non il giusto rilievo formale della forza e della destinazione di essa forza.

Considerate il carattere potente di quel sia $9 \mathrm{~B}^{54}$ [239|240] che adoperai nell'ultima offensiva. Considerate il carattere grazioso di quel sva $5^{55}$ che maravigliò Vienna. Nell'uno e nell'altro è raggiunto un tipo singolare di bellezza conveniente, come in un oggetto dell'industria antica, come in una lanterna del Caparra, come in un violino del Guarnieri.

Già, durante la guerra, affermammo il primato nella costruzione dei nostri apparecchi da battaglia. Nessuno degli Stati alleati o avversi era riuscito a costruirne e a usarne di così robusti e ingenti. In condizioni infauste, nuove forme, nuove qualità, nuove misure erano via via create dai costruttori per adattare gli apparecchi a nuovi servizii. L'arte degli antichi maestri italiani conduceva la mano degli operai nel pulire cèntine e travi, nel sagomare ali e timoni, nell'inflettere fusoliere e carlinghe. Tutte le potenze dei motori erano provate di grado in grado, fino alla massima di circa settecento cavalli. Con un solo motore andammo a Vienna e ne tornammo. Con un solo motore io e Natale Palli venimmo dalla fronte dell'Aisne al Po in poco

53 Da p. 233 a 239 D’Annunzio rievoca i compagni delle passate imprese.

54 È la seconda versione del ricognitore-bombardiere biplano monomotore prodotto dalla Società Italiana Aviazione (SIA), che divenne in seguito FIAT Aviazione.

55 È un aereo analogo al precedente, prodotto dalla società Ansaldo su progetto di Umberto Savoja e Rodolfo Verduzio. Dalle iniziali dei progettisti e della società deriva l'acronimo SVA che designò una decina di versioni di questo aereo di grande successo: compì, tra l'altro, non solo il volo su Vienna (lo SVA 10 di D'Annunzio è conservato al Vittoriale), ma anche il raid Roma-Tokyo di Arturo Ferrarin. 
più di tre ore. Con un solo motore seguiremo l'itinerario dell'Estremo Oriente per tappe cotidiane di circa duemila chilometri. [...] Fra breve una macchina del medesimo tipo [sia 9 B], ma capace d'un volo continuo di quattromila chilometri, porterà nel giro della sua elica l'astro dell'Italia nova ai nostri fratelli del Brasile e dell'Argentina, che l'invocano e l'aspettano. ${ }^{56}$

Non ci sono ancòra nella terra lontana campi battuti per l'atterrata delle grandi aquile? Ma ci sono [241|242] fiumi laghi mari, ci sono foci e porti, per l'arrivata dei grandi àlbatri [... D'Annunzio si ricollega qui a un colloquio con il generale Sailer, che gli parlava così della gente del Carso: "Chi può dubitare delle sorti di questa nostra razza inesauribile e incoercibile?" ${ }^{27}$ ].

Di quella buona materia umana una parte rimarrà attaccata alla sua terra; ma una parte s'involerà verso l'avventura e la conquista, una parte metterà le ali, $[244 \mid 245]$ intraprenderà le mille e mille vie azzurre, andrà di là dai mari $\mathrm{e}$ dai deserti e dalle montagne senza più temere l'ostacolo, aprirà i tramiti senz'orme e le rotte senza scìa verso quell'Oriente estremo dove giunsero con lenta fretta $i$ "tre latini"."

Liberiamoci dall'Occidente che non ci ama e non ci vuole. Volgiamo le spalle all'Occidente che ogni giorno più si sterilisce e s'infetta e si disonora in ostinate ingiustizie e in ostinate servitù. Separiamoci dall'Occidente degenere che, dimentico d'aver contenuto nel suo nome "lo splendore dello spirito senza tramonto", è divenuto un'immensa banca giudea in servizio della spietata plutocrazia transatlantica.

L'Italia che "sola è grande e sola è pura", l'Italia delusa, l'Italia tradita, l'Italia povera si volga di nuovo all'Oriente dove fu fiso lo sguardo de' suoi secoli più fieri. Non ode l'appello degli Arabi e degli Indi oppressi appunto da quei giusti che tengono la nostra Malta e ci strappano la nostra Fiume? Ad appello d'amore risposta d'amore, che non può essere se non alata, cioè spiritale. Le ali secondano oggi il senso vero della vita, che è la bramosia di ascendere per fatica e dolore alla conquista dello spirito.

56 Da San Paolo era giunta una delle prime sottoscrizioni per Fiume, come D'Annunzio ricorda nel discorso del 3 dicembre 1919: Agli Italiani di San Paolo, in: Gabriele D’Annunzio (1932b), La penultima ventura, Libro secondo, 157-158.

57 Da questo punto il testo viene riportato sino alla fine del discorso, cioè da p. 242 a p. 244 . Nel testo, D'Annunzio si ricollega a un colloquio con il generale Sailer, che gli parlava così della gente del Carso: “Chi può dubitare delle sorti di questa nostra razza inesauribile e incoercibile?”.

58 Marco Polo (1254-1324) viaggiò in Oriente con il padre Niccolò e con lo zio Matteo, come viene ricordato anche nel discorso in onore di Shimoi Harukichi. 
Oggi le "primavere sacre" si propagano per l'aria come il polline. Non v'è impedimento che le arresti, non v'è distanza che le affanni. Se il tempo è ringiovanito, lo spazio è riassunto. Lo stil novo del mondo [245|246] è lo scorcio. Come il Mantegna scorciava una figura, ecco che una guerra scorcia la storia, ecco che un'ala umana scorcia i più lunghi itinerarii dei mercatori, $\mathrm{i}$ più vasti peripli dei navigatori, e d'un tratto ci fa finitimi al Cataio di Marco Polo o alla Primavista di Sebastiano Caboto. ${ }^{59}$

L'Italia sia maestra anche di questi scorci aerei. L'arte del Tintoretto, che serrava in pochi palmi di tela la veemenza della folgore, passi alle calotte degli emisferi e alle carte degli atlanti.

"Si vola! Si vola!" L'annunzio di oggi sotto il cielo di pace è come l'annunzio di allora sotto il cielo di guerra.

I divieti sono aboliti, le restrizioni sono infrante. In questi otto mesi tetri di armistizio i vecchi capi sedentarii hanno opposto alle nostre impazienze: "Perché volete rischiare la vita? Perché volete consumare una tanto costosa materia? A che pro? Restate tranquilli a terra".

Avevamo il primato nella costruzione, avevamo il primato dell'arditezza. Ciascuno dei nostri apparecchi aveva fatto un lavoro per cento, ciascuno dei nostri piloti aveva fatto un lavoro per mille. Avevamo lottato contro i capi e contro il nemico. Avevamo vinto i capi e il nemico.

Ed ecco, finita la guerra, eravamo condannati a [246|247] marcire nell'ozio dei campi come in principio, quando il primo comandamento era di volare il meno possibile, di fare il meno possibile, di rischiare il meno possibile. Finita la guerra, rimaneva sospeso sui nostri coppi di cuoio ${ }^{\mathbf{6 0}}$ quest'ordine rigido: "Non è lecito volare se non per necessità belliche". E le necessità belliche si riducevano a ruminare e a ingoiare le umiliazioni cotidiane che ci infliggeva la Conferenza. Il sangue di Francesco Baracca, di Ugo Niutta, di Maurizio Pagliano, di Ignazio Lanza, di tanti altri eroi

59 A Sebastiano Caboto (1484-1557) è attribuita la scoperta di Terranova, "Primavista", o Terra Prima Vista.

60 "Celata senza cresta, gronda e visiera; specie di morione semplice e liscio, usato da soldati e marinai. D'Annunzio, II-921: 'Ti saluto, | a capo del naviglio tuo di corsa, | o duca dell'Egeo Marco Sanuto. | Sul tuo coppo di ferro splende l'Orsa"' (Salvatore Battaglia (1992), Grande dizionario della lingua italiana, s.v. 'Coppo': è l'undicesimo di 14 significati di 'coppo'). 
severi, era barattato con le lacrimette del "leguleio molle". ${ }^{61}$ Quella era la rugiada di pace per le nostre ali rattoppate che sapevano la guazza delle notti adriatiche!

Compagni, non è più proibito volare. Anzi da oggi la sentenza marina diventa celeste: "volare necesse est, vivere non est necesse". E il comandante della Prima Squadriglia Navale S. A. ha l'onore di dirvi che basteranno duecento siluranti aeree ad aver ragione di tutta la massa navale britannica nel nostro Mediterraneo.

Come càmpano nei ritrovi solitarii di Tripoli e di Bengasi le nostre belle squadriglie? Oziano ancòra? Risognano gli inseguimenti e i combattimenti nel cielo del Piave e del Grappa? Perché gli aquilotti restano prigionieri nelle gabbie roventi? Perché guatano verso il sud e non spiccano il volo? Mandate un'aquila [247|248] romana a liberarli. Consegnate a ciascuno il segno di Roma, il nome d'Italia. Fate che vadano a cercare i nuovi cittadini d'Italia nei villaggi e negli attendamenti più remoti. Fate che annientino il deserto di sabbia, fate che annientino il deserto di sale. Fate che fra Tripoli e Murzucco, fra Tripoli e Tummo la via gialla si muti in via cerulea e che l'oasi di Cufra veda giungere nel vento le ali tricolori come le bandiere tese della nuova Patria. Congiungete la Tripolitania all'Eritrea, la Cirenaica alla Somalia. I vittoriosi del cielo carsico, del cielo veneto, del cielo istriano mandateli a consolare i morti di Adua.

Lasciate che gli aviatori abbiano la lor sola guida nel loro istinto, come le rondini, come le cicogne, come le gru. Lasciateci andare, lasciateci osare. Lasciateci seguire i nostri destini, di mèta in mèta, di morte in morte, di gloria in gloria. Lasciateci il nostro privilegio di combattenti che possono e vogliono ancòra vincere, che possono e sanno ancòra morire. Quando le Fiamme nere, le Fiamme verdi, le Fiamme cremisi saranno disperse, si raduneranno tuttavia le Fiamme blu ${ }^{62}$ per andare "più alto e più oltre" verso l'ignoto, per tentare l'intentato, per osare l'inosabile.

Andiamo! Tentiamo! Osiamo!

61 Vittorio Emanuele Orlando (1860-1952), che rappresentò l'Italia alla Conferenza di pace di Parigi del 1919, fu anche un illustre giuspubblicista, docente in varie università italiane: di qui lo sprezzante appellativo datogli da D'Annunzio.

62 Riferimento alle mostreggiature che, nell'uniforme, individuano l'arma di appartenenza. Le fiamme a una, due o tre punte, individuavano gli Arditi (Fiamme nere), gli Alpini (Fiamme verdi), i Bersaglieri (Fiamme cremisi) e l'Aviazione (Fiamme blu). 
Quando io dicevo una di queste tre parole, tutti balzavano in piedi, laggiù, sotto le tettoie piene di [248|249] rombo; e sùbito i cuori toccavano la mèta proposta. Pareva che sùbito all'orizzonte periglioso i cuori fraterni facessero un astro rosso: l'astro umano infallibile, segnale sicuro di tutte le rotte tremende.

C'è qui qualcuno che si ricordi di quella sera grigia, nel campo di San Pelagio, ${ }^{63}$ quando per la prima volta, dissipando col gesto il fumo delle sigarette e delle malinconie, io proposi la rotta dell'Estremo Oriente?

Piovigginava. Ci opprimeva la tristezza dell'ozio costretto, il tedio della vana attesa, l'ambascia della vittoria mutilata e agonizzante, l'odio meschino delle oche per le aquile. A un tratto una finestra si spalancava su l'infinito. Il bel Rischio dal profilo tagliente si appoggiava al davanzale, e mirava lontano.

Dissi a Natale Palli: "Bisogna che andiamo a Tokio, in dieci o dodici tappe".

Tutti vibravano, tutti erano pronti. Pareva che tutti avessero già nell'anima il medesimo sogno, e che io non fossi se non l'indovino e l'interprete improvviso. "Andiamo!". E tutti volevano partire sùbito; come quando il condottiero dà il segnale a uno stormo di migratori e l'aria è mossa da un solo frullo concorde.

L'Oriente! L'antica magìa trasfigurava il nostro ricovero di legno in una pagoda di Delhi o in una casa da tè; e ciascuno già si sceglieva la sua baiadera e la sua geisha. E c'era là chi aveva poco più [249|250] di diciassette anni, come Marco Polo quando Nicolò e Matteo fratelli lo presero con loro e lo condussero a Layas, nella baia di Scanderun, ${ }^{64}$ di dove le carovane partivano per il paese mirabile.

Era dolce fantasticare. Avevamo dinanzi a noi su la mensa le piccole mele verdi che si gettano ai porci. "La sera del terzo giorno discenderemo a Basra, sul golfo Persico, e andremo a cercare i migliori datteri del mondo ne' suoi palmeti che fanno vento alle stelle. A mezzo del sesto giorno discenderemo presso la porta di Aladino, in una città morta del Gran Mogol, e ci dissete-

63 Lo scomodo campo di volo di San Pelagio, vicino a Monselice nel Veneto, fu la base della squadriglia "Serenissima" per il volo su Vienna. Il volo venne pianificato nella vicina Villa Zaborra Castello di San Pelagio, oggi sede di un Museo dell'aria e dello spazio, con un settore dedicato al volo su Vienna.

64 Scanderun o Alessandretta (in turco İskenderun), città portuale che oggi fa parte della Turchia. 
remo con l'uva moscata che si matura sui monti del Cabul, grossa come uova di colombe, conservata in una scatola di foglie spesse cucita con fili esatti di gramigna. E quanta pena avremo a salvare gli apparecchi dall'insolenza delle scimmie che salteranno su da le rovine come i 'liquidatori' e gli 'alienatori' degli 'uffici'!"

Gli occhi cesii di Natale Palli s'illuminavano come se riflettessero lo splendore del Tai-Mahal. Poi la sua fronte di ventitré anni si corrugava come la fronte stessa della saggezza meditabonda. Diceva: "Bisogna che il motore ci renda da mille e cinquecento a duemila chilometri al giorno, divisi in due tappe". Considerava la carta, davanti a sé. Soggiungeva: "Da Calcutta a Mandalay ci sono ottocento cinquanta chilometri. Da Mandalay a Hanoi, novecento cinquanta. [250|251] Bisogna farli in una sola giornata, con una sosta intermedia per riempire i serbatori”.

Già meditava la prova, da San Pelagio a Parigi, da Parigi a Roma in una sola giornata, per superare il previsto. E si proponeva di precedere ad ogni costo il rivale di Francia. Pensava che in quel momento il suo dovere d'Italiano gli comandasse di conficcare un'altra spina del valore italiano nel cuore geloso di chi, credendo al miracolo della Marna, negava i miracoli del Piave e di Vittorio Veneto.

Gli dicevo sorridendo: "Invece di scendere clamorosamente su una brutta terrazza delle Galeries Lafayette, come il caro Védrines, ${ }^{65}$ noi scenderemo in una sera di luna verso il fiume Giumma, ${ }^{66}$ sopra l'immensa loggia di marmi bianchi, nella reggia silenziosa del Gran Mogol, nella sala scoperta delle udienze dov'è il trono di marmo nero; e la notte sarà così chiara che distingueremo nel bianco i disegni sottili d'oro, d'argento, d'onice, di turchese e di porfido". ${ }^{67}$

65 L'aviatore Jules Védrines (1881-1919), nonostante il divieto della polizia, accettò la sfida delle Galeries Lafayette di atterrare su una terrazza di 28 metri per 12, sull'edificio delle Galeries stesse verso Boulevard Haussmann. Realizzò l'impresa il 19 gennaio 1919, ricevendo il premio messo in palio e la multa prevista dalla polizia. Una stele sul tetto ricorda l'aviatore che "a pour la première fois réalisé l'atterrissage d'un avion sur le toit d'une maison dans une grande ville ici même".

66 Il testo si riferisce al fiume Yamuna presso il Taj Mahal in India. Il nome "Giumma" per questo fiume nella stessa località, Agra, compare anche in Guido Gozzano: "Oggi, costeggiando le rive del Giumma, contemplo dal basso il maniero ciclopico” (Guido Gozzano (1917), Verso la cuna del mondo, 178).

67 Anche questa evocazione - tanto nell'ambiente quanto nella toponomastica - sembra ispirata alle pagine che Guido Gozzano dedica ad Agra: l'immacolata, nel suo Verso la cuna 
Fu questa l'ultima favola che raccontai all'eroe fanciullo. Era il pomeriggio del 19 marzo. Nel commiato egli lasciò non so che bagliore su la mia soglia, come se la predestinazione fosse già fatto divino.

Non calò egli sopra il candore dei marmi regali, in una sera calda di Agra; ma nella solitudine della neve perpetua, in una mattina di tormenta. Visse due [251-252] lunghi giorni con sé, col suo sacrifizio e col suo coraggio. Per due giorni fu più che umano, prima di farsi divino in eterno. Poi si addormentò col capo sul braccio, come si sarebbe addormentato accanto a me, in un vespro di bragia, presso Giaipur la Rosea o presso Benares la Santa, all'ombra dell'insegna di San Marco dipinta su la nostra fusoliera ormeggiata in un campo color di grogo ${ }^{68}$ sparso di corvi nerazzurri.

Il suo ultimo pensiero fu per la madre che lo portò, degna di portarlo. Ma sono certo che il penultimo fu per me, e che di me pensò: "Egli andrà, proseguirà. Questa è la prima tappa”.

Per lui, come per ogni spirito eroico, "il sogno è fratello dell'atto" e anche la morte non è se non un atto creatore, il più misterioso e virtuoso degli atti creatori.

Quando nel pomeriggio d'agosto atterrammo sul campo di San Pelagio tornando da Vienna, non c'eravamo ancora liberati dei nostri calzari e dei nostri camauri, ${ }^{69}$ che il Capo ci domandò nella prima commozione: "Che cosa possiamo fare per voi?”. Pronti rispondemmo, con un sol cuore: "Mandarci a Berlino".

Se domandassimo al suo spirito presente: "Che cosa possiamo noi fare per te?" egli risponderebbe: "Andare alla mèta che mi fu proposta e promessa".

Andremo. [252-253]

del mondo del 1917. Ad Agra si trova anche il Taj Mahal, mausoleo fatto costruire da un sovrano della dinastia Moghul, e richiamato nel testo di D'Annunzio poco sopra.

68 Salvatore Battaglia (1992), Grande dizionario della lingua italiana: da 'grogo' rinvia a 'gruogo': "Zafferano, croco”. "Color del gruogo: color giallorosso”, e adduce come esempio questo stesso passo di D’Annunzio (s.v. 'Gruogo').

69 "Berretto di velluto rosso aderente al capo e calzato fin sulle orecchie, portato dal papa", e adduce come esempio un passo di D'Annunzio: "vestiremmo le nostre pellicce, proveremmo le nostre armi, metteremmo i nostri camauri lanuti, i nostri calzari di pelle" (Salvatore Battaglia (1992), Grande dizionario della lingua italiana s.v. Camauro). Nel presente testo D’Annunzio sembra usarlo come sinonimo di 'coppo', di cuffia in cuoio da aviatore.) 
Egli era una volontà di vittoria. La sua carne non era stata messa al mondo se non per servire una volontà di vittoria. E quel che di lui non è morto, quel che di lui non poteva morire, e non potrà mai morire, è la volontà di vittoria.

Egli divenne un piccolo cadavere livido nel lenzuolo di neve; ${ }^{70} \mathrm{ma}$ in tutta l'Alpe non v'era picco che fosse acuminato ed eccelso come la sua volontà di vittoria. E in tutta l'Italia non v'era luogo che fosse tanto spiritualmente italiano quanto quel poco di suolo apparito di sotto la neve sciolta dall'ultimo calore del suo piccolo corpo e secondo la forma esatta del suo piccolo corpo.

L'alpigiano che lo trovò e lo palpò, disse fra sé: "È morto". Ma il compagno che primo si chinò su la sua faccia immobile, disse in sé: "Ha vinto".

Se, com'egli divenne un piccolo cadavere livido, io mi riducessi a un mucchio di carboni e di scorie in una valletta della Mesopotamia o su la riva sinistra del Gange un rimasuglio di rogo tra i roghi, prima di raggiungere la mèta prefissa, io avrei vinto; perché altri ineluttabilmente porterebbe più oltre la mia volontà di vittoria. $\mathrm{E}$ in tutta l'Italia non vi sarebbe sepolcro che fosse tanto italiano quanto quel mucchio solingo.

E dico che questa fede mia e di pochi, o di molti, è infinitamente più forte di tutte le dottrine radicate [253-254] nel ventre e di tutte le menzogne radicate nella viltà. Per ciò non può non vincere.

L'Ala liberata d'Italia si leva contro tutte le potenze avverse dello spirito. Per ciò la salute è in essa.

Fu già detto come l'ombra della macchina alata sia simile all'ombra del legno di sacrifizio e di salvazione.

Le sue doppie ali traverse, fra la prua e i timoni, formano la croce cruenta. E v'è un canto dei morti, v'è un canto degli immortali, che la confessano. Tutti i nostri martiri si levano gridando: Credo. ${ }^{71}$ Ecco che il grido si ripercuote da questo romanissimo campo in tutti i campi d'Italia.

70 L'aereo di Natale Palli incappò nella medesima tempesta che poco prima aveva obbligato Arturo Ferrarin a rinunciare a quello stesso raid. Palli precipitò con l'aereo ma si salvò, errò durante due giorni per le montagne innevate e morì congelato.

71 Questa parola è anche il titolo del discorso con cui D’Annunzio conclude il 1919 e traccia il bilancio dell'anno fiumano: Credo [XXXI decembre MCMXIX], in: Gabriele D'AnNunzio (1932b), La penultima ventura, Libro secondo, 205-212. "Versaglia vuol dire decrepitezza, infermità, ottusità, dolo, baratto, e ferocia che guarda con gli occhi sbarrati della paura. Ronchi vuol dire giovinezza, bellezza, ardimento, sacrifizio gioioso, mira lontana, novità 
O amore d'Italia, o amore di terra lontana, andremo, tenteremo, oseremo. Nulla via invia. Fu la parola della Comina ${ }^{72}$ compagni. Era scritta sulla mia prua liscia. Ve ne ricordate? È oggi la parola di Centocelle.

Quando i tre Polo, i "tre latini", si partirono da Acri pel maraviglioso viaggio, recavano un'ampolla d'olio del Santo Sepolcro, considerato dai divoti e dai convertiti come tutela contro ogni periglio e come rimedio contro ogni male.

Così potessimo noi portare un'ampolla di sangue dei nostri martiri, che ci riscaldasse nel gelo, che c'illuminasse nel buio e nel dubbio, che ci sanasse da ogni pensiero impuro, che ci rinnovasse in ogni [254|255] ora il coraggio, che c'inspirasse in ogni ora il sacrifizio, che ci preparasse in ogni ora a ben morire, che in ogni alba c'infondesse una nuova speranza, che ogni sera evocasse sul nostro patimento e su la nostra stanchezza di figli fragili il soffio divino dell'Italia eterna!

\section{b) Il saluto di Gabriele D'Annunzio al "fratello samurai" Shimoi Harukichi (1920)}

Il 2 febbraio 1920 Gabriele D'Annunzio pronunciò un discorso in onore di Shimoi Harukichi, qui riportato per intero. Poiché esso contiene alcuni riferimenti che oggi potrebbero risultare oscuri, per non interromperne la lettura si anticipano alcuni chiarimenti: nel contesto del discorso dannunziano il lettore ritroverà quindi le frasi che qui di seguito vengono citate tra virgolette.

profonda" (207). Come i legionari romani, "in mezzo a questo campo trincerato abbiamo posto le fondamenta d'una città di vita, d'una città novissima" (208). "Non v'è luogo della terra dove l'anima umana sia più libera e più nuova che su questa riva. Compagni, alla fine dell'anno mirabile, celebriamo questa creazione e preserviamo questo privilegio" (211). D’Annunzio così conclude: "A chi la forza? A noi! Ardisco e non ordisco. E nella nostra insegna il ragnatelo è rotto dal pugnale, e il nodo è tagliato dalla spada netta. Così fu ieri. Non altrimenti sarà domani. Compagni fedeli a me fedele, non conosciamo noi né i trenta denari, né la rinnegazione. Domani, al limitare del nuovo anno, prima che il gallo canti, vogliamo balzare tutti in piedi gridando: Credo."

72 La Comina, nei pressi di Pordenone, fu la prima scuola italiana di aviazione civile, inaugurata il 7 agosto 1910. Con lo scoppio della guerra e data la sua vicinanza al fronte, fu trasformata in scuola militare di volo. Vi operò tanto D'Annunzio quanto la "Squadra della Comina, specializzata in operazioni ad alto rischio, con il motto Cominus et Eminus Ferit, "da lontano e da vicino ferisce", risalente a Luigi XII di Francia: si riteneva che l'istrice - raffigurato nella sua impresa - potesse anche lanciare gli aculei. È vicina all'attuale base aerea di Aviano. 
D'Annunzio, evocando "quell'Impero dove è tuttora atteso il rombo delle mie ali", si riferisce al volo dall'Italia al Giappone, progettato con Shimoi ma poi non realizzato perché entrambi coinvolti nell'avventura di Fiume.

"Il campo di San Nicolò del Lido", a Venezia, fu un campo d'aviazione costruito nel 1915 per esigenze belliche. Dal 1918 vi operò la $1^{a}$ Squadriglia Navale, una squadriglia aerea speciale della Regia Marina comandata da Gabriele d'Annunzio con il grado di maggiore, che aveva coniato il motto "Sufficit animus". La squadriglia venne disciolta nel 1919 alla fine della Guerra.

La frase "avendomi egli mandato un dono di poesia" si riferisce con ogni probabilità al volumetto di poesie tradotte dal giapponese e curato nel 1917 da Shimoi e da Gherardo Marone (1891-1962), ${ }^{73}$ avvocato e letterato italo-argentino che di Shimoi condivideva la passione per la letteratura, ma non l'indirizzo politico, perché fu un antifascista militante e firmò il manifesto degli intellettuali antifascisti promosso da Benedetto Croce. La raccolta di poesie giapponesi è dedicata "A Paolo Argira", pseudonimo con cui firmava le sue poesie la compagna di Marone, Fiorina Centi. Uno pseudonimo noto nella cerchia dei letterati: Ungaretti chiude una sua cartolina a Marone con le parole "Salutatemi Paolo Argira".

La vittoria militare del Giappone sul "Figlio del Cielo", cioè sull'imperatore della Cina, coronò la Prima guerra sino-giapponese nel 1894-95, sancendo il tramonto della Cina come potenza continentale e la supremazia del Giappone sull'Asia. Il "Cesare slavo" (con un riferimento all'etimologia che vuole la parola russa "zar" derivata da "Caesar") venne sconfitto nella Guerra russo-giapponese del 1904-05, con la quale il Giappone si affermò come potenza mondiale.

La "sudicia mazza agitata dal porcaro serbo" è la minaccia d'invasione di Fiume da parte dei serbi, le cui pressioni nelle trattative di pace avevano portato a non assegnare Fiume all'Italia, generando la situazione in cui si era inserito il colpo di mano di D'Annunzio e dei suoi legionari. Pietro I Karadordević, re di Serbia dal 1903, è chiamato "porcaro serbo" perché, per liberarsi dalla dipendenza

73 Акıко Yosano et al. (1917), Poesie giapponesi. La seconda edizione, arricchita di nuovi autori, venne pubblicata nel 1927 con il titolo Lirici giapponesi. Harukichi Shimoi (2019), Un samurai a Fiume, 135-139; nei Documenti in appendice al volume è riprodotto (135-136) un brano tratto dall'Introduzione anonima ma scritta da Harukichi Shimoi e Gerardo Marone al volume: Aкıко Yosano et al. (1917), Poesie Giapponesi, seguito da: Alcuni giudizi sulle Poesie giapponesi di Harukichi Scimoi e Gerardo Marone (136-139), "pubblicati in forma anonima a guisa di conclusione nell'Antologia della Diana, Libreria della Diana (Stabilimento Tipografico Silvio Morano), Napoli 1918, p. 192 s..”

74 Giuseppe Ungaretti (2015), Da una lastra di deserto, 8. 
economica con l'Austria-Ungheria, sottoscrisse un'unione doganale con la Bulgaria. L'Austria-Ungheria reagi con un embargo sulle carni suine della Serbia e la Serbia rispose potenziando la sua produzione, in una competizione che venne chiamata la "guerra dei maiali" e che durò fino al 1911. Alla fine della Prima guerra mondiale, il Regno dei Serbi, Croati e Sloveni (di cui divenne re Pietro I di Serbia) venne riconosciuto dalla Conferenza di Pace di Parigi del 1919 e durò fino al 1929, quando conflui nel Regno di Jugoslavia.

"La parola superba di Okuma": Ōkuma Shigenobu (1838-1922), primo ministro del Giappone nel 1898 e nel 1914-16, era favorevole all'importazione della cultura europea in Giappone. Il passo contenuto nel testo che segue era già presente nell'Orazione al popolo di Milano in morte di Giosuè Carducci del 24 marzo 1907. Li si legge infatti: "Organati e serrati in unità impenetrabile, essi tendono non soltanto alla signoria dell'Asia ma dell'intero Pacifico. La loro cupidigia guarda alle Filippine, all'Indocina, alle Indie olandesi, ad Hawai. È nota la parola superba di Okuma: 'L'Europa è decrepita. Noi raccoglieremo le sue eredità'. Ma io dico che l'Europa non è decrepita. Anzi dico che la sua febbre laboriosa non è se non una febbre di giovinezza, non è se non un anelito verso l'apparizione di una vita più libera e più alta”. A questo punto dell'Orazione del 1907 D'Annunzio rivolgeva lo sguardo altrove: "Guardate la Germania". Il testo per Shimoi è solo leggermente diverso, perché quando D'Annunzio scrisse l'orazione su Carducci l'Italia non aveva ancora subito la "vittoria mutilata" del 1918; per questo nel saluto a Shimoi D'Annunzio inserisce nel 1920 una frase che manca nel testo del 1907: "Quale esempio per l'immiserita e avvilita Italia dell'altra sponda, che indietreggia davanti all'ombra di una sudicia mazza agitata dal porcaro serbo!"?

D'Annunzio - tanto qui, quanto nel precedente discorso, alla nota 58, p. 272 parla di "tre latini" giunti nell'Oriente estremo "or è sette secoli" perché Marco Polo (1254-1324) viaggiò in Oriente con il padre Niccolò e con lo zio Matteo.

Infine, l'Alalà" che conclude il saluto a Shimoi - solo o completato con l'interiezione latina eja eja eja - è il grido di battaglia d'origine greco-classica adottato da D'Annunzio e dai legionari fumani, e poi onnipresente nel ventennio fascista. Caduto in disuso dopo il fascismo, la sua forza evocativa del passato regime è tale che anche ai giorni nostri è stato usato come titolo per una storia del fascismo. ${ }^{\mathbf{7 6}}$

75 Gabriele D’Annunzio (2013), Orazione al popolo di Milano in morte di Giosuè Carducci.

76 Giampaolo Pansa (2014), Eia Eia Alalà. Controstoria del fascismo; nelle edizioni successive con il sottotitolo: Nascita del fascismo.

II. Italia 


\section{Gabriele D'Annunzio, Saluto all'ospite d'Oriente ${ }^{77}$}

Noi vogliamo stasera onorare in Fiume d'Italia un ospite dell'Estremo Oriente venuto a noi con un gruppo di grandi pensieri come quegli stormi di gru chiarosonanti che fendono il cielo del suo paese. Vogliamo salutare a questa nostra mensa di guerra un messaggero del Sol Levante, un inviato di quell'Impero dove è tuttora atteso il rombo delle mie ali che qui brucia il perpetuo rogo.

Se il lungo volo non può portarmi laggiù è certo che Haru-Kici Scinoi $[s i c]$ è stato qui portato da un lungo volo.

E, se fino a ieri egli ebbe nel suo piccolo petto un grande cuore italiano, ha oggi sotto la stella di Fiume un ardentissimo cuore fiumano. E già ama le nostre dolci isole del Carnaro come quelle del suo innumerevole arcipelago.

Credo di aver raccontato il mio incontro patetico con lui, in [60|61] un battello veloce e rombante, attraverso la laguna grigia, poco dopo la sciagura di Caporetto.

Egli mi aveva chiesto di visitare il campo di San Nicolò del Lido, dove io avevo le mie ali e le mie armi.

Parlavamo dell'Italia dolorosa, parlavamo del nostro sacrifizio, del nostro sangue, dei giorni disperati e delle speranze invitte. Se ne ricorda Scimoi? [sic]

Vidi a un tratto due lacrime vive sgorgare dai suoi sconosciuti occhi di straniero. E subitamente lo riconobbi fratello, come tutti lo riconosciamo qui. E il cuore mi si aperse.

$\mathrm{E}$, un giorno della seguente primavera ansiosa, avendomi egli mandato un dono di poesia, io gli ricordai quelle lacrime improvvise e gli scrissi: "Nessun poeta della tua stirpe compose mai strofa su rugiada più celeste di quel tuo pianto".

Così noi stasera sentiamo che dall'Oriente non ci viene soltanto la luce ma anche l'amore. Splendet et ardet.

77 Questo Saluto, datato 2 febbraio 1920, è contenuto nel volume stampato in occasione del trentennale della marcia di Ronchi: Gabriele D'Annunzio, Saluto all'ospite d'Oriente. Non è invece in: Gabriele D’Annunzio (1932a), La penultima ventura, Libro primo, né in Gabriele D'Annunzio (1932b), La penultima ventura, Libro secondo, a cura dell'Istituto nazionale per l'edizione di tutte le opere di Gabriele D’Annunzio, perché i testi ivi pubblicati terminano con Credo del 31 dicembre 1919. Con minime differenze grafiche è anche in Harukichi Shimoi (2019), Un samurai a Fiume, 208-210, tratto da "La Vedetta d'Italia”, Fiume, 4 febbraio 1920. 
Più di una volta io ho gridato: Liberiamoci dall'Occidente che non ci ama e non ci vuole. Volgiamo le spalle all'Occidente che ogni giorno più si sterilisce e s'infetta e disonora in ostinate ingiustizie e in ostinate servitù. Separiamoci dall'Occidente degenere che, dimentico d'aver contenuto nel suo nome lo splendore dello spirito senza tramonto, è divenuto un'immensa banca in servizio della spietata plutocrazia transatlantica.

L'Italia delusa, l'Italia tradita, l'Italia povera si volga di nuovo all'Oriente dove fu fisso lo sguardo de' suoi secoli più fieri. Il popolo italiano fu sempre il più sagace dei migratori. Quell'Asia, che oggi gli è contesa dalla perfida avarizia altrui, fu sempre alla mercé dei suoi ardiri. Ma non importa che gli sia contesa. "Teneo te, Asia" è una parola romana da rendere italica.

Quale fatto storico è comparabile in grandezza alla resurrezione asiatica, al subitaneo ringiovanimento che rinnova la sacra Asia, la regione dall'ampia e sublime unità?

Ecco che un impero quasi immobile, quello del Sol Levante, dà [61|62] esempio inaudito d'una trasformazione che sembra piuttosto una creazione dal profondo.

Coloro i quali vinsero il Figlio del Cielo e il Cesare slavo, oggi aspirano a tutte le conquiste. Organati e serrati in unità impenetrabile, essi tendono non soltanto alla signoria dell'Asia ma dell'intero Pacifico. La loro forza guarda alle Filippine, all'Indocina, alle Indie olandesi, ad Hawai.

Quale esempio per l'immiserita e avvilita Italia dell'altra sponda, che indietreggia davanti all'ombra di una sudicia mazza agitata dal porcaro serbo!

È nota la parola superba di Okuma: "L'Europa è decrepita. Noi raccoglieremo le sue eredità".

$\mathrm{Ma}$ io dico che l'Europa non è decrepita. Anzi dico che la sua febbre laboriosa non è se non una febbre di giovinezza, non è se non un anelito verso l'apparizione di una vita più libera e più alta.

Dove una tanta febbre batte più forte? Nel polso di Fiume. Dove le forme di una vita novissima cominciano a disegnarsi? Su questa riva del Carnaro.

Da Fiume d'Italia Porta dell'Oriente salutiamo la luce di quell'Oriente estremo dove, or è sette secoli, giunsero con lenta fretta i "tre latini". Beviamo alla fraternità di questo ospite luminoso che ci sa dire come le più belle aurore non siano ancora nate.

La nostra è prossima. Prope est.

Alalà.

2 febbraio 1920 .

II. Italia 
II.2. Stalin e Bobbio sulla propaganda di pace (1952-53).

Con due lettere inedite tra Mucchi e Bobbio

Il movimento dei Partigiani della Pace è stato appena accennato nel precedente testo, perché oggi ha una rilevanza soltanto storica, mentre negli anni Cinquanta fu un energico attore politico e, quindi, oggetto di appassionati dibattiti. Un esempio delle diverse opinioni su questo movimento si ricollega ai tre testi esaminati in questa appendice. La rivista "Rinascita" del Partito Comunista Italiano pubblicò uno scritto di Stalin contenente una valutazione sui Partigiani della Pace $^{78}$ (qui commentato a II.2.a): esso rappresentava quindi la posizione ufficiale dell'Unione Sovietica su quel movimento. La lettura di quell'articolo suscitò $i$ fondati dubbi di Norberto Bobbio, che prese criticamente posizione in un saggio sulla rivista "Occidente"79 (qui commentato a II.2.b). In un incontro a Parigi Bobbio discusse di questi due scritti con l'amico Gabriele Mucchi, pittore e comunista ortodosso ma non settario. Dopo quell'incontro $i$ due amici riaffrontarono il tema in due lunghe lettere finora inedite (qui riprodotte integralmente a II.2.c), che riassumono in modo a mio giudizio esemplare le due opposte posizioni non solo sui Partigiani della Pace, ma sull'intero problema della pace nel pieno della Guerra fredda.

Tutti questi documenti videro la luce nel clima politico del primo decennio del dopoguerra. Esso è troppo vicino per essere insegnato nelle scuole e troppo lontano per emergere dai ricordi di famiglia, quindi è oggi poco noto ai non specialisti; perciò un richiamo sintetico a quei fatti ormai lontani può collocare nella giusta prospettiva il tono e gli argomenti dei testi di Stalin e di Bobbio, nonché delle due lettere di Mucchi e di Bobbio.

Quando nel 1947-49 vennero approvate le tre costituzioni pacifiste, tutti avevano ben presenti tanto gli orrori appena passati della guerra tradizionale, della guerra per bande e della controguerriglia quanto il terrore incombente della guerra atomica appena sperimentata. Per questo, in quegli anni, Bobbio voleva destare

78 Le osservazioni di Stalin sui Partigiani della Pace costituiscono il $\$ 6$ di Problemi economici del socialismo nell'URSS, in: Rinascita, ottobre 1952, supplemento al n. 9, poi riprodotto in forma ciclostilata in Iosif STALIN (1972), Problemi economici del socialismo nell'URSS e ristampato nel 1976 con uno scritto di Emilio Sereni del 1953, introduzione di Franco Botta. Le citazioni sono tratte da quest'ultima edizione.

79 Norberto Bobbio (1952), Pace e propaganda di pace. Ristampato in Norberto Bobbio (1955), Politica e cultura, 72-83; le citazioni nel testo sono tratte da quest'ultima pubblicazione. 
una "coscienza atomica" che rendesse consapevoli del rischio di estinzione che correva l'intera umanità in caso di olocausto atomico: ${ }^{80}$ coscienza che oggi si è molto affievolita, nonostante la proliferazione delle armi atomiche in mani spesso poco affidabili. ${ }^{\mathbf{1}}$

La Guerra fredda tra gli Alleati occidentali e $i$ sovietici faceva temere uno scontro militare imminente: dal 1946 al 1954 i francesi erano impegnati nella Guerra di Indocina (che nel 1955 si trasformò nella guerra americana del Vietnam); nel 1949 la vittoria dei comunisti cinesi aveva portato alla formazione della Repubblica Popolare Cinese, mentre la guerra di Corea scoppiata nel 1950 sembrava preludere a uno scontro in Europa. La strategia statunitense mirava al "contenimento" dell'espansione comunista nel timore che essa si abbattesse sull'Europa occidentale, dal momento che quella orientale era già entrata nella sfera della diretta influenza sovietica con la creazione delle democrazie popolari.

L'Europa occidentale, indebolita e frammentata, muoveva $i$ primi passi verso strutture comuni con il Consiglio d'Europa nel 1949, con la CECA nel 1951, fino all'Euratom nel 1957 e alla Comunità Economica Europea (CEE) nel 1958. In particolare, sotto l'egida della Nato, fondata nel 1949, dal 1950 si tentò di organizzare un esercito comune, cioè una Comunità Europea di Difesa (CED). A questa Comunità $i$ singoli Stati avrebbero conferito una divisione del proprio esercito nazionale, con l'eccezione della Germania, il cui riarmo era avversato dalla Francia: la Germania avrebbe dovuto creare una sua divisione da includere nella $C E D$, senza però ricostruire il suo esercito nazionale. Un riflesso di questa incertezza di collocamento affiora nel dibattito costituente tedesco con il problema dell'eventuale inclusione di cittadini tedeschi in un esercito straniero in caso di guerra (cfr. p. 320). Il progetto della CED, dopo molte ostilità e diffidenze, ${ }^{\mathbf{2}}$ falli nel 1954 a causa del voto contrario del Parlamento francese. La protezione dell'Europa era dunque affidata ai singoli (ma ancora deboli) eserciti nazionali del dopoguerra e alla forte presenza militare statunitense soprattutto nell'area del

80 Mario G. Losano (2018), Norberto Bobbio, 359-369.

81 "According to SIPRI [Stockholm International Peace Research Institute], the worldwide total inventory of nuclear weapons as of 2018 stood at 14,465, of which 3,750 were deployed with operational forces", SIPRI Yearbook 2018 (www.sipriyearbook.org) Armaments, Disarmament and International Security, (https:/www.sipri.org/sites/default/files/ SIPRIYB18c06.pdf).

82 Per esempio: Unità di tutto il popolo contro la C.E.D. e il riarmo tedesco. Per la pace e l'indipendenza nazionale (1952?). Questo giornale (Tribuna della Pace) era l'organo del Comitato provinciale dei Partigiani della Pace di Bologna.

II. Italia 
possibile primo impatto, cioè in Germania. Il timore di una guerra sul suolo europeo si andò attenuando a partire dal 1953, quando con la morte di Stalin iniziò il lento disgelo dell'Unione Sovietica.

Il movimento dei Partigiani della Pace - fondato nel 1949 e concluso nel 1956 - coincise quindi con gli anni della massima tensione mondiale ed europea. Oggi, dopo oltre un settantennio di pace in Europa, questo grande movimento pacifista è dimenticato dai più, mentre alcuni studi storici ne hanno messo in luce l'importanza (ma anche le contraddittorie caratteristiche): infatti la bomba atomica esigeva un ripensamento anche del pacifismo. ${ }^{83}$ Questo movimento venne promosso dal Cominform nel 1947 e l'Unione Sovietica fu la principale artefice sia della sua espansione presso $i$ vari partiti comunisti europei, sia del congresso inaugurale a Parigi del 20-24 aprile 1949. Il pacifismo dei Partigiani della Pace è quindi connotato anche da un deciso antiamericanismo. ${ }^{84}$ In Italia parteciparono alle attività di quel movimento, per quanto egemonizzato dall'URSS, anche numerosi pacifisti non comunisti, dai socialisti ai cristiani, ${ }^{\mathbf{8 5}}$ dai liberali ai repubblicani.

La presenza degli italiani al Congresso di Parigi dei Partigiani della Pace fu eccezionalmente alta, a ulteriore riprova di quanto il problema della pace fosse allora sentito. Il diario del principale organizzatore, il dirigente comunista Emilio Sereni, ne offre una testimonianza diretta: "Invece dei 300 delegati previsti per l'Italia (ed erano già molti, più che per ogni altro paese), a Parigi ne ho condotti 1200", con i conseguenti problemi dei posti in treno, in albergo e al congresso (problemi che nel viaggio obbligarono Sereni a fare "il bigliettaio, il controllore, il poliziotto e il cassiere"); a Parigi, i posti per gli italiani al Congresso vennero portati da 300 a 450: "di più non se ne potevano chiedere, altrimenti il Congresso si sarebbe trasformato da internazionale in italiano". La "delegazione italiana cosi numerosa ha costituito uno degli avvenimenti centrali del congresso: non per caso ho ottenuto che Nenni fosse il primo relatore, che un italiano (che poi sono stato io) fosse uno dei cinque oratori al grande meeting di Buffalo" (cioè allo stadio

83 Questa differenza rispetto ai pacifismi prebellici è esaminata in una ricerca di Sondra Cerrai (2011), I partigiani della pace in Italia.

84 Questo tema è affrontato da Giulia Bentivoglio (2002), Antiamericanismo in Italia nel secondo dopoguerra. Cfr. inoltre TANJA SARPIERI (2002), Il pacifismo nella politica internazionale della sinistra italiana.

85 Ivano Granata (1993), Don Mazzolari e il movimento dei Partigiani della Pace (1950-1952). Va ricordato anche Don Andrea Gaggero, che negli anni Sessanta ebbe un ruolo importante a fianco del pacifista Aldo Capitini: cfr. Sondra Cerrai (2011), I partigiani della pace in Italia, 82. 
parigino, cosi soprannominato dopo la tournée del "Wild West" di Buffalo Bill) e che l'italiano fosse una lingua ufficiale del Congresso. Insomma, l'Italia entrò "per il portone d'onore" perché "solo sul terreno delle energie popolari l'Italia può essere forte". 86

Questa pluralità di partecipanti finiva per rendere meno unidirezionali $i$ toni propagandistici promossi dall'URSS, e questo conferiva al movimento italiano una sua caratteristica peculiare. ${ }^{87}$ L'autrice dello studio già ricordato si muove infatti lungo "il difficile crinale della doppia identità di questo movimento, non tacendone (ed anzi analizzandola) l'origine strumentale e l'organizzazione fideistica ma cercando di vedere soprattutto gli elementi di innovazione e di creazione di qualcosa di diverso e di 'buono in sé' come direbbero certi filosofi hegeliani". ${ }^{\mathbf{8}}$

In Italia, secondo un detto comune, le tre organizzazioni più capillarmente presenti sul territorio erano $i$ carabinieri, le parrocchie e le cellule del partito comunista, quindi anche nei centri di piccole dimensioni operavano gruppi di Partigiani della Pace. La disseminazione di allora è ancora oggi attestata dalla grande quantità di pubblicazioni propagandistiche stampate in tutta la penisola e reperibili nelle biblioteche.

I Partigiani della Pace propugnavano anche la rivoluzione comunista o, almeno, sociale; però una rivoluzione raramente è pacifica e questa peculiarità del movimento - anzi, questa contraddizione - è al centro delle due lettere di Mucchi e Bobbio che verranno esaminate a p. 297-303. Entrambe le lettere vennero scritte nel febbraio del 1953, cioè nel periodo in cui era più acuta la percezione del pericolo d'una guerra, e subito dopo il Congresso dei Popoli, svoltosi a Vienna il 5-12 dicembre 1952, al quale, come si vedrà nella lettera di Mucchi, la partecipazione non si limitò ai soli Partigiani della Pace.

86 Emilio Sereni (2015), Diario (1946-1952); sul congresso mondiale dei Partigiani della Pace cfr. pp. 147-149, da cui sono tratte le citazioni nel testo. Da questo diario, pubblicato postumo, traspare la rigorosa ortodossia di Sereni, non meno che nel diario e nelle lettere, anch'essi inediti, della moglie: "E' il Partito il vero protagonista delle pagine di Marina”, Амвrogio Donini (1955), Prefazione, 10 (nella clandestinità, "Marina” era il nome di Xenia, la moglie di Sereni).

87 Ruggero Giacomini (1984), I partigiani della pace. "Questo libro, che può rivelarsi utilissimo per le informazioni offerte e per la puntuale cronaca degli avvenimenti che caratterizzarono il movimento dei Partigiani della Pace fino al 1953 [...], ha il grave difetto di essere chiaramente 'di parte' e di prendere per ora colato tutto ciò che veniva declamato sulla pubblicistica del movimento", Sondra Cerrai (2011), I partigiani della pace in Italia, Introduzione.

88 Sondra Cerrai (2011), I partigiani della pace in Italia, Introduzione.

II. Italia 
a) Stalin, i "Partigiani della Pace" e l'imperialismo bellicista

All'inizio degli anni Cinquanta l'URSS progettava una nuova stesura del manuale ufficiale di economia politica e nel febbraio del 1952 Stalin prese posizione sui materiali preparatori. Lo scritto di Stalin affronta quindi il vasto problema di che cosa sia l'economia politica secondo il marxismo sovietico. In questo contesto si pone il problema della convivenza o dello scontro fra capitalismo e socialismo e, in particolare, il paragrafo 6 si occupa della Questione della inevitabilità delle guerre fra i paesi capitalistici, ${ }^{\mathbf{8 9}}$ nel cui contesto Stalin esamina il movimento dei Partigiani della Pace.

Di fronte all'affermazione che "le guerre tra i paesi capitalistici abbiano cessato di essere inevitabili" Stalin risponde: "Questi compagni sbagliano" (p. 90). Gli Stati Uniti hanno "messo al passo" $i$ principali Stati capitalistici, che però "tenteranno di sottrarsi alla schiavitu americana e di porsi sulla strada di uno sviluppo autonomo". I primi Stati a imboccare questa via potrebbero essere l'Inghilterra e la Francia, per conservare il controllo sui loro mercati e sulle materie prime. Gli Stati sconfitti, "la Germania (occidentale) e il Giappone", erano grandi potenze prebelliche e perciò pensare che non vogliano riguadagnare la loro autonomia "significa credere nei miracoli" (p. 91). La guerra tra paesi capitalistici avrà luogo (come è avvenuto nella Seconda guerra mondiale) "perché, mentre la guerra fra i paesi capitalistici pone solo la questione del predominio di determinati paesi capitalistici su altri paesi capitalistici, la guerra contro l'URSS deve invece necessariamente porre la questione dell'esistenza del capitalismo stesso"; inoltre, afferma Stalin, $i$ paesi capitalistici "tengono conto della politica pacifica dell'Unione Sovietica" (p. 92) e sanno quindi che non verranno da essa attaccati.

A questo punto Stalin affronta la questione centrale: "Si domanda quale garanzia esista che la Germania e il Giappone non si rimettano nuovamente in piedi e non tentino di sottrarsi alla schiavitu americana e di vivere una propria vita autonoma? Penso che non esistano garanzie di questo genere. Ma da ciò deriva che l'inevitabilità delle guerre tra paesi capitalistici continua a sussistere" (p. 93). Di fronte a questa inevitabilità prende posizione il movimento per la pace, che "ba per scopo di sollevare le masse popolari alla lotta per mantenere la pace, per scongiurare una nuova guerra mondiale. Per conseguenza, esso non persegue lo scopo di rovesciare il capitalismo e di instaurare il socialismo - esso si limita a

89 In Iosif Stalin (1972), Problemi economici del socialismo nell'URSS: nell'edizione del 1976 il \$ 6 è alle pp. 89-94; nell'edizione del 1972, alle pp. 40-46: cfr. supra, nota 78, p. 284. 
perseguire i fini democratici della lotta per mantenere la pace" (p. 93). In certi luoghi e in certe circostanze potrebbe verificarsi che il movimento per la pace si trasformi "in lotta per il socialismo, ma questo non sarebbe più l'attuale movimento per la pace, bensi un movimento per rovesciare il capitalismo" (p. 94).

Il confine tra movimento rivoluzionario e movimento pacifista sembra cosi fondarsi sulla netta distinzione tra il pacifismo del mondo comunista e il bellicismo del mondo capitalistico, e Stalin conclude asserendo l'inevitabilità delle guerre tra Stati capitalistici, ma non pronunciandosi invece sulla possibile guerra fra $i$ due blocchi: nel suo testo, "l'inevitabilità delle guerre fra i paesi capitalistici" diventa ambiguamente "l'inevitabilità delle guerre" tout court.

Questa frase finale colpisce Bobbio, che la cita per esteso nel suo articolo (esaminato qui di seguito: II.2.b), perché essa gli sembra confermare l'ambiguità insita nel movimento dei Partigiani della Pace:

La cosa più probabile, - scrive Stalin, - è che l'attuale movimento per la pace, inteso come movimento per mantenere la pace, in caso di successo porterà a scongiurare una guerra determinata, a rinviarla per un certo tempo, a mantenere per un certo tempo una pace determinata, a costringere alle dimissioni un governo guerrafondiaio sostituendolo con un altro governo, disposto a salvaguardare per un certo tempo la pace. Questa, naturalmente, è una cosa buona. Anzi, è una cosa ottima. Tuttavia questo non basta per eliminare l'inevitabilità delle guerre fra i paesi capitalistici. Non basta, perché, nonostante tutti questi successi del movimento per la difesa della pace, l'imperialismo continua a sussistere, conserva le sue forze, e per conseguenza continua a sussistere l'inevitabilità delle guerre. Per eliminare l'inevitabilità delle guerre, è necessario distruggere l'imperialismo. ${ }^{90}$

b) Bobbio: qual è la pace dei "Partigiani della Pace"?

Il testo di Stalin venne pubblicato in vista del "Congresso dei popoli", organizzato a Vienna per il 12 dicembre $1952 .{ }^{91}$ Poiché vi avrebbero partecipato anche movimenti e persone non appartenenti al movimento dei "Partigiani della Pace", Bobbio lo commenta criticamente per "avviare un discorso in cui, all'urto violento e sterile

90 Norberto Bobbio (1955), Politica e cultura, 76, corsivi di Bobbio (cfr. supra, nota 79, p. 285): le citazioni nel testo sono tratte da quest'ultima pubblicazione; in Stalin è presente solo il primo corsivo: IosIf STALIn (1976), Problemi economici del socialismo nell'URSS, 94.

91 Congresso mondiale dei popoli per la pace, Vienna, 5-12 dicembre 1952 (1953). Il sito di Lombardia Beni Culturali indica: Atti a stampa del Congresso dei popoli per la pace (Vienna, 12-19 dicembre 1952), http://www.lombardiabeniculturali.it/archivi/unita/MIUD14658A/, ma non accessibili (25 marzo 2018).

II. Italia 
delle accuse, si sostituisca uno scambio di argomenti storici e logici suscettibili di un ulteriore esame". ${ }^{2}$ Il contesto in cui questi eventi si svolgevano venne cosi sintetizzato nel 1955 da Bobbio stesso: "Nel 1951 si diceva 'politica dei blocchi'; nel 1953 si cominciò a dire 'coesistenza'; ora si parla di 'distensione", e la distensione esige "un dialogo continuo, sincero, vivace e fecondo tra le parti in conflitto" ( $p .10)$.

Il desiderio di dialogo traspare anche dal tono critico ma rispettoso della sua analisi, tuttavia oggi molti non ricordano più quanto aspri fossero $i$ toni da una parte e dall'altra e quanto inconsueto fosse invece il pacato atteggiamento di Bobbio. Basterà confrontare le citazioni di Bobbio che seguono con due esempi, tratti l'uno dal "campo" sovietico e l'altro da quello anticomunista. Un testo sovietico definisce cosi la parola 'pacifismo':

"Movimento borghese di opposizione a tutte le guerre. Mascherandosi ipocritamente dietro la parola d'ordine del pacifismo, $i$ reazionari si oppongono alle guerre di liberazione nazionale, alle guerre rivoluzionarie, alle guerre civili ed alle altre guerre aventi lo scopo di difendere il popolo dagli attacchi stranieri, di liberare il popolo dalla schiavitù capitalista, di liberare i paesi coloniali e sottoposti all'oppressione capitalista. La politica dei pacifisti concorre alle guerre imperialiste, aggressive ed ingiuste."93

Gli argomenti degli anticomunisti sono opposti, ma il tono è lo stesso. Però la cosa ancor più sorprendente è che l'autore delle righe che seguono è Enzo Enriques Agnoletti, allievo di Calamandrei, partigiano, membro del Partito d'Azione, liberalsocialista, direttore de "Il Ponte". Insomma, una biografia parallela a quella di Bobbio che però non si rispecchia nello stile di questa polemica:

"Ci proponiamo in questo opuscolo di studiare e smascherare una delle maggiori mistificazioni della nostra epoca.

Per mezzo dell'appello di Stoccolma e di quello più recente di Praga, attraverso l'intera attività svolta dai Partigiani della Pace e coronata da un congresso che doveva aver luogo a Sheffield e che ripiegò su Varsavia - riavvicinandosi cosi al suo clima di origine - il

92 Norberto Bobbio (1955), Politica e cultura, 72.

93 Questa definizione è tratta da I.V. Liekhin, F. N. Petrov (1949), Dizionario sovietico delle parole straniere, 484, e viene posta in esergo al volume anticomunista [Enzo EnRIQues Agnolettr] [1951?], Chi sono i partigiani della pace. Come autore di quest'ultimo scritto, l'elenco degli opuscoli alla fine di un altro volumetto indica Enzo Enriques Agnoletti. Dal Congresso internazionale per la libertà della cultura (1951) prese origine il Comitato e, poi, l'Associazione italiana per la libertà della cultura, che pubblicò - accanto a quello qui citato - opuscoli dei maggiori intellettuali europei, da Eugenio Montale a Thomas Mann: il tono spesso troppo polemico degli scritti era il frutto amaro di tempi inveleniti, e non deve trarre in inganno sulla serietà di molti degli argomenti addotti. 
comunismo staliniano non ha mai cessato di monopolizzare a profitto della politica sovietica la grande parola della pace. Tutti $i$ testi, tutti $i$ fatti, tutti $i$ documenti riuniti confermano come l'iniziativa chiamata di Stoccolma e l'attività dei Combattenti o Partigiani della Pace hanno Mosca per punto di partenza, il Cominform per stato maggiore mal camuffato, gli uomini di fiducia dello stalinismo internazionale per veri responsabili.

Dopotutto, nulla impedirebbe alla Russia sovietica di fare la campagna per la pace né agli uomini di buona volontà di seguirla, se non si scoprisse fin dall'inizio che sotto questo grottesco camuffamento non si vuole servire la pace ma bensi persuadere il mondo che un grande Stato militare e autoritario è la sola potenza al mondo che sia in possesso delle virtù pacifiste.

Lo scopo di questa falsa offensiva di pace non è solamente quello di preparare una non-resistenza all'aggressione in quei paesi nei quali esistono le libertà individuali e collettive, e dunque di rendere possibile la guerra, essa tende anche a corrompere una delle piu antiche e solide tradizioni del pensiero democratico. Essa mira infatti a distruggere la nostra convinzione che il pericolo della guerra provenga da quei governi i quali esercitano un potere assoluto e si sottraggono al controllo e alla vigilanza dell'opinione pubblica". ${ }^{44}$

Le conclusioni di Enriques Agnoletti sui Partigiani della Pace coincidono nella sostanza, ma non certo nella forma, con le argomentazioni di Bobbio:

"Il movimento dei Partigiani della Pace nasconde a malapena ed al massimo per gli ingenui di essere una organizzazione sussidiaria, una filiale del comunismo totalitario. Basta leggere la stampa russa per constatare che appunto cosi il Cremlino considera questa organizzazione, la quale serve semplicemente da cortina fumogena per nascondere il superriarmo e le operazioni dei satelliti (vedi i fatti di Corea)." ${ }^{25}$

L'argomentazione di Bobbio si fonda sul relativismo dei valori: la pace è "un fine ultimo soltanto per chi ritiene che la vita sia un bene supremo", mentre invece chi, per esempio, vede il bene supremo nella libertà è disposto a rischiare e anche a perdere la vita pur di conservare la libertà. In particolare, in questa "gerarchia di fini e di valori" (p. 73), la pace è desiderabile per chi ha già raggiunto altri obiettivi fondamentali, come la libertà e il benessere: "La pace è essenzialmente conservatrice" (p. 73, corsivo di Bobbio). Partendo da questo punto di vista, "sorge il legittimo sospetto che non possa essere legittimamente pacifista chi non ha interesse a mantenere lo status quo" (p. 74). Per questo Bobbio crede nel pacifismo dei socialdemocratici della Seconda internazionale, perché essi accettavano la società in cui vivevano e si proponevano di trasformarla con le riforme, $e$

94 [Enzo Enriques Agnoletti] [1951?], Chi sono i partigiani della pace, 3-4. La guerra atomica è uno dei temi centrali: Da Hiroshima all'appello di Stoccolma, ovvero quando i partigiani della Pace erano i partigiani della bomba atomica (15-19); L'URSS contraria a una legalità internazionale in materia atomica (37-38).

95 [Enzo Enriques Agnoletti] [1951?], Chi sono i partigiani della pace, 4.

II. Italia 
non con la rivoluzione. Quel movimento socialdemocratico e riformista era stato distrutto dal nazi-fascismo e bisognava "costruire il comunismo" su scala mondiale con la rivoluzione, come in Cina, come in Vietnam. Quindi l'ideale della sinistra che continuava a ispirarsi alla Terza Internazionale (benché disciolta nel 1943) non poteva più essere il pacifismo, anche se, mentre Bobbio scriveva quelle pagine, $i$ partiti e $i$ movimenti che si richiamavano alla Terza Internazionale si proclamavano pacifisti e costituivano il nerbo dei "Partigiani della Pace".

Per questo gli avversari dei "Partigiani della Pace" vedevano, per usare le parole di Bobbio, "qualcosa di ambiguo in un movimento pacifista che viene promosso e sostenuto dai seguaci di note teorie rivoluzionarie, cioè di teorie che pongono l'ideale della giustizia al di sopra dell'ideale della pace" ( $p$. 74). Bobbio precisa che essi "non costituiscono un movimento pacifista in senso generico", bensi un "movimento realistico e non utopistico" ( $p .75)$, cioè un movimento che parte dalla concreta situazione storica in cui opera: poiché in quegli anni di Guerra fredda si contrapponevano due blocchi, i Partigiani della Pace lottavano per la pace fra $i$ due blocchi. Bobbio formula con queste parole il nucleo della sua critica, che piacque pochissimo alle sinistre di allora:

"Ma perché essi [i Partigiani della Pace] ritengono che sia bene che regni la pace oggi fra $i$ due blocchi? Perché la guerra minaccerebbe di distruggere le conquiste sociali raggiunte nei paesi del socialismo. Ma allora è chiaro che anche per questo nuovo movimento della pace, sebbene sia costituito in gran parte da aderenti a teorie rivoluzionarie, la pace ha una funzione essenzialmente conservatrice. Là dove la rivoluzione è avvenuta, il rivoluzionario diventa necessariamente fautore della conservazione dello status quo. E poiché per garantire lo status quo, occorre un periodo di pace, non c'è nessuna contraddizione nel fatto che il rivoluzionario aderisca a un movimento per la pace” ( $p .75$, corsivo di Bobbio).

Tuttavia l'assenza di guerra fra $i$ due blocchi consolida anche il regime capitalistico negli Stati non socialisti: qui Bobbio riporta il lungo passo di Stalin pubblicato su "Rinascita" (e citato a p. 290). Secondo Bobbio in questo passo Stalin afferma che il fine supremo non è la pace, ma la distruzione dell'imperialismo. Quindi, conclude Bobbio, "la mèta cui tendono i Partigiani della Pace non è un fine ultimo ma strumentale, non è una soluzione definitiva, ma solamente interlocutoria” (p. 76), in attesa della rivoluzione mondiale.

Di fronte alla dicotomia della Guerra fredda, i Partigiani della Pace affermano che, dei due blocchi, "l'uno, quello sovietico, è pacifico, l'altro, quello americano, è bellicista", mentre altri pacifisti (per esempio, in Occidente) sostengono che "dei due blocchi, quello sovietico è più bellicoso di quello americano": però "queste persone sono automaticamente escluse dal novero dei Partigiani della Pace” ( $p .77)$, come 
infatti avvenne per i rappresentanti dell'eterodossa Jugoslavia. ${ }^{\mathbf{9 6}}$ Ora, se entrambe le correnti possono essere d'accordo sul perseguimento della pace (accordo sugli atteggiamenti), possono però discordare su che cosa sia la pace (dis-accordo sulle credenze). Si può cioè essere d'accordo sull'atteggiamento pacifista, ma la pace verrà perseguita con mezzi diversi senza un accordo sulle convinzioni ("credenze") concernenti le cause della guerra, cioè se gli uni credono che la causa delle guerre sia l'imperialismo americano, e gli altri no. In pratica, $i$ Partigiani della Pace non possono ammettere nelle loro file chi si dice pacifista, ma non condanna l'imperialismo americano: per questa ragione, secondo Bobbio, "il loro disaccordo non riguarda la desiderabilità della pace, ma soltanto certi giudizi di fatto relativi alle cause di guerra", e quindi "non possono essere considerati come bellicisti" ( $p$. 78). Individuata la causa delle guerre nell'imperialismo americano, il movimento dei Partigiani della Pace "è (mi si permetta il bisticcio) partigiano, nel senso che tra le varie parti del conflitto o meglio tra le due grandi parti in conflitto, ha preso decisamente partito in favore dell'una contro l'altra" (p. 79). Questo venir meno dell'imparzialità mina l'efficacia del movimento: "Curiosi pacieri i Partigiani della Pace. Essi si offrono di ristabilire la pace tra $i$ contendenti. Ma dichiarano sin dall'inizio senza alcuna reticenza che dei due contendenti l'uno ha ragione e l'altro ha torto, che la pace si può salvare soltanto mettendosi da una parte sola" ( $p .79)$. Viene cosi meno l'imparzialità del giudice e, quindi, l'efficacia del suo verdetto.

In realtà $i$ Partigiani della Pace si pongono non come arbitri o pacieri, ma come difensori dei popoli minacciati dalla bellicosità dell'imperialismo (che è solo americano). "Si può, ecco il punto, - si chiede Bobbio - eliminare l'imperialismo americano con atti di pace, senza compiere atti di guerra (anche se di guerra giusta)?" (p. 81). Il paciere mira al "raddrizzamento di una causa compromessa"; il "difensore della giusta causa" mira invece al "raddrizzamento di una situazione capovolta". In conclusione, "i Partigiani della Pace devono dire chiaramente quale dei due [compiti] vogliono esercitare, perché non si possono esercitare tutti e due insieme" (p. 81).

Indubbiamente, ammette Bobbio, "la realtà è più complessa di qualche sillogismo". Però per lui-pur scusandosi "se anche qui tiriamo fuori qualche sillogis-

96 Il rifiuto della firma dei "titini", in: [Enzo Enriques Agnoletti] [1951?], Chi sono i partigiani della pace, 42: l'appello di Stoccolma ("in linea di principio aperto a tutti") "non può venir firmato dal partito comunista jugoslavo, il quale firmerebbe volentieri se gli organizzatori della mascherata di Stoccolma non rifiutassero con orrore la sua adesione."

II. Italia 
mo" (p. 82) - è contraddittorio il fatto che i Partigiani della Pace sostengano la possibilità della coesistenza pacifica fra $i$ due blocchi, cioè la possibilità di un compromesso. Non è possibile conciliare "il principio della coesistenza dei due blocchi con quello dell'imperialismo come unica causa di guerra" (p. 82). O l'America è pericolosa, e "allora non si vede come sia possibile la coesistenza"; o l'URSS può coesistere con l'America, e "allora vuol dire che l'America non è più pericolosa" (p. 82). In ciò consiste "l'ambigua natura del movimento", che asserisce contemporaneamente che la pace è un fine ultimo, però lo usa come fine strumentale. Chiarito questo punto - sia pur con "qualche sillogismo" che, come vedremo, gli verrà rimproverato dall'amico Mucchi - Bobbio ritorna all'argomentazione di Stalin:

"Anche su questo punto le parole di Stalin nell'articolo citato sgomberano il campo dagli equivoci e, rivelando la vera natura del movimento, ne circoscrivono, in modo che può sembrare preoccupante, l'efficacia. Dal passo sopra riportato infatti abbiamo appreso che il fine supremo della politica dei paesi del socialismo è la distruzione dell'imperialismo e che il movimento dei Partigiani della Pace non è in grado di raggiungere questo fine supremo. Da questa tesi manca un breve passo per concludere che per raggiungere il fine supremo occorre un'azione diversa da quella dei Partigiani della Pace. E quale può essere quest'azione? Ci soccorre ancora una frase di Stalin: 'Pù darsi che, per un concorso di circostanze, la lotta per la pace si sviluppi in certe zone trasformandosi in lotta per il socialismo, ma questo non sarebbe più l'attuale movimento per la pace, bensì un movimento per rovesciare il capitalismo"” (p. 83).

Bobbio ha messo in corsivo questa parte della frase di Stalin perché, nell'interpretazione che quest'ultimo ne dà, l'azione pacifista, "essenzialmente conservatrice, deve trasformarsi in un'azione rivoluzionaria ('rovesciamento'), vale a dire - non bisogna aver paura delle parole - in un'azione di guerra” (p. 84).

\section{c) Mucchi e Bobbio: prospettive divergenti sui "Partigiani della Pace"}

Al saggio di Bobbio su "Occidente" si riferiscono le due lettere inedite trascritte qui di seguito: la prima del pittore Gabriele Mucchi (1899-2002), favorevole al movimento per la pace egemonizzato dall'Unione Sovietica e dai partiti comunisti, la seconda di Bobbio, che espone gli argomenti critici contro quella monopolizzazione del pacifismo, non ovviamente contro il pacifismo stesso. Le due lettere sono conservate presso il Centro Studi Piero Gobetti, a Torino. ${ }^{97}$

97 Centro Studi Piero Gobetti, Archivio Norberto Bobbio, fasc. 3000 (Carteggio): Gabriele Mucchi a Norberto Bobbio, Milano, 3 febbraio 1953, due facciate dattiloscritte con corre- 
L'incontro del filosofo Bobbio con Gabriele Mucchi è legato a Torino, perché li nacque Mucchi e li ritornava presso la sua guida spirituale, lo zio Annibale Pastore, che era stato anche il professore di flosofia teoretica con cui Bobbio aveva conseguito nel 1933 la sua seconda laurea. Mucchi, laureato in ingegneria, fu richiamato come ufficiale di artiglieria in entrambe le guerre mondiali, ma dopo l'8 settembre 1943 si uni ai partigiani e si iscrisse al partito comunista. Dopo la guerra abitò a Berlino Est, dove insegnò nella locale accademia di belle arti. A Bobbio lo univano dunque le radici torinesi e l'antifascismo militante, non però il comunismo che Mucchi professò sino alla fine della sua lunga vita. ${ }^{\mathbf{9 8}}$

Nella sua lettera Mucchi menziona la rivista Realismo. Mensile di arti figurative, che egli contribui a fondare a Milano nel giugno 1952. Dal 1952 al 1955 ogni numero si compone di regola di otto pagine nell'ampio formato di un quotidiano; dal 1955 la nuova serie cambia grafica, assumendo le dimensioni più ridotte, tipiche della rivista mensile, ma termina già l'anno dopo. I fascicoli ospitano scritti e riproduzioni di pittori e scultori di sinistra che si ispirano al realismo, però non al realismo sovietico: "Traiamo le origini dalla cultura dell'antifascismo e della Liberazione. La continuiamo nella nostra occasione storica, moderni perché attuali e legati ai problemi della nostra terra e del nostro popolo"99 Mucchi condivide questo indirizzo politico ed estetico.

Un ricordo scritto da di Renato Guttuso ci riporta all'ambiente artistico milanese, con i suoi entusiasmi e con le sue difficoltà, in cui si muoveva anche Mucchi durante la guerra:

"Ho con Mucchi un vecchio debito, di quelli che non si dimenticano. E risale agli anni '35-'36, anni nei quali appresi, nel modo più concreto possibile, che cosa significa miseria, freddo, fame, disperazione. Da Mucchi trovai sempre un uovo, o cinque lire. E non io solo, altri amici e colleghi artisti e scrittori bussavamo a quella porta di Via Rugabella, e ne uscivamo sollevati.

Ma il mio debito non è solo di questo genere: in casa di Mucchi ci si vedeva con Birolli, Manzù, Tomea, Sassu, Cantatore e Quasimodo e i De Grada, regolarmente un giorno ogni settimana; spesso io restavo li a dormire su una branda nello studio al caldo, perché il mio scantinato di via Guglielmo Pepe spremeva acqua dalle mura. Discutevamo di pittura e di

zioni manoscritte alla fine; Norberto Bobbio a Gabriele Mucchi, Torino, 10 febbraio 1953, 3 facciate dattiloscritte: per un errore materiale, questa lettera di Bobbio porta la data "1952", ma è chiaramente la risposta alla lettera di Mucchi della settimana prima.

98 Ulteriori notizie su Bobbio e Mucchi, nonché sul loro vasto carteggio, sono in Mario G. Losano (2018), Norberto Bobbio, 400-403; cfr. inoltre Gabriele Mucchi (1994), Le occasioni perdute.

99 In: Realismo. Mensile di arti figurative (1952, giugno), n. 1, p. 1.

II. Italia 
antifascismo, vedevamo libri e riviste, gettavamo un'occhiata sull'Europa proibita (era il tempo dell'aggressione all'Etiopia e delle sanzioni). Si parlava con Sassu di Diego Rivera, si polemizzava sulla pittura sovietica che s'era vista qualche tempo prima alla Biennale di Venezia, si iniziava in un modo incerto e appassionato un discorso che doveva poi svilupparsi e precisarsi negli anni futuri". Un ambiente di sinistra ma non conformista: "I comunisti, e Mucchi lo è senza riserve, hanno il dovere di non essere rozzi, schematici, approssimativi, di non essere come farebbe comodo al nemico che noi fossimo".100

Questo era dunque l'ambiente in cui operava il corrispondente di Bobbio negli anni in cui i due discutevano sulla contraddittoria natura dei Partigiani della Pace.

Gabriele Mucchi a Norberto Bobbio, Milano, 3 febbraio $1953 .{ }^{101}$

Carissimo Bobbio,

È già tanto tempo che ti voglio scrivere, ma tu sai come succede... Ora sono a letto con l'influenza e trovo la buona occasione.

Prima di tutto: ti rimando 500 L. delle 1500 che mi hai dato a Parigi. Distrattamente mi son fatto dare da te un abbonamento per l'estero: l'essere a Parigi mi ha indotto in quest'errore.

Secondo: spero che avrai ricevuto i numeri passati di Realismo. Avrai già pensato male di noi, di me. No. Adesso sta uscendo un numero doppio, dove troverai certamente qualcosa di interessante e anche qualche cosa di mio. Esce in ritardo, e non so se riusciremo a riacchiappare la periodicità col prossimo numero. È un lavoro fatto, per forza di cose, troppo "artigianalmente". Leggerai anche un appello, steso da me, ai lettori, pregati di comprensione e di aiuto. Spero che anche tu ci darai una mano, anche se il nostro giornale avrà qualche punto che non condividi - se non altro per la convinzione che c'è dentro. ${ }^{102}$

100 Renato Guttuso (1954), La nuova esperienza di Mucchi. Mucchi si ricollega a questo saggio in Gabriele Mucchi (1955), Pittura e antifascismo.

101 Intestazione dattiloscritta: "Gabriele Mucchi, Milano, 501 Viale Montesanto 3, Tel. 638755 - 3 II 1953”. Alcune abbreviazioni sono state tacitamente completate nella trascrizione dell'originale dattiloscritto.

102 Il "numero doppio" di Realismo. Mensile di arti figurative cui fa riferimento Mucchi è in realtà il "n. 5-6-7, novembre 1952 - gennaio 1953", di 12 pagine. Pubblica in prima pagina l'appello scritto da Mucchi, ma firmato "La Redazione": "Anche questo numero di 'Realismo' - numero doppio - esce con ritardo sul previsto" perché "non può ancora permettersi il lusso di quel personale di redazione retribuito che servirebbe a risolvere con un lavoro metodico e continuato il problema dell'esattezza nella periodicità". Il "qualcosa di mio” cui accenna Mucchi è l'ampio articolo Gabriele Mucchi (1952), Del manierismo. 
Terzo: ho letto con molto interesse il tuo opuscolo "Pace e propaganda di pace". ${ }^{103}$ La tua argomentazione è molto fine intelligente e anche, salvo in ultimo, simpatica. Ho l'impressione che ti abbia portato anche più in là di dove tu stesso volevi andare. (Dico questo perché secondo me tu sei il tipo di un... partigiano della Pace!) La cosa più vera mi pare che sia là dove tu dici "La realtà è più complessa di qualche sillogismo". È più complessa e anche più semplice dei tuoi intelligenti sillogismi. Forse è diversa.

I Partigiani della Pace vogliono (v. Appello del Congresso dei Popoli di Vienna, che come ti ebbi a dire non è più il Congresso dei Partigiani della Pace soltanto) "che sia abbandonato il ricorso alla forza a profitto della negoziazione” e doman[d]ano che U.S.A - U.R.S.S. - Cina - Inghilterra Francia aprano le negoziazioni. Il Congresso indica ciò come necessario per "metter fine alla tensione internaz. e preservare il mondo da disastri maggiori”. Questo è il documento principe. Naturalmente in altri documenti essi domandano la non-rimilitarizzazione della Germania e del Giappone, il ritiro di tutte le truppe straniere, la cessazione del fuoco in Corea, Malesia, Viet Nam, la libertà dei popoli coloniali a scegliere il loro destino ecc. Ma come vogliono queste cose? per negoziazione immediata. E non domandano di essere loro a negoziare. Non vogliono essere, loro stessi, pacieri. Non è vero che "si offrono per ristabilire la pace fra i contendenti" - ma semplicemente chiamano i contendenti alla pace. Li chiamano ponendoli davanti alle loro responsabilità: non per le vie diplomatiche tradizionali, ma attraverso una nuova diplomazia, che non è più nemmeno diplomazia, ma è: voce dei popoli. Il Congresso dei Popoli di Vienna è stato semplicemente: il Congresso dei popoli. È l'organizzazione che sostituisce l'ONU per il fatto che qui ci sono uomini semplici delegati di popoli, mentre là ci sono i diplomatici, delegati di governi. La nuova diplomazia o non diplomazia parte dal fatto che i popoli, in sé, non hanno interesse a farsi guerra. Da ciò deriva che pure le nazioni, là dove sia il popolo ad ${ }^{104}$ avere il potere, non hanno più interesse a farsi guerra. (Perciò noi diciamo che l'avvento del popolo al potere sarà un bene per la maggioranza degli uomini e [1|2] finirà con essere un bene finale per tutti.)

103 È l'estratto dell'articolo di Bobbio, Pace e propaganda di pace; cfr. nota 79, p. 285.

104 Nel dattiloscritto: "da". 
Qui tu arriverai col tuo stringatissimo ragionare e arriverai perfino a dimostrarmi, come fai nel tuo scritto, che chi vuole la pace in realtà vuole la guerra. Come fare, caro Bobbio? Ce lo siamo già detto anche a Parigi: poiché anche attraverso i ragionamenti più acuti non si arriva alla verità assoluta, bisogna scegliere fra lo scetticismo inevitabile, nell'un caso, e una qualche verità che si ritiene giusta e sulla quale si decide di basare i propri atti, nell'altro caso. Per me la verità è quella che dice che il capitalismo porta all'imperialismo e che l'imperialismo sbocca anche senza volerlo (e ti ammetto senz'altro che una quantità di capitalisti siano amanti amantissimi della pace), per forza, nella guerra. La mia esperienza (breve, in confronto alla Storia...) mi conferma questa verità. Succederà un giorno che paesi socialisti si attacchino fra loro? Non voglio aspettare gli anni della morte per vedere se la mia verità era valida o no, e magari morire col dubbio... Perciò lotto per la pace dei popoli sapendo che quella è la pace per tutti, lotto nel modo, come posso, che mi sembra giusto e che è, ti assicuro, molto bello.

E qui devo dirti che a mio giudizio il tuo studio cade proprio all'ultima pagina, là dove concludi mettendo in cattiva luce i Partigiani della Pace (che "dicono e disdicono", che hanno un'“ambigua natura", che alla luce delle parole di Stalin (male interpretate) rivelerebbero "la reale natura del movimento" (circoscrivendone in modo preoccupante l'efficacia) là dove dici: "...l'azione di pace... deve trasformarsi in un'azione rivoluzionaria ('rovesciamento') vale a dire - non bisogna aver paura delle parole - in un'azione di "guerra".

Qui il fine dialettico ti fa fare confusione sul concetto di guerra. Diventa un sofisma: il "vero partigiano della Pace nella situazione del tiranno è partigiano della guerra" come tu dici. Tali sono stati i rivoluzionari francesi che hanno creato la civiltà che ci ha nutriti, tali i patrioti che hanno combattuto per l'unità d'Italia e degli altri paesi in quei tempi, tali i rivoluzionari russi che hanno creato il primo paese socialista, tali i partigiani del '43-'45 ecc. ecc.? Tali tutti quelli che hanno fatto guerra all'ingiustizia per la giustizia? Ma questi non sono fautori di guerra, e non sono io che lo dico.

Io sto con loro - altrimenti con chi dovrei stare? con chi ha scatenato la Prima e la Seconda guerra mondiale, o con chi scatenerà la nuova guerra capitalista?

Tu, con chi vuoi stare? È tutto qui il problema: soli, al di sopra del bene e del male? No, sennò si finisce con lo stare semplicemente con chi dirige la baracca nel momento attuale: oggi americani e Vaticano. 
Salutami molto caramente tua Moglie e a te una viva stretta di mano e grazie per avermi dato l'occasione di questa chiacchierata.

Tuo Mucchi

Dimenticavo: nemmeno il primo passo staliniano a mio giudizio è interpretato bene. Non dice, come dici tu, "che la pace è una cosa buona anzi ottima, ma non rappresenta il fine supremo, e che il fine supremo è la distruzione dell'imperialismo". Dice invece: le varie cose che otterrà il movimento per la pace sono cose buone anzi ottime, ma non bastano a eliminare le guerre fra $i$ paesi capitalisti ("tuttavia questo non basta per eliminare l'inevitabilità delle guerre fra i paesi capitalistici") - (tutta l'argomentazione di Stalin in questo scritto è tesa a dimostrare che avverranno guerre fra $\mathrm{i}$ paesi capitalisti). Dunque: 1 . le guerre avverranno a causa dell'imperialismo fra i paesi capitalisti; 2 . la pace invece si manterrà tra i paesi socialisti; 3 . la pace potrà esserci anche fra $i$ paesi capitalisti, e quindi fra tutti, se sarà distrutto l'imperialismo. Questo non significa che la pace non sia il fine supremo e che il fine supremo sia invece la distruzione dell'imperialismo! Questo è l'unico mezzo possibile perché anche i paesi capitalisti arrivino alla pace, cioè al fine supremo: pace per tutti. ${ }^{105}$

Norberto Bobbio a Gabriele Mucchi, Torino, 10 febbraio $1953^{106}$

Caro Mucchi,

Ti ringrazio della tua attentissima lettura del mio opuscolo sulla pace e delle tue osservazioni. Non potevi darmi miglior prova della tua amicizia che con questa lettera che esamina e discute con tanto scrupolo e pazienza le mie tesi.

Ti rispondo non con una difesa ma semplicemente con qualche chiarimento.

105 Il passo da "Questo non significa" a "pace per tutti" è aggiunto a mano in fondo alla pagina, proseguendo poi sul margine destro.

106 Su carta intestata "Università degli Studi di Torino, Facoltà di Giurisprudenza" con la data "Torino, 10 febbraio 1952": il dattiloscritto indica "1952" per un palese errore di battitura, cfr. nota 97, p. 296. 
1) Quando io dico che il partigiano della pace può trasformarsi in un partigiano della guerra (comincio da questo punto che più ti ha colpito) voglio dire semplicemente che data l'impostazione iniziale del movimento (l'America è il tiranno, la Russia il liberatore) il vero obiettivo non può essere quello della pace, ma è quello - come sempre in una situazione in cui si contrappone il tiranno ai suoi oppressori ${ }^{\mathbf{1 0 7}}$ - della guerra. Ciò non vuol avere nelle mie parole un significato peggiorativo o dispregiativo (ho sempre infatti parlato di guerra giusta), ma semplicemente mettere in rilievo il contrasto che esiste tra il chiamarsi partigiani della pace e sostenere una politica che non può arrivare alla pace (eliminazione dell'imperialismo) se non attraverso alla guerra. Non dico dunque che i partigiani della pace siano fomentatori di guerra. Constato semplicemente che se si pone come termine finale della propria azione la distruzione dell'imperialismo, bisogna avere il coraggio di dire che questo fine non può essere raggiunto che con la guerra e non illudersi e illudere gli altri parlando di pace. Da quando le classi dirigenti americane parlano di liberazione degli stati sovietizzati, posso comprendere qualcuno che dica che questo è un fine nobilissimo, ma non potrei comprendere che qualcuno dicesse che è un fine [1|2] pacifico.

2) Prendo atto con soddisfazione del fatto che nel Congresso dei Popoli i partigiani della pace abbiano insistito più sulla linea della coesistenza e della negoziazione che su quella dell'antiamericanismo. E ciò che tu mi dici mi viene confermato dal discorso inaugurale di Joliot Curie ${ }^{\mathbf{1 0 8}}$ (che ho letto in quei giornali che mi hai dato e di cui ti ringrazio ancora), che potrei sottoscrivere. Secondo me questa è l'unica via possibile per un movimento che voglia avere una qualche efficacia come movimento per la pace. Mi pare dunque che il mio articolo scritto prima del Congresso e in occasione di esso metteva il dito sul punto giusto, vale a dire sul fatto che non si può chiedere la coesistenza pacifica e nello stesso tempo continuare a dire che dei due blocchi che dovrebbero coesistere uno solo è quello bellicista.

3) Che le nazioni dove il popolo governa non si facciano più la guerra è una vecchia divisa dei pacifisti. Mazzini profetava che [non] ci sarebbero più

107 Verosimilmente non "oppressori” (come nel dattiloscritto), ma "avversari".

108 Il fisico francese Frédéric Joliot-Curie (1900-1958) ricevette nel 1934, con la moglie Irène, il premio Nobel per la chimica; partecipò alla resistenza francese; nel 1950 venne privato dei suoi incarichi perché comunista e ricevette il Premio Stalin per la Pace per la sua attività di presidente del congresso mondiale dei Partigiani della Pace del 1949. 
state guerre quando alla santa Alleanza dei Re si sarebbe sostituita la Santa Alleanza dei Popoli. Furono fatti i popoli e mai guerre furono più terribili. Si disse che non erano i popoli che decidevano ma i borghesi coi loro parlamenti. Ma dobbiamo credere proprio che passando da un regime borghese ad uno socialista siano i popoli a decidere? Nel regime socialista britannico è ancora il parlamento; in quello sovietico è la direzione del partito. Perché le direzioni di partito di due paesi socialisti non dovrebbero scatenare la guerra? Il conflitto Stalin-Tito insegni.

Questo per dire che la guerra è sempre possibile sino a che ci saranno stati, siano feudali, borghesi o proletari. L'unica cosa seria che può fare un movimento di partigiani della pace è di mettersi in mezzo ai litiganti (stati) per cercare di diminuirne l'attrito. Se questo è stato fatto al Congresso di Vienna vuol dire che si è imboccata ${ }^{109}$ la via giusta. Ma guai se questa voce contro i governi [2|3] per uno scambio (di cui tu non sei del tutto immune) tra la voce dei popoli e quella degli stati cosiddetti popolari si trasformasse in una voce contro un solo governo. Tutti i governi in quanto tali (e lo dico senza indignarmi perché è una legge sociale) considerano la guerra come un mezzo per conservarsi e sopravvivere. La lotta contro la guerra si fa solo mettendosi contro i governi [,] contro tutti i governi, anche contro quelli delle repubbliche popolari. Se deve essere voce di popolo come tu dici [,] sia contro le "cricche" imperialistiche americane e anche contro le direzioni dei partiti degli stati sovietici.

4) Quanto al tuo invito a decidersi, con cui la tua lettera si chiude, rispondo che la mia decisione è proprio quella di non stare né di qua né di là, o meglio per dirlo con la formula della nostra società europea di cultura $^{110}$ e di qua e di là. Credo che [sia] l'unica posizione consona alla situazione dell'uomo di cultura che vede tante magagne (e anche tanti valori) da tutte e due le parti e si rifiuta di distinguere il mondo in eletti e reprobi (come purtroppo la maggior parte dei comunisti da un lato [e] la 'cricca' di Eisenhower dall'altro hanno già fatto).

Grazie delle 500 lire e tanti cordiali saluti.

[Senza firma, perché copia dattiloscritta realizzata con la carta carbone.]

109 Nel dattiloscritto: "imbroccata".

110 Bobbio si riferisce alla Società Europea di Cultura, diretta da Umberto Campagnolo, di cui facevano parte tanto Bobbio quanto Mucchi. 


\section{Germania: Il ripudio della guerra e il riarmo nelle costituzioni dei due Stati tedeschi}

La divisione postbellica del territorio tedesco portò all'emanazione di due costituzioni: una per le tre zone occupate dagli Alleati occidentali, l'8 maggio 1949, ed una per la zona occupata dai sovietici, il 7 ottobre 1949. I due testi costituzionali nascevano sotto la pressione di ideologie contrapposte, ma si richiamavano a una comune eredità nazionale dalla quale prendevano le distanze. Quindi, sia pure con una terminologia e con un tono diversi, nelle due costituzioni affiorano alcuni temi legati a una storia oggi spesso dimenticata: dall'una e dall'altra parte della Cortina di Ferro, $i$ tedeschi sentivano incombere una nuova guerra e, inoltre, temevano l'arruolamento di tedeschi in eserciti non tedeschi; erano incerti sull'ambito territoriale in cui le norme avrebbero dovuto essere applicate e ciascuno Stato evitava di indicare la Germania per lui "altra" con il suo nome ufficiale, per non pregiudicare un'eventuale unificazione, che avverrà oltre quarant'anni dopo.

Per la Germania occidentale viene qui presentata una scelta di passi tratti dai verbali delle riunioni dei costituenti (\$III.1), che documentano come nel dibattitto costituente il linguaggio politico andasse assumendo forme sempre piu giuridiche, per cristallizzarsi infine nei singoli articoli della Legge Fondamentale della Germania occidentale. Della Germania orientale vengono invece tradotti alcuni articoli della costituzione e due brevi testi legislativi (III. 2 e 3), uno interamente dedicato alla difesa della pace e l'altro alla creazione dell'Armata Popolare Nazionale. Questo duplice riarmo contrapposto creava due eserciti tedeschi che operavano in alleanze militari ostili l'una all'altra, la Nato e il Patto di Varsavia, e quindi per tutti $i$ tedeschi il timore d'una guerra in generale includeva anche lo spettro d'una possibile guerra civile.

Questa contrapposizione appare chiara nella definizione che un'enciclopedia della DDR forniva nel 1964 dell'Armata Popolare Nazionale di quello Stato: "La fondazione della Armata Popolare Nazionale (NVA) rispondeva all'esigenza dei lavoratori di proteggere con forza le conquiste del socialismo e si rese necessaria sulla base della rimilitarizzazione della Germania occidentale, della sua entrata nella Nato e della creazione della Bundeswehr, cioè dell'esercito federale". ${ }^{111}$

111 Meyers Neues Lexikon in acht Bänden (1964), s.v. Volksarmee. 
III.1. Il ripudio della guerra nel dibattito costituente tedesco-federale (1948)

I documenti sulla genesi dell'attuale costituzione della Germania federale esigono una lettura particolare: è soprattutto scoprendo la filigrana nascosta dietro le parole che si percepisce la penombra incerta in cui viveva la Germania dell'immediato dopoguerra, in cui tutto era stato perso e ben poco ricostruito; in cui gli amici di oggi erano $i$ nemici di ieri, in cui non si sapeva di preciso quale territorio corrispondesse alla parola "Germania"; in cui si erano appena spenti i fuochi della guerra calda e già si avvertiva l'alito gelido della Guerra fredda. Né i padri costituenti né l'uomo della strada potevano intuire se quella penombra incerta era solo il crepuscolo di un passato sinistro o era già l'annuncio di un'alba serena. L'unica speranza era che il far parte d'una fine potesse già essere un inizio.

Quest'atmosfera di incerte certezze traspare anche dalle pieghe della verbalizzazione neutra e stringata dei dibattiti costituenti, dove poche parole quasi nascoste evocano quelle ombre che settant'anni fa gravavano sul presente e sul futuro e che, quindi, oggi rischiano di sfuggire a chi non ha più la percezione del mondo di allora. Sono rivelatrici, ad esempio, le esitazioni per indicare l'ambito di applicazione territoriale dei testi normativi. Poiché non era più chiaro che cosa significasse "Germania", le soluzioni proposte facevano riferimento al territorio o agli abitanti, anche per lasciarne aperta l'applicazione a futuri riaccorpamenti della nazione smembrata: ad esempio, si sostituisce 'l'espressione 'inclusione della Germania' con 'inclusione del suo territorio" negli enti sovranazionali (cfr. infra, nota 126, p. 310).

'Territorio' ormai neppure più autonomo nelle sue decisioni perché sotto lo stretto controllo degli Alleati. Un costituente, nel proporre di "includere anche qualcosa sulla produzione di armi", aggiunge: "Si tratta di qualcosa che ci viene ordinato dall'esterno" (cfr. infra, p. 313). A proposito degli articoli costituzionali pacifisti un altro costituente constata: "Questo capitava ai tempi in cui gli americani erano terribilmente interessati alla questione. Siccome oggi non se ne interessano più di tanto, lasciamo cadere questo articolo!" (cfr. infra, p. 319). Inoltre si era attenti alla "reazione della stampa americana alle disposizioni previste dal Progetto del Herrenchiemsee" sul rifiuto della guerra, perché agli occhi degli americani quello era il "biglietto da visita" della nuova Germania (cfr. infra, III.1, c). Infine, oggi risulta enigmatica la richiesta che "ai tedeschi sia proibito di prestare servizio militare sotto la bandiera di uno Stato straniero" (cfr. infra, nota 137, p. 322): eppure nell'incipiente Guerra fredda e sotto l'occupazione straniera per non pochi tedeschi - che non avevano ancora un loro esercito - l'essere chiamati

III. Germania 
dagli americani a combattere contro $i$ sovietici era una possibilità non astratta. Non era chiaro al comando di chi sarebbe stata sottoposta l'unità tedesca nelle forze armate europee, in quella CED che poi non venne realizzata (cfr. p. 286). Un costituente sottolineava che l'articolo contro la guerra poteva rivelarsi utile anche a questo fine: "Se ora però cancelliamo il passo in questione, si dirà che spianiamo la strada a un militarismo americano, perché permetteremmo alla popolazione di prestare servizio sotto gli americani" (cfr. infra, p. 326).

Invece i tempi non erano ancora maturi per regolare l'obiezione di coscienza. La vivace discussione su questo argomento (cfr. infra, III.5) non portò a un corrispondente articolo nella costituzione del 1949, che comunque prevedeva che nessuno potesse essere obbligato al servizio militare (art. 4, c. 3m GG). Nel 1956 la Germania federale istitui il servizio militare obbligatorio. Solo una ventina d'anni dopo, con la modifica costituzionale del 24 giugno 1968, la materia venne dettagliatamente regolata dal nuovo articolo 12 a, composto da sei lunghi commi sul servizio militare e civile obbligatorio. Le trasformazioni di questo articolo riflettono anche l'evoluzione della posizione femminile nella società tedesca. Infatti nella formulazione originaria l'art. 12a riguardava soltanto "gli uomini a partire dai diciotto anni compiuti" (c. 1) e affermava che le donne "in nessun caso possono prestare servizio in armi" (c. 4). In seguito a una sentenza della Corte di Giustizia Europea che asseriva il carattere discriminatorio di quella norma, la modifica costituzionale del 2000 portò all'attuale disposizione, secondo cui le donne "in nessun caso possono essere obbligate a prestare servizi armati” (c. 4). Dal 2011 è stato abolito il servizio di leva obbligatorio e la Bundeswehr è organizzata su base volontaria.

Con la fine della guerra, l'Impero Tedesco si era trasformato in un territorio frammentato e occupato dalle truppe vincitrici. Gli Alleati temevano una Germania di nuovo unita, quindi avevano imposto uno Stato federale riesumando $i$ Länder preunitari, ma non la troppo vasta Prussia, che era stata smembrata e che non sussisteva più neppure come nome. Nel primo dopoguerra $i$ nuovi Stati della Federazione, $i$ Länder, erano stati $i$ primi a darsi delle costituzioni democratiche, mentre l'incerto futuro complessivo della Germania postbellica rendeva difficile fissare un globale assetto costituzionale per un paese diviso in quattro zone d'occupazione (che vedevano però le tre zone occupate dagli Alleati occidentali contrapposte alla Zona di Occupazione Sovietica), con Berlino regolata da uno statuto specifico, con territori ceduti alla Polonia e all'Unione Sovietica e, infine, con il destino della Sarre ancora incerto e destinato a concludersi solo nel 1956 con la sua unione alla Repubblica federale ormai consolidata. 
Per non compromettere una futura unificazione della Germania, come si è detto, si evitò di creare un' "assemblea costituente" per dare una "costituzione" a un territorio cosi fluido, ma si preferi istituire una "convenzione costituzionale" (Verfassungskonvent) per dare una "legge fondamentale" (Grundgesetz) alle tre zone occupate dagli Alleati occidentali, che sarebbero cosi divenute la Repubblica Federale di Germania, contrapposta alla Repubblica Democratica Tedesca, che ebbe una sua vita separata - anche costituzionalmente - dal 1949 al 1990.

Gli 11 presidenti dei Länder occidentali convocarono una "convenzione costituzionale" - o "Verfassungskonvent auf Herrenchiemsee" - che si riuni dal 10 al 23 agosto 1948 nel castello di Ludwig II su un'isola lacustre in Baviera, la Herreninsel nel Chiemsee: qui prese origine il progetto di costituzione e il catalogo dei diritti fondamentali che servi di base al successivo lavoro costituente del Consiglio Parlamentare (Parlamentarischer Rat) e che, infine, conflui nella Legge Fondamentale ${ }^{112}$ ancora oggi in vigore.

Il Parlamentarischer Rat venne eletto dagli undici parlamenti (Landtage) dei Länder occidentali e si riuni a Bonn dal $1^{\circ}$ ottobre 1948, sotto la presidenza di Adenauer, per preparare un testo costituzionale sulla base dei "Frankfurter Dokumente" dei tre Alleati occidentali e del progetto elaborato dal "Verfassungskonvent auf Herrenchiemsee". Era composto da 65 membri a pieno titolo e da 5 membri soltanto consultivi in rappresentanza di Berlino, il cui status ne faceva un tertium che non stava né da una parte né dall'altra e che quindi - non solo in questo caso osservava ma non decideva. Il testo approvato dal Parlamentarischer Rat l'8 maggio 1949 (con dodici voti contrari) e, successivamente, dai parlamenti dei Länder occidentali e dai tre governatori militari occidentali, entrò in vigore il 23 maggio 1949: a partire da questa data esso divenne la costituzione (o, piu propriamente, la "Legge Fondamentale") della Repubblica Federale di Germania e, dopo l'unificazione nel 1990, venne estesa anche ai territori dell'ex Repubblica Democratica Tedesca.

I testi tradotti qui di seguito sono tratti dai protocolli delle sedute di questi organi costituenti, pubblicati dal Parlamento Federale (Bundestag). ${ }^{113}$ I protocolli sono ricchi di note con rinvii interni, con richiami a documenti e a precisazioni: vengono qui riportate solo le note ritenute utili per il lettore odierno, mentre le note del traduttore sono tra parentesi quadra.

112 Peter März, Heinrich Oberreuter (eds.) (1999), Weichenstellung für Deutschland?

113 Der Parlamentarische Rat: 1948-1949. Akten und Protokolle (1975-2009).

III. Germania 
a) "Diritto internazionale e diritto federale"114

Le pagine che seguono provengono dal "Rapporto del Sottocomitato I" (Bericht des Unterausschusses I, uno dei tre Sottocomitati del Verfassungskonvent), ${ }^{\mathbf{1 1 5}}$ che affronta $i$ problemi di principio, quindi anche il rapporto fra diritto internazionale e diritto federale (Völkerrecht und Bundesrecht). In quest'ultima tematica ricade il rifiuto della guerra, perché esso è strettamente connesso con l'entrata della Federazione "in un sistema di sicurezza collettiva che le garantisca la pace".

I. - La Commissione era unanimemente dell'opinione che le regole generali del diritto internazionale dovessero essere parte integrante del diritto federale, e precisamente nel senso che esse devono generare direttamente diritti e doveri per gli abitanti del territorio nazionale (cittadini e stranieri). La formulazione adottata è diversa dall'art. 4 della Costituzione di Wei$\operatorname{mar}^{116}$ al fine di evitare le controversie che nell'epoca di Weimar hanno avuto un ruolo fatale. ${ }^{117}$ Inoltre questa formulazione deve esprimere il fatto che il popolo tedesco ha la volontà di vedere nel diritto internazionale qualcosa di più che un ordinamento le cui norme vincolano soltanto gli Stati in quanto tali.

La Sottocommissione propone unanimemente di includere il seguente articolo C nella Legge Fondamentale: Le norme generali del diritto internazionale sono parte integrante del diritto federale e producono direttamente diritti e doveri per tutti gli abitanti del territorio federale. ${ }^{\mathbf{1 1 8}}$

114 [Peter Bucher (ed.) (1981), Der Verfassungskonvent auf Herrenchiemsee, 206-208. La Einleitung del volume contiene la dettagliata storia di questi primi passi costituzionali (VII-CXXXV), poiché circolava già un progetto bavarese: Nr. 1. Bayerischer Entwurf eines Grundgesetzes für den Verfassungskonvent, 1-52.]

115 Peter Bucher (ed.) (1981), Der Verfassungskonvent auf Herrenchiemsee, LXXV-LXXXII: Unterausschuß I: Grundsatzfragen (Präambel, Namensgebung, gebietliche Ausdehnung und Gliederung, Quelle der Staatsgewalt, Gerichtshof zur Sicherung der verfassungsmäßig gewährleisteten Rechte). I suoi componenti sono elencati alle pp. LXXIII-LXXIV [del testo originale tedesco].

116 Art. 4 della Costituzione di Weimar: "Le regole generalmente riconosciute del diritto internazionale sono in vigore come parte integrante e vigente del diritto imperiale”. [Nota 59 dell'originale]

117 Di parere contrario Apelt, p. 145: "Per quanto è dato di vedere, nella prassi statale l'art. 4 non ha svolto alcuna funzione particolare e, in ogni caso, non ne è derivata alcuna difficoltà". [Nota 60 dell'originale]

118 Proposta di Carlo Schmid nella $2^{\mathrm{a}}$ seduta della Sottocommissione: "Le regole generalmente riconosciute del diritto internazionale sono parte integrate del diritto federale e 
II. - La Sottocommissione ritiene inoltre concordemente che la Legge Fondamentale debba prevedere che la Federazione - con una legge approvata a maggioranza qualificata - possa trasferire diritti sovrani a enti interstatali. Si deve così facilitare l'istituzione di organismi internazionali che (con effetto sul territorio degli Stati partecipanti) devono essere creati per occuparsi di eventi che finora erano lasciati esclusivamente alle singole sovranità nazionali. Il popolo tedesco esprime la volontà di rinunciare in futuro alla guerra come mezzo della politica e a trarne le debite conseguenze. Tuttavia, per non essere preda indifesa della violenza altrui, ha bisogno che il territorio federale venga incluso in un sistema di sicurezza collettiva che gli garantisca la pace. È opinione concorde del Comitato che la Federazione debba essere pronta - nell'interesse della pace e di un durevole ordine dei rapporti europei - ad approvare le limitazioni ai suoi poteri sovrani derivanti dai rapporti all'interno di tale sistema. La Commissione è consapevole che così si impone un impegno preventivo al popolo tedesco, ma è tuttavia del parere che, dopo quanto è avvenuto in nome del popolo tedesco, sia opportuno quest'impegno preventivo come conseguenza dei corrispondenti impegni degli altri Stati partecipanti.

La Sottocommissione decide unanimemente di includere la seguente proposta di articolo D nella Legge Fondamentale:

generano direttamente diritti e doveri per tutti gli abitanti del territorio federale". Nelle parole "generalmente riconosciute" Nawiasky vedeva "la fonte di una possibile controversia. Più volte la scienza giuridica ha sostenuto che lo Stato che si appella al diritto internazionale deve averlo approvato. Pertanto egli raccomanda di dire soltanto "le regole" o "i princìpi". Beyerle temeva che "venissero così recepite anche tutte le teorie del diritto internazionale" [...]. È infatti sempre dubbio in quale misura una frase è adatta a esprimere un riconoscimento. Si pone così il quesito se ciò che è stato stabilito a Norimberga sia riconosciuto anche da noi e venga accettato come diritto internazionale. Si potrebbe anche pensare di dire "le regole giuridiche (Rechtssätze) del diritto internazionale". Nawiasky riteneva che quest'espressione fosse troppo specialistica e proponeva "le regole generali del diritto internazionale". Carlo Schmid voleva anche "che venissero inclusi anche i princìpi di diritto naturale del diritto internazionale, che sono l'espressione del livello di civiltà di un'epoca": "Sarebbe una novità se noi ora dichiarassimo: Noi consideriamo il diritto internazionale non come un diritto che obbliga lo Stato soltanto (ma non il singolo dentro lo Stato), bensì come un diritto universale, che attraverso la crosta dello Stato giunge fino all'individuo, conferendogli diritti immediati e imponendogli doveri immediati”. [Nota 61 dell'originale] 
"La Federazione può trasferire per legge $i$ diritti di sovranità a istituzioni interstatuali. $^{\mathbf{1 1 9}}$

In particolare, nell'interesse del mantenimento della pace, può inserire il proprio territorio in un sistema di sicurezza collettiva e, sulla base della reciprocità, accettare quelle limitazioni della propria sovranità, grazie alle quali si può raggiungere e garantire un ordine pacifico e duraturo dei rapporti europei. ${ }^{\mathbf{2 0}}$

Tale legge richiede la maggioranza del numero legale dei membri del Bundesrat e del Bundestag."

III. Infine la Commissione ha ritenuto che la Legge Fondamentale debba contenere una norma che punisca le azioni intraprese nell'intento di turbare la coesistenza pacifica dei popoli e soprattutto le azioni poste in essere con l'intenzione di preparare una guerra. In futuro il diritto federale deve offrire la possibilità richiamare alle loro responsabilità le persone la cui attività, a partire dal suo territorio, sia intenzionalmente diretta a minacciare pericolosamente la pace, si tratti sia di riarmo segreto sia di istigazione militaristica e nazionalistica.

La Commissione è inoltre unanimemente d'accordo sul fatto che le persone colpevoli di questi delitti debbano essere escluse, dopo la condanna, dalla tutela garantita in base ad alcuni diritti fondamentali.

Per l'articolo E si propone la seguente formulazione: Le azioni intraprese con la finalità di turbare la coesistenza pacifica dei popoli e, in particolare, di preparare una guerra, devono essere punite. ${ }^{\mathbf{1 2 1}}$

119 Proposta di Carlo Schmid, che si riferiva all'ONU. [Nota 64 dell'originale]

120 Nella seconda seduta della Sottocommissione, Kordt aveva dapprima proposto: "Il popolo tedesco, riconoscendo che la sua esistenza e il suo sviluppo possono essere realizzati soltanto con l'inclusione della Germania in un sistema di sicurezza collettiva, è disposto in condizioni di reciprocità ad approvare quelle limitazioni della propria sovranità nazionale che possono garantire un ordine pacifico e durevole dei rapporti europei”. Formulazione di Dankwerts: "La Federazione può accettare limitazioni della propria sovranità se viene inclusa in un sistema di sicurezza collettiva, con il quale può essere garantito un ordine pacifico e durevole dei rapporti europei”. Queste proposte vennero così riassunte da Beyerle: "In particolare, nell'interesse del mantenimento della pace mondiale, [il popolo tedesco] può accettare l'inclusione della Germania in un sistema di sicurezza collettiva [...]". Su proposta di Suhr, Carlo Schmid sostituì l'espressione "inclusione della Germania" con "inclusione del suo territorio". [Nota 65 dell'originale]

121 Proposta di Carlo Schmid nella $2^{\text {a }}$ seduta della Sottocommissione I. Al dubbio espresso da Drexelius nella $7^{a}$ seduta della Sottocommissione I "con riferimento all'azione di truppe dell'ONU e dell'ingresso in un patto militare”, Beyerle rispose che "azioni di preparazione 
b) I rapporti di diritto internazionale della Federazione ${ }^{\mathbf{1 2 2}}$

All'unanimità viene proposto il seguente articolo:

Con una legge la Federazione può trasferire propri diritti sovrani a istituzioni interstatali.

In particolare, nell'interesse del mantenimento della pace, può inserire il proprio territorio in un sistema di sicurezza collettiva e può approvare limitazioni della propria sovranità, grazie alle quali si possa raggiungere e mantenere un ordine pacifico e durevole dei rapporti europei.

Tale legge richiede la maggioranza del numero legale dei membri del Bundesrat e del Bundestag.

Ripudio della guerra.

Infine la Convenzione ritiene che la Legge Fondamentale debba contenere una norma che punisca le azioni intraprese con l'intenzione di turbare la coesistenza pacifica dei popoli e, soprattutto, tutte le azioni intraprese al fine di preparare una guerra. Il diritto della Federazione deve in futuro offrire la possibilità di perseguire le persone la cui attività è intenzionalmente diretta a mettere in pericolo la pace, si tratti sia di riarmo segreto sia di istigazione militaristica e nazionalistica. Dopo la condanna, le persone colpevoli di questi delitti devono essere escluse dalla tutela garantita da alcuni diritti fondamentali.

L'articolo proposto a questo proposito ha la seguente formulazione:

Sono punite le azioni compiute con l'intenzione di turbare la pacifica convivenza dei popoli e, in particolare, di preparare una guerra.

alla guerra $[\ldots]$ sono punibili solo quando vengono intraprese al fine di turbare la coesistenza pacifica dei popoli”. [Nota 67 dell'originale]

122 [Il testo è tratto da Peter Bucher (ed.) (1981), Der Verfassungskonvent auf Herrenchiemsee, 517-518: Nr. 14. Verfassungsausschuß der Ministerpräsidentenkonferenz der westlichen Besatzungsmächte - Bericht über den Verfassungskonvent auf Herrenchiemsee vom 10. bis 23. August 1948. Nel Darstellender Teil si descrivono i problemi dello Stato federale futuro (segue l'intero progetto della Legge Federale) e, in particolare, i suoi problemi di diritto internazionale.] 
c) Le Regole del diritto internazionale e Legge Fondamentale (art. 22 CHE [Chiemseer Entwurf]) ${ }^{123}$

Passiamo ora agli articoli sul diritto internazionale. In proposito il Dr. Eberhard ha presentato una proposta. La cosa forse migliore è che egli stesso ci esponga un panorama generale della problematica. Poi potremo discutere sulla redazione dei singoli articoli.

Dr. Eberhard: Permettetemi anzitutto di esporre le ragioni per cui presento delle integrazioni alle proposte contenute nel Progetto del Herrenchiemsee. La mia proposta, distribuita in forma ciclostilata, si fonda su lunghe consultazioni con i collaboratori dell'Ufficio per la Pace.

A mio avviso, nel loro insieme questi articoli sono un biglietto da visita tedesco verso il mondo esterno. Inoltre, verso l'interno, possono avere un effetto educativo perché rendono chiara la nostra disponibilità ad accogliere le regole generali del diritto internazionale come parte integrante del diritto federale. Il fatto che producano effetti anche all'estero risulta chiaro dalla reazione della stampa americana alle disposizioni previste dal Progetto del Herrenchiemsee. Il "New York Herald Tribune" parla di una pietra miliare nella trasformazione del pensiero tedesco in pensiero europeo.

Penso però che si possa andare ancora oltre al Progetto del Herrenchiemsee che - nell'art. 2, c. 2 - autorizza la Federazione a includere il proprio territorio in un sistema di sicurezza collettiva nell'interesse del mantenimento della pace. Vorrei qui aggiungere anche la disponibilità a sottomettersi a un sistema di arbitrato internazionale. Anche nell'art. $25 \mathrm{mi}$ parrebbe importante una modifica. In base ad esso, la cessione o la permuta di parti del territorio federale sono efficaci soltanto se le modifiche sono approvate dalla corrispondente popolazione. Qui si dovrebbe dire: "Cessione e permuta di parti del territorio statale tedesco", per riferirsi non soltanto al territorio federale, ma anche ai Länder che oggi non sono parte del territorio federale.

Infine propongo un'integrazione all'Art. 26. L'Art. 26 prevede la punizione delle azioni intraprese nell'intento di turbare la convivenza pacifica dei popoli e, in particolare, di preparare una guerra. Ritengo che qui si debba

123 [Eberhard Pikart, Wolfram Werner (eds.) (1993), Ausschuß für Grundsatzfragen, 315-317. "Il Comitato per le Questioni di Principio è uno dei numerosi livelli di incontro e discussione del Parlamentarischer Rat”, IX (“... war der Ausschuß für Grundsatzfragen nur eine von mehreren Gesprächs- und Diskussionsebene des Parlamentarischen Rates”).] 
includere anche qualcosa sulla produzione di armi e munizioni. Si tratta di qualcosa che ci viene ordinato dall'esterno. Ma dobbiamo essere noi stessi a sottomettere la produzione delle armi a un controllo tedesco, riservando i particolari a un'apposita legge. Invece dovrebbe essere espresso il principio che ne è alla base: ciò ha anche una rilevanza di politica interna. Non credo che le SA e le SS avrebbero potuto armarsi se la Costituzione di Weimar avesse contenuto una disposizione che vietasse il trasporto, l'importazione, l'immagazzinamento e la circolazione di armi e munizioni.

Presidente, Dr. v. Mangoldt: Credo che ci sia poco da aggiungere sull'argomento. Se non vi sono richieste di intervento, possiamo passare alla discussione dei singoli articoli. La disposizione più importante riguarda le regole generali del diritto internazionale, le quali sono elementi costitutivi del diritto federale e generano direttamente diritti e doveri per tutti gli abitanti del territorio federale.

Dr. Eberhard: L'art. 28 ha un precursore nell'art. 4 della Costituzione di Weimar. Questa disposizione non ebbe alcuna rilevante importanza pratica, ${ }^{124}$ mentre noi qui diciamo chiaramente (come anche a proposito dei diritti fondamentali) che queste norme producono immediatamente diritti e doveri per tutti gli abitanti del territorio federale, quindi non solo per il Governo federale. Ho in mente il Patto Kellogg, ${ }^{125}$ il patto di rifiuto della guerra, che nel nostro caso vincolerebbe direttamente i cittadini. La formulazione "tutti gli abitanti del territorio federale" è stata scelta nel progetto del Herrenchiemsee per includere anche gli stranieri e le DP [Displaced Persons]. ${ }^{\mathbf{1 2 6}}$

Dr. Schmid: Sappiamo perché l'art. 4 della Costituzione di Weimar non ha praticamente prodotto alcun effetto nei tribunali. L'espressione "le regole generalmente riconosciute del diritto internazionale” offriva a ogni giudice

124 [Cfr. nota 116, p. 308; Costituzione di Weimar (1919), Art. 4: "I principi fondamentali riconosciuti dal diritto delle genti hanno valore di parti integranti del diritto tedesco".]

125 Il Patto Kellogg, sul rifiuto della guerra, fu firmato da 15 Stati a Parigi il 27 agosto 1928; Cfr. Hans Wehberg (1953), Krieg und Eroberung. [Nota 14 dell'originale]

126 [Il termine 'Displaced Persons' (DP) indica i profughi dell'Europa orientale e gli ex internati nei campi nazisti che, subito dopo la guerra, si trovavano in Europa soprattutto nei centri di raccolta ("DPs Camps") di Germania, Austria e Italia. Una parte di loro poté emigrare negli Stati Uniti grazie al Displaced Persons Act del 1948. Cfr. Mark Wyman (1998), DPs: Europe's Displaced Persons. 1945-1951.] 
la possibilità di affermare che questo e quello non era generalmente riconosciuto e che quindi poteva disinteressarsene.

Sappiamo inoltre che gli studiosi di diritto internazionale e la magistratura del Reichsgericht sostenevano decisamente la "teoria della trasformazione". In base ad essa il diritto internazionale ha soltanto un destinatario, cioè lo Stato. Esso attribuisce diritti e doveri soltanto agli Stati, e i singoli individui possono dedurre un diritto non dal diritto internazionale, ma soltanto da quello nazionale. Quindi finché il diritto internazionale non viene trasformato in diritto nazionale - con un atto legislativo o per consuetudine - l'individuo non può richiamarsi al diritto internazionale né può essere obbligato in base al diritto internazionale. Questa era la teoria internazionalistica largamente dominante nel XIX e nel XX secolo: e non soltanto in Germania, ma in forma particolarmente estrema in Germania. Il defunto professor Zorn ${ }^{127}$ ha prodotto effetti catastrofici. In Inghilterra la situazione era diversa. Nella teoria del diritto gli inglesi sono più generali. Ad esempio, non hanno mai avuto la differenza tra diritto amministrativo e il resto del diritto. Per loro anche il diritto internazionale era soltanto un prolungamento del Common Law. Solo più tardi, entrati in contatto più stretto con il pensiero giuridico europeo, la situazione mutò leggermente. In ogni caso in questo campo l'Inghilterra è sempre stata più avanzata e - nell'ambito del diritto internazionale generale - ha sempre attribuito direttamente diritti e doveri all'individuo. Il principio di diritto canadese "Common Law Canadian Law" è solo una creazione recente.

Sarebbe bene seguire questo esempio. La trasformazione del diritto internazionale in diritto statale per via legislativa non è qualcosa di necessario "in sé”, o per natura o per ragione, ma è una convenzione su cui si sono trovati d'accordo professori e uomini di Stato. Perché allora non spingere un po' oltre questa convenzione, anzi dissolverla e sostituirla con una nuova, in base alla quale il diritto internazionale deve essere direttamente parte del diritto nazionale, in modo che da esso derivino direttamente doveri e diritti per l'individuo? Naturalmente ciò non varrebbe per il diritto internazionale

127 Philipp Zorn (1850-1928), autore di numerosi scritti sulle due conferenze di pace dell'Aia; p. es. Die internationale Schiedgerichtsbarkeit, Hannover 1913. [Il titolo completo è Philipp Zorn (1913), Friedens- und Kriegsbündnisse. Lo scritto Die internationale Schiedgerichtsbarkeit venne poi ripubblicato nella rivista Das Recht nel 1917 (come estratto: Helwing, Hannover 1917).] 
pattizio, quando cioè gli Stati sottoscrivono trattati su specifici argomenti. Non intendo dire questo. Mi riferisco alle regole generali del diritto internazionale. Mi spingerei anzi a sostenere che queste regole generali varrebbero anche se ad esse seguisse un accordo internazionale. Una formulazione dettagliata di queste regole generali è, per esempio, la normativa dell'Aia sulla guerra terrestre. Non si tratta di un diritto configurato in modo particolare, ma della fissazione contrattuale di un generale diritto internazionale consuetudinario.

Siamo così giunti a parlare non di regole del diritto internazionale generalmente riconosciute, ma soltanto di regole generali. Esse devono essere dichiarate direttamente parte integrante del diritto federale e da esse devono scaturire direttamente doveri e diritti per gli abitanti del territorio federale. Ma attenzione: non soltanto per i cittadini dello Stato federale; anche lo straniero che abita da noi deve potersi richiamare direttamente al diritto internazionale nei suoi rapporti con le autorità. Nella nostra costituzione del Württemberg e Baden siamo andati persino oltre e, nell'ambito del diritto degli stranieri, abbiamo escluso il diritto di rappresaglia. ${ }^{\mathbf{1 2 8}}$

d) La turbativa della pace fra i popoli

(art. 31; art. 26 CHE [Chiemseer Entwurf]) ${ }^{\mathbf{1 2 9}}$

Passiamo ora all'art. 31.

Dr. Eberhard: L'art. 31 corrisponde all'art. 26 [sul rifiuto della guerra] del Progetto del Herrenchiemsee. È stato recepito alla lettera.

Pres. Dr. v. Mangoldt: Mi sembra puramente declamatorio. Che cosa vuol dire? Abbiamo sempre insistito sul fatto che le disposizioni costituzionali sono direttamente diritto vigente. Ho dei dubbi: indicazioni ai tribunali le conosciamo già dalla Costituzione di Weimar, e sono un gravame spiacevole.

Dr. Schmid: La Costituzione del Württemberg e Baden definisce queste azioni semplicemente come incostituzionali.

128 Art. 46, c. 2 della Costituzione del Württemberg e Baden del 28 novembre 1946: "I diritti attribuiti agli stranieri dal diritto internazionale possono da lui esser fatti valere anche se non sono previsti dal diritto nazionale". [Nota 18 dell'originale]

129 Nr. 15, Zwölfte Sitzung des Ausschusses für Grundsatzfragen. 15. Oktober 1948, in: EBerhard Pikart, Wolfram Werner (eds.) (1993), Ausschuß für Grundsatzfragen, 331-332. 
Pres. Dr. v. Mangoldt: È già meglio che dire che quelle azioni vengono punite. Ritengo che potremmo accettare quella formulazione.

Dr. Eberhard: Ritorno alla mia proposta di dire a questo punto anche qualcosa sulla produzione, il trasporto, l'importazione e la circolazione di armi e munizioni. Sembra un po' macchinoso, ma se vogliamo sottolineare l'apertura alla pace dobbiamo anche dire qualcosa in proposito.

Dr. [Hellmuth, sic,] von Weber: Ma è proprio necessario che diciamo qualcosa al riguardo?

Dr. Eberhard: Le Potenze occupanti possono naturalmente importare armi. Questo è un altro problema, e non possiamo vietarlo nella nostra costituzione.

Dr. Heuss: Mi disturba un po' il concetto impreciso di "armi e munizioni”. Penso per esempio alle munizioni da caccia.

Pres. Dr. v. Mangoldt: Forse possiamo dire in generale "materiale bellico", oppure "Armi, munizioni e materiale bellico d'ogni tipo".

Dr. Pfeiffer: Penso che possa bastare: "Materiale bellico d'ogni tipo".

Dr. Eberhard: Non credo che "materiale bellico" sia meglio di "armi e munizioni”. Quest'ultima formulazione escluderebbe anche che una specie di SA ["Sturmabteilung", il primo gruppo paramilitare nazionalsocialista] potesse di nuovo armarsi. Per una guerra civile ci sono armi efficaci che non si possono definire "materiale bellico".

Dr. Pfeiffer: Invece della prolissa elencazione "importare, esportare" non sarebbe più semplice dire: "non possono essere prodotte né messe in circolazione"?

Dr. Eberhard: Così però non si colpirebbe il trasporto attraverso il territorio federale.

Pres. Dr. v. Mangoldt: Potremmo forse dire: "Materiale bellico d'ogni tipo non può essere prodotto, né trasportato né messo in circolazione senza l'autorizzazione del Governo federale. Disposizioni specifiche sono regolate per legge”.

Avremmo così portato a termine il nostro compito, ad eccezione degli art. 24 e 27 , che riguardano la bandiera federale e la nuova struttura territoriale. Forse la commissione incaricata della redazione può presentarci una proposta. Chiuderei qui la seduta odierna. 
e) Discussione e decisione delle petizioni: l'obiezione di coscienza ${ }^{\mathbf{1 3 0}}$

Signora Dr. [Helene] Weber: La petizione della Baronessa von MinnigerodeAllerberg contiene un Appello alla pace. Dobbiamo decidere come procedere con queste petizioni. In varie petizioni il popolo scrive che ha nostalgia della pace e che noi dobbiamo impegnarci affinché si realizzi la pace e affinché l'idea della pace e il pacifismo vengano in qualche mondo ancorati nella costituzione.

Pres. Dr. v. Mangoldt: Non possiamo gravare altre commissioni con queste richieste. Possiamo solo inserirle nell'ordine del giorno.

Signora Dr. [Helene] Weber: Anche un'altra petizione di Lemke contiene un appello alla pace. Vi si pone il problema se la guerra sia un crimine. La lettera non contiene nulla di significativo e anche in questo caso si può solo metterla all'ordine del giorno.

In un'altra petizione (Worell) non soltanto si chiede la pace, ma si mette anche in rilievo l'ovvio diritto di ogni cittadino all'obiezione di coscienza. In più petizioni si sottolinea che la costituzione dovrebbe contenere un articolo sull'obiezione di coscienza.

Dr. Heuss: In proposito c'è anche una comunicazione del Frauenbund. ${ }^{\mathbf{1 3 1}}$ Ho già detto una volta quanto io apprezzi che, con straordinaria discrezione, nessuno dei presenti si sia pronunciato su questo argomento.

Signora Dr. [Helene] Weber: Stavo solo per dire una mezza parola sulla petizione di quest'organizzazione femminile, quando Lei ha emesso un sonoro grido di spavento. Lei, Dr. Heuss, ha detto: "Per carità di Dio!"

Dr. Heuss: Si pone il problema se qui vogliamo giungere a un dibattito di principio su questo tema. Io sono decisamente contrario a farlo. Ha già avuto luogo nei singoli parlamenti dei Länder. L'Assia ha incluso qualcosa del genere nella sua costituzione. ${ }^{\mathbf{1 3 2}}$

130 Nr. 20, Fünfzehnte Sitzung des Ausschusses für Grundsatzfragen. 27. Oktober 1948, in: Eberhard Pikart, Wolfram Werner (eds.) (1993), Ausschuß für Grundsatzfragen, 417-423.

131 [Si tratta del "Berliner Frauenbund 1945 e.V.': cfr. nota 139, p. 322.]

132 È l'art. 69 della Costituzione dell'Assia del 1946. [Nota 18 dell'originale] ['L'Assia si dichiara a favore della pace, della libertà e della comprensione fra i popoli. La guerra è ripudiata. - È incostituzionale ogni azione diretta a preparare una guerra" (Hessen bekennt sich zu Frieden, Freiheit und Völkerverständigung. Der Krieg ist geächtet. - Jede Handlung, die mit der Absicht vorgenommen wird, einen Krieg vorzubereiten, ist verfassungswidrig).] 
(Zinn: "La guerra viene ripudiata")

Pres. Dr. v. Mangoldt: Ci siamo occupati del tema nella redazione dell'art. $31 \mathrm{e}$ siamo giunti alla conclusione che non vogliamo andare oltre a quanto già abbiamo detto nell'art. 3 .

Dr. Heuss: Sono del tutto d'accordo. Ma dobbiamo avere le idee chiare in proposito. Di recente un giornalista mi ha chiesto qual è la mia posizione al riguardo. E io gli ho risposto onestamente che sono contrario all'inclusione di un articolo sull'obiezione di coscienza. Devono averlo pubblicato da qualche parte. Adesso sto ricevendo lettere che ci invitano a fare in modo che, in caso di guerra, tutti gli organi legislativi si arruolino nei battaglioni d'assalto: e allora il popolo sarebbe d'accordo. La gente si occupa in generale seriamente del pro e del contro. Nel Parlamento del Württemberg abbiamo avuto un ampio dibattito su questo tema e abbiamo votato una legge che è stata approvata mentre io non ero presente. ${ }^{133}$ Avrei protestato energicamente. Quella legge è stata difesa anche da uno dei miei amici.

Wunderlich: Il ripudio generale della guerra e l'obiezione individuale di coscienza sono due temi radicalmente distinti.

Signora Dr. [Helene] Weber: Nella petizione che ho presentato si parla sempre di obiezione di coscienza, e non di ripudio della guerra.

Pres. Dr. v. Mangoldt: Ce ne siamo già occupati in questa Commissione. Dobbiamo chiederci come procedere di fronte a queste richieste. Queste persone devono ricevere una comunicazione?

Wunderlich: Ogni persona deve ricevere una risposta.

Pres. Dr. v. Mangoldt: Alle persone è stato provvisoriamente risposto che la loro richiesta è stata ricevuta e che verrà qui discussa. Ora devono ricevere una risposta definitiva.

Dr. Heuss: Non si deve dare nessuna risposta definitiva. Poi la gente la pubblica nei giornali, e partono le polemiche. È corretto ringraziare la gente per l'interesse e informarla che le loro comunicazioni sono state incluse come materiale per i dibattiti.

133 Il parlamento del Württemberg e Baden non ha emanato una legge sul ripudio della guerra. Probabilmente il Dr. Heuss si riferiva all'Iniziativa di legge dei quattro gruppi parlamentari sul rifuto di prestare servizio militare in armi, in Verhandlungen des WürttembergBadischen Landtages, 88. Sitzung, 4. März 1948, p. 1672; 73. Sitzung, 22. April 1948, pp. 1789-1993. [Nota 20 dell'originale] 
Pres. Dr. v. Mangoldt: In generale ho fatto così fin dall'inizio.

Dr. Heuss: Dovrebbe bastare. Per alcune persone l'obiezione di coscienza è divenuta un tema centrale, una questione di fede. Se a noi pare sufficiente, sono d'accordo con l'articolo che dichiara punibili le preparazioni della guerra tanto ideali quanto oggettive. Però la gente non ne sarà soddisfatta perché vuole vedere dichiarato come diritto individuale il diritto di rifiutare il servizio militare in guerra. In base alle mie conoscenze storiche, il servizio militare in guerra è un dovere anche nelle democrazie. È quindi infelice fissare in una costituzione democratica il principio che ognuno può rifiutarsi anche se si tratta di una guerra di difesa. Nel progetto di costituzione predisposto da Carlo Schmid avevamo la frase patetica: "La guerra non è un mezzo della politica". ${ }^{\mathbf{1 3 4}}$ Tutti erano d'accordo. Io invece nel parlamento del Land mi sono opposto con un lungo discorso, dicendo: "Ritengo che il diritto di difesa dei württemberghesi contro i bavaresi, se questi ultimi decidono di attaccarli, sia un dovere nazionale; non ci penso neppure ad accettare questa norma senza oppormi”. Questo naturalmente non l'ho detto nel parlamento del Land, quindi non dovete preoccuparvi. Non sono poi così privo di tatto. Infine lì abbiamo respinto quella frase nella votazione pubblica. Questo capitava ai tempi in cui gli americani erano terribilmente interessati alla questione. Siccome oggi non se ne interessano più di tanto, lasciamo cadere questo articolo! Anche in questa sede non fornirò nessuna spiegazione individuale sul fatto che noi l'abbiamo respinta. Direi soltanto: consideriamola come documentazione. È un problema molto serio.

Pres. Dr. v. Mangoldt: La difficoltà consiste anche in questo: dobbiamo assolutamente avere una polizia. Questa polizia, che si occupa dell'ordine interno, può avere una sua funzione anche nel caso di un attacco dall'esterno. Se si accetta come principio il diritto di rifiutare il servizio in armi, non si può istituire una polizia. Ci sono momenti di transizione che possono condurre a difficoltà straordinarie.

Wunderlich: Personalmente sono un sostenitore dell'obiezione di coscienza: ci tengo a dirlo apertamente. Io ho vissuto di persona come nel Terzo

134 Progetto provvisorio di una costituzione per il Nord-Württemberg e il Nord-Baden del 24 aprile 1946, in: Frank R. Pfetsch (ed.) (1986), Verfassungsreden und Verfassungsentwürfe, 353 s. [Nota 21 dell'originale] 
Reich sono stati trattati gli "Ernste Bibelforscher" ${ }^{135}$ quando questa gente venne fucilata a schiere; e ho visto con quale coraggio queste persone sono morte per la loro fede. A questi gruppi di persone deve essere offerta una possibilità, come è stato fatto anche in Inghilterra. ${ }^{\mathbf{1 3 6}}$ Perché dobbiamo esser da meno dell'Inghilterra, perché dovremmo essere meschini su questo problema? A ciò si aggiunge un'ulteriore considerazione. Oggi ci viene molto spesso fatto presente che potrebbe capitare che una potenza straniera reclutasse dei tedeschi per un qualche suo fine. In presenza di questa eventualità, non dovrebbe essere sancito almeno il diritto individuale all'obiezione di coscienza, indipendentemente da chi (e se) ne fa o non ne fa uso? Su questi due argomenti decisivi si fonda il mio voto favorevole all'obiezione di coscienza.

Zinn: Bisognerebbe accordare questo diritto a coloro che rifiutano il servizio militare in guerra a causa di un profondo convincimento. Sono consapevole del fatto che, se lo si scrive lapidariamente nella costituzione, la cerchia di quelli che vi si possono realmente richiamare per motivi morali è effettivamente molto ristretta, mentre sarà molto estesa la cerchia di chi vi si richiama soltanto per imboscarsi. Oggi è difficile indicare questo confine in una disposizione costituzionale. Lo si può fare soltanto con una legge molto prudente, come hanno fatto gli inglesi. L'ambito di chi può aspirare a questo privilegio, in Inghilterra, è stato concretamente limitato attraverso una specie di procedura di controllo.

Pres. Dr. v. Mangoldt: Si dimentica sempre che non si può regolare tutto con la costituzione, ma che ci sono anche le leggi.

Zinn: Non riconosco a nessuno il diritto di fare l'imboscato, a meno che non possa richiamarsi a una giustificazione morale interiore. Sarei contrario, se fosse necessario.

Wunderlich: È chiaro che non voglio concedere indiscriminatamente questo diritto.

135 [Gli "Ernste Bibelforscher" (o Testimoni di Geova) "vennero perseguitati crudelmente dal 1933 al 1945. Secondo recenti valutazioni circa diecimila di loro vennero arrestati, e di essi quattro-cinquemila morirono nelle prigioni e nei lager nazisti” (Michael H. Kater (1969), Die Ernsten Bibelforscher im Dritten Reich, 181). Nei Lager, secondo la manìa classificatoria dei nazisti, i Testimoni di Geova erano contraddistinti dal triangolo viola.]

136 Durante la Seconda guerra mondiale l'Inghilterra riconobbe l'obiezione di coscienza non soltanto per seri motivi religiosi e d'altro genere, ma ammise anche l'esenzione da tutti i servizi governativi per ragioni fondate sulle proprie convinzioni. Cfr. HeInRICH Kipp (1952), Das Grundrecht der Kriegsdienstverweigerung; KarSten BredemeIER (1991), Kriegsdienstverweigerung im Dritten Reich. [Nota 23 dell'originale] 
Dr. Heuss: Condivido del tutto l'opinione del collega Zinn. Rispetto soprattutto le persone che rifiutano il servizio militare per motivi religiosi e che per questo hanno accettato di subire gravi punizioni. Storie orrende come quelle dei nazisti non si erano prima mai verificate. Per quanto ne so, prima i Mennoniti sono stati assegnati alla sanità, quindi non dovevano combattere con le armi. La legge dei Mennoniti proibisce di uccidere esseri umani e per questo li si era assegnati alla sanità, dove si sono anche distinti. Dai tempi della Prima guerra mondiale ricordo un uomo (che del resto era un bravo commilitone) che non aveva mai sparato perché la sua religione gli proibiva di uccidere. Però questo problema va risolto in sede legislativa, e non costituzionale. Quando tutte le potenze del mondo sanciscono doveri militari ecc., la nostra dichiarazione, se la includiamo nella costituzione, diviene un po' ridicola perché non siamo in grado di operare militarmente.

Zinn: Si potrebbe dire soltanto: "Il diritto al rifiuto del servizio militare in armi per ragioni di coscienza è regolato dalla legge federale”. È il massimo cui ci si può spingere perché implica il riconoscimento del principio.

Dr. Heuss: Il problema del combattimento in armi è divenuto oggi del tutto obsoleto, perché la produzione di bombe o la costruzione di carri armati è un'attività bellica ben superiore a quella di chi sorveglia il ponte con un fucile. I primi hanno un'influenza ben più rilevante sulla condotta della guerra.

Pres. Dr. v. Mangoldt: Proporrei di risolvere questo problema con la formulazione: "La commissione ha dibattuto a fondo la questione, senza però poter giungere alla decisione di includere una corrispondente prescrizione tra i diritti fondamentali o nella parte generale che li segue immediatamente". Di più non possiamo dire.

Dr. Heuss: Per me è già fin troppo. Non darei alcuna risposta a queste persone.

Pres. Dr. v. Mangoldt: Non voglio dare una risposta a queste persone, voglio solo mettere a verbale una nota per la Commissione Principale. Su ciò siamo d'accordo. (Nessuna presa di posizione contraria.)

Signora Dr. [Helene] Weber: C’è poi la risoluzione di un parlamento giovanile del Mecklemburgo, che si ricollega a questi problemi. (Segue una lettura parziale.) ${ }^{\mathbf{1 3 7}}$

137 Petizione Nr. 148 del Kröpeliner Jugend-Parlament (Z 5/107, Bl. 250). Si tratta di una risoluzione adottata con 21 voti favorevoli, 2 voti contrari e 12 astensioni: "Il $4^{\circ}$ Parlamento Giovanile di Kröpelin, riunito il 4 ottobre 1948 a Kröpelin (Mecklemburgo), il cui 
La si può trattare come la precedente.

Pres. Dr. v. Mangoldt: Proprio così.

Signora Dr. [Helene] Weber: Un'altra petizione ci giunge dalla zona occupata dai Sovietici. È diretta al Parlamentarischer Rat e anche al Deutscher Volksrat di Berlino, nonché al parlamento del Mecklemburgo: questo va forse tenuto presente.

Un'altra petizione ci giunge dagli esperantisti. Vi si chiede l'inclusione del diritto al rifiuto del servizio militare in armi per ragioni di coscienza dunque, con questa limitazione - nella futura costituzione federale e in quella del Land Renania Nord-Vestfalia; nonché l'inclusione nell'insegnamento scolastico dell'esperanto come lingua ausiliaria. Alla prima domanda abbiamo appena risposto. La seconda richiesta riguarda non noi, ma i Ministeri della Cultura dei singoli Länder.

Pres. Dr. v. Mangoldt: Sul primo punto proporrei di procedere come abbiamo fatto per le precedenti petizioni. Per il secondo punto, proporrei di includerlo all'ordine del giorno, perché è un problema culturale.

Signora Dr. [Helene] Weber: Un'altra petizione viene dalla Società per la Pace (Friedensgesellschaft) ${ }^{\mathbf{1 3 8}}$ di Amburgo, che chiede una legge sul ripudio della guerra. (Segue una lettura parziale.) Non so se abbiamo detto qualcosa di definitivo sul ripudio della guerra.

Pres. Dr. v. Mangoldt: Il ripudio della guerra è contenuto nell'art. 31. Per il resto procediamo come per le petizioni precedenti.

Signora Dr. [Helene] Weber: Ora viene la petizione già ricordata dal Dr. Heuss del Frauenbund di Berlino del 1947. ${ }^{139}$ (Segue una lettura parziale.)

Präsidium è formato da giovani di tutti i partiti e organizzazioni, invita a fare in modo che, nel trattare le questioni costituzionali, ai tedeschi sia proibito di prestare servizio militare sotto la bandiera di uno Stato straniero. Inoltre deve essere garantita l'esenzione da ogni pena a tutti i cittadini tedeschi che, in caso di guerra, si rifiutino di prestare il servizio militare. Motivazione: Riconosciamo il servizio in guerra solo come azione di autodifesa. Non c'è autodifesa né nel servizio in armi sotto una bandiera straniera, né in una guerra d'aggressione del proprio Stato. In questo tipo di servizio vediamo una disponibilità all'uccisione volontaria che è punibile in base alle umane concezioni giuridiche. La gioventù di tutti i popoli va esortata ad attivarsi per l'idea dell'obiezione di coscienza e ad essere vigile contro ogni istigazione alla guerra" (Kriegshetze). [Nota 24 dell'originale]

138 [La "Deutsche Friedensgesellschaft", fondata nel 1892, venne rifondata nel 1946 e svolse un'attiva propaganda a favore del ripudio della guerra e dell'inclusione dell'obiezione di coscienza nelle costituzioni dei Länder e nella Legge Fondamentale.]

139 Petizione del 9 settembre 1946 (ciclostilata come Drucks. Nr. 121): "Il Berliner Frauenbund 1947 prega i delegati del Parlamentarischer Rat di presentare i seguenti punti alla 
Abbiamo già risolto il terzo punto con l'art. 29. Il secondo punto ricade nella competenza dei Länder. La promozione della pace è uno dei grandi compiti culturali. Il primo punto - sull'obbligo diretto o indiretto ad uccidere - è connesso con la già discussa questione della guerra.

Dr. Kleindinst: Vi ricade anche il problema dell'eutanasia e dell'eliminazione delle vite indegne di essere vissute. ${ }^{\mathbf{1 4 0}}$

Pres. Dr. v. Mangoldt: Si ripresenta ancora una volta la questione se nell'art. 2 - che nel contesto generale produce ora un effetto un po' infelice - deve in qualche modo essere incluso anche il diritto alla vita. Dobbiamo discuterne in una seconda lettura. L'art. 2 ("L'essere umano è libero") e l'art. 3 ("La libertà della persona è intangibile”) stanno l'uno accanto all'altro senza che se ne veda chiaramente la differenza.

Zinn: Ci avevo già pensato. "L'essere umano è libero" è soltanto una dichiarazione. Anche la frase "Tutte le persone sono uguali davanti alla legge” non ha alcun significato giuridico. In realtà nell'art. 1 dovrebbe essere incluso qualcosa che si riferisca complessivamente alla dignità umana. Gli altri articoli devono avere un contenuto giuridico, tutto il resto deve rientrare nell'art. 1.

Signora Dr. [Helene] Weber: Sul Punto 1 possiamo dire che il Comitato sui Princìpi se ne occuperà ancora in una seconda lettura.

Pres. Dr. v. Mangoldt: Si occuperà quindi dell'art. 1 e dell'art. 2.

seduta plenaria dell'Assemblea dei Delegati: (1) Nessun cittadino può essere costretto a compiere azioni che conducano direttamente o indirettamente alla morte di persone. Dall'uso di questo diritto non deve derivare alcun svantaggio. Di conseguenza questa libertà deve essere inclusa tra gli inalienabili diritti fondamentali di ogni cittadino. (2) Ogni governo è tenuto ad appoggiare ogni movimento e attività che miri a una pace duratura. Vanno predisposte le risorse a ciò necessarie. (3) La Germania è disponibile - in condizioni di parità con tutti gli altri Stati contraenti - a partecipare a una comunità europea o mondiale di Stati. Quindi, in condizioni di parità, a un certo momento la Germania è disposta a cedere parti della propria sovranità a un'istanza sovranazionale in vista della pacifica organizzazione del mondo". [Nota 28 nell'originale. Cfr. anche supra, p. 312]

140 [Sono i temi della politica eugenetica del nazionalsocialismo, che portarono fra l'altro all'Olocausto. Il termine 'vita indegna di essere vissuta' (lebensunwertes Leben) è nel titolo dell'opera del giurista Karl Binding e dello psicologo Alfred Hoche: KarL Binding, Alfred Hoche (1920), Die Freigabe der Vernichtung lebensunwerten Lebens (con il titolo La liberalizzazione della soppressione della vita senza valore, in: Ernesto De Cristofaro, Carlo Saletti (eds.) (2012), Precursori dello sterminio, 42-89]. 
Zinn: Nella costituzione dell'Assia abbiamo una disposizione che si occupa della pena di morte: ${ }^{\mathbf{1 4 1}}$ il diritto alla vita può essere cancellato soltanto dalla sentenza di un tribunale. Sarebbe antiestetico recepire una simile disposizione.

f) Incostituzionalità delle azioni che preparano una guerra; divieto delle armi (art. 29b e 29c) ${ }^{\mathbf{1 4 2}}$

Dr. Eberhard: Possiamo completare l'esame dell'art. 29b? La Commissione dei Principi ha sottoposto alla Commissione Principale la seguente formulazione: Le azioni intraprese con l'intenzione di turbare la coesistenza pacifica dei popoli sono incostituzionali. La Commissione Principale si è dichiarata d'accordo in linea di principio, respingendo invece la formulazione del Comitato per la Redazione, che riguardava soltanto la guerra d'aggressione. $\mathrm{Ma}$, come ha detto il collega Kaufmann, nella costituzione del Württemberg c'è una bella frase. ${ }^{143}$ Gli ho detto che essa è uguale al nostro art. 31. Egli ricorda che nei lavori preparatori della Costituzione del Württemberg e Baden si trovava solo una frase: "La guerra non è un mezzo della politica". Egli ha poi avanzato la proposta conciliante di non far iniziare l'articolo indicando un'azione negativa: "Viene rifiutata la guerra come mezzo per $i$ conflitti tra i popoli". Non mi è sembrato male continuare con la frase positiva.

Dr. Bergsträsser: Lo respingo: è solo una dichiarazione.

Dr. Heuss: Non ci si guadagna molto.

Dr. Eberhard: La Commissione Principale ha interrotto la votazione.

141 Art. 21 della Costituzione dell'Assia del $1^{\circ}$ dicembre 1946. [Nota 29 nell'originale] [“Chi è stato dichiarato colpevole di un'azione punibile può essere privato della libertà e dei diritti civili (o in essi limitato) sulla base delle leggi penali e di una sentenza giudiziaria. Chi ha commesso reati particolarmente gravi può essere condannato a morte" (Ist jemand einer strafbaren Handlung für schuldig befunden worden, so können ihm auf Grund der Strafgesetze durch richterliches Urteil die Freiheit und die bürgerlichen Ehrenrechte entzogen oder beschränkt werden. Bei besonders schweren Verbrechen kann er zum Tode verurteilt werden).]

142 Nr. 39. Dreißigste Sitzung des Ausschußes für Grundsatzfragen. 6. Dezember 1948, in: Eberhard Pikart, Wolfram Werner (eds.) (1993), Ausschuß für Grundsatzfragen, $852-854$.

143 Art. 47 della Costituzione del Württemberg e Baden del 28 novembre 1946: "Ogni azione intrapresa con l'intenzione di turbare la collaborazione pacifica tra i popoli e, in particolare, di preparare una guerra, è incostituzionale". [Nota 19 dell'originale] 
Pres. Dr. Mangoldt: Dal momento che dobbiamo scegliere una formulazione, dobbiamo prendere quella del Patto Kellogg e dire: "La guerra viene ripudiata" ["Der Krieg wird geächtet"]. È già divenuto un terminus technicus, altrimenti si dovrebbe dire: "viene rifiutata".

Heile: "La guerra viene ripudiata come mezzo della politica". Se veniamo attaccati e ci difendiamo, non si tratta di politica, ma di legittima difesa: e questo naturalmente non lo vogliamo "ripudiare".

Dr. Heuss: Carlo Schmid era molto orgoglioso dell'espressione: "La guerra non è un mezzo della politica", ma gli ho detto che è solo una dichiarazione.

Pres. Dr. v. Mangoldt: Allora era molto meglio la redazione originaria: "Azioni intraprese con l'intenzione di..."

Dr. Eberhard: Questa frase non gode di grandi simpatie; discutiamone un momento. Del resto il Comitato dei Principi potrebbe dire che vuol mantenere la formulazione originaria.

Pres. Dr. v. Mangoldt: Allora diciamo: “... intrapresa con l'intenzione ... di turbare”. Mi sembra che sia già un po' meglio.

Dr. Eberhard: Solo una parola sul secondo capoverso. Si era proposto di cancellarlo, però non ci si è riusciti perché il collega Dr. v. Mangoldt non era presente nel Comitato Principale. Gli altri colleghi del suo gruppo non si sono decisi abbastanza in fretta ad approvare la mia richiesta: e ora, così com'è formulato, non va bene. Dobbiamo scrivere almeno: "armi adatte alla condotta della guerra", e non: "armi destinate alla condotta della guerra".

Dr. v. Mangoldt: Ora è diventato l'art. 29c del Comitato Principale.

Dr. Eberhard: Ma il Governo federale non può occuparsi dell'intenzione di produrre armi adatte alla guerra.

Pres. Dr. v. Mangoldt: Avevamo detto che saremmo stati d'accordo se il tutto venisse cancellato.

Walter: Si tratta solo di teoria.

Dr. Bergsträsser: Se il Governo federale lo approva, diviene un'autorizzazione.

Dr. Heuss: Nella situazione odierna, mi parrebbe insensato cancellarlo, perché si direbbe: "A settembre l'avete incluso, ma a dicembre siete dell'opinione che non sia più d'attualità". In questo momento suona quasi come una presa di posizione politica. Ritengo giusto dire "adatte", invece di "destinate". Ma non cancellerei l'articolo in questione. 
Pres. Dr. v. Mangoldt: Io l'avrei formulato come propone Lei, ma il collega Dr. Eberhard aveva sostenuto che lo si poteva fare soltanto di fronte a una breve richiesta, spontanea e comune, dei partiti. Però mi sembra giusto dire "adatte", invece di "destinate".

Heile: Nei congressi internazionali, per esempio poco tempo fa a Roma, si è detto che i tedeschi si trovano in una situazione che li costringe a prendere posizione sulla questione della partecipazione a un sistema internazionale di difesa. Si è detto addirittura che in Germania l'organizzazione militare era pienamente in corso. Noi non sappiamo nulla in proposito. Se ora però cancelliamo il passo in questione, si dirà che spianiamo la strada a un militarismo americano, perché permetteremmo alla popolazione di prestare servizio sotto gli americani.

Pres. Dr. v. Mangoldt: Allora siamo d'accordo: mettiamo "adatte", invece di "destinate". Quindi: nell'art. $29 c$ nella redazione del Comitato Principale diciamo "armi adatte" e non "armi destinate".

Non avrebbe molto senso continuare a discutere. La nostra prossima seduta può avere luogo dopo quella del Comitato Principale, se quest'ultima non dura oltre le 18. Dichiaro sospesa la seduta. 
g) La redazione finale degli articoli sul ripudio della guerra

\begin{tabular}{|c|c|}
\hline $\begin{array}{l}\text { Redazione finale del Verfassungskonvent } \\
\text { auf Herrenchiemsee }\end{array}$ & $\begin{array}{l}\text { Legge Fondamentale per la Repubblica } \\
\text { Federale tedesca ( } 23 \text { maggio 1949) }{ }^{145}\end{array}$ \\
\hline $\begin{array}{l}\text { Articolo } 29 a \\
\text { (1) La Federazione può trasferire per leg- } \\
\text { ge alcuni diritti sovrani a enti interstatali. } \\
\text { (2) Per il mantenimento della pace la } \\
\text { Federazione può aderire a un sistema di } \\
\text { reciproca sicurezza collettiva: la Federa- } \\
\text { zione acconsentirà a quelle limitazioni } \\
\text { della propria sovranità che producono e } \\
\text { assicurano un ordine pacifico e durevole } \\
\text { in Europa e tra i popoli del mondo. } \\
\text { (3) Per regolare i conflitti interstatuali la } \\
\text { Federazione aderirà a un arbitrato gene- } \\
\text { rale, comprensivo, obbligatorio e interna- } \\
\text { zionale. }\end{array}$ & $\begin{array}{l}\text { Articolo } 25 \text { [Diritto internazionale e } \\
\text { diritto federale] } \\
\text { Le regole generali del diritto internazio- } \\
\text { nale sono parte integrante del diritto } \\
\text { federale. Esse prevalgono sulle leggi e } \\
\text { fanno sorgere diritti e doveri immediati } \\
\text { per gli abitanti del territorio federale. }\end{array}$ \\
\hline $\begin{array}{l}\text { Articolo } 29 b \\
\text { Le azioni atte a turbare la coesistenza } \\
\text { pacifica dei popoli e intraprese a questo } \\
\text { fine sono vietate e da punire. }\end{array}$ & $\begin{array}{l}\text { Articolo } 26 \text { [Divieto di preparare una } \\
\text { guerra di aggressione] } \\
\text { (1) Le azioni che possono turbare la } \\
\text { pacifica convivenza dei popoli e intra- } \\
\text { prese con tale intento, in particolare al } \\
\text { fine di preparare una guerra offensiva, } \\
\text { sono incostituzionali. Tali azioni devono } \\
\text { essere perseguite penalmente. } \\
\text { (2) Le armi da guerra possono essere } \\
\text { prodotte, trasportate e messe in commer- } \\
\text { cio soltanto con l'autorizzazione del } \\
\text { Governo federale. I particolari sono sta- } \\
\text { biliti da una legge federale. }\end{array}$ \\
\hline $\begin{array}{l}\text { Articolo } 29 c \\
\text { (1) Le armi da guerra possono essere pro- } \\
\text { dotte, trasportate e messe in circolazione } \\
\text { soltanto con l'autorizzazione del Governo } \\
\text { federale. } \\
\text { (2) Ulteriori disposizioni sono regolate } \\
\text { dalla legge federale. }\end{array}$ & \\
\hline
\end{tabular}

144 È la redazione finale che il Verfassungskonvent auf Herrenchiemsee sottopose al Parlamentarischer Rat: 40. Allgemeiner Redaktionsausschuß, Art. 1-29, in: Eberhard Pikart, Wolfram Werner (eds.) (1993), Ausschuß für Grundsatzfragen, 890-891.

145 Questa traduzione proviene dal sito: http://www.consiglioveneto.it/crvportal/BancheDati/ costituzioni/de/zGermania_sin.pdf. 
III.2. Repubblica Democratica Tedesca: la Costituzione (1949) e la Legge a tutela della pace (1950)

La costituzione della Repubblica Democratica Tedesca del 7 ottobre $1949^{\mathbf{1 4 6}}$ si richiama alla specifica concezione del pacifismo già illustrata a proposito dei Partigiani della Pace (cfr. p. 201 e $i$ testi sull'Italia nell'Appendice II,2). Essa non menziona direttamente o indirettamente né $i$ problemi delle forze armate, né quelli della condotta di un'eventuale guerra perché nel 1949 le forze armate della RDA non erano ancora state istituite.

Il Preambolo della costituzione del 1949 stabilisce che la RDA ha il dovere "di garantire la pace". Questo dovere è poi specificato nell'art. 5: "1) Le norme generalmente riconosciute del diritto internazionale impegnano i pubblici poteri nonché tutti $i$ cittadini. 2) È dovere dei poteri pubblici vegliare al mantenimento e alla difesa delle relazioni amichevoli con tutti $i$ popoli. 3) Nessun cittadino potrà partecipare ad azioni di guerra tendenti ad opprimere un popolo".

L'art. 6 (già richiamato alla nota 157, p. 59) vieta le attività di propaganda che possono condurre a tensioni interne o estere: "1) Tutti i cittadini godono di uguaglianza di diritti dinanzi alla legge. 2) Qualsiasi propaganda diffamatoria o di boicottaggio contro istituzioni e organismi democratici, qualsiasi incitamento all'assassinio di uomini politici democratici, qualsiasi manifestazione di odio religioso, razziale e contro altri popoli, qualsiasi propaganda militarista e bellicista e tutte le altre azioni dirette contro l'eguaglianza dei diritti costituiscono dei delitti ai sensi del codice penale. L'esercizio dei diritti democratici nello spirito della Costituzione non costituisce una propaganda diffamatoria. 3) Le persone condannate per siffatti reati non possono occupare funzioni né nei servizi pubblici né nei posti direttivi dell'attività economica e culturale. Esse perdono qualsiasi diritto elettorale attivo e passivo".

I reati sopra elencati "costituiscono dei delitti ai sensi del codice penale": ma nel 1949, al momento dell'entrata in vigore della Costituzione della RDA, in tutta l'area tedesca vigeva ancora il codice penale imperiale del 1871, con le successive modificazioni. L'elaborazione del nuovo "Codice penale della Repubblica Democratica Tedesca" durò fino al 1968, e nel frattempo dovette essere emanata nel 1957 una legge penale complementare più compatibile con la nuova situazione politica.

146 Gesetz über die Verfassung der Deutschen Demokratischen Republik, in: Hans-Ulrich Носнваuм (ed.) (1958), Staats- und verwaltungsrechtliche Gesetze, 25-78. 
Sui reati previsti dalla costituzione il testo del 1957 contiene precisazioni che sarà opportuno esaminare più avanti, dopo le leggi cronologicamente anteriori.

L'articolo 112 della Costituzione elenca le materie di esclusiva competenza legislativa, fra le quali non figura nessuna materia relativa alle forze armate o allo stato di belligeranza perché in quegli anni la RDA era uno Stato occupato e disarmato. Gli unici riferimenti alla guerra si riferiscono al passato, "ai danni di guerra e alle spese d'occupazione e alle indennità o restituzioni" e "alle vittime del nazismo".

Art. 112 - Alla Repubblica spetta il potere esclusivo di legiferare in merito: - alle relazioni con l'estero; - al commercio estero; - alle dogane; nonché all'unità del territorio doganale e commerciale, e alla libertà di circolazione delle merci; - alla nazionalità e alla libertà di circolazione degli individui; - all'immigrazione e all'emigrazione; - all'estradizione; ai passaporti e ai diritti degli stranieri, all'anagrafe; al diritto civile; - al diritto penale; - all'ordinamento della giustizia e alla procedura giudiziaria; - al diritto del lavoro; - ai trasporti; - alle poste, alle trasmissioni, alla radiotrasmissioni; - al cinematografo e alla stampa; - alla moneta; - ai pesi e misure e alla loro verifica; - alle assicurazioni sociali; - ai danni di guerra e alle spese d'occupazione e alle indennità o restituzioni; - alle vittime del nazismo.

Nel primo dopoguerra, il timore di un nuovo conflitto e la minaccia nucleare avevano suscitato un intenso dibattito sulla conservazione della pace, cui la Repubblica Democratica Tedesca partecipò l'anno dopo la costituzione emanando una legge con misure a favore della pace. Il suo carattere pacifista presenta non solo le peculiarità già esaminate a proposito del movimento comunista per la pace, ma anche un contenuto ambiguo, perché quella lotta per la pace prevede pene molto dure contro chi dissente dalla politica dello Stato comunista. La lettura di questo testo deve dunque tenere presente questo doppio e conflittuale registro: da un lato, la lotta per la pace; dall'altro la repressione del dissenso interno.

Legge a tutela della pace del 15 dicembre $1950 .{ }^{\mathbf{1 4 7}}$

La politica aggressiva dei governi imperialistici degli Stati Uniti, della Gran Bretagna e della Francia, che mira a una nuova carneficina mondiale, minaccia di coinvolgere il popolo tedesco in una nuova e criminosa guerra civile. La rimilitarizzazione della Germania occidentale e l'impegno nel risuscitare il militarismo e l'imperialismo tedeschi rappresentano un grave pericolo per l'esistenza e per il futuro della nazione tedesca e per la pace e la sicurezza in Europa. Solo attraverso la democrazia e la pace si può raggiungere e garantire l'unità e l'indipendenza della Germania. La politica

147 Gesetz zum Schutze des Friedens vom 15. Dezember 1950, in: Hans-Ulrich Hochbaum (ed.) (1958), Staats- und verwaltungsrechtliche Gesetze, 168-172 (GBl, 1199). 
imperialistica di coinvolgimento della Germania occidentale in una nuova guerra criminosa e fin dall'inizio destinata all'insuccesso costituisce una minaccia per il nostro popolo e per la nostra patria.

La nazione deve essere liberata da questa minaccia. Il mantenimento della pace costituisce l'interesse nazionale più urgente, insieme con il sostegno a tutte le forze democratiche e patriottiche dell'intero popolo tedesco.

La propaganda bellicistica degli imperialisti anglo-americani e dei loro complici rappresenta una seria messa in pericolo per la pace europea e per l'amicizia del popolo tedesco con tutti i popoli pacifisti del mondo.

La propaganda bellicistica, qualunque forma essa assuma, è uno dei più gravi delitti contro l'umanità.

Di conseguenza la Volkskammer, ${ }^{[\mathbf{1 4 8}]}$ in conformità con gli articoli 5 e 6 della costituzione, approva la seguente

"Legge a tutela della pace".

Art. 1. Chi vilipende altri popoli o razze e istiga contro di loro o al loro boicottaggio per turbare i rapporti pacifici tra i popoli e per coinvolgere il popolo tedesco in una nuova guerra, viene punito con la detenzione e, nei casi gravi, con la prigione. ${ }^{\mathbf{1 4 9}}$

Art. 2. (1) Chi propaganda un'azione aggressiva, in particolare una guerra d'aggressione, $o$ istiga in altro modo alla guerra, e chi arruola, induce o incita dei tedeschi a partecipare ad azioni di guerra che mirano all'oppressione di un popolo, viene punito con la detenzione e, nei casi gravi, con la prigione.

(2) In pari misura viene punito chi arruola dei tedeschi per la legione straniera francese o per analoghe unità militari straniere e per truppe mercenarie, ovvero istiga a farne parte.

Art. 3. (1) Chi fa propaganda per il risorgere dell'aggressivo militarismo tedesco e dell'imperialismo ovvero per l'inclusione della Germania in un blocco militare aggressivo, viene punito con la detenzione e, nei casi gravi, con la prigione.

148 La "Volkskammer", Camera del Popolo, era il parlamento della DDR, ma non è paragonabile al parlamento d'una democrazia occidentale perché è parte d'un sistema politico a partito unico. Ecco la sua definizione fornita dalla più diffusa delle enciclopedie della DDR, Meyers Neues Lexikon in acht Bänden (1964), vol. 8, alla voce "Volkskammer": "Massimo organo rappresentativo del popolo e organo supremo del potere statale della DDR. La Camera del Popolo viene eletta direttamente dal popolo in libere elezioni democratiche, organizzate dal Fronte Nazionale, e incarna la sovranità del popolo guidato dalla classe lavoratrice. Attraverso il Fronte Nazionale unisce tutte le forze politiche della società sotto la guida della classe lavoratrice. Viene eletta ogni quattro anni e ad ogni elezione almeno un terzo dei deputati è sostituito da nuovi candidati."

149 Pene detentive in vigore nella DDR: Gefängnis, detenzione da 1 giorno a 5 anni; Zuchthaus: prigione (o penitenziario) da 1 a 15 anni o ergastolo. 
(2) In egual misura viene punito chi istiga contro accordi di diritto internazionale che mirano a mantenere e a consolidare la pace e l'evoluzione della Germania sulla base della democrazia e della pace, nonché chi istiga alla violazione di tali accordi per coinvolgere la Germania in azioni belliche aggressive.

Art. 4. Chi esalta o fa propaganda per l'uso di armi atomiche e di altre armi di distruzione di massa, come armi velenose, radioattive, chimiche e batteriologiche, viene punito con la detenzione e, nei casi gravi, con la prigione.

Art. 5. Chi, al servizio della propaganda bellicista, addita al dispregio o al discredito il movimento per il mantenimento e il consolidamento della pace, ovvero incita contro i partecipanti alla lotta per la pace o li fa perseguitare, viene punito con la detenzione e, nei casi gravi, con la prigione.

Art. 6. (1) Nei casi di violazione particolarmente grave degli art. da 1 a 5 della presente legge la pena della prigione non è inferiore ai cinque anni, ovvero è a vita.

(2) In particolare, un caso è considerato particolarmente grave se la fattispecie viene messa in essere su incarico diretto di Stati i cui organi o agenzie praticano l'istigazione alla guerra o una politica aggressiva contro popoli pacifici. In tali casi può essere comminata anche la pena di morte.

Art. 7. È punibile anche la preparazione o il tentativo delle fattispecie degli articoli da 1 a 6.

Art. 8. (1) In base alla presente legge, in aggiunta a ogni pena può essere comminata una pena pecuniaria di ammontare illimitato.

(2) Inoltre può essere riconosciuta la confisca (Einziehung) totale o parziale del patrimonio del reo. Se il reo è condannato a morte, all'ergastolo o alla detenzione non inferiore a cinque anni, il suo intero patrimonio viene confiscato.

Art. 9. (1) Se il reo è condannato a una pena detentiva (Zuchthaus) in base a questa legge, la sentenza deve anche comminare la perdita pro tempore o perpetua dei seguenti diritti: 1') l'impiego pubblico o l'attività in posizione direttiva nella vita economica e culturale; 2') la capacità elettorale attiva e passiva.

(2) Se il reo è condannato a una pena minore, possono essere comminate le sanzioni indicate al punto 1 '.

Art. 10. (1) Un procedimento contro la violazione della presente legge può essere aperto soltanto su istanza del Procuratore Generale della Repubblica Democratica Tedesca.

(2) Competente per il procedimento è il Tribunale Supremo della Repubblica Democratica Tedesca. Il Procuratore Generale può presentare l'accusa davanti a un altro tribunale o incaricarne il Procuratore Generale di uno dei Länder della Repubblica Democratica Tedesca.

(3) Il Tribunale Supremo della Repubblica Democratica Tedesca è competente anche nei casi in cui la fattispecie è compiuta da un cittadino tedesco non nel 
territorio della Repubblica Democratica Tedesca, anche se il reo non ha alcun domicilio o abituale dimora nel territorio della Repubblica Democratica Tedesca.

Art. 11. Il Consiglio dei Ministri della Repubblica Democratica Tedesca emana le misure attuative della presente legge.

Art. 12. La presente legge entra in vigore il 16 dicembre 1950.

Berlino, 15 dicembre 1950.

Il Presidente della Volkskammer pubblica la presente legge in data venti dicembre millenovecentocinquanta.

Berlino, il ventidue dicembre millenovecentocinquanta.

Il Presidente della Repubblica Democratica Tedesca

Wilhelm Pieck

Nel quinquennio successivo alla Legge a tutela della pace giunse a maturazione la ricostituzione delle forze armate in entrambi gli Stati tedeschi, nel 1955 nella Germania occidentale e nel 1956 nella Germania orientale. Nel settembre 1955, qualche mese prima della legge istitutiva delle forze armate della Repubblica Democratica Tedesca, la Legge per il completamento della Costituzione ${ }^{\mathbf{1 5 0}}$ integrava il testo costituzionale con le disposizioni indispensabili per inserire le forze armate nella struttura istituzionale di quello Stato:

\1. L'articolo 5 della Costituzione della Repubblica Democratica Tedesca viene completato come segue: "Il servizio a difesa della patria e delle conquiste dei lavoratori è un onorato dovere nazionale dei cittadini della Repubblica Democratica Tedesca”.

\2. L'articolo 112 della Costituzione della Repubblica Democratica Tedesca viene completato come segue: "Alla Repubblica spetta il compito di legiferare sulla difesa militare della patria e sulla protezione della popolazione civile".

\3. L'organizzazione del servizio per la difesa militare della patria e per la protezione della popolazione civile è regolata con decreto del Consiglio dei ministri.

150 Gesetz zur Ergänzung der Verfassung vom 26. September 1955, in: Hans-Ulrich Hochbaum (ed.) (1958), Staats- und verwaltungsrechtliche Gesetze, 79-80. 


\section{III.3. La legge istitutiva dell'Armata Popolare Nazionale (NVA) della DDR (1956) ${ }^{151}$}

Per realizzare la legislazione annunciata dal $\mathbb{} 2$ sopra citato, la Repubblica Democratica Tedesca istitui il 15 gennaio 1956 tanto le proprie forze armate quanto il Ministero della difesa. Sino alla costruzione del muro di Berlino l'Armata Popolare Nazionale (Nationale Volksarmee) si fondò sul servizio militare volontario, a differenza della Bundeswehr tedesco-occidentale. Venne disciolta il 2 ottobre 1960.

\section{La legge istitutiva dell'Armata Popolare Nazionale (NVA)} della Repubblica Democratica Tedesca (1956).

La protezione dello Stato dei Lavoratori e dei Contadini, nonché dei risultati conseguiti dai lavoratori e la tutela del loro pacifico lavoro, sono doveri elementari del nostro Stato democratico, sovrano e pacifista. La rinascita del militarismo aggressivo nella Germania occidentale e l'istituzione dell'esercito mercenario ${ }^{\mathbf{1 5 2}}$ tedesco- $^{-}$ occidentale costituiscono una costante minaccia per il popolo tedesco e per tutti i popoli d'Europa.

Per aumentare la capacità difensiva e per salvaguardare la nostra Repubblica Democratica Tedesca, sulla base dell'art. 5 e dell'art. $112^{153}$ della Costituzione della Repubblica Democratica Tedesca, la Volkskammer approva la seguente legge:

151 Gesetz über die Schaffung der Nationalen Volksarmee und des Ministeriums für Nationale Verteidigung vom 18. Januar 1956 [Legge istitutiva dell'Armata Nazionale Popolare della Repubblica Democratica Tedesca], in: Hans-Ulrich Hochbaum (ed.) (1958), Staats- und verwaltungsrechtliche Gesetze, 81-82.

152 "Westdeutsche Söldnerarmee", cioè la Bundeswehr, "armata mercenaria" perché la Repubblica Federale Tedesca era entrata nella NATO il 6 maggio 1955. Nei testi degli Stati comunisti e in quelli di ispirazione comunista in Occidente quando si parla dell'“avversario di classe" non sono rari questi sconfinamenti dal linguaggio giuridico a quello propagandistico: così 'guerrafondiaio' sta per 'bellicistico', 'complice' o 'manutengolo' (Helfershelfer) per 'alleato'.

153 Art. 5 Cost. DDR: “1) Le norme generalmente riconosciute del diritto internazionale impegnano i pubblici poteri nonché tutti i cittadini. 2) È dovere dei poteri pubblici vegliare al mantenimento e alla difesa delle relazioni amichevoli con tutti i popoli. 3) Nessun cittadino potrà partecipare ad azioni di guerra tendenti ad opprimere un popolo". L'art. 112 Cost. DDR - "Alla Repubblica spetta il potere esclusivo di legiferare in merito" [...] - elenca una lunga serie di competenze, in cui non si accenna alla difesa: gli unici riferimenti alla guerra si riferiscono al passato: legislazione in merito "ai danni di guerra e alle spese d'occupazione e alle indennità o restituzioni; - alle vittime del nazismo (http:// www.dircost.unito.it/cs/pdf/19491007_germaniaRepubblicaDemocratica_ita.pdf). 
Art. 1.

(1) Viene istituita una "Nationale Volksarmee" [Armata Popolare Nazionale].

(2) La "Nationale Volksarmee" è formata dalle forze armate di terra, di mare e di cielo necessarie alla difesa della Repubblica Democratica Tedesca. La consistenza numerica delle forze armate è limitata in conformità con le esigenze della protezione del territorio della Repubblica Democratica Tedesca, della protezione dei suoi confini e della difesa aerea.

Art. 2.

(1) Viene istituito un "Ministerium für Nationale Verteidigung" [Ministero della Difesa Nazionale].

(2) Il "Ministerium für Nationale Verteidigung" organizza e guida la "Nationale Volksarmee" (forze armate di terra, di mare e di cielo) sulla base e in applicazione delle leggi, decreti e decisioni della Volkskammer e del Consiglio dei Ministri della Repubblica Democratica Tedesca.

(3) I compiti del "Ministerium für Nationale Verteidigung" sono stabiliti dal Consiglio dei Ministri.

Art. 3.

La presente legge entra in vigore con la sua pubblicazione (Verkündung).

Il Presidente della Volkskammer, a nome del Präsidium della Volkskammer, pubblica la presente legge approvata il ventun gennaio millenovecentocinquantasei.

Berlino, il ventiquattro gennaio millenovecentocinquantasei.

Il Presidente della Repubblica Democratica Tedesca Wilhelm Pieck

I reati penali contro la pace, sopra ricordati a proposito dell'articolo 6 della Costituzione, vennero regolati nel 1957 con la legge che - in attesa dell'emanazione del nuovo codice penale della Repubblica Democratica Tedesca - adeguava ai tempi nuovi alcune parti del codice penale imperiale ancora in vigore: si tratta della Legge per il completamento del codice penale (Strafergänzungsgesetz) del dicembre $1957 .^{154}$ Nella Sezione sui Reati contro lo Stato e l'attività dei suoi organi, due articoli riguardano $i$ reati menzionati nella costituzione:

154 Gesetz zur Ergänzung des Strafgesetzbuches (Strafergänzungsgesetz) vom 11. Dezember 1957, in: Strafgesetzbuch und andere Strafgesetze. Textausgabe mit Anmerkungen und Sachregister (1960), pp. n.n.: la sequenza delle pagine è sostituita da quella degli articoli. 
S19. Propaganda antistatale e provocazione. (1) Chi:

1. fa propaganda o apologia del fascismo e del militarismo, ovvero incita contro altri popoli o razze;

2. incita contro lo Stato degli Operai e dei Contadini [la Repubblica Democratica Tedesca], contro i suoi organi, contro le organizzazioni sociali o contro un singolo cittadino a causa delle sue attività statali o sociali, ovvero a causa della sua appartenenza a un'istituzione statale o a un'organizzazione sociale, passa alle vie di fatto o minaccia azioni violente, è punito con una pena detentiva (Gefängnis) non inferiore a tre mesi. Il tentativo è punibile.

(2) Parimenti viene punito chi produce scritti o altri oggetti di tale contenuto, ovvero li introduce o diffonde a scopo provocatorio.

(3) In casi gravi - specialmente se l'azione è compiuta su incarico degli enti o degli individui menzionati al $\$ 14$ [Spionaggio], oppure se si tratta di un'azione pianificata

- si condanna alla prigione (Zuchthaus). ${ }^{155}$

[...]

$\$ 25$. Favoreggiamento di un reato contro lo Stato.

(1) Il favoreggiamento accordato dopo la commissione di un reato contro lo Stato per cui è prevista la prigione (Zuchthaus) è punito con la detenzione (Gefängnis).

(2) Il favoreggiamento non viene punito se è da addebitare al coniuge, a fratelli o sorelle o a parenti diretti o adottivi del reo o di un correo, al fine di evitargli la pena.

Queste due norme del 1957 rivelano come la tutela della pace o la limitazione delle forze armate fosse passata in secondo piano rispetto alle esigenze di repressione proprie d'uno Stato autoritario.

155 Sui due tipi di pena detentiva (Zuchthaus e Gefängnis) cfr. nota 149, p. 330.

III. Germania 



\section{Elenco degli scritti citati}

Abe determined to revise Constitution (2018, March 26), in: "The Japanese News" by Yomiuri Shimbun, Monday, 1

Abe, Shinzō (2013), Utsukushii kuni e [Verso un paese meraviglioso], Tokyo: Bungei Shunju, prima edizione 2006

Abegg, Lily (1936), Yamato. Der Sendungsglaube des japanischen Volkes, Frankfurt a.M.: Sozietäts-Verlag, $285 \mathrm{pp}$.

Adorno, Theodor W. (1970), Erziehung nach Auschwitz [Vortrag im Hessischen Rundfunk, 18.4.1966], in: Adorno, Theodor W., Erziehung zur Mündigkeit. Vorträge und Gespräche mit Hellmut Becker 1959-1969, Gerd Kadelbach (ed.), quinta edizione, Frankfurt a.M.: Suhrkamp, 92-109 (estratti in: Die Zeit 01/ 1993, https://www.zeit.de/1993/01/erziehung-nach-auschwitz)

Adorno, Theodor W. (2019), Aspekte des neuen Radikalismus. Ein Vortrag, Berlin: Suhrkamp, $87 \mathrm{pp}$.

Aga-Rossi, Elena (2016), Cefalonia. La resistenza, l'eccidio, il mito, Bologna: Il Mulino, $252 \mathrm{pp}$.

[Agnoletti, Enzo Enriques], [1951?], Chi sono i partigiani della pace, Roma: Comitato italiano per la libertà della cultura, $43 \mathrm{pp}$.

Agostini, Giulia (2015), Intrecci letterari tra Italia e Giappone nel Novecento: i casi di Mishima e D’Annunzio, da https://www.academia.edu/31084334/Intrecci_ letterari_tra_Italia_e_Giappone_nel_Novecento_i_casi_di_Mishima_e_DAnnunzio

Allemagne, Italie, Japon: les fascismes (1982), in: le débat, 21, 192 pp.

Allinson, Gary D. (1997), Japan's Postwar History, Ithaca (NY): Cornell University Press, XIV-208 pp.

Alloatti, Franca (2004), La carta di guerra non è un fenomeno isolato, in: Coarelli, Rossella (ed.), Istruiti e laboriosi: gli anni della ricostruzione, 73-88

Amitrano, Giorgio, lucia Caterina, Giuseppe De Marco (eds.) (2005), Studi in onore di Luigi Polese Remaggi, Napoli: Università degli Studi di Napoli L'Orientale, XIV-478 pp.

Anders, Günther (1961), Essere o non essere. Diario di Hiroshima e Nagasaki, Torino: Einaudi, XVII-290 pp.

Ando, Junko (2002), Japan und die Preußische Verfassung, in: Krebs, Gerhard (ed.), Japan und Preußen, München: Iudicium, 163-184

Antoni, Kuaus J. (1991), Der himmlische Herrscher und sein Staat. Essay zur Stelle des Tenno im modernen Japan, München: Judicium, 250 pp.

Aristarco, Guido (1996), Il cinema fascista: il prima e il dopo, Bari: Edizioni Dedalo, 302 pp. 
Auer, James E. (1990), Article 9 of Japan's Constitution: From Renunciation of Armed Force 'Forever' to the Third Largest Defense Budget of the World, in: Law and Contemporary Problems, 53:2, 171-187

Augeri, Nunzia (2014), L'estate della libertà. Le repubbliche partigiane e zone libere, Roma: Carocci, 191 pp.

Auswärtiges Амт (ed.) (1928), Materialien zum Kriegsächtungspakt, Berlin: Reichsdruckerei, $81 \mathrm{pp}$.

Avagliano, Mario, Marco Palmieri (2009), Gli internati militari italiani. Diari e lettere dai Lager nazisti, 1943-1945, con prefazione di Giorgio Rochat, Torino: Einaudi, LXIV-338 pp.

Avagliano, Mario, Marco Palmieri (2019), Dopoguerra. Gli italiani fra speranze e illusioni (1945-1947), Bologna: Il Mulino, 496 pp.

Avagliano, Mario, Marco Palmieri (2020), I militari italiani nei lager nazisti: una resistenza senz'armi (1943-1945), Bologna: Il Mulino, 457 pp.

Ayçoberry, Pierre (1982), Franz Neumann, Behemoth, in: le débat, 21, 178-191

Bacigalupi, Marcella, Piero Fossati (1986), Da plebe a popolo. L'educazione popolare nei libri di scuola dall'Unità d'Italia alla Repubblica, Firenze: La Nuova Italia, $282 \mathrm{pp}$.

Bailer-Galanda, Brigitte (ed.) (1996), Die Auschwitzleugner. „Revisionistische“ Geschichtslüge und historische Wahrheit, Berlin: Elephanten Press, 396 pp.

Baird, JaY W. (ed.) (1972), From Nuremberg to My Lai, Lexington (Mass.): Heath, $292 \mathrm{pp}$.

Barile, Paolo (1947), La magistratura si ribella alle leggi?, in: Il Ponte, 3:11-12, 1067-1074

Bartolini, Giulio (2002), Le modifiche al codice penale militare di guerra a seguito della missione italiana in Afghanistan, in: La Comunità Internazionale, $171-203$

Bästlein, Klaus (2009), “Nazi-Blutrichter als Stützen des Adenauer-Regimes”. Die DDR-Kampagnen gegen NS-Richter und -Staatsanwälte, die Reaktionen der bundesdeutschen Justiz und ihre gescheiterte "Selbstreinigung" 1957-1968, in: Bästlein, Klaus et al. (2009), Beiträge zur juristischen Zeitgeschichte der $D D R$, Berlin: Berliner Landesbeauftragter für die Unterlagen des Staatssicherheitsdienstes der Ehemaligen DDR, 53-93

Bästlein, Klaus, Annette Rosskopf, Falco Werkentin (2009), Beiträge zur juristischen Zeitgeschichte der DDR, Berlin: Berliner Landesbeauftragter für die Unterlagen des Staatssicherheitsdienstes der Ehemaligen DDR, 94 pp.

Battaglia, Achille (ed.) (1955), Dieci anni dopo. 1945-1955. Saggi sulla vita democratica italiana, Bari: Laterza, XII-598 pp.

Battaglia, Achille (1955), Giustizia e politica nella giurisprudenza, in: Battaglia, Achille (ed.), Dieci anni dopo. 1945-1955. Saggi sulla vita democratica italiana, Bari: Laterza, 319-408

Battaglia, Roberto (1947), Il riconoscimento dei partigiani, in: Il Ponte, 3:11-12, 1001-1014

Battaglia, Salvatore (1992), Grande dizionario della lingua italiana, Torino: Utet 
Beer, Lawrence W., Hiroshi Itoh (eds.) (1996), The Constitutional Case Law in Japan. 1970 through 1990, Seattle: University of Washington Press, XIV-668 pp.

Belladonna, Simone (2015), Gas in Etiopia. I crimini rimossi dell'Italia coloniale, Vicenza: Neri Pozza, 286 pp.

Bentivoglio, Giulia (2002), Antiamericanismo in Italia nel secondo dopoguerra: dal Piano Marshall al movimento dei Partigiani della pace, Relatore Petracchi Giorgio, tesi di laurea difesa nell'anno accademico 2001-02 e conservata nella Biblioteca dell'Archivio Centrale dello Stato, Roma, 200 pp.

Benz, Wolfgang (1978), Amerikanische Besatzungsherrschaft in Japan 1945-1947, in: Vierteljahrshefte für Zeitgeschichte, 26:2, 265-346

Benz, Wolfgang, Jörg Leuschner (eds.) (1993), Geeinte Nation - Geteilte Geschichte. Die deutsche Gesellschaft nach der Wiedervereinigung, Salzgitter: Archiv der Stadt Salzgitter, $122 \mathrm{pp}$.

Beonio-Brocchieri, Paolo (1996), Storia del Giappone, Milano: Mondadori, 177 pp.

Beretta, Lia (2013), Hirohito in Italia. Diari, Moncalieri: Centro Interuniversitario di Ricerche sul "Viaggio in Italia", $250 \mathrm{pp}$.

Bergamini, David (1971), Japan's Imperial Conspiracy, London - New York: Heinemann, 2 voll.

Berger, Franz Severin, Christiane Holler (1994), Trümmerfrauen. Alltag zwischen Hamstern und Hoffen, Wien: Ueberreuter, 204 pp.

Berkofsky, Axel (2004, 26 aprile), Japanisches Militär in Irak. Neudefinition japanischer Sicherheitspolitik?, Konrad-Adenauer-Stiftung, Auslandsinformationen, da https:/www.kas.de/c/document_library/get_file?uuid=d14d56b6-1415-b22d3f55-6721ff7f1289\&groupId=252038

Berkofsky, Axel (2005), Die neuen Amerikanisch-Japanischen Leitlinien für Verteidigungskooperation. Implikationen für Japans regionale Sicherheitspolitik, Münster: Lit, 400 pp.

Berkofsky, Axel (2010), Japan's Post-War Constitution. Origins, Protagonists and Controversies, in: Il Politico, 2, 5-26

Berkofsky, Axel (2012), A Pacifist Constitution for an Armed Empire. Past and Present of Japanese Security and Defence Policies, Milano: Angeli, 319 pp.

Berndt, Jacqueline (2003), Eine zeitlos schöne Nation. Das „Neue Geschichtslehrbuch“ als Bildergeschichte, in: Richter, Steffi, Wolfgang Höpken (eds.), Vergangenheit im Gesellschaftskonflikt, 191-124

Bessel, Richard (ed.) (1996), Fascist Italy and Nazi Germany. Comparisons and Contrasts, Cambridge: Cambridge University Press, XIV-242 pp.

Biagi, Enzo (2008), Io c'ero. Un grande giornalista racconta l'Italia del dopoguerra, Milano: Rizzoli, VIII-522 pp.

Bianchi D’Espinosa, Luigi (1947), Il “caso Pilotti”, in: Il Ponte, 3:11-12, 1108-1115

Bianco, Dante Livio (1947), Partigiani e C.L.N. davanti ai tribunali civili, in: Il Ponte, 3:11-12, 1033-1040

Bieber, Hans-Joachim von (2014), SS und Samurai: Deutsch-Japanische Kulturbeziebungen 1933-1945, München: Iudicium, 1311 pp. 
Binding, Karl, Alfred Hoche (1920), Die Freigabe der Vernichtung lebensunwerten Lebens. Ibr Maß und ibre Form, Leipzig: Meiner, 62 pp.

Bisson, Thомаs A. (1954), Zaibatsu Dissolution in Japan, Berkeley: University of California Press, XI-314 pp.

Bittner, Wolfgang (2017, 7 aprile), Verbot der Vorbereitung eines Angriffskrieges, in: Hintergrund. Das Nachrichtenmagazin, da https:/www.hintergrund.de/politik/ inland/verbot-der-vorbereitung-eines-angriffskrieges/

Blessin, ErICH (1967-1969), Bundesentschädigungsschlußgesetz. Kommentar zu der Neufassung des Bundesentschädigungsgesetzes, München: Beck, 2 voll.

Bobbio, Norberto (1952), Pace e propaganda di pace, in: Occidente. Rassegna mensile di studi politici, n. 5, 344-352, ristampa in: Bobbio, Norberto (1955), Politica e cultura, Torino: Einaudi, 72-83

Bobbio, Norberto (1955), Politica e cultura, Torino: Einaudi, 282 pp.

Bobbio, Norberto (1979), Il problema della guerra e le vie della pace, Bologna: Il Mulino, 209 pp.

Bobbio, Norberto (1989), Il Terzo assente. Saggi e discorsi sulla pace e sulla guerra, Pietro Polito (ed.), Milano: Sonda, 236 pp.

Bobbio, Norberto (1997), Autobiografia, Roma - Bari: Laterza, 274 pp.

Bocca, Giongio (2005), Una repubblica partigiana. Ossola, 10 settembre - 23 ottobre 1944, Milano: Il Saggiatore, 2005, III-138 pp., prima edizione 1964

Boccini, Luciano (1940), Il problema dei danni di guerra, Bologna: Zanichelli, $\mathrm{X}-286 \mathrm{pp}$.

Bonanate, Luigi (2018), Art. 11, Roma: Carocci, 143 pp.

Bork, Henrik (1999, 11 marzo), Geschichtsklitterung in Sprechblasen, in: Die Zeit, 8, anche: http://www.zeit.de/1999/11/199911.manga_.xml

Boßmann, Dieter (1982), "Was ich über Adolf Hitler gehört habe ...”. Folgen eines Tabus: Auszüge aus Schüler-Aufsätzen von heute, Frankfurt a.M.: Fischer, 359 pp.

Botteri, Inge (2010), La donna tra modernità e fascismo, in: Annali di storia dell'educazione e delle istituzioni scolastiche, 17, 13-29

Bracci, Mario (1947), Come nacque l'amnistia, in: Il Ponte, 3:11-12, 1090-1107

Bracht, Mary Lynn (2018), Figlie del Mare, Milano: Longanesi, 371 pp.

Bracht, Mary Lynn (2018, 4 maggio), "Svelo il Giappone delle ipocrisie che abusò di 200mila bimbe-schiave", intervista di Michela Ravalico, in: Libero, 25

Brackman, Arnold C. (1989), The Other Nuremberg. The Untold Story of the Tokyo War Crimes Trials, London: Collins, VIII-482 pp.

Braunbuch. Kriegs- und Naziverbrecher in der Bundesrepublik und in Westberlin. Staat, Wirtschaft, Verwaltung, Armee, Justiz (1965). Nationalrat der Nationalen Front des Demokratischen Deutschland / Dokumentationszentrum der Staatlichen Archivverwaltung der DDR (eds.), Berlin: Staatsverlag der Deutschen Demokratischen Republik, 387 pp.

Breccia, Gastone (2019), Corea, la guerra dimenticata, Bologna: Il Mulino, 392 pp.

Brecher, W. Puck (2017), Honored and dishonored guests. Westerners in wartime Japan, Cambridge (Mass.): Harvard University Press, XIV-370 pp. 
Bredemeier, Karsten (1991), Kriegsdienstverweigerung im Dritten Reich. Ausgewählte Beispiele, Baden-Baden: Nomos, 235 pp.

Brodesser, Hermann-Josef, Bernd Josef Fehn, Tilo Franosch, Wilfried Wirth (2000), Wiedergutmachung und Kriegsfolgenliquidation. Geschichte, Regelungen, Zablungen, München: Beck, XVII-251 pp.

Brondino, Michele, Alfonso Di Giovine (eds.) (1987), Colonialismo e neocolonialismo nei libri di storia per le scuole medie inferiori e superiori, Milano: Angeli, 245 pp.

Brooker, Paul (1991), The Faces of Fraternalism. Nazi Germany, Fascist Italy, and Imperial Japan, Oxford: Clarendon Press, VIII-397 pp.

Brunori, Maurizio (1993), Il Giappone. Storia e civiltà del Sol Levante, Milano: Mursia, $312 \mathrm{pp}$.

Bucher, Peter (ed.) (1981), Der Verfassungskonvent auf Herrenchiemsee (Der Parlamentarische Rat, vol. 2), Boppard am Rhein: Boldt, CXXXXV-676 pp.

Cajani, Luigi (2003), Italien und der Zweite Weltkrieg in den Schulgeschichtsbüchern, in: Cornelissen, Christoph et al. (eds.), Erinnerungskulturen, 269-284

Calamandrei, Piero (1947), Restaurazione clandestina, in: Il Ponte, 3:11-12, 959-968

Cammelli, Giongio (1950), Aritmetica per le scuole di avviamento professionale, Milano: Signorelli, 275 pp., riprodotto in: CoArelli, Rossella (ed.) (2004), Istruiti e laboriosi: gli anni della ricostruzione, $239 \mathrm{pp}$.

Campagnolo, Stefano (ed.) (2018), Si faccia un articolo di fondo ... Il regime fascista, Farinacci e il ventennio a Cremona, Cremona: Biblioteca Statale, $221 \mathrm{pp}$.

Canosa, Romano (1978), Le sanzioni contro il fascismo. Processi ed epurazioni a Milano negli anni 1945-47, Milano: Mazzotta, 155 pp.

Canosa, Romano (1999), Storia dell'epurazione in Italia. Le sanzioni contro il fascismo 1943-1948, Milano: Baldini \& Castoldi, 465 pp.

Caprara, Maurizio (2016, 11 agosto), Presto riapriremo l'ambasciata a Tripoli. E aiuteremo Serraj, in: Corriere della Sera, 5

Capristo, Annalisa (2002), L'espulsione degli ebrei dalle accademie italiane, Torino: Zamorani, XIV-405 pp.

Caracciolo, Davide (2008), La ignominiosa alleanza. Il contributo mafioso alla vittoria alleata in Sicilia, in: Instoria, 7, da http:/www.instoria.it/home/vittoria _alleata_sicilia.htm

Carlassare, Lorenza (1999), Costituzione italiana e guerra "umanitaria", in: Dogliani, Mario, Stefano Sicardi (eds.), Diritti umani e uso della forza, 23-29

Carlassare, Lorenza (2013, 11 febbraio), L'art. 11 della Cost. nella visione dei Costituenti, in: Costituzionalismo.it, da https://www.costituzionalismo.it/lart11-cost-nella-visione-dei-costituenti/

Carnevale, Paolo (ed.) (2004), Guerra e Costituzione. Atti del Convegno dell'Università degli studi Roma Tre, Roma 12 aprile 2002, Torino: Giappichelli, VII-314 pp. 
Caroli, Rosa (2010), Storia e storiografia in Giappone. Dai crimini di guerra ai criminali di guerra, in: Contini, Giovanni et al. (eds.), Memoria e rimozione, 91-108

Caroli, Rosa, Francesco Gatti (2017), Storia del Giappone, Roma - Bari: Laterza, XXXVI-365 pp.

Casadio, Quinto (1967), Gli ideali pedagogici della Resistenza, Bologna: Alfa, 207 pp.

Cassero, Riccardo (2004), Le veline del Duce. Come il fascismo controllava la stampa, Milano: Sperling \& Kupfer, 178 pp.

Cassese, Antonio (1975), Commento all'art. 11 della Costituzione, in: Branca, Giuseppe (ed.), Commentario alla Costituzione. Principi fondamentali, artt. 1-12, Bologna - Roma: Zanichelli - Foro Italiano, 566

Cassese, Antonio (2001), International Law, Oxford: Oxford University Press, XVII-469 pp.

Centro Militare di Studi Strategici (1990), I movimenti pacifisti e antinucleari in Italia, 1980-1988, Roma: Rivista Militare, 354 pp., anche: https://issuu.com/ rivista.militare1/docs/11_-_i_movimenti_pacifisti_e_antinu

Cerrai, Sondra (2011), I partigiani della pace in Italia. Tra utopia e sogno egemonico, Limena: Libreriauniversitaria, tesi di dottorato dell'Università di Pisa, 303 pp.

Chiari, Bernhard (ed.) (2010), Auslandseinsätze der Bundeswehr, Paderborn - München: Schöningh, 324 pp.

Chickering, Roger (1975), Imperial Germany and a World without War. The Peace Movement and German Society, 1892-1914, Princeton (N.J.): Princeton University Press, XIV-487 pp.

Chiesa, Pasquale (2012), L'algoritmo del perdono, in: Raggi, Barbara, Baroni di razza, 9-17

Ciapparoni la Rocca, Teresa (2005), Gli scrittori italiani e il Giappone, in: Amitrano, Giorgio et al. (eds.), Studi in onore di Luigi Polese Remaggi, $105-132$

Cignoni, Francesco (2018), Le "veline" di Farinacci nel Fondo Bacchetta della Biblioteca statale di Cremona, in: Campagnolo, Stefano (ed.), Si faccia un articolo di fondo, 40-68

Coarelli, Rossella (ed.) (2001), Dalla scuola all'Impero. I libri scolastici del fondo Braidense (1924-1944), Milano: Viennepierre, 206 pp.

Coarelli, Rossella (ed.) (2004), Istruiti e laboriosi: gli anni della ricostruzione. I libri scolastici del fondo della Braidense, 1945-1953, Milano: Viennepierre, 239 pp.

Coarelli, Rossella (2004), Il secondo dopoguerra (1945-1953). La defascistizzazione dei libri di testo, in: Coarelli, Rossella (ed.), Istruiti e laboriosi: gli anni della ricostruzione, $131-159$

Coarelli, Rossella (2010a), Da "Bertoldo" a "Settebello". Donne e morale di regime: l'autarchia e la guerra, in: Annali di storia dell'educazione e delle istituzioni scolastiche, 17, 31-38

Coarelli, Rossella (2010b), Riviste femminili nate durante il ventennio fascista, in: Annali di storia dell'educazione e delle istituzioni scolastiche, 17, 105-116 
Coccoli, Carlotta (2017), Monumenti violati. Danni bellici e riparazioni in Italia nel 1943-1945: il ruolo degli Alleati, Firenze: Nardini, 431 pp.

Coffrini, Fabrice (2019, 7 gennaio), Obras roubadas por nazistas desafiam museu, in: O Estado de S. Paulo, C4

Cohen, David (s.d.), Transitional Justice in Divided Germany after 1945, Berkeley: War Crimes Studies Center, 25 pp., da https://www.ocf.berkeley.edu/ wcsc/ wp-content/uploads/Papers/cohen-trans-justice-germany.pdf

Congresso mondiale dei popoli per la pace, Vienna, 5-12 dicembre 1952 (1953), Cremona: Pizzorni, $10 \mathrm{pp}$.

Contini, Giovanni, Filippo Focardi, Maria Petricioli (eds.) (2010), Memoria e rimozione. I crimini di guerra del Giappone e dell'Italia, Roma: Viella, 226 pp.

Coppellotti, Celestino (1936), Evoluzione della Germania hitleriana. Con tutti i documenti dal riarmo al $1^{\circ}$ aprile 1936, Milano: Editoriale Arte e Storia, 76 pp.

Corazza, Heinz (1944), Die Samurai. Ritter des Reiches in Ehre und Treue, mit einem Vorwort des Reichsführers SS und Chefs der deutschen Polizei Heinrich Himmler, Berlin: Zentralverlag der NSDAP, 31 pp. (Sonderdruck aus dem „Schwarzen Korps“ - 76.-100. Tausend)

Cornelissen, Christoph, Lutz Klinkhammer, Wolfgang Schwentker (eds.) (2003), Erinnerungskulturen. Deutschland, Italien und Japan seit 1945, Frankfurt a.M.: Fischer, 368 pp.

Costa, Pietro (2018), Art. 10, Roma: Carocci, 135 pp.

Costanzo, Ezıo (2006), Mafia e Alleati. Servizi segreti americani e sbarco in Sicilia. Da Lucky Luciano ai sindaci "uomini d'onore”, Catania: Le Nove Muse Editrice, $253 \mathrm{pp}$.

Coticchia, Fabrizio (2014), La guerra che non c'era. Opinione pubblica e interventi militari italiani dall'Afghanistan alla Libia, Milano: Università Bocconi, 156 pp.

Courtade, Francis, Pierre Cadars (1975), Geschichte des Films im Dritten Reich, München: Hanser, 335 pp.

Crisafulli, Edoardo, Maria Katia Gesuato (eds.) (2011), Una lingua per amica. L'Italiano nostro e degli altri. Atti della X settimana della Lingua Italiana nel Mondo, Tokyo: Istituto Italiano di Cultura, $198 \mathrm{pp}$.

La crisi della Resistenza (1947), in: Il Ponte, 3:11-12, 953-1121 pp.

Crome, Erhard (2008, agosto), Kriegsächtung - aktuell und drängend, in: "RLS [Rosa-Luxemburg-Stiftung] Standpunkte”, pp. 1-4

D'Annunzio, Gabriele (1932a), La penultima ventura, Libro primo: Il sudore di sangue, [Milano]: Istituto nazionale per la edizione di tutte le opere di Gabriele D’Annunzio, 333 pp.

D’Annunzio, Gabriele (1932b), La penultima ventura, Libro secondo: L'urna inesausta, [Milano]: Istituto nazionale per la edizione di tutte le opere di Gabriele D’Annunzio, IX-211 pp.

D'Annunzio, Gabriele (1932c), Credo [XXXI decembre MCMXIX], in: D'Annunzio, Gabriele (1932b), La penultima ventura, Libro secondo, 205-212 
D’Annunzio, Gabriele (1932d), L'ala d'Italia è liberata. Discorso agli aviatori di Centocelle [IX luglio MCMXIX], in: D'Annunzio, Gabriele (1932a), La penultima ventura, Libro primo, 231-255

D’Annunzio, Gabriele (1949a), La penultima ventura. Discorsi e messaggi scelti (15 gennaio '19 - 20 marzo '24), [Milano]: Associazione Amici del Vittoriale, 233 pp.

D’Annunzio, Gabriele (1949b), Saluto all'ospite d'Oriente, in: D'Annunzio, Gabriele (1949a), La penultima ventura. Discorsi e messaggi scelti, 60-62

D’Annunzio, Gabriele (1949c), Al legionario Alceste De Ambris, in: D’Annunzio, GABriele (1949a), La penultima ventura. Discorsi e messaggi scelti, 213-219

D’Annunzio, Gabriele (2009), La Carta del Carnaro e altri scritti su Fiume, Fressura, Marco, Karlsen, Patrick (eds.), Roma: Castelvecchi, XVII-168 pp.

D'Annunzio, Gabriele (2013), Orazione al popolo di Milano in morte di Giosuè Carducci (24 marzo 1907), in: D'Annunzio, Gabriele, L'allegoria dell'autunno, a cura di Annamaria Andreoli e Giorgio Zanetti, Milano: Mondadori (e-Meridiani), http://www.intratext.com/IXT/ITA3506/_PA.HTM

Da Frè, Giuliano (2018, 2 gennaio), Portaerei per il Giappone?, in: Rivista Italiana Difesa, da http://www.portaledifesa.it/index phppag,3_id,2096_arg,3_npp,2_ npag, $14 . h \mathrm{html}$

DANiels, Roger (1983), Japanese American, wartime relocation of, in: Kodansha Encyclopedia of Japan, Tokyo: Kodansha, vol. 4, 17-18

Daniels, Roger (1983), War Relocation Authority, in: Kodansha Encyclopedia of Japan, Tokyo: Kodansha, vol. 8, 228

Daniels, Roger (1983), Wartime Relocation Centers, in: Kodansha Encyclopedia of Japan, Tokyo: Kodansha, vol. 8, 228

De Cristofaro, Ernesto, Carlo Saletti (eds.) (2012), Precursori dello sterminio: Binding e Hoche all'origine dell'"eutanasia" dei malati di mente in Germania, Verona: Ombre Corte, 92 pp.

De Giorgi, Fulvio (2004), Nel "cantiere scolastico" della Repubblica, in: Coarelli, Rossella (ed.), Istruiti e laboriosi: gli anni della ricostruzione, 13-72

De Guttry, Andrea (1997), Le missioni delle forze armate italiane fuori area. Profili giuridici della partecipazione nazionale alle peace support operations, Milano: Giuffrè, 153 pp.

De Vergottini, Giuseppe (2004), Guerra e Costituzione. Nuovi conflitti e sfide alla democrazia, Bologna: Il Mulino, $350 \mathrm{pp}$.

De Vincentiss, Mauro (2011, 25 settembre), Recensione de: Patrizia Pacini. La costituente: storia di Teresa Mattei, in: Patria Indipendente, pp. 40-41, anche: http://anpi.it/media/uploads/patria/2011/40-43_LIBRI.pdf

Deaglio, Enrico (2009), Patria 1978-2008, Milano: Il Saggiatore, 939 pp.

Deaglio, Enrico (2017), Patria 1967-1977, Milano: Feltrinelli, 637 pp.

Decker, Gunnar (2015), 1965, der kurze Sommer der DDR, München: Hanser, 493 pp.

Di Capua, Giovanni (2005), Il biennio cruciale (luglio 1943-giugno 1945). L'Italia di Charles Poletti, Soveria Mannelli: Rubbettino Editore, 458 pp. 
Di Feo, Gianluca (2018, 21 giugno), Libia, la guerra segreta dei droni partiti da Sigonella, in: La Repubblica, pp. 1-2

Di Francesco, Tommaso (2019, 14 aprile), La nostra Libia dimenticata, in: Il Manifesto, da https://ilmanifesto.it/la-nostra-libia-dimenticata-2/

Di Giovine, Alfonso (1987), Considerazioni in margine alla ricerca, in: Brondino, Michele, Alfonso Di Giovine (eds.), Colonialismo e neocolonialismo, 125-134

Di Russo, Marisa (2005), Influenze e suggestioni letterarie dall'incontro tra Oriente e Occidente. D’Annunzio in Giappone, in: Amitrano, Giorgio et al. (eds.), Studi in onore di Luigi Polese Remaggi, 175-197

Di Stasi, Lawrence (2004), Una Storia Segreta. The Secret History of Italian American Evacuation and Internment during World War II, Berkeley: Heyday Books, XXII-327 pp.

Doehring, Karl, Bernd Josef Fehn, Hans Günter Hockerts (2001), Jahrhundertschuld, Jahrhundertsühne. Reparationen, Wiedergutmachung, Entschädigung für nationalsozialistisches Kriegs- und Verfolgungsunrecht, München: Olzog, 142 pp.

Dogliani, Mario (1999), Diritti dell'uomo: principi universali o ideologia?, in: Dogliani, Mario, Stefano Sicardi (eds.), Diritti umani e uso della forza, 43-47

Dogliani, Mario, Stefano Sicardi (eds.) (1999), Diritti umani e uso della forza. Profili di diritto costituzionale interno e internazionale, Torino: Giappichelli, VIII-287 pp.

Domenico, Roy Palmer (1991), Italian Fascists on Trial: 1943-1948, Chapel Hill (NC): University of North Carolina Press, XVII-295 pp.

Donat, Walter (1943a), Deutschland und Japan. Eine Einführung, in: Donat, Walter et al., Das Reich und Japan, 5-14

Donat, Walter (1943b), Der deutsche und der japanische Reichsgedanke, in: Donat, Walter et al., Das Reich und Japan, 105-131

Donat, Walter et al. (1943), Das Reich und Japan. Gesammelte Beiträge, Berlin: Junker und Dünnhaupt, $211 \mathrm{pp}$.

Donini, Ambrogio (1955), Prefazione, in: Sereni, Marina, I giorni della nostra vita, $7-12$

Donne e fascismo. Immagini e modelli educativi (2010), in: Annali di storia dell'educazione e delle istituzioni scolastiche, 17, 13-116 pp.

Eco, Umberto (2018), Il fascismo eterno, Milano: La Nave di Teseo, 51 pp.

Edsel, Robert M. (2009), Monuments Men. Allied Heroes, Nazi Thieves, and the Greatest Treasure Hunt in History, London: Arrow, XVI-473 pp.

Egbert, Lawrence D., Paul A. Joosten (eds.) (1947-1949), Der Prozess gegen die Hauptkriegsverbrecher vor dem internationalen Militärgerichtshof: Nürnberg, 14. November 1945 - 1. Oktober 1946. Gemäß den Weisungen des Internationalen Militärgerichtshofes vom Sekretariat des Gerichtshofes unter der Autorität des Obersten Kontrollrats für Deutschland veröffentlicht, Nürnberg: Internationaler Militärgerichtshof, 42 voll.

Ehrenburg, Ilya (1953), Partigiano della Pace, in: Realismo. Mensile di arti figurative, numero speciale a 16 pagine, $9-10,3$ 
EıcкHOfF, BÄrbel et al. (1988), Restauration im Recht, Jahrbuch für Sozialökonomie und Gesellschaftstheorie (Hochschule für Wirtschaft und Politik Hamburg), Opladen: Westdeutscher Verlag, 232 pp.

Elm, Ludwig (1991), Nach Hitler, nach Honecker. Zum Streit der Deutschen um die eigene Vergangenheit, Berlin: Dietz, 208 pp.

Erasmo da Rotterdam (2013), Adagi, Lelli, Emanuele (ed.), Milano: Bompiani, LXXVIII-3011 pp.

Ferencz, Benjamin B. (1986), Lohn des Grauens. Die Entschädigung jüdischer Zwangsarbeiter, Frankfurt a.M.: Campus, 288 pp., prima edizione: Less than Slaves. Jewish forced labor and the quest for compensation (1979), Cambridge (Mass.) London: Harvard University Press, XXII-249 pp.

Ferrarin, Arturo (1921), Il mio volo Roma-Tokio, Torino: ARP, 89 pp.

Ferrarin, Arturo (1929), Voli per il mondo. Con presentazione di Benito Mussolini, con 134 illustrazioni, 3 carte geografiche e un autografo, Milano: Mondadori, XV-282 pp., ristampa 1942

Ferretti, Valdo (1983), Il Giappone e la politica estera italiana 1935-1941, Milano: Giuffrè, XI-254 pp.

FISCH, Jörg (1992), Reparationen und Entschädigungen nach dem Zweiten Weltkrieg, München: Beck, 359 pp.

Fischer, Torben (2009), Exildebatte, in: Fischer, Torben, Matthias N. Lorenz (eds.), Lexikon der "Vergangenheitsbewältigung" in Deutschland, 48-50

Fischer, Torben, Matthias N. Lorenz (eds.) (2009), Lexikon der "Vergangenheitsbewältigung" in Deutschland. Debatten- und Diskursgeschichte des Nationalsozialismus nach 1945, seconda edizione, Bielefeld: transcript, 395 pp.

Flechtheim, Alfred (2015), Raubkunst und Restitution, Bambi, Andrea, Axel Drecoll (eds.), Berlin: De Gruyter Oldenbourg, XVIII-303 pp.

Floreanini, Gisella (1955), Si impedisca il riarmo della Germania di Bonn!, discorso pronunciato alla Camera dei Deputati nella seduta del 17 dicembre 1954, Roma: Tipografia della Camera dei deputati, $21 \mathrm{pp.}$

FoA, Vittorio (1947), La crisi della Resistenza prima della Liberazione, in: Il Ponte, 3:11-12, 982-993

Focardi, Filippo (2010), Criminali a piede libero. La mancata "Norimberga italiana", in: Contini, Giovanni et al. (eds.), Memoria e rimozione, 187-202

Foljanty-Jost, Gesine (1979), Schulbuchgestaltung als Systemstabilisierung in Japan, Bochum: Brockmeyer, 157 pp.

Foresti, Fabio (ed.) (2003), Credere, obbedire, combattere. Il regime linguistico del Ventennio, Bologna: Pendragon, 154 pp.

Forni, Lorena, Tiziana Vettor (eds.) (2017 [ma 2018]), Sicurezza e libertà in tempo di terrorismo globale, Torino: Giappichelli, XVI-286 pp.

Forno, Mauro (2005), La stampa nel Ventennio. Strutture e trasformazioni nello Stato totalitario, Soveria Mannelli: Rubbettino, XVI-304 pp.

Fortunio, Tommaso (1946), La legislazione definitiva sulle sanzioni contro il fascismo. Delitti fascisti, epurazione, avocazione. Commento, dottrina, giurisprudenza, Roma: Nuove Edizioni Jus, 224 pp. 
Fortunio, Tommaso (1948), Revisione e revoca dell'epurazione. Testo e commento del Decreto Legislativo 7 febbraio 1948, n. 48, Roma: Universal, 32 pp.

Fraenkel, Ernst (1983), Il doppio Stato. Contributo alla teoria della dittatura, Introduzione di Norberto Bobbio, Torino: Einaudi, XXIX-266 pp.

Franz-Willing, Georg (1991), Umerziehung: die De-Nationalisierung besiegter Völker im 20. Jahrhundert, Coburg: Nation Europa, 270 pp.

Frei, Norbert (ed.) (2003), Carriere. Le élites di Hitler dopo il 1945, Torino: Bollati Boringhieri, $297 \mathrm{pp}$.

Friedländer, SaUl (1982), De l'antisémitisme à l'extermination. Esquisse historiographique, in: le débat, 21, 131-150

Galante Garrone, Carlo (1947), Guerra di liberazione (dalle galere), in: Il Ponte, 3:11-12, 1041-1066

Gallige Sprechblasen (1999), in: Der Spiegel, 26, 170, anche: https://magazin.spiegel. de/EpubDelivery/spiegel/pdf/13880890

Galluzzo, Marco (2016, 11 agosto), Reparti speciali a difesa degli 007 già sul terreno. Così Palazzo Chigi ha dato il via al "suo" piano, in: Corriere della Sera, 3

García SaÉz, José Antonio (2016), Kelsen versus Morgenthau. Paz, política y derecho internacional, Madrid: Centro de Estudios Políticos y Constitucionales, $511 \mathrm{pp}$.

García SaÉz, José Antonio (2019), Pacifismo Jurídico, in: Eunomía. Revista de la Cultura de la Legalidad, 220-234

Gatti, Francesco (1997), Il fascismo giapponese, Venezia: Cafoscarina, 313 pp.

Gautier, Judith (1884), Poëmes de la libellule. Traduit du japonais d'après la version littérale de M. Saionzi, Paris: Gillot (non paginé)

Gergolet, Mara (2019, 7 gennaio), Tokyo prepara l'isola-trincea, in: Corriere della Sera, 13

Gerra, Ferdinando (1974), L'impresa di Fiume, Prefazione di Alberto M. Ghisalberti, Milano: Longanesi, vol. 1: Fiume d'Italia, 323 pp.; vol. 2: La Reggenza italiana del Carnaro, 346 pp.

Gesellschaft: Ein japanisches Geschichtslehrbuch für die 6. Klasse der Grundschule. Vierter Abschnitt: Vom Mandschurischen Zwischenfall (1931) bis zur Gegenwart (2006), Aus dem Japanischen von David Baumgart (Kleine Reihe 49), Berlin: MoriÔgai-Gedenkstätte der Humboldt-Universität, 64 pp.

Getreuer, Peter (1986), Der verbale Pazifismus. Die Verteidigung Japans 1972-1983 in demoskopischen Befunden, Wien: Institut für Japanologie, 667 pp.

Giacomini, Ruggero (1984), I partigiani della pace. Il movimento pacifista in Italia e nel mondo negli anni della prima guerra fredda, Prefazione di Enzo Santarelli, Milano: Vangelista, 322 pp., in sintesi: http:/www.resistenze.org/sito/te/cu/st/ cust3c06.htm

Il Giappone ritira le sue truppe dall'Iraq (2006, 20 giugno), in: Corriere della Sera, da http:/www.corriere.it/Primo_Piano/Esteri/2006/06_Giugno/20/ritiro_giappone.shtml

Gigli, Silvia (2017, aprile), Dacia Maraini: prigioniera in Giappone, in: informatore Unicoopfirenze, 4, 8-9, anche: http://www.coopfirenze.it/informatori/notizie/ dacia-maraini-prigioniera-in-giappone 
Gimbel, John (1990), Science, Technology, and Reparations: Exploitation and Plunder in Postwar Germany, Stanford (Cal.): Stanford University Press, XV-280 pp.

Ginsborg, Paul (2006), Storia d'Italia dal dopoguerra a oggi, Torino: Einaudi, XII-622 pp.

Ginzburg, Natalia (1963), Lessico famigliare, Torino: Einaudi, 217 pp.

Ginzburg, Natalia (2016), Le piccole virtù. Lessico famigliare, Torino: La Stampa, $311 \mathrm{pp}$.

Godau-Schüttke, Klaus-Dettlev (2001), Von der Entnazifizierung zur Renazifizierung der Justiz in Westdeutschland, in: forum historiae iuris (fhi), da https://forhistiur.de/2001-06-godau-schuttke/?1=de\#str21

Goncourt, Edmond de (1881), La maison d'un artiste, Paris: Charpentier, 2 voll.

Goodman, Carl F. (2008), The Rule of Law in Japan. A Comparative Analysis. Second Revised Editon, The Hague: Kluwer, XI-586 pp.

Gordon, Andrew (1983), Reparations for Southeast Asia, in: Kodansha Encyclopedia of Japan, Tokyo: Kodansha, vol. 6, 302

Goschler, Constantin (1992), Wiedergutmachung. Westdeutschland und die Verfolgten des Nationalsozialismus, München: Oldenburg, 343 pp.

Goschler, Constantin (2005), Schuld und Schulden. Die Politik der Wiedergutmachung für NS-Verfolgte seit 1945, Göttingen: Wallstein, 543 pp.

Gozzano, Guido (1917), Verso la cuna del mondo. Lettere dall'India (1912-1913), Milano: Treves, XV-264 pp.

Granata, Ivano (1993), Don Mazzolari e il movimento dei Partigiani della Pace (1950-1952), in: Il Risorgimento. Rivista di storia del Risorgimento e storia contemporanea, 1, 29-54

Gribaudi, Gabriella (2016), Combattenti, sbandati, prigionieri. Esperienze e memorie di reduci della Seconda guerra mondiale, Roma: Donzelli, XVIII-238 pp.

Grimm, Hans (1932), Volk obne Raum, München: Langen, 1352 pp.

Der große Ploetz. Die Daten-Enzyklopädie der Weltgeschichte (1998), 32a edizione, Darmstadt: Wissenschaftliche Buchgesellschaft, XXXI-2047 pp.

Grosser, Dieter, Stephan Bierling, Beate Neuss (eds.) (2015), Bundesrepublik und DDR 1969-1990 (Deutsche Geschichte in Quellen und Darstellungen, vol. 11), Stuttgart: Reclam, 422 pp.

Grossi, Alessia (2017, 4 novembre), La sabbia che ricoprì le vergogne della Storia, in: Il Fatto Quotidiano, 17

Gründler, Gerhard E., Arnim von Manikowsky (1967), Das Gericht der Sieger. Der Prozess gegen Göring, Hess, Ribbentrop, Keitel, Kaltenbrunnen und andere, Oldenburg: Stalling, 288 pp.

Guerri, Giordano Bruno (2009), Filippo Tommaso Marinetti. Invenzioni, avventure e passioni di un rivoluzionario, Milano: Mondadori, 334 pp.

Guerri, Giordano Bruno (2019), Disobbedisco. Cinquecento giorni di rivoluzione: Fiume 1919-1920, Milano: Mondadori, 550 pp.

Guttuso, Renato (1954), La nuova esperienza di Mucchi, in: Realismo. Mensile di arti figurative, 19,7

346 Elenco degli scritti citati 
Guy, George F. (1981), The Defence of General Yamashita, in: Supreme Court Historical Society - Yearbook 1981, 52-67

HaAsch, Günther (ed.) (2000), Bildung und Erziehung in Japan. Ein Handbuch zur Geschichte, Philosophie, Politik und Organisation des japanischen Bildungswesens von den Anfängen bis zur Gegenwart, Berlin: Colloquium, 460 pp.

Hansen, Janine (1997), Arnold Fancks „Die Tochter des Samurai“. Nationalsozialistische Propaganda und japanische Filmpolitik, Berlin: Harrassowitz, IX-178 pp.

Hasberg, Wolfgang (ed.) (2008), Modernisierung im Umbruch. Geschichtsdidaktik und Geschichtsunterricht nach 1945, Berlin - Münster: Lit, 412 pp.

Hasebe, Yasuo (2015, 24 dicembre), The End of Constitutional Pacifism in Japan?, in: IACL-AIDC BLOG, da https://blog-iacl-aidc.org/new-blog/2018/5/27/analysis -the-end-of-constitutional-pacifism-in-japan?rq=Yasuo\%20Hasebe

Haski, Pierre (2019, 14 giugno), Chi vuole scatenare una guerra nello stretto di Hormuz, in: (Internazionale), https:/www.internazionale.it/opinione/pierrehaski/2019/06/14/petroliere-stretto-hormuz

Haushofer, Karl (1923), Japan und die Japaner. Eine Landeskunde, Leipzig - Berlin: Teubner, VI-166 pp.

Henderson, Dan F. (ed.) (1968), The Constitution of Japan. Its First Twenty Years 1947-1967, Seattle: University of Washington Press, 323 pp.

Herbst, Ludolf, Constantin Goschler (eds.) (1989), Wiedergutmachung in der Bundesrepublik Deutschland, München: Oldenbourg, 428 pp.

Herde, Peter (1983), Italien, Deutschland und der Weg in den Krieg im Pazifik 1941, Wiesbaden: Steiner, 105 pp.

Herde, Peter (2000), Der Japanflug. Planungen und Verwirklichung einer Flugverbindung zwischen den Achsenmächten und Japan 1942-1945, Stuttgart: Steiner, 303 pp.

Herrmann, Anne-Kathrin (2009), Karl Jaspers: Die Schuldfrage, in: Fischer, Torben, Matthias N. Lorenz (eds.), Lexikon der "Vergangenheitsbewältigung" in Deutschland, 44-45

Нirohito (1964), Neujahrsbotschaft des Tenno vom 1. Januar 1946, in: Aus Politik und Zeitgeschichte, B 40-41/64, 23-24

Hirsch, Ernst (1982), Aus Kaisers Zeiten durch die Weimarer Republik in das Land Atatürks. Eine unzeitgemässe Biographie, München: Schweizer, 354 pp.

Hochbaum, Hans-Ulrich (ed.) (1958), Staats- und verwaltungsrechtliche Gesetze der Deutschen Demokratischen Republik, Berlin (Ost): VEB Deutschen Zentralverlag, 777 pp.

Hockerts, Hans Günter, Claudia Moisel, Tobias Winstel (eds.) (2006), Grenzen der Wiedergutmachung. Die Entschädigung für NS-Verfolgte in West und Osteuropa 1945-2000, Göttingen: Wallstein, 876 pp.

Hoffend, Andrea (1998), Zwischen Kultur-Achse und Kulturkampf. Die Beziehungen zwischen 'Drittem Reich' und faschistischem Italien in den Bereichen Medien, Kunst, Wissenschaft und Rassenfrage, Frankfurt a.M.: Lang, XIV-508 pp.

Hofmann, Reto (2015), The Fascist Effect. Japan and Italy, 1915-1952, Ithaca - London: Cornell University Press, X-203 pp. 
Höft, Andrea (2009), Nationalsozialismus im Schulunterricht, in: Fischer, Torben, Matthias N. Lorenz (eds.), Lexikon der "Vergangenheitsbewältigung" in Deutschland, 172-176

Holian, Timothy J. (1996), The German-Americans and World War II. An Ethnic Experience, New York: Lang, XII-243 pp.

Hook, Glenn D., Gavan McCormack (2001), Japan's Contested Constitution. Documents and Analysis, London: Routledge, VIII-212 pp.

Horwitz, Solis (1950), The Tokyo Trial, in: International Conciliation, 465, 473-584

I lavoratori tedeschi contro il riarmo della Germania (1951), conferenza operaia europea contro la rimilitarizzazione della Germania, Berlino, 23-25 marzo 1951, Torino: Tipografia Teatrale, $21 \mathrm{pp}$.

IACCH, Franco (2018, 28 novembre), Il Giappone sta per annunciare le portaerei difensive, in: Il Giornale, da http://www.ilgiornale.it/news/mondo/giapponesta-annunciare-portaerei-difensive-1608425.html

Die Idee der Kriegsächtung in Amerika (1925), in: Die Friedens-Warte, 25:10, 301-303

Ienaga, Saburō (1970), The Historical Significance of the Japanese Textbook Lawsuit, in: Bulletin of Concerned Asian Scholars, 2:4, 2-12

Ienaga, Saburō (1978), The Pacific War, 1931-1945. A Critical Perspective of Japan's Role in World War II, New York: Panteon Books, XVI-316 pp.

Ienaga, SAburō (2001), Japan's Past, Japan's Future: One Historian's Odyssey, Lanahm: Rowman and Littlefield, X-203 pp.

Igari, Hiromi (2000), Die Schulbuchkontroverse um die Geschichtsdarstellung in Japan, in: Jahrbuch für Antisemitismusforschung, 9, 270-280

Inoue, Куоко (1991), MacArthur's Japanese Constitution. A Linguistic and Cultural Study of Its Making, Chicago: University of Chicago Press, XII-378 pp.

Insolvibile, Isabella (2010), Kos 1943-1948. La strage, la storia, Napoli: Edizioni Scientifiche Italiane, $300 \mathrm{pp}$.

Isensee, Josef, Paul Kirchiof (eds.) (1997), Handbuch des Staatsrechts der Bundesrepublik Deutschland, vol. 9, Heidelberg: Müller, XLVI-1346 pp.

L'Italia ripudia la guerra: non rendiamo più facile dichiararla (2015, 27 gennaio), in: Rete della Pace - Rete Italiana per il Disarmo - Sbilanciamoci, da http://www.dis armo.org/rete/a/41247.html

Iто, Нirobumi (1889), Commentaries on the Constitution of the Empire of Japan, Tokyo: Igirisu-horitsu gakko, 22nd year of Meiji, XIII-259 pp.

Japan: NATO's longest-standing partner in the Asia-Pacific (2013, 13-16 aprile), NATO, da http://www.nato.int/cps/en/natohq/news_99788.htm?selectedLocale=en

Jasper, Gotthard (1989), Die disqualifizierten Opfer. Der Kalte Krieg und die Entschädigung für die Kommunisten, in: Herbst, Ludolf, Constantin Goschler (eds.), Wiedergutmachung in der Bundesrepublik Deutschland, 361-384

Jaspers, Karl (1946), Die Schuldfrage. Von der politischen Haftung Deutschlands, Heidelberg: Lambert Schneider, 106 pp., anche: (2015), München - Zürich: Piper, 99 pp., edizione tascabile, con postfazione del 1962 
Jaspers, Karl (1990), Freiheit und Wiedervereinigung. Über Aufgaben der deutschen Politik, con prefazione di Willi Brandt, München - Zürich: Pieper, 125 pp.

Jeismann, Karl-Ernst (ed.) (1982), Deutschland und Japan im Spiegel ihrer Schulbücher, Braunschweig: Georg-Eckert-Institut für Internationale Schulbuchforschung, Diesterweg, 123 pp.

Jemolo, Arturo Carlo (1947), La battaglia che non fu data, in: Il Ponte, 3:11-12, 969-981

Kades, Charles (1989), The American Role in Revising Japan's Imperial Constitution, in: Political Science Quarterly, 104:2, 215-247

Karatani, Kojin (2006), Sekai kyowakoku he. Shugi, neshon, kokka wo koete [Verso la repubblica mondiale. Capitale, nazione e oltre la nazione], Tokyo: Iwanami Shoten, 236 pp.

Karatani, Kojin (2016), Kenpo no muishiki [L'inconscio e la Costituzione], traduzione di Pier Giorgio Girasole, Tokyo: Iwanami Shoten, 199 pp., giunto alla quinta edizione 2017

Karlsch, RaIner (1993), Allein bezahlt? Die Reparationsleistungen der SBZ/DDR 1945-1953, Berlin: Links, 302 pp.

Karlsch, Rainer, Agnes Tandler (2001), Ein verzweifelter Wirtschaftsfunktionär? Neue Erkenntnisse über den Tod Erich Apels 1965, in: Deutschland-Archiv, 34, 50-64

Kasahara, Toikushi (2010), Il massacro di Nanchino e la struttura del negazionismo politico in Giappone, in: Contini, Giovanni et al. (eds.), Memoria e rimozione, 33-41

Kasza, Gregory J. (1988), The State of the Mass Media in Japan, 1918-1945, Berkeley: University of California Press, XVI-336 pp.

Kater, Michael H. (1969), Die Ernsten Bibelforscher im Dritten Reich, in: Vierteljahrshefte für Zeitgeschichte, 17:2, 181-218

Kelsen, Hans (2020), L'annessione dell'Austria al Reich tedesco e altri scritti (1918-1931), traduzione e cura di Fernando D’Aniello, Torino: Nino Aragno Editore, XLIV-95 pp.

Kersevan, Alessandra (2008), Lager italiani. Pulizia etnica e campi di concentramento fascisti per civili jugoslavi 1941-1943, Roma: Nutrimenti, 287 pp.

Kipp, Heinrich (1952), Das Grundrecht der Kriegsdienstverweigerung, in: Festschrift für Wilhelm Laforet anläßlich seines 75. Geburtstags, München: Isar Verlag, 83-106

Kishi, Nobusuke (1983), Kishi Nobusuke Kaikoroku [Memorie di Kishi Nobusuke], Tokyo: Kōsaidō, 643 pp.

Kittel, Manfred, Horst Möller (2006), Die Beneš-Dekrete und die Vertreibung der Deutschen im europäischen Vergleich, in: Vierteljabrshefte für Zeitgeschichte, $54: 4,541-581$

Klee, Ernst (1991), Persilscheine und falsche Pässe. Wie die Kirchen den Nazis halfen, Frankfurt a.M.: Fischer, 191 pp.

Klein, Angelika (ed.) (1987), Der Friedensgedanke in Politik und Traditionsverständnis der DDR, Halle (Saale): Martin-Luther-Universität Halle-Wittenberg, 142 pp. 
Klein, Gabriella B. (1986), Politica linguistica del fascismo, Bologna: Il Mulino, 234 pp.

Kleßmann, Christoph, Hans Misselwitz, Günter Wichert (eds.) (1999), Deutsche Vergangenheit - eine gemeinsame Herausforderung. Der schwierige Umgang mit der doppelten Nachkriegsgeschichte, Berlin: Links, 338 pp.

Koellreutter, Отto (s.d.), Das Wesen der Spruchkammern und der durch sie durchgeführten Entnazifizierung. Ein Rechtsgutachten. Als Manuskript gedruckt, [s.l.], $32 \mathrm{pp}$.

Koellreutter, Отto (1939), National Socialism and Japan, in: Contemporary Japan, $8: 2,194-202$

Koellreutter, Отто (1940), Das politische Gesicht Japans, Berlin: Heymanns, 63 pp., seconda edizione 1943, 61 pp.

Koellreutter, Отto (1941), Der heutige Staatsaufbau Japans, Berlin: Junker und Dünnhaupt, $28 \mathrm{pp}$.

Koellreutter, Отto (1944), Die politische Entwicklung des heutigen Japans, Berlin: De Gruyter, 159 pp.

Koellreutter, Отto [1954], Die Entnazifizierung, eine Sünde wider Recht und Ehre, Vortrag gehalten am 5. September 1954 auf dem 2. Internierten-Treffen in Landau/Pfalz, Landau/Pfalz: Gepel, 14 pp.

Kolko, Joyce, Gabriel Kolko (1972), The Limits of Power. The World and Unites States Foreign Policy, 1945-1954, New York: Harper \& Row, XII-820 pp.

Kosaka, Masataka (1982), A History of Postwar Japan, foreword by Edwin O. Reischauer, Tokyo: Kodansha International, $281 \mathrm{pp}$.

Kosexi, Sноichi (1998), The Birth of Japan's Postwar Constitution, Boulder: Westview Press, XII-259 pp.

Krebs, Gerhard (ed.) (1994), Formierung und Fall der Achse Berlin-Tokyo, München: Iudicium, $256 \mathrm{pp}$.

Krebs, Gerhard (ed.) (2002), Japan und Preußen, München: Iudicium, 356 pp.

Kriegsverbrecherprozesse, in: Brockhaus-Enzyklopädie (2006), 21a edizione, Leipzig Mannheim: Brockhaus, vol. 15, 757-759

Kubaczek, Martin, Masahiko Tsuchiya (eds.) (2005), “Bevorzugt beobachtet”. Zum Japanbild in der zeitgenössischen Literatur, München: Iudicium, 283 pp.

Kuhn, Hans-Werner, Peter Massing, Werner Skuhr (1993), Politische Bildung in Deutschland. Entwicklung - Stand - Perspektiven, seconda edizione ampliata, Opladen: Leske - Budrich, 447 pp.

Kühnhardt, Ludger, Gerd Leutenecker, Martin Rupps (eds.) (1996), Die doppelte deutsche Diktaturerfahrung. Drittes Reich und DDR - ein historisch-politikwissenschaftlicher Vergleich, Frankfurt a.M.: Lang, 330 pp.

Kultusministerkonferenz (1978), Behandlung des Nationalsozialismus im Unterricht (Beschluss der Kultusministerkonferenz vom 20.4.1978), in: Sammlung der Beschlüsse der Ständigen Kultusministerkonferenz, Grundwerk, terza edizione, Köln 1982: Link, 553 pp.

$350 \quad$ Elenco degli scritti citati 
Kultusministerkonferenz (1980), Empfehlung zur Behandlung des Widerstandes in der NS-Zeit im Unterricht (Beschluss der Kultusministerkonferenz vom 4.12.1980), https://www.gesetze-bayern.de/Content/Document/BayVV_2230_1 _1_1_1_3_UK_184/true

Kumagai, Naoko, David Noble (2016), The Comfort Women. Historical, Political, Legal, and Moral Perspectives, Tokyo: International House of Japan, XI-229 pp.

Kurasawa, Аiко (2010), Romusha: la memoria più crudele dell'occupazione giapponese in Indonesia, in: Contini, Giovanni et al. (eds.), Memoria e rimozione, $56-65$

Kuroda, Tаміко (1994), Aufarbeitung oder Restauration? Geschichtsbewusstsein und "Vergangenheitsbewältigung" in Japan, in: Geschichte Lernen, 38:3, 7-9

Kuss, Horst (2008), Neue Wege - alte Ziele?, in: Hasberg, Wolfgang (ed.), Modernisierung im Umbruch. Geschichtsdidaktik und Geschichtsunterricht nach 1945, Berlin - Münster: Lit, 293-296

Labanca, Nicola (ed.) (1992), Fra sterminio e sfruttamento. Militari internati e prigionieri di guerra nella Germania nazista (1939-1945), Firenze: Le Lettere, VII-361 pp.

Labanca, Nicola (ed.) (2000), La memoria del ritorno. Il rimpatrio degli Internati militari italiani (1945-1946), Firenze: Giuntina, LXX-352 pp., edizione fuori commercio

Labanca, Nicola (ed.) (2003), La Libia nei manuali scolastici italiani: 1911-2001, Roma: Istituto Italiano per l'Africa e l'Oriente, $235 \mathrm{pp}$.

Labanca, Nicola (2010), Compensazioni, passato coloniale, crimini italiani. Il generale e il particolare, in: Contini, Giovanni et al. (eds.), Memoria e rimozione, 127-161

Lamberti, Maria Mimita (1985), Giapponeserie dannunziane, in: Gallotta, Aldo, Ugo Marazzi (eds.), La conoscenza dell'Asia e dell'Africa in Italia nei secoli XVIII e XIX, vol. 2, Napoli: Istituto Universitario Orientale, 295-319

Lanna, Noemi (2016), Il duplice dissenso di Oda Makoto: il pacifismo come critica al vittimismo, in: Migliore, Maria Chiara, Antonio Manieri, Stefano Romagnoli (eds.), Il dissenso in Giappone. La critica al potere in testi antichi e moderni, Ariccia: Aracne, 171-189

Legnani, Massimo [1968], Politica e amministrazione nelle Repubbliche Partigiane. Studio e documenti, Milano: Istituto Nazionale per la Storia del Movimento di Liberazione, $173 \mathrm{pp}$.

Lehndorff-Felsko, Angelika (1981), Der KPD-Verbotsprozeß 1954 bis 1956. Wie es dazu kam, sein Verlauf, die Folgen, Frankfurt a.M.: Verlag Marxistische Blätter, 220 pp.

Leo, Annette (1993), Die Auseinandersetzung mit der nationalsozialistischen Vergangenheit in der DDR, in: Benz, Wolfgang, Jörg Leuschner (eds.), Geeinte Nation - Geteilte Geschichte. Die deutsche Gesellschaft nach der Wiedervereinigung, Salzgitter: Archiv der Stadt Salzgitter, 45-53

Levi, Riccardo (1947), L'azione economica e sociale dei C.L.N. dell'Alta Italia, in: Il Ponte, 3:11-12, 994-1000 
Li Gotti, Claudio (2008), Gli americani a Licata. Dall'amministrazione militare alla ricostruzione democratica, Civitavecchia: Prospettiva editrice, $108 \mathrm{pp}$.

Libia e Iraq, forze speciali italiane sul terreno: lo scoop del Fatto confermato dal documento trasmesso al Copasir (2016, 10 agosto), in: Il Fatto Quotidiano, da http:/www.ilfattoquotidiano.it/2016/08/10/libia-forze-speciali-italiane-sul-terrenolo-scoop-del-fatto-confermato-dal-copasir/2967379/

Libri scolastici, digitalizzazione all'italiana (2012, febbraio 12), in: Lim e dintorni, da http:/lavagna.wordpress.com/2012/02/12/libri-scolastici-digitalizzazione-allitaliana/

Liekhin, L. V., F. N. Petrov (1949), Dizionario sovietico delle parole straniere, Mosca: Edizioni di Stato

Linhart, Sepp (2005), Das heroische Japan. Deutschsprachige Japan-Literatur zwischen 1933 und 1945, in: Kubaczek, Martin, Masahiko Tsuchiya (eds.), "Bevorzugt beobachtet". Zum Japanbild in der zeitgenössischen Literatur, München: Iudicium, 41-65

Llewelyn, James, David Walton, Gen Kinkawa (2009), A Pacifist State in a Hostile Region. Japan and Post-War Conflict in Southeast Asia, New York: Nova Science Publishers, XIV-234 pp.

Losano, Mario G. (1960, 15 marzo), Giustizia impunita, in: Libera critica. Mensile di studenti medi e universitari, 3, pp. 1 e 4

Losano, Mario G. (1963), L'aeroporto non c'entra, in: Il Ponte, 19:12, 1648-1649

Losano, Mario G. (1964), La Germania spiegata agli studenti, in: Il Ponte, 20:3, 391-392

Losano, Mario G. (1965), Il diritto alla sberla, in: Il Ponte, 21:1, 135-136

Losano, Mario G. (1973), Tre consiglieri giuridici europei e la nascita del Giappone moderno, in: Materiali per una storia della cultura giuridica, 3:1, 517-667

Losano, Mario G. (1984), La recezione dei modelli giuridici europei nella realtà giapponese: Hermann Roesler (1834-1894), in: Sociologia del diritto, 11:3, 29-44

Losano, Mario G. (2001), Il diritto libero di Theodor Sternberg dalla Germania al Giappone, in: Sociologia del diritto, 2, 115-154

Losano, Mario G. (2003), Recensione a Tratta, prostituzione forzata e diritto internazionale. Il caso delle "donne di conforto" di Maria Clara Maffei, in: Diritto, immigrazione e cittadinanza, 2, 249-251

Losano, Mario G. (2009a), Ernst Hirsch (1902-1985). Dall'europeizzazione del diritto in Turchia alla sociologia giuridica in Germania, Parte I. La formazione e l'esilio di Ernst Hirsch, in: Materiali per una storia della cultura giuridica, 39:1, 159-182

Losano, Mario G. (2009b), Ernst Hirsch (1902-1985). Dall'europeizzazione del diritto in Turchia alla sociologia giuridica in Germania, Parte II. Ernst Hirsch sociologo del diritto, in: Materiali per una storia della cultura giuridica, 39:2, 341-373

Losano, Mario G. (2011), La geopolitica del Novecento. Dai Grandi Spazi delle dittature alla decolonizzazione, Milano: Bruno Mondadori, 336 pp. 
Losano, Mario G. (2016a), Alle origini della filosofia del diritto in Giappone. Il corso di Alessandro Paternostro a Tokyo nel 1889. In appendice: A. Paternostro, Cours de philosophie du droit, 1889, Torino: Lexis, XI-246 pp., anche: https://www.acade mia.edu/35729992/Mario_G._Losano_Alle_origini_della_filosofia_del_diritto_ in_Giappone._Il_corso_di_Alessandro_Paternostro_a_Tokyo_nel_1889._In_ appendice_A._Paternostro_Cours_de_philosophie_du_droit_1889_Lexis_Torino 2016_XI-246_pp, (2011) http://opacplus.bsb-muenchen.de/search?isbn=978-388008-006-5

Losano, Mario G. (2016b), Il portoghese Wenceslau de Moraes e il Giappone ottocentesco. Con 25 sue corrispondenze nelle epoche Meiji e Taisho (1902-1913), Torino: Lexis, XXVII-569pp., anche: https://www.academia.edu/35755566/Il_porto ghese_Wenceslau_de_Moraes_e_il_Giappone_ottocentesco_Con_25_sue_corri spondenze_nelle_epoche_Meiji_e_Taisho_1902-1913_Lexis_Torino_2016_XXV II-569_pp

Losano, Mario G. (2016c), Lo spagnolo Enrique Dupuy e il Giappone ottocentesco. In appendice: Enrique Dupuy, La transformación del Japón en la era Meiji, 1867-1894, Torino: Lexis, XXIII-407 pp., anche: https://www.academia.edu/ 35755644/Enrique_Dupuy_e_Giappone_XIX_sec._In_appendice_Enrique_ Dupuy_La_transformaci\%C3\%B3n_del_Jap\%C3\%B3n_en_la_era_Meiji_ 1867-1894_Lexis_Torino_2016_XXIII-407_pp

Losano, Mario G. (2017a), Il rifiuto della guerra nelle costituzioni postbelliche di Giappone, Italia e Germania, in: Moita, Luís, Luís Valença Pinto (eds.), Espaços económicos e espaços de segurança, Lisboa: Observare - Universidade Autónoma de Lisboa, 71-87, in forma più estesa, 71-125: https://www.aca demia.edu/35729327/Il_rifiuto_della_guerra_nelle_costituzioni_postbelliche_ di_Giappone_Italia_e_Germania_in_Lu\%C3\%ADs_Moita_Lu\%C3\%ADs_Valen \%C3\%A7a_Pinto_eds._Espa\%C3\%A7os_econ\%C3\%B3micos_e_espa\%C3\%A7os _de_seguran\%C3\%A7a_pp._71-125

Losano, Mario G. (2017b), Guerre ibride, omicidi mirati, droni: conflitti senza frontiere e senza diritto, in: Forni, Lorena, Tiziana Vettor (eds.), Sicurezza e libertà in tempo di terrorismo globale, Torino: Giappichelli, 19-38

Losano, Mario G. (2018), Norberto Bobbio. Una biografia culturale, Roma: Carocci, $510 \mathrm{pp}$.

Lowe, KeIth (2014), Der wilde Kontinent. Europa in den Jahren der Anarchie 1943-1950, Stuttgart: Klett-Cotta, 526 pp.

LUTHER, JöRg (1999), Il giudice costituzionale può fermare la guerra? Una sentenza del Bundesverfassungsgericht sull'intervento militare in Kosovo, in: Dogliani, Mario, Stefano Sicardi (eds.), Diritti umani e uso della forza, 85-89

Lutz, Dieter S. (1982), Krieg und Frieden als Rechtsfrage im Parlamentarischen Rat 1948-1949. Wertentscheidung, Auslegungsmethodik, Dokumentation, BadenBaden: Nomos, 125 pp.

Luyssen, Johanna (2019, 16 ottobre), Adorno, icône de la lutte contre l'extrême droite, in: Libération, 13, https://www.liberation.fr/planete/2019/10/15/adornoicone-de-la-lutte-contre-l-extreme-droite_1757831 
Lyon, Cherstin M. (2012), Prisons and Patriots: Japanese American Wartime Citizenship, Civil Disobedience, and Historical Memory, Philadelphia: Temple University Press, XIII-239 pp.

MacArthur, Douglas (1964), Reminiscences, New York: McGraw-Hill, 438 pp.

MacArthur, Douglas (1966), Reports of General MacArthur. 1st Supplement: MacArthur in Japan. The Occupation. Military Phase, Charles A. Willoughby (ed.), Washington, DC: U.S. Government Printing Office, 2 voll.

Macdonald, Stuart (1991), Science, Technology, and Reparations: Exploitation and Plunder in Postwar Germany by John Gimbel, in: Prometeus. Critical Studies in Innovation, 9:1, 155-156

Maffei, Maria Clara (2002), Tratta, prostituzione forzata e diritto internazionale. Il caso delle "donne di conforto", Milano: Giuffrè, 155 pp.

Magi, Laura (2010), Fino a che punto l'Italia ha risarcito i danni alle vittime di dei crimini commessi durante la Seconda guerra mondiale, in: Contini, Giovanni et al. (eds.), Memoria e rimozione, 203-217

Major, Patrick (1998), The Death of the KPD. Communism and Anti-Communism in West Germany 1945-1956, Oxford: Clarendon Press, XIV-335 pp.

Maki, John (1964), Court and Constitution in Japan. Selected Supreme Court Decisions 1948-1960, Seattle: University of Washington Press, XLVI-445 pp.

Maki, John M. (ed.) (1980), Japan's Commission on the Constitution: the Final Report, Seattle: University of Washington Press, X-413 pp.

Maltarich, Bill (2005), Samurai und Supermen. National Socialist Views of Japan, Oxford u.a.: Lang, 406 pp.

Mancino, Pietro (2019, 21 febbraio), Il Colle graziò il comunista assassino, in: Libero, 9

ManN, Erika (1938), School for Barbarians. Education under the Nazis. Introduction by Thomas Mann, New York: Modern Age Books, 159 pp., ristampa 2014: Mineola (N.Y.): Dover Publications, 159 pp.

Mann, Erika (2011), Zehn Millionen Kinder. Die Erziehung der Jugend im Dritten Reich, Reinbek bei Hamburg: Rowohlt, 220 pp.

Mann, Thomas (1938), Introduction, in: ManN, EriKa, School for Barbarians, 5-9

Mann, Thomas (1997), Die deutschen KZ [Radiobotschaft, 8.5.1945], in: Mann, Thomas, Essays, vol. 6: Meine Zeit 1945-1955, Kurzke, Hermann, Stephan STACHORSKI (eds.), Frankfurt a.M.: Fischer, 11-13

Maraini, Dacia (1973), E tu chi eri? Interviste sull'infanzia, Milano: Bompiani, 269 pp.

Maraini, Dacia (1978), Mangiami pure, Torino: Einaudi, 86 pp.

Maraini, Dacia (1993), Bagheria, Milano: Rizzoli, 167 pp.

Maraini, Dacia (2001), La nave per Kobe. Diari giapponesi di mia madre, Milano: Rizzoli, 259 pp.

Maraini, Fosco (1988), Ore giapponesi. Nuova edizione, Milano: Dall'Oglio, 523 pp.

Maraini, Fosco (2001), Case, amori, universi, Milano: Mondadori, 703 pp., prima edizione 1999 
MARTin, Bernd (1970), Restauration - die Bewältigung der Vergangenheit in Japan, in: Zeitschrift für Politik, 17:2, 153-170

Maruyama, Masao (1982), Théorie et psychologie de l'ultra-nationalisme, in: le débat, 21, 105-130

Maruyama, Masao (1990), Le radici dell'espansionismo. Ideologie del Giappone moderno, Torino: Fondazione Agnelli, XVIII-369 pp.

Marxen, Klaus (2001), Die Bestrafung von NS-Unrecht in Ostdeutschland, in: Marxen, Klaus et al. (eds.), Der Umgang mit Kriegs- und Besatzungsunrecht in Japan und Deutschland, 159-176

Marxen, Klaus, Koichi Miyazawa, Gerhard Werle (eds.) (2001), Der Umgang mit Kriegs- und Besatzungsunrecht in Japan und Deutschland, Berlin - Baden-Baden: Berlin-Verlag - Nomos, 237 pp.

März, Peter, Heinrich Oberreuter (eds.) (1999), Weichenstellung für Deutschland? Der Verfassungskonvent von Herrenchiemsee, München: Olzog, 222 pp.

Marzo Magno, Alessandro (2017), Missione grande bellezza. Gli eroi e le eroine che salvarono $i$ capolavori italiani saccheggiati da Napoleone e da Hitler, Milano: Garzanti, 309 pp.

Masaru, Tonomura (2015), Die Erinnerung an die Kolonien im Japan der Nachkriegszeit. Unter besonderer Berücksichtigung der Probleme im Zusammenhang mit der Herrschaft über Korea, Halle-Wittenberg: Martin-Luther-Universität, 30 pp.

Mason, Tiм (1982), Banalisation du nazisme? La controverse actuelle sur l'interprétation du national-socialisme, in: le débat, 21, 151-166

Matsumura, TAKao (2010), L'Unità 731 e la guerra batteriologica dell'esercito giapponese, in: Contini, Giovanni et al. (eds.), Memoria e rimozione, 67-77

Maunz, Theodor, Günter Dürig (1984), Grundgesetz - Kommentar, vol. V: Art. 70-99, München: Beck

Mazziotti di Celso, Manlio (1993), Lezioni di diritto costituzionale, vol. 2: La Costituzione italiana. Precedenti storici. Principi fondamentali e rapporti civili, Milano: Giuffrè, X-365 pp.

[Menzel, Johanna M.] (1957), Der geheime deutsch-japanische Notenaustausch zum Dreimächtepakt, in: Vierteljahrshefte für Zeitgeschichte, 2, 182-193

Mercuri, Lamberto (1988), L'epurazione in Italia 1943-1948, Cuneo: L'Arciere, 275 pp.

Mesmer, Philippe (2018, 18 dicembre), Le Japon muscle les capacités offensives des Forces d'autodéfense, in: Le Monde, da https:/www.lemonde.fr/international/ article/2018/12/18/le-japon-muscle-les-capacites-offensives-des-forces-d-autodefense _5399222_3210.html

Meyer, Dennis (2009), Entnazifizierung, in: Fischer, Torben, Matthias N. Lorenz (eds.), Lexikon der "Vergangenheitsbewältigung" in Deutschland, 18-19

Meyers Lexikon (1936-1942), ottava edizione, 9 voll., Leipzig - Wien: Bibliographisches Institut

Meyers Neues Lexikon in acht Bänden (1964), Leipzig: VEB Bibliographisches Institut, vol. 8 
Middlebrooks, William C. (2008), Beyond Pacifism. Why Japan Must Become a "Normal” Nation, Westport (Conn.): Praeger Security International, XVII-155 pp.

Migliore, Maria Chiara, Antonio Manieri, Stefano Romagnoli (eds.) (2016), Il dissenso in Giappone. La critica al potere in testi antichi e moderni, Ariccia: Aracne, $256 \mathrm{pp}$.

Mrhr, Anja (2018), Regime Consolidation and Transitional Justice. A Comparative Study of Germany, Spain and Turkey, Cambridge: Cambridge University Press, IX-468 pp.

Minami, Keita (2017, 27 novembre), Kempo 9-jo no genzai ishiki rutsu wa "Tokugawa no heiwa" - shisoka Karatani Kojin [Una coscienza reale dell'articolo 9 e le radici nella "Pax Tokugawa". Il parere del pensatore Karatani Kojin], in: Mainichi Shimbun (edizione di Tokyo del mattino), da https://mainichi.jp/articles/ 20171127/ddm/004/070/020000c

Minear, Richard H. (1971), Victors' Justice. The Tokyo War Crimes Trial, Princeton $(\mathrm{NJ})$ : Princeton University Press, XV-229 pp.

Minear, Richard H. (1983), War Crimes Trials, in: Kodansha Encyclopedia of Japan, Tokyo: Kodansha, vol. 8, 223-225

Minear, Richard H. (2008), Foreword, in: Nozaki, Yoshiko, War Memory, Nationalism and Education, I-XX

Minerbi, Alessandra (1999), La scuola dei barbari: un documento sulla Germania nazista, in: Qualestoria, 2, 186-216

Ministry of Foreign Affairs, Japan (2007), Japan's Contribution to Afghanistan Working on the frontline in the war on terrorism, da http://www.mofa.go.jp/ region/middle_e/afghanistan/pamph0703.pdf

Moita, Luís, Luís Valença Pinto (eds.) (2017), Espaços económicos e espaços de segurança, Lisboa: Observare - Universidade Autónoma de Lisboa, 751 pp.

Molodiakov, Vassili (2017, 7 settembre), Who Is the Author of Article 9 of the Constitution of Japan?, in: nippon.com, da http://www.nippon.com/en/ column/g00269/

Monducci, Francesco (2015, 27 luglio), Guerra e pace nei manuali scolastici italiani, in: Novecento.org, Pensare la didattica, da http://www.novecento.org/pensare -la-didattica/guerra-e-pace-nei-manuali-scolastici-italiani-1314/

Montanelli, Indro (2002), Soltanto un giornalista. Testimonianza resa a Tiziana Abate, Milano: Rizzoli, 357 pp.

Montino, Davide (2005), Le parole educate: libri e quaderni tra fascismo e Repubblica, Milano: Selene, 270 pp.

Moore, Ray A., Donald L. Robinson (1998), The Constitution of Japan. A Documentary History of its Framing and Adoption, Princeton: Princeton University Press, CD-ROM

Moore, Ray A., Donald L. Robinson (2002), Partners for Democracy. Crafting the New Japanese State under MacArthur, New York: Oxford University Press, XIV-449 pp.

Morgenthau, Henry (1945), Germany is our Problem, New York: Harper, XIII-239 pp.

356 Elenco degli scritti citati 
Morra, Francesco (1941), Numeri e figure. Matematica per la scuola media, Milano: Trevisini, $121 \mathrm{pp}$.

Mossdorf, Отto (1943), Der soldatische Charakter des deutschen und japanischen Volkes, in: Donat, Walter et al., Das Reich und Japan, 95-104

Mucchi, Gabriele (1952), Del manierismo e di due mostre dei manieristi, in: Realismo. Mensile di arti figurative, 5-6-7, 4

Mucchi, Gabriele (1955), Pittura e antifascismo, in: Realismo. Mensile di arti figurative, Nuova Serie, 2, 53-55

Mucchi, Gabriele (1994), Le occasioni perdute. Memorie 1899-1993, Prefazione di Norberto Bobbio, Milano: L'Archivolto, 651 pp.

Müller, Reinhard (2010), Brunolf Baade und die Lufffahrtindustrie der DDR - die wahre Geschichte des Strahlverkehrsflugzeuges 152, Erfurt: Sutton, 449 pp.

MüNCH, PhilıpP (2015), Die Bundeswehr in Afghanistan. Militärische Handlungslogik in internationalen Interventionen, Freiburg im Breisgau: Rombach Verlag, XII-433 pp.

Munzert, Maria (2009), Neue Antisemitismuswelle, in: Fischer, Torben, Matthias N. LoRenz (eds.), Lexikon der "Vergangenheitsbewältigung" in Deutschland, $85-87$

Mura, Guido (2004a), Esempi di grafica del ventennio nei libri scolastici, in: Coarelli, Rossella (ed.), Istruiti e laboriosi: gli anni della ricostruzione, 63-73

Mura, Guido (2004b), Oltre l'immagine. La grafica dei libri scolastici negli anni della ricostruzione, in: Coarelli, Rossella (ed.), Istruiti e laboriosi: gli anni della ricostruzione, 111-130

Muramatsu, Mariko (1996), Il buon suddito del Mikado: D'Annunzio japonisant, Milano: Archinto, $132 \mathrm{pp}$.

Muramatsu, Mariko (2011), Outa occidentale di Gabriele D’Annunzio, ovvero quando la metrica giapponese plasma la poesia italiana, in: Crisafulli, Edoardo, Maria Katia Gesuato (eds.), Una lingua per amica, 75-84, anche: https://utcp.c.u-tokyo.ac.jp/publications/pdf/CollectionUTCP11_Muramatsu_ 04.pdf

Nagy-Talavera, Nicholas M. (2001), The Green Shirts and the Others. A History of Fascism in Hungary and Romania, Iaşi: Center for Romanian Studies, 548 pp.

Nakai, Akio (1988), Die "Entmilitarisierung" Japans und die "Entnazifizierung" Deutschlands nach 1945 im Vergleich, in: Beiträge zur Konfliktforschung, 2, 5-21

Nakath, Detlef, Siegfried Prokop, Rosa-Luxemburg-Stiftung Brandenburg (eds.) (2010), DDR-Geschichte: Bilder \& Zerrbilder. Siegfried Prokop zum 70. Geburtstag, Berlin: Karl Dietz, 340 pp.

Napoli, Franco Felice (1996), Villa Wolkonsky, 1943-1988. Il lager nazista di Roma, Como: Europa Edizioni, 256 pp.

Narita, Ryuichi (2003), Geschichte schreiben in Schulbüchern. Revisionismus, Konstruktivismus, und das Subjekt der Geschichte, in: Richter, StefFi, Wolfgang Höpken (eds.), Vergangenheit im Gesellschaftskonflikt, 119-131 
NATO and Japan conduct first ever joint counter-piracy drill (2014, 25 settembre), NATO, da http:/www.nato.int/cps/en/natohq/news_113373.htm?selectedLocale=en

Natta, Alessandro (1992), Reducismo o silenzio?, in: Labanca, Nicola (ed.), Fra sterminio e sfruttamento, 327-332

Natta, Alessandro (1997), L'altra Resistenza. I militari italiani internati in Germania. Introduzione di Enzo Collotti, Torino: Einaudi, XXXIV-141 pp.

Negri, Gugliemo, Silvano Simoni (1990), Le Costituzioni inattuate, Roma: Colombo, XXIV-109 pp.

Nenni, Pietro (1978), Vento del Nord. Giugno 1944 - giugno 1945, Torino: Einaudi, LXXI-418 pp.

Neo-Gōmanism Manifesto Special - on War, in: Wikipedia, L'enciclopedia libera, da https://en.wikipedia.org/wiki/Neo_G\%C5\%8Dmanism_Manifesto_Special_\%E2 \%80\%93_On_War

Neumann, Franz L. (1942), Behemoth. The Structure and Practice of National-Socialism 1933-1944, London - New York: Victor Gollancz, 419 pp., seconda edizione 1944

Niehuss, Merith, Ulrike Lindner (eds.) (2012), Besatzungszeit, Bundesrepublik und DDR 1945-1969 (Deutsche Geschichte in Quellen und Darstellungen, vol. 10), Stuttgart: Reclam, 478 pp.

Nibon kokan shuan bunsho [Raccolta dei principali trattati internazionali del Giappone] (1985), Tokyo: Hara Shobo, 1186 pp.

Nihonkoku-kenpo wo kokumin wa do mukaetaka [Come i giapponesi hanno percepito la Costituzione] (1997), Tokyo: Kōbunken, 319 pp.

Nish, IAN (2011), The Japanese in War and Peace, 1942-1948. Selected documents from a translator's in-tray, Folkestone: Global Oriental, XII-454 pp.

Nitsch, Carlo (2014), Renato Treves esule in Argentina. Sociologia, filosofia sociale, storia. Con documenti inediti e la traduzione di due scritti di Treves, Torino: Accademia delle Scienze, 239 pp. (Memoria della Classe di Scienze Morali, Storiche e Filologiche, Serie V, vol. 38, fasc. 2)

Niven, William John (2002), Facing the Nazi Past. United Germany and the Legacy of the Third Reich, London: Routledge, XXII-266 pp.

Nozaki, Yosнiко (2008), War Memory, Nationalism and Education, in Postwar Japan: 1945-2007. The Japanese Textbook Controversy and Ienaga Saburo's Court Challenges, London: Routledge, XX-198 pp.

Occupation of Japan, in: New World Encyclopedia, da http://www.newworldencyclopedia.org/entry/Occupation_of_Japan

Office of the United States Chief of Counsel for Prosecution of Axis Criminality (1946-1948), Nazi Conspiracy and Aggression, Washington (DC): U.S. Government Printing Office, 8 voll., cui seguono Supplements (2 voll.), Opinion and Judgement (1 vol.)

Oliva, Gianni (2018), La grande storia della Resistenza. 1943-1948, Torino: Utet, $527 \mathrm{pp}$.

$358 \quad$ Elenco degli scritti citati 
Onuki, Атsuкo (2003), Instrumentalisierung der Geschichte und Nationaldiskurse. Das Beispiel der Schulbuchdiskussion in Japan, in: Richter, STEFfi, Wolfgang Höpken (eds.), Vergangenheit im Gesellschaftskonflikt, 133-149

Oppler, Alfred C. (1961), Sunagawa Case. Its Legal and Political Implications, in: Political Science Quarterly, 76:2, 241-263

Oppler, Alfred C. (1976), Legal Reform in Occupied Japan. A Participant Looks Back, Princeton (NJ): Princeton University Press, XVII-345 pp.

Oppler, Alfred C. (1983), Sunagawa Case, in: Kodansha Encyclopedia of Japan, Tokyo: Kodansha, vol. 7, 274-275

Osten, Philipp (2003), Der Tokioter Kriegsverbrecherprozeß und die japanische Rechtswissenschaft, Berlin: Berliner Wissenschafts-Verlag, 218 pp.

Ostwald, Paul (1941), Deutschland und Japan. Eine Freundschaft zweier Völker, Berlin: Junker und Dünnhaupt, 157 pp.

Otтo, Carsten (1999), Japans bewaffneter Pazifismus: Die Sicherheitspolitik einer wirtschaftlichen Supermacht seit dem Ende des Ost-West-Konfliktes, in: Asien, 73, 7-28

Pacini, Patrizia (2009), Teresa Mattei, una donna nella storia. Dall'antifascismo militante all'impegno in difesa dell'infanzia, Firenze: Consiglio Regionale della Toscana, 220 pp., anche: http://www.consiglio.regione.toscana.it/upload/CPO/ pubblicazioni/pub75.pdf

Pacini, Patrizia (2011), La Costituente: storia di Teresa Mattei, Milano: Altraeconomia, 222 pp.

Pal, Radhabinod (1953), International Military Tribunal for the Far East: Dissentient Judgment, Calcutta: Sanyal, XVII-701 pp.

Pala, Elena (2010), Le “donne di Salò" nelle pagine de "La Domenica del Corriere": tra focolare e patria (1943-1945), in: Annali di storia dell'educazione e delle istituzioni scolastiche, 17, 87-98

Pansa, Giampaolo (2002), I figli dell'Aquila, Milano: Sperling \& Kupfer, XI-385 pp.

Pansa, Giampaolo (2003), Il sangue dei vinti. Quello che accadde in Italia dopo il 25 aprile [1945], Milano: Sperling \& Kupfer, X-380 pp.

Pansa, Giampaolo (2005), Sconosciuto 1945, Milano: Sperling \& Kupfer, XII-476 pp.

Pansa, Giampaolo (2006), La grande bugia, Milano: Sperling \& Kupfer, X-468 pp.

Pansa, Giampaolo (2007), I gendarmi della memoria, Milano: Sperling \& Kupfer, XVI-503 pp.

Pansa, Giampaolo (2012), La guerra sporca dei partigiani e dei fascisti, Milano: Rizzoli, 445 pp.

Pansa, Giampaolo (2014), Eia Eia Alalà. Controstoria del fascismo, Milano: Rizzoli, 375 pp., edizioni successive con il sottotitolo: Nascita del fascismo

Pansa, Giampaolo (2019), Quel fascista di Pansa, Milano: Rizzoli, 235 pp.

Pantaleone, Michele (1978), Mafia e politica, Torino: Einaudi, XIV-258 pp.

Der Parlamentarische Rat: 1948-1949. Akten und Protokolle (1975-2009), herausgegeben vom Deutschen Bundestag und vom Bundesarchiv unter Leitung von Rupert Schick und Friedrich P. Kahlenberg, Boppard am Rhein: Boldt, 14 voll. 
Passarelli, Gianluca, Dario Tuorto (2012), Lega e Padania. Storia e luoghi delle camicie verdi, Bologna: Il Mulino, 229 pp.

Pautasso, Guido Andrea (2019), Prefazione. Harukichi Shimoi, Samurai a Fiume e profeta del fascismo in Giappone, in: Shimoi, Harukichi, Un samurai a Fiume, 13-131

Pedroletti, Brice (2016, 16 agosto), Pékin et Manille cherchent l'apaisement en mer de Chine, in: Le Monde, 4

Pekar, Thomas (2008), Held und Samurai. Zu den ideologischen Beziehungen zwischen Japan und Nazi-Deutschland, in: Archiv für Kulturgeschichte, 90:2, 437-448

Peretti-Griva, Domenico Riccardo (1947), Il fallimento dell'epurazione, in: Il Ponte, 3:11-12, 1075-1081

Pfetsch, Frank R. (ed.) (1986), Verfassungsreden und Verfassungsentwürfe. Länderverfassungen 1946-1953, Frankfurt a.M.: Lang, 660 pp.

Piccigallo, Philip R. (1979), The Japanese on Trial. Allied War Crimes Operations in the East 1945-1951, Austin: University of Texas Press, XV-292 pp.

Pieri, Piero, Giorgio Rochat (2002), Badoglio, Maresciallo d'Italia, Milano: Mondadori, XVII-617 pp., aggiornamento dell'edizione 1974, Torino: Utet, VIII-914 pp.

Pikart, Eberhard, Wolfram Werner (eds.) (1993), Ausschuß für Grundsatzfragen (Der Parlamentarische Rat, vol. 5/I-II), Boppard am Rhein: Boldt, vol. 5/I: LXVIII-520 pp., vol. 5/II: 5566 pp.

Pingel, Falk (1994), Nationalsozialismus und Holocaust in westdeutschen Schulbüchern, in: Steininger, Rolf, Ingrid Böhler (eds.), Der Umgang mit dem Holocaust in Europa - USA - Israel, Köln - Weimar: Böhlau, 221-232

Plehwe, Friedrich-Karl von (1980), Als die Achse zerbrach. Das Ende des DeutschItalienischen Bündnisses im Zweiten Weltkrieg, Wiesbaden: Limes Verlag, 291 pp.

Poggiolini, Ilaria (2004), Resa incondizionata e istituto monarchico: il caso del Giappone al termine del secondo conflitto mondiale, in: Tesoro, Marina (ed.), Monarchia, tradizione, identità nazionale, 35-47

Porth, Kenneth L. (2010), Transcending Law. The Unintended Life of Article 9 of the Japanese Constitution, Durham: Carolina University Press, XVI-288 pp.

Pötzl, Norbert F. (2019, 5 gennaio), Räuberlohn, in: Der Spiegel, 2, 36-38

Pouthier, Jean-Luc (1982), National-syndicalisme et totalitarisme dans l'Italie fasciste, in: le débat, 21, 167-177

Powles, Cyril H. (1999), Pacifism in Japan, 1918-1945, in: Brock, Peter, Thomas P. Socknat (eds.), Challenge to Mars. Essays on Pacifism from 1918 to 1945, Toronto: University of Toronto Press, 427-439

Predieri, Alberto (1947), Garibaldini e partigiani, in: Il Ponte, 3:11-12, 1015-1032

Pritchard, R. John, Sonia M. Zaide (eds.) (1987), The Tokyo War Crimes Trial: [The Comprehensive Index and Guide to the Proceedings of the International Military Tribunal for the Far East], New York: Garland, 5 voll.

Procacci, Giuliano (2003), La memoria controversa. Revisionismi, nazionalismi e fondamentalismi nei manuali di storia, Cagliari: AM\&D, 143 pp. 
Pugliese, Pasquale (2013, 15 marzo), Ispiratevi a Teresa Mattei, cancellate gli F35, "Vita Bookazine", da http://www.vita.it/it/blog/disarmato/2013/03/15/ispiratevi-a-teresa-mattei-cancellate-gli-f35/3640/

Pustau, Eduard von, Moriguchi Okanouye-Kurota (1936), Japan und Deutschland, die beiden Welträtsel. Politische, wirtschaftliche und kulturelle Entwicklung, Berlin: Deutscher Verlag für Politik und Wirtschaft, 223 pp.

RADler, Max (1946), Schwarz wird weiss oder mechanische Entnazifizierung, in: Der Simpl, 1, 63, da http://digi.ub.uni-heidelberg.de/diglit/simpl1946/0063/image

Raggi, Barbara (2012), Baroni di razza. Come l'università del dopoguerra ha riabilitato gli esecutori delle leggi razziali, Roma: Editori Internazionali Riuniti, 216 pp.

Raimondo, Sergio, Valentina De Fortuna, Giulia Ceccarelli (eds.) (2017), Bushido as allied: The Japanese warrior in the cultural production of Fascist Italy (1940-1943), in: Revista de Artes Marciales Asiáticas (RAMA), 12:2, 82-100

Ramaioli, Federico Lorenzo (2016a), Addio alle armi: l'articolo 9 della Costituzione giapponese, in: Rivista della Cooperazione Giuridica Internazionale, 53, 100-127

Ramaioli, Federico Lorenzo (2016b), Disarmo e riarmo nella costituzione giapponese, in: Orientalia Parthenopea, 26, 101-134, anche: https:/www.academia. edu/31667175/Disarmo_e_riarmo_nella_Costituzione_giapponese

Ramaioli, Federico Lorenzo (2017), Il nazional-capitalismo degli zaibatsu come forma di governance, in: Rivista della Cooperazione Giuridica Internazionale, $55,85-110$

Rastelli, Achille (2000), Bombe sulla città. Gli attacchi aerei alleati. Le vittime civili a Milano, Milano: Mursia, 258 pp.

Ravagli, Giovanni (1947), La mancata avocazione dei profitti di regime, in: Il Ponte, 3:11-12, 1082-1089

Recchioni, Massimo (2011), Il tenente Alvaro, la Volante rossa e $i$ rifugiati politici italiani in Cecoslovacchia, Roma: DeriveApprodi, 219 pp.

Recchioni, Massimo (2013), Francesco Moranino, il comandante "Gemisto". Un processo alla Resistenza, Roma: DeriveApprodi, 187 pp.

Reel, A. Frank (1949), The Case of General Yamashita, Chicago: University of Chicago Press, VI-324 pp.

Reichel, Karl Ferdinand (1953), Japans “Nationale Polizei-Reserve”, in: Militärpolitisches Forum, 1, 41-42; 2, 30-34

Reichelt, Julian (2010), Ruhet in Frieden, Soldaten! Wie Politik und Bundeswehr die Wahrheit über Afghanistan vertuschten, Köln: Fackelträger, 217 pp.

Relations with Japan (2016, 7 aprile), NATO, da http:/www.nato.int/cps/en/natohq/ topics_50336.htm

Reparti italiani nella guerra all'Isis. Ma il parlamento non ne sa nulla (2016, 30 luglio), in: Il Fatto Quotidiano, 1

Richter, Steffi (2003), Zurichtung der Vergangenheit als Schmerzlinderung in der Gegenwart, in: Richter, Steffi, Wolfgang Höpken (eds.), Vergangenheit im Gesellschaftskonflikt, 1-26 
Richter, Steffi, Wolfgang Höpken (eds.) (2003), Vergangenheit im Gesellschaftskonflikt. Ein Historikerstreit in Japan, Köln: Böhlau, VI-240 pp.

Rieder, Bruno (1984), Die Entscheidung über Krieg und Frieden nach deutschem Verfassungsrecht. Eine verfassungshistorische und verfassungsdogmatische Untersuchung, Berlin: Duncker \& Humblot, 418 pp.

Righi, Stefano (2014), La città illuminata. L'intuizione di Giuseppe Colombo, l'Edison e l'elettrificazione dell'Italia, Milano: Rizzoli, $319 \mathrm{pp}$.

Robinson, Greg (2009), A Tragedy of Democracy. Japanese Confinement in North America, New York: Columbia University Press, VIII-397 pp.

Rochat, Giongio (1970), Inchiesta sui testi per l'insegnamento della storia contemporanea nella scuola italiana, in: Il Movimento di Liberazione, n. 101, 3-67

Roesler, Hermann (1984), Berichte aus Japan (1879-1880), Losano, Mario G. (ed.), Milano: Unicopli, XXVII-398 pp., anche: http://daten.digitale-sammlungen.de /db/0010/bsb00106246/images/

RoESLER, Jörg (2010), Zwischenfall unterwegs oder Vorbote vom Ende? Erich Apels Tod und das Schicksal des NÖS, in: NaKath, Detlef et al. (eds.), DDRGeschichte, 186-208

Röhl, Wilhelm (1963), Die japanische Verfassung, Frankfurt a.M.: Metzner, 273 pp.

Röling, Bernard V. A., ChristiaAn F. Rüter (eds.) (1977), The Tokyo Judgement. The International Military Tribunal for the Far East (IMTFE) 29 April 1946 - 12 November 1948, Amsterdam: University Press Amsterdam, 3 voll.

Romeike, SANya (2016), Transitional Justice in Germany after 1945 and after 1990, Nürnberg: International Nuremberg Principles Academy, 79 pp., anche: https://www.nurembergacademy.org/fileadmin/media/pdf/news/Transitional Justice_in_Germany.pdf

Rötzer, Florian (2017, 7 gennaio), $\mathbb{} 80$ StGB “Vorbereitung eines Angriffskriegs” ist seit 1. Januar 2017 gestrichen, in: Telepolis, da https://www.heise.de/tp/features /80-StGB-Vorbereitung-eines-Angriffskriegs-ist-seit-1-Januar-2017-gestrichen-359 0763.html

Rudnik, Carola S. (2009), Doppelte Vergangenheitsbewältigung, in: Fischer, Torben, Matthias N. Lorenz (eds.), Lexikon der "Vergangenheitsbewältigung" in Deutschland, 275-279

Ruiru, Li (2016, 13 luglio), Vi spiego perché non accettiamo questo verdetto, in: Corriere della Sera, 18

SAGI, Nana (1981), Die Wiedergutmachung für Israel, Stuttgart: Seewald, 261 pp.

Saitta, Armando (1957), Il cammino umano. Corso di storia ad uso dei licei, Firenze: La Nuova Italia, 955 pp.

Salandra, Antonio (1930), L'intervento (1915). Ricordi e pensieri, Milano: Mondadori, $385 \mathrm{pp}$.

Salomon, Ernst von (1951), Der Fragebogen, Hamburg: Rowohlt, 807 pp.

SAlomon, ERnst von (2018), Un destino tedesco: l'autobiografia di uno scrittore ribelle condannato da Weimar, incarcerato da Hitler, processato dagli Americani, Milano: Oaks, 227 pp.

Salvemini, Gaetano (1947), Ottimismo, in: Il Ponte, 3:11-12, 953-958 
Samarani, Guido (2010), Il massacro di Nanchino e la guerra di resistenza antigiapponese in Cina (1937-1945), in: Contini, Giovanni et al. (eds.), Memoria e rimozione, $79-90$

SAntevecchi, Guido (2016, 10 luglio), Un rischio il Giappone che archivia il pacifismo, in: Corriere della Sera, 29

Santevecchi, Guido (2016, 11 luglio), Giappone, Abe più forte. Ora può cambiare la costituzione pacifista, in: Corriere della Sera, 17

Santevecchi, Guido (2016, 13 luglio), "La Cina non ha diritti sulle isole contese": lo schiaffo dell'Onu, la rabbia di Pechino, in: Corriere della Sera, 18

SARPIERI, TANJA (2002), Il pacifismo nella politica internazionale della sinistra italiana, negli anni della guerra fredda. Limiti e prospettive dell'azione social-comunista all' $i$ nterno del movimento dei Partigiani della Pace, Relatrice Patrizia Dogliani, tesi di laurea difesa nell'anno accademico 2001-02 e conservata nella Biblioteca dell'Università di Bologna, Campus di Forli, 143 pp.

Schaller, Michael (1985), The American Occupation of Japan. The Origins of the Cold War in Asia, New York: Oxford University Press, XII-351 pp.

Scherrer, Philipp (2010), Das Parlament und sein Heer. Das Parlamentsbeteiligungsgesetz, Berlin: Duncker \& Humblot, 403 pp.

Schmidt-Sinns, Dieter (1980), Der Nationalsozialismus als didaktisches Problem. Beiträge zur Behandlung des NS-Systems und des deutschen Widerstands im Unterricht, relazioni tenute nel convegno Fachtagung zur Behandlung von Nationalsozialismus und Widerstand im Schulunterricht, Berlin, 7-10 novembre 1978, Bonn: Bundeszentrale für politische Bildung, $226 \mathrm{pp}$.

Schönbach, Karsten Heinz (2015), Die deutschen Konzerne und der Nationalsozialismus, 1926-1943, Berlin: Trafo, 658 pp.

Schöpfel, Ann-Sophie (2013), La voix des juges français dans les procès de Nuremberg et de Tokyo. Défense d'une idée de justice universelle, in: Revue d'histoire, 249, 101-114

Schreiber, Gerhard (1990), Die italienischen Militärinternierten im deutschen Machtbereich 1943-1945. Verraten, verachtet, vergessen, München - Wien: Oldenbourg, $642 \mathrm{pp}$.

SChreIber, Gerhard (1992), I militari italiani internati nei campi di concentramento del Terzo Reich. Traditi, disprezzati, dimenticati, Roma: Ufficio Storico dello Stato Maggiore dell'Esercito, 903 pp.

SCHröder, Florian (2005), Das parlamentarische Zustimmungsverfahren zum Auslandseinsatz der Bundeswehr in der Praxis, Köln - München: Heymanns, 358 pp.

Schwarz, Jürgen, Armin A. Steinkamm (eds.) (1993), Rechtliche und politische Probleme des Einsatzes der Bundeswehr "out of area". Protokoll und Dokumentation eines Symposiums der Universität der Bundeswehr München am 12. und 13. Dezember 1991, Baden-Baden: Nomos, 295 pp.

Schwarz, Walter (ed.) (1974-1987), Die Wiedergutmachung nationalsozialistischen Unrechts durch die Bundesrepublik Deutschland, München: Beck, 6 voll.

Schwegmann, Christoph (ed.) (2011), Bewährungsproben einer Nation. Die Entsendung der Bundeswehr ins Ausland, Berlin: Duncker \& Humblot, XXI-234 pp. 
SChweIzer, Katja (1999), Täter und Opfer in der DDR. Vergangenheitsbewältigung nach der zweiten deutschen Diktatur, Münster: Lit, XXI-255 pp.

Schwentker, Wolfgang (2003), Die Grenzen der Entzauberung. Zur Rolle des Tenno in Staat und Gesellschaft Japans nach 1945, in: Cornelissen, Christoph et al. (eds.), Erinnerungskulturen, 123-136

Scimoi, Harukici [Shimoi, Harukichi] (1919), La guerra italiana vista da un giapponese, con introduzione di Giuseppe de Lorenzo, autografi di Gabriele D’Annunzio [e altri], epilogo di Gherardo Marone, seconda edizione, Napoli: Libreria della Diana, 97 pp.

Seidel, Hans-Christoph (ed.) (2007), Zwangsarbeit im Europa des 20. Jahrhunderts. Bewältigung und vergleichende Aspekte, Essen: Klartext, 253 pp.

Seifer, Hubertus (1971), Die Reparationen Japans. Ein Beitrag zum Wandel des Reparationsproblems und zur wirtschaftlichen Entwicklung Japans nach 1945, Opladen: Westdeutscher Verlag, 208 pp.

Sereni, Emilio (2015), Diario (1946-1952), introduzione e cura di Giorgio Vecchio, Roma: Carocci, 207 pp.

Sereni, Marina (1955), I giorni della nostra vita, quarta edizione, Roma: Editori Riuniti, 229 pp.

Setta, Sandro (1993), Profughi di lusso. Industriali e manager di Stato dal fascismo alla epurazione mancata, Milano: Angeli, 128 pp.

Setta, SAndro (2005), L’Uomo Qualunque. 1944-1948, Roma - Bari: Laterza, $\mathrm{XV}-342 \mathrm{pp}$.

Severgnini, Nathascia (2018, 4 maggio), Schiave dei soldati, un racconto che è vero (e il mondo non lo sa), in: Corriere della Sera, 46

Seymour, Robert L. (1974-1975), Japan's Self-Defense: The Naganuma Case and Its Implications, in: Pacific Affairs, 47:4, 421-436

Shimoi, Harukichi (1938, aprile), D’Annunzio no yokogao [Profilo di D’Annunzio], in: Kaiz, 459-460

Shimoi, Harukichi (2015), Buio sotto il faro. Scritti di un giapponese a Napoli: Shimoi Harukichi, Piscopo, Ugo (ed.), Napoli: Guida, 254 pp.

Shimoi, Harukichi (2019), Un samurai a Fiume, Pautasso, Guido Andrea (ed.), Sesto San Giovanni (Milano): Oaks, 300 pp.

Sicardi, Stefano (1999), I mille volti della guerra, la Costituzione e il diritto internazionale, in: Dogliani, Mario, Stefano Sicardi (eds.), Diritti umani e uso della forza, 95-112

SieP, Julia (2011), Nationalisierte Mütterlichkeit als Phänomen der Moderne: Frauenzeitschriften in Japan, Deutschland und Italien in den 1930er Jahren, München: Meidenbauer, $342 \mathrm{pp}$.

Silvestri, Mario (2002), Dal riarmo della Germania alla catastrofe, 1933-1946 (La decadenza dell'Europa occidentale, vol. 2), Milano: BUR, 920 pp.

SIPRI Yearbook 2018 (www.sipriyearbook.org), Armaments, Disarmament and International Security, da https://www.sipri.org/sites/default/files/SIPRIYB18c06.pdf

Sircana, Giuseppe (1996), Ferrarin, Arturo, in: Dizionario Biografico degli Italiani, Roma: Treccani, vol. 46, 682-684 
Skya, Walter A. (2009), Japan's Holy War. The Ideology of Radical Shinto Ultranationalism, Durham - London: Duke University Press, XII-387 pp.

Smiatacz, Carmen (2015), Ein gesetzlicher Schlussstrich? Der juristische Umgang mit der nationalsozialistischen Vergangenheit in Hamburg und Schleswig-Holstein 1945-1960. Ein Vergleich, Münster: LIT Verlag, 486 pp.

Soh, Chunchee Sarah (2008), The Comfort Women. Sexual Violence and Postcolonial Memory in Korea and Japan, Chicago: University of Chicago Press, XXVIII-352 pp.

Sontag, Ernst (1950-51), Die Deutschen Spruchgerichte in der britischen Zone, in: Die Friedenswarte, 51-64

Spang, Christian W. (2000), Karl Haushofer und die Geopolitik in Japan. Zur Bedeutung Haushofers innerhalb der deutsch-japanischen Beziehungen nach dem Ersten Weltkrieg, in: Diekmann, Irene, Peter Krüger, Julius H. Schoeps (eds.) (2000), Geopolitik. Grenzgänge im Zeitgeist, vol. 1.2: 1945 bis zur Gegenwart, Potsdam: Verlag für Berlin-Brandenburg, 591-629

Spang, Christian W. (2013), Karl Haushofer und Japan. Die Rezeption seiner geopolitischen Theorien in der deutschen und japanischen Politik, München: Iudicium, $1008 \mathrm{pp}$.

Spinosa, Antonio (2005), D’Annunzio, il poeta armato, Milano: Mondadori, 344 pp.

Spoerer, Mark (2007), Zwangsarbeitsregimes im Vergleich. Deutschland und Japan im Ersten und im Zweiten Weltkrieg, in: Seidel, Hans-Christoph (ed.), Zwangsarbeit im Europa des 20. Jahrhunderts, 187-226

Spulcioni, Gianni (2014), L'organizzazione del consenso nel ventennio fascista. Dall'ufficio stampa al Ministero della cultura popolare, Ariccia: Aracne, $403 \mathrm{pp}$.

Stalin, Iosif (1972/1976), Problemi economici del socialismo nell'URSS, Milano: Cooperativa Editrice Distributrice Proletaria, 124 pp., ristampa: Problemi economici del socialismo nell'URSS (1976), con uno scritto di Emilio Sereni del 1953, introduzione di Franco Botta, Bari: De Donato, 204 pp.

Stam, Arthur (2003), The Diplomacy of the "New Order": The Foreign Policy of Japan, Germany and Italy: 1931-1945, Soesterberg: Aspekt, $112 \mathrm{pp}$.

STEIndorf, JoAснім (2007), Waffenrecht. Waffengesetz, Beschussgesetz, Kriegswaffenkontrollgesetz einschließlich untergesetzlichem Regelwerk und Nebenbestimmungen, München: Beck, XIV-1058 pp.

Steinmeier fordert stärkeres Engagement der Bundeswehr (2017, 16 giugno), in: Die Welt, da https://www.welt.de/politik/deutschland/article165595858/Steinmeier-fordert-staerkeres-Engagement-der-Bundeswehr.html

Stella, Gianfranco (1993), Rifugiati a Praga. I partigiani italiani rifugiati a Praga, [s.l.]: SO.ED.E., 190 pp.

Stella, Gianfranco (2018), Compagno mitra. Saggio storico sulle atrocità partigiane, [s.l., s.n.], 606 pp.

Stöcker, Helene (1926), Das Problem der Kriegsächtung, in: Die Friedens-Warte, $26: 5,144-147$

Strafgesetzbuch und andere Strafgesetze. Textausgabe mit Anmerkungen und Sachregister (1960), sesta edizione, Berlin (Ost): VEB Deutscher Zentralverlag, 554 pp. 
Sugita, Yoneyuki (2003), Pitfall or Panacea. The Irony of US Power in Occupied Japan, 1945-1952, New York: Routledge, XX-222 pp.

SüHL, Klaus (ed.) (1994), Vergangenheitsbewältigung 1945 und 1989. Ein unmöglicher Vergleich? Eine Diskussion, Berlin: Volk und Welt, 215 pp.

Tagore, Rabindranath (1917), Nationalism, London: MacMillan, 135 pp., seconda edizione 1918: https:/www.gutenberg.org/files/40766/40766-h/40766-h.htm

Tagore, Rabindranath (1923), Nazionalismo, Lanciano: Carabba, V-139 pp.

Tagore, Rabindranath (2012a), Nacionalismo, Madrid: Taurus, 102 pp.

Tagore, Rabindranath (2012b), El nacionalismo en Japón, in: Tagore, Rabindranath, Nacionalismo, Madrid: Taurus, 7-41

Takada, Yasumasa, Aкinisa Ota [redazione del Yomiuri Shimbun] (2018, March 26), LPD quest to revise top law buffeted by Moritomo scandal, in: "The Japanese News" by Yomiuri Shimbun, Monday, 3

ТАкеdA, Kiуоко (1988), The Dual-Image of the Japanese Emperor, London: MacMillan, $183 \mathrm{pp}$.

Takemae, Eiji (2002), The Allied Occupation of Japan, New York: Continuum, XLV-751 pp.

Takeuchi, Keirchi (2004), La politica dell'identità nazionale nel Giappone moderno: analisi dei manuali di geografia, in: Tesoro, Marina (ed.), Monarchia, tradizione, identità nazionale, 215-224

Tanaka, Hideo (1987), The Conflict between Two Legal Traditions in Making the Constitution of Japan, in: Ward, Robert E., Sakamoto Yoshikazu (eds.), Democratizing Japan, 107-132

Taylor, Telford (1970), Nuremberg and Vietnam. An American Tragedy, Chicago: Quadrangle Books, 224 pp.

Tesoro, Marina (ed.) (2004), Monarchia, tradizione, identità nazionale. Germania, Giappone e Italia tra Ottocento e Novecento, Milano: Bruno Mondadori, 233 pp.

Tesoro, Marina (2004), Introduzione, in: Tesoro, Marina (ed.), Monarchia, tradizione, identità nazionale, 1-6

Tolzmann, Don Heinrich (ed.) (1995-1998), German-Americans in the World Wars, München: Saur, 5 voll.

Tönnies, Ilse (ed.) (1950), Beiträge zur Gesellungs- und Völkerwissenschaft. Professor Dr. Richard Thurnwald zu seinem 80. Geburtstag gewidmet, Berlin: Mann, 477 pp.

Toscano, Mario (ed.) (1988), L'abrogazione delle leggi razziali in Italia, 1943-1987. Reintegrazione dei diritti dei cittadini e ritorno ai valori del Risorgimento. Prefazione di Giovanni Spadolini, Roma: Bardi, 265 pp.

Tosi, Rosanna (1999), La guerra: il dovere di ripudiarla e l'interesse ad evitarla, in: Dogliani, Mario, Stefano Sicardi (eds.), Diritti umani e uso della forza, 113-116

Trento, Francesco (2016), La guerra non era finita. I partigiani della Volante Rossa, Roma - Bari: Laterza, V-199 pp.

Trials of War Criminals Before the Nuernberg Military Tribunals under Control Council Law no. 10 (1949-1953), Washington (DC): U.S. Government Printing Office, 15 voll. 
Trindade, Hélgio (1974), Integralismo. O fascismo brasileiro na década de 30, Porto Alegre - São Paulo: Univeridade Federal do Rio Grande do Sul - Difusão Européia do Livro, 388 pp.

Truzzi, Silvia (2018, 15 aprile), L'articolo 11 e i limiti alle guerre "travestite", in: Il Fatto Quotidiano, 5

U.S. Department of Justice (2001, novembre), Report to the Congress of the United States: A Review of the Restrictions on Persons of Italian Ancestry During World War II, Washington, D.C., IX-44 pp.

Ugai, Nobushige, Herbert Zachert (1964), Ideal und Realität der japanischen Verfassung, in: Aus Politik und Zeitgeschichte, B 40-41/64, 3-7

Ugai, Nobushige, Herbert Zachert (eds.) (1964), Die japanische Verfassung, in: Aus Politik und Zeitgeschichte, B 40-41/64, 24 pp.

Umeda, Kinji (1997a), Sengo minshu-shugi undo no nagare to Nihon koku-kempo no seitei [Lo sviluppo del movimento democratico nel dopoguerra e la promulgazione della costituzione giapponese], in: Rekishi Kyoikusha Kyogikai [History Educationalist Conference of Japan], Nibonkoku-kenpo wo kokumin wa do mukaetaka [Come i giapponesi hanno percepito la Costituzione], Tokyo: Kōbunken [s.p.]

UMEDA, KINJI (1997b), Ima manabitai kingendai rekishi [La storia contemporanea che si vuole conoscere], Tokyo: Kyoikushiryo editore, 397 pp.

Ungaretti, Giuseppe (2015), Da una lastra di deserto. Lettere dal fronte a Gherardo Marone. A cura di Francesca Bernardini Napoletano, Milano: Mondadori, XXXI, $264 \mathrm{pp}$.

Unità di tutto il popolo contro la C.E.D. e il riarmo tedesco. Per la pace e l'indipendenza nazionale (1952?), in: Tribuna della Pace, supplemento al n. 2, anno $4^{\circ}$

Urach, Albrecht von (1940), Ostasien. Kampf um das kommende Großreich, con 35 fotografie, Berlin: Steiniger, $191 \mathrm{pp}$.

Urach, Albrecht von (1942), Das Geheimnis japanischer Kraft, Berlin: Zentralverlag der NSDAP, 95 pp.

Urach, Albrecht von (1943), Die Neuordungsprobleme Japans und Deutschlands, in: Donat, Walter et al., Das Reich und Japan, 188-211

Vagnini, Alessandro (2015), L'Italia e l'imperialismo giapponese in Estremo Oriente. La missione del Partito Nazionale Fascista in Giappone e nel Manciukuò, Ariccia: Aracne, 249 pp.

Vannuccini, Vanna, Francesca Predazzi (2019), Piccolo viaggio nell'anima tedesca, 12a edizione, Milano: Feltrinelli, $144 \mathrm{pp}$.

VARI, Filippo (1999), La "vecchia” Costituzione e la "nuova" guerra: breve analisi della "crisi del Kosovo", in: Dogliani, Mario, Stefano Sicardi (eds.), Diritti umani e uso della forza, 117-137

Venturini, Maria (1953), Per non tornare indietro! Contro il riarmo della Germania, Roma: Associazione Nazionale Perseguitati Politici Italiani Antifascisti (A.N.P.P.I.A.), 70 pp.

Vercelli, Claudio (2013), Il negazionismo. Storia di una menzogna, Roma - Bari: Laterza, XI-216 pp. 
Vicentini, Luigi (1924), Mussolini veduto dall'estero, Milano: Barion Editore, 159 pp. Vinciguerra, Mario (1947), Un equivoco, in: Il Ponte, 3:11-12, 1116-1121

Visco, Antonio (1943), La riparazione dei danni di guerra alla popolazione civile, Milano: Bocca, 297 pp.

Vollnhals, Clemens (1989), Entnazifizierung und Selbstreinigung im Urteil der evangelischen Kirche. Dokumente und Reflexionen 1945-1949, München: Kaiser, $243 \mathrm{pp}$.

Vollnhals, Clemens (ed.) (1991), Entnazifizierung. Politische Säuberung und Rehabilitierung in den vier Besatzungszonen 1945-1949, München: DTV, 359 pp.

Vor 25 Jahren: Bundesverfassungsgericht billigt Auslandseinsätze (2019, 8 luglio), Bundeszentrale für politische Bildung, da http:/www.bpb.de/politik/hintergrund-aktuell/188072/20-jahre-parlamentsvorbehalt-10-07-2014

WAHL, RaIner (ed.) (2008), Verfassungsänderung, Verfassungswandel, Verfassungsinterpretation. Vorträge bei Deutsch-Japanischen Symposien in Tokyo 2004 und Freiburg 2005, Berlin: Duncker \& Humblot, 508 pp.

Ward, Robert E., Frank Joseph Shulman (1974), The Allied Occupation of Japan 1945-1952. An Annotated Bibliography of Western-Language Materials, Chicago: American Library Association, XX-867 pp.

Ward, Robert E., Sakamoto Yoshikazu (eds.) (1987), Democratizing Japan. The Allied Occupation, Honolulu: University of Hawaii Press, XV-456 pp.

Watanabe, Harumi (2010), Come condividere in modo razionale la memoria dei fatti storici. Considerazioni sul massacro di Nanchino, in: Contini, Giovanni et al. (eds.), Memoria e rimozione, 109-118

Weber, Petra (2000), Justiz und Diktatur. Justizverwaltung und politische Strafjustiz in Thüringen 1945-1961, München: Oldenbourg, XII-524 pp.

Wehberg, Hans (1953), Krieg und Eroberung im Wandel des Völkerrechts, Frankfurt a.M.: Metzner, 134 pp.

Weiler, Berndt (1999, 7 agosto), Kobayashi - und wie er die Welt sieht. Ein Manga-Zeichner läuft Sturm gegen Kritiker aus Deutschland und präsentiert seine Sicht der japanischen Vergangenheit, in: Die Welt, 16

Weis, Julia (2009), Karl Jaspers: Freiheit und Wiedervereinigung, in: Fischer, Torben, Matthias N. Lorenz (eds.), Lexikon der "Vergangenheitsbewältigung" in Deutschland, 156-157

Welch, David (2001), Propaganda and the German Cinema 1933-1945, London: Tauris, X-352 pp.

Weniger, Erich (1949), Neue Wege im Geschichtsunterricht, Frankfurt a.M.: SchulteBulmke, 106 pp., prima edizione 1946

Werte, Gerhard (2001), Die Bestrafung von NS-Unrecht in Westdeutschland, in: MarXen, Klaus et al. (eds.), Der Umgang mit Kriegs- und Besatzungsunrecht in Japan und Deutschland, 137-157

Wetzler, Peter (1989), Kaiser Hirohito und der Krieg im Pazifik. Zur politischen Verantwortung des Tenno in der modernen japanischen Geschichte, in: Vierteljahrshefte für Zeitgeschichte, 37:4, 611-644 
Whitney, Courtney (1956), MacArthur, his Rendez-Vous with History, New York: Knopf, XI-547-XIII pp.

Wide-mouthed frog (2013, 20 agosto), in: The Economist, da https:/www.economist. com/asia/2013/08/10/wide-mouthed-frog

Winkler, Christian G. (2011), The Quest for Japan's New Constitution. An Analysis of Visions and Constitutional Reform Proposals 1980-2009, London: Routledge, XXI-215 pp.

Winstel, Tовіаs (2006), Verhandelte Gerechtigkeit. Rückerstattung und Entschädigung für jüdische NS-Opfer in Bayern und in Westdeutschland, München: Oldenbourg, $426 \mathrm{pp}$.

Wolfder (1934), Il riarmo della Germania e le moderne forze militari, in: Rassegna italiana, 193, 6 pp. (estratto)

Wolfe, Robert (ed.) (1984), Americans as Proconsuls. United States Military Government in Germany and Japan, 1944-1952, Carbondale: Southern Illinois University Press, XXVI-563 pp.

Woller, Hans (1996), Die Abrechnung mit dem Faschismus in Italien 1943 bis 1948, München: Oldenbourg, VI-436 pp.

Wrobel, Hans (1989), Verurteilt zur Demokratie. Justiz und Justizpolitik in Deutschland 1945-1949, Heidelberg: Decker und Müller, XII-400 pp.

Wuessing, Fritz et al. (1951), Wege der Völker. Geschichtsbuch für deutsche Schulen, Berlin - Hannover: Berthold Schulz

Wyman, Mark (1998), DPs: Europe's Displaced Persons. 1945-1951, Ithaca - London: Cornell University Press, $257 \mathrm{pp}$.

Yagyu, Kunichika (2003), Der Yasukuni-Schrein im Japan der Nachkriegszeit. Zu den Nachwirkungen des Staatsshinto, in: Cornelissen, Christoph et al. (eds.), Erinnerungskulturen, 243-253

Yamaguchi, YASushi (1976), Faschismus als Herrschaftssystem in Japan und Deutschland. Ein Versuch des Vergleichs, in: Geschichte in Wissenschaft und Unterricht, 27, 89-99

Yano, Hisashi (2010), I lavoratori forzati delle colonie giapponesi. Un confronto con il caso tedesco, in: Contini, Giovanni et al. (eds.), Memoria e rimozione, 43-54

Yasumasa Takada, Aкinisa Ota [redazione del "Yomiuri Shimbun"] (2018, 26 marzo), LPD quest to revise top law buffeted by Moritomo scandal, "The Japanese News" by Yomiuri Shimbun, 3

Yosano, Акiko, Suikei Maeta, Nobutsuna Sasaki, Tekkan Yosano (1917), Poesie giapponesi, traduzione di Harukichi Shimoi e Gherardo Marone, Napoli: Ricciardi, 78 pp., seconda edizione, arricchita di nuovi autori: Lirici giapponesi (1927), scelti e tradotti da Harukichi Scimoi e Gherardo Marone, Lanciano: Carabba, XIII-103 pp.

Yoshimi, Yoshiaki (2003), Das Problem der "Trostfrauen", in: Richter, Steffi, Wolfgang Höpken (eds.), Vergangenheit im Gesellschaftskonflikt, 97-117

Zachert, Herbert (1950), Shinto und Staatsführung im neuen Japan, in: TönNies, ILSE (ed.), Beiträge zur Gesellungs- und Völkerwissenschaft. Professor Richard Thurnwald zu seinem 80. Geburtstag gewidmet, Berlin: Mann, 463-468 
Zambarbieri, Annibale (2004), Una doppia fedeltà. I cattolici giapponesi tra culto imperiale e culto cristiano, in: Tesoro, Marina (ed.), Monarchia, tradizione, identità nazionale, 157-182

Zimmermann, Clemens (2007), Medien im Nazionalsozialismus. Deutschland, Italien und Spanien in den 1930er und 1940er Jahren, Köln: Böhlau, 316 pp.

Zimmermann, Hannah (2012), Geschichte obne Zeitgeschichte. Eine Untersuchung von zwei Jahren Geschichtsunterricht in den frühen 1960er Jahren, München: Utz, $162 \mathrm{pp}$.

Zorn, Philipp (1913), Friedens- und Kriegsbündnisse. Die internationale Schiedsgerichtsbarkeit. Die Idee des ewigen Friedens, in: Anschütz, Gerhard (ed.), Handbuch der Politik, vol. 2, Berlin - Leipzig: Rothschild, 795-803, ristampa: Die internationale Schiedsgerichtsbarkeit (1917), in: Das Recht, 5-20 (come estratto: Hannover 1917: Helwing) 


\section{Indice analitico}

Questo indice analitico rinvia alle principali parole-chiave contenute nel testo, omettendo quelle pressoché onnipresenti, come ad esempio i nomi dei tre Stati dell'ex Asse. Inoltre non sono incluse le parole-chiave contenute nei titoli di libri e articoli.

La parola-chiave si trova alla pagina indicata dal numero che la segue (p. es.: "Abissinia, 75."); se essa si trova soltanto nella nota a piè di pagina, il numero è seguito da “n." (p. es.: “111 n.”); se essa si trova tanto nella pagina quanto in nota, il numero è seguito da "e n.” (p. es.: "111 e n.”); se essa è presente anche nella pagina contigua, sia nel testo sia in nota, il numero è seguito da "s." (p. es.: "111 s."); se essa è presente in più di due pagine contigue, sia nel testo sia in nota, viene indicata la pagina iniziale e quella finale (p. es.: "111-114"). Se due o più termini sono sinonimi o correlati, si rinvia al lemma principale con l'indicazione "vedi" (p. es.: "Cartelli monopolistici, vedi Konzern; Zaibatsu, in Giappone."). Se un termine è concettualmente collegato con uno o più altri termini, si rinvia all'altro termine (o agli altri termini) con l'indicazione "vedi anche" (p. es. "Caso Auerbach, 158; vedi anche: Antisemitismo.").

I segni speciali - p. es. $\ddot{a}, c ̧, \breve{s}$ e simili - vengono inseriti nella sequenza dei normali caratteri alfabetici.

Abissinia, 75.

Acri, 279.

Adua, 274.

Aeroplano, aereo, -i, 16, 20-22, 51 s., 120 e n., 137 s., 165, 182, 190, 213, 231, 236, 241, 243, 270 s., 274,

278-280, 332.

- di Gabriele D’Annunzio, 271 e n. Vedi anche: Aviazione (Aeronautica); "Baade 152".

Afghanistan, 82, 164 s., 208, 232.

Agra, $276 \mathrm{~s}$.

Aia, L', 79, 190, 248, $312 \mathrm{~s}$.

Alalà, grido di battaglia, 24, 281, 283.

Albania, 54, 75 s., 112, 114.

Algeri, 55.

“Allied Council for Japan”, 175.

"Allied Military:

- Currency" (AM-Lire), $56 \mathrm{n}$.

- Government of Occupied Territories” (AMGOT), $55 \mathrm{~s}$.
Alitalia, primo volo postbellico, 193. Amburgo, 320.

America Latina, 7, 35.

AMGOT, vedi "Allied Military Government of Occupied Territories".

AM-Lire, vedi "Allied Military Currency".

Analfabetismo spirituale, e democrazia, 148, 153.

Anschluss, $9 \mathrm{~s}$.

Antifascismo, antifascista, -i, 80, 86, 88, 90, 92, 127, 151, 160, 194, 201 s., 205 s., 280, 295 s.; vedi anche: Resistenza.

Antisemitismo, 81, 157 s.; vedi anche: Caso Auerbach; Razza.

Approvvigionamento, 117, 253:

- petrolifero, 51, 189.

Vedi anche: Cibo.

Arbitrato internazionale, 190, 310, 325. 
Arditi, corpo militare, 18, 20 n., $275 \mathrm{n}$.

Argentina, 272.

Arma, -i, 39, 42, 52, 90, 165, 184, 195, 203, 212, 219, 226, 306, 317, 319 s., 322-325:

- atomica, vedi Atomica, bomba; Bombardamento, atomico.

- chimica, 77, 166, 329.

- produzione di, 227-229, 305, 311, 314, 319.

Vedi anche: Guerra, batteriologica; Missile; "Unità 731".

Armata Popolare Nazionale, vedi Volksarmee, Nationale (NVA, DDR).

Arruolamento, in eserciti stranieri, 303, 305, 318, 320 n., 324.

"Ashida Amendment", revisione costituzionale, $179 \mathrm{~s}$.

Asse, 15, 105, 163, 196, 199; vedi anche: Patto d'Acciaio; Patto Tripartito.

Assia, costituzione dell' -, 315 e n., 322 e $n$.

Atomica, -he, bomba, -e, 2, 42, 45, 83 s., 189, 191, 201, 210 s., 284 s., 291 e n., 329:

inventario mondiale delle -, $285 \mathrm{n}$.

Auschwitz, lager, 159 s., 162.

Austria, austriaco, -i, 9, 112, 105, 112, 219, 259, 311 n.; vedi anche: Austroungarico, Impero.

Austro-ungarico, Impero, 9, 281; vedi anche: Austria.

Autodifesa, 179, 182, 187 s., 320 n.:

- collettiva, 61, 187 s., 259, 269;

vedi anche: Sicurezza collettiva.

- individuale, 61, 186.

Vedi anche: Forze di Autodifesa

(Giappone).

Aviano, base aerea, 279 n.; vedi anche: Comina, La.

Aviazione (Aeronautica), 20 s., 120, 241 s., 271 n., 275, 279 s.; vedi anche: Aeroplano.
"Baade 152", aereo, 120.

Baden-Württemberg, vedi Württemberg e Baden.

Bakufu, 172; anche: Governo militare.

Base, -i, militare, -i, 60, 138.

Basilea, 156.

Basra (Bassora), 275.

Batavia, 72.

Battaglione Verde, pseudo-partigiani, $88 \mathrm{~s}$.

Baviera, 9, 54, 98 n., 126, 194, 221 s., 305.

BCOF, vedi "British Commonwealth Occupation Force".

Belgio, 59 n., 221.

Benares, 277.

Bengasi, 274.

Berlino, 32, 37 s., 58 s., 94, 125, 220 n., 277, 295, 304 s., 320, 330, 332: muro di -, 59, 120 n., 211, 331.

Berna, $86 \mathrm{n}$.

Berufsverbot, interdizione professionale, $220 \mathrm{n}$.

Birmania, 58.

Bologna, $285 \mathrm{n}$.

Bolscevismo, 26, 33, 39 e n.; vedi anche: Comunismo.

Bombardamento, -i, 15-17, 112, 133 n., 166, 193 s.:

- atomico, 45; vedi anche: Atomica, bomba.

Bonn, 230, 305.

Brandenburgo, 199.

Brasile, 21, 25, 88, 272 e n. volo fino al,- 21 e n.

Briga e Tenda, cessione di -, 58 .

Brindisi, 195.

"British Commonwealth Occupation Force" (BCOF), $46 \mathrm{~s}$.

Brunei, 190 e n.

Bundesgrenzschutz (Germania Federale), 181 n.; vedi anche: Riarmo.

Bundespolizei (Germania Federale), 181 n.; vedi anche: Riarmo. 
Bundeswehr (Germania Federale), 181 n., 225 n., 230, 232 e n., 302, 304, 331 e n.:

- come "armata mercenaria”, $331 \mathrm{n}$. Vedi anche: Riarmo; Wehrmacht, abolizione della -.

Bulgaria, 107, 201, 281.

Burma, 109.

Bushido, 8, $17 \mathrm{n}$.

Byakkotai ("Tigre Bianca"), 24 s.; vedi anche: Tokyo, colonna romana.

Calcutta, 276.

Cambogia, 77, 109, 186, 229 s.

Camera:

- dei Fasci e delle Corporazioni, 54.

- del Popolo (DDR), $328 \mathrm{n}$.

Camicie:

- Nere, 11, 24, 88; vedi anche: Fascismo.

- Verdi, 88 s.; vedi anche: Integralismo.

Campo, -i, di concentramento, 35, $155,159,161$ : religione nei,$- \quad 197 \mathrm{~s}$.

Canada, 71, $108 \mathrm{n}$.

Canton (Cina), 21.

Capitolazione, 45, 69, 82, 137, 154, 161, 236; vedi anche: Resa.

Caporetto, 18, 282.

Carnaro, 282 s.:

Carta del -, 39 e n.; vedi anche: Fiume.

Carnia, repubblica partigiana, 146.

Cartelli monopolistici, vedi Konzern; Zaibatsu, in Giappone.

Casa Imperiale (Giappone), 246; vedi anche: Imperatore.

Casale Monferrato, 270.

Caserta, resa di -, 45.

Caso Auerbach, 158; vedi anche: Antisemitismo.

Cassibile, resa o armistizio di -, 44.

Castelfranco, 91.

Cattolicesimo, Cattolico, -i, 8 s., 90 , 152; vedi anche: Cristiano, -i;
Democrazia Cristiana (DC); Papa; Vaticano.

Cecoslovacchia, 92, 123 e n.

Cefalonia, eccidio di -, 196 e n.

Cibo:

"Giornata del" - (Giappone), 253 s. mancanza di -, 48, 128, 249, 251-254.

Cina, $15,30,37,44,51-53,58$, 71-73, 77, 81 n., 108 s., 133, 135 s., 174, 182, 185, 187, 189-191, 201, 206 n., 236 s., 260, 262, 280, 292, 297; vedi anche: Repubblica Popolare Cinese.

Cinecittà (Roma), 14.

Cinegiornale, 149; vedi anche: "Film Luce"; "Settimana Incom"; Cinema.

Cinema (cinematografia), $12 \mathrm{n}$., 14 e n., 143, 327; vedi anche:

Propaganda; Radio.

Cirenaica, 151, 274; vedi anche: Libia.

Classi di valutazione del crimine, 66, 101-103.

Coblenza, 221.

Codice, -i:

- penale, 59, 87, 101, 143, 207, 212 e n., 224, 326.

- - della DDR, $326 \mathrm{~s}$.

Coesistenza pacifica, 295, 302, 308 s., $322,325$.

"Colomba della pace" (Picasso), 201.

Colonia, sinagoga di -, 158.

Colonialismo: $30,74,135,150$ :

- giapponese, 28; vedi anche: "Sfera di Co-prosperità della Grande Asia Orientale".

Colpa, quattro dimensioni della (Jaspers), 155.

Colonialismo, vedi Colonia.

Colonia, -ie, Colonizzazione, 7, 9 s., 28,31 e n., 57 s., 70, 72-75, 114, 132, 206, 248, 260, 290, 297:

- Giappone come potenza anticoloniale, $14,30,135,142-144,150 \mathrm{~s}$. 
Vedi anche: Decolonizzazione; Guerre, di liberazione nazionale.

Comfort women, 73 n., 77, 78 e n., 110 s., 135 s., 143 s.; vedi anche: Indennizzo; Prostituzione coatta; Risarcimento.

Comina, La, scuola di volo, 279 e n.; vedi anche: Aviano.

Cominform, 286, 291.

Comitato, -i:

- "di Liberazione Nazionale" (CLN, Italia), 88, 90, 148 n., 194 e n.

- "Parlamentare per la Sicurezza della Repubblica” (Copasir), $215 \mathrm{s.}$

"Commissariato, Alto, per le Sanzioni contro il Fascismo", 88 e n., 93.

"Commissione Alleata di Controllo" (Kontrollrat), 94, 99-101, $147 \mathrm{n}$.

Common Law, 178, 312.

Comunismo, 4, 59, 91 s., 123 s., 127, 142, 149, 155 n., 176, 194 n., 197, 200 n., 203 e n., 207, 220 e n., 231 n., 250 s., 253 s., 256, 284-289, 291-296, 300 s., 327, 331 n.; vedi anche: Marxismo; Socialismo.

Conferenza:

- dei Ministri dell'Istruzione (Germania), 157 s., $160 \mathrm{~s}$.

- del Cairo, 237 e n., 239.

- dell'Aja, 248.

- delle Sei Potenze, Londra, 221.

- di Parigi, 274 n., 281.

- di Postdam, 107, 235.

- di Yalta, 60.

“Comunità Europea di Difesa” (CED), 285, 304.

Congresso:

- dei Partigiani della Pace, Parigi, 201 s., 287, $302 \mathrm{n}$.

- dei Popoli, Vienna, 201, 287, 289, 297, 300.

- del Partito Liberaldemocratico

(Giappone), 262 s., 267.

Conservatorismo, conservatore, -i, 96, 137 s., 143 s., 171, 202, 265:
- giapponese, 18, 50, 179, 185 s., 249.

“Containment", del comunismo, 184. "Convenzione costituzionale" (Verfassungskonvent), 222, 305, 309.

Copasir, vedi "Comitato Parlamentare per la Sicurezza della Repubblica”.

"Co-prosperità", vedi "Sfera di Co-prosperità della Grande Asia Orientale”.

Córdoba (Argentina), 115.

Corea, 2, 31, 57 s., 79, 81, 109, 135, 144, 173, 176, 181, 201, 237 n., 248, 260, 285, 291, 297:

- del Nord, 44, 52 s., 185, 189, 191, $261 \mathrm{~s}$.

- del Sud, 51, 136, 187.

“Corpo Volontari della Libertà, organizzazione antifascista, $148 \mathrm{n}$.

Corte (o Tribunale):

- Costituzionale:

--(Germania), 124, 127, 157, 229-232.

--(Giappone), 166 n., 181-184.

--(Italia), 116 n., 209.

- di Giustizia Europea, 304.

- Penale Internazionale, 215 e n.

- Permanente di Arbitrato sulla Legge del Mare, 190.

- Suprema, degli USA, 79.

Coscrizione obbligatoria, 195, $211 \mathrm{e}$ n., 260; vedi anche: Obiezione di coscienza; Servizio militare.

Costa Rica, 40 e n.

Costituzione, -i, 2:

- della Repubblica Spagnola, 38 e n., 208.

- di Costa Rica, 40 n.

- italiana, 41 e n., 56 n., 179, 193, 204-209; vedi anche: Statuto Albertino.

- Meiji, 7 n., 32, 43, 48 s., 54, 170, 174, 176 s., $256 \mathrm{n}$.

- postbelliche, 1-4, 38, 40.

- tedesca, vedi Legge, Fondamentale (GG, Germania). 
Vedi anche: Repubblica di Weimar.

Cremona, 12.

Crimea, annessione della -, 225.

Crimine, -i, 224, 315:

- contro l'umanità, 143 n., 215.

- delle dittature, 3, 162.

- di guerra, 11, 44, 63-89, 100,

128, 144, 151, 172, 174, 177, 215,

235, 237 s., 242, 251, 253.

valutazione del - (cinque classi), 66, 101-103.

Cristianesimo, cristiano, -i, 7 s., 97, 221, 286:

- e libri scolastici, 139.

Vedi anche: Cattolicesimo;

Democrazia Cristiana (DC);

Vaticano.

“Croce Rossa Italiana”, 2 n.

Croci Frecciate, Ungheria, 88.

Cuba, crisi di -, 166.

Cyberwar, 53; vedi anche: Guerra, ibrida.

Dalmazia, 114.

DDR, vedi Repubblica Democratica Tedesca.

Debito, -i, di guerra, 119, 121; vedi anche: Riparazione, -i.

Decolonizzazione, 135, 206 e n.; vedi anche: Colonia; Guerre, di liberazione nazionale.

Defascistizzazione, 80,147 n., 149 s.; vedi anche: Epurazione.

Delhi, 275.

Democristiano, -i, 86, 93, 116, 147, 207; vedi anche: Partito, Democrazia Cristiana (DC).

Demontage, 107 e n., 117 s., 121; vedi anche: Riparazione, -i.

Denazificazione, 58 n., 60 s., 61 n., 80 s., 94-105, 155:

- e ri-nazificazione, $97 \mathrm{~s}$.

Vedi anche: Epurazione;

"Spruchkammer".

Desistenza e Resistenza, 93.
Detenzione, 89, 92, 194, 224, 329:

- (Gefängnis) nella DDR, 328 s., 333; vedi anche: Prigione o Penitenziario (Zuchthaus) nella DDR.

Diaoyu, isole, 51, 191; vedi anche: Senkaku.

"Diciotti”, nave con migranti, 92.

Direttiva (n.24 e n. 38) e denazificazione, 100 .

Diritto, -i:

- alla vita, 321 e n.

- fondamentali, 46, 157, 221, 237, 240, 307, 308 s., 311, 319, 321.

- internazionale, 59-61, 71, 202, 204, 209 s., 223, 225 s., 306-313, 325 s., 329, 331.

-- e consuetudine, 209 e n., 313.

- - e diritto nazionale, 312, 325.

- naturale e - positivo, $78 \mathrm{~s}$.

Vedi anche: Common Law.

Disarmo, 61, 179, 207, 217, 220, 235, 238, 241; vedi anche: Pace; Pacifismo.

Displaced persons, vedi Profugo.

Dittatura, -e, 3, 8, 11-15, 33, 37 n., 63 s., 157, 248 n.; vedi anche: Fascismo;

Nazionalsocialismo; Stalinismo.

Divinità, della dinastia imperiale

(Giappone), 10 s., 43, 69, 133, 170 s., 179, 255; vedi anche: Imperatore, del Giappone.

Dodecaneso, 57.

Dolchstosslegende, vedi "Leggenda della pugnalata alle spalle".

Donna, -e, 143 n., 304:

- "delle macerie", 17.

- e Costituente italiana, 203 e n.

- "Enfranchisment of women"

(Giappone), 173.

- nelle dittature, 13 s., 155.

Tribunale Internazionale delle -, $143 \mathrm{n}$.

Vedi anche: Comfort women;

"Frauenbund, Berliner"; Stampa femminile.

Drone, -i, 213 e n.; vedi anche: Guerra, ibrida. 
Dual:

- States, 11.

- use, 227.

Duce, 13; vedi anche nell'Indice dei nomi: Mussolini.

- della Romania, 89.

Ebrei, 81, 108, 115 e n., 119, 122 s., 125 s., 157 s., 176 n.:

- in Giappone, 81 e n.

Vedi anche: "Manifesto della razza"; Questione ebraica; Razza; Tribunale della Razza.

Educazione civica, insegnamento dell' -, 137, 159.

Egeo, mare, 55, 114, 196, $273 \mathrm{n}$.

Eja, vedi Alalà, grido di battaglia.

Elba, isola, $138 \mathrm{n}$.

Emirati Arabi, 213.

Epurazione, -i, 3, 49, 58 n., 63 s., 68 e n., 79 s., 82-91, 93-95, 100, 102 s., 105 s., 116, 125, 128-130, 147, 156, 162, 173:

questionario per l' - , 82, $95 \mathrm{~s}$.

Vedi anche: Defascistizzazione;

Denazificazione.

Erfurt, 120.

Ergastolo, 83, 87, 92 e n., 207, 224, $328 \mathrm{~s}$.

Eritrea, 75, 151, 274.

ERP, vedi "European Recovery Programm".

"Esercito di Transizione" (Italia), $199 \mathrm{~s}$.

Esotismo, 18, 22, 270.

Etiopia, 54, 75 s., 112, $296 \mathrm{n}$.

Eugenetica nazista, 81, 321 e n.

"European Recovery Programm"

(ERP), 50 n.; vedi anche: "Piano

Marshall".

"Far Eastern Commission"

(USA), 108 s., 175, 255.

Fascismo, fascista, -i, 13 s., 17 s., 25 ,

54, 85-88, 92 s., 113, 116, 145-149,

151, 203, 270, 281 e n., 292, 333:

- eterno o Ur-fascismo, 106.
- in Giappone, 11 n., 18, 24 s., 248; vedi anche: Militarismo.

Vedi anche: Antifascismo; Nazifascismo; Nazionalsocialismo; Totalitarismo.

Fedeltà, come valore-guida, 24 s., 27, 82, 87, 113, 145, 152.

Ferrari, prototipo postbellico, 193.

Filippine, isole, 51, 58, 70, 72, $108 \mathrm{~s}$., 190, 281, 283.

"Film Luce", 14; vedi anche: Cinema; Cinegiornale.

Firenze, 55.

Fiume, 18-26, 269 s., 272, 280-283:

- Lega di, 9.

Libera Città di -, 39.

- Questione fiumana, 19 e $\mathrm{n}$.

Vedi anche: Carta del Carnaro.

Foochow (Cina), 21.

Formosa, 57; vedi anche: Taiwan.

Forze di Autodifesa (Giappone), 49, 51, 53, 165, 179 s., 182, 184 s., 189 s., 258 s., 261, 263-265, 268 s.; vedi anche: National Police Reserve.

Fosse Ardeatine, eccidio delle -, 92.

Francia, 7, 51, 55-58, 71 s., 76, 94, 108 n., 123 e n., 166, 201, 213, 221, 226, 248, 276, 279 n., 285, 288, 297, 327.

Francoforte, 159:

- "Frankfurter Dokumente", e costituente tedesca, 221.

Scuola di -, 15, 60, 159.

"Frauenbund, Berliner", 315 e n., 320 e n.; vedi anche: Obiezione di coscienza.

Führer, 13; vedi anche nell'Indice de $i$ nomi: Hitler, Adolf.

Futurismo, futurista, 39 e n., 269 s.

Galeries Lafayette, volo, 276 e n.

Gange, 278.

Gefängnis, vedi Detenzione nella DDR.

Genocidio, 215 n.; vedi anche: Cri-

mine, di guerra.

Genova, $16,52 \mathrm{n}$. 
Geografia, insegnamento della -, 74, 131-133, 149.

Geopolitica, 1, 28 s., 37 e n., 185, 188, 211.

Germania, indeterminatezza del territorio, $303 \mathrm{~s}$.

GG, vedi Legge Fondamentale.

Giaipur (Jaipur), 277.

Gibuti, 51.

"Giornata del cibo" (Giappone), 254; vedi anche: Cibo.

"Gioventù Italiana del Littorio", e scuola, $145 \mathrm{~s}$.

Giumma (Yamuna), fiume, 276 e n.

"Giustizia e Libertà", organizzazione antifascista, 91.

Golan, alture del -, $186 \mathrm{n}$.

Governo militare alleato:

- e Germania, 95, 118.

- e Giappone, 49, 108, 172; vedi anche: Bakufu.

- e Italia, 56, 147.

Gran Bretagna, 7, 15, 58, 71, 75, 81 n., 108 n., 123 n., 128, 132, 166, 221, 248, 327; vedi anche: Regno Unito.

“Gran Consiglio del Fascismo", 54, 194.

Grande Guerra, 18 n., 21, 269; vedi anche: Prima guerra mondiale.

Grappa, battaglia del -, 274.

Grecia, 57, 75 s., 112, 123 e n., 196.

Griglia di valutazione del crimine (cinque classi), 66, 101-103.

"Grundgesetz", vedi Legge, Fondamentale (GG, Germania).

Guam, isola, 58.

Guardia di Ferro (Romania), 88 s.; vedi anche: Integralismo.

Guerra, -e: - batteriologica, 73, 78, $141 \mathrm{n}$., 329; vedi anche: "Unità 731" (Giappone).

- Boshin, 24, $260 \mathrm{n}$.

- civile, 44, 54 s., 72, 92, 153, 194, 233, 302, 314, 327.

- "dei maiali", 281.
- del Golfo, 142, 165, 186, 208, 229.

- del Kosovo, 43 e n., 165, 208-210, 225, 231.

- di Corea, 2 e n., 79, 82, 109, 135, 176, 181, 285.

- di liberazione nazionale, $135 \mathrm{n}$., 142 e n., 206 s., 290; vedi anche:

Decolonizzazione.

- fra Stati capitalisti, 288 s., 299 s.

- fredda, 1, 42, 58, 62, 64, 71, 79, 85, 97 e n., 104, 116, 126 s., 134, 153, 156, 158, 164, 220 s., 284 s., 292, $303 \mathrm{~s}$.

- ibrida, 1, 164 e n., 179, 212, 227; vedi anche: Cyberwar, "Out of area". - partigiana, vedi Partigiano, -i.

Prima - mondiale, vedi Prima guerra mondiale.

- russo-giapponese, 70, 269, 280.

Seconda - mondiale, vedi Seconda guerra mondiale.

- umanitarie, vedi Guerre, ibride.

Guerriglia, 45, 165, 284; vedi anche: Resistenza.

Haiku, uta, vedi Esotismo.

Haiti, $186 \mathrm{n}$.

Hanoi, 276.

Herrenchiemsee, e costituzione tedesca, 222, 303, 305 s., 310 s., 313, 325.

Hiroshima, 16, 42, 169, 210, 249.

Hokkaido, isola, 44, 81 n., 237, 239, 247.

Honshu, isola, 81 n., 237, 239.

Humanitarian warfare, vedi Guerra, ibrida.

IMI, vedi Internati Militari Italiani. Imperatore:

- della Cina, 280.

- del Giappone, 8, 10 s., 24-26, 29, 32, 43 s., 46-48, 69 s., 78 e n., 82, 85, 133, 170 s., 174 s., 179, 194, 235 s., 238, 240 s., 248, 250-251, 255 s., 259-261, 268; vedi anche: 
Divinità, della dinastia imperiale;

Tenno.

Imperialismo, imperialista, -i, 32, 201, 257, 288 s., 290, 292-294,

298-301, $327 \mathrm{~s}$.

Impressionismo (pittura), vedi Esoti-

smo.

Inconscio, e rifiuto della guerra (Giappone), 257-261.

Indennizzo, -i: $35,106,108,113-115$, 123-127, 128, 245:

- 'al contrario', 116.

Vedi anche: Risarcimento.

India, 30 s., 77, 109, 135 n., 142, 206.

Indie Olandesi, 72, 281, 283.

Indocina, 72, 281, 283, 285; vedi anche: Vietnam.

Indonesia, 58, 108 n., 109, 111, $186 \mathrm{n}$.

Industria bellica, 16 s., 39, 107, 181, 226.

Inghilterra, vedi Gran Bretagna.

INSMLI, vedi "Istituto Nazionale per la

Storia del Movimento di Libera-

zione in Italia”.

Integralismo (Brasile), 25 e n., 88.

Internamento, 17, 34-36, 96, 100, 113, 172 n., 197, 311 n.; vedi anche:

Stranieri nemici; "Internati Militari Italiani” (IMI).

"Internati Militari Italiani” (IMI), 196-199; vedi anche: Internamento.

"International Military Tribunal", vedi Norimberga, processo di -; Tokyo, processo di -; Tribunale, militare.

Internazionale, Seconda e Terza, $291 \mathrm{~s}$.

Internet, mass-media, 130, 188:

- e libri scolastici, 162.

Iran, 165, 188.

Iraq, 165 s., 186, 188, 208, 213, 216 s.; vedi anche: Guerra del Golfo.

Ishigaki, isola, 51, 189-191.

Israele, 64, 123, 126, 159.

Istituto:

- “Fritz Bauer”, 162.
- LUCE ('L'Unione Cinematografica Educativa”), 14; vedi anche: Cinema.

- "Nazionale per la Storia del Movimento di Liberazione in Italia” (INSMLI), $147 \mathrm{n}$.

Ius ad bellum, 2, 61, 179; vedi anche:

Sovranità nazionale.

Izumo, portarei “difensiva”, $52 \mathrm{~s}$.

Jaipur, vedi Giaipur.

Japonisme, vedi Esotismo.

"Japonização”, "Japonización” (Porto-

gallo e Spagna), 23 e n.

Judo, arte marziale, 18.

Jugoslavia, 56 s., 75 s., 77, 94, 112, 123

e n., 128, 281, 293 e n.

Kokutai, essenza della nipponicità, 133, 250 e n., 252, 255 s.

Komintern, 142.

Kontrollrat, vedi "Commissione Alleata di Controllo".

Konzerne, cartelli in Germania 173 n., 226; vedi anche: Zaibatsu.

Kos (Coo), eccidio di -, 196 e n.

Kosovo, vedi Guerra del -.

$\mathrm{KPD}$, vedi Partito, Kommunistische Partei Deutschlands.

"Kriegsächtung" (rifiuto), nella costituzione tedesca, 204, 223 e n., 218, 309, 316 e n., 320 e n., 325; vedi anche: "Ripudio", nella costituzione italiana.

Kröpelin (Mecklemburgo), 319 n.

Kurili, isole, 44.

Kyoto, 22, 24, 247, 254.

Kyushu, isola, 81 n., 237, 239.

Lager, vedi Campo di concentramento. Langhe, repubblica partigiana, 146.

Laos, 77, 109.

Lavoro, -i, forzati, 31, 108, 110, 197.

Lealtà democratica, 35,78 : - e diritto al risarcimento, 227. 
Lega:

- delle Nazioni, 61.

- di Fiume, 9.

- Nord, partito (Italia), 89 e $n$.

Legge, -i:

- "a Tutela della Pace" (DDR), 4, 59, 326-330.

- "applicativa dell'art. 26, c. 2 della Grundgesetz”, 228.

- Fondamentale (GG, Germania), 4, 9 s., 41 e n., 61-63, 219, 221-224, 228 s., 233, 302, 305-310, 320 n., 325.

- razziali, 115 s., 125, 175.

- "sul controllo delle armi da guerra" (Germania), 228.

"Leggenda della pugnalata alle spalle" (Dolchstosslegende), 176.

Leningrado, 201.

Libertà, $30,41,57,129,157,193,198$, 202, 204 s., 207, 237, 240, 242 s., 248 s., 291-293, 298, 315, 321 s., 327:

- di espressione, 140.

- di impresa, e riarmo, $227 \mathrm{~s}$.

- provvisoria, 89. Vedi anche: "Corpo Volontari della -"; "Giustizia e -".

Libia, 75, 108, 150-152, 213 e n., 217, 233 e n.; vedi anche: Cirenaica; Tripoli; Tripolitania.

Libri scolastici, $3,63,68,74$, 128-161:

- e approvazione ministeriale, 136, 140.

scandalo dei -, 132.

Vedi anche: Geografia, insegnamento della -; Internet; Scuola; "Visione liberale della storia".

Limitazione, -i, 243, 333:

- di sovranità, $40 \mathrm{s.}$, 56, $163 \mathrm{~s}$., 166 n., 193, 202, 205, 208 s., 230, 232 s., 307-309, 320, 325.

Linea Gotica, 55.

Livorno, 55.
"Londoner Beschlüße", e federalismo tedesco, 221.

Londra, 30, 221: Patto di -, 19 e n. Trattato di -, 121.

Ludwigsburg, Ufficio per il perseguimento dei crimini nazisti, 67 e $\mathrm{n}$.

Lussemburgo, 59 n., 123n, 221.

Macao, $206 \mathrm{n}$.

Madama Butterfly (opera), 25; vedi anche: Fedeltà.

"Magic", apparecchio decrittatore, 15 e $\mathrm{n}$.

Malesia, 58, 70, 190, 297.

Malta, isola, 272.

Manciukuò, 30, 38 n., 109, 249; vedi anche: Manciuria.

Manciuria, 57 s., 69, 169, 171, 237 n.; vedi anche: Manciukuò.

Mandalay, 276.

Manga, fumetti, 143-145.

Manifesto:

- degli intellettuali antifascisti, 280.

- dei Futuristi, 39 e n.

- del Congresso della Pace, 201.

- "del Partito Comunista", 198.

- “dell'Arrogantismo” (Giappone), 145.

- "della Razza”, 54, 116; vedi anche: Ebrei; Questione ebraica; Razza; Tribunale della Razza.

Manuali scolastici, vedi Libri scolastici.

Marbury vs. Madison (USA), 182; vedi anche: "Suzuki Decision".

Marcia su Roma, 19, 54, 87; vedi anche: Fascismo.

Marxismo, 50, 288; vedi anche: Comunismo; Socialismo.

Mass-media, vedi Internet.

Matsumoto Draft, revisione costituzionale, 178.

Mauthausen, lager, 197.

Mecklemburgo, parlamento giovanile del,$- \quad 319 \mathrm{~s}$. 
Meiji, epoca (era), 29, 43, 47 s., 70 n., 81, 131 e n., 144, 169, 172, 260; vedi anche: Costituzione, Meiji.

Mennoniti, e obiezione di coscienza, 319.

Mesopotamia, 278.

Milano, 16 e n., 19, 93, 145 n., 296.

Militarismo, 169 n., 333:

- americano, 305, 324.

- giapponese, 8, 15, 26 s., 44, 46, 61, 81, 85, 97, 101, 133, 164, 169 s., 170, 236, 239 s., 242.

- tedesco, 327, 329, 331.

Ministry of Defense (Giappone), 184.

Missile, -i, 51 s., 62, 166, 184 s., 189 s., 190 s.; vedi anche: Arma.

Missioni di pace, vedi Guerre, ibride.

Modernizzazione del Giappone, 10, 132, 283; vedi anche: "Japonização".

Monferrato, repubblica partigiana, 146.

Monselice, $275 \mathrm{n}$.

Moritomo, caso (o scandalo), 262-267.

Mosca, 101, 118, 201 n., 255, 291.

"Mostra Internazionale d'Arte Cinematografica" (Venezia), 14; vedi anche: Cinema.

Mozambico, 186.

“Naganuma Case”, e articolo 9 (Giappone), 184 s.; vedi anche: "Sunagawa Case", "Suzuki Decision".

Nagasaki, 16, 42, 169, 210, 249.

Nagoya, 36, 252.

Nanchino, massacro di -, 69 e n., 73, 82, 136, 141 e n., 143.

Napoli, 20:

- (Regio) Istituto Orientale, $18 \mathrm{s.}$, $26 \mathrm{e} \mathrm{n}$.

National Police Reserve (Giappone), 49 s., 84, 180-182, 184; vedi anche: Forze di Autodifesa.

Nationalsozialistische Deutsche Arbeiterpartei, NSDAP, 100, 157; vedi anche: Nazionalsocialismo; Partito nazionalsocialista.
NATO ("North Atlantic Treaty Organization”), 43, 52 n., 62, 152, 163-166, 201, 208 s., 220, 229-231, 285, 302, $331 \mathrm{n}$.

Nazi-fascismo, 86, 90, 292; vedi anche: Nazionalsocialismo; Fascismo.

Nazionalsocialismo (nazionalsocialista, -i), 9, 11, 14-16, 25-29, 33, 35, 44, 60 s., 64 s., $67,74,81,88,94-105,112,117$, 119, 122-130, 142, 154-162, 194, 197 s., 236 n., 250 n., 311 n., 314, 318 s., $321 \mathrm{n}$.

Vedi anche: Fascismo, Militarismo, Nazi-fascismo; Terzo Reich; Totalitarismo.

Nazioni Unite (ONU), 2, 43, 61, 76, 153, 165 s., 182 s., 186, 208 s., 210 n., 213, 224-226, 230, 239 s., 242, 248, 262, 297, $308 \mathrm{n}$.

Nazismo, nazista, -i, vedi Nazionalsocialismo.

Negazionismo:

- dell'Olocausto, 143 s., 161.

- in Giappone, 73, 143.

Nepal, $186 \mathrm{n}$.

"Neues Ökonomisches System" (NÖS), $121 \mathrm{n}$.; vedi anche: Trattato economico URSS-DDR.

Neutralità, 8, 207.

New York, 176.

Norimberga, processo di -, 3, 64-69, 73 s., 76-79, 101, 162, 196 n., 307.

Norvegia, 123 e n.

Notte dei Cristalli, 160.

Nuova Zelanda, 71, $108 \mathrm{n}$.

NVA (Nationale Volksarmee, DDR), vedi Armata Popolare Nazionale.

Obiezione di coscienza, 211, 304, 315-318, 320 n.; vedi anche: Coscrizione obbligatoria; Servizio militare.

Oder-Neisse, linea, 58.

Okinawa, 16, 137 s., 191, 247, 249: - e "suicidio di massa", 141 e n. 
Olanda, 59 n., 71 s., 108 n., 123 n., 165, 221.

Olocausto, 143, 158-162, $321 \mathrm{n}$.

ONU, vedi Nazioni Unite.

Opere d'arte, sottratte, $122 \mathrm{~s}$.

“Ordinanza 110" e denazificazione, 99.

"Organization for the Prohibition of Chemical Weapons”, 166.

"Organizzazione delle Nazioni Unite", vedi Nazioni Unite.

Osaka, 267.

“Ospedale 68" (Corea), 2 e n.

Ossola, repubblica partigiana, 146.

"Out of area”, 209, 214, 225, 229 s., 232 n.; vedi anche: Guerra, ibrida.

Outa, uta, haiku, vedi Esotismo.

Pace, 2, 4 s., 38-42, 53, 56 s., 59, 63, 67, 86, 107 s., 112, 128, 137, 161, 169, 174, 180, 193, 200, 203, 207, 210, 214 n., 217, 220, 225, 227 s., 236, 238 s., 240, 244, 245, 248, 257, 260, 269-274, 284-315, 320 s., 325-333: - come dono alla comunità, 261 . mantenimento della -, 56, 101, 153, 163, 182 s., 201 s., 205, 211 s., 223. missione, -i, di -, 1, 187, 208, 230, 232 e n.

- separata (Italia), 194; vedi anche: Armistizio.

trattato di -, 40, 45, 67, 72, 76, 83, 107, 109, 200, 212.

Vedi anche: "Colomba della -";

Partigiani della -.

Pacifismo, 3, 153, 164, 174, 186, 189, 202, 219, 223, 227, 257, 286,

290-294, 315, 326:

- giuridico, 40 e n.

- verbale, Giappone, 171.

Vedi anche: Disarmo.

Paesi capitalisti, vedi Stati capitalisti.

Palazzo del Cinema (Venezia), 14; vedi anche: Cinema.

Palermo, $55 \mathrm{n}$.

Papa, 8, 277 n.; vedi anche: Cattolicesimo.
Papua Nuova Guinea, 58.

Paracelso, isole, $190 \mathrm{n}$.

"Parlamentarischer Rat" (consiglio parlamentare), 305 .

Partigiano, -i, 55, 85, 88, 90-92, 94, 113, 115, 146 e n., 194-196, 203.

- della Pace, 4, 59, 201 s., 220, 284-301, 326.

- e pseudo-partigiani, 88.

Vedi anche: Resistenza.

Partito:

- Alternative für Deutschland (AfD), 105.

- Comunista, 124, 127, 155, 200 n., 207, 219, 253 s., 294 n., 302.

-- Giapponese, 176, 250 s., 253 s., 256.

-- Italiano, 91 n., 149, 194 n., 197, 200 n., 207, 219, 284, 287, 294.

- - Jugoslavo, $293 \mathrm{n}$.

- d'Azione, 92 n., 194 n., 290.

- Democratico del Lavoro (PDL, Italia), $194 \mathrm{n}$.

- Democratico Nazionale del Giappone (CDPJ), $264 \mathrm{~s}$.

- Democrazia Cristiana (DC, Italia), 194 n., 200 n.; vedi anche: Cattolico, -i; Cristiano, -i; Vaticano.

- Deutsche Reichspartei (DRP), 158.

- Imperiale Socialista (SRP), 157.

- Komeito (Partito della Giustizia, Giappone), 188, 258, 267 s.; vedi anche: Sokka Gakkai.

- Kommunistische Partei Deutschlands (KPD), 127, 220 e n.

- - e lealtà democratica, 127.

- "L'uomo qualunque", 86 e n., $206 \mathrm{n}$.

- Liberaldemocratico (LDP, Giappone), 143, 185 s., 189, 233, 258, 262 s., 265-268.

- Liberale Italiano (PLI, Italia), $194 \mathrm{n}$.

- Nazionale Fascista, 12, 54, 146. 
- Nazionalsocialista, 11, 27, 98 s., 102, 157; vedi anche: NSDAP, Nationalsozialistische Deutsche Arbeiterpartei; Nazionalsocialismo.

- neonazista (Nationaldemokratische Partei Deutschlands, NPD), 105.

- Socialdemocratico (SPD, Germania), 221, 230.

- Socialismo, del -, Democratico (PDS, Germania), 231 e n.

- Socialista (PSI, Italia), $200 \mathrm{n}$.

- Socialista di Unità proletaria (PSIUP, Italia), $194 \mathrm{n}$.

- Sozialistische Einheitspartei Deutschlands (SED), 124, $155 \mathrm{n}$., 220, $231 \mathrm{n}$.

Patto:

- Anticomintern, 37, 79.

- Atlantico, vedi NATO.

- Briand-Kellogg, 38 e n., 61, 202 s., 223, 248, 311 e n., 323. - d'Acciaio, 54; vedi anche: Asse.

- Tripartito, 1-3, 8, 25, 30, 32 s., 37 s., 40, 44, 54, 56 s., 73, 80, 108, 128, 169, 171, 177, 194, 196, 233.

Paulskirche, parlamento della -, $61 \mathrm{n}$.

Peace keeping, Peace restoring, vedi

Guerra, ibrida.

Pena:

- detentiva, due tipi, 328 s., 333.

- di morte, 56, 87, 207, 322, 329.

Pesaro, 55.

Pescara, 193, $195 \mathrm{~s}$.

Pescadores, isole, $237 \mathrm{n}$.

"Petersberger Wende", vedi Svolta di Petersberg.

Piano:

- Marshall, 5, 50 n., 119; vedi anche: "European Recovery Programm" (ERP).

- Morgenthau, 117 e n.

- Pauley, $108 \mathrm{~s}$.

Piave, battaglia del -, 274, 276.

Pistoia, 113.

Polizia, -e, e riarmo, vedi Riarmo, e polizie.
Polonia, 10, 54, 58, 123 e n., 201 n., 306.

Portaerei "difensive", $52 \mathrm{s.}$

Portogallo, 7:

- e "japonização", 23 e n.

Potsdam:

Conferenza di -, 107, 235.

- "Declaration", 44, 48, 67 s., 98 s., 100 n., 108, 171, 173, 177 s., 235-238, 256.

--sua interpretazione, 178.

Praga, 92, 201, 290:

Primavera di -, $120 \mathrm{n}$.

Premio Nobel, 30, 161, $302 \mathrm{n}$.

Prescrizione, dei delitti nazisti, 159.

Prigione o Penitenziario (Zuchthaus) nella DDR, 328 e n., 333 e n.; vedi anche: Detenzione (Gefängnis) nella DDR.

Prigionieri:

- di guerra, 35 s., 70, 72 s., 76, 89, 123, 135 n., 172, 193, 195, 199, 235, 237, 242.

- politici, 173, 249.

Vedi anche: Internati Militari Italiani.

Prima e seconda guerra del Golfo, 142, 165, 186, 208, 229.

Prima guerra mondiale, 9, 18 s., 26, 31, 35, 39, 61, 84, 106 s., 121, 151, 157, 176 n., 219 n., 223, 248, 257, 281, 319; vedi anche: Grande Guerra.

Prima guerra sino-giapponese, 70, 144, 280.

Primavista (o Terranova), 273 e n. Profugo, -hi (Displaced persons), 58, 114, $311 \mathrm{n}$.; vedi anche: Rifugiato.

Propaganda, 12, 14, 45, 59, 70, $101 \mathrm{n}$., 133, 138 n., 142, 147, 203, 284 e n., 297, 320 n., 326, 328 s., 331, 333.

Prostituzione, 193: - coatta, 73, 76, 78, 110, 143; vedi anche: Comfort women.

Provenza, 196.

Prussia (prussiano, -i), 9, 27, 131, 169 n., 175 n., 304. 
Purge categories, 68 e n., 83 n.; vedi anche: Valutazione del crimine.

Quaccheri, 8 .

Questione ebraica, $36 \mathrm{n}$.; vedi anche: Ebrei; "Manifesto, della razza"; Razza; Tribunale della razza.

Radikalenerla $\beta$, decreto contro gli estremisti, $220 \mathrm{n}$.

Radio, e politica, 12 n., 14, 160, 170 s., 195, 203, 327; vedi anche: Cinema; Propaganda.

Raubkunst, vedi Opere d'arte, sottratte. Razza, razziale, -i, 81 s., 101 n., 108, 157, 237, 243, 272 e n., 326, 328, 333:

leggi -, 54, 59, 115-117, 125, 175. Vedi anche: Antisemitismo; Ebrei; "Manifesto della -"; Tribunale della -.

"Regime (Il) fascista", giornale, 12. Regno:

- d'Italia, 7, 54, 116, 193-196, 200.

- dei Serbi, Croati, Sloveni, 281.

- "del Sud" (Italia), 44, $86 \mathrm{n}$.

- di Sardegna, 54.

- Unito, 236, 248; vedi anche: Gran Bretagna.

Reich, 124:

- e Kokutai, 250 n., 252; vedi anche: Kokutai.

Terzo -, 9 e n., 26, 161, 196, 198, $317 \mathrm{~s}$.

Repubblica, -he:

- Democratica Tedesca (DDR), 4, 58, 101 n., 119, 307, 326-333; vedi anche: Seconda dittatura tedesca.

- di Vichy, 72.

- di Weimar, 9s., 14, 32, 54, 61 n., 128, 154, 176 s., 306 e n., 311 e n., 313.

- Federale di Germania (o Tedesca), 9, 41 n., 58, 119, 121, 123 s., 139, 154, 219, 222, 224, 306 s., 325, $331 \mathrm{n}$.

- partigiane, 146.
- Popolare Cinese, 47, 72, 190 n., 285; vedi anche: Cina.

- Sociale Italiana (RSI), 44, $54 \mathrm{s.,}$ 85, 113, 146 s., 193 s., 198 s.; vedi anche: Salò, Repubblica di.

Resa, 147:

- di Cassibile e Caserta (Italia), 44, 196, 199.

- incondizionata (Giappone), 44 s., 83, 138, 170-172, 235, 237-241, 246, 249.

Vedi anche: Capitolazione.

Resistenza, 42, 55, 77, 86 e n., 88, 92-94, 148 s., 161, 195, 198 s., 201, 205, 300 n.:

- e desistenza, 93.

Riarmo, 46-50, 175, 180 s., 186, 199, 223, 235, 252, 286 e n., 290, 303 s., 309, 327, 330:

- duplice in Germania, 219 s.

- e polizie, 181 n., 317; vedi anche per la Germania: Bundesgrenzschutz; Bundespolizei; Bundeswehr; Nationale Volksarmee; Volkspolizei, Kasernierte; per il Giappone: Forze di Autodifesa; National Police Reserve; per l'Italia: "Esercito di Transizione".

Rifugiato, -i, 104, 153; vedi anche: Profugo.

Riparazione, -i, 3, 59, 63, 75-77, 106-128, 158, 162, 235, 237, 243, 245; vedi anche: Debito, -i, di guerra; Demontage; Risarcimento.

"Ripudio" della guerra, nella costituzione italiana, 41, 56, 202-204, 207, 209, 211, 223; vedi anche: "Kriegsächtung", nella costituzione tedesca.

Risarcimento, -i, 113 s., 124-126, 158: diritto soggettivo al -, 76 s., 114. esclusione dal -, $127 \mathrm{~s}$. Vedi anche: Comfort women; Indennizzo; Demontage; Riparazione.

Rivoluzione, 39, 154, 287, 292 s.: 
- d'Ottobre, 248; vedi anche:

Unione Sovietica.

Ro-Ber-To, 38 e n.; vedi anche: Patto

Tripartito.

Rodi, 197.

Rom, deportati, 126.

Romania, 89, 107.

Ronchi, dei Legionari, $278 \mathrm{n}$.

Rote Armee Fraktion, 159.

Ruanda, 77.

Russia, 44, 53, 55, 58, 144, 166, 185, 194, 213, 225, 291, 300; vedi anche: Unione Sovietica.

SA (Sturmabteilung, paramilitari nazisti), 311, 314.

Sacro Romano Impero, $61 \mathrm{n}$.

Saipan, isola, e suicidio di massa, 138 e n.

Sakhalin, isola, 44, 57.

Salò, Repubblica di -, 13, 36, 54 s., 195-197, 199; vedi anche: Repubblica Sociale Italiana.

Salomone, isole, 58 .

Samurai, 24, 33 n., 260:

- e SS nazionalsocialiste, 27 s., 169 n. "fratello -", 17, 19, 24, 269, 279; vedi anche nell'Indice dei nomi: Shimoi, Harukichi. mito dei -, 26-28.

San Francisco, vedi Trattato di -.

San Nicolò del Lido, campo di volo, 282.

San Paolo (São Paulo, Brasile), 272 n.

San Pelagio, campo di volo, 275 e n.

Sandbostel, lager, 198.

Sarre (Saarland), e Germania, 304.

Sassonia, 118.

Savoia, 270.

Scanderun (Alessandretta), 275 e n.

SBZ, Sowjetische Besatzungszone, vedi Zona di Occupazione Sovietica.

SCAP, vedi "Supreme Command of the Allied Powers".

Scarborough Shoal, secca occupata dalla Cina, 190.

Schio, strage di -, $91 \mathrm{~s}$.
Schleswig-Holstein, $102 \mathrm{~s}$.

Schutzstaffel, vedi SS.

Sciara Sciatt, battaglia di -, 75 .

Scuola, 2, 74, 129 s., 133, s., 139,

145-147, 152, 161, 163, 172:

- di Francoforte, 15, 60.

- Riforma Bottai, 147.

Vedi anche: Libri scolastici.

Seconda dittatura tedesca, 154, 162; vedi anche: Repubblica Democratica Tedesca (DDR).

Seconda guerra mondiale: $1,5,7 \mathrm{n}$., 19, 21, 26 n., 35, 38 e n., 44 s., 54, 69 s., 75 s., 79, 83, 106 s., 114, 121, 131 n., 135, 141 s., 152 s., 157, 163, 165, 169, 190 n., 206, 219 s., 229, 232, 248, 262, 288, 298, $318 \mathrm{n}$.

Seconda guerra sino-giapponese, 15 , 69, 82.

“Security Laws" (Giappone), e articolo 9, 186, $188 \mathrm{~s}$.

Sengoku, epoca degli Stati combattenti, $260 \mathrm{n}$.

Senkaku, isole, 51 s., 191; vedi anche: Diaoyu.

Serbia, 208, 280.

Servizio militare, 199, 207, 211, 303 s., 317-320, 331; vedi anche:

Coscrizione obbligatoria; Obiezione di coscienza.

"Settimana Incom", 149; vedi anche: Cinema; Cinegiornale.

Seul, 2.

"Sfera di Co-prosperità della Grande Asia Orientale", 28, 31, 58, 70, 72, 110, 135 n., $142 \mathrm{~s}$.

Sheffield, 290.

Shikoku, isola, 81 n., 237, 239.

Shinto, shintoismo, - di Stato, 132, 170 e $n$.

Shogun (Shogunato), 260 e n.:

- "dagli occhi azzurri", 46, 172;

vedi anche nell'Indice dei nomi:

MacArthur, Douglas.

Showa, epoca, $131 \mathrm{n}$.

Sicilia, 56, 193, 213. 
Sicurezza collettiva, 207, 223, 230, 232, 306-310, 325; vedi anche:

Autodifesa; Sovranità nazionale.

Sigonella, base militare, 213.

Singapore, 58, 70 s., 105 n., 139 n., 189.

Sinti, deportati, 126.

Siria, 166, 189, $225 \mathrm{n}$.

Socialismo, socialista, -i, 50, 86, 92 n., 94, 105, 133, 181, 194 n., 200 n., 207, 231, 251 n., 253 s., 284, 286, 288 s.:

Stati -, 9, 292, 294, 298 s., 301.

Vedi anche: Comunismo; Marxismo.

Società:

- delle Nazioni, 9, 54.

- Europea di Cultura, 301 e n.

- per la Pace, Amburgo, 320 e n.

Sokka Gakkai, 258 n.; vedi anche:

Komeito (Partito della Giustizia, Giappone).

Solidarietà, 33,202 :

- meccanica, 34.

- nazionale, 25.

Somalia, 75, 186 n., 229 s., 274.

Sovranità:

- militare, 61, $63 \mathrm{n}$.

- nazionale, 2, 40 s., 56, 60, 84, 163, 166 n., 171, 178, 185, 190, 193, 202, 205 s., 208 s., 223, 225, 230, 232 s., 237, 239, 259, 307-309, 321, 325, 328.

Vedi anche: Limitazione, di sovranità; Sicurezza collettiva.

Spagna, 7, 12 n., 23, 38 e n., 51, 203: - e "japonización", 23 e n.

Spazio: "Grande -"; Popolo senza -; vitale; vedi Geopolitica.

SPD, vedi Partito, Socialdemocratico (Germania).

Spratly, isole, $51,190 \mathrm{n}$.

"Spruchkammer", organo per la denazificazione, 95-97.

Sri Lanka, 51.

SS, paramilitari nazisti, e Samurai, 27 s., 169 n., 311.
Stalinismo, 98, 291; vedi anche: Dittatura.

Stampa femminile, 13.

Stati capitalisti, guerra fra -, 288 s., 290, $298 \mathrm{~s}$.

Stati Uniti d'America (USA, U.S.), 7 , 11 n., 15 e n., 34 s., 44 s., 47, 58, 67, 69-72, 74, 78-81, 83, 95 n., 108 s., $111,113,117,128,139,164-166$, 170 s., $174-176,182-184,186-188$, 199, 206, 208 s., 221, 236-242, 248, 270, 288, 311 n., 327.

Vedi anche: USA, U.S.

Statuto Albertino, 54, 56.

Stoccolma, appello di -, 290 e n., $293 \mathrm{n}$.

Stranieri nemici, 34 e n.; vedi anche: Internamento; Internati Militari Italiani (IMI).

"Strategia in avanti" (Vorwärtsstrategie), 62.

Stretto di Hormuz, e articolo 9 (Giappone), $188 \mathrm{~s}$.

Sudeti, 58 .

Suicidio, -i, 121, 158, 174:

- di massa, vedi Okinawa; Saipan.

- e resa giapponese, 170.

- e unità "Tigre Bianca", 24.

"Sunagawa Case", e articolo 9 (Giappone), 182-184; vedi anche: "Naganuma Case", "Suzuki Decision”.

"Supreme Command of the Allied Powers" (SCAP), 49, 68, 82, 175 n., 176, 238.

"Suzuki Decision", e articolo 9 (Giappone), 181 s.; vedi anche: "Naganuma Case", "Sunagawa Case".

Svizzera, 123 n., 146, 153 n.

Svolta di Petersberg, e interventi militari, 230.

Tachikawa, base aerea, 182; vedi anche: "Sunagawa Case".

Taisho, epoca, 84, $131 \mathrm{n}$.

Taiwan, 51, 58, 72, 190, 237 n.; vedi anche: Formosa. 
Taj Mahal, $276 \mathrm{~s}$.

Teheran, 188.

Tenda e Briga, cessione di -, 58 .

Tenno, 10 s., 31, 45, 170; vedi anche: Imperatore del Giappone.

Terranova (o Primavista), $273 \mathrm{n}$.

Territorio:

- dei Länder, 10.

- del Reich, 9, 56.

- e invasione/occupazione, $45 \mathrm{~s}$, 56 s., 72, 89, 122, 138, 166, 180, 196, 216, 219-221, 236, $303 \mathrm{~s}$.

- nazionale, 58, 72, 75, 107, 114, 117, 119 s., 156, 162, 223, 288, 306-313, 325, 327, 330, 332.

- - e internamento, $34 \mathrm{~s}$.

Terrorismo, 68, 153, 164 n., 211, 213, 233: - islamico, 167.

Testimoni di Geova, e Terzo Reich, 318 e. n.

Texas, 111, 139.

Tobruk (Libia), 213.

Tokugawa:

- epoca, 27, 131 n., 257 s., 260 e n.

pax -, 257-260; vedi anche: In-

conscio.

Vedi anche: Shogunato.

Tokyo:

bombardamento di -, $16,133 \mathrm{n}$.

colonna romana a,- 25 .

processo di -, 3, 44, 64-69, $73 \mathrm{~s}$., 77, 79, 82-85, 101, 142-144, 162.

università di - , 26, 177, 257.

volo fino a -, 20-22, 269 s., 275.

Vedi anche: Patto Tripartito; Ro-

Ber-To.

Torino, 16, 42, 115, 294 s., 299 e n.

Totalitarismo, totalitario, 15, 44, 129, 145, 248, 291:

- “teoria del -”, 156 s., 159.

Vedi anche: Fascismo; Nazio-

nalsocialismo; Stalinismo.

Traduzione simultanea, inizi, 79 .
Trattato, -i: 37 e n., 40, 49, 67, 75-77, 120 s., 163 s., 166 n., 182-184, 200, 212, $226 \mathrm{n} .:$

- di San Francisco, 45, 83, 107, 109, 183.

- di Versailles, 106 s., 121, 279 n.

- economico URSS-DDR, 121 e n.; vedi anche: "Neues Ökonomisches System" (NÖS).

- US-Japan Security Treaty, $182 \mathrm{~s}$. Vedi anche: Nato.

“Tre latini”, 272, 279, 281, 283; vedi anche nell'Indice dei nomi: Polo, Marco.

Treunbrietzen, fabbrica, 199 e $\mathrm{n}$. Tribunale, -i, 74, 76 s., 82, 89, 96 s., 98-100, 102 s., 114, 124, 127, 140, 143 n., 157, 180, 182 s., 190, 229-232, 311, 315, 322, 329 s.:

- della Razza, 116 n.; vedi anche: Ebrei; "Manifesto della Razza"; Questione ebraica; Razza. - militare, 57, 64-69, 73 s., 83-87, 142 s., 196, 207; vedi anche:

Norimberga, processo di -; Tokyo, processo di -.

- Penale Internazionale, 79.

Trieste, $52 \mathrm{n}$.:

e "Territorio Libero di -" (Zona A), $56 \mathrm{n}$.

Tripoli, Tripolitania, 151, 213, 274; vedi anche: Libia.

Turchia, turco, -hi, 125 e n., 150 s., $166,229,275 \mathrm{n}$.

"Ufficio per il perseguimento dei crimini nazisti”, Ludwigsburg, 67 e n.

UNCLOS, vedi "United Nations Convention of the Law of the Sea".

Ungheria, 107, 123 e n., 201 n.; vedi anche: Austro-ungarico, Impero.

Unione Sovietica, 10, 21, 46 s., 58, 71 s., 75 s., 80 s., 83,108 s., 112 , 117-121, 128, 165, 169, 182, 184, 
201 s., 206, 221, 259, 284-288, 294, 304; vedi anche: Russia.

“Unità 731” (Giappone), 73 n., 78, 141 n.; vedi anche: Guerra batteriologica.

"United Nations Convention of the Law of the Sea" (UNCLOS), 190.

URSS, vedi Russia; Unione Sovietica.

USA, U.S., 35, 44, 46, 53, 71 s., 108, 165, 172, 182, 193, 201, 208; vedi anche: Stati Uniti d'America.

Valutazione del crimine (cinque classi), 66, 101-103.

Varsavia, 290:

- Patto di, 220, 302.

Vaticano, 89, 298; vedi anche: Cattolicesimo; Papa.

"Veline" e propaganda, 12 s., 146.

Venezia, città, 14, 280, 296; vedi anche: Cinema.

Venezia Giulia, 57, 114.

"Verfassungskonvent" (costituente), e costituzione tedesca, 222, 307-309, $311,325$.

Versaglia (Versailles), 278 n.; vedi anche: Trattato di Versailles.

Vichy, Repubblica di -, 72.

Vienna, 131, 201, 270 s., 275 n., 277, 287, 289 e n., 297, 301; vedi anche: Volo su -.

Vietnam, 71, 82, 109, 138, 285, 293, 297; vedi anche: Indocina.

"Visione liberale della storia", 135, 137, 142-144; vedi anche: Libri scolastici.

Vite "indegne di essere vissute", 81, 321 e $n$.

"Volante Rossa”, 91e n.
Volksarmee, Nationale (NVA, DDR), 181 n., 302, 331 s.; vedi anche: Riarmo.

Volkskammer, Parlamento della DDR, 328 e n., 330-332.

Volkspolizei, Kasernierte (DDR), 181 n.; vedi anche: Riarmo.

Volo:

- a Tokyo (D’Annunzio), 20-22, 269-271, 280, 282.

- in Brasile, 21 e n., 272.

- su Vienna (D’Annunzio), 270 s., 275 n., 277.

Wake, isola, 58.

Weimar, vedi Repubblica, di Weimar.

Wehrmacht, abolizione della -, $181 \mathrm{n}$.

Woody, isola, $190 \mathrm{n}$.

Württemberg e Baden, costituzione del Land, 313 e n., 316 s., 322 e n.

Yamuna, vedi Giumma.

Yokohama, 52, 71.

Zaibatsu, cartelli in Giappone, 172 s., 244; vedi anche: Konzern.

Zona, -e, di occupazione (Germa-

nia), 58:

- Americana, 60 s., 66, 95 s., 101, $125 \mathrm{~s}$.

- Britannica, 95, 102 s., 117.

- Francese, 95, 102, 117.

- Sovietica (SBZ), 58, 66, 94, 96, 101, 117-119, 304; vedi anche:

Repubblica Democratica Tedesca (DDR).

Vedi anche: Trieste, Zona A.

Zuchthaus, vedi Prigione o Penitenziario nella DDR. 



\section{Indice dei nomi}

Questo indice elenca i nomi delle persone menzionate nel testo e nelle note, omettendo i nomi menzionati nei titoli di libri e articoli.

Il nome si trova alla pagina indicata dal numero che lo segue (p. es.: "Arendt, Hannah, 15."); se il nome si trova soltanto nella nota a piè di pagina, il numero è seguito da "n.” (p. es.: “111 n.”); se il nome si trova tanto nella pagina quanto in nota, il numero è seguito da "e n." (p. es.: "111 e n.”); se nome è presente anche nella pagina contigua, sia nel testo sia in nota, il numero è seguito da "s." (p. es.: “111s.”); se il nome è presente in più di due pagine continue, sia nel testo sia in nota, viene indicata la pagina iniziale e quella finale (p. es.: “111-114").

Abe, Shinzo, 185 s., 189 s., 258, 262 s., 266 e n.

Abegg, Lily, 28 e n.

Adenauer, Konrad, 80, 98, 305.

Adorno, Theodor W., 105 e n., 160 e n. Aga-Rossi, Elena, $196 \mathrm{n}$.

Agnoletti, vedi Enriques Agnoletti,

Enzo.

Agostini, Giulia, 22 n.

Al Sarraj, Fayez, 217.

Allinson, Gary D., $2 \mathrm{n}$.

Alloatti, Franca, 148 n.

Amado, Jorge, 201.

Ambris, Alceste de, 39 e n.

Ambrosio, Vittorio, 195.

Anders, Günther, 210 e n.

Antonescu, Ion, 89.

Antoni, Klaus J., 133 n.

Anya, Masaki, 247.

Apel, Erich, 121 e n.

Apelt, Hermann, $306 \mathrm{n}$.

Aragon, Louis, 201.

Ardemagni, Mirko, 113.

Arendt, Hannah, $15 \mathrm{~s}$.

Argira, Paolo, pseudonimo di Fiorina

Centi, 280.

Aristarco, Guido, $14 \mathrm{n}$.

Asakawa, Tamotsu, 247.
Ashida, Hitoshi, 179, 249.

Auer, James E., 184.

Auerbach, Philipp, 158.

Augeri, Nunzia, $146 \mathrm{n}$.

Avagliano, Mario, 193 n., 199 n.

Ayçoberry, Pierre, $16 \mathrm{n}$.

Azzariti, Gaetano, $116 \mathrm{n}$.

Baade, Brunolf, 120 e n.

Bacigalupi, Marcella, 146 n., 152 n.

Badoglio, Pietro, 55, 76, 151, 194-196.

Bailer-Galanda, Brigitte, $160 \mathrm{n}$.

Baird, Jay W., $71 \mathrm{n}$.

Balbo, Italo, 21.

Baracca, Francesco, 273.

Barile, Paolo, $94 \mathrm{n}$.

Bartolini, Giulio, $212 \mathrm{n}$.

Bästlein, Klaus, $104 \mathrm{n}$.

Battaglia:

- Achille, $94 \mathrm{n}$.

- Roberto, $94 \mathrm{n}$.

- Salvatore, 204 n., 273 n., 277 s.

Bauer, Fritz, 162.

Beer, Lawrence W., $181 \mathrm{n}$.

Belladonna, Simone, $151 \mathrm{n}$.

Bentivoglio, Giulia, 286 n. 
Benz, Wolfgang, 47 n., 57 n., 68 n., 83 n., 175 n.

Beonio-Brocchieri, Paolo, $2 \mathrm{n}$.

Beretta, Lia, $15 \mathrm{n}$.

Bergamini, David, $69 \mathrm{n}$.

Berger, Franz Severin, $17 \mathrm{n}$.

Bergsträsser, Ludwig, $322 \mathrm{~s}$.

Berkofsky, Axel, 171 n., 174 n., 186 n.

Berlinguer, Enrico, 197.

Berlusconi, Silvio, 75 .

Bernard, Henri, 65 n., 77 s.

Bernardi, Guido, $92 \mathrm{n}$.

Berndt, Jacqueline, 136 n., 145 n.

Bessel, Richard, $8 \mathrm{n}$.

Beyerle, Konrad, 307 s., 309 n.

Biagi, Enzo, 13 e n., 91 e n.

Bianchi d'Espinosa, Luigi, $94 \mathrm{n}$.

Bianco, Dante Livio, $94 \mathrm{n}$.

Bieber, Hans-Joachim von, $27 \mathrm{n}$.

Binding, Karl, $321 \mathrm{n}$.

Birolli, Renato, 295.

Bisson, Thomas A., $173 \mathrm{n}$.

Bittner, Wolfgang, $225 \mathrm{n}$.

Bobbio, Norberto, 4, 115, 202, 206, 210 e n., 269, 284 s., 287, 289-299, $301 \mathrm{n}$.

Bocca, Giorgio, 146 n.

Boccini, Luciano, $114 \mathrm{n}$.

Bonanate, Luigi, $204 \mathrm{n}$.

Bonaparte, Napoleone, 113, 204,

Bonomi, Ivanoe, 85 s., 93.

Bork, Henrik, $144 \mathrm{n}$.

Boßmann, Dieter, 160 e n.

Botta, Franco, $284 \mathrm{n}$.

Bottai, Giuseppe, 147.

Botteri, Inge, $13 \mathrm{n}$.

Bracci, Mario, $94 \mathrm{n}$.

Brach, Mary Lynn, $110 \mathrm{~s}$.

Brackman, Arnold C., 65 n., 78 n.

Brandt, Willy, 120.

Breccia, Gastone, $2 \mathrm{n}$.

Brecher, W. Puck, 36 n.

Bredemeier, Karsten, $318 \mathrm{n}$.

Brežnev, Leonid Il'ič, 121.

Brodesser, Hermann-Josef, 118.

Brondino, Michele, 74 n., $130 \mathrm{n}$.
Brooker, Paul, 33 s.

Brunori, Maurizio, $172 \mathrm{n}$.

Bucarelli, Palma, 113.

Bucher, Peter, 306 n., 309 n.

Caboto, Sebastiano, 273 e n.

Cadars, Pierre, $14 \mathrm{n}$.

Cajani, Luigi, 145 n.

Calamandrei, Piero, 94 e n., 290.

Calvino, Italo, 91.

Cammelli, Giorgio, $150 \mathrm{n}$.

Campagnolo:

- Stefano, $12 \mathrm{n}$.

- Umberto, $301 \mathrm{n}$.

Campi, Carlo Azelio, $91 \mathrm{n}$.

Canosa, Romano, 86 n., 93 n.

Cantatore, Domenico, 295.

Caparra, Niccolò il Grosso, detto il,$- \quad 271$.

Capitini, Aldo, $286 \mathrm{n}$.

Caprara, Maurizio, $216 \mathrm{n}$.

Capristo, Annalisa, $115 \mathrm{n}$.

Caracciolo, Davide, $55 \mathrm{n}$.

Carducci, Giosuè, 281.

Carlassare, Lorenza, 167 n., 208 n.

Carnevale, Paolo, $211 \mathrm{n}$.

Caroli, Rosa, 2 n., 70 n., 143 s.

Carsten, Otto, $164 \mathrm{n}$.

Casadio, Quinto, $146 \mathrm{n}$.

Cassero, Riccardo, $12 \mathrm{n}$.

Cassese, Antonio, 207 n., 210 n.

Caviglia, Enrico, 19.

Centi, Fiorina (pseudonimo: Paolo

Argira), 280.

Ceseri, Antonio, 199 n.

Cerrai, Sondra, 286 s.

Chandra Bose, Subhas, $135 \mathrm{n}$.

Chiang Kai-shek, 190 n., 323.

Chiari, Bernhard, $232 \mathrm{n}$.

Chichibu, (Yasuhito, principe -), 24.

Chickering, Roger, $219 \mathrm{n}$.

Chiesa, Pasquale, $116 \mathrm{n}$.

Churchill, Winston, 235.

Ciapparoni La Rocca, Teresa, $22 \mathrm{n}$.

Cignoni, Francesco, $12 \mathrm{n}$.

Clooney, George, 113 n. 
Coarelli, Rossella, 13 n., 145-150.

Coccoli, Carlotta, $112 \mathrm{n}$.

Coffrini, Fabrice, 123 n.

Cohen, David, $65 \mathrm{n}$.

Comencini, Luigi, 196.

Conti, Ettore, $38 \mathrm{n}$.

Conti Pertanini, Mario, $151 \mathrm{n}$.

Contini, Giovanni, 69 n., 74 n., 108 n.

Coppellotti, Celestino, 219 n.

Corazza, Heinz, $28 \mathrm{n}$.

Cornelissen, Christoph, $33 \mathrm{n}$.

Costa, Pietro, $204 \mathrm{n}$.

Costanzo, Ezio, $55 \mathrm{n}$.

Coticchia, Fabrizio, 165 n.

Courtade, Francis, $14 \mathrm{n}$.

Cramer, Myron C., 78.

Croce, Benedetto, 280.

Crome, Erhard, $223 \mathrm{n}$.

D'Aniello, Ferdinando, $10 \mathrm{n}$.

D'Annunzio:

- Gabriele, 9, 17-24, 26, 39 e n., 88 s., 269-274, 277-282.

- Gabriellino, $88 \mathrm{~s}$.

Da Frè, Giuliano, $53 \mathrm{n}$.

Dankwerts, Justus, $308 \mathrm{n}$.

De Ambris, Alceste, vedi Ambris, Alceste de.

De Gasperi, Alcide, 86, 116, 208.

De Gaulle, Charles, $56 \mathrm{n}$.

De Giorgi, Fulvio, 146 n., 148 s., $150 \mathrm{n}$.

De Grada, Raffaele, 295.

De Guttry, Andrea, 212 n.

De Vergottini, Giuseppe, 39 s., $211 \mathrm{n}$.

De Vincentiis, Mauro, $203 \mathrm{n}$.

Deaglio, Enrico, $2 \mathrm{n}$.

Decker, Gunnar, 120 s.

Di Capua, Giovanni, $55 \mathrm{n}$.

Di Feo, Gianluca, 213 n.

Di Francesco, 233 n.

Di Giovine, Alfonso, 74 n., 130 e n.

Di Russo, Marisa, $23 \mathrm{~s}$.

Di Stasi, Lawrence, 35 n.

Doehring, Karl, 119 n.
Dogliani, Mario, 43 n., 207 n., 209 e n., $231 \mathrm{n}$.

Donat, Walter, 27 s., $250 \mathrm{n}$.

Donati, Benvenuto, 115.

Donini, Ambrogio, $287 \mathrm{n}$.

Donnedieu de Vabres, Henri, $77 \mathrm{~s}$.

Douhet, Giulio, 16.

Drexelius, Hermann, 308 n.

Dürig, Günter, 122 n.

Durkheim, Émile, 34.

Duterte, Rodrigo (Filippine), $190 \mathrm{n}$.

Eberhardt, Fritz, 310 s., 313-315, 322-324.

Eco, Umberto, 106 e n.

Edano, Yukio, 264.

Edsel, Robert M., 113 n.

Egbert, Lawrence D., $66 \mathrm{n}$.

Ehrenburg (Erenburg, Il'ja Grigor'evič), 201 e $n$.

Eichmann, Adolf, 64, 159, 162.

Eickhoff, Bärbel, $99 \mathrm{n}$.

Einaudi, Giulio, 201.

Einstein, Albert, 201.

Eisenhower, Dwight D., 183, 301.

Elm, Ludwig, $154 \mathrm{n}$.

Enriques Agnoletti, Enzo, 290 s., 293.

Erasmo da Rotterdam (Desiderius Erasmus Roterodamus), $4 \mathrm{~s}$.

Farge, Oliver (?), 201.

Farinacci, Roberto, 12.

Ferencz, Benjamin B., $123 \mathrm{n}$.

Ferrarin, Arturo, 20 s., 271 n., 278 n.

Ferretti, Valdo, $15 \mathrm{n}$.

Fisch, Jörg, 106, 109-112, 155.

Fischer, Torben, $155 \mathrm{n}$.

Flechtheim, Alfred, $122 \mathrm{n}$.

Floreanini, Gisella, $220 \mathrm{n}$.

Focardi, Filippo, 69 n., 74 n.

Foljanty-Jost, Gesine, 139-141.

Ford, Gerald, 34.

Foresti, Fabio, $12 \mathrm{n}$.

Forno, Mauro, $12 \mathrm{n}$.

Fortunio, Tommaso, 87.

Fossati, Piero, 146 n., 152 n. 
Fraenkel, Ernst, $11 \mathrm{n}$.

Franz-Willing, Georg, $60 \mathrm{n}$.

Frei, Norbert, $116 \mathrm{n}$.

Freud, Sigmund, 257, 259.

Fried, Alfred Hermann, 219 n.

Friedländer, Saul, 33 n.

Fuji, Shinichi, 32.

Fujioka, Nobukatsu, 135, 137, 142 s.

Furuya, Naoki, 247.

Gabrielli, Noemi, 113.

Gaggero, don Andrea, $286 \mathrm{n}$.

Galante Garrone, Carlo, 94 n.

Galluzzo, Marco, 216 n.

Gandhi, Mohandas Karamchnad, 135 n., 153.

García Saéz, José Antonio, 40 n.

Gatti, Francesco, 2 n., 8 n.

Gautier:

- Judith, $22 \mathrm{n}$.

- Théophile, $22 \mathrm{n}$.

Genovese, Vito, $55 \mathrm{n}$.

Gentile, Giovanni, 145.

Gentiloni, Paolo, $216 \mathrm{n}$.

Gergolet, Mara, 51 n., 190 n.

Gerra, Ferdinando, 19-20.

Getreuer, Peter, $171 \mathrm{n}$.

Gheddafi, Muhammar, 75 .

Giacomini, Ruggero, 201 n., 288 n.

Gigli, Silvia, $36 \mathrm{n}$.

Gimbel, John, $117 \mathrm{n}$.

Ginsborg, Paul, 2 n.

Ginzburg, Natalia, 42 e n., 201.

Girasole, Pier Giorgio, 3, 247 e n., 249 n., 252 n., 257 n., 268 n.

Gliozzi, Ettore, $151 \mathrm{n}$.

Godau-Schüttke, Klaus-Dettlev, 97 n.

Goebbels, Joseph, 14.

Goncourt, Edmond de, 22 e n.

Gonella, Guido, 147.

Goodman, Carl F., $181 \mathrm{n}$.

Goschler, Constantin, $123 \mathrm{~s}$.

Gozzano, Guido, $276 \mathrm{n}$.

Granata, Ivano, $286 \mathrm{n}$.

Graziani, Rodolfo, 76, 151.

Gribaudi, Gabriella, 195 n.
Grimm:

- Fratelli, 223.

- Hans, $30 \mathrm{n}$.

Grosser, Dieter, $2 \mathrm{n}$.

Grossi, Alessia, 199 n.

Gründler, Gerhard E., 65 n.

Guareschi, Giovanni, 197.

Guarino, Antonio, 149.

Guarnieri, Giuseppe, detto del Gesù, 271.

Guerri, Giordano Bruno, 9 n., 39 n.

Gurlitt, Cornelius e Hildebrand, 122.

Guttuso, Renato, 201, 295 e n.

Guy, George F., $71 \mathrm{n}$.

Haasch, Günther, 131 n., 133 n.

Hamano, Jo, $253 \mathrm{n}$.

Hansen, Janine, $14 \mathrm{n}$.

Hasebe, Yasuo, $187 \mathrm{~s}$.

Haski, Pierre, 189 n.

Hata, Nagami, 247.

Haushofer, Karl, 28 s., 37 e n., 135.

Hayashi, Fusao, 135.

Hegel, Georg Wilhelm Friedrich, 257.

Heile, Wilhelm, $323 \mathrm{~s}$.

Henderson, Dan F., $170 \mathrm{n}$.

Herde, Peter, 15 n., 22 n.

Herrmann, Anne-Kathrin, $155 \mathrm{n}$.

Heuss, Theodor, 314-320, $322 \mathrm{~s}$.

Higashikuni, Naruhito, 174, 250.

Himmler, Heinrich, $27 \mathrm{~s}$.

Hiranuma, Kiichiro, 255.

Hirohito, imperatore, 15, 24, 47 s., 69, 78 e n., 250, 252, $255 \mathrm{~s}$.

Hirsch, Ernst, 125 e n.

Hitler, Adolf, 27, 32, 44, 54, 112 s., 157, 159 s., 162, 175, 194.

Hochbaum, Hans-Ulrich, 59 n., 326 s., $330 \mathrm{~s}$.

Hoche, Alfred, $321 \mathrm{n}$.

Hockerts, Hans Günter, 123 n.

Hoffend, Andrea, $12 \mathrm{n}$.

Hofmann, Reto, 11 n., 17 s., 20 e n., 25 n., 113 n.

Höft, Andrea, 160-162.

Holian, Timothy J., 35 n. 
Holler, Christiane, $17 \mathrm{n}$.

Hook, Glenn D., 179 n., 185 n.

Höpken, Wolfgang, $136 \mathrm{n}$.

Horwitz, Solis, $67 \mathrm{n}$.

Hosoda, Hiroyuki, 264, 266.

Hueck, Alfred, 125.

Huysum, Jan van, 122.

Iacch, Franco, $52 \mathrm{n}$.

Ichinohe, Fujio, 247.

Ienaga, Saburo, 68, 73 n., 134, 138-141, 152.

Igari, Hiromi, $142 \mathrm{n}$.

Ikeda, Ichiro, 247.

Inoue, Kyoko, $170 \mathrm{n}$.

Insolvibile, Isabella, $196 \mathrm{n}$.

Isensee, Josef, $124 \mathrm{n}$.

Ito:

- Hirobumi, 49 e n., 139.

- Satoru, 247.

Itoh, Hiroshi, $181 \mathrm{n}$.

Jasper, Gotthard, $126 \mathrm{s.}$

Jaspers, Karl, 155 e n.

Jeismann, Karl-Ernst, $131 \mathrm{n}$.

Jemolo, Arturo Carlo, $94 \mathrm{n}$.

Joliot-Curie:

- Frédéric, 201, 300 e n.

- Irène, $300 \mathrm{n}$.

Joosten, Paul A., 66 n.

Kades, Charles, 178 e n.

Kant, Immanuel, 257, 262.

Kappler, Herbert, 92.

Karatani, Kojin, 257 s., 262.

Karlsch, Rainer, 117-121.

Kasahara, Toikushi, $69 \mathrm{n}$.

Kasza, Gregory J., 12 n.

Katayama, Tetsu, 249.

Kater, Michael H., $318 \mathrm{n}$.

Kawai, Kazuo, 83 e n.

Kawashima, Takane, 247.

Kaufmann, Theophil, 322.

Keller, Georges, 123.

Kersevan, Alessandra, 75 n.
Kim:

- Hak-sun, 111.

- Jong-un, 211 e n.

King, Martin Luther, 153.

Kipp, Heinrich, 318 n.

Kirchhos, Paul, 124 n.

Kitagawa, Kazuo, 267.

Kitamura, Tokoku, 257 e n.

Kittel, Manfred, $58 \mathrm{n}$.

Klee, Ernst, $95 \mathrm{n}$.

Klein:

- Angelika, $220 \mathrm{n}$.

- Gabriella B., 12 n.

Kleindinst, Ferdinand, 321.

Kleßmann, Christoph, $154 \mathrm{n}$.

Kobayashi, Yoshinori, 136, 143-145.

Koellreutter, Otto, 27-30, 32 e n., 81, $96 \mathrm{s.}$

Koike, Yuriko, 265.

Koizumi, Junichiro, 136, 165 s.

Kolko, Joyce e Gabriel, 173 n.

Komaki, Kaoru, 247.

Konoe, Fumimaro, 29, 174, 251.

Kordt, Theodor (Theo), $308 \mathrm{n}$.

Kosaka, Masataka, $2 \mathrm{n}$.

Koseki, Shoichi, 170 n., 172 n., 247, $255 \mathrm{n}$.

Krebs, Gerhard, 8 n., 169 n.

Kuhn, Hans-Werner, $154 \mathrm{n}$.

Kühnhardt, Ludger, 154 n.

Kumagai, Naoko, $78 \mathrm{n}$.

Kurasawa, Aiko, $73 \mathrm{n}$.

Kuroda, Tamiko, 134 n.

Labanca, Nicola, 75 n., 150-152, $199 \mathrm{n}$.

Lamberti, Maria Mimita, 23 n.

Lanna, Noemi, $171 \mathrm{n}$.

Lanz, Hubert, $196 \mathrm{n}$.

Lanza, Ignazio, 273.

Legnani, Massimo, $146 \mathrm{n}$.

Lehndorff-Felsko, Angelika, $220 \mathrm{n}$.

LeMay, Curtis, 16.

Leo, Annette, $154 \mathrm{n}$.

Levi:

- Alessandro, 115. 
- G., 201.

- Riccardo, $94 \mathrm{n}$.

Li, Ruiyu, $190 \mathrm{n}$.

Li Gotti, Claudio, $55 \mathrm{n}$.

Liekhin (Lëchin), Ivan V., 290 n.

Lindner, Ulrike, $2 \mathrm{n}$.

Linhart, Sepp, $27 \mathrm{n}$.

Llewelyn, James, $171 \mathrm{n}$.

Lombardo-Radice, Giuseppe, 145.

Losano, Mario G., 1 n., 7 n., 16 n., 23 n., 28 n., 37 n., 43 n., 78 n., 90 n., 98 n., 125 n., 135 n., 213 n., 227 n., 285 n., 295 n.

Lowe, Keith, 193 n.

Luciano, Lucky, $55 \mathrm{n}$.

Ludwig II, di Baviera, 305.

Luther, Jörg, $231 \mathrm{n}$.

Lutz, Dieter S., $62 \mathrm{n}$.

Luyssen, Johanna, $105 \mathrm{n}$.

Lyon, Cherstin, M., $35 \mathrm{n}$.

MacArthur, Douglas, 19 n., 45, 46, 48 s., 67-70, 108, 170 e n., 172-175, 177-179, 237 s., 255, 259.

Maffei, Maria Clara, $78 \mathrm{n}$.

Magi, Laura, 75-77.

Magini, Publio, $22 \mathrm{n}$.

Major, Patrick, $220 \mathrm{n}$.

Maki, John M., 170 n., $181 \mathrm{n}$.

Maltarich, Bill, $27 \mathrm{n}$.

Mambrino, re immaginario, 271.

Mangoldt, Hermann von, 311, 313-321, $323 \mathrm{~s}$.

Manikowsky, Arnim von, $65 \mathrm{n}$.

Mann:

- Erika, $128 \mathrm{~s}$.

- Thomas, 129 e n., 154 s., $290 \mathrm{n}$.

Mantegna, Andrea, 273.

Manzù, Giacomo, 297.

Maraini:

- Dacia, 36 e n.

- Fosco, 16 e n., 35 s., 45 s., 113, 138 n., 170 e n., 172 s.

Marinetti, Filippo Tommaso, 39 e n.

Marone, Gherardo, 280 e n.

Maruyama, Masao, 33 n., 70 n., 73 n.
Martin, Bernd, $81 \mathrm{n}$.

Marx, Karl, 198, 257.

Marxen, Klaus, $64 \mathrm{~s}$.

März, Peter, 305 n.

Marzo Magno, Alessandro, $113 \mathrm{n}$.

Masaru, Tonomura, 32 n., 135 n.

Mason, Tim, $33 \mathrm{n}$.

Matisse, Henri, 201.

Matsudaira, Setsuko, 24.

Matsui, Ichiro, 265.

Matsumoto, Joji, 174, 178, 255.

Matsumura, Takao, $73 \mathrm{n}$.

Matsuoka, Yosuke, 37.

Mattei, Teresa, 203 e n.

Maunz, Theodor, $122 \mathrm{n}$.

Mazziotti di Celso, Manlio, $207 \mathrm{n}$.

Mazzolari, don Primo, $286 \mathrm{n}$.

Mazzoleni, Emil, 186 n.

McCormack, Gavan, 179 n.

McLure, Robert A., 60.

Menzel, Johanna M., $37 \mathrm{n}$.

Mercuri, Lamberto, $86 \mathrm{n}$.

Mesmer, Philippe, $51 \mathrm{n}$.

Meyer, Dennis, $95 \mathrm{n}$.

Middlebrooks, William C., 185 n.

Migliore, Maria Chiara, $171 \mathrm{n}$.

Mihr, Anja, $65 \mathrm{n}$.

Minami, Keita, $257 \mathrm{n}$.

Minear, Richard H., 65 n., 71 n., 83 n., $138 \mathrm{~s}$.

Minerbi, Alessandra, $129 \mathrm{n}$.

Minnigerode-Allenberg, Freifrau von, 315.

Minobe, Tatsukichi, 133.

Miyamoto, Taro, $250 \mathrm{n}$.

Möller, Horst, $58 \mathrm{n}$.

Molodiakov, Vassili, $177 \mathrm{n}$.

Monducci, Francesco, $153 \mathrm{n}$.

Montale, Eugenio, $290 \mathrm{n}$.

Montanelli, Indro, 19 e n., 26 n., 92 e n.

Montino, Davide, 13 n., $80 \mathrm{n}$.

Moore, Ray A., $172 \mathrm{n}$.

Moranino, Francesco, $91 \mathrm{~s}$.

Morgenthau:

- Hans Joachim, $40 \mathrm{n}$.

- Henry, 99 s., 117 e n. 
Mori, Arinori, $131 \mathrm{s.}$

Moritomo, 262-267; vedi nell'Indice analitico: Caso Moritomo.

Mossdorf, Otto, $27 \mathrm{n}$.

Mucchi, Gabriele, 202, 284 s., 287, 294-296, 299, $301 \mathrm{n}$.

Müller, Reinhard, $120 \mathrm{n}$.

Münch, Philipp, $165 \mathrm{n}$.

Munzert, Maria, $158 \mathrm{n}$.

Mura, Guido, 150.

Muramatsu, Mariko, $22 \mathrm{n}$.

Murphy, Frank, 71 n., 79.

Mussolini, Benito, 10, 12, 18-20, 24-26, 54, 85, 106, $193 \mathrm{~s}$.

Nagy-Talavera, Nicholas M., 89 n.

Nakae, Chomin, 257 e n.

Nakai, Akio, 44 e n., 46, 81 s., 84 s., $106 \mathrm{~s}$.

Nakanishi, Inosuke, 249.

Napoli, Franco Felice, 89 n.

Narita, Ryuichi, $137 \mathrm{n}$.

Natta, Alessandro, 197 e n.

Nawiasky, Hans, 307 n.

Negri, Guglielmo, 39 n.

Nenni, Pietro, 86 e n., 88, 201, 286.

Neumann, Franz L., 15 s.

Neruda, Pablo, 201.

Niehuss, Merith, $2 \mathrm{n}$.

Nimitz, Chester, 16.

Nish, Ian, 193 n.

Nishio, Kanji, 136 s.

Nitsch, Carlo, $115 \mathrm{~s}$.

Nitti, Francesco Saverio, 206 e n.

Niutta, Ugo, 273.

Niven, William John, $154 \mathrm{n}$.

Noble, David, $78 \mathrm{n}$.

Nobusuke, Kishi, 84, 183.

Nosaka, Sando, 254.

Nozaki, Yoshiko, 69 n., 139 n., 141 n.

Oberreuter, Heinrich, $305 \mathrm{n}$.

Oda, Makoto, $171 \mathrm{n}$.

Okanouye-Kurota, Moriguchi, $26 \mathrm{n}$.

Okuma, Shigenobu, 281, 283.

Oliva, Gianni, 146 n., 194 s.
Ōmori, Hisao, 247.

Onuki, Atsuko, $137 \mathrm{n}$.

Oppler, Alfred, 46 n., 175 s., 181, $183 \mathrm{n}$.

Orlando, Vittorio Emanuele, $274 \mathrm{n}$.

Osten, Philipp, 73 s., 177 n.

Ostwald, Paul, $26 \mathrm{n}$.

Ota, Akihisa, $265 \mathrm{n}$.

Ott, Eugen, 37.

Pacini, Patrizia, 203 n.

Pagliano, Maurizio, 273.

Pal, Radhabinod, $78 \mathrm{n}$.

Pala, Elena, $14 \mathrm{n}$.

Palli, Natale, 270 s., 275 s., 278 n.

Palmieri, Marco, 193 n.

Pansa, Giampaolo, 90 s., 93, 281 n.

Pantaleone, Michele, $55 \mathrm{n}$.

Parri, Ferruccio, 85, $148 \mathrm{n}$.

Passarelli, Gianluca, $89 \mathrm{n}$.

Pastore, Annibale, 295.

Pauley, Edwin W., $108 \mathrm{~s}$.

Pautasso, Guido Andrea, $18 \mathrm{n}$.

Pavese, Cesare, 42.

Pedroletti, Brice, $190 \mathrm{~s}$.

Pekar, Thomas, $27 \mathrm{n}$.

Pende, Nicola, 116.

Peretti-Griva, Domenico, $93 \mathrm{~s}$.

Perfecto, Gregorio, $71 \mathrm{n}$.

Petrov, Fedor Nikolaevič, 290 n.

Pfeffer, Nathaniel, $174 \mathrm{n}$.

Pfeiffer, Anton, 314.

Pfetsch, Frank R., 317 n.

Picasso, Pablo, 201 e n.

Piccigallo, Philip R., 67 n., 71 s., 79.

Pieri, Piero, $194 \mathrm{n}$.

Pietro I Karađorđević, re di Serbia, 280.

Pikart, Eberhard, 310 n., 313 n., 315 n., 322 n., 325 n.

Pingel, Flak, $158 \mathrm{n}$.

Plehwe, Friedrich-Karl von, $8 \mathrm{n}$.

Poggiolini, Ilaria, $45 \mathrm{n}$.

Poletti, Charles, $55 \mathrm{n}$.

Polo:

- Marco, 58 n., 272 s., 275, 279, 281. 
- Niccolò e Matteo, 58 n., 272 n., 281; vedi anche nell'Indice analitico: "Tre latini".

Polverelli, Gaetano, 12.

Porth, Kenneth L., $171 \mathrm{n}$.

Pötzl, Norbert F., 122 n.

Powles, Cyril H., $171 \mathrm{n}$.

Predazzi, Francesca, $62 \mathrm{n}$.

Predieri, Alberto, $94 \mathrm{n}$.

Priebke, Erich, 92.

Pritchard, R. John, $67 \mathrm{n}$.

Procacci, Giuliano, 128 n., 134-136.

Pugliese, Pasquale, $203 \mathrm{n}$.

Pustau, Eduard von, $26 \mathrm{n}$.

Quasimodo, Salvatore, 201, 295.

Radler, Max, $61 \mathrm{n}$.

Raggi, Barbara, $116 \mathrm{n}$.

Raimondo, Sergio, $17 \mathrm{n}$.

Ramaioli, Federico Lorenzo, 171 n., 173.

Ramos, Fidel (Filippine), $190 \mathrm{n}$.

Rastelli, Achille, $16 \mathrm{n}$.

Ravà, Adolfo, $115 \mathrm{~s}$.

Ravalico, Michela, $111 \mathrm{n}$.

Ravagli, Giovanni, $94 \mathrm{n}$.

Recchioni, Massimo, 76 n., 89 n., $92 \mathrm{n}$.

Reel, A. Frank, 71 n.

Reichel, Karl Ferdinand, 50 n.

Reichelt, Julian, $232 \mathrm{n}$.

Richter, Steffi, 136 e n.

Rieder, Bruno, 61-63.

Righi, Stefano, $38 \mathrm{n}$.

Rivera, Diego, 295.

Robinson:

- Donald L., $172 \mathrm{n}$.

- Greg, 35 n.

Rochat, Giorgio, 152 n., 194 n.

Roesler:

- Hermann, 7 n., 32, 43.

- Jörg, $121 \mathrm{n}$.

Röhl, Wilhelm, 49 e n.

Röling, Bernard V.A., 67 s., 78 n.

Romeike, Sanya, $65 \mathrm{n}$.
Rötzer, Florian, $225 \mathrm{n}$.

Rousseau, Jean-Jacques, $257 \mathrm{n}$.

Roy Palmer, Domenico, 90 n., 93 n.

Rudnik, Carola S., $154 \mathrm{n}$.

Ruini, Meuccio, 202, $204 \mathrm{~s}$.

Russo Perez, Guido, 206.

Rüter (Rueter), Christiaan F., 67 n., $78 \mathrm{n}$.

Rutledge, Wiley, $71 \mathrm{n}$.

Sagawa:

- Michio, 252.

- Nobushisa, 263.

Sagi, Nana, $123 \mathrm{n}$.

Sailer, Emilio, 272.

Saito, Chunichi, 247.

Saitta, Armando, 152 e n.

Salandra, Antonio, 19 e n.

Saletti, Carlo, $321 \mathrm{n}$.

Salgado, Plínio, 25.

Salomon, Ernst von, 96 e n.

Salvemini, Gaetano, $94 \mathrm{n}$.

Salvini, Matteo, 92 e n.

Samarani, Guido, 69 n.

Santevecchi, Guido, 189 s.

Sanudo, Marco, 273 n.

Saragat, Giuseppe, 92 e n.

Sarpieri, Tanja, $286 \mathrm{n}$.

Sassu, Aligi, 295.

Savoja, Umberto, $271 \mathrm{n}$.

Schaller, Michael, $45 \mathrm{n}$.

Scherrer, Philipp, $231 \mathrm{n}$.

Schmid, Carlo, 306-308, 311, 313, 317, 323.

Schmidt, Eike, 122.

Schmidt-Sinns, Dieter, $160 \mathrm{n}$.

Schönbach, Karsten Heinz, 173 n.

Schöpfel, Ann-Sophie, 77-79.

Schreiber, Gerhard, $198 \mathrm{n}$.

Schröder, Florian, $231 \mathrm{n}$.

Schwarz:

- Jürgen, $232 \mathrm{n}$.

- Walter, $123 \mathrm{~s}$.

Schwegmann, Christoph, $232 \mathrm{n}$.

Schweizer, Katja, 162.

Schwentker, Wolfgang, $133 \mathrm{n}$. 
Seidel, Hans-Christoph, 110.

Seifer, Hubertus, 108.

Sereni:

- Emilio, 284 n., 286 e n.

- Marina, 42 n., $286 \mathrm{n}$.

Setta, Sandro, 86 n., 90 n., 206 n.

Severgnini, Nathascia, $111 \mathrm{n}$.

Seymour, Robert L., $181 \mathrm{n}$.

Shidehara, Kijuro, 48, 172, 174 s., 250 s., 253-256.

Shigemitsu, Mamoru, 82.

Shigeru, Ishiba, 268.

Shimoi, Harukichi, 17-29, 269 s., 272 n., 279-282.

Shindo, Toyo, 247.

Shiratori, Toshio, 177 e n.

Shoichi, Koseki, 247, 255 n.

Shostakovich (Šostakovič, Dmitrij

Dmitrievič), 201.

Shulman, Frank Joseph, $170 \mathrm{n}$.

Sicardi, Stefano, 43 n., 207, 231.

Siep, Julia, $12 \mathrm{~s}$.

Silvestri, Mario, $220 \mathrm{n}$.

Simoni, Silvano, $39 \mathrm{n}$.

Sircana, Giuseppe, $21 \mathrm{n}$.

Skya, Walter A., $8 \mathrm{n}$.

Smiatacz, Carmen, $103 \mathrm{n}$.

Soh, Chunchee Sarah, $78 \mathrm{n}$.

Sonnino, Sydney, 19.

Spang, Christian W., 29 n., 135 n.

Spinosa, Antonio, $20 \mathrm{n}$.

Spoerer, Mark, 110.

Spulcioni, Gianni, $12 \mathrm{n}$.

Stalin, Josif (Josif Vissarionovič

Džugašvili), 4, 197, 202, 248, 269,

284-286, 288 s., 292, 294, 298-301.

Stam, Arthur, $11 \mathrm{n}$.

Stein, Lorenz von, 131.

Steindorf, Joachim, $228 \mathrm{n}$.

Steinkamm, Armin A., $232 \mathrm{n}$.

Steinmeier, Frank-Walter, 225 e n.,

Stella, Gianfranco, $92 \mathrm{~s}$.

Sternberg, Theodor, 125 e n.

Stöcker, Helene, $223 \mathrm{n}$.

Stoph, Willi, 120.

Sugita, Yoneyuki, $45 \mathrm{n}$.
Sühl, Klaus, 154 n.

Suhr, Otto, $308 \mathrm{n}$.

Suttner, Bertha von, 219 n.

Suzuki:

- Hidemi, 3, 262.

- Mosaburo, 181.

- Yasuzo, 251 e n.

Tagore, Rabindranath, 23, $30 \mathrm{~s}$.

Takada, Yasumasa, $265 \mathrm{n}$.

Takahashi, Hirofumi, 247.

Takano, Iwaburo, 251 e n.

Takayanagi, Masui, $255 \mathrm{n}$.

Takeda, Kiyoko, $11 \mathrm{n}$.

Takemae, Eiji, $45 \mathrm{n}$.

Takeuchi, Keiichi, 35 n., 132 s., 135 n.

Tamaki, Yuichiro, 265.

Tanaka, Hideo, 178 e n.

Tandler, Agnes, $121 \mathrm{n}$.

Taylor, Telford, $71 \mathrm{n}$.

Tesio, Federico, 271.

Tesoro, Marina, $10 \mathrm{~s}$.

Tito, Josip Broz, 301.

Togliatti, Palmiro, 203 n., 207.

Tojo, Hideki, 85.

Tolzmann, Don Heinrich, 35 n.

Tomea, Fiorenzo, 295.

Tonomura, Masaru, $32 \mathrm{n}$.

Toscano, Mario, $115 \mathrm{n}$.

Tosi, Rosanna, $208 \mathrm{n}$.

Trainine, A. N. [Trajnin, Aron N.], 60.

Trenta, Elisabetta, $233 \mathrm{n}$.

Trento, Francesco, $91 \mathrm{n}$.

Treves, Renato, 115 s., 125.

Trindade, Hélgio, $25 \mathrm{n}$.

Truman, Harry S., 108, 173, 235.

Trump, Donald, 211 e n.

Truzzi, Silvia, $167 \mathrm{n}$.

Tsukamoto, Seiichi, 247.

Tuorto, Dario, $89 \mathrm{n}$.

Ugai, Nobushige, 49 n., $180 \mathrm{n}$.

Ulbricht, Walter, $155 \mathrm{n}$.

Umeda, Kinji, 247-249, 252 n., 256.

Ungaretti, Giuseppe, 280 e n. 
Urach, Albrecht, 27 n., 30 e n., 33 n., $142 \mathrm{n}$.

Vagnini, Alessandro, $15 \mathrm{n}$.

Vannuccini, Vanna, $62 \mathrm{n}$.

Vansittan, Robert, $117 \mathrm{n}$.

Védrines, Jules, 276 e n.

Venturini, Maria, $220 \mathrm{n}$.

Vercelli, Claudio, $160 \mathrm{n}$.

Verduzio, Rodolfo, $271 \mathrm{n}$.

Vicentini, Luigi, $24 \mathrm{n}$.

Vinciguerra, Mario, $94 \mathrm{n}$.

Visco, Antonio, $114 \mathrm{n}$.

Vittorini, Elio, 201.

Vollnhals, Clemens, 60 n., 95 s.

Volpe, Gioacchino, 151.

Wahl, Rainer, $185 \mathrm{n}$.

Walther, Felix, 323.

Ward, Robert E., $170 \mathrm{n}$.

Washburne, Carleton W., 147, 152.

Watanabe:

- Harumi, 69 n., 144 n.

- Kenji, 247.

Weber, Dr. Helene, 314-316, 319-321.

Weber, Dr. Hellmuth (sic) von, 314.

Weber, Petra, $96 \mathrm{n}$.

Wehberg, Hans, $311 \mathrm{n}$.

Weiler, Berndt, $144 \mathrm{n}$.

Weiss, Julia, $155 \mathrm{n}$.

Welch, David, $14 \mathrm{n}$.

Weniger, Erich, 156 e n.

Werner, Wolfram, 310 n.,313 n.,315 n., 322 n., 325 n.
Werte, Gerhard, $64 \mathrm{n}$.

Wetzler, Peter, $70 \mathrm{n}$.

Whitney, Courtney, 178 e n., 255.

Wiesel, Elie, 161.

Winkler, Christian G., 185 n.

Winstel, Tobias, $126 \mathrm{n}$.

Wittgens, Fernanda, 113.

Wolfder, $219 \mathrm{n}$.

Wolfe, Robert, $47 \mathrm{n}$.

Woller, Hans, $87 \mathrm{n}$.

Worell (autore di una petizione), 315.

Wrobel, Hans, $105 \mathrm{n}$.

Wunderlich, Hans, 316-318.

Wyman, Mark, $313 \mathrm{n}$.

Yagyu, Kunichika, $8 \mathrm{n}$.

Yamaguchi, Yasushi, $8 \mathrm{n}$.

Yamakawa, Hitoshi, 254 e n.

Yamashita, Tomoyuki, 70 s., 79 n.

Yano, Hisashi, $73 \mathrm{n}$.

Yasuhito, principe, vedi Chichibu.

Yosano, Akiko, $280 \mathrm{n}$.

Yoshida, Shigeru, 172, 177, 254-256, 259.

Yoshimi, Yoshiaki, $136 \mathrm{n}$.

Zachert, Herbert, 49 n., 170 n., 180 n.

Zaide, Sonia M., $67 \mathrm{n}$.

Zambarbieri, Annibale, 1 n., $8 \mathrm{n}$.

Zimmermann:

- Clemens, $12 \mathrm{n}$.

- Hannah, $159 \mathrm{n}$.

Zinn, August, 316, 318 s., 321 s.

Zorn, Philipp, 312 e n. 


\section{About the Author}

Mario G. Losano (1939) is an emeritus Professor of Legal Philosophy; currently Professor at the University of Turin and the University of MilanoBicocca; a member of the Academy of Sciences, Turin; Affiliate Researcher at the Max Planck Institute for European Legal History, Frankfurt am Main. Moreover, he has authored more than 60 books and 600 academic articles, and his writings have been translated into 12 languages. Principal works include: Corso di informatica giuridica, 3 vol. (1984); I grandi sistemi giuridici (2000); Sistema e struttura nel diritto, 3 vol. (2002); La geopolitica del Novecento (2011); Norberto Bobbio. Una biografia culturale (2018). Full bibliography: www.mariolosano.it. 



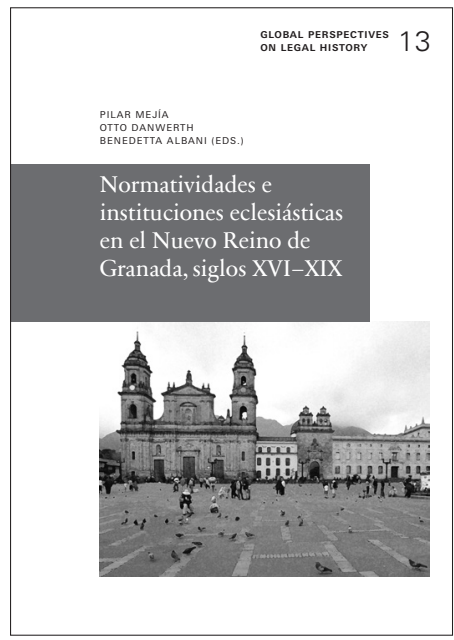

Pilar Mejía, Otto Danwerth, Benedetta Albani (eds.)

Normatividades e instituciones eclesiásticas en el Nuevo Reino de Granada, siglos XVI-XIX

Global Perspectives on Legal History 13

Frankfurt am Main: Max Planck Institute for European Legal History 2020.

288 S., € 21,08 D

ISBN 978-3-944773-24-7

eISBN 978-3-944773-25-4

Open Access Online Edition:

http://dx.doi.org/10.12946/gplh13

Ecclesiastical institutions and actors were essential for the formation of normative orders in early modern Ibero-America. In a series of seminars, organised by the Max Planck Institute for European Legal History in Mexico City, Lima, Bogotá and São Paulo, scholars from different disciplines presented innovative studies on the history of religious normativity and its practices. Based upon the Bogotá colloquium, the present volume focuses on the New Kingdom of Granada (16th-19th centuries). 
Global Perspectives on Legal History is a book series edited and published by the Max Planck Institute for European Legal History, Frankfurt am Main, Germany. As its title suggests, the series is designed to advance the scholarly research of legal historians worldwide who seek to transcend the established boundaries of national legal scholarship that typically sets the focus on a single, dominant modus of normativity and law.

The series aims to privilege studies dedicated to reconstructing the historical evolution of normativity from a global perspective.

It includes monographs, editions of sources, and collaborative works. All titles in the series are available both as premium print-on-demand and in the open-access format.

\section{Released volumes}

13 | Pilar Mejía, Otto Danwerth, Benedetta Albani (eds.)

Normatividades e instituciones eclesiásticas en el Nuevo Reino de Granada, siglos XVI-XIX

12 Otto Danwerth, Benedetta Albani, Thomas Duve (eds.)

Normatividades e instituciones eclesiásticas en el virreinato del Perú, siglos XVI-XIX

11 | Massimo Brutti, Alessandro Somma (eds.)

Diritto: storia e comparazione.

Nuovi propositi per un binomio antico

10 | Gunnar Folke Schuppert

The World of Rules.

A Somewhat Different Measurement of the World

9 | Guido Pfeifer, Nadine Grotkamp (eds.)

Außergerichtliche Konfliktlösung in der Antike.

Beispiele aus drei Jahrtausenden

8 | Elisabetta Fiocchi Malaspina

L'eterno ritorno del Droit des gens di Emer de Vattel (secc. XVIII-XIX).

L'impatto sulla cultura giuridica in prospettiva globale

7 | Víctor Tau Anzoátegui

El Jurista en el Nuevo Mundo.

Pensamiento. Doctrina. Mentalidad 
6 | Massimo Meccarelli, María Julia Solla Sastre (eds.)

Spatial and Temporal Dimensions for Legal History.

Research Experiences and Itineraries

5 | Benedetta Albani, Otto Danwerth, Thomas Duve (eds.)

Normatividades e instituciones eclesiásticas en la Nueva España, siglos XVI-XIX

4 | Osvaldo Rodolfo Moutin

Legislar en la América hispánica en la temprana edad moderna.

Procesos y características de la producción de los Decretos del Tercer Concilio

Provincial Mexicano (1585)

3 | Thomas Duve, Heikki Pihlajamäki (eds.)

New Horizons in Spanish Colonial Law.

Contributions to Transnational Early Modern Legal History

2 | María Rosario Polotto, Thorsten Keiser, Thomas Duve (eds.)

Derecho privado y modernización.

América Latina y Europa en la primera mitad del siglo XX

1 | Thomas Duve (ed.)

Entanglements in Legal History: Conceptual Approaches

More information on the series and forthcoming volumes: http://global.rg.mpg.de 

MAX PLANCK INSTITUTE

FOR EUROPEAN LEGAL HISTORY

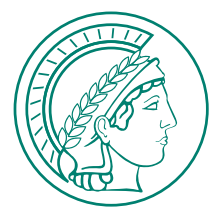

The three defeated Axis powers - Japan, Italy and Germany - incorporated a prohibition on wars of aggression into their democratic constitutions. This book covers the years of the Nuremberg and Tokyo Trials and the constituent assemblies of 1947-49 through to current debates on the adaptation of the pacifist articles in line with new "humanitarian" wars. Aspects relating to the birth of the three countries' constitutions are treated in great detail in three appendices.

\section{http://global.rg.mpg.de}

ISBN 978-3-944773-26-1

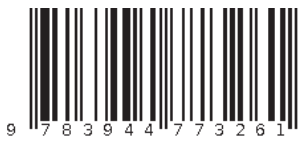

